Adolescent medicine and general pediatrics I

\section{Concurrent session}

\section{2:45 PM}

\section{Thursday, January 20, 2022}

\section{\#1 QUALITY IMPROVEMENT INCREASES COMMUNITY HOSPITAL SMOKE EXPOSURE SCREENING, EDUCATION, AND REFERRAL}

${ }^{1}$ E Daniel ${ }^{*},{ }^{2,3} \mathrm{MY}$ Hamline. ${ }^{1}$ Sutter Health, Sacramento, CA; ${ }^{2}$ UC Davis Children's Hospital, Sacramento, $C A ;{ }^{3}$ Adventist Health Lodi Memorial, Lodi, CA

\subsection{6/jim-2022-WRMC.1}

Purpose of Study Tobacco use starts young and is the leading cause of preventable disease, disability, and death in the United States. Secondhand smoke increases ear and respiratory infections, asthma attacks, and risk of Sudden Unexpected Infant Death. Few smoking cessation studies in inpatient pediatrics are formal quality improvement projects and most are at academic institutions. We sought to increase smoke exposure screening, smoking cessation education, and referrals in our community hospital pediatric population. By improving screening and documentation, we anticipate increased provider awareness and smoking cessation interventions.

Methods Used All pediatric ward, newborn nursery, and Level II nursery admissions were eligible. Interventions were education on smoke exposure screening and Helpline referrals, standardizing documentation for screening and discharge instructions, visual reminders, and Helpline wallet cards.

The primary outcome measure was monthly percentage of pediatric inpatients screened for smoke exposure. Secondary outcomes were percentage of pediatric inpatients screening positive for smoke exposure who received discharge instructions or who received a Helpline referral (self or family member). Length of stay (LOS) was monitored as a balancing measure.

Outcome measures were analyzed with statistical process control in SPC for Excel. Baseline and intervention periods for LOS were compared with t-tests.

Summary of Results We increased baseline average smoke exposure screening rates from $14 \%$ to $73 \%$, meeting criteria for special cause variation (figure 1). Education on smoke exposure avoidance increased from $5 \%$ to $57 \%$. Helpline referrals increased from $0 \%$ to $21 \%$. There was no significant change in length of stay.

Conclusions Pediatrician-led smoking cessation interventions are feasible and effective in community hospital pediatric units with no significant impact on length of stay.

\section{\#2 $\quad$ PILOMATRICOMA IN A CHILD WITH TURNER} SYNDROME: A RARE ENTITY

IS Horowitz, K Tappin*. Valley Children's Healthcare, Madera, CA

10.1136/jim-2022-WRMC.2

Purpose of Study Pilomatricoma is a rare, skin neoplasm that is often confused with dermoid or brachial cleft cysts. Julian

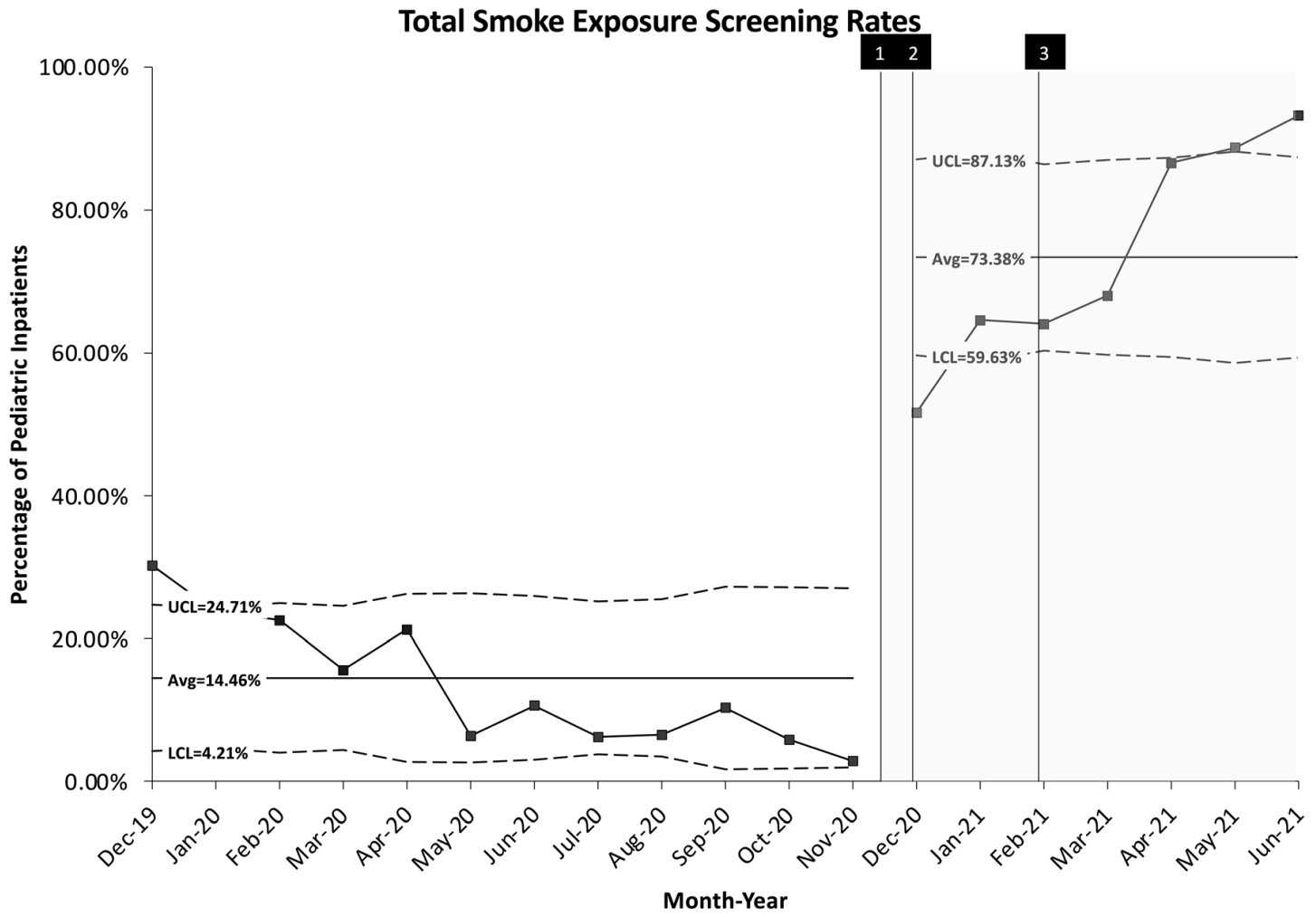

Abstract \#1 Figure 1 Vertical lines are timing of interventions. 1) Monthly Pediatrician education started 2) EMR documentation standardized, visual reminders posted 3 ) Helpline wallet cards available.

$\mathrm{UCL}=$ Upper Control Limit, $\mathrm{Avg}=$ Average, $\mathrm{LCL}=$ Lower Control Limit

Baseline: Dec 2019-Nov 2020. Intervention: Dec 2020-June 2021 
et al., reported that pilomatricomas are commonly misdiagnosed pre-operatively in up to $75 \%$ of cases.

We report the case of a child with Turner's syndrome with a pilomatricoma that was diagnosed on biopsy. We review the histopathologic features and emphasize its association with Turner's syndrome.

Methods Used Case Report.

Summary of Results 2 year old female with Turner Syndrome presented with a progressive mass above her right upper lip for 6 months.

On exam, she was well-appearing with phenotypical features of Turner's syndrome. A $0.5 \times 0.5 \mathrm{~cm}$ erythematous, verrucous, well circumscribed, nontender, mobile lesion was noted above her lip.

She underwent complete excision of the mass without complication. Excisional biopsy revealed the presence of viable basaloid cells and shadow cells confirming the diagnosis of pilomatricoma.

Conclusions A pilomatricoma, otherwise known as pilomatrixomas, are benign subepidermic tumors of the hair follicle matrix. The lesions occur on the face and neck with a mean age of onset between 5.8- 7 years old.

Lesions are usually asymptomatic but, inflammation and ulceration can occur. The most common clinical presentation is a firm, subcutaneous lesion with an irregular surface. The overlying skin may be red, blue, or display the tent sign. Studies have reported the initial development of pilomatricomas in children with Turner's syndrome. The exact cause of this is unknown although animal studies suggest a genetic component.

Histopathologically, a pilomatricoma appears as a mass composed of viable basaloid cells, shadow cells, calcification, and ossification. The mainstay of treatment of a pilomatricoma is complete surgical excision as the lesions do not regress spontaneously. Early excision within 12 months of diagnosis is associated with better cosmetic outcomes. Recurrence and malignant transformation is rare.

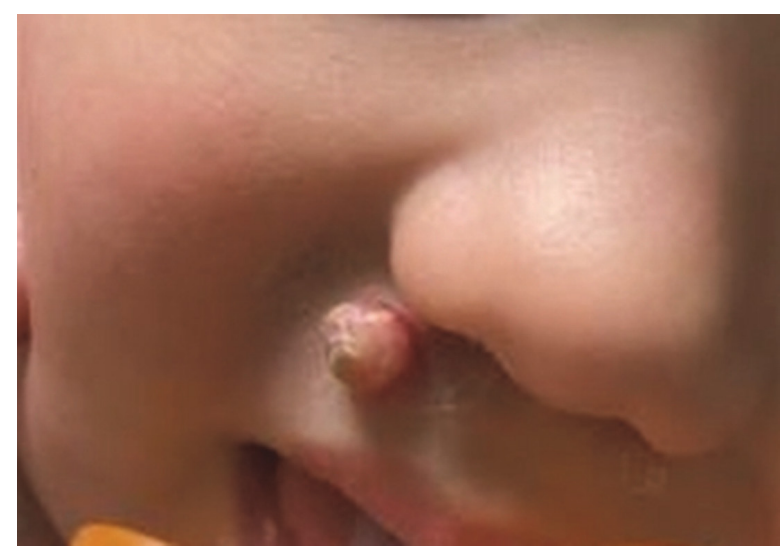

\section{Abstract \#2 Figure 1}

This case highlights the importance of considering pilomatricoma as a cause of solitary skin nodules, especially when on the head, neck or upper extremities. Additionally, physicians caring for children with Turner syndrome should be aware of the prevalence of pilomatricoma in this population.
\#3 1 MENTAL HEALTH HOPSCOTCH: IMPROVING ADOLESCENT MENTAL HEALTH ONE HOP AT A TIME

${ }^{1}$ AS Rodriguez*, ${ }^{1} \mathrm{M}$ Flores, ${ }^{1} \mathrm{~T}$ Fildes, ${ }^{1} \mathrm{~J}$ Charbonnet, ${ }^{1} \mathrm{~K}$ Colwell, ${ }^{2} \mathrm{R}$ Kinman. ${ }^{1}$ Fresno High School, Fresno, CA; ${ }^{2}$ University of California San Francisco Fresno, Fresno, CA

\subsection{6/jim-2022-WRMC.3}

Purpose of Study Fresno High Women 's Alliance students continue to collaborate with UCSF Fresno pediatricians to create community action research projects on topics of adolescent concern using a 'youth as partner' approach. Given the social isolation and increasing depression students noticed amongst themselves and their peers due to COVID19, the Women's Alliance teens chose to focus this last year on improving mental health amongst their peers. Mental Health Hopscotch was chosen for its simplicity and ease of use. The fact that it was created by an adolescent in response to the COVID19 pandemic provided further impetus for its use.

Methods Used Students collaborated with the school's Social Emotional Wellness and Support team, choosing to do their mental health intervention during National Mental Health Month. They created a Mental Health 'Sunshine' at their school entrance, chalking positive affirmations in a sun-shaped diagram for all to see, and chalked a Mental Health Hopscotch on the sidewalk in the front of the school. Silicone bracelets with motivational quotes and mental health awareness pencils, stickers, and mini buttons were handed out to those who completed the Hopscotch. A QR code linked to Google Forms was used to survey students who completed the Hopscotch.

Summary of Results 42 students were surveyed. $12 \%$ of students reported their average stress level was 'just right', 38\% reported they could 'handle' their stress, 21\% felt that they were 'getting stressed', 19\% reported they were 'starting to lose it', and 10\% described their stress as 'getting out of control'. Students primarily dealt with stress by listening to music (31\%), exercising (19\%), and sleeping (14\%). Half of students surveyed reported difficulty sleeping at night, while $90 \%$ of students felt that doing Mental Health Hopscotch helped boost their mood.

Conclusions Although only a limited number of students were surveyed due to restricted numbers of students present on campus, the majority of students felt stressed with half the students reporting difficulty sleeping at night. Mental Health Hopscotch provided a simple, quick, yet no-cost approach to boost students' mood, thus empowering teens concerned about the mental health of their peers to stage a mental health intervention on their own school grounds.

\section{\#4 DAYLIGHT SAVINGS AND PEDIATRIC EMERGENCIES}

${ }^{1,2} \mathrm{~V}$ Wong * ${ }^{1,2}$ P Enarson, ${ }^{1,2} \mathrm{~J}$ Lee. ${ }^{1}$ The University of British Columbia Faculty of Medicine, Vancouver, BC, Canada; ${ }^{2} B C$ Children's Hospital, Vancouver, BC, Canada

\subsection{6/jim-2022-WRMC.4}

Purpose of Study Daylight Savings Time (DST) is a biannual time change where during 'spring forward,' clocks are set forward one hour, potentially resulting in sleep deprivation for much of society. During 'fall back,' the opposite occurs. Circadian rhythm disruption has been shown to have effects on 
Incidence Ratios Excluding Infectious/Fever (2011-2019)

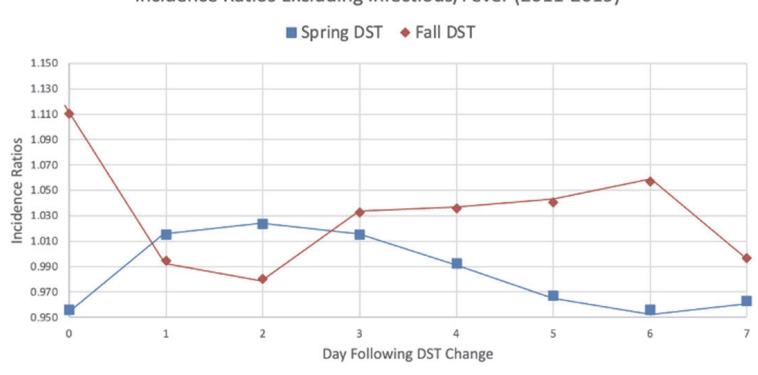

Abstract \#4 Figure 1

cardiovascular, neuropsychiatric, metabolic, immune-related and accidental events in adults. A 2018 study showed increased emergency department (ED) visits for adults in the time after DST. These findings have not been verified in pediatric populations and if extant, may have implications on managing ED patient volume and expectations. We hypothesized that the large-scale sleep deprivation following spring time change would result in increased ED presentations, particularly among certain presentations (neurologic, psychiatric, accidental/traumatic) that may be especially susceptible to sleep deprivation, and that the fall time change would have an opposite effect.

Methods Used We retrospectively collected and analyzed the primary medical complaint of all children (0-16 years) presenting to BC Children's Hospital ED in the 2 weeks before and 3 weeks after the biannual DST time changes during 2011 to 2019. Incidence ratios (IR) of ED presentations were calculated over day 0 (day of time change) to day 7. IRs were calculated for all presentations and broken down by specialty.

Summary of Results After excluding infectious presentations, the IR was increased during the first week following spring time change: Monday by $6 \%$, Tuesday by $7 \%$ and Wednesday by $6 \%$, though the results were statistical insignificant $(p>0.05)$. There were significant decreases $(p<0.05)$ after the fall time change: Monday by $12 \%$, Tuesday by $13 \%$ and Wednesday by $8 \%$.

Conclusions Following the seasonal time change in the spring, there were increases in IR, though this did not reach statistical significance. There were significant decreases in IR during the three days following the fall shift. Together these findings suggest that the widespread sleep deprivation at spring time change results in adverse health effects among children, while extra sleep in the fall time change may be protective.

Circadian rhythm disruption from DST in the children shows potentially important effects on emergency visits and further study can lead to better patient care and ED preparation. This may be informative in developing policy regarding the need for DST.

\section{\#5 THE RELATIONSHIP BETWEEN SELF-PERCEIVED HEALTH STATUS AND CHRONIC CONDITION, ACCESS TO CARE, AND AWARENESS OF CHRONIC CONDITION AMONG ADULTS WITH HISTORY OF ADVERSE CHILDHOOD EXPERIENCES}

L Trinh*, S Teklehaimanot, M Shaheen. Charles Drew University of Medicine and Science, Los Angeles, CA

10.1136/jim-2022-WRMC.5
Purpose of Study Adults with a history of adverse childhood experiences (ACEs) are at increased risk for chronic disease and, thus, poor health outcomes. Yet, the effect of chronic disease awareness on health outcomes in adults with ACEs has not been examined. The objective of this study was to determine the relationship between general health status and chronic disease, access to care, and awareness of chronic condition among adults with history of ACEs.

Methods Used Data from the 2019 Behavioral Risk Factor Surveillance System were analyzed. Descriptive statistics were used to determine the prevalence of ACEs, chronic disease, healthcare access, chronic disease awareness, general health status, and population characteristics. Bivariate analysis using Chi-squared test was performed on history of ACEs by all independent variables and general health status by all independent variables. Multivariable logistics regression was used to determine the relationship between general health status and ACEs, adjusting for chronic disease, healthcare access, chronic disease awareness, and demographics.

Summary of Results Of the 78,112 respondents, 63\% reported being exposed to at least one ACE. History of ACEs was associated with high prevalence of chronic diseases $(p<0.002)$, lower healthcare coverage $(p<0.0001)$, lower chronic disease awareness $(p<0.006)$, and report of fair/poor general health status $(p<0.0001)$. In adjusted analyses, adults with 2,3 , and $\geq 4$ ACE events had 1.24, 1.22, and 1.45 times increased odds of reporting fair/poor health status compared to those reporting no ACEs.

Conclusions Adults with history of ACEs face barriers to achieving good health. There is a need to expand ACEs screening in primary settings so that early intervention can improve general health outcomes.

\section{\#6 1 CHILDHOOD OBESITY, SOCIAL DETERMINANTS OF HEALTH, SOCIAL VULNERABILITY AND HOSPITALIZATION}

J Johnson*, M Baum. Loma Linda University School of Medicine, Loma Linda, CA

\subsection{6/jim-2022-WRMC.6}

Purpose of Study The prevalence of overweight adolescents has increased dramatically over the last decade. Although previous research has demonstrated the contribution of diet, exercise habits, and parenting on obesity rates, this early study addresses the relationship between hospitalization, social vulnerability index, social determinants of health, and childhood obesity.

Methods Used We studied children between the ages of 5 and 17.9 years, seen in inpatient $(n=39)$ and outpatient $(n=35)$ settings at healthcare facilities affiliated with Loma Linda University Health between January 2020 through June 2021. We collected Body Mass Index scores (BMI) and demographic information. A standardized questionnaire gathered social determinants of health information available in the electronic health record. Using home addresses, we identified the Social Vulnerability Index score (SVI) (Flanagan, 2011) associated with their census tract (Center for Disease Control, 2018). The SVI ranks each tract on four main themes: socioeconomic status, household composition and disability, minority status and language, and housing and transportation.

Summary of Results The most significant comparison were the differences in risk between the inpatient $(n=39)$ and 
Abstract \#6 Table $1 \mathrm{BMI} \leq$ 85th percentile = normal weight or underweight, BMI > 85th percentile = overweight or obese (Kuczmarski, 2002). *Hisp = person of Cuban, Mexican, Puerto Rican, South/Central American, or other culture regardless of race (US Census Bureau, 2011).

\begin{tabular}{|c|c|c|c|c|c|}
\hline & Inpatient & & Outpatient & & $p$ value \\
\hline \multirow[t]{2}{*}{ Age } & 5-13.9 yr & $14.0-18.0$ & $5-13.9 \mathrm{yr}$ & $14.0-18.0$ & \\
\hline & 19 & 20 & 12 & 23 & 0.209 \\
\hline \multirow[t]{2}{*}{ Ethnicity } & Non Hisp & Hisp* & Non Hisp & Hisp* & \\
\hline & 12 & 27 & 8 & 27 & 0.444167 \\
\hline \multirow[t]{2}{*}{ Sex } & Female & Male & Female & Male & \\
\hline & 23 & 16 & 26 & 9 & 0.164419 \\
\hline \multirow[t]{2}{*}{ Language } & English & Spanish & English & Spanish & \\
\hline & 31 & 8 & 29 & 6 & 0.711721 \\
\hline \multirow[t]{2}{*}{ BMI } & $\leq 85$ th percentile & $>85$ th percentile & $\leq 85$ th percentile & $>$ 85th percentile & \\
\hline & 23 & 16 & 16 & 19 & 0.254005 \\
\hline $\mathrm{SDOH}$ & No Risk & Risk & No Risk & Risk & \\
\hline SVI & 21 & 18 & 13 & 22 & 0.150006 \\
\hline Social Connection & 14 & 13 & 4 & 14 & 0.046854 \\
\hline Financial Risk & 12 & 16 & 22 & 3 & 0.000624 \\
\hline Transportation & 27 & 3 & 29 & 1 & 0.300623 \\
\hline Tobacco & 31 & 2 & 25 & 2 & 0.83518 \\
\hline Food Insecurity & 16 & 14 & 24 & 4 & 0.00773 \\
\hline Stress & 17 & 12 & 24 & 7 & 0.117752 \\
\hline Physical Activity & 8 & 21 & 11 & 20 & 0.511064 \\
\hline
\end{tabular}

outpatient $(\mathrm{n}=35)$ populations. Inpatients were more likely to experience Social Connection risk $(\mathrm{p}=0.046)$, Financial risk $(\mathrm{p}=0.0006)$, and Food Insecurity $(\mathrm{p}=0.0077)$ than outpatients.

When comparing between patients living in the upper quartile $(n=40)$ and the lower three quartiles $(n=34)$ of SVI, there was little difference in BMI, Financial risk, Food Insecurity risk, Transportation risk, Physical Activity risk and Stress risk. There was similarly little difference when comparing age groups of younger versus older children.

Conclusions There are significant disparities in risk between pediatric inpatient and outpatient populations. Steps could be taken to identify inpatients with reduced resources to improve food insecurity, social connection risk, and financial risk. Although SVI is important for understanding the context of each patient, every family has unique social determinants and risks that could be addressed by physicians.

\section{\#7 $\quad$ SUBJECTIVE SOCIAL STATUS AND FOOD INSECURITY RELATIONSHIP WITH WEIGHT-SPECIFIC QUALITY OF LIFE AMONG LATINO ADOLESCENTS AND BETWEEN SEXES}

${ }^{1} \mathrm{~A}$ Penghat ${ }^{*},{ }^{2} \mathrm{~KB}$ Vander Wyst, ${ }^{3} \mathrm{~A}$ Peña, ${ }^{3} \mathrm{~A}$ Williams, ${ }^{3} \mathrm{SL}$ Ayers, ${ }^{3,4} \mathrm{GQ}$ Shaibi. ${ }^{1}$ Midwestern University Arizona College of Osteopathic Medicine, Glendale, $A Z$; ${ }^{2}$ Midwestern University, Glendale, AZ; ${ }^{3}$ Arizona State University, Phoenix, AZ; ${ }^{4}$ Phoenix Children's Hospital, Phoenix, AZ

\subsection{6/jim-2022-WRMC.7}

Purpose of Study Social status and food insecurity (FiS) may contribute to health disparities among youth. This study aimed to evaluate whether subjective social status (SSS) and FiS are associated with weight-specific quality of life (wQoL) among Latino youth with obesity. We further explored whether relationships differed by sex.

Methods Used One-hundred and forty-one Latino youth (47\% male; mean age: $15.3 \pm 0.9$ ) with obesity completed surveys to assess SSS, perceived FiS, and wQoL (self, social, and environment). Separate linear regression models were performed to examine the relationship between SSS \& FiS with wQoL, after controlling for sex and BMI percentile. Data were then stratified by sex to determine Pearson's $r$ for wQoL \& SSS and wQoL \& FiS.

Summary of Results Mean total $(64.1 \pm 24.9)$, self $(57.3 \pm 29.3)$, social (69.5 \pm 25.6$)$, and environmental $(60.5 \pm 26.3)$ wQoL, with males reporting higher total, self, and environmental wQoL as compared to females (all $\mathrm{p}<0.05$ ) after controlling for BMI. Over one-third of the cohort indicated very low food insecurity (36.2\%) or marginal food insecurity (34\%), which did not differ by sex. Despite no sex differences in SSS society (mean diff $=-0.074, \mathrm{p}=0.77$ ) or SSS school (mean diff $=-0.354, \quad p=0.28$ ), there was a positive relationship between SSS school and all scales of wQoL regardless of sex (all $\mathrm{p}<0.01)$. SSS school was a significant predictor of total wQoL $(\beta=4.24, p<0.001)$ and the self $(\beta=3.49, p=0.008)$, social $(\beta=4.40, p<0.001)$, and environment $(\beta=4.57$, $\mathrm{p}<0.001)$ subscales. SSS school explained $9 \%$ of the variance in total, social, and environment wQoL, and $4 \%$ of the variance in self wQoL. There was an inverse relationship between FiS and all scales of wQoL, particularly for those experiencing marginal and high FiS (all $\mathrm{p}<0.03$ ). This correlation was stronger for males for all wQoL scales at all levels of FiS except high FiS. Marginal FiS was a significant predictor of total $(\beta=-12.94, p=0.006)$, self $(\beta=-12.48, p=0.029)$, social $(\beta=-12.06, p=0.013)$, and environment $(\beta=-14.66, p=0.003)$ wQoL after controlling for sex and BMI percentile. High FiS was a significant predictor of total $(\beta=-22.46, p<0.001)$, self $(\beta=-18.56, p=0.016)$, social $(\beta=-24.16, p<0.001)$, and environment $(\beta=-22.76, p<0.001)$ wQoL after controlling for sex and BMI percentile. Marginal and high FiS explained $8 \%$ of the variance in total, social, environmental wQoL, and 3\% of the variance in self wQoL.

Conclusions Among Latino youth with obesity, social status is associated with wQoL while food insecurity is inversely associated with wQoL. 
\#8 MEDICAL STUDENT KNOWLEDGE AND PERCEPTIONS OF PEDIATRIC OBESITY MANAGEMENT

K Eckert, S Thomas, M Buls, M Cox*, E Chowdry, B Scott. University of Nevada Reno School of Medicine, Reno, NV

\subsection{6/jim-2022-WRMC.8}

Purpose of Study The prevalence of pediatric obesity continues to increase. In Nevada, approximately $40 \%$ of youth are overweight or obese and $70 \%$ of these children will remain overweight as adults. Traditional medical school curriculum does not adequately prepare students to counsel families on this subject. The purpose of this study was to evaluate the progression of third year medical student knowledge base involved in managing obesity in children and adolescents over the course of the pediatric clinical curriculum.

Methods Used 63 medical students in the third year clinical clerkship curriculum at the University of Nevada, Reno School of Medicine were given a survey to evaluate their knowledge on pediatric obesity and perceptions surrounding treatment before a six-week pediatric clerkship. During the clerkship, students received instruction on the diagnosis and treatment of obesity. At the conclusion of the clerkship, students took a post-survey to assess knowledge gained during the experience. Paired samples T-test and Chi-square tests were used to assess differences between pre- and post- surveys.

Summary of Results After the clerkship, there was an increase in the mean score in knowledge and comfort level in recommending a treatment program for overweight/obese children between pre- $(\mathrm{M}=1.97, \mathrm{SD}=0.91)$ and post-tests $(\mathrm{M}=3.55$, $\mathrm{SD}=0.89) \quad(\mathrm{t}(62)=10.25, \quad \mathrm{p}<0.0001)$. There was also an increase in the mean score in their ability to effectively counsel overweight/obese children between pre- $(M=2.40$, $\mathrm{SD}=0.90)$ and post-tests $(\mathrm{M}=3.86, \mathrm{SD}=0.77) \quad(\mathrm{t}(62)=10.33$, $\mathrm{p}<0.0001)$. In addition, there was an increase in students believing in the overall efficacy of counseling in the treatment for overweight/obese children and adults between the pre$(\mathrm{M}=2.91, \mathrm{SD}=0.75)$ and post-tests $(\mathrm{M}=3.34, \mathrm{SD}=0.76)(\mathrm{t}(62)$ $=3.23, \mathrm{p}=0.0019$ ).

Conclusions The curriculum improved student knowledge and understanding of pediatric obesity. In particular, perceived comfort and ability to counsel patients and families about obesity prevention and treatment increased. As obesity continues to be a challenge, expansion of medical student education in this area is imperative to address this problem.

\section{Cardiovascular I}

\section{Concurrent session}

\section{2:45 PM}

\section{Thursday, January 20, 2022}

\section{\#9 1-YEAR OUTCOMES OF PATIENTS UNDERGOING HEART TRANSPLANT DURING THE COVID PANDEMIC}

${ }^{1} \mathrm{G}$ Esmailian, ${ }^{*}{ }^{2} \mathrm{~N}$ Patel, ${ }^{2} \mathrm{~T}$ Singer-Englar, ${ }^{2} \mathrm{~S}$ Kim, ${ }^{2} \mathrm{M}$ Hamilton, ${ }^{2} \mathrm{~J}$ Kobashigawa. ${ }^{1} T$ The George Washington University School of Medicine and Health Sciences, Washington, DC; ${ }^{2}$ Cedars-Sinai Smidt Heart Institute, Los Angeles, CA
Purpose of Study The ongoing COVID-19 pandemic has brought considerable challenges to heart transplantation. Most notably, towards the start of the pandemic, changes in the outpatient care of post-transplant patients were brought forward at our center to further prevent the spread of the virus and protect highly immunosuppressed recipients. For example, blood draws for immunosuppression adjustments were conducted at home and early coronary angiograms were eliminated. Video visits were conducted for post-operative months 7, 9, and 11. Despite these changes in management, formal analyses on the impact of these changes on heart transplant recipient outcomes have yet to be conducted. Now over one year removed from the pandemic's onset, we sought to examine if the modifications in outpatient care impacted 1-year outcomes of patients transplanted during the start of the COVID-19 pandemic.

Methods Used Between March 6 and September 1, 2020, we assessed 50 heart transplant patients transplanted during the COVID-19 pandemic. These patients were compared to patients who were transplanted during the same months between 2011 and $2019(n=482)$. Endpoints included subsequent 1-year survival, 1-year freedom from cardiac allograft vasculopathy (CAV: stenosis $\geq 30 \%$ ), 1-year freedom from any-treated rejection, 1-year freedom from acute cellular rejection, 1-year freedom from antibody-mediated rejection, hospital and ICU length of stay, and 1-year freedom from non-fatal major adverse cardiac events (NF-MACE: MI, new CHF, PCI, ICD/pacemaker, or stroke).

Summary of Results Patients transplanted during the COVID19 pandemic had similar outcomes compared to those of patients transplanted in years prior to the pandemic. There were no significant differences in hospital and ICU length of stay between the two groups. There were also no significant differences in 1-year survival, 1-year freedom from CAV, 1year freedom from any treated rejection, and 1-year freedom from acute cellular or antibody mediated rejection between both groups. Patients transplanted during the pandemic had a significantly higher 1-year freedom from NF-MACE.

\begin{tabular}{|c|c|c|c|}
\hline & $\begin{array}{l}\text { Patients } \\
\text { Transplanted During } \\
\text { COVID Pandemic } \\
(n=50)\end{array}$ & $\begin{array}{l}\text { Patients } \\
\text { Transplanted Prior to } \\
\text { CoVID Pandemic } \\
(n=482)\end{array}$ & P-Value \\
\hline 1-Year Survival & $94.0 \%$ & $90.5 \%$ & 0.438 \\
\hline 1-Year Freedom from CAV & $100.0 \%$ & $96.5 \%$ & 0.192 \\
\hline $\begin{array}{l}\text { 1-Year Freedom from NF- } \\
\text { MACE }\end{array}$ & $98.0 \%$ & $86.9 \%$ & 0.024 \\
\hline $\begin{array}{l}\text { 1-Year Freedom from Any } \\
\text { Treated Rejection }\end{array}$ & $90.0 \%$ & $84.2 \%$ & 0.262 \\
\hline $\begin{array}{l}\text { 1-Year Freedom from Acute } \\
\text { Cellular Rejection }\end{array}$ & $90.0 \%$ & $92.1 \%$ & 0.658 \\
\hline $\begin{array}{l}\text { 1-Year Freedom from } \\
\text { Antibody-Mediated } \\
\text { Rejection }\end{array}$ & $94.0 \%$ & $94.8 \%$ & 0.845 \\
\hline $\begin{array}{l}\text { Average Length of Hospital } \\
\text { Stay (Days) }\end{array}$ & $22.96 \pm 37.19$ & $17.21 \pm 19.71$ & 0.079 \\
\hline $\begin{array}{l}\text { Average Length of ICU Stay } \\
\text { (Days) }\end{array}$ & $11.06 \pm 20.61$ & $8.31 \pm 8.19$ & 0.069 \\
\hline
\end{tabular}


Conclusions Despite necessary changes being made to posttransplant care to mitigate the spread of COVID-19 and protect an immunosuppressed population, heart transplantation during the COVID-19 pandemic appears safe with 1-year outcomes comparable to years prior.

\section{\#10 COVID PANDEMIC AND SOCIAL MITIGATIONS IMPROVE OUTCOMES FOR HEART TRANSPLANT PATIENTS}

C Washington, S Kim*, N Patel, T Singer-Englar, M Hamilton, J Kobashigawa. Cedars-Sinai Smidt Heart Institute, Los Angeles, CA

\subsection{6/jim-2022-WRMC.10}

Purpose of Study The COVID pandemic affected how our medical staff treated our heart transplant (HTx) patients during this period of time. Patients were seen virtually via telemedicine and patients self-isolated at home. What we do not know is the impact of this treatment during the COVID pandemic on HTx outcomes. As patients were self-isolating, it is possible that medication and medical compliance were increased and there may have been a decrease in non-COVID infection rates as exposure was minimized due to patients selfisolating. None of these factors have been assessed prior and, thus, we reviewed our large, single center patient population for this study.

Methods Used Between March 2020 and September 2020, we assessed 55 HTx patients who were transplanted during this period of time and followed for 6 months. Patients were selfisolating and had every other clinic visit changed to a virtual visit to minimize exposure to COVID. Endpoints for this study included 6-month survival, re-hospitalization, number of non-COVID infections (defined as the need for intravenous antibiotics), any treated rejection (ATR), and maintenance of therapeutic immunosuppressive blood levels. The study patients were then compared to a control group of the previous three years, averaging each year at the same time points of March 13, 2017, March 13, 2018, and March 13, 2019, and followed for 6 months. Each group was averaged and then compared to the study group.

Summary of Results The study group (during the COVID pandemic) demonstrated a significant decrease in re-hospitalization in the first 6 months following HTx compared to the control group. There was a numerical decrease in non-COVID infectious complications. There was no difference in survival and freedom from any-treated rejection episodes between the two

\begin{tabular}{llll} 
Abstract \#10 & Table 1 & \\
\hline & $\begin{array}{l}\text { HTx Patients Transplanted } \\
\text { between Mar.-Sep. 2020 } \\
(\mathbf{n}=55)\end{array}$ & $\begin{array}{l}\text { HTx Patients Transplanted } \\
\text { between Mar.-Sept. } \\
\text { 2017-2019 } \\
(\mathbf{n}=169)\end{array}$ & $\begin{array}{l}\text { P- } \\
\text { Value }\end{array}$ \\
\hline $\begin{array}{l}\text { 6-Month Survival } \\
\text { 6-Month Freedom }\end{array}$ & $92.7 \%$ & $94.1 \%$ & 0.733 \\
$\begin{array}{l}\text { from ATR } \\
\text { Rehospitalization } \\
\text { within 6 Months }\end{array}$ & $16.4 \%(9)$ & $91.1 \%$ & 0.981 \\
$\begin{array}{l}\text { Non-COVID } \\
\text { Infection within 6 }\end{array}$ & $10.9 \%(6)$ & $37.9 \%(64)$ & 0.003 \\
Months & & $18.9 \%(32)$ & 0.323 \\
\hline
\end{tabular}

groups. Reasons for rehospitalization included infections, various cardiac and renal issues, malaise, and fever.

Conclusions The COVID pandemic demonstrated that self-isolation and virtual visits resulted in fewer hospitalizations possibly due to less infectious complications. This implies that perhaps stricter restrictions for community exposure might benefit HTx patients in the 6 months following transplantation.

\section{\begin{tabular}{|l|l}
$\# 11$ & SEX DISPARITIES IN HEART TRANSPLANT WAITLIST
\end{tabular} TIME FOLLOWING THE DONOR HEART ALLOCATION POLICY CHANGE}

${ }^{1}$ I Sindha* , ${ }^{2} \mathrm{~T}$ Singer-Englar, ${ }^{2} \mathrm{~S}$ Kim, ${ }^{2} \mathrm{~N}$ Patel, ${ }^{2} \mathrm{M}$ Hamilton, ${ }^{2} \mathrm{~J}$ Kobashigawa. ${ }^{1}$ University of California Los Angeles, Los Angeles, CA; ${ }^{2}$ Cedars-Sinai Smidt Heart Institute, Los Angeles, $C A$

\subsection{6/jim-2022-WRMC. 11}

Purpose of Study There are many reports in organ transplantation that demonstrate that there are sex discrepancies in waitlist urgent status, time on the heart transplant waitlist, waitlist mortality. There are no differences in men versus women in terms of heart disease and in terms of mortality for these two sexes. It would be expected that both men and women would have similar percentages as urgent status, especially after the new donor heart allocation policy change took place in October 2018. We chose to assess our male and female patients to establish whether there exists a difference in patients listed as urgent status on the HTx waitlist.

Methods Used Between November 2018 and December 2020 (after donor heart allocation change in October 2018), we assessed 276 patients on the HTx waitlist. Patients were followed for 6 months and censored after they were transplanted or removed from the waitlist. Percent of patients of each sex listed as urgent status (status 1, 2, 3) was recorded. Mortality on the waitlist, waitlist time, and removal from the waitlist due to being too sick were secondary endpoints.

Summary of Results After the donor heart allocation policy change in October 2018, women were significantly less likely to be listed as urgent status compared to men. For those patients listed as urgent status, there was no significant difference in mortality for women versus men on the HTx waitlist. The waitlist time was shorter for men compared to women (see table 1).

Conclusions There appears to be a sex disparity for women being less likely to be listed as urgent status on the HTx waitlist. Further studies are needed to determine whether this difference has a biologic mechanism or whether there is selection bias and/or treatment bias present in their care.

\begin{tabular}{llll} 
Abstract \#11 Table 1 & & \\
\hline Endpoints & $\begin{array}{l}\text { Female patients } \\
(\mathbf{n}=\mathbf{7 3})\end{array}$ & $\begin{array}{l}\text { Male patients } \\
(\mathbf{n}=\mathbf{2 0 3})\end{array}$ & p-value \\
\hline 6-month survival & $98.6 \%$ & $96.6 \%$ & 0.249 \\
6-month freedom from severe illness & $98.6 \%$ & $96.1 \%$ & 0.189 \\
Urgent status at listing & $28.8 \%$ & $49.3 \%$ & 0.003 \\
Urgent status at removal & $37.0 \%$ & $59.1 \%$ & 0.002 \\
Average time on waitlist (days) & $124.4 \pm 164.7$ & $108.3 \pm 181.9$ & 0.546 \\
\hline
\end{tabular}




\section{\#12 ARE OVERSIZED DONOR HEARTS ASSOCIATED WITH} POOR OUTCOME AFTER HEART TRANSPLANTATION?

${ }^{1} \mathrm{M}$ Rubio, ${ }^{2} \mathrm{~N}$ Patel ${ }^{*},{ }^{2} \mathrm{~S}$ Kim, ${ }^{2} \mathrm{~T}$ Singer-Englar, ${ }^{2} \mathrm{M}$ Hamilton, ${ }^{2} \mathrm{~J}$ Kobashigawa. ${ }^{1}$ University of California Los Angeles, Los Angeles, CA; ${ }^{2}$ Cedars-Sinai Smidt Heart Institute, Los Angeles, $C A$

\subsection{6/im-2022-WRMC.12}

Purpose of Study In heart transplantation (HTx), donor-torecipient size matching has been done by height and weight. More recently, predicted heart mass (PHM) has been found to be more clinically useful to reflect outcome. Using PHM, it has been demonstrated that under-sizing a donor heart for a larger recipient with high pulmonary artery pressures leads to increased mortality. It has recently been noted in the International Society for Heart and Lung Transplantation (ISHLT) registry that there may be increased risk in placing an oversized donor heart using weight into a smaller recipient. This clinical outcome has not been established using PHM. We sought to address this question in our large, single center experience using PHM.

Methods Used Between January 2010 and June 2020, we assessed 588 donor-to-recipient donor heart matches. We used PHM to assess whether there were outcome differences when the donor hearts were oversized. We divided the donor-torecipient PHM ratio into two categories: normal (90-110\%, $\mathrm{n}=524$ ), and markedly oversized (greater than $140 \%, \mathrm{n}=64$ ). Outcomes included 1-year survival, freedom from 1-year rejection (acute cellular rejection [ACR], antibody-mediated rejection [AMR]), freedom from cardiac allograft vasculopathy (CAV: stenosis $\geq 30 \%$ ), freedom from cardiac dysfunction (defined as LVEF $\leq 40 \%$ ), and freedom from non-fatal major adverse cardiac events (NF-MACE: myocardial infarction, new congestive heart failure, percutaneous coronary intervention, implantable cardioverter defibrillator/pacemaker implant, stroke).

Summary of Results Markedly oversized donor hearts using PHM compared to normal matching showed no difference in 1-year survival, freedom from 1-year ACR, freedom from CAV, freedom from NF-MACE, and freedom from cardiac dysfunction. There was a significantly lower 1-year freedom from AMR in the markedly oversized donor heart group which is due to more women recipients (sensitized due to previous pregnancies) in this group $(71 \%$ oversized group vs. $20 \%$ normal group).

\section{Abstract \#12 Table 1}

\begin{tabular}{llll}
\hline & $\begin{array}{l}\text { Oversized PHM } \\
{[>140 \%](n=64)}\end{array}$ & $\begin{array}{l}\text { Normal PHM } \\
{[90-110 \%](n=524)}\end{array}$ & P-Value \\
\hline Mean PHM & $154.86 \pm 15.44$ & $99.95 \pm 5.53$ & $<0.001$ \\
1-Year Survival & $93.8 \%$ & $90.6 \%$ & 0.420 \\
1-Year Freedom from CAV & $96.9 \%$ & $96.6 \%$ & 0.852 \\
1-Year Freedom from NF-MACE & $95.3 \%$ & $87.2 \%$ & 0.065 \\
1-Year Freedom from ACR & $92.2 \%$ & $91.8 \%$ & 0.899 \\
$\begin{array}{l}\text { 1-Year Freedom from AMR } \\
\text { 1-Year Freedom from Right }\end{array}$ & $82.8 \%$ & $94.8 \%$ & $<0.001$ \\
Heart Failure & $100.0 \%$ & $100.0 \%$ & - \\
1-Year Freedom from Cardiac & $98.4 \%$ & & 0.669 \\
Dysfunction & & $99.0 \%$ & 0.706 \\
1-Year Freedom from Pulmonary & $93.8 \%$ & & \\
Hypertension & & $95.0 \%$ & \\
\hline
\end{tabular}

Conclusions Markedly oversized donor-to-recipient matching using PHM does not result in poor outcomes after heart transplantation. This has potential to expand the donor pool, particularly for smaller patients.

\section{\#13 NEW APPROACH TO MYOCARDIAL INFARCT SIZING USING LONG AXIS CARDIAC MAGNETIC RESONANCE IMAGING TO IMPROVE IN VIVO VALIDITY}

${ }^{1} \mathrm{~J}$ Ref*, ${ }^{2} \mathrm{~S}$ Daugherty, ${ }^{1} \mathrm{IR}$ Chinyere, ${ }^{1} \mathrm{~J}$ Zeng, ${ }^{2} \mathrm{~J}$ Lancaster, ${ }^{3} \mathrm{~L}$ LD, ${ }^{3} \mathrm{~F}$ Kenneth, ${ }^{1} \mathrm{~B}$ Mark, ${ }^{1} \mathrm{G}$ Adrian, ${ }^{2} \mathrm{~J}$ Koevary, ${ }^{2} \mathrm{~S}$ Goldman, ${ }^{4} \mathrm{R}$ Avery. ${ }^{1}$ The University of Arizona College of Medicine Tucson, Tucson, AZ; ${ }^{2}$ The University of Arizona Sarver Heart Center, Tucson, AZ; ${ }^{3}$ Banner University Medical Center Tuscon, Tucson, AZ; ${ }^{4}$ Northwestern University Feinberg School of Medicine, Chicago, IL

\subsection{6/jim-2022-WRMC.13}

Purpose of Study The AHA 17-segment model is the preferred clinical method to define LV MI size in CMR imaging, although can be subjective. We propose a novel measurement technique based on long-axis (LAX) CMR from a porcine model of MI to improve accuracy and reproducibility of infarct volume quantification. Data were collected from MRI exams and endpoint organ harvesting for histopathologic analysis.

Methods Used Yucatan mini swine were subjected to 90-minute ischemia/reperfusion of the left anterior descending (LAD) coronary artery. Six-months after infarction, two observers evaluated four infarct sizing methods: myocardial contouring of post-mortem heart slices, contouring using cardiac MRI, AHA 17-segment model analysis and novel LAX MRI infarct sizing.

Novel LAX MRI infarct sizing was done in routine 2-, 3and 4-Chamber LAX LGE MRIs. The precise length of MI could be measured in each plane. These measurements provide exact anatomic location of MI along each wall of the heart relative to the apex.

Total heart length (THL) from apex to base is measured in the 2-Chamber LAX MRI to standardize processing and provide a more accurate LV infarct percentage, since not every heart is the exact same size and infarct percentage is calculated relative to specific anatomy.

Infarct length measurements from each of the 3 LAX planes are summated and divided by THL to produce a LongAxis Ratio (LAR) value. LAR values were plotted against MRI contoured infarct size to produce a table that approximates LV infarct percentage using the LAR value.

Summary of Results LV infarct sizes ranges were $1.6 \%$ $25.8 \%(\mathrm{n}=10)$ of the left ventricle using reference standard histopathologic infarct sizing. Intraclass correlations (ICC) were calculated between two observers and averaged due to high similarity, ICC $>.900$. A t-test of .0006 and Bland-Altman plots show statistically significant differences in 17-segment model infarct size compared to histopathologic analysis while no significant difference was found when compared to our novel method with 0.8198 . Linear correlation showed an $\mathrm{R}^{2}$ of 0.9111 between MRI contoured infarct size and our novel MRI infarct sizing model to predict infarct size as a percentage while the $\mathrm{R}^{2}$ of the $17-\mathrm{Seg}$ model is 0.8197 . A representative $\mathrm{MRi}$ from a patient is shown was produced to demonstrate the clinical relevance of this approach.

Conclusions AHA 17-segment model provides inferior assessment of LV infarct size compared to proposed LAX infarct sizing suggesting it maybe a robust and easily implementable quantitative assessment of LV infarct size in advanced imaging. 
\#14 THE IMPORTANCE OF NATURAL HISTORY DATA COLLECTION AS DEMONSTRATED IN DANON DISEASE ECHOCARDIOGRAM ANALYSIS

${ }^{1} \mathrm{G}$ Storm*, ${ }^{1} \mathrm{~K}$ Boynton, ${ }^{1} \mathrm{~A}$ Barnard, ${ }^{2} \mathrm{E}$ Eshraghian, ${ }^{2} \mathrm{~K}$ Hong, ${ }^{1} \mathrm{~L}$ Mestroni, ${ }^{2} \mathrm{E}$ Alder, ${ }^{1} \mathrm{M}$ Taylor. ${ }^{1}$ CU SOM, Aurora, CO; ${ }^{2}$ UCSD, La Jolla, CA

\subsection{6/jim-2022-WRMC. 14}

Purpose of Study With over 7,000 rare diseases affecting 1 in 10 Americans, longitudinal data describing the clinical course of rare diseases is essential to understand disease natural history and prepare for studies of novel treatments. Traditionally, published data associated with these diseases is limited to cross-sectional times of diagnosis and major events making inferences on the progression and trajectory of various phenotypes difficult. A prime example is Danon Disease, a rare genetic cardiac disease with a malignant outcome of death or need for heart transplantation in most males.

Methods Used To mitigate the lack of longitudinal natural history knowledge in Danon patients, a retrospective clinical database was developed using the REDCap database program. All current and past medical history for Danon patients enrolled internationally was collected and entered into the database by two research sites. To date, this registry has enrolled over 100 patients, with roughly equal representation of males and females. This includes the collection of data on over 550 echos.

Summary of Results To demonstrate the power of this natural history study, echocardiogram data were collected over time and examined for trends in ejection fraction (EF) of the patients' hearts over the age during that time of their illness. Data on the eight patients with the largest number of longitudinal EF data points, prior to transplant, were extracted and graphed. The data revealed that EF drops off much earlier, at almost half the age in males compared to females and that

\section{Females}
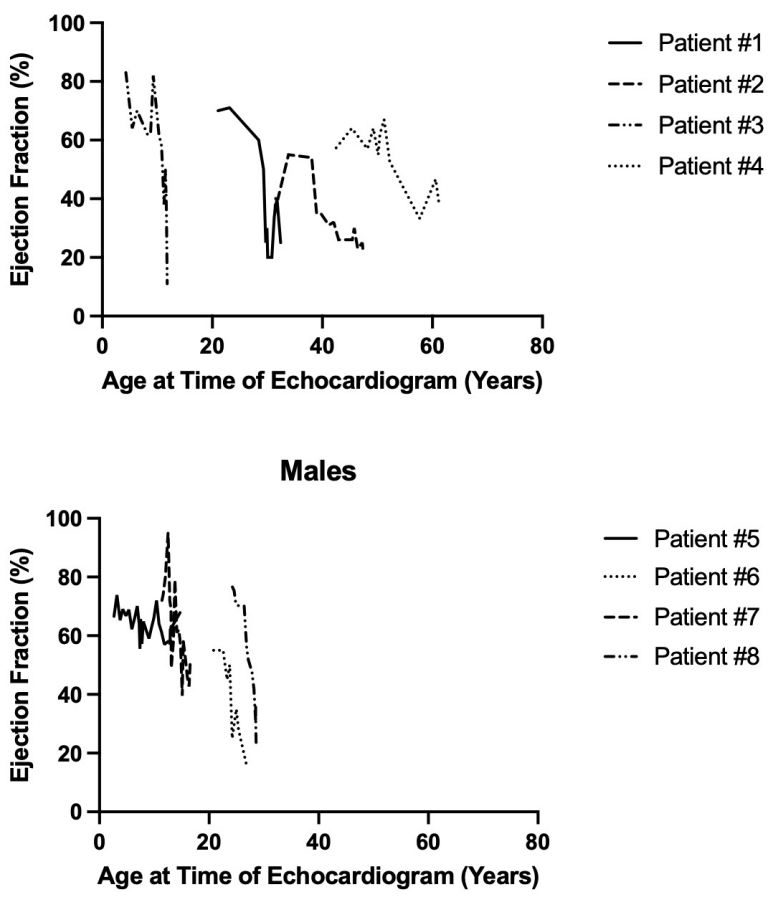

Abstract \#14 Figure 1 substantial variation in EF is present for subjects, rather than a smooth, gradual decline.

Conclusions As this natural history data continues to be analyzed, further analysis will be done that aims to look at the progression of heart disease in Danon patients prior to transplant. These trends will be imperative in not only understanding the progression of the disease to drive best clinical practices, but also to utilize as controls in clinical trials for potential treatments of Danon Disease.

\section{$\# 15$ LIFESTYLE COUNSELING FOR VETERANS WITH SYMPTOMATIC HEART FAILURE}

${ }^{1} \mathrm{C}$ Taylor*, ${ }^{1} \mathrm{~A}$ Edmeade, ${ }^{1} \mathrm{CE}$ Walters, ${ }^{2} \mathrm{~J}$ Patel. ${ }^{1}$ Loma Linda University School of Medicine, Loma Linda, CA; ${ }^{2}$ VA Loma Linda Healthcare System, Loma Linda, CA

\subsection{6/jim-2022-WRMC.15}

Purpose of Study Lifestyle counseling has shown to be effective in modifying health behaviors and reducing cardiovascular risk in healthy patients. However, data supporting effectiveness of lifestyle counseling in patients with symptomatic heart failure is limited. This quality improvement study hypothesizes that lifestyle counseling focusing on sleep, activity, nutrition, medication adherence, and self-care will be associated with improvements in health behaviors in veterans with heart failure.

Methods Used This study screened a selected cohort of patients with symptomatic heart failure from the Loma Linda VA Heart Failure program. The study included 5 counseling sessions over a 9 week interval. In week 1, pre-intervention surveys were completed using the following validated surveys: Pittsburgh Sleep Quality Index, Veterans Specific Activity Questionnaire, Mini Nutritional Assessment, Eight-Item Morisky Medication Adherence Scale, and Self-Care of Heart Failure Index. During weeks 2 through 8, three counseling sessions focusing on nutrition, sleep, exercise, and self-care were tailored to each patient following best practices from LLVA Heart Failure Program. In week 9, post-intervention surveys were completed.

Summary of Results Out of 112 screened, 49 (44\%) agreed to participate. Each patient was given a baseline score during the pre-intervention surveys during week 1. Baseline Pittsburgh Sleep Quality Index average score was 8.74 with post-intervention survey average of 7.68. Lower scores on the Pittsburgh Sleep Quality Index demonstrate higher quality sleep habits and sleep quality. For the Veterans Specific Activity Questionnaire baseline average score was 4.36 and post-intervention was 4.55 . This questionnaire ranked activities according to metabolic equivalent of task from 1 to 13 . At baseline in the Mini Nutritional Assessment patients reported an average score of 11.38. After lifestyle counseling, patients reported an average score of 12.40. The Mini Nutritional Assessment scoring system had a maximum of 14 with normal nutritional status ranging from 12 to 14 , at risk of malnutrition from 8 to 11 , and malnourished scores with score under 7 . The Eight-Item Morisky Medication Adherence Scale had baseline and post-interventions average scores of 5.51 and 6.30, respectively.

Conclusions All survey data indicated positive changes in lifestyle from pre to post surveys. Lifestyle counseling may improve health behaviors in patients with symptomatic heart failure. 


\section{AND STROKE RISK}

M Kapadia*, P Jagadish, M Hutchinson, $\mathrm{H}$ Lee. The University of Arizona College of Medicine Tucson, Tucson, AZ

\subsection{6/jim-2022-WRMC.16}

Purpose of Study To review the literature on stroke risk in patients with atrial fibrillation (AF) undergoing electroconvulsive therapy (ECT) as well as anticoagulation recommendations.

Methods Used Two authors independently performed a literature review of PubMed, searching for 'electroconvulsive therapy and atrial fibrillation.' The resulting articles and their references were reviewed for relevance to $\mathrm{AF}$ and stroke risk. Summary of Results Rozig, et al. (2018) found that ECT is not associated with an increased risk of new or recurrent stroke. Among 23 studies, we found that post-ECT cardioversion of AF to normal sinus rhythm occurred in 2 cases. Neither was associated with stroke, though this finding may limited by low sample size. However, ECT has induced AF in at least 6 cases without stroke.

ECT requires fewer joules than does cardioversion for atrial fibrillation, and current is applied to the brain for ECT versus the heart for synchronized cardioversion. The mechanism of $\mathrm{AF}$ induction/cardioversion is a catecholamine surge and varied hemodynamic changes.

Because of the rarity of stroke in ECT patients, routine anticoagulation prior to ECT is controversial. Furthermore, direct electrical stimulation of the brain risks hemorrhagic stroke after ECT.

In patients with existing $\mathrm{AF}$ who are not anticoagulated, an alternative means of reducing cardioversion risk may involve modulation of post-ECT hemodynamic changes by beta-blockade. Beta blockade may exacerbate bradycardia in some while reducing reflex tachycardia in others and may even decrease in seizure length, thus, lowering the efficacy of ECT.

Conclusions The risk of inducing stroke by cardioversion of $\mathrm{AF}$ in the setting ECT is very low despite a documented risk of cardioversion. Routine anticoagulation prior to ECT remains controversial. Imaging modalities such as echocardiography or mitigation of hemodynamic effects may further reduce the risk of stroke in these patients. Areas for further study are precise assessment of cardioversion and stroke risk in AF patients and the effect of routine beta-blockade on cardioversion risk.

\section{\#17 CRITICAL ROLE OF SPECIALIZED PRO-RESOLVING MEDIATORS IN ATHEROSCLEROSIS}

S Rangarajan*, P Rangchaikul, MM Radwan. Western University of Health Sciences, Pomona, $C A$

\subsection{6/jim-2022-WRMC. 17}

Purpose of Study Coronary artery disease is the leading cause of death worldwide with over 17.9 million deaths per year according to WHO. There has been a vast amount of research done in understanding the causes, and therefore the treatments of this disease. Our body's inflammatory processes have been identified as a nidus of the elaborate process that ultimately leads to life-threatening cardiovascular events. However, research around understanding how the body puts an end to such naturally occurring inflammation i.e., resolution of this inflammation, is gaining traction and has shed light into new avenues for future management of $\mathrm{CV}$ diseases. In this narrative review we discuss the pathophysiological and molecular mechanisms of atherosclerosis including inflammation, apoptosis and efferocytosis, the recent development in the understanding of a new class of molecules called Specialized Proresolving Mediators (SPMs), and the impact of such findings in the realm of cardiovascular treatment options.

Methods Used We searched the MEDLINE database, PubMed restricting ourselves to original research articles as much as possible, and analyzed papers published in the last 20 years on the complex pathophysiology of atherosclerosis and the role of resolvins.

Summary of Results Specialized Pro-resolving Mediators (SPMs) is a class of molecules that acts as strong local modulators of acute inflammation. It is further classified into resolvins (E and D series), maresins, protectins, and lipoxins. Resolvins mediate resolution of inflammation through a variety of actions. Some of them are reduced chemotaxis of neutrophils by blocking the action of $\mathrm{LTB}_{4}$, a strong neutrophil chemoattractant, reduced PMN chemotaxis by effecting changes in their actin polymerization, downregulation of leukocyte integrin activation thereby reducing their response to platelet activation factor (PAF), a potent pro-inflammatory cytokine. Maresins are involved in converting pro-inflammatory phenotype of macrophage M1 to pro-resolving $\mathrm{M} 2$, in reducing superoxide production by $\mathrm{TNF} \alpha$ and nuclear translocation of $\mathrm{p} 65$, which together result in a reduction of pro-inflammatory $\mathrm{NF \kappa B}$ pathway. Protectins act in a pro-resolving manner by downregulating certain markers for chemotaxis such as Vascular Cell Adhesion Molecule (VCAM-1) and Monocyte Chemoattractant Protein (MCP-1). Lipoxins are shown to facilitate resolution by stopping further recruitment of neutrophils, inducing nonphlogistic migration and induction of macrophages to clear apoptotic neutrophils.

Conclusions We expect to see further research in translating these findings to bedside clinical trials in the treatment of conditions with a pathophysiological basis of inflammation such as coronary artery disease, asthma, periodontal disease, etc.

\section{Endocrinology and metabolism I}

\section{Concurrent session}

\section{2:45 PM}

\section{Thursday, January 20, 2022}

\section{\#18 CORONARY ARTERY CALCIFICATION SCORING IN TRANSGENDER INDIVIDUALS}

T Duro*, E Choi, P Kapsner. The University of New Mexico, Albuquerque, NM

\subsection{6/jim-2022-WRMC.18}

Purpose of Study To assess risk factors and the prevalence of coronary artery disease (CAD) using coronary artery calcium (CAC) scoring in transgender individuals receiving gender affirming hormone therapy (GAHT).

Transgender individuals are treated with cross sex hormone therapy. These hormones alter metabolic profiles and may be 
associated with risk factors for CAD. Little data is available on the atherosclerotic vascular risk due to GAHT in transgender individuals. CAC Scores have been validated as a noninvasive method to assess risk for cardiovascular events in the general population.

Methods Used This is a pilot study assessing feasibility of obtaining baseline risk profiles and CAC scores in transgender patients over the age of 18 , in order to establish a baseline risk assessment and the prevalence of CAD in this population. Patients with risk factors other than smoking and family history were excluded. Difference in CAC Scores were compared to those in The Coronary Artery Risk Development in Young

\begin{tabular}{lll} 
Abstract \#18 Table 1 & \multicolumn{2}{l}{ Baseline characteristics } \\
\hline & $\begin{array}{l}\text { Transwomen } \mathbf{n = 2 5} \\
\mathbf{n}(\%) \text { or median (IQR) }\end{array}$ & $\begin{array}{l}\text { Transmen } \mathbf{n}=22 \\
\mathbf{n}(\%) \text { or median (IQR) }\end{array}$ \\
\hline Age & $40(14)$ & $38(11)$ \\
History of smoking & $14(56 \%)$ & $16(73 \%)$ \\
Current smoker & $7(28 \%)$ & $2(9 \%)$ \\
GAHT Duration, yr & $3.2(11.9)$ & $5.5(10.5)$ \\
Family history of CAD & $2(8 \%)$ & $6(27 \%)^{*}$ \\
BMI & $24.8(7.3)$ & $29.2(6.2)$ \\
SBP & $119.0(18)$ & $118.0(16)$ \\
DBP & $78.0(15)$ & $77.5(9)$ \\
HbA1c & $5.3(0.3)$ & $5.3(0.4)$ \\
Triglycerides & $126.0(92)$ & $102.5(110)$ \\
Cholesterol & $182.0(38)$ & $182.0(41)$ \\
HDL & $57.0(34)$ & $47.5(30)$ \\
LDL & $91.0(29)$ & $105.5(36)$ \\
hsCRP & $1.1(1.5)$ & $1.7(2.5)$ \\
Total testosterone & $26.0(131)$ & $623.5(741) *$ \\
Estradiol & $91.2(167)$ * & $35.5(22.5)$ \\
\hline
\end{tabular}

${ }^{*}$ Statistically significant as determined by $p$ value $<0.05$

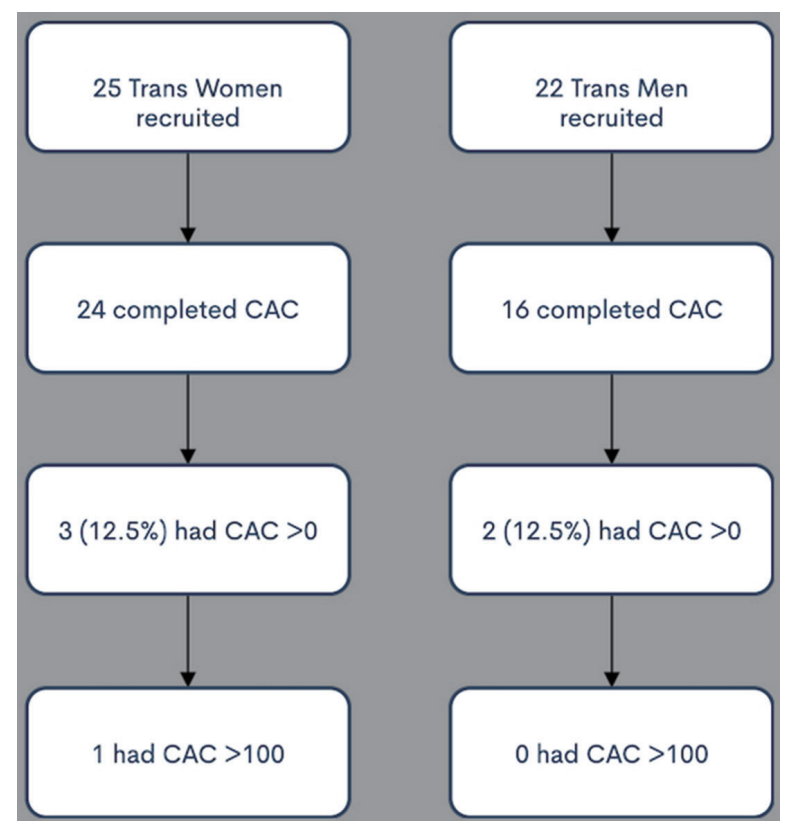

Abstract \#18 Figure 1
Adults (CARDIA) study. Baseline characteristics were compared using t-tests.

Summary of Results Out of 25 transwomen recruited, 24 completed CAC. $3 / 24(12.5 \%)$ had CAC $>0$. One had CAC $>100$. Out of 22 transmen recruited, 16 completed CAC. 2/ $16(12.5 \%)$ had CAC $>0$. None had CAC $>100$. CAC scores did not correlate with the presence of risk factors. Patient data in table 1 .

Conclusions Overall, $12.5 \%$ of transgender people on GAHT had positive CAC scores. This is similar to the findings of the CARDIA study where the prevalence of positive CAC scores in the general population was $11.7 \%$. There was a higher percentage of current smokers in transwomen who also had higher triglycerides, but a lower LDL. Transmen had a higher family history of CAD, percentage having ever smoked, and a higher hsCRP. This cross-sectional study shows that obtaining CAC scores in transgender patients is feasible and that the prevalence of positive CAC scores appears to be similar to that of the general population. A larger, longitudinal study will be performed to expand on these findings.

\section{\#19 BEYOND BINDING: KEY QUESTIONS REGARDING THE ROLE OF SHBG IN MALE REPRODUCTION}

K Schuppe*, K Roberts. Washington State University Elson S Floyd College of Medicine, Spokane, WA

\subsection{6/jim-2022-WRMC. 19}

Purpose of Study Human Sex Hormone Binding Globulin (SHBG) is a homodimeric glycoprotein encoded by the SHBG gene on chromosome $17 \mathrm{p} 13.1$. The gene is predominantly expressed in the liver and SHBG is secreted into the blood whereas, in murine species where much of our understanding comes from, the ortholog of SHBG is expressed primarily in Sertoli cells. SHBG binds both steroidal and nonsteroidal ligands with high affinities and has been traditionally viewed as functioning as a reservoir and means of transport for steroids in serum. SHBG has also been implicated in androgen uptake by cells and as a signaling molecule with a cell surface receptor on target cells. More recently a germ cell-specific form has been identified in the human testis, raising questions about a role for SHBG in germ cell function and fertilization. The objective of this review was to determine aspects of SHBG function that require further investigation.

Methods Used A systematic search of the literature, using NCBI and other databases compiling peer-reviewed publications, was conducted using keywords: Sex Hormone Binding Globulin; SHBG; SHBG Receptor; SHBG Isoform; SHBG Expression; Megalin; Androgen Binding Protein; ABP. Additional papers were found via citations, both forward and back. The focus of the review was on literature published in the last 25 years, prioritizing the most recent research. Studies were excluded where SHBG function, structure, genetic regulation was not a topic of investigation, such as studies focused on genome-wide analysis, SNP's, and SHBG in the context of an upstream pathology.

Summary of Results In total 95 publications were identified and kept for producing either new experimental evidence or analysis of prior data that provided new insights into the role of SHBG on reproduction. The literature search converged on studies investigating SHBG's steroid-binding properties, 
protein-protein and protein-receptor interactions, novel germ cell forms of SHBG, and clinical findings from both normal and natural mutant phenotypes. The findings from these studies were summarized and gaps in our knowledge of SHBG's role in reproductive function were identified

Conclusions Four unanswered questions regarding SHBG/ABP function emerged from review of the literature. 1) Is SHBG essential in the development and regulation of the reproductive system? 2) What is the identity of the cell surface receptor for SHBG? A number of studies have demonstrated specific binding and subsequent signaling, yet no receptor has been identified. 3) Does the site of SHBG synthesis and secretion matter? 4) What is the function of the germ cell form of SHBG? Germ cells have the second-highest level of expression of SHBG, but we know very little about it. These questions seem key to future research on the role of SHBG in reproduction.

\section{\#20 SUPRASELLAR AND SELLAR MENINGIOMA IN PREGNANCY PRESENTING WITH WORSENING VISUAL DEFICITS AND PANHYPOPITUITARISM}

${ }^{1} \mathrm{SM}$ Laies*, ${ }^{2} \mathrm{~F}$ Fuh, ${ }^{1} \mathrm{C}$ Lovato. ${ }^{1}$ The University of New Mexico, Albuquerque, NM; ${ }^{2}$ University of California Los Angeles Health System, Montecito, CA

\subsection{6/jim-2022-WRMC.20}

Case Report Meningiomas account for 13\% of CNS tumors during pregnancy and are predominantly low grade WHO I tumors. Case series have reported rapid growth of these tumors during pregnancy, particularly during the second and third trimester, likely due to factors including intra-tumoral hypervascularity and tumor growth due to high sex hormone levels and the presence of progesterone receptors on tumor cells. As a result, patients can present with neurologic and visual field (VF) deficits and in rare cases, herniation and coma. We report a case of a pregnant patient with worsening of VF deficits and panhypopituitarism due to a meningioma in the suprasellar and sellar region. A 36-yearold woman G4P3 at 20 weeks, was transfered to our facility for neurosurgical intervention for acute worsening of VF deficits. She initially presented to her ophthalmologist with a 4-month history of headaches and right-sided vision loss and was found to have a sellar mass on imaging. She then began to have nausea and vomiting, weakness, and orthostasis which necessitated admission. The patient was given i.v. hydrocortisone (HC) due to a suspicion for adrenal insufficiency with improvement in symptoms. However, after receiving steroids, she developed polydipsia and polyuria. On arrival to our facility, the patient was noted to have urinary output (UO) of up to 6 liters per day with a low urine osmolality of $53 \mathrm{mosm} / \mathrm{Kg}(50-600 \mathrm{mosm} / \mathrm{Kg})$ and serum $\mathrm{Na}$ as high as $147 \mathrm{mmol} / \mathrm{L}(134-144 \mathrm{mmol} / \mathrm{L})$. She was given desmopressin (DDAVP) with improvement in serum $\mathrm{Na}$ and UO. Biochemical testing also revealed secondary hypothyroidism and L-thyroxine (L-T4) was initiated. An MRI of the brain on admission showed a $3.6 \mathrm{~cm}$ lobulated suprasellar and sellar mass with mass effect on the optic chiasm. Formal VF testing showed complete VF loss on the right and temporal loss on the left. After review of the patient's case by a multidisciplinary team consisting of Neurosurgery, MFM, and Endocrinology it was decided to proceed with surgery. The patient underwent left pterional craniotomy and resection of the suprasellar component of the mass with decompression of the optic chiasm. Pathology showed a meningioma, WHO Grade I. Post-operatively, VF deficits improved and on discharge from the hospital, she was continued on DDAVP, L-T4 and HC. This is a rare case of a meningioma resulting in hormonal deficiencies in the anterior and posterior pituitary along with VF deficits which likely developed due to the location and rapid growth of the tumor in the setting of pregnancy. The decision for surgery during pregnancy should be based on clinical presentation and should involve a multidisciplinary team to determine the best management that limits complications to the mother and fetus.

\section{\#21 UNRAVELING THE MYSTERY OF THYROTOXICOSIS IN A PATIENT WITH PANHYPOPITUITARISM}

AT Chuang * BW Hager, M Bouchonville. University of New Mexico Health Sciences Center, Albuquerque, NM

10.1136/jim-2022-WRMC.21

Case Report In the setting of panhypopituitarism, hypothyroidism develops as a result of TSH deficiency rather than a primary insult to the thyroid gland. We describe a case of a patient with acquired panhypopituitarism who developed severe thyrotoxicosis, which persisted despite discontinuation of replacement thyroid hormone.

A 24-year-old Hispanic female with panhypopituitarism following a craniopharyngioma resection in 2010 presented with severe thyrotoxicosis. She initially presented with anxiety and palpitations and was observed to have a mild free T4 (FT4) elevation, which prompted a reduction in her replacement dose of levothyroxine. With progression of her FT4 elevation, the levothyroxine was discontinued and she was ultimately referred to adult endocrinology when clinical presentation worsened. Upon further evaluation, she reported weight loss of more than thirty pounds, palpitations, insomnia, fatigue, muscle weakness, bilateral hand tremors, nausea, vomiting, and dizziness. She confirmed a period of several months without levothyroxine. Heart rate was $109 \mathrm{bpm}$, no proptosis was noted, thyroid examination revealed no thyromegaly, no tremors were appreciated, and there were no findings suggestive of heart failure. FT4 at that time was $7.6 \mathrm{ng} / \mathrm{dL}$ (reference 0.7$1.6 \mathrm{ng} / \mathrm{dL}$ ). Thyroid peroxidase antibody and $\mathrm{TSH}$ receptor antibody levels were negative. Thyroid ultrasound demonstrated an atrophic left thyroid lobe; no nodules were identified. Thyroid uptake was low at $1.8 \%$ at 24 hours. This unexpected finding prompted a thyroglobulin (TG) level to distinguish between subacute thyroiditis or an ectopic source of thyroid tissue versus a low TG state such as factitious thyrotoxicosis. TG was low at $3.3 \mathrm{ng} / \mathrm{ml}$, prompting a pharmacy query, which revealed that she was continuing to fill levothyroxine prescriptions at three pharmacies.

A more comprehensive review of her behavioral health history revealed persistent depressive disorder, PTSD, psychogenic nonepileptic seizures, and numerous recent psychosocial stressors. We approached her care in a non-confrontative manner by presenting a range of possible explanations for her clinical and laboratory findings and sharing our recommended treatment. 
This case highlights an unexpected presentation of factitious thyrotoxicosis secondary to surreptitious use of levothyroxine in a patient with acquired panhypopituitarism, and the stepwise evaluation that led to this conclusion. We discuss the strategies implemented in managing this patient and review approaches to patients with factitious disorders.

\section{\#22 FEASIBILITY OF EXTENDING SLEEP IN A PILOT SAMPLE OF TEENS WITH OBESITY AND SHORT SLEEP: THE REM STUDY}

${ }^{1} \mathrm{AE}$ Bowen ${ }^{*},{ }^{2} \mathrm{~S}$ Simon, ${ }^{2} \mathrm{M}$ Cree-Green, ${ }^{2} \mathrm{KJ}$ Nadeau, ${ }^{2} \mathrm{~J}$ Kaar. ${ }^{1}$ Children's Hospital Colorado, Aurora, CO; ${ }^{2}$ University of Colorado Anschutz Medical Campus, Aurora, CO

\subsection{6/jim-2022-WRMC.22}

Purpose of Study Insufficient sleep duration is common among adolescents and may contribute to insulin resistance, dysglycemia, and precursors to type 2 diabetes (T2D). Youth-onset T2D has devasting long-term effects, and thus prevention strategies for adolescents at risk for T2D are needed. Increasing total sleep time (TST) may be one such strategy. We tested the feasibility of a 4-week sleep extension intervention for adolescents treated within an outpatient weight management clinic.

Methods Used High school students aged 14-19 years with insufficient sleep ( $<8$ hours) on school nights were recruited during the academic year. Exclusion criteria included T2D, medications that affect sleep, and a schedule that precluded participants from adhering to sleep extension (e.g., night shift employment). Following sleep monitoring at home for 1 week, a revised sleep schedule was collaboratively created with a target of increasing time in bed (TIB) by 2 hours. Participants followed this schedule for 2 weeks (W2), returned to the clinic to discuss barriers to adherence to the prescribed schedule, and then followed the revised schedule for another two weeks (W4). Feasibility was assessed by adherence to wearing sleep watch and submitting sleep diaries, improvement in TIB and TST, and self-reported barriers to following prescribed sleep schedule. Data are reported as averages \pm sd or medians ( $\min , \max$ ).

Summary of Results A total of 6 participants have been recruited for the study to-date; however, 2 were withdrawn due to COVID-19 school closures when shifted to online learning. Participants $(n=4)$ were aged $17.0 \pm 0$ years, $100 \%$ Hispanic, with a BMI percentile of $98.4 \pm 0.9 \mathrm{~kg} / \mathrm{m}^{2}$. All participants completed the intervention; 99.4\% adhered to Actigraphy and $65.6 \%$ to the sleep diary. At baseline, the average TIB was 7.3 \pm 1.6 hours and TST was $5.8 \pm 1.5$ hours. Compared to baseline, W2 hours of TIB $[1.2(-0.1,2.6)]$ and TST [1.2 $(-0.4,2.1)]$ increased and W4 hours of TIB $[0.1(-1.8,5.3)]$ and TST $[0.7(-1.5,4.1)]$ increased. Increased TIB was primarily achieved by shifting bedtimes earlier, while waketimes remained relatively consistent. Barriers to adherence included homework, extracurricular activities, and parents' and youths' variable work schedules.

Conclusions Initial findings indicate a 4-week sleep extension intervention in adolescents with short sleep seeking treatment for weight management is feasible. Participants increased TIB and TST by a median of 1.2 hours. However, additional strategies are needed to maintain such improvements. School and community efforts to delay high school start times may benefit youth at risk for T2D by enabling them to increase TST.
\#23 RELATIONSHIP BETWEEN HIGH CARBOHYDRATE AND VITAMIN D INTAKE ON SLEEP QUALITY AMONG ADULT POPULATION OF UNITED STATES

C Brown*, M Shaheen, K Schrode. Charles Drew University of Medicine and Science, LoS Angeles, CA

10.1136/jim-2022-WRMC.23

Purpose of Study Quality of sleep depends on diet as carbohydrate or vitamin D. Sleep quality is challenging to study due to cost, resources, and availability of research subjects. There is a gap in the literature examining the impact of carbohydrates and vitamin $\mathrm{D}$ intakes on sleep quality. We aim to examine the association between carbohydrate and vitamin D intakes and sleep quality among adult US population.

Methods Used We analyzed data from the National Health and Nutrition Examination Survey 2007-2014. Carbohydrate and vitamin D intakes were categorized into three groups based on the distribution. Sleep quality was assessed using hours of sleep, have sleep problem and doctor diagnosed sleep disorder. We used chi square and multiple logistic regression to analyze the data considering the design and sample weight. Summary of Results Of the 16,415 adults, 35\% had high carbohydrate intake (>283 gm), 31\% had low vitamin D intake, $36 \%$ slept $<7$ hours/day, $27 \%$ reported sleep problem, and $9 \%$ had sleep disorder. There was no relation between the high carbohydrate intake and low vitamin $\mathrm{D}$ intake and the hours of sleep $(p>0.05)$. However, high vitamin $D$ intake was associated with sleep disorders and troubled sleep $(p<0.05)$ adjusting for the confounding variables. Minority, divorced/ widowed, overweight/obese, smokers, with diabetes, kidney disease, depression were more likely to have low sleep hours relative to the other groups $(\mathrm{p}<0.05)$.

Conclusions Our study indicated no association between carbohydrate and vitamin D intakes and sleep quality. Longitudinal prospective studies are needed to examine factors associated with quality of sleep and their mechanisms.

\section{\#24 AUTOIMMUNE HEPATITIS PRESENTING AS FULMINANT HEPATIC FAILURE}

NC Trang*, AS Gill, K Radicic, N Karapetians, S Mishra. Kern Medical Center, Bakersfield, $C A$

10.1136/jim-2022-WRMC.24

Purpose of Study To present an interesting case with an atypical presentation of a rare disease.

Methods Used A single patient case report was conducted after IRB approval.

Summary of Results Autoimmune hepatitis (AIH) is a chronic inflammatory disease of the liver that typically presents with the presence of AIH-related antibodies. There are two types of AIH: type 1 is associated with anti-smooth muscle antibody (anti-SMA) and type 2 associated with anti-liver/kidney microsome type 1 antibody or anti-liver cytosol type 1 antibody. About $25 \%$ of patients with $\mathrm{AIH}$ are asymptomatic. Rarely, patients with AIH present with features of fulminant hepatic failure with rapidly progressive liver impairment, coagulopathy, and hepatic encephalopathy or coma.

We present a case of a 63-year-old female who presents to the hospital with altered mental status (AMS) of one day duration. Her labs were significant for acute kidney injury (AKI), elevated liver function tests, and urinalysis consistent 
with urinary tract infection (UTI). Computerized tomography (CT) abdomen and pelvis showed bilateral pyelonephritis. Urine cultures grew pan-sensitive Escherichia coli, and she was treated with 10 days of Ceftriaxone. During this course, renal function worsened with uremic-range BUN and she became oliguric, thus hemodialysis was initiated with good response in both renal indices and mental status. Patient was also found to have coagulopathy with a worsening PT/INR of 32.2/3.24. Further workup of AKI revealed proteinuria and positive atypical p-ANCA and anti-SMA, raising concern for autoimmune disease in both kidneys and liver.

Conclusions Our patient's symptoms originally raised concerns for pyelonephritis secondary to a UTI. Despite a full course of antibiotics, her kidney function continued to decline until receiving dialysis. She was also determined to have fulminant hepatitis with coagulopathy followed by positive autoimmune antibodies. This course leads us to believe that the etiology of her encephalopathy was secondary to autoimmune liver and kidney disease.

The main interest of this case report lies in autoimmune hepatitis secondary to atypical P-ANCA and anti-SMA presenting as fulminant hepatic failure in the setting of AKI. Atypical P-ANCA seems to be more specific for autoimmune hepatitis than the typical P-ANCA [1]. Anti-SMA antibodies found in about $50 \%$ of type $1 \mathrm{AIH}$ [1]. Both nephrology and gastroenterology recommended renal/liver biopsy for definitive diagnosis which will be done outpatient.

\section{\#25 DIABETIC NEUROPATHIC CACHEXIA - AN UNCOMMON MIMIC}

${ }^{1}$ JS Gilbert ${ }^{*},{ }^{2} \mathrm{~K}$ McCloskey, ${ }^{3} \mathrm{P}$ Gulani, ${ }^{3} \mathrm{~A}$ Outon. ${ }^{1}$ University of Colorado, Denver, CO; ${ }^{2}$ Beth Israel Deaconess Medical Center, Boston, MA; ${ }^{3}$ Jacobi Medical Center, Bronx, NY

\subsection{6/jim-2022-WRMC.25}

Case Report A 52-year-old man with poorly controlled type 2 diabetes presented with four months of watery diarrhea. During this period, he also noticed an unintentional 80-pound weight loss, a 'burning' sensation on his anterior thighs, and new onset depression and anxiety. He had no recent history of fever, chills, or night sweats. He was not prescribed insulin, though glipizide was added to his diabetes regimen about six months prior. Physical examination revealed a cachectic man with a BMI of $18.4 \mathrm{~kg} / \mathrm{m} 2$, normal vital signs, and an unremarkable rest of exam. Laboratory evaluation was notable for a white blood cell count of $21.8 \times 10^{\wedge} 3$ cells $/ \mathrm{mm} 3$, an anion gap of 26, and a glucose level of $280 \mathrm{mg} / \mathrm{dL}$. He also had a CRP of $147.1 \mathrm{mg} / \mathrm{dL}$ and an HbA1C of $19.3 \%$. Serology for HIV-1, HIV-2, HBsAg, and HCV were all negative, and a fecal fat test was normal. Chest $\mathrm{x}$-ray was clear, a transthoracic echocardiogram showed no valvular vegetations, and CT scans of his chest, abdomen, and pelvis were completely normal.

This patient's concerning cluster of symptoms provoked an extensive workup to rule out cancer, infection, and malabsorptive syndromes. However, this patient's presentation matched an uncommon neuropathy syndrome found in diabetes. Diabetic neuropathic cachexia (DNC) is an extremely rare neuropathy - only 36 cases have been reported in the literature but its unique cluster of symptoms often incites a search for a hidden malignancy or insidious infection. DNC most commonly presents in a type- 2 diabetic after initiation of an oral anti-hyperglycemic medication with profound weight loss, mood symptoms, symmetric peripheral neuropathy, and painful limb paresthesias. Management of this neuropathy is directed at improving glycemic control, as most patients recover within one to two years with improvement in $\mathrm{A} 1 \mathrm{C}$, though some can suffer residual neurologic deficits.

\section{\#26 UPPER EXTREMITY DEEP VENOUS THROMBOSIS SECONDARY TO THYROTOXIC PERIODIC PARALYSIS WITH ACCOMPANYING HYPERPHOSPHATEMIA AND SEVERE HYPOMAGNESEMIA}

J Rahesh*, L Al-Sukhni, B Quraishi, T Naguib. Texas Tech University Health Sciences Center, Lubbock, TX

\subsection{6/jim-2022-WRMC.26}

Case Report A 20 year old male with history of drug abuse and osteochondritis presented to the emergency department with bilateral leg weakness and feet numbness since the morning when he woke up unable to move his legs or his back and fell out of bed, after which he noticed that he had feeling in his legs but not his feet. He was found to be profoundly hypokalemic at $1.6 \mathrm{mmol} / \mathrm{L}$ and hypomagnesemic at $1.5 \mathrm{mg} / \mathrm{dL}$. Phosphate levels were not taken but calcium was $9.0 \mathrm{mg} / \mathrm{dL}$. These values were verified by a redraw and repeat chemistry. The patient denied nausea, vomiting, and diuretic use, but did report lose bowel movements for long months due to self-diagnosed IBS. He also reported alcohol use of 4 6 beers 1-2 times a week for years. The patient was admitted for potassium and magnesium intravenous replacement. Chest X Ray, MRI of the cervical spine, CT scan of the brain, and drug panel were all negative.

On day 1 of hospital stay the patient's electrolyte levels improved, with a potassium of $3.6 \mathrm{mmol} / \mathrm{L}$ a magnesium of $1.8 \mathrm{mg} / \mathrm{dL}$, a phosphate of $4.0 \mathrm{mg} / \mathrm{dL}$, and a calcium of 8.9 $\mathrm{mg} / \mathrm{dL}$. On day 2 , he reported improved strength after working with physical therapy but did not feel at baseline. His labs indicated a potassium of $3.6 \mathrm{mmol} / \mathrm{L}$, a magnesium of 1.8 $\mathrm{mg} / \mathrm{dL}$ a phosphate of $5.0 \mathrm{mg} / \mathrm{dL}$ and a calcium of $9.3 \mathrm{mg} / \mathrm{dL}$. His SARS-CoV2 antigen, blood, and urine cultures were all negative. A negative TTG IgA test ruled out Celiac disease.

On day 3, potassium was $4.4 \mathrm{mmol} / \mathrm{L}$, and magnesium was $1.7 \mathrm{mg} / \mathrm{dL}$, and a newly elevated phosphate of $5.7 \mathrm{mg} / \mathrm{dL}$ was noted. Calcium was $9.0 \mathrm{mg} / \mathrm{dL}$. Right arm edema and pain at the PICC line site prompted removal of the line but an ultrasound showed an occlusive DVT. A heparin drip was ordered, and a V/Q scan was negative. An asymptomatic run of ventricular tachycardia prompted an echocardiogram and troponin evaluation, but both were negative.

On day 4 his labs revealed a low TSH of $<0.01$ an hypomagnesemia of $1.8 \mathrm{mg} / \mathrm{dL}$. Potassium was within normal limits. Phosphate was still elevated at $5.5 \mathrm{mg} / \mathrm{dL}$ and calcium was $10.0 \mathrm{mg} / \mathrm{dL}$.

On day 5 a low TSH of $<0.01$ was confirmed once again, elevated T3 $9.99 \mathrm{pg} / \mathrm{ml}$ and T4 $2.86 \mathrm{ng} / \mathrm{dL}$ were also noted. Potassium at this time was $4 \mathrm{mmol} / \mathrm{L}$, magnesium was within normal limits, phosphate was elevated at $7.0 \mathrm{mg} / \mathrm{dL}$, and calcium was $9.5 \mathrm{mg} / \mathrm{dL}$. A PTH was also measured at this time and was found to be normal at $36.7 \mathrm{pg} / \mathrm{mL}$. Ventricular tachycardia was attributed to hyperthyroidism, and a diagnosis of thyrotoxic periodic paralysis was made. An ultrasound of the neck showed a hypervascular thyroid consistent with Graves' 
disease or thyroiditis. Thyroid stimulating immunoglobulin was drawn, and the patient began a regimen of $10 \mathrm{mg}$ methimazole 3 times daily.

On day 6 the patient had a potassium of $3.8 \mathrm{mmol} / \mathrm{L}$, phosphate of $6.2 \mathrm{mg} / \mathrm{dL}$, calcium of $9.6 \mathrm{mg} / \mathrm{dL}$ and a magnesium of $2.3 \mathrm{mg} / \mathrm{dL}$. On day 7 the patient was discharged.

\section{Healthcare delivery research I}

\section{Concurrent session}

\section{2:45 PM}

\section{Thursday, January 20, 2022}

\section{\#27 DIABETES PREVENTION AT WORK: 10-YEARFOLLOW-UP OF GLYCEMIC CONTROL AND SERUM LIPOPROTEINS IN HEALTHY WORKERS WITH AND WITHOUT HEALTH COACHING AND MONETARY INCENTIVES}

1,2LW Raymond*. 'Atrium Health, Charlotte, NC; ${ }^{2}$ University of North Carolina System, Chapel Hill, NC

\subsection{6/jim-2022-WRMC.27}

Purpose of Study Knowing of the success of lifestyle changes in the Diabetes Prevention Program, we wondered if similar results could be achieved in a workplace setting with the collaboration of an employer in the Charlotte, NC region.

Methods Used We compared diabetes incidence and serum lipoprotein concentrations in two groups. Cohort 1 comprised 504 workers (mean age 49, 88\% males). Cohort 2 comprised 131 workers (age 52, 85\% males). Cohort 1 received health coaching by a physician assistant or nurse practitioner who encouraged regular exercise, healthy weight, carbohydrate limitation, smoking abstinence, and blood pressure control. They compared baseline values of $\mathrm{HbA} 1 \mathrm{c}$ with those associated with prediabetes and diabetes, $\geq 5.7$ and $\geq 6.5$, respectively. Cohort 1 workers also received up to $\$ 800$ per year, based on the above lifestyle choices as well as HbA1c and lipoprotein levels. The latter were also measured in Cohort 2, but no incentives or health coaching were provided, other than individual letters containing their blood test results.

Summary of Results Diabetes developed over 10 years in 59 Cohort 1 participants compared to 93 expected (chi-squared $=8.56, \mathrm{p}=0.003)$ on the basis of initial HbA1c values (Zhang X et al., Diabetes Care 2010;33:1665). Workers with prediabetes decreased from 192 to 141 . In Cohort 2, five participants were diagnosed with diabetes, the same number as

\begin{tabular}{|c|c|c|c|c|c|c|}
\hline & \multicolumn{4}{|l|}{ Cohort 1} & \multicolumn{2}{|l|}{ Cohort 2} \\
\hline & Initial & Follow-up & $p$ value & Initial & Follow-up & $p$ value \\
\hline Cholesterol & $191 \pm 39$ & $183 \pm 40$ & 0.0001 & $195 \pm 37$ & $187 \pm 35$ & 0.027 \\
\hline LDL & $121 \pm 34$ & $106 \pm 36$ & 0.0001 & $127 \pm 33$ & $113 \pm 30$ & 0.0001 \\
\hline HDL & $42 \pm 12$ & $48 \pm 14$ & 0.0001 & $49 \pm 13$ & $53 \pm 14$ & 0.004 \\
\hline Triglycerides & $144 \pm 95$ & $152 \pm 97$ & 0.21 & $98 \pm 55$ & $107 \pm 61$ & 0.157 \\
\hline
\end{tabular}

expected. However, workers with prediabetes increased from 28 to 36.

Serum lipoproteins improved in both cohorts (table 1).

Conclusions Preventing diabetes benefits individual workers and their families. Employers who share in the health care costs of their workforce also stand to benefit substantially from diabetes prevention, as these costs are $\$ 9,601$ per year higher in persons with this condition. Health coaching and monetary incentives were associated with improved glycemic control as well as lower lipoproteins. Only the latter improvement was found in the group of workers not provided either of the above interventions in this retrospective analysis. Further prospective observations may identify the respective roles of coaching and monetary incentives. Whether these improvements are associated with better outcomes in cardiovascular end points in these workers would also be of great importance.

\section{\#28 MEDICAL STUDENT PERCEPTIONS OF INTIMATE PARTNER VIOLENCE SCREENINGS IN IN-PERSON VS TELEMEDICINE SETTINGS}

${ }^{1} \mathrm{~J} \mathrm{Lai}{ }^{*},{ }^{1} \mathrm{~A}$ Cheng, ${ }^{1,2} \mathrm{AL}$ Nelson, ${ }^{1} \mathrm{M}$ Fraix, ${ }^{1} \mathrm{M}$ Hudson. ${ }^{1}$ Western University of Health Sciences, Pomona, CA; ${ }^{2}$ University of California Los Angeles, Los Angeles, CA

\subsection{6/jim-2022-WRMC.28}

Purpose of Study Intimate Partner Violence (IPV) is a public health crisis that impacts $25 \%$ of women and $10 \%$ of men in the US, totaling 43 million women and 38 million men. IPV screenings traditionally occurred at doctor offices, which was to be a safe space, but with the transition to telemedicine, screening is done at home. This study sought to identify potential educational and practice gaps in care surrounding IPV screenings in different settings. We aimed to understand medical students' general experiences (personal and professional), attitudes, and perceptions of IPV screening.

Methods Used To assess student experiences with IPV screenings as both patients and clinicians-in-training, two separate IRB-approved surveys were created and beta-tested for Western U COMP/COMPNW medical students. One for those who had clinical rotations and the other for those who had not. Questions included personal experiences with in-person and telemedicine IPV screenings, how screenings were conducted, and their perceived importance. Fourth-year students were asked additional questions regarding their experiences observing patients being screened. We used descriptive analysis of the responses to determine the frequency of IPV screening and the modalities in which IPV screening was conducted in in-person and telemedicine environments.

Summary of Results 170 students participated in the study for a response rate of 13\%: 140 students from the non-clinical cohort and 30 students from the clinical cohort. Overall, $36.9 \%$ of students, who had been seen for an in-person appointment, reported they had been screened for IPV, while only $12.5 \%$ of students seen in telemedicine appointments were screened. Among those with in-person appointments, screening was by written survey (31.7\%), online survey $(10 \%)$, and via verbal screen $(58.3 \%)$. However, among those seen via telemedicine, screening was conducted by online survey $(30 \%)$ and by verbal screening (70\%). Healthcare staff who administered IPV screens shifted from medical assistants (MAs) (35.6\%), doctors (35.6\%) and nurses (26.7\%) for in- 
person screenings to MAs (28.6\%), doctors (28.6\%) and receptionists $(28.6 \%)$ in the telemedicine screenings. In the clinical cohort, $56 \%$ of students observed in-person IPV screenings during rotations, compared to $8.33 \%$ of students with telemedicine experience who observed IPV screenings via telemedicine.

Conclusions Medical students reported IPV screening was decreased in the telemedicine setting, which could increase the risk of under detection. Virtual screening was more frequently done verbally and conducted by less trained personnel. This study is unique because it provides the perspective of medical students as both patients and healthcare providers in training and demonstrates both educational and practice gaps in this new environment.

\section{\#29 TDAP VACCINE UPTAKE AND ATTITUDES AMONG ENGLISH AND SPANISH SPEAKING PREGNANT PATIENTS}

${ }^{1} \mathrm{E}$ Jimenez ${ }^{*},{ }^{2,3} \mathrm{H}$ Stohl, ${ }^{1} \mathrm{AL}$ Nelson. ${ }^{1}$ Western University of Health Sciences, Pomona, $C A$; ${ }^{2}$ Harbor-UCLA Medical Center, Torrance, CA; ${ }^{3}$ University of California Los Angeles David Geffen School of Medicine, Los Angeles, CA

\subsection{6/jim-2022-WRMC.29}

Purpose of Study Despite the recommendations of The American College of Obstetrics and Gynecology, and the health benefits that administering the T-dap vaccine can have on prenatal patients and their newborns, nearly $45 \%$ of prenatal patients do not receive the vaccine. We surveyed English and Spanish speaking prenatal patients, to measure uptake and assess patient knowledge and attitudes of the Tdap vaccine. We compared the responses of English and Spanish speaking patients to identify if inconsistencies existed.

Methods Used Using an IRB approved protocol, we surveyed low-income patients at Harbor UCLA Medical Center Obstetrics clinic in Torrance, CA for 7 weeks in Summer 2021. Patients were at least 18 years of age and $\geq 32$ weeks gestational age. Upon obtaining verbal consent, we administered a 31-question beta-tested survey in their preferred language, English or Spanish.

Summary of Results The response rate achieved by the survey was $97 \%$. There was a total of 98 participants, 80 of which answered the survey in English and 18 in Spanish. 49\% of subjects were Hispanic/Latino, 35\% African American, 3\% Caucasian and $13 \%$ were of other ethnicities. Most of our patients (67\%) were between the ages of 20-30. 69\% of English-speaking patients received the vaccine and $79 \%$ of Spanish speaking patients also received the vaccine. Among all our 98 participants there was an overall Tdap vaccine uptake of $70 \%$. The most common reason for refusal of the Tdap vaccine among English speaking patients was due to safety concerns for their baby (47\%), concerns for themselves $(47 \%)$ or because they believed that they did not need the vaccination (47\%). While, only $4 \%$ of English-speaking patients denied the vaccine because they were unaware they required it, this was the most common reason for refusal of the vaccine by Spanish speaking patients (66\%).

Conclusions Overall, $70 \%$ of patients received the Tdap, higher than national averages, but still short of the goal, leaving substantial numbers of women and their newborns unprotected. Both uptake and reasons for refusal of Tdap vaccine differed among English and Spanish speaking patients. This may suggest a need to look more closely at the how Tdap vaccine information is presented in both languages to ensure that the same information or information more relevant to different groups, is being relayed to all prenatal patients.

\section{\#30 EQUITY THROUGH THE END-OF-LIFE: ADVANCING PALLIATIVE CARE FOR NORTHERN CALIFORNIA'S RURAL UNDERSERVED}

R George*, S Gill. UC Davis School of Medicine, Sacramento, CA

\subsection{6/jim-2022-WRMC.30}

Purpose of Study Interdisciplinary research has shown palliative care improves patient experience by addressing adverse symptoms and psychosocial needs during complex and life-limiting illness. The benefits of early inclusion of palliative care in medical management extends to caregivers, providers, and health systems. Additionally, the integrated interprofessional model of palliative care equips it for addressing health disparities for populations carrying a disproportionate burden of illness. Rural communities are among the systemically marginalized and underserved, and as such are poised to benefit from the advancement of palliative care. While prior research assessed challenges faced by rural palliative care providers, an analysis of the scope and equity of palliative care services for Northern California's rural underserved has not been undertaken. Further, this study identifies areas of underservice, analyzes corresponding population demographics, and underlines opportunities for addressing specific health disparities.

Methods Used This research included the collection and analysis of publicly available data pertaining to populations and palliative care organizations of Northern California. Population data sources included the Health Resources and Services Administration and the United States Census Bureau. Palliative care organization data sources included the Center to Advance Palliative Care and the National Hospice and Palliative Care Organization. Data were correlated to identify statistically significant factors pertaining to palliative care access, as well as benchmarks for determining underserved status within the region.

Summary of Results Statistically significant correlations with key population, organizational, and access stratification data identified 13 factors found to be consistent with a county being underserved relative to palliative care access in the region. Several of these factors also represent risk for a county remaining underserved due to economic and organizational infrastructure deficits. Based on these factors, 19 of the 29 counties included in this study qualified as underserved and are at risk for remaining underserved. Additionally, strong evidence exists that protective factors enhancing palliative care access include increased population diversity across race, sex, and age groups. Significant opportunities were identified for expanding the scope, access, and equity of palliative care services based on these findings.

Conclusions Our analysis provides a data-driven approach to improving the access and equity of palliative care services in rural Northern California. A collaborative, community-based, approach to the early inclusion of palliative care in medical management has the possibility of significantly improving health outcomes for medically underserved populations. Additionally, understanding how increased population diversity acts as a protective factor is worthy of further study. 
\#31 BEYOND THE BLACKOUT: THE FAR REACHING IMPACT OF TRAUMATIC BRAIN INJURY ON THE HYPOTHALAMIC-PITUITARY-GONADAL-AXIS AND REPRODUCTIVE FUNCTION OF NAVAL SPECIAL OPERATIONS FORCES

${ }^{1} \mathrm{~K}$ Schuppe*, 'MG Johnston, ${ }^{2} \mathrm{CC}$ Lyons. 'Washington State University Elson S Floyd College of Medicine, Spokane, WA; ${ }^{2}$ Naval Medical Center San Diego, San Diego, CA

\subsection{6/jim-2022-WRMC.31}

Purpose of Study Traumatic brain injury (TBI), is a common injury amongst veterans who have served in Iraq and Afghanistan. With the number of veterans from these conflicts now approaching 3 million, it is estimated that approximately $20 \%$ have suffered at least one TBI. Beyond the structural trauma, TBI may also lead to transient, or even permanent, pituitary insufficiency. Of particular interest are the consequences of TBI-induced hypogonadotropic-hypogonadism (HG) on short and long-term health. TBI clearly presents economic implications for the nation, related both to direct medical expenses and indirect costs. The purpose of this review is to summarize the knowledge about post-TBI hypopituitarism, its screening and treatment recommendations, costs, with a special focus on the potential impacts of post-traumatic hypogonadism on naval special operators.

Methods Used We utilized DoD, CDC, and NIH datasets on TBI's, alongside Endocrine Society and AUA Guidelines, and finally NCBI and Google Scholar searches of the following key terms: pituitary dysfunction, traumatic brain injury, hypogonadotropic hypogonadism, hypogonadism treatment, TBI screening, hormone therapy, fertility, special operations, special forces. The aforementioned sources were used to roughly predict prevalence of post-TBI Hypogonadism in special operators, it's potential costs and consequences, and carry over its standards for screening and treatment.

Summary of Results According to recent estimates, the range of TBI amongst all servicemen serving in Iraq or Afghanistan, taken together from 2000 to 2016, runs from 11-23\%. Persistent hypogonadotropic hypogonadism following TBI meanwhile, amongst the general population, is predicted to fall within the wide range of $8-41 \%$. It was found that hypogonadism was associated with incidence of PTSD among other physiologic consequences such as sexual dysfunction, osteoporosis, and neurodegeneration. Recent literature has proposed post-TBI pituitary dysfunction screening and therapy among the general public, but little for post-deployment special forces or veterans specifically.

Conclusions While we can crudely postulate from comparisons to other groups, there is a distinct lack of recent data on TBI's in naval special forces. But, what is clear is the connection between TBI's and subsequent pituitary dysfunction, in which hypogonadotropic hypogonadism is likely the second most common subtype. Taken together, further retrospective and prospective studies are needed to further investigate hypogonadotropic pituitary dysfunction after TBI's acquired in the line of duty by naval special forces servicemen with the purpose of establishing screening guidelines for these servicemen and ultimately provide appropriate treatment algorithms for the preservation of their quality of life, fertility, and protection against comorbid disease.

\section{\#32 QUANTIFYING BURDEN OF CARE FOR CLEFT LIP AND PALATE PATIENTS FROM THE PATIENT, PROVIDER, AND SYSTEM PERSPECTIVES}

${ }^{1,2} \mathrm{~A}$ Buchel ${ }^{*},{ }^{2,3} \mathrm{E}$ Wells-Durand, ${ }^{2,3} \mathrm{M}$ Bucevska, ${ }^{2,3} \mathrm{R}$ Courtemanche, ${ }^{2,3} \mathrm{~J}$ Staples, ${ }^{2,3} \mathrm{R}$ Thomson, ${ }^{2,3} \mathrm{DJ}$ Courtemanche, ${ }^{2,3} \mathrm{~S}$ Palm, ${ }^{2,4} \mathrm{~T}$ Gibson, ${ }^{2,4} \mathrm{~A}$ Loo, ${ }^{2,3} \mathrm{JS}$ Arneja. ${ }^{1}$ The University of British Columbia Faculty of Arts, Vancouver, BC, Canada; ${ }^{2} B C$ Children's Hospital, Vancouver, BC, Canada; ${ }^{3}$ The University of British Columbia Faculty of Medicine, Vancouver, BC, Canada; ${ }^{4}$ The University of British Columbia Faculty of Dentistry, Vancouver, $B C$, Canada

\subsection{6/jim-2022-WRMC.32}

Purpose of Study Management of cleft lip and palate (CLP) is complex and multidisciplinary, and can last until adulthood. This study aims to describe the healthcare utilization burden of care (BoC) for the management of patients with non-syndromic CLP by identifying a provider burden, characterizing an interaction burden, and calculating an economic burden associated with their health system interactions.

Methods Used This study is designed as a retrospective chart review between January 1, 1999 and April 30, 2021 of patients with non-syndromic CLP cared for at British Columbia (BC) Children's Hospital. Healthcare utilization data for inpatient, outpatient, and emergency encounters were extracted from paper and electronic health records. Community outpatient data were obtained from affiliated specialists. Bottom-up micro-costing was utilized for hospital costing, our tariff guide was utilized for provider reimbursement, and zip code was utilized to calculate patient costs.

Summary of Results Our results indicate that 58 patients identified with cleft lip and palate had a mean of 156.4 healthcare interactions (consults/follow-ups/surgeries) between the ages of 0-18 years. The distribution of healthcare interactions was 92.4\% outpatient, $7.3 \%$ inpatient, and $0.3 \%$ emergency. Patients had a mean of 11.4 surgical procedures, for which the primary services are plastic surgery (5.6), surgical ENT (2.6), oral surgery (1.2) and dentistry (1.2). The remainder consisted of emergency room visits $(0.5)$ and interactions with outpatient speciality services including orthodontics (78.2), plastic surgery (14.7), and ENT (13.5) most frequently. Costing data will ultimately be provided at the physician, patient and system levels.

Conclusions Patients born with non-syndromic CLP have a high frequency of healthcare encounters, suggesting a substantial BoC. These findings will inform parents, and motivate the development of more efficient healthcare systems to maximize patient access to care by right sizing resources (provider, support infrastructure, property/plant/equipment).

\section{\#33 THE EFFECTIVNESS OF A LOW-COST SIMULATION- BASED MODULE FOR TEARCHING ULTRASOUND- GUIDED FINE NEEDLE ASPIRATION OF THYROID NODULES}

${ }^{1} \mathrm{H}$ Huber* ${ }^{*}{ }^{1} \mathrm{~S}$ Thevuthasan, ${ }^{2} \mathrm{~T}$ Robinson, ${ }^{1,3} \mathrm{M}$ Sardesai, ${ }^{1,3} \mathrm{~T}$ Tylee. ${ }^{1}$ University of Washington School of Medicine, Seattle, WA; ${ }^{2}$ University of Wyoming, Laramie, WY; ${ }^{3}$ Harborview Medical Center, Seattle, WA

\subsection{6/jim-2022-WRMC.33}

Purpose of Study High rates of thyroid nodules in the population often require fine needle aspiration (FNA) to rule out 
thyroid cancer. Competency guidelines for residents and fellows to properly perform FNAs are lacking, leading to these essential skills being taught on the job. Simulation based trainings offered by professional societies are effective but often require travel and are expensive. We created a brief hands-on module designed to be 1-2 hours in length to introduce trainees to basic thyroid ultra-sound (US) and US-guided FNA to improve trainees' comfort with the procedures. This study evaluated whether participating in this module improves the comfort of resident and fellows with thyroid US and FNA prior to performing the procedure on patients while being cost- and time-effective.

Methods Used A hands-on training module for US-guided FNAs was developed and offered yearly for 6 years to residents and fellows at Harborview Medical Center. The models used were purchased from Northwestern Medical Center for $\$ 25$ each and one model was used for each session. 40 presurveys and 26 post-surveys were collected directly before and after the module. Participants were primarily otolaryngology residents $(n=15,11)$ and endocrinology fellows $(n=13,8)$ with varying experience. The surveys assessed their comfort level performing US-guided FNAs on a scale of 1 to 5, with 5 being most comfortable and able to perform independent of supervision. The surveys also assessed their comfort with interpreting thyroid US, and long- and short-axis US-guided FNA. Effectiveness was assessed by calculating the change in comfort using population averages, regardless of specialty on the postsurvey compared with the baseline level. Significance was determined using a permutation test.

Summary of Results On average, participants' comfort with US-guided FNA increased by $1.19(\mathrm{p}=0.0006)$ on the $1-5$ scale, comfort level with long- and short-axis US-guided FNA increased by 1.54 and 1.51 , and comfort with interpreting thyroid US improved by 0.97 . For endocrinology fellows and otolatyngology residents specifically, their change in comfort level for performing US-guided FNA increased by 1.02 and 1.17 .

Conclusions Overall, residents and fellows showed an improvement in comfort level after completion of the module. This improvement was not only evident in performing US-guided FNA but also with FNA technique and US interpretation. Although comfort does not equivalate skill, at $\$ 25$ per session our module is a promising alternative to costly and time-consuming simulations courses, which often cost upwards of $\$ 700$ per individual. These training modules can be executed in most residency and fellowship training programs to provide accessible training of these important skills.

\section{\#34 PRE-OPERATIVE ISTOP HUDDLE FOR IMPROVED TRANSITION OF CARE OF PEDIATRIC AND NEONATAL CRITICAL CARE PATIENTS}

\footnotetext{
${ }^{1,2,3} \mathrm{M}$ Nolan ${ }^{*},{ }^{2,3,4} \mathrm{~A}$ Hadley, ${ }^{1} \mathrm{M}$ Baserga, ${ }^{3} \mathrm{C}$ Pries, ${ }^{3} \mathrm{~A}$ Nicholson, ${ }^{3} \mathrm{M}$ Harrison, ${ }^{3,5} \mathrm{~N}$ Dinh, ${ }^{3,4} \mathrm{E}$ Prentice, ${ }^{3} \mathrm{~K}$ Miller, ${ }^{3,4}{ }^{4}$ Huntington, ${ }^{3} \mathrm{~B}$ Huizinga, ${ }^{2,3,6}{ }^{6}$ Durkin. ${ }^{1}$ University of Utah Health, Salt Lake City, UT; ${ }^{2}$ Michigan State University, Grand Rapids, MI; ${ }^{3}$ Helen DeVos Children's Hospital, Grand Rapids, MI; ${ }^{4}$ Spectrum Health Medical Group, Grand Rapids, MI; ${ }^{5}$ Neonatal Associates, PHC, Grand Rapids, Ml; ${ }^{6}$ Pediatric Surgeons of West Michigan, PC, Grand Rapids, MI
}

\subsection{6/jim-2022-WRMC.34}

Purpose of Study Patients admitted to the pediatric or neonatal intensive care units (PICU or NICU) at Helen DeVos Children's Hospital in Grand Rapids, MI, prior to their surgery are taken directly from the PICU or NICU to the operating room (OR). Therefore, these patients do not undergo routine preoperative (pre-op) checklists in the pre-op holding area. A critical care pediatric patient underwent a wrong-sided surgery, highlighting the need for a standardized approach to improve completion of the pre-op checklist and communication between the ICU and surgery teams in the perioperative period.

Methods Used Using quality improvement methodology, the NICU, PICU, pediatric surgery, and pediatric hospital medicine teams completed an A3 form and performed a gap analysis. To address the concerns identified in the root cause analysis, we developed a bedside team huddle composed of ICU, surgery, and anesthesia teams to be performed in the ICU prior to the patient being taken to the OR. We created the acronym iSTOP to outline the components of the pre-op huddle: (i) introductions; (S) surgical procedure to be performed; (T) any tubes, lines, or drains; (O) ongoing plan/intra-operative plan; (P) post-operative care and pain management plan.

Summary of Results Over the course of 90 days, 24 pre-op bedside iSTOP huddles were convened for ICU patients requiring surgery. All team members were present and all key elements of iSTOP were reviewed in over $90 \%$ of instances. Surgical site was appropriately marked $100 \%$ of the time, and pre-op checklist was completed at least $80 \%$ of the time. During this time period, there were zero serious safety events for ICU-to-OR patients.

Conclusions The iSTOP huddle improved completion rate of the pre-op checklist and enhanced care team communication and patient safety surrounding care transitions between ICU and surgical departments. This huddle format can be extended to incorporate other bedside procedures within the ICUs and other areas of the hospital.

\section{\#35 IMPACT OF A PRE-OPERATIVE CHECKLIST ON SURGICAL DELAYS IN COMPLEX SPINE SURGERY}

${ }^{1} \mathrm{LN}$ DePledge ${ }^{*},{ }^{2} \mathrm{CE}$ Drolet, ${ }^{2} \mathrm{~K}$ Nold, ${ }^{2} \mathrm{~S}$ Hermanson, ${ }^{2} \mathrm{PK}$ Louie, ${ }^{1,2} \mathrm{R}$ Sethi. ${ }^{1}$ University of Washington School of Medicine, Seattle, WA; ${ }^{2}$ Virginia Mason Medical Center, Seattle, WA

\subsection{6/jim-2022-WRMC.35}

Purpose of Study Adult spinal deformity surgery is associated with high rates of perioperative adverse events (AE). To minimize the risk of AEs, patients must undergo a multitude of various labs, imaging, procedures, and evaluations before surgery. This process can be complicated for both patients and providers, which can lead to surgical delays. To address this problem, Virginia Mason Neuroscience Institute created a comprehensive preoperative checklist, detailing all necessary aspects of surgical optimization. The goal of this study was to evaluate the impact of a comprehensive preoperative checklist on surgical delays in patients undergoing adult spinal deformity surgery. We hypothesized that checklist-directed optimization would reduce the number of surgical delays and need for postoperative intensive care.

Methods Used Appointed members of the complex spine surgery team were tasked with coordinating surgical optimization using a checklist from $9 / 1 / 20$ to $8 / 1 / 21$ ( $n=51$ ). Complex spine surgeries (to treat adult spinal deformity) between $1 / 1$ / $18-8 / 31 / 20$ ( $\mathrm{n}=142)$ were not medically optimized via checklist and thus served as a historical control. Indications for surgery including infection, tumor, and urgent/emergent 
cases were excluded. Surgeries that were delayed due to COVID, or those that deviated from the established care pathway were also excluded. Impact of the checklist on the frequency of pre-/peri-operative delays and need for postoperative intensive care were investigated. Chi-square analysis was used to interpret these data.

Summary of Results Of 235 patients scheduled for complex spine surgery, 193 met our criteria. Checklist-directed surgical optimization did not significantly reduce surgical delays, with $19.0 \%$ of surgeries experiencing a delay in the historical control group compared to $15.7 \%$ in the study group ( $\mathrm{p}=$ 0.38 ). However, patients in the study group were less likely to require postoperative intensive care $(11.1 \%)$ compared to the control group $(25.3 \%)$ ( $\mathrm{p}=0.031)$.

Conclusions Checklist directed pre-surgical optimization was instituted at a single, high-volume spine surgery center. Although this intervention did not reduce the number of surgical delays, it does have the potential to increase patient safety, as use of the checklist was associated with reduced need for postoperative intensive care. Further research on ways to improve interdisciplinary coordination for preoperative optimization to reduce surgical delays is needed to maximize patient safety and minimize AEs.

\section{Immunology and rheumatology I}

\section{Concurrent session}

\section{2:45 PM}

\section{Thursday, January 20, 2022}

\section{\#36 SARS-COV-2 IMMUNE COMPLEXES ELICIT TISSUE FACTOR EXPRESSION ON HUMAN PERIPHERAL BLOOD MONOCYTES}

1,2J Plagenz* ${ }^{*}{ }^{2} \mathrm{~T}$ Peters, ${ }^{1,2}{ }^{2} \mathrm{~S}$ Pincus, ${ }^{1,2} \mathrm{~N}$ Meissner. ${ }^{1}$ University of Washington School of Medicine, Seattle, WA; ${ }^{2}$ Montana State University Bozeman, Bozeman, MT

\subsection{6/jim-2022-WRMC.36}

Purpose of Study Innate and adaptive immune responses may play a role in severe complications of SARS-CoV-2 infection (COVID-19). The formation of virus-antibody immune complexes may result in aberrant activation of innate immune cells, including circulating monocytes. Thromboembolic complications are a hallmark of severe COVID-19, in which a hypercoagulable state is observed. Currently, the mechanism of this is poorly understood. Tissue factor, also known as Coagulation Factor III, is key to activating the clotting cascade; it is constitutively expressed extra-vascularly but can be upregulated on circulating monocytes during inflammation. Conditions that predispose patients to severe COVID-19, such as metabolic syndrome, are associated with elevated plasma levels of endotoxin. We postulate that aberrant inflammatory activation of monocytes via SARS-CoV-2/antibody immune complexes, in tandem with endotoxin, can upregulate tissue factor and induce hypercoagulability.

Methods Used Immune complexes were formed by mixing inactivated SARS-CoV-2 with Bamlanivimab (Bam), a therapeutic monoclonal antibody specific for the spike receptor-binding domain of SARS-CoV-2. Antibodies to a different domain of the spike were used to capture immune complexes, which were then detected using biotin/avidin. Effects on monocyte cell-surface expression of tissue factor were investigated using flow cytometry. Human peripheral blood mononuclear cells were cultured with SARS-CoV-2, Bam, endotoxin, and combinations of the three. Monocytes were identified by forward/ side scatter and CD14 expression.

Summary of Results SARS-CoV-2 immune complexes were readily detectable by immunoassay. Immune complexes were also stable under different storage conditions. These complexes increased endotoxin-induced tissue factor expression on monocytes to a greater degree than did endotoxin alone. Incubation with neither Bam nor SARS-CoV-2 alone induced tissue factor expression.

Conclusions Antibody-mediated mechanisms are key in clearing SARS-CoV-2 infection. Our results show that the formation of virus-antibody immune complexes may also result in aberrant activation of innate immune cells, including circulating monocytes, leading to tissue factor upregulation. These results may aid in understanding the hypercoagulable state seen in SARSCoV-2 infection. The next step is to evaluate the effect of immune complexes on in-vitro coagulation by using tissue factor-induced Factor Xa activity assays.

\section{\#37 HOW THE TFH RESPONSE IN NEONATES FED BREAST MILK DEVOID OF MATERNAL ANTIBODIES IMPACTS THE COMPOSITION AND FUNCTION OF INTESTINAL MICROBES}

${ }^{1}$ IT Vanteru*, ${ }^{2} \mathrm{~B}$ Wang, ${ }^{2} \mathrm{~S}$ Torres. 'University of Washington School of Medicine, Seattle, WA; ${ }^{2}$ Fred Hutchinson Cancer Research Center, Seattle, WA

\subsection{6/jim-2022-WRMC.37}

Purpose of Study Breast milk is essential to the health and development of children. In addition to nutrients, breastmilk contains immune-modulating factors, including antibodies that protect infants from infections. However, breastfeeding is not available for all women and children. While infant formula is designed to meet growing infants' basic nutrition needs, it does not contain factors like antibodies. We discovered that in the absence of breastmilk antibodies, mice develop increased levels of CD4 $\mathrm{T}$ follicular helper ( $\mathrm{Tfh}$ ) cells and germinal center (GC) B cells in gut draining lymphoid tissues. However, in germ-free mice that were also deficient in maternal antibodies (matAbs), the Tfh and GC B cell levels closely reflected antibody-sufficient neonates' levels. These results suggest the significance of the microbiota in the role of Tfh and GC B cell responses. Tfh cells are essential for maintaining host-microbe homeostasis, and dysregulated increases in Tfh cells can alter the microbiota composition, potentially causing colitis. An indispensable role of the microbiota is to prevent the spread of pathogenic infections through colonization resistance. We hypothesize that the increase in Tfh and GC B cells in pups lacking maternal antibodies will alter the intestinal microbiota and its function.

Methods Used To understand how the Tfh cells can alter the intestinal microbiota, we treated half of the mice that are sufficient and deficient in maternal antibodies with anti-ICOSL, which effectively dampens the Tfh cell expression. We then infected all the mice with Salmonella typhimurium, a bacterial pathogen. Fecal samples were collected daily for 5 days. On day 5 post-infection, fecal samples, cecum, and liver were harvested to determine infection burden. 
Summary of Results Our data indicates no significant difference in colony-forming units (CFU) between the matAb sufficient and deficient groups, signifying that the presence of maternal antibodies does not change the susceptibility to S. typhimurium infection. In addition, there was no difference seen between the groups treated with anti-ICOSL versus control, indicating that early life Tfh cells giving rise to GC B cells producing T-dependent antibodies do not play a role in conferring differential resistance to $S$. typhimurium. This trend was seen when examining both localized and systemic infection across all three organs.

Conclusions Although neonates that do not receive matAbs in breastmilk have increased numbers of Tfh and GC B cells which have the potential to produce antibodies and change the microbiota composition of the gastrointestinal tract, there was no difference in S.typhimurium infectivity between mice transiently devoid of Tfh cells versus control. To continue exploring the role of colonization resistance, further research is needed to determine how Tfh cells influence host-microbe interactions and subsequently its change in infection susceptibility.

\section{\#38 CONSERVATIVE MANAGEMENT OF KNEE OSTEOARTHRITIS UTILIZING BONE MARROW ASPIRATE CONCENTRATE}

${ }^{1}$ B Leiby ${ }^{*},{ }^{2} \mathrm{~GB}$ Foremny, ${ }^{2} \mathrm{~J}$ Hawley, ${ }^{2} \mathrm{~J}$ Galloway, ${ }^{3} \mathrm{~J}$ Willford, ${ }^{2,4} \mathrm{JC}$ McGinley. ${ }^{1}$ University of Washington School of Medicine, Seattle, WA; ${ }^{2}$ The McGinley Clinic, Casper, WY; ${ }^{3}$ WWAMI Medical Education Program, Laramie, WY; ${ }^{4}$ University of Washington School of Medicine, Seattle, WA

\subsection{6/jim-2022-WRMC.38}

Purpose of Study Bone marrow aspirate concentrate (BMAC), along with conservative patient management, offers a minimally invasive option in treating chronic pain from knee osteoarthritis. Knee osteoarthritis affects $35 \%$ of adults aged 65 years and older. BMAC has been shown to decrease inflammation and improve cartilage signal on MRI. We hypothesize BMAC injections, along with conservative care, will provide short- and long-term relief of pain associated with knee osteoarthritis.

Methods Used A retrospective chart review was conducted to identify patients with knee osteoarthritis who received BMAC injections and conservative care in our clinic from November 2013 to November 2019. Under CT and ultrasound guidance, $60 \mathrm{cc}$ of bone marrow was aspirated from the posterior iliac crest. Each $60 \mathrm{cc}$ sample of aspirate was centrifuged, concentrated to $10 \mathrm{cc}$, and injected into the knee joint under sonographic guidance. Patients were non-weight bearing utilizing crutches and a compartment specific off-loading brace for 3 weeks after the procedure and partial weight bearing with just the brace for an additional 3 weeks. All NSAIDs were held 10 days prior and 3 months following the procedure. A $0-10$ patient self-reported pain scale was used as the primary outcome. Secondary outcomes included adverse events and additional treatments. Pain scores were collected on the day of treatment and fixed timepoints up to 3 years post-injection. A 2-tailed Wilcoxon signed rank test with a .05 alpha level was used to determine statistical significance between differences in reported pain level at each follow-up compared to baseline.

Summary of Results Forty-seven patients (71 knees, 26 males, 21 females) with an average age $64 \pm 9$ years, received BMAC injections and conservative management. These patients were followed for 3 years post treatment (mean follow-up 30.1 \pm 11.0 months). Reported pain level was significantly reduced 3 weeks post-injection compared to baseline (47 patients, 71 knees; mean $\Delta-2.0$ points; $\mathrm{p}<.001$; table 1 ). Pain continued to decrease up to 3 years post-injection compared to baseline (37 patients, 54 knees; mean $\Delta-3.9$ points; $\mathrm{p}<.001)$. No adverse events were reported. Thirteen patients (17 knees) subsequently received additional treatments including injections (10 patients, 12 knees). Three patients (5 knees) underwent knee arthroplasty. Zero patients underwent repeat BMAC injections during the 3-year follow-up period.

Conclusions BMAC injections, along with conservative management, represents a safe, effective, and minimally invasive treatment option for treating knee osteoarthritis pain for up to 3 years. Few patients in our study progressed to knee arthroplasty suggesting this approach to be a viable alternative to surgery.

\section{\#39 PREVALENCE OF FRAILTY AND ASSOCIATED FACTORS IN A NATIONAL OBSERVATIONAL COHORT OF RHEUMATIC DISEASES}

${ }^{1} \mathrm{C}$ Chang* ${ }^{*}{ }^{1} \mathrm{~N}$ Singh, ${ }^{1} \mathrm{~J}$ Andrews, ${ }^{2} \mathrm{~K}$ Wipfler, ${ }^{3} \mathrm{~S}$ Lieber, ${ }^{4} \mathrm{SE}$ Sattui, ${ }^{5,6} \mathrm{JF}$ Baker, ${ }^{7} \mathrm{P}$ Katz, ${ }^{2} \mathrm{~K}$ Michaud, ${ }^{1,8} \mathrm{~K}$ Wysham. ${ }^{1} \mathrm{UW}$, Seattle, WA; ${ }^{2} \mathrm{FORWARD}$, Wichita, KS; ${ }^{3} \mathrm{HSS}$, New York, NY; ${ }^{4}$ UPitt, Pittsburgh, PA; ${ }^{5}$ UPenn, Philadelphia, PA; ${ }^{6}$ VHA, Philadelphia, PA; ${ }^{7}$ UCSF, San Francisco, $C A ;{ }^{8} \mathrm{VHA}$, Seattle, WA

\subsection{6/jim-2022-WRMC.39}

Purpose of Study Frailty is associated with disability and early mortality and may be reversible. It is accelerated in patients with certain rheumatic musculoskeletal diseases (RMDs). The prevalence of and disease-specific factors associated with frailty across multiple RMDs is unknown.

Methods Used Data were acquired from FORWARD, The National Databank for Rheumatic Diseases, an observational longitudinal US registry with biannual patient questionnaires. Frailty was measured by self-reported measure: the FRAIL scale, which queries 5 items: 1) fatigue, 2) resistance (climbing stairs), 3) ambulation, 4) illnesses, and 5) loss of weight and categorizes those with $\geq 3$ items as frail. Those with missing RMDs or frailty variables were excluded $(\mathrm{N}=117)$. Prevalence of frailty across RMDs was described. Multivariable logistic regression was performed to identify variables independently

Abstract \#38 Table 1 Mean reported pain scores

\begin{tabular}{|c|c|c|c|c|c|c|c|c|c|}
\hline Time & $\begin{array}{l}\text { Baseline } \\
n=71\end{array}$ & $\begin{array}{l}3 \text { weeks } \\
n=71\end{array}$ & $\begin{array}{l}6 \text { weeks } \\
n=71\end{array}$ & $\begin{array}{l}3 \text { months } \\
\mathrm{n}=71\end{array}$ & $\begin{array}{l}6 \text { months } \\
n=71\end{array}$ & $\begin{array}{l}1 \text { year } \\
n=63\end{array}$ & $\begin{array}{l}1.5 \text { years } \\
\mathrm{n}=58\end{array}$ & $\begin{array}{l}2 \text { years } \\
n=58\end{array}$ & $\begin{array}{l}3 \text { years } \\
n=54\end{array}$ \\
\hline Mean Pain Score & 5.6 & $3.6^{*}$ & $3.2^{*}$ & $3.1^{*}$ & $2.3^{*}$ & $1.9^{*}$ & $1.8^{*}$ & $1.8^{*}$ & $1.7^{*}$ \\
\hline
\end{tabular}


associated with frailty in the entire cohort and stratified by RMDs.

Summary of Results 3,348 individuals were included and 1,084 were frail (32\%). RMDs evaluated were rheumatoid arthritis (71\%), osteoarthritis (OA) (16\%), fibromyalgia (5\%), systemic lupus erythematous (SLE) (4\%), other connective tissue diseases (CTDs) (2\%), spondylarthritis (1\%), and vasculitis (1\%). Frail participants were older $(69.8 \pm 10.6)$ compared to nonfrail $(66.3 \pm 11.7)$ and had a higher prevalence of obesity $(52 \%$ vs. $31 \%)$. The distribution of frailty was equal across RMDs $(\sim 33 \%)$ except vasculitis and CTDs, which had a lower prevalence of frailty $(20 \%$ and $26 \%$, respectively). Ambulation and fatigue were the most common frailty components across RMDs. In the primary multivariable model evaluating the entire cohort, increasing age $(\mathrm{OR}=1.05$ [95\%CI 1.04-1.06], female sex $(\mathrm{OR}=1.74$ [95\%CI 1.57-1.95]), overweight $(\mathrm{OR}=1.49 \quad[95 \% \mathrm{CI} \quad 1.17-1.89])$ and obesity $(\mathrm{OR}=$ $3.04[95 \% \mathrm{Cl} 2.42-3.82])$, prior fracture $(\mathrm{OR}=1.87$ [95\%CI 1.56-2.26]), increased disease activity $(\mathrm{OR}=1.24 \quad$ [95\%CI $1.18-1.30])$, and pain $(\mathrm{OR}=1.11$ [95\%CI 1.07-1.16]) had significant independent associations with frailty (table 1). Biologic use was associated with lower odds of frailty $(\mathrm{OR}=0.78[95 \%$ CI 0.64-0.96]). Among the RMDs, SLE was associated with an increased odds of frailty with $\mathrm{OA}$ as the reference $(\mathrm{OR}=1.70$ [95\%CI 1.02-3.03]). Overall, disease-specific associations were similar to the primary multivariable model with obesity and disease activity maintaining statistical significance in most models.

Abstract \#39 Table 1 Multivariable logistic regression evaluating factors associated with frailty in the entire cohort $(\mathrm{N}=2947)$

\begin{tabular}{|c|c|c|c|}
\hline Variables & OR & $95 \% \mathrm{Cl}$ & $p$-value \\
\hline Age (years) & 1.05 & $1.04-1.06$ & $<0.001$ \\
\hline Male sex & 0.74 & $0.57-0.95$ & 0.017 \\
\hline \multicolumn{4}{|l|}{ BMI: } \\
\hline -Underweight & 1.60 & $0.87-2.93$ & 0.131 \\
\hline -Normal weight & ref & - & - \\
\hline -Overweight & 1.49 & $1.17-1.89$ & 0.001 \\
\hline -Obese & 3.04 & $2.42-3.82$ & $<0.001$ \\
\hline \multicolumn{4}{|l|}{ Primary Rheumatic } \\
\hline \multicolumn{4}{|l|}{ Diagnoses: } \\
\hline -Rheumatoid arthritis & 1.18 & $0.91-1.54$ & 0.217 \\
\hline -Systemic lupus & 1.76 & $1.02-3.03$ & 0.042 \\
\hline \multicolumn{4}{|l|}{ erythematous } \\
\hline -Osteoarthritis & ref & - & - \\
\hline -Fibromyalgia & 0.98 & $0.65-1.48$ & 0.936 \\
\hline -Spondylarthritis & 1.29 & $0.37-4.44$ & 0.687 \\
\hline -Vasculitis & 0.57 & $0.20-1.64$ & 0.296 \\
\hline -Connective Tissue & 1.45 & $0.75-2.77$ & 0.268 \\
\hline \multicolumn{4}{|l|}{ Diseases* } \\
\hline Fracture ever & 1.87 & $1.56-2.26$ & $<0.001$ \\
\hline Disease duration (years) & 1.01 & $1.00-1.01$ & 0.055 \\
\hline Disease severity & 1.24 & $1.18-1.30$ & $<0.001$ \\
\hline Pain Scale & 1.11 & $1.07-1.16$ & $<0.001$ \\
\hline \multicolumn{4}{|l|}{ Medication use: } \\
\hline -DMARD use & 0.93 & $0.77-1.13$ & 0.452 \\
\hline -Biologic Use & 0.78 & $0.64-0.96$ & 0.019 \\
\hline -Prednisone dose & 1.01 & $1.00-1.03$ & 0.142 \\
\hline
\end{tabular}

-DMARD: disease modifying antirheumatic drug. *Connective tissue diseases: Sjogrens, scleroderma, mixed connective tissue disease and myositis
Conclusions Frailty is common among RMDs affecting nearly 1 in 3 participants. Obesity, prior fracture and a diagnosis of SLE had the highest associations with frailty. Future work is needed to identify factors that predict frailty onset and potential interventions to treat frailty within RMDs.

\section{\#40 ADJUNCT THERAPIES FOR PEMPHIGUS DISEASE: A SYSTEMATIC REVIEW}

${ }^{1} \mathrm{~K}$ Nguyen*, ${ }^{2} \mathrm{~S}$ Worswick. 'Western University of Health Sciences, Pomona, CA; ${ }^{1}$ University of Southern California, Los Angeles, CA

\subsection{6/jim-2022-WRMC.40}

Purpose of Study Pemphigus vulgaris (PV) and IgA Pemphigus are mucocutaneous autoimmune diseases that commonly present as painful blisters eroding the skin of the face, trunk, scalp, groin, and axillae in affected patients. The pathogenesis of pemphigus disease stems from autoantibodies against desmosomal proteins essential to maintaining keratinocyte adhesion. A histopathologic exam may reveal a reduction in desmosomal cadherin proteins and epidermal acantholysis. Currently, there is no cure for pemphigus, though corticosteroids and steroid-sparing agents are commonly used to control the proliferation of lesions and prevent disease progression. Frequently used non-steroidal agents include mycophenolate mofetil, azathioprine, IVIG and rituximab. Despite these treatment options, patients often succumb to long-term corticosteroid complications. Less oft-used therapies include dapsone and sulfasalazine for $\mathrm{PV}$, and colchicine for IgA pemphigus which offer potential steroid-sparing alternatives with fewer adverse effects, however, their efficacies has not been clearly established. The objective of our systematic review is to investigate the use of dapsone, sulfasalazine, and colchicine in the treatment of PV and IgA pemphigus.

Methods Used We searched the PubMed database using the search terms: 'dapsone' 'sulfasalazine' 'pemphigus vulgaris' 'colchicine' 'IgA pemphigus disease.' Our inclusion criteria included published articles written in English between 19702021 exploring the use of dapsone, colchicine or sulfasalazine for pemphigus, and included case series, retrospective studies, and randomized control trials. Our exclusion criteria eliminated reports with fewer than three patients, and review articles. 275 articles were identified, of which 27 relevant studies were eligible. 15 studies were excluded after screening, resulting in 12 remaining studies.

Summary of Results 46 (63\%) out of 73 patients responded to dapsone, suggestive of its efficacy as either a monotherapy for or as a part of combination therapy for PV. In 65 patients receiving sulfasalazine adjunct therapy, 61 (94\%) achieved clinical remission. Adequate data is lacking regarding colchicine therapy for pemphigus as the current literature only reports four IgA pemphigus patients treated with this agent.

Conclusions More research is required to elucidate an effective and safe therapy for individuals burdened with pemphigus disease. Certainly, the rarity of this condition and the difficulty in finding adequate control groups present a major barrier for holding clinical trials on alternative therapies. Going forward, dermatologists may consider the use of dapsone or sulfasalazine adjuvant therapy in PV patients to slowly lower corticosteroid use as lesions begin to diminish and to prevent relapse of cutaneous flare ups for patients in remission. 
\#41 THE ADJUNCTIVE THERAPY FOR MYCOBACTERIUM TUBERCULOSIS INFECTION IN TYPE 2 DIABETES MELLITUS

${ }^{1} \mathrm{C}$ Sisliyan*, ${ }^{2} \mathrm{~A}$ Beever, ${ }^{2} \mathrm{~N}$ Kachour, ${ }^{1} \mathrm{~J}$ Owens, ${ }^{2} \mathrm{~K}$ Sasaninia, ${ }^{3} \mathrm{~A}$ Kalloli, ${ }^{4} \mathrm{~W}$ Khamas, ${ }^{3} \mathrm{~S}$ Subbian, ${ }^{1,2} \mathrm{~V}$ Venketaraman. ${ }^{1}$ Western University of Health Sciences College of Osteopathic Medicine of the Pacific, Pomona, CA; ${ }^{2}$ Western University of Health Sciences, Pomona, CA; ${ }^{3}$ Rutgers New Jersey Medical School, Newark, NJ; ${ }^{4}$ Western University of Health Sciences, Pomona, CA

\subsection{6/jim-2022-WRMC.41}

Purpose of Study Type 2 Diabetes Mellitus (T2DM) is an inflammatory disease that can alter the immune response resulting in several physiological manifestations. Glutathione (GSH), a thiol required to maintain intracellular redox state homeostasis, is classically deficient among individuals with T2DM. Glutathione also appears to be pertinent in the immune response against Mycobacterium tuberculosis (Mtb) infection. In our previous studies, we have identified L-GSH's direct opposing effects against oxidative damage as well as its immune enhancing effects in HIV+ patients. We explored for similar effects in T2DM which also involves inflammatory and infectious states that could potentiate the replication of $M t b$ and further diminish the immune response. Specifically, our study aims to further elucidate GSH's role in the granulomatous effector response. In this study, we attempted to uncover whether GSH deficiency in diabetic mice $(\mathrm{db} / \mathrm{db})$ impairs the formation of granulomas and the granulomatous effector response to further the understanding of the detailed mechanism of $M t b$ pathogenesis and the potential for novel therapies against the disease brought on by the infection.

Methods Used $\mathrm{Db} / \mathrm{db}$ mice were infected with $M t b$ and treated with one of 3 regimens, either: 1) an optimal dose of rifampicin (RIF), 2) a suboptimal dose of RIF, or 3) a suboptimal dose of RIF in addition to reduced form of GSH encapsulated in liposomes (L-GSH). 3 male and 3 female mice were sacrificed for each group over the span of $3 \mathrm{~h}, 2,4$ wks, 6 wks, and 8 wks post-treatment to study the collective effects of LGSH and RIF in Mtb infection. Granuloma samples from each group were formalin-fixed and analyzed accordingly. We are currently measuring the survival of $M t b$ along with the levels of cytokines, free radicals and GSH in untreated, RIF-treated and RIF+GSH-treated $\mathrm{db} / \mathrm{db}$ mice.

Summary of Results We expect to obtain the data from the aforementioned assays shortly.

Conclusions If our data shows a marked elevation of immune defensive cytokines and granuloma formation with concurrent reduction in $M t b$ survival and free radical production in LGSH treated mice, then we can support our hypothesis that GSH enhances the granulomatous effector response against Mtb infection in T2DM. In addition, if we observe greater immune responses in $\mathrm{RIF}+\mathrm{GSH}$ treated $\mathrm{db} / \mathrm{db}$ mice, we may further explore the use of GSH as an adjunct therapy in $M t b$ infection in T2DM.

\section{\#42 ACTINIC GRANULOMA: A RARE CASE OF SUN DAMAGE}

${ }^{1} \mathrm{~K}$ Nguyen*, ${ }^{2} \mathrm{C}$ Wong, ${ }^{2} \mathrm{E}$ Nguyen. ${ }^{1}$ Western University of Health Sciences, Pomona, $C A$; ${ }^{2}$ Riverside Community Hospital, Riverside, CA

10.1136/jim-2022-WRMC.42

Case Report A 53-year-old female with a past medical history of anxiety presented to the dermatology clinic with a pruritic eruption for six weeks. She had been gardening without gloves the day prior to the onset of the eruption. A review of systems was inconclusive. Her examination revealed diffuse red papules coalescing into plaques with mild scales involving the scalp, face, neck, torso, and upper and lower extremities including palms and soles, and sparing the ears, bilateral axillae, elbows, and knees. Her biopsy revealed solar elastosis and abundant multinucleated foreign body giant cells with ingested elastic fibers. The patient's clinical presentation and histopathology was consistent with a diagnosis of actinic granuloma (AG). Her treatment included $20 \mathrm{mg}$ of prednisone PO QAM for one month along with fluticasone $0.05 \%$ face cream BID and triamcinolone $0.1 \%$ cream BID applied to the affected skin on the body. After one month, all lesions flattened except for post-inflammatory erythema macules. Sun avoidance and daily sunscreen use was also recommended. At the most recent follow up, her lesions resolved demonstrating the efficacy of corticosteroid treatment.

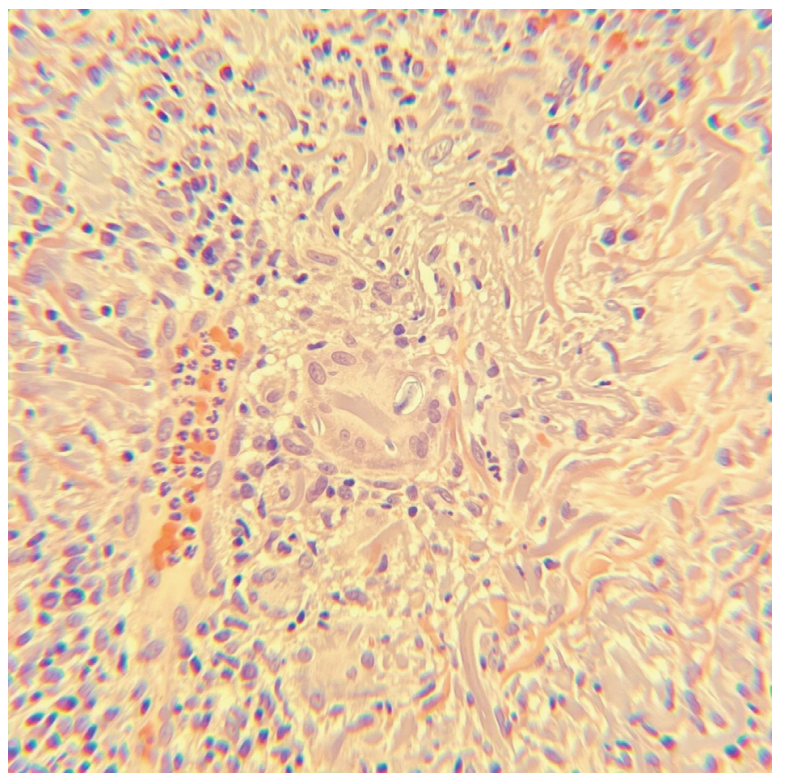

Abstract \#42 Figure 1 Solar elastosis and abundant multinucleated foreign body giant cells with ingested elastic fibers

AG is a rare skin eruption with an unknown pathogenesis, however, it is proposed that a sun-induced inflammatory response attracts giant cells to form granulomas and degrade elastic material. Lesions begin as multiple small pink papules and nodules that coalesce into demarcated, annular plaques and a hypopigmented center to form the classic ring shape. Actinic elastosis surrounds the outer annulus ring, with histiocytes and giant cells within the raised border, and the innermost central zone filled with minimal to absent elastic fibers. Lesions are commonly found on the forehead, neck, extremities, and hands. Our patient differed from the typical presentation in that she described intense pruritus associated with her eruption.

\section{\#43 COMPLETE CONGENITAL HEART BLOCK IN A NEONATE}

S Benke*, S Sukumaran. Valley Children's Hospital, Madera, CA

10.1136/jim-2022-WRMC.43 
Case Report Congenital heart block (CHB) in neonates is associated with high morbidity and mortality. $\mathrm{CHB}$ generally occurs due to the presence of maternal autoantibodies of the $\mathrm{Ro} / \mathrm{La}$ family or cardiac defects.

We describe a neonate born with $\mathrm{CHB}$ who was found to have neonatal lupus erythematosus (NLE).

Methods Used Case Report

Summary of Results A term female infant was born by cesarean delivery at 37 weeks to a 24-year-old healthy primigravida. At delivery, the baby's heart rate was 55 beats per minute. The patient was admitted to the neonatal intensive care unit for further evaluation and management of fetal bradycardia.

Electrocardiogram demonstrated third-degree atrioventricular (AV) block and fetal echocardiogram showed a ventricular rate of 60-65 beats per minute and an atrial rate of 116-128 beats per minute. There was good ventricular function without evidence of hydrops.

Physical exam revealed a term, well-appearing infant female with bradycardia but normal S1 and S2 without murmurs. The remainder of her examination was within normal limits. Laboratory evaluation of the infant and the mother showed positive anti-SSA/Ro and anti-SSB/La antibodies.

The baby was diagnosed with NLE and CHB. The infant's heart rate was monitored closely but she maintained a heart rate greater than 60 beats per minute and hence was discharged home. A pacemaker was scheduled for placement as an outpatient.

Conclusions NLE is a rare acquired autoimmune disorder that occurs due to passive placental transfer of maternal autoantibodies to SSA/Ro and/or SSB/La. Anti-SSA/Ro autoantibodies are found in about $85-90 \%$ of mothers of neonates with $\mathrm{CHB}$, and studies of pregnancies in anti-SSA/Ro positive mothers estimated the risk of $\mathrm{CHB}$ to be $1-5 \%$.

Cardiac involvement in NLE is usually irreversible and characterized by second- or third-degree CHB. A ventricular rate of less than 55 beats per minute, hydrops fetalis, or atrioventricular valve regurgitation indicate poor fetal prognosis.

This case emphasizes the importance of considering NLE in infants with fetal bradycardia, congenital AV block or arrhythmias and evaluating the mother and infant for autoantibodies to SSA/Ro and/or SSB/La. We also highlight the need for early referral to cardiology and possible pacemaker implantation in infants who do not respond to medical therapies alone.

\section{\#44 OBJECTIVE CHARACTERIZATION OF OSTEOPATHIC LYMPHATIC PUMP TECHNIQUE EFFICACY USING SERUM COVID-19 ANTIBODY LEVELS FOLLOWING VACCINATION}

A Comer ${ }^{*}$, E Lee, E Martinez, B Loveless, P Crone, S Fuchs, H Szurmant, J Sanchez. Western University of Health Sciences, Pomona, CA

\subsection{6/jim-2022-WRMC.44}

Purpose of Study Lymphatic Pump Technique (LPT) is an Osteopathic Manual Medicine technique involving external pressure to various lymphatic structures with the goal of improving lymph drainage. Because of the leukocyte content of lymph, LPT is often indicated for use as an adjuvant therapy in patients with acute or chronic infections. Previous studies have primarily characterized LPT efficacy in terms of clinical or symptomatic outcomes, typically with rather small cohorts of subjects, which has been a criticism of the technique. To address the limitations of past studies, we present a study in which the serum concentration of anti-spike protein COVID-19 antibodies are measured in 100 subjects following treatment with LPT (experimental group) and 100 subjects without LPT treatment (control group) in conjunction with COVID-19 vaccination. The ongoing study is designed to follow the subjects for one year after the first COVID-19 vaccine.

Methods Used Subjects were split into treatment or control groups in a double-blinded randomized process. Participants returned for blood draws with the following schedule based on the day of their first vaccination: day 0 (1rst vaccine), day 7, day 21 (2nd vaccine), days 28, 35, 90, 182 and, 365. In the treatment branch, LPT was performed on the day of each vaccination and the following day for a total of 4 treatments. Blood draws were performed immediately prior to both vaccine administration and treatment with LPT. Blood samples were processed, and serum biobank is created. Serum antispike antibody levels are to be determined using quantitative ELISA. All recruited participants were over the age of 18 and were not vaccinated. The study was approved by the WesternU Institutional Review Board.

Summary of Results Recruitment has been successful and is ongoing. Currently, 96 participants have been recruited. 12 participants have dropped out for various reasons, leaving 84 continuing participants. To address attrition, compensation has been changed from a total of $\$ 100$ to $\$ 200$, dispersed as $\$ 25$ per blood draw. Attrition rate before the compensation change was $30 \%(6 / 20)$ but reduced to $7.9 \%(6 / 76)$ after the change. Total retention rate is $87.5 \%$ with $53.6 \%(45 / 84)$ of participants having completed the 5 th blood draw. Additionally, the diversity of the participant population is promising with significant representation of LatinX individuals (55.9\%) and females (58.3\%).

Conclusions The ongoing study is still recruiting participants, however significant progress has been made, with 84 participants currently on board. Additionally, retention and the diversity of the participant population are both promising. The first set of serum samples is soon to be subjected to ELISA antibody testing and results are forthcoming.

\section{Infectious diseases I}

\section{Concurrent session}

\section{2:45 PM \\ Thursday, January 20, 2022}

\section{\#45 ANTI-DEPRESSANTS AND COVID-19 SEVERITY: A
RETROSPECTIVE STUDY OF HOSPITALIZED ADULT
PATIENTS}

${ }^{1} \mathrm{SH}$ Rauchman*, ${ }^{2} \mathrm{~S}$ Mendelson, ${ }^{1} \mathrm{C}$ Rauchman, ${ }^{3} \mathrm{~A}$ Pinkhasov, ${ }^{3} \mathrm{U}$ Kasselman, ${ }^{3} \mathrm{AB}$ Reiss, ${ }^{1}$ Fresno Institute of Neuroscience, Fresno, CA; ${ }^{2}$ Providence Holy Cross Medical Center, Mission Hills, CA; ${ }^{3}$ NYU Long Island School of Medicine, Mineola, NY

\subsection{6/jim-2022-WRMC.45}

Purpose of Study The SARS-CoV2 virus continues to have devastating consequences worldwide. Though vaccinations have 
helped to reduce the impact of the virus, new strains still pose a threat to the unvaccinated, and to a lesser extent vaccinated, individuals. Therefore, it is imperative to identify treatments to reduce the severity of Covid-19. Recently, acute use of selective serotonin reuptake inhibitor (SSRI) antidepressants in COVID+ patients has been shown to reduce the severity of symptoms compared to placebo. Since SSRIs are a widely used anti-depressant, the aim of this study was to determine whether COVID+ patients already on SSRI treatment upon admission to the hospital had reduced mortality compared to COVID+ patients not on chronic SSRI treatment.

Methods Used A retrospective observational study design was used. Electronic medical records of 9,044 patients with a laboratory-confirmed diagnosis of Covid-19 from 03/2020 to 03/ 2021 from six hospitals were queried for demographic information, admission date; discharge date and disposition; length of stay; admission diagnoses; medications on admission; comorbidities; age; gender; ethnicity; admission to ICU; ventilator use; supplemental oxygen; oxygen saturation; discontinuation of antidepressant medications upon ICU admission.

Using $\mathrm{R}$, a logistic regression model was run with mortality as the outcome and SSRI status as the exposure. An adjusted logistic regression model was run to account for $\mathrm{R}$ age category, gender, and race. All tests were considered significant at $\mathrm{p}$ of 0.05 or less.

Summary of Results In this sample, no patients admitted on SSRIs had them discontinued. This is consistent with current recommendations. There was no significant difference in the odds of dying between COVID+ patients on chronic SSRIs vs COVID + patients not taking SSRIs, after controlling for age category, gender, and race. The odds of COVID+ patients on chronic SSRIs dying was 0.90 (95\%CI: 0.74, 1.09; $\mathrm{n}=832$ ) compared to COVID+ patients not on SSRIs $(p=0.29$; $\mathrm{n}=8211$ ).

Conclusions In times of pandemics due to novel infectious agents it is difficult, but critical to evaluate safety and efficacy of drugs that might be repurposed for treatment. This large sample size of 9,044 patients suggests that there will be no significant benefit to use of SSRIs to decrease mortality rates for hospitalized patients with Covid-19 who are not currently on SSRI medications. This study shows the utility of large clinical databases in addressing the urgent issue of determining what commonly prescribed drugs might be useful in treating COVID-19.

\section{\#46 T-CELL AND ANTIBODY RESPONSES TO MRNA VACCINATION IN SARS-COV-2-CONVALESCENT SUBJECTS}

${ }^{1} \mathrm{~S}$ Wanner*, 'VL Campbell, 'S Selke, 1,2DM Koelle. 'University of Washington School of Medicine, Seattle, WA; ${ }^{2}$ Fred Hutchinson Cancer Research Center, Seattle, WA

\subsection{6/jim-2022-WRMC.46}

Purpose of Study Natural and vaccine-induced immunity are important for SARS-CoV-2 control. We evaluated SARS-CoV2-specific $\mathrm{T}$ cell-mediated immune responses in COVID-19 survivors followed through vaccination. We compared $\mathrm{T}$ cell tests from Oxford Immunotec (OI) with an in-house laboratorydeveloped test (LDT). Each used peptides covering Spike (S) and non-vaccine proteins within SARS-CoV-2. We hypothesized that $\mathrm{T}$ cell responses to $\mathrm{S}$ will increase after mRNA vaccination. We compared vaccine immune boost in persons previously hospitalized vs. non-hospitalized for COVID-19, and the relationship between $\mathrm{T}$ cell and neutralizing antibody $(\mathrm{nAb})$ responses.

Methods Used 20 subjects (median age 62.7, 50\% female, most White) with PCR-confirmed SARS-CoV-2 infection donated plasma and peripheral blood mononuclear cells (PBMCs). Samples were from a median of 49 days after recovery from COVID-19 (V0), just prior to the 1st vaccination (E01), and 2-4 weeks after each mRNA dose (E02 and E03). $\mathrm{T}$ cell responses were measured by interferon-gamma enzyme-linked immunospot assays (ELISPOT). We compared our LDT assay using the $\mathrm{S}$ and nucleocapsid $(\mathrm{N})$ proteins with the OI assay including the $\mathrm{S}, \mathrm{N}$, and matrix (M) proteins. $\mathrm{nAb}$ levels were measured by fluorescence inhibition. Linear regression was used to assess correlation between tests. Wilcoxon matched-pairs signed rank tests were used to evaluate differences in immune responses over time. Mann-Whitney tests were used to examine differences between hospitalized and non-hospitalized groups.

Summary of Results Strong correlation was noted between LDT and OI results for $S$ protein at each time point $(\mathrm{rho}=$ $0.88,0.85,0.60$, and 0.77 , respectively). Between V0 and E02 (median of 327 days) and V0 and E03 (median of 345.5 days), there were significant increases in S-specific $\mathrm{T}$ cell responses $(p=0.0005$ and 0.0006 , respectively). No additional boost between E02 and E03 $(p=0.54)$ was observed. Low level (V0) responses to $\mathrm{N}$ and $\mathrm{M}$ were not boosted with vaccination. No significant difference in S-specific $\mathrm{T}$ cell responses between hospitalized and non-hospitalized groups were noted. For both hospitalized and non-hospitalized persons, nAb levels increased significantly after 1 st dose of vaccine $(p=<0.0001)$, with no additional nAb increase after the 2nd dose. No correlation between $\mathrm{nAb}$ and $\mathrm{S}$-specific $\mathrm{T}$ cell responses at either V0 or E03 was noted.

Conclusions The OI assay is suitable for assessing $\mathrm{T}$ cell responses to SARS-CoV-2 mRNA vaccines. T cell responses to $\mathrm{N}$ and $\mathrm{M}$ did not boost, as expected. In this cohort, primary infection severity did not impact vaccine responses 9 months later. $\mathrm{nAb}$ and $\mathrm{T}$ cell response increases were complete after one dose. This indicates that a second dose may not be needed, at least if given 3-4 weeks after the first in persons recovering from COVID-19 in the prior 9 months.

\section{\#47 ASSESSMENT OF MATERNAL AND INFANT ANTIBODY RESPONSE TO COVID-19 AND TRANSPLACENTAL TRANSFER RATIOS AT LABOR \& DELIVERY}

${ }^{1} \mathrm{C}$ Liu*, ${ }^{1} \mathrm{M}$ Cambou, ${ }^{1} \mathrm{~T}$ Mok, ${ }^{1} \mathrm{VM}$ Fajardo, ${ }^{1} \mathrm{D}$ Battacharya, ${ }^{1} \mathrm{~T}$ Kerin, ${ }^{1} \mathrm{FJ}$ Ibarronda, ${ }^{2} \mathrm{~T}$ Fuller, 'S Paiola, ${ }^{1} \mathrm{G}$ Aldrovandi, ${ }^{1} \mathrm{R}$ Rao, ${ }^{1} \mathrm{O}$ Yang, ${ }^{1} \mathrm{~K}$ Nielsen-Saines. ${ }^{1}$ University of California Los Angeles David Geffen School of Medicine, Los Angeles, CA; ${ }^{2}$ University of California Los Angeles, Los Angeles, CA

\subsection{6/jim-2022-WRMC.47}

Purpose of Study Newborn protection from infection is dependent on both neonatal innate immune responses \& transplacental transfer of maternal antibodies (abs). Better understanding of maternal SARS-CoV-2 ab responses during labor \& delivery (L\&D) can help evaluate maternal risks for infection, dynamics of placental transfer, and neonatal 
vulnerability. Limited data describes how COVID-19 severity shapes maternal \& infant ab responses. Stratification by severity can help characterize the protection to the newborn. The purpose of this study is to investigate maternal SARSCoV-2 ab concentrations during L\&D by disease severity, and compare infant $\mathrm{ab}$ responses at birth when exposed to varying severity in utero.

Methods Used This project is part of the prospective observational cohort study COVID19 Outcomes in Mother-Infant Pairs, analyzing mother-infant dyads in the US \& Brazil. Serology of 101 pregnant women in Los Angeles (delivery: April 15, 2020-May 28, 2021) were analyzed and confirmed SARS CoV-2 PCR+ during pregnancy. Maternal blood at L\&D, cord blood, and infant blood at birth were analyzed by ELISA for IgA, IgG \& IgM (anti-spike receptor binding domain).

Summary of Results For 101 women, 72 had matched cord blood \& 86 infant specimens. $76 \%$ of women produced all 3 anti-SARS-CoV-2 IgG, IgM, and IgA; 93\% had at least one positive ab class; $5 \%$ had no detectable abs. Infant serum at birth contained only IgG and no IgM or IgA. With increased duration between onset of infection \& delivery, maternal IgG levels waned, and conversely, transplacental transfer ratios increased $\left(\mathrm{R}^{2}=0.27\right)$. Maternal IgG levels increased with disease severity. A significant increase in infant IgG levels was observed in children born to symptomatic mothers vs asymptomatic mothers $(\mathrm{p}<0.0001)$. A trend towards more robust $a b$ responses was observed in infants with severe/critical COVID19 exposure in utero $(\mathrm{p}=0.07)$.

Abstract \#47 Table 1 Demographics and clinical characteristics of mother-infant dyads infected with SARS-CoV-2 during pregnancy

\begin{tabular}{ll}
\hline Maternal Demographics and Medical History & All Women (N = 101) \\
\hline Age, Median (Range) & $33(16-42)$ \\
Race/Ethnicity & No. (\%) \\
Latina & $46(45.5)$ \\
White & $27(26.7)$ \\
Black/African American & $8(7.9)$ \\
Asian/Other & $20(19.8)$ \\
Insurance & No. $(\%)$ \\
Public & $36(35.6)$ \\
Private & $65(64.4)$ \\
Gravidity, Median (Range) & $2(1-10)$ \\
CoVID-19 Severity & No. $(\%)$ \\
Asymptomatic & $14(13.9)$ \\
Mild/Moderate & $76(75.2)$ \\
Severe/Critical & $11(10.9)$ \\
Gestational Age at Diagnosis & No. (\%) \\
First Trimester & $10(9.9)$ \\
Second Trimester & $39(38.6)$ \\
Third Trimester & $52(51.5)$ \\
Diagnosis Date-to-Delivery Interval, Median (IQR), Days & $62(32-120)$ \\
Medical History Prior to Pregnancy & No. (\%) \\
Any Comorbidities & $52(51.5)$ \\
Obesity (Pre-Pregnancy BMI $>30)$ & $32(31.7)$ \\
Diabetes Mellitus (Not Gestational) & $3(3.0)$ \\
Congenital Heart Disease & $5(5.0)$ \\
Asthma & $12(11.9)$ \\
\hline & \\
\hline
\end{tabular}

Conclusions Our findings demonstrate how altered maternal responses across distinct COVID-19 disease severity categories influence neonatal protection against SARS CoV-2.

\section{\#48 BIOMARKERS PREDICTIVE OF MORTALITY IN COVID-19 PATIENTS WITH DIAGNOSED HEART FAILURE}

${ }^{1} \mathrm{~T}$ Nguyen*, ${ }^{1} \mathrm{JY}$ Hwang, ${ }^{1} \mathrm{C}$ Lee, ${ }^{2} \mathrm{~T}$ Buck, ${ }^{2} \mathrm{~K}$ Mun, ${ }^{2} \mathrm{TT}$ Vanteru, ${ }^{2} \mathrm{~A}$ Lu, ${ }^{2} \mathrm{D}$ Tirschwell, ${ }^{1}$ A Kim. 'Washington State University Elson S Floyd College of Medicine, Spokane, WA; ${ }^{2}$ University of Washington School of Medicine, Seattle, WA

\subsection{6/jim-2022-WRMC.48}

Purpose of Study The purpose of this study is to identify common biomarkers and biosignals in COVID-19 patients with heart failure that are associated with increased risk of in-hospital mortality. COVID-19 is associated with worse outcomes in patients with pre-existing comorbidities, such as heart failure $(\mathrm{HF})$. Biomarkers such as B-type natriuretic peptide, troponin, and interleukin- 6 have been elevated in patients with HF and COVID-19 and may provide insight on the severity of disease but may not be collected in all patients. The exact association between patients with prior HF and the biomarkers commonly utilized is limited and should be evaluated further. Our study evaluates biosignals and biomarkers that may be predictive of mortality in COVID-19 patients with history of HF.

Methods Used All patients included were 18 years of age or older, diagnosis of COVID-19 was confirmed by PCR test or hospital clinical criteria and were hospitalized in the University of Washington (UW) Medicine hospital systems between February 2020 to December 2020. The data was collected as part of a national effort for the American Heart Association COVID-19 CVD Registry. The biosignals that were analyzed include temperature, heart rate, respiratory rate, diastolic blood pressure, and systolic blood pressure. The biomarkers include admission white blood cell (WBC) count, platelets, serum creatinine, AST $(\mathrm{u} / \mathrm{L})$, ALT $(\mathrm{u} / \mathrm{L})$, and lymphocyte count. Patient data also tracked previous medical history and disposition at discharge. A LASSO multivariate regression model was used to identify the variables most predictive of mortality among patients with heart failure.

Summary of Results The study included 54 of the 393 COVID-19 patients (13.7\%) with previous diagnosis of heart failure (46\% male, mean age 77). Our model estimates that for each standard deviation unit above average (z-score), patients with previously diagnosed heart failure were $13 \%$ more likely to die due to COVID-19 ( $p=0.021)$. Among patients with prior heart failure, each z-score increase for WBC count and serum creatinine increased risk of mortality by $3.5 \%(p=.043)$ and $5.7 \%(p=.046)$, respectively.

Conclusions Our data suggests that there may be significance in monitoring WBC count and serum creatinine levels among COVID-19 patients with prior heart failure. The WBC count and serum creatinine have a stronger relationship to mortality in patients with prior heart failure compared to those without heart failure. Immune response may be reduced in heart failure patients which can account for the decreased WBC count, but further studies are needed to elucidate the exact mechanism and relationship. The results of this study may provide a roadmap to triage heart failure patients based on admission lab values in the COVID-19 environment. 
\#49 ABSTRACT WITHDRAWN

\section{\#50 ASSOCIATIONS OF CONGENITAL SYPHILIS INCIDENCE AND THE FEMALE AGRICULTURAL WORKER POPULATION IN CALIFORNIA}

${ }^{1} \mathrm{~J}$ Fang ${ }^{*},{ }^{1} \mathrm{R}$ Silva, ${ }^{1} \mathrm{~K}$ Pinkerton, ${ }^{1,2} \mathrm{D}$ Sankaran. 'University of California Davis, Davis, $C A$; ${ }^{2}$ Adventist Health and Rideout, Marysville, CA

\subsection{6/jim-2022-WRMC.49}

Purpose of Study The national rate of congenital syphilis (CS) has dramatically increased recently. It remains unknown if the children of the agricultural worker population (AWP) are more susceptible to CS in California. Identifying subpopulations vulnerable to transmitting CS may inform the design of intervention efforts. Thus, this study set out to determine whether CS incidence rates are associated with the female AWP in California.

Methods Used Data from all 58 California counties were retrospectively obtained from the California Department of Public Health and United States Department of Agriculture regarding CS incidence per 100,000 live births and female AWP from December 2014 and December 2018. Female AWP per county was estimated according to the national proportion of female to male agricultural workers provided by the Department of Agriculture. Data was analyzed using geographical information systems mapping and Pearson's correlation coefficient $(r)$ tests.

Summary of Results The average statewide CS incidence was 68.2 cases per 100,000 live births in 2018. CS incidence and female AWP were concentrated heavily in California's agricultural Central Valley, with a few coastal exceptions (figure 1 AC). CS incidence and female AWP were moderately but significantly correlated $(r=0.343 ; 95 \%$ confidence interval = $0.093-0.552 ; p<0.001$ ) (figure 1D).

Conclusions Our findings provide evidence that California counties with a higher incidence of CS tend to be home to a greater number of female agricultural workers than counties with low incidence of CS. Given these findings, this study suggests the urgent need to implement culturally appropriate and enduring prenatal healthcare interventions that prioritize treatment of maternal syphilis and prevention of CS in female AWP.

\section{\#51 CHARACTERIZATION OF THE HIGHLY DIVERSE GENOMIC REGIONS OF AN IMPORTANT AFRICAN VECTOR OF SCHISTOSOMIASIS}

${ }^{1} \mathrm{R}$ Burd ${ }^{*},{ }^{1} \mathrm{~J}$ Cayton, ${ }^{2} \mathrm{~T}$ Pennance, ${ }^{3} \mathrm{JA}$ Tennessen, ${ }^{2} \mathrm{M}$ Steinauer, ${ }^{4} \mathrm{~F}$ Rawago, ${ }^{4} \mathrm{M}$ Odiere, ${ }^{4} \mathrm{G}$ Owino. ${ }^{1}$ Western University of Health Sciences College of Osteopathic Medicine of the Pacific, Pomona, CA; ${ }^{2}$ Western University of Health Sciences College of Osteopathic Medicine of the Pacific-Northwest, Lebanon, OR; ${ }^{3}$ Harvard University $T H$ Chan School of Public Health, Boston, MA; ${ }^{4}$ Kenya Medical Research Institute, Kisumu, Kenya

\subsection{6/jim-2022-WRMC.50}

Purpose of Study Schistosomiasis is a neglected tropical disease impacting the health of millions of humans primarily in regions of poverty. Freshwater snails are obligate vector hosts of the flatworm parasites (schistosomes) that cause this disease. Our research goal is to uncover the genetics underlying immunity of snails to schistosomes so that novel control
A

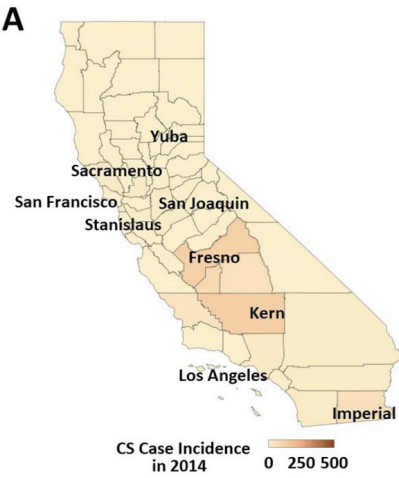

C

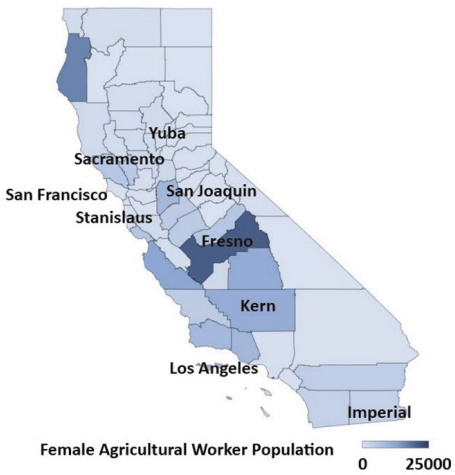

B

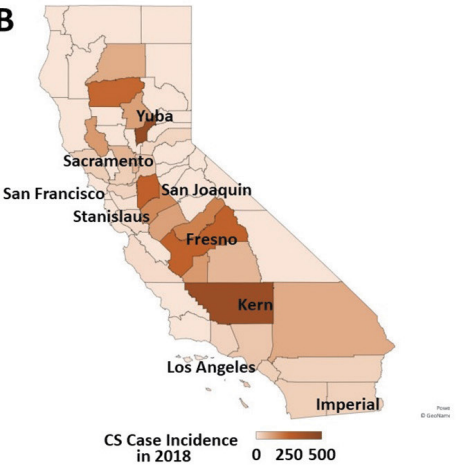

D

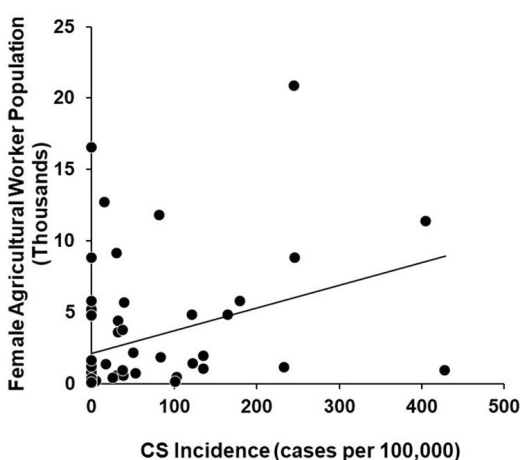

Abstract \#50 Figure 1 Geographical distributions of CS incidence rates (A: in 2014, B: in 2018) per 100,000 live births, and agricultural worker populations (C: in 2017). Correlation between CS Incidence (2018) and female AWP (2017) $(r=0.343 ; 95 \% \mathrm{Cl})=0.093-0.552 ; p<0.001)(\mathrm{D})$. CS congenital syphilis 
strategies may be developed to prevent human infection. Pathogen recognition receptors (PRRs) are part of the first line of defense against pathogens. They are hypothesized to be under balancing selection due to selection pressure on pathogens to evolve novel epitopes to evade immune recognition and on host receptors to detect pathogens. Thus, PRRs and other immune loci are expected to be among the most diverse regions of the genome. Using whole genome data from an important

African snail vector, Biomphalaria sudanica, we hypothesize that diverse regions of the genome will be enriched with immune related loci, and that we can identify novel PRRs through annotation of these regions.

Methods Used Five B. sudanica strains (collected: Lake Victoria, Kenya) were sequenced using the PacBio and Illuminapaired-endreads. Mean inter-line diversity was calculatedacross the genomes and segmented into smaller windows $(10-100 \mathrm{~kb})$. Each window with a mean inter-line diversity value $>1 \%$, had up to $1 \mathrm{Mb}$ of surrounding nucleotides annotated and transmembrane domains (TMDs) identified using predictive software. To determine if TMD peptides were over-represented in high- diversity regions of the genome, we compared the proportion of TMD peptides against the proportion of those in 30 randomly assigned contig regions.

Summary of Results 67 of 6815 windows met our nucleotide diversity threshold of $1 \%$ divergence. 421 of 818 immunesuspected peptides were identified to have TMDs, over-represented in regions of high diversity when compared to randomized control regions, supporting our hypothesis. Immunerelated genes associated with Schistosoma resistance in other species including PTC2 and GRC were also identified using this bioinformatic approach.
Conclusions Our findings support the diversity-based approach to identifying PRRs which successfully identified known $B$. glabrata PRRs and novel PRRs in B. sudanica genomes. Our established list of candidate genes for pathogen recognition will provide a foundation guiding resistance studies, gene knockout and GWAS with Biomphalaria species.

\section{\#52 IS VITAMIN D DEFICIENCY ASSOCIATED WITH MORE SEVERE COVID-19 INFECTION?}

C Ko*1 , B Bajoua ${ }^{1}$, A Garg ${ }^{1}$, C Metz ${ }^{1}$, M Shean ${ }^{1}$, Talebi Dolouei I ${ }^{1}$, M To $^{1}$, B Afghani ${ }^{1,2}$ ${ }^{1}$ University of California Irvine, Irvine, $C A ;{ }^{2}$ Children's Hospital of Orange County, Orange, $C A$

\subsection{6/jim-2022-WRMC.51}

Purpose of Study Vitamin D has been shown to be helpful in preventing certain respiratory tract infections, but the link between COVID-19 severity and vitamin D levels remains unclear. The purpose of this study was to clarify the relationship between COVID-19 severity and vitamin D levels through a literature review.

Methods Used A systematic literature review using PubMed and Google Scholar was conducted. Our inclusion criteria included those studies that a) measured vitamin D levels, b) included both a control and subject group, c) measured the association between COVID-19 severity and vitamin D levels, and d) took into account other potential COVID-19 related comorbidities among controls versus subjects groups. Studies that measured only prevention of COVID-19 infection (positivity rate) or mortality were excluded.

Abstract \#52 Table 1 Studies evaluating the association between vitamin D level and COVID-19 disease severity

\begin{tabular}{|c|c|c|c|c|}
\hline $\begin{array}{l}\text { Author, year, } \\
\text { and location }\end{array}$ & $\begin{array}{l}\text { Controls: number }(\mathrm{N}) \text { and } \\
\text { mean or median age (yrs) }\end{array}$ & $\begin{array}{l}\text { Subjects: number }(\mathrm{N}) \text { and } \\
\text { mean or median age (yrs) }\end{array}$ & $\begin{array}{l}\text { Serum } 25(\mathrm{OH}) \mathrm{D} \text { concentration } \\
\text { in controls vs. subjects, } \mathrm{p} \text { - } \\
\text { value }\end{array}$ & Outcome based on comorbidities and vitamin D levels, p-value \\
\hline $\begin{array}{l}\text { Radujkovic, 2020, } \\
\text { Germany }\end{array}$ & $\begin{array}{l}\text { Controls: COVID-19 outpatients, } \\
\mathrm{N}=92, \text { Median age }=56\end{array}$ & $\begin{array}{l}\text { Subjects: COVID-19 inpatients, } \\
\mathrm{N}=93, \text { Median age }=63\end{array}$ & $\begin{array}{l}\text { Median: } 18.6 \text { vs. } 14.6 \mathrm{ng} / \mathrm{mL} \text {, } \\
\mathrm{p}=0.001\end{array}$ & $\begin{array}{l}\text { Adjusted by age, gender, and comorbidities, VDD ( }<12 \mathrm{ng} / \mathrm{mL} \text { ) was } \\
\text { associated with invasive mechanical ventilation and death (HR } 6.12 \\
\text { and } 14.73 \text {, respectively. } p<0.001 \text { for both). }\end{array}$ \\
\hline Luo, 2020, China & $\begin{array}{l}\text { Controls: Non-severe COVID-19, } \\
\mathrm{N}=261 \text {, Median age }=54.0\end{array}$ & $\begin{array}{l}\text { Subjects: Severe COVID-19, } \\
\mathrm{N}=74 \text {, Median age }=62.5\end{array}$ & $\begin{array}{l}\text { Median: } 11.0 \text { vs. } 9.24 \mathrm{ng} / \mathrm{mL} \text {, } \\
\mathrm{p}<0.0001\end{array}$ & $\begin{array}{l}\text { Adjusted by sex, age, comorbidities, BMI, smoking status, and vitamin } \\
\text { D status, VDD ( }<12 \mathrm{mg} / \mathrm{L}) \text { was associated with COVID-19 severity (OR } \\
2.72,95 \% \mathrm{Cl} 1.23-6.01, \mathrm{p}=0.014) \text {, but VDD was not associated with } \\
\text { mortality ( } \mathrm{p}=1.0) \text {. }\end{array}$ \\
\hline $\begin{array}{l}\text { Karahan, 2021, } \\
\text { Turkey }\end{array}$ & $\begin{array}{l}\text { Controls: Moderate COVID-19, } \\
\mathrm{N}=47 \text {, Mean age }=56.1\end{array}$ & $\begin{array}{l}\text { Subjects: Severe-critical COVID- } \\
19, \mathrm{~N}=102, \text { Mean age }=67.0\end{array}$ & $\begin{array}{l}\text { Median: } 26.3 \text { vs. } 10.1 \mathrm{ng} / \mathrm{mL} \text {, } \\
\mathrm{p}<0.001\end{array}$ & $\begin{array}{l}\text { 25(OH)D levels significantly predictive of in-hospital mortality } \\
\text { (multivariate analysis: } \mathrm{OR} 0.927,95 \% \mathrm{Cl} 0.875-0.982, \mathrm{p}=0.01 \text { ). }\end{array}$ \\
\hline Jain, 2020, India & $\begin{array}{l}\text { Controls: Asymptomatic COVID- } \\
19, \mathrm{~N}=91 \text {, Mean age }=42.34\end{array}$ & $\begin{array}{l}\text { Subjects: Severely ill COVID-19, } \\
\mathrm{N}=63 \text {, Mean age }=51.41\end{array}$ & $\begin{array}{l}\text { Mean: } 27.89 \text { vs. } 14.35 \mathrm{ng} / \mathrm{mL} \text {, } \\
p=0.0001\end{array}$ & $\begin{array}{l}\text { Fatality rate for VDD }(<20 \mathrm{ng} / \mathrm{mL}) \text { higher than that for normal } 25(\mathrm{OH}) \\
\text { D }(21.1 \% \text { vs. } 31.1 \% \text {, p-value } \mathrm{NR}) \text {. }\end{array}$ \\
\hline $\begin{array}{l}\text { Macaya, 2020, } \\
\text { Spain }\end{array}$ & $\begin{array}{l}\text { Controls: Non-severe COVID-19, } \\
\mathrm{N}=49, \text { Median age }=63\end{array}$ & $\begin{array}{l}\text { Subjects: Severe COVID-19, } \\
\mathrm{N}=31 \text {, Median age }=75\end{array}$ & $\begin{array}{l}\text { Median: } 19 \text { vs. } 13 \mathrm{ng} / \mathrm{mL} \text {, } \\
\mathrm{p}=0.145\end{array}$ & $\begin{array}{l}\text { Adjusted by age, gender, obesity, cardiac disease, and CKD, VDD } \\
\text { (<20 ng/mL) did not significantly predict higher risk of developing } \\
\text { severe COVID-19 (OR 3.2, 95\% Cl 0.9-11.4, } \mathrm{p}=0.07) \text {. }\end{array}$ \\
\hline Ye, 2020, China & $\begin{array}{l}\text { Controls: Mild/moderate COVID- } \\
19, \mathrm{~N}=50, \text { Median age }=39\end{array}$ & $\begin{array}{l}\text { Subjects: Severe/critical COVID- } \\
19, N=10, \text { Median age }=65\end{array}$ & $\begin{array}{l}\text { Median: } 22.64 \text { vs. } 15.28 \mathrm{ng} / \mathrm{mL} \text {, } \\
\mathrm{p}<0.05\end{array}$ & $\begin{array}{l}\text { Multivariate analysis including VDD, age, sex, renal failure, diabetes, } \\
\text { and HTN: Statistically significant association between VDD (<20 ng/mL) } \\
\text { and severe/critical COVID-19 disease (OR 15.18, 95\% Cl 1.23-187.45). }\end{array}$ \\
\hline $\begin{array}{l}\text { Kerget, 2020, } \\
\text { Turkey }\end{array}$ & $\begin{array}{l}\text { Controls: COVID- } 19 \text { without } \\
\text { ARDS, } N=53 \text {, Mean age }=38.3\end{array}$ & $\begin{array}{l}\text { Subjects: COVID- } 19 \text { with ARDS, } \\
\mathrm{N}=35 \text {, Mean age }=67.9\end{array}$ & $\begin{array}{l}\text { Mean: } 21.8 \text { vs. } 16.8 \mathrm{ng} / \mathrm{mL} \text {, } \\
\mathrm{p}=0.102\end{array}$ & NR \\
\hline Campi, 2021, Italy & $\begin{array}{l}\text { Controls: Severely symptomatic } \\
\text { COVID- } 19 \text { hospital ward admits, } \\
\mathrm{N}=49 \text {, Mean age }=68.82\end{array}$ & $\begin{array}{l}\text { Subjects: Severely symptomatic } \\
\text { COVID-19 ICU admits, } \mathrm{N}=54 \text {, } \\
\text { Mean age }=63.67\end{array}$ & $\begin{array}{l}\text { Mean: } 22.4 \text { vs. } 14.4 \mathrm{ng} / \mathrm{mL} \text {, } \\
\mathrm{p}=0.0003\end{array}$ & $\begin{array}{l}25(\mathrm{OH}) \mathrm{D} \text { levels inversely correlated with subsequent need for ICU } \\
\text { admission in severely symptomatic patients (RR } 0.989,95 \% \mathrm{CI} 0.981- \\
0.997, p=0.011 \text { ), and also inversely correlated with in-hospital } \\
\text { mortality }(\mathrm{p}=0.002 \text { ). }\end{array}$ \\
\hline
\end{tabular}

Units for vitamin $\mathrm{D}$ levels in two studies (Luo et al. and Ye et al.), originally reported in $\mathrm{nmol} / \mathrm{L}$, were converted to $\mathrm{ng} / \mathrm{mL}$ in order to achieve consistency in the units across all studies. $25(\mathrm{OH})$

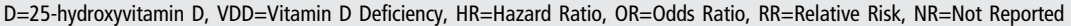


Summary of Results Eight studies satisfied our inclusion criteria (see table 1 below). A majority of studies showed significantly lower vitamin D levels in the more severe COVID-19 subjects compared to control patients with less severe COVID-19. Most studies that assessed COVID-19 outcomes based on vitamin D deficiency (VDD) found VDD to be associated with worse outcomes, including more severe disease and increased mortality. The limitations of this review include inter-study variability in the co-morbidities included in multivariate analysis, variability in the definition of VDD among different studies, and a lack of information on vitamin D supplements and other treatments before infection or during hospitalization in several studies. Finally, a causal relationship could not be assessed because all studies were observational, and information on the vitamin D levels before hospital admission, during healthy state, was not available in a majority of studies.

Conclusions There may be an association between lower vitamin D levels and more severe COVID-19 disease. However, larger longitudinal studies that not only measure vitamin D levels pre-COVID-19 disease but also take into account all variables, such as comorbidities and treatments that could affect disease severity, are warranted.

\section{\#53 THE USE OF VAGINAL PROBIOTICS SUPPOSITORIES IN PREVENTION OF RECURRENT URINARY TRACT INFECTIONS IN ADULT WOMEN: A LITERATURE REVIEW}

${ }^{1} \mathrm{D}$ Devineni* ${ }^{*},{ }^{1}$ I Bade, ${ }^{1} \mathrm{C}$ Diyakonov, ${ }^{1} \mathrm{E}$ Lee, ${ }^{1} \mathrm{~N}$ Lulla, ${ }^{1} \mathrm{~K}$ Parang, ${ }^{1} \mathrm{~S}$ Soni, ${ }^{1,2} \mathrm{~B}$ Afghani. ${ }^{1}$ University of California Irvine School of Medicine, Irvine, $C A ;{ }^{2}$ Children's Hospital of Orange County, Orange, $C A$

\subsection{6/jim-2022-WRMC.52}

Purpose of Study Urinary tract infections (UTIs) are one of the most common bacterial infections in women. Concerns over the effectiveness of antibiotics in preventing recurrent UTIs, due to antibiotic resistance and the adverse effects of antibiotics on healthy microbiota, have raised the necessity to investigate reliable non-antibiotic treatments for preventing recurrent UTIs. It has been proposed that probiotics or lactobacillus may be effective in preventing infections by restoring the normal vaginal flora. The purpose of this study is to investigate the effectiveness of vaginal probiotic suppositories for prevention of recurrent UTIs in adult women.

Methods Used A systematic literature review was conducted through databases such as PubMed and Google Scholar. Only studies that were published after 1990, and compared use of vaginal probiotics with a control group in adult women with recurrent UTI were included. Studies with follow-up period of $<6$ months were excluded.

Summary of Results We found 5 studies that fit our inclusion criteria (See table 1). In majority of the studies, the frequency of recurrent UTIs was lower in patients who received probiotic vaginal suppositories when compared to controls. However, there was great variability among the studies with respect to the probiotic formulation as well as treatment dose and frequency. The applications were intermittent and varied from daily to weekly to monthly. The probiotic species used in different studies included L. Rhamnosus, L. Fermentum, and L. Crispatus. The sample sizes were small and did not divide the patients into different categories based on risk factors or co-morbidities. In addition, the bacterial cause of UTI was not mentioned in majority of the studies. Mild side effects were noted in both probiotic and control group, and included increased vaginal discharge, vaginal odor, mild irritation and dysuria.

Conclusions Our review suggests a promising role for use of intermittent vaginal probiotic suppositories for prevention of recurrent UTIs in adult women. Larger prospective studies with longer follow-up period are needed to determine the optimal probiotic dosage and frequency in different groups of patients with recurrent UTI.

\section{Neonatology general I}

\section{Concurrent session}

\section{2:45 PM}

\section{Thursday, January 20, 2022}

\section{\#54 NUCLEATED RED BLOOD CELL EMERGENCE-TIME IN NEWBORN LAMBS FOLLOWING A DOSE OF DARBEPOETIN ALFA}

${ }^{1,2} \mathrm{TM}$ Bahr* ${ }^{2} \mathrm{~K}$ Albertine, ${ }^{1,2} \mathrm{RD}$ Christensen, ${ }^{2} \mathrm{MJ}$ Dahl, ${ }^{2} \mathrm{~A}$ Rebentisch, ${ }^{2} \mathrm{E}$ Dawson, ${ }^{2} \mathrm{E}$ Major, ${ }^{2} \mathrm{H}$ Foreman, ${ }^{2} \mathrm{D}$ Headden, ${ }^{2} \mathrm{Z}$ Vordos, ${ }^{2} \mathrm{~A}$ Nabi, ${ }^{2} \mathrm{~L}$ Pettet, ${ }^{2} \mathrm{P}$ Badrov, ${ }^{2} \mathrm{C}$ Addison, ${ }^{2} \mathrm{D}$ Christensen. ${ }^{1}$ Intermountain Healthcare, Salt Lake City, UT; ${ }^{2}$ University of Utah Health, Salt Lake City, UT

\subsection{6/jim-2022-WRMC.53}

Purpose of Study A high nucleated red blood cell (NRBC) count in a neonate at birth has been suggested as a biomarker for fetal hypoxia. However, it is not clear if it indicates when

Abstract \#53 Table 1 Studies on the efficacy of intravaginal probiotics in preventing recurrent UTIs

\begin{tabular}{|c|c|c|c|c|c|}
\hline $\begin{array}{l}\text { First author's last name, Year of } \\
\text { Publication, Location of study }\end{array}$ & Name of Probiotic & $\begin{array}{l}\text { Duration and frequency of } \\
\text { usage (intravaginal) }\end{array}$ & $\begin{array}{l}\text { Number of Subjects } \\
\text { and controls }\end{array}$ & $\begin{array}{l}\text { Frequency of UTIs in } \\
\text { subjects vs controls }\end{array}$ & $P$ value \\
\hline Reid, 1992, Canada & $\begin{array}{l}\mathrm{L} \text { casei rhamnosus and } \mathrm{L} \\
\text { fermentum }\end{array}$ & $\begin{array}{l}\text { Twice a week for } 2 \text { weeks then } \\
\text { once monthly }\end{array}$ & 19 vs 21 & $21 \%$ vs $47 \%$ & $\mathrm{p}<0.05$ \\
\hline Baerheim, 1994, Norway & $\mathrm{L}$ casei rhamnosus & Twice a week for 26 weeks & 25 vs 22 & Incidence ratio $=1.4$ & $\begin{array}{l}95 \% \mathrm{Cl}=0.88 \text { to } \\
1.98(\mathrm{p}>0.05)\end{array}$ \\
\hline Reid, 1995, Canada & $\begin{array}{l}\text { L. rhamnosus and L- } \\
\text { fermentum }\end{array}$ & Once a week 1 year & $\begin{array}{l}55 \text { subjects, baseline } \\
\text { was control }\end{array}$ & $\begin{array}{l}\text { Episodes decreased from } 6 \text { to } \\
1.6 / y r\end{array}$ & $p<0.001$ \\
\hline Uehara, 2006, Tokyo & L. Cirspatus (Lactin-V) & Every 2 days for 1 year & $\begin{array}{l}9 \text { Subjects, baseline was } \\
\text { control }\end{array}$ & $\begin{array}{l}\text { Recurrences decreased from } \\
5.0 / \mathrm{yr} \text { to } 1.3 / \mathrm{yr}\end{array}$ & $p=0.0007$ \\
\hline Stapleton, 2011, USA & L. Cirspatus (Lactin-V) & 5 days, then weekly for 10 weeks & 50 vs 50 & $15 \%$ vs $27 \%$ & $\mathrm{p}<0.01$ \\
\hline
\end{tabular}



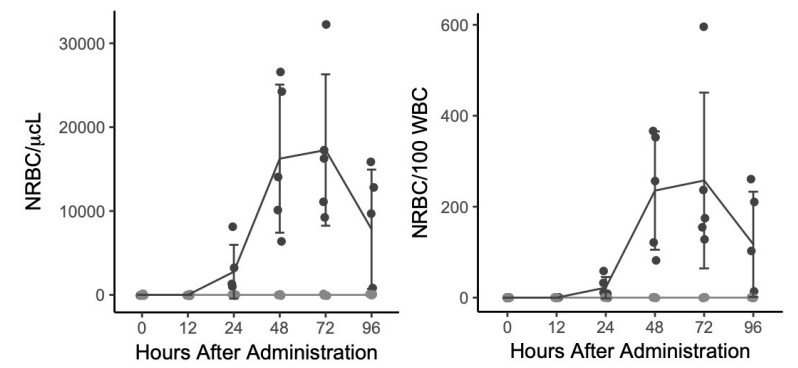

Abstract \#54 Figure $1 \mathrm{NRBC} / \mu \mathrm{L}$ (left) and NRBC/100 WBC (right), before (time 0 ) and at intervals following darbepoetin administration to five term lambs, as well as values in five similarly instrumented control lambs. Values from the darbepoetin recipients are shown by a solid black circle and those from the controls by a solid gray circle.

the hypoxia occurred. We aimed to measure the time between administering a high-dose of darbepoetin, simulating the marked rise in erythropoietin that follows a hypoxic event, and the first appearance of NRBC in the blood. Limited observations of this interval, the 'NRBC emergence-time,' in human neonates suggest it is greater than 24 hours.

Methods Used We obtained serial blood counts on ten newborn lambs; five dosed with darbepoetin $(10 \mu \mathrm{g} / \mathrm{kg})$ and five placebo controls, to assess the NRBC emergence-time.

Summary of Results The first appearance of NRBC was at 24 h (mean \pm SD, $2757 \pm 3210 \mathrm{NRBC} / \mu \mathrm{L}$ vs. $0 / \mu \mathrm{L}$ in controls). Peak was $48-72 \mathrm{~h}(16,758 \pm 8434 / \mu \mathrm{L}$ vs. $0 / \mu \mathrm{L}$ in controls), followed by fewer at 96 hours $(7823 \pm 7114 / \mu \mathrm{L} v s$. $0 / \mu \mathrm{L}$ in controls). Similarly, reticulocytes peaked at 48-72 h $(113,094$ $\pm 3210 / \mu \mathrm{L}$ vs. $10,790 \pm 5449 / \mu \mathrm{L}$ in controls), with no changes in platelets or leukocytes.

Conclusions The NRBC emergence time in newborn lambs is similar to reports from newborn humans. By extrapolation, if a neonate has a high NRBC at birth, the erythropoietic stimulus likely occurred within the interval 24 to 96 hours prior to birth.

\section{\#55 REFERENCE INTERVALS FOR END-TIDAL CARBON MONOXIDE OF PRETERM NEONATES}

${ }^{1} \mathrm{TR}$ Christensen*, ${ }^{2} \mathrm{~S}$ Pakdeeto, ${ }^{1} \mathrm{TM}$ Bahr, ${ }^{1} \mathrm{E}$ Gerday, ${ }^{1} \mathrm{M}$ Sheffield, ${ }^{3} \mathrm{~K}$ Christensen, ${ }^{2} \mathrm{~S}$ Supapannachart, ${ }^{2} \mathrm{P}$ Nuntnarumit, ${ }^{2} \mathrm{~S}$ Sukwiset, ${ }^{1} \mathrm{RK}$ Ohls, ${ }^{1} \mathrm{RD}$ Christensen. ${ }^{1}$ University of Utah Health, Salt Lake City, UT; ${ }^{2}$ Mahidol University Faculty of Medicine Ramathibodi Hospital, Bangkok, Thailand; ${ }^{3}$ Brigham Young University, Provo, UT

\subsection{6/jim-2022-WRMC.54}

Purpose of Study Every molecule of heme metabolized to bilirubin releases one molecule of carbon monoxide (CO). On that basis, hemolysis can be detected and quantified by measuring $\mathrm{CO}$ in exhaled breath. We constructed reference intervals for end-tidal carbon monoxide (ETCOc) levels of neonates 28-34 weeks gestation to assess the hemolytic rate. New instrumentation allows providers to non-invasively measure ETCOc in preterm neonates with low tidal volumes and breathing rates up to $70 \mathrm{bpm}$. Reference intrevals for term and late preterm neonates exist, but until now none have been created for preterm neonates.

Methods Used Prospective four-NICU study in Bangkok, Thailand, and Utah, USA. Neonates born between 28-34 weeks and up to 28 days old were eligible. Once informed consent was received, a modified CoSense ETCOc analyzer was used

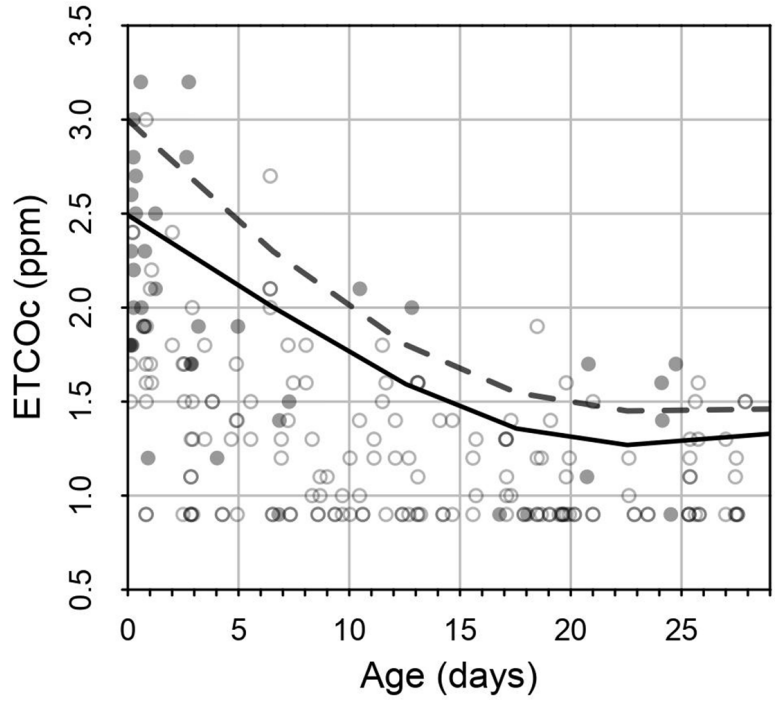

Abstract \#55 Figure 1 The dashed line and the grey circles are preterm infants from Bangkok. The solid line and open circles are preterm infants from Utah, USA

to record results. Data from the CoSense devices were linked to patient charts to obtain demographic information.

Summary of Results Values from days one through 28 were charted and upper ( $>95$ th percentile) reference interval limits were calculated. During the entire 28 days, the ETCOc upper reference intervals of babies in Bangkok were higher than those in Utah $(\mathrm{p}<0.01)$. No differences were found due to sex, or earliest vs. latest gestation at birth (both $\mathrm{p}>0.1$ ). Preterm neonates in Bangkok and Utah had higher ETCOc values during the first 48 hours after birth than thereafter $(p<0.01)$. Conclusions Using the reference interval chart we created, the hemolytic rate of preterm infants $\geq 28$ weeks can be assessed. This identification allows us to focus subsequent testing to find the cause of the hemolysis, administer more intensive phototherapy, and to assure consistent in-and out-patient follow-up to those with hemolytic jaundice.

\section{\#56 ANEMIC AT BIRTH}

${ }^{1,2} \mathrm{TM}$ Bahr*, ${ }^{2} \mathrm{LM}$ Shelley, ${ }^{1} \mathrm{ER}$ Henry, ${ }^{2} \mathrm{RK}$ Ohls, ${ }^{1,3} \mathrm{~S} \mathrm{Li},{ }^{1} \mathrm{~S}$ Illstrup, ${ }^{1,2} \mathrm{RD}$ Christensen. ${ }^{1}$ Intermountain Healthcare, Salt Lake City, UT; ${ }^{2}$ University of Utah Health, Salt Lake City, UT; ${ }^{3}$ University of Utah, Salt Lake City, UT

\subsection{6/jim-2022-WRMC.55}

Purpose of Study Using ten years of multihospital data, we identified neonates with 'severe anemia at birth', defined by a hemoglobin/hematocrit within the first six hours after birth below the 1st percentile. We determined whether caregivers recognized anemia within the first 24 hours after birth, the probable cause of the anemia, treatment given, and whether review suggested a different cause of anemia than listed in the medical record.

Methods Used Data from neonates born 2011-2020 were obtained from the Intermountain Healthcare Data Warehouse. We reviewed records of all infants with severe anemia at birth. We then categorized the cause as either; hemorrhage, hemolysis, hypoproduction, a combination of etiologies, or unable to determine. 

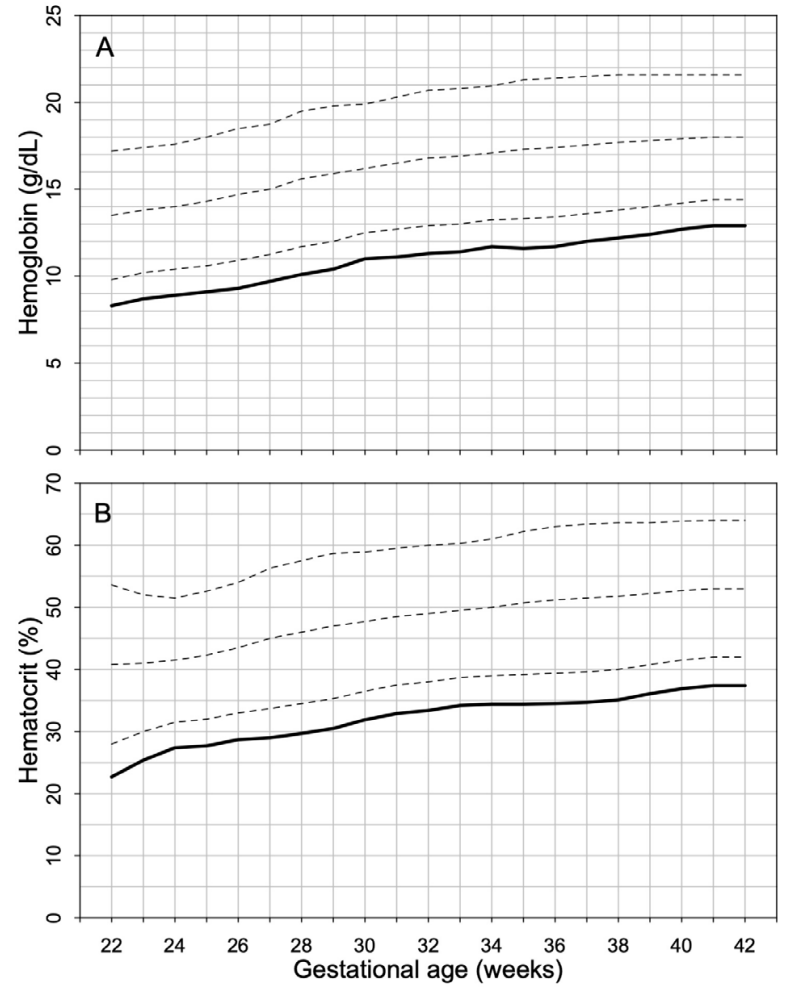

Abstract \#56 Figure 1 Reference intervals for hemoglobin (A) and hematocrit (B) of neonates on the day of birth by gestational age. The bold line indicates the 1st percentile reference interval, below which we label neonates as having 'severe anemia at birth'. Dashed lines show the 5 th percentile, median, and 95th percentiles.

Summary of Results Of 299,927 live births 344 had severe anemia. In $153(44.5 \%)$ the anemia was unrecognized during the first 24 hours. The lowest hemoglobin/hematocrit values were among those with hemorrhage vs. hemolysis $(\mathrm{P}<0.013)$ or vs. hypoproduction $(\mathrm{P}<0.001)$. In infants with severe anemia secondary to hemorrhage, abruption/other perinatal event and fetomaternal hemorrhage (FMH) were the most likely etiologies. DIC was a common hemolytic cause of anemia, with $85 \%$ of DIC cases coincident with hemorrhagic anemia.

Conclusions Severe anemia at birth often went unrecognized on the first day. Earlier recognition may be facilitated by an electronic medical record-associated hemoglobin/hematocrit nomogram, with values $<1$ st percentile clearly identified.

\section{\#57 FETOMATERNAL HEMORRHAGE: EVIDENCE FROM A MULTIHOSPITAL HEALTHCARE SYSTEM THAT UP TO 40\% OF SEVERE CASES ARE MISSED}

${ }^{1} \mathrm{NR}$ Carr*, ${ }^{2} \mathrm{ER}$ Henry, ${ }^{1,2} \mathrm{TM}$ Bahr, ${ }^{1} \mathrm{RK}$ Ohls, ${ }^{1} \mathrm{~J}$ Page, ${ }^{1} \mathrm{SJ}$ Ilstrup, ${ }^{1} \mathrm{RD}$ Christensen. ${ }^{1}$ University of Utah Health, Salt Lake City, UT; ${ }^{2}$ Intermountain Healthcare, Salt Lake City, UT

\subsection{6/jim-2022-WRMC.56}

Purpose of Study We previously reported fetomaternal hemorrhage $(\mathrm{FMH})$ in $1 / 9160$ births, and only one neonatal death from FMH among 219,853 births. Recent reports indicate $\mathrm{FMH}$ is not uncommon among stillbirths. Consequently, we speculated we were missing cases among early neonatal deaths. We began a new FMH initiative to determine the current incidence.

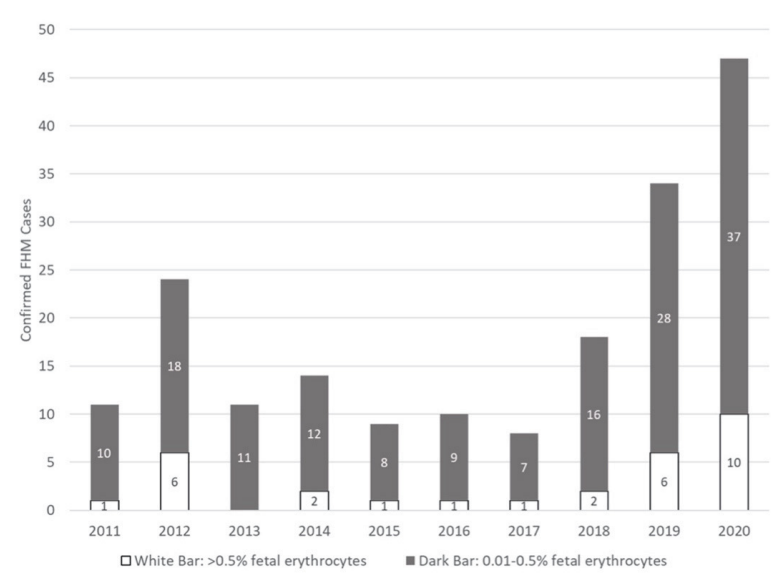

Abstract \#57 Figure 1

Methods Used We analyzed births from 2011 to 2020 where FMH was diagnosed. We also evaluated potential cases among neonates receiving an emergent transfusion just after birth, whose mothers were not tested for FMH.

Summary of Results Mong 297,403 births, 1375 mothers were tested for FMH (1/216 births). Fourteen percent tested positive (1/1599 births). Of those, we found 25 with clinical and laboratory evidence of FMH adversely affecting the neonate. Twenty-one received one or more emergency transfusions on the day of birth; all but two lived. We found 17 others who received an emergency transfusion on the day of birth where FMH was not tested for, but was likely; eight of those died. The 42 severe (proven + probable) cases equate to $1 / 7081$ births. We judged that 10 of the 42 had an acute $\mathrm{FMH}$, and in the others it likely had more than a day before birth.

Conclusions We estimate that we fail to diagnose $>40 \%$ of our severe FMH cases. Needed improvements include; 1) education to request maternal FMH testing when neonates are born anemic, 2) education on false negative FMH tests, 3) improved FMH communications between neonatology, obstetrics, and blood bank.

\section{\#58 IRON FOLIC ACID SUPPLEMENTATION AS A PREDICTOR OF ANEMIA IN PREGNANT WOMEN IN RURAL INDIA}

B Tomlin*, P Brahmbhatt, B Fassl, JW Thomas, A Judkins. University of Utah Health, Salt Lake City, UT

\subsection{6/jim-2022-WRMC.57}

Purpose of Study Perinatal anemia is a massive global public health burden with an estimated global prevelance of approximately $40 \%$. Severe anemia increases the risk of maternal mortality and can adversely effect fetal development. Adequate correction of anemia is essential for a healthy pregnancy and infant, but universal screening and monitoring is not the care standard in most LMICs. In lieu of universal screening and treatment, providing access to Iron Folic Acid (IFA) tabs is considered an effective and cost-efficient intervention to prevent and treat anemia of pregnancy. However, despite widespread availability of IFA tabs, anemia prevalence continues to be high and the presence of IFA programs may falsely reassure clinicians that patients taking them have adequate hemoglobin. 
Methods Used The study took place at Mota Fofalia Community Health Center (MF-CHC) in Gujarat, India operated by a public-private partnership. The University of Utah operates an academic global health program in collaboration with MF-CHC and assists the health center in sustainable capacity building in maternal-child health. As part of a communitybased antenatal care (ANC) program, we recruited a cohort of pregnant women from the surrounding community to complete a standardized nutrition and health survey and participate in scheduled prenatal visits according to WHO and Indian ANC guidelines which include measurement of vital signs and ANC guideline-based interventions. At each ANC visit, a blood hemoglobin level was drawn and each participant was asked if they are currently taking IFA or Albendozole, an antiparisitic.

Summary of Results A total of 501 women were included in the study. 448 (89\%) report taking IFA and 53 (11\%) report not taking IFA. The average hemoglobin for those taking IFA was $10.11 \mathrm{~g} / \mathrm{dL}$ (IQR 9.3-11.1) with an average gestational age at screening of 23.0 weeks while the average of those not taking was $10.41 \mathrm{~g} / \mathrm{dL}$ (IQR 9.8-11.6) $(\mathrm{p}=.28)$ with an average gestational age of 10.9 weeks. In the group taking IFA tablets, $97 \%$ were also taking Albendozole while only $21 \%$ of mothers not taking IFA tablets were taking Albendozole.

Conclusions In areas with a high prevalence of anemia, patient compliance with standard IFA antenatal therapy is not a an adequate indicator of intervention. While it appears many mothers begin taking IFA as they become pregnant, the presence of readily available IFA therapy to the community is not sufficient in addressing perinatal anemia.

\section{\#59 1 THE VALIDITY OF POINT-OF-CARE HEMOGLOBIN MEASUREMENTS IN NEONATES}

${ }^{1}$ AF Ahmed* ${ }^{*}$ 'JP Wang, ${ }^{2} \mathrm{R}$ Ramanathan, ${ }^{2} \mathrm{AM}$ Yeh. ${ }^{1}$ LAC+USC Medical Center, USC, LoS Angeles, CA; ${ }^{2}$ Keck School of Medicine, USC, Los Angeles, CA

\subsection{6/jm-2022-WRMC.58}

Purpose of Study Hemoglobin ( $\mathrm{Hb}$ ) analysis among patients in the Neonatal Intensive Care Unit (NICU) is essential in diagnosing anemia, a common problem among neonates of low birth weight or preterm infants. This same population is especially at risk for iatrogenic anemia, given the need for frequent laboratory analysis while having a small blood volume. $\mathrm{Hb}$ measured by way of point-of-care (POC) meters or blood gas analyzers, which each require 1-2 drops of blood per reading, could prevent significant phlebotomy in the NICU as compared to the Complete Blood Count (CBC), which requires 0.6 to 1 milliliter. This study aims to determine the validity of POC and blood gas $\mathrm{Hb}$ measurements as compared to $\mathrm{Hb}$ values from the $\mathrm{CBC}$.

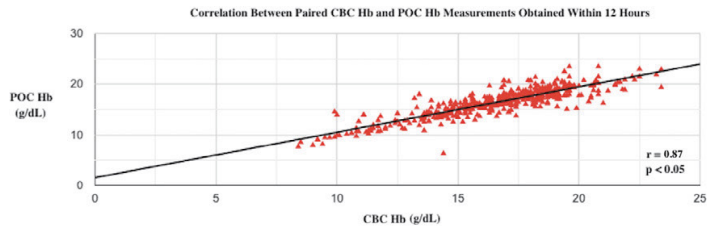

Abstract \#59 Table 1 Correlation between CBC HB and paired $\mathrm{POC} / \mathrm{blood}$ gas $\mathrm{Hb}$

\begin{tabular}{lllll}
\hline & $\begin{array}{l}\text { Sample } \\
\text { size }\end{array}$ & $\begin{array}{l}\text { Mean } \\
\text { Difference }\end{array}$ & $\begin{array}{l}\text { Standard Deviation } \\
\text { of Mean Difference }\end{array}$ & $\begin{array}{l}\text { Pearson } \\
\text { Coefficient }(r), \\
\text { p value }\end{array}$ \\
\hline $\begin{array}{l}\text { CBC vs POC Gas } \\
\text { within 12 hours }\end{array}$ & 488 & 1.1 & 1.0 & $0.89, \mathrm{p}<0.05$ \\
CBC vs POC Gas & 243 & 1.3 & 1.0 & $0.83, p<0.05$ \\
$\begin{array}{l}\text { between 12-24 } \\
\text { hours }\end{array}$ & & & & \\
$\begin{array}{l}\text { CBC vs POC Hgb } \\
\text { within 12 hours }\end{array}$ & 477 & 1.0 & 1.0 & $0.87, p<0.05$ \\
CBC vs POC Hgb & 290 & 1.3 & 1.2 & $0.75, p<0.05$ \\
between 12-24 & & & & \\
hours & & & & \\
\hline
\end{tabular}

Methods Used This is a retrospective study of patients admitted to the LAC+USC Medical Center NICU between January 2020 and April 2021 with paired $\mathrm{Hb}$ measurements from the laboratory-run $\mathrm{CBC}$ and either $\mathrm{POC} \mathrm{Hb}$ from HemoCue B 201 or blood gas $\mathrm{Hb}$ from Gem Premier 5000. Qualifying data was divided into groups based on time between different blood draws, the first comprising of measurements collected within 12 hours of each other, the second of measurements collected between 12 and 24 hours of each other. POC or blood gas $\mathrm{Hb}$ and $\mathrm{CBC} \mathrm{Hb}$ measurements collected over 24 hours from each other were excluded. T-tests were used for analysis of continuous, normally distributed variables. Regression analysis was performed to determine the relationship between paired $\mathrm{Hb}$ measurements. Statistical significance was set at $\mathrm{p}<0.05$.

Summary of Results We identified 250 subjects with qualifying paired $\mathrm{Hb}$ values from the $\mathrm{CBC}$ and $\mathrm{POC} \mathrm{Hb}$ or blood gas $\mathrm{Hb}$. There were 488 paired $\mathrm{CBC}$ and blood gas $\mathrm{Hb}$ samples in the $<12$ hour group, and 243 paired samples in the 12-24 hour group. There were 479 paired CBC and POC Hb samples in the $<12$ hour group, and 290 paired samples in the 12-24 hour group (table 1). Correlation coefficient (R) for the $\mathrm{CBC}$-blood gas $\mathrm{Hb}$ groups were 0.89 in the $<12$ hour group and 0.83 in the $12-24$ hour group. Correlation coefficient for the CBC-POC $\mathrm{Hb}$ groups were 0.87 in the $<12$ hour group and 0.75 in the 12-24 hour group (figure 1).

Conclusions There was a strong correlation between paired POC $\mathrm{Hb}$ or blood gas $\mathrm{Hb}$ and $\mathrm{CBC} \mathrm{Hb}$ values obtained within 12 hours of one another. Our results show that POC $\mathrm{Hb}$ or blood gas $\mathrm{Hb}$ should be considered as alternatives for $\mathrm{CBC} \mathrm{Hb}$. The patients in the NICU would benefit in prevention of iatrogenic anemia. Prospective studies with age- or weight-based grouping and planned pairing within pre-defined time periods would be beneficial in determining whether the correlation persists between POC and $\mathrm{CBC} \mathrm{Hb}$ measurements in the NICU

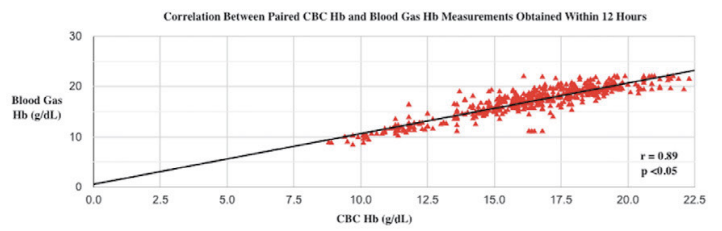


\#60 ALTERNATIVE WAYS OF ACQUIRING BILIRUBIN MEASUREMENT IN PRETERM AND TERM INFANTS ADMITTED TO NEONATAL INTENSIVE CARE UNIT

D Cho*, K Ramm, L Barton, R Ramanathan, M Biniwale. LAC+USC Medical Center, Los Angeles, CA

\subsection{6/jim-2022-WRMC.59}

Purpose of Study Measuring bilirubin levels in infants admitted to neonatal intensive care unit is done to avoid hyperbilirubinemia and bilirubin toxicity. Practitioners strive to minimize blood tests to reduce patient discomfort and iatrogenic anemia. To minimize blood draws in the monitoring of bilirubin levels, we assessed the accuracy of alternative methods of measurement via blood gas analyzers and transcutaneous bilirubin monitoring.

Methods Used Using serum bilirubin as a gold standard, we analyzed the accuracy of simultaneous measurements from blood gas analyzer and transcutaneous monitoring. The accuracy of blood gas and transcutaneous bilirubin measurements was ascertained with correlation coefficient and by calculating mean differences between the serum bilirubin levels and the two alternative methods.

Summary of Results Study consisted of 86 patients with gestational ages 24 to 41 weeks. The correlation coefficient for serum bilirubin vs transcutaneous measurements was $\mathrm{r}=$ $0.893(\mathrm{p}<0.00001)$. The correlation coefficient was $\mathrm{r}=$ $0.9283(\mathrm{p}<0.00001)$ for preterm infants, and $\mathrm{r}=0.8392$ $(p<0.000013)$ for term infants. The mean difference between serum bilirubin vs transcutaneous measurements was 0.45 with a standard deviation of $1.55 \mathrm{mg} / \mathrm{dL}$. The correlation coefficient for serum bilirubin vs blood gas bilirubin was $r=0.959$ $(\mathrm{p}<0.00001)$. The correlation coefficient was $\mathrm{r}=0.9291$ $(\mathrm{p}<0.00001)$ for preterm infants, and $\mathrm{r}=0.9742$ $(\mathrm{p}<0.00001)$ for term infants. The mean difference between

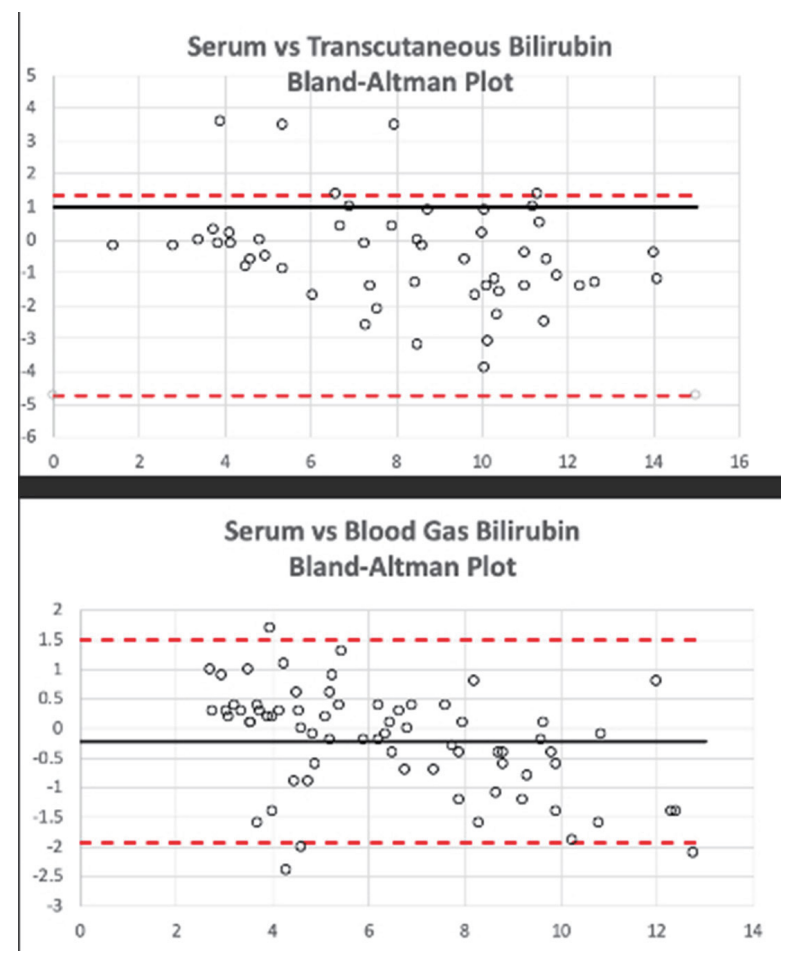

Abstract \#60 Figure 1 serum bilirubin vs blood gas bilirubin was 0.21 with a standard deviation of $0.87 \mathrm{mg} / \mathrm{dL}$.

Conclusions Both transcutaneous and blood gas analyzer bilirubin levels had a strong correlation with serum levels, with bilirubin using blood gas analyzer being slightly more accurate. We plan to continue collecting bilirubin data for a total of 6 months. If accuracy of transcutaneous or blood gas analyzer bilirubin measurements are acceptable, we plan to pursue these alternative methods of bilirubin measurements over the following 6 months and assess to what extent we were able to minimize blood draws.

\section{\#61 1 PREDICTORS OF HOME OXYGEN THERAPY IN VERY LOW BIRTH WEIGHT INFANTS}

Y Shao*, A Hisey, N Nanduri, K Ramm, C Marquez, L Barton, R Ramanathan, M Biniwale. Los Angeles County University of Southern California Medical Center, Los Angeles, CA

\subsection{6/jim-2022-WRMC.60}

Purpose of Study Very low birth weight (VLBW) infants with prolonged respiratory morbidities such as bronchopulmonary dysplasia (BPD) may need to be discharged home while receiving oxygen therapy. The risk factors causing prolonged respiratory support vary for these infants. The study was performed to characterize factors associated with VLBW infants who require oxygen therapy at discharge.

Methods Used Data on all VLBW infants was gathered from the electronic medical record between the years of 2009 and 2021 retrospectively with IRB approval obtained prior. Oxygen therapy was statistically analyzed using SPSS Version 28 statistical software against early neonatal outcomes such as ventilation, intubation, chest compressions, or surfactant, or against common neonatal morbidities, including IVH, BPD, ROP, and patent ductus arteriosus (PDA).

Summary of Results Of 560 VLBW infants, 144 (25.7\%) were discharged home on oxygen. Significant maternal risk factors

Abstract \#61 Table 1 Home oxygen therapy requirement against common neonatal outcomes

\begin{tabular}{|c|c|c|c|}
\hline & $\begin{array}{l}\text { Infants requiring } \\
\text { home oxygen }\end{array}$ & $\begin{array}{l}\text { Infants not requiring } \\
\text { home oxygen }\end{array}$ & p-value \\
\hline Mean Birth Weight (g) & 795 & 1077 & $<0.001$ \\
\hline $\begin{array}{l}\text { Mean Gestational Age } \\
\text { (weeks) }\end{array}$ & 26 & 29 & $<0.001$ \\
\hline $\begin{array}{l}\text { Histologic } \\
\text { Chorioamnionitis (\%) }\end{array}$ & 19.5 & 6.5 & 0.003 \\
\hline $\begin{array}{l}\text { Intubation in Delivery } \\
\text { Room (\%) }\end{array}$ & 56.0 & 30.8 & $<0.001$ \\
\hline Chest Compressions (\%) & 18.0 & 7.0 & $<0.001$ \\
\hline Surfactant Therapy (\%) & 50.7 & 39.4 & 0.024 \\
\hline $\begin{array}{l}\text { Invasive Ventilation at } 24 \\
\text { hours (\%) }\end{array}$ & 70.3 & 24.9 & $<0.001$ \\
\hline $\begin{array}{l}\text { Duration of Invasive } \\
\text { Ventilation (days) }\end{array}$ & 33 & 9 & $<0.001$ \\
\hline PDA (\%) & 84.7 & 52.8 & $<0.001$ \\
\hline $\begin{array}{l}\text { PDA Requiring Surgery } \\
\text { (\%) }\end{array}$ & 41.9 & 6.5 & $<0.001$ \\
\hline Severe IVH (\%) & 8.1 & 1.5 & 0.014 \\
\hline BPD (\%) & 91.4 & 35.5 & $<0.001$ \\
\hline Length of Stay (days) & 81 & 59 & $<0.001$ \\
\hline
\end{tabular}


included histologic chorioamnionitis, intubation, and chest compressions at delivery. These infants had lower gestational age as well as lower birth weight. These infants were more likely to require surfactant, invasive ventilation at 24 hours, and receive additional ventilatory support including high-frequency oscillatory ventilation or jet ventilation (table 1). Infants who were discharged with home oxygen therapy were significantly more likely to have BPD and hemodynamically significant patent ductus arteriosus (PDA) requiring surgery. Associated comorbidities included retinopathy of prematurity (ROP) requiring treatment, severe intraventricular hemorrhage $(\mathrm{IVH})$, and increased length of stay in NICU (table 1). Regression analysis revealed lower birth weight, longer duration of invasive ventilation, PDA requiring surgical intervention, and BPD to be the most significant predictors.

Conclusions The need for high-frequency ventilation such as jet and oscillatory ventilation, as well as chest compressions, is associated with home oxygen need at discharge. Additionally, VLBW infants who required home oxygen therapy were more likely to have needed invasive ventilation, delivery room intubation, and surfactant therapy. They were also more likely to have common neonatal morbidities such as BPD, severe IVH, severe ROP, and PDA.

\section{\#62 CONSEQUENCES OF MATERNAL BREAST MILK ANTIBODIES ON THE ABUNDANCE OF INTESTINAL MICROBES IN NEONATES}

1,2 $\mathrm{HL}$ Wolf*, 1,2 B Wang, ${ }^{2} \mathrm{~J}$ Schwensen, ${ }^{2} \mathrm{M}$ Koch. ${ }^{1}$ University of Washington School of Medicine, Seattle, WA; ${ }^{2}$ Fred Hutchinson Cancer Research Center, Seattle, WA

\subsection{6/jim-2022-WRMC.61}

Purpose of Study Breast milk is an important contributor of the neonatal microbiome. Studies have associated breastfeeding with a decreased risk of acquiring inflammatory bowel disorders later in life. As breastfeeding is not possible for all mothers and children, gaining a mechanistic understanding of this process can lead to the development of early-life interventions that foster beneficial host-microbiota relationships.

We have previously shown that breast milk antibodies are important for maintaining mucosal homeostasis. Mice deficient in breast milk antibodies exhibit perturbations in mucosal immunity, including elevated $\mathrm{T}$ follicular helper (Tfh) cell and germinal center (GC) B cell responses in the gut-associated lymphoid tissues. We hypothesize that the Tfh and GC B cell response generated by the neonate in the absence of breast milk antibodies target resident mucosal bacteria and lead to long-term alterations in the abundance of gut-microbiota.

Methods Used We extracted DNA from intestinal-wall associated microbes and quantified the abundance using quantitative-PCR of the bacterial 16s rRNA. We looked at both the small intestinal wall microbes and large intestinal wall microbes at ages 3, 5 and 11 weeks in maternal antibody sufficient or deficient pups. To explore the effects of Tfh cells and GC B cells, we treated half of each group with anti-Inducible T-cell Co-Stimulator Ligand (anti-ICOSL) antibody, which blocks Tfh and GC B cell formation. We used a paired T-test to determine the significance of our results.

Summary of Results We found no significant difference in the abundance of wall-associated microbes across all four groups in the small or large intestine. These data suggest that the breast milk antibody dependent response as well as the Tfh- cell dependent response do not alter the abundance of wallassociated microbes.

Conclusions Studies are ongoing to determine if breast milk antibodies affect intestinal microbe composition. Limitations to this study may include the drinking water our mice were exposed to throughout the duration of this experiment. Due to mouse husbandry standards operations within our institution our mice were given acidified water which we have recently discovered to significantly alter their microbial composition. Our goal is to repeat this experiment with non-acidified water as we suspect any potential differences in the microbial abundance may have been masked by the effects of acidified water.

\section{Neonatology pulmonary I}

\section{Concurrent session}

\section{2:45 PM}

\section{Thursday, January 20, 2022}

\section{\#63 EFFECTS OF GESTATIONAL LONG-TERM HYPOXIA ON THE COLLAGEN MATRIX OF FETAL SHEEP PULMONARY ARTERIES}

${ }^{1} \mathrm{RC}$ Torres Chavez*, ${ }^{2} \mathrm{C}$ Gheorghe, ${ }^{1} \mathrm{~L}$ Zhang, ${ }^{2} \mathrm{M}$ Yellon $\mathrm{S},{ }^{2} \mathrm{~S}$ Wilson. ${ }^{1}$ Loma Linda University School of Medicine, Loma Linda, CA; ${ }^{2}$ Loma Linda University Department of Basic Sciences, Loma Linda, CA

\subsection{6/jim-2022-WRMC.62}

Purpose of Study Gestational long-term hypoxia (gLTH) is a significant stressor that leads to multiple diseases including pulmonary hypertension. Evidence indicates that gLTH causes oxidative stress and inflammation, which changes cell structure and function. These effects are driven by changes in cellular metabolism, protein expression, and transcriptional regulation. Our proteomic data show that gLTH leads to vascular remodeling and specifically to reduction of collagen 1A1, 1A2, and $3 \mathrm{~A} 1$, though the data do not delineate where in the arterial wall these changes are occurring. We hypothesized that gLTH causes loss of collagen in all arterial layers which was tested by visualizing and quantifying the collagen content in different layers of fetal pulmonary arteries.

Methods Used Fetal sheep pulmonary arteries from normoxic and gLTH environments were obtained and stained with picrosirius red dye (PSR) to visualize collagen in captured images of arterial biopsies by assessing the optical density (OD) of birefringence from polarized light. Fluorescence microscopy was used to capture images of the arterial samples. Data from Image $\mathrm{J}$ analysis of $\mathrm{OD}$ birefringence, inversely related to crosslinked collagen, of the various vascular layers and treatments were evaluated by analysis of variance.

Summary of Results The image analysis showed a significant decrease in optical density, and therefore enhanced crosslinking, in the adventitia compared to the media for all samples belonging to either normoxic or gLTH groups. However, there was no significant difference in optical density of the adventitial versus medial layers between vessels from normoxic and gLTH fetuses.

Conclusions The results indicate that optical density quantification can be used to detect substantial differences in collagen 
and crosslinked structure between the medial and adventitial layers. The findings also raise the possibility that neither collagen nor its crosslinked structure may be affected by gLTH. The data provides evidence that this technique needs refinement to properly visualize the locations where modest changes in expression may occur. Secondarily, the inability of PSR stain to distinguish among certain types of collagen subtypes leaves open the possibility that a shift in the type of collagen may affect biomechanical processes that are associated with pulmonary vascular development or gLTH. The PSR red staining method may not have been definitive, but this study is an important steppingstone towards developing an experimental strategy of visualizing modifications in vascular collagen isoform expression that complement contemporary analytical quantification techniques that provide unique insight into vascular structure and function.

\section{\#64 MESENCHYMAL STROMAL CELL EXTRACELLULAR VESICLES IMPROVE ALVEOLAR FORMATION IN MECHANICALLY VENTILATED PRETERM LAMBS}

${ }^{1} E$ Major*, ${ }^{1} \mathrm{~A}$ Rebentisch, ${ }^{1} \mathrm{E}$ Dawson, ${ }^{1} \mathrm{H}$ Foreman, ${ }^{1} \mathrm{D}$ Headden, ${ }^{1} \mathrm{Z}$ Vordos, ${ }^{2} \mathrm{MJ}$ Dahl, ${ }^{3} \mathrm{D}$ Null, ${ }^{3} \mathrm{~A}$ Mitsialis, ${ }^{4} \mathrm{~S}$ Kourembanas, ${ }^{1} \mathrm{~K}$ Albertine. ${ }^{1}$ The University of Utah School of Medicine, Salt Lake City, UT; ${ }^{2}$ University of California Davis, Davis, CA; ${ }^{3}$ University of California Davis Health System, Sacramento, CA; ${ }^{4}$ Harvard Medical School, Boston, MA

\subsection{6/jim-2022-WRMC.63}

Purpose of Study Bronchopulmonary dysplasia (BPD) is histopathologically characterized alveolar simplification in preterm infants who are chronically mechanically ventilated. Mesenchymal stromal cell extracellular vesicles (MEx) treatment improved alveolar formation in mouse neonatal hyperoxia models of BPD. We tested the hypothesis that MEx will improve alveolar formation in chronically mechanically ventilated preterm lambs.

Methods Used Preterm lambs (128d; term $\sim 150 \mathrm{~d}$; $\sim 28 \mathrm{w}$ human gestation) were exposed to antenatal steroids, perinatal surfactant, and resuscitated and supported by mechanical ventilation for 6-7d (Drager VN500, SIMV). Physiological targets were $\mathrm{PaO}_{2} 60-90 \mathrm{mmHg}, \mathrm{PaCO}_{2} 45-60 \mathrm{mmHg}, \mathrm{O}_{2}$ saturation $88-92 \%$, pH 7.25-7.35. One group was treated with $\mathrm{MEx}$ $\left(60 \times 10^{6}\right.$ cell equivalents; $\left.10 \mathrm{~mL} ; \mathrm{n}=8 ; 4 \mathrm{~F} 4 \mathrm{M}\right)$ at hours of life 6 and 78 (iv); the control group received vehicle (MEx diluent in saline; $10 \mathrm{~mL} ; \mathrm{n}=8 ; 4 \mathrm{~F} 4 \mathrm{M}$ ). We used morphometry and stereology to quantify structural indices of alveolar formation, and immunoblot to quantify apoptosis (cleaved caspase 3) and proliferation (PCNA).

Summary of Results Radial alveolar count and secondary septal volume density were significantly greater $(* \mathrm{p}<0.05)$ in the MEx-treated group compared to the control group (figure $1 \mathrm{~A}$ and B). Distal airspace wall thickness was significantly narrower in the MEx-treated group compared to the control
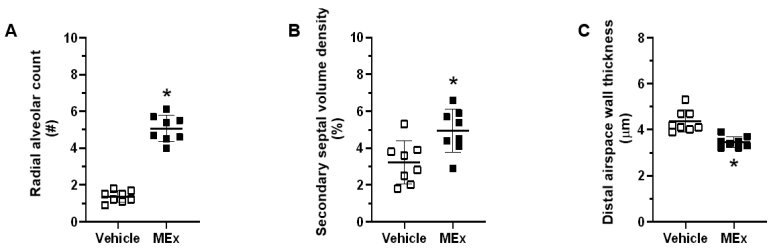

Abstract \#64 Figure 1 Quantitative morphological results show that alveolar formation is signficantly better (* $p$ group (figure 1C). Normalized cleaved caspase 3 protein abundance was not different between the MEx-treated and control groups $(0.71 \pm 0.05$ vs $0.69 \pm 0.04$, respectively). Normalized PCNA protein abundance was significantly lower in the MExtreated group versus the control group $(0.43 \pm 0.05$ vs 0.55 \pm 0.05 , respectively). No differences were detected between females and males.

Conclusions We conclude that MEx improved alveolar formation in chronically mechanically ventilated preterm lambs. We speculate that MEx may be an effective therapy to promote normal structural development of the lung in preterm infants who require mechanical ventilation and are at-risk of developing BPD.

\section{\#65 REGENERATIVE RESPONSE OF ALVEOLAR TYPE 2 CELLS TO A GENETICALLY-INDUCED MOUSE PHENOCOPY OF BRONCHOPULMONARY DYSPLASIA}

${ }^{1,2}$ GA Kohbodi* ${ }^{1,2} \mathrm{~F}$ Gao, ${ }^{1,2} \mathrm{C} \mathrm{Li},{ }^{1,2} \mathrm{~N}$ Peinado, ${ }^{1} \mathrm{R}$ Ramanathan, ${ }^{1,2} \mathrm{P}$ Minoo. ${ }^{1}$ Los Angeles County University of Southern California Medical Center, Los Angeles, CA; ${ }^{2}$ University of Southern California Keck School of Medicine, Los Angeles, CA

\subsection{6/jim-2022-WRMC.64}

Purpose of Study In adult lung, alveolar type II cells (AT2s) serve as facultative stem cells. They proliferate in response to injuries to regenerate and repair the alveoli. There is lack of information on whether AT2s in immature lungs undergoing alveologenesis, such as those of preterm neonates may act as resident stem cells and undergo proliferation in response to injuries that cause BPD. Genetic inactivation of both TGF $\beta$ receptors in secondary crest myofibroblasts (SCMF) arrested alveologenesis causing a BPD phenocopy. Alveolar arrest was accompanied by decreased number of SCMF and AT2s, thus suggesting cross-communication between the two cell types during alveologenesis. To determine the mechanism, we quantified AT2 cell numbers in control and mutant lungs at postnatal days 7 and 14 (PN7 and PN14) during alveologenesis.

Methods Used A total of 12 mouse lungs, (control, $n=6$, and mutant, $n=6)$ were examined at PN7 and PN14 ( $n=3$ for each control and mutant.) Immuno-histochemistry and immuno-fluorescence were performed on multiple samples of lung tissues. AT2s were identified as SPC positive cells. Proliferating AT2s (pAT2s) were identified as SPC; KI67 double positive cell. To correct for hypoplasia in BPD samples, all results were normalized against total lung cells, identified as DAPI positive.

Summary of Results In PN7 lungs, the ratio of AT2s/total cells (SPC+/DAPI+) was higher in mutant vs control (AT2s: $10.77 \%$ vs 8.58 , respectively) likely due to reduction in DAPI + cells that included reduced SCMF. The pAT2s remained unchanged (pAT2: mutant $0.47 \%$ vs control 0.54\%). In contrast, in PN14 lungs, both total AT2s, and pAT2s decreased in the mutant lungs vs control (total AT2s: $8.62 \%$ vs $10.54 \%$, respectively) and (pAT2: $0.50 \%$ vs $0.93 \%$, respectively) indicating that proliferation of both SCMF and AT2s has decreased.

Conclusions In mutant lungs, TGF $\beta$ receptors inactivation decreases SCMF numbers, while AT2s are unaffected in early phases of BPD-like pathogenesis. As the phenotype and loss of SCMF become more established and widespread, inhibition of AT2 proliferation becomes measurable. Two conclusions are derived from these observations: 1) targeted SCMF have a regulatory impact on AT2 proliferation, which is a 
regenerative response to injury. 2) Despite important differences between mouse phenocopy and human BPD, similar dynamics may occur in the lungs of preterm infants who develop BPD. Response of the endogenous stem cells (i.e. AT2s) in the lung to initial injuries may be governed by an orthologous mesenchymal cell type (SCMF-like) and their communications with AT2 stem cells.

Supported by NHLBI, NIH and the Hastings Foundation

\section{\#66 MESENCHYMAL STROMAL CELL EXTRACELLULAR VESICLES IMPROVE RESPIRATORY SYSTEM PHYSIOLOGICAL OUTCOMES IN MECHANICALLY VENTILATED PRETERM LAMBS}

${ }^{1}$ A Rebentisch*, 'E Dawson, 'E Major, 'H Foreman, 'D Headden, 'Z Vordos, ${ }^{1} M J$ Dahl, ${ }^{2} \mathrm{D}$ Null, ${ }^{3} \mathrm{~A}$ Mitsialis, ${ }^{3} \mathrm{~S}$ Kourembanas, ${ }^{1} \mathrm{~K}$ Albertine. ${ }^{1}$ The University of Utah School of Medicine, Salt Lake City, UT; ${ }^{2}$ University of California Davis, Davis, CA; ${ }^{3}$ Harvard Medical School, Boston, MA

\subsection{6/jim-2022-WRMC.65}

Purpose of Study Mesenchymal stromal cell extracellular vesicle (MEx) treatment has therapeutic efficacy in murine neonatal hyperoxia models of bronchopulmonary dysplasia (BPD). Whether MEx will be beneficial in chronically ventilated preterm neonates is unknown. We tested the hypothesis that MEx will improve respiratory system physiological outcomes in chronically mechanically ventilated preterm lambs.

Methods Used Preterm lambs (128d; term $\sim 150 \mathrm{~d}$; $\sim 28 \mathrm{w}$ human gestation) were exposed to antenatal steroids, perinatal surfactant, and resuscitated and supported by mechanical ventilation for 6-7d (Drager VN500, SIMV). Physiological targets were $\mathrm{PaO}_{2} 60-90 \mathrm{mmHg}, \mathrm{PaCO}_{2} 45-60 \mathrm{mmHg}, \mathrm{O}_{2}$ saturation 88-92\%, pH 7.25-7.35. One group was treated with $\mathrm{MEx}$ $\left(60 \times 10^{6}\right.$ cell equivalents; $\left.10 \mathrm{~mL} ; \mathrm{n}=8 ; 4 \mathrm{~F} 4 \mathrm{M}\right)$ at hours of life 6 and 78 (iv); the control group received vehicle (MEx diluent in sterile saline; $10 \mathrm{~mL} ; \mathrm{n}=8 ; 4 \mathrm{~F} 4 \mathrm{M}$ ). We report daily physiological outcomes for respiratory severity score (RSS), oxygenation index (OI), Arterial-alveolar (A-a) gradient, and oxygen saturation/ $/ \mathrm{FiO}_{2}(\mathrm{~S} / \mathrm{F})$ ratio. Liver and kidney function tests were assessed.

Summary of Results MEx-treated preterm lambs were $\sim 1 \mathrm{~d}$ younger (* $\mathrm{p}<0.05)$ and weighed less $\left(^{*}\right)$ at delivery than control lambs (figure $1 \mathrm{~A}$ and $\mathrm{B}$ ). MEx-treated lambs tolerated enteral feeding and maintained weight (*) whereas control lambs were less tolerant of enteral feedings and lost weight over 7d (figure 1B). RSS, OI, and A-a gradient were lower for MEx-treated group (*) compared to the control group. S/ F ratio was higher for the MEx-treated group (*) compared to the control group. Neither liver nor kidney toxicity was detected. Differences were detected between females and males.

Conclusions We conclude that MEx improved respiratory system physiological outcomes in chronically mechanically ventilated preterm lambs. We speculate that MEx may be an effective therapy for appropriate functional development of the lung in preterm infants who require mechanical ventilation.

\section{\#67 OXIDIZED PHOSPHOLIPID NEUTRALIZING ANTIBODY AMELIORATES HYPEROXIA INDUCED LUNG INJURY}

${ }^{1}$ W Tang ${ }^{*},{ }^{1,2} X$ Sun, ${ }^{1} \mathrm{JL}$ Witztum, ${ }^{1} \mathrm{C}$ Glass, ${ }^{1} \mathrm{E}$ Sajti. ${ }^{1}$ University of California San Diego, La Jolla, CA; ${ }^{2}$ The University of Texas Health Science Center at San Antonio, San Antonio, TX

\subsection{6/jim-2022-WRMC.66}

Purpose of Study Oxidized phospholipids (OxPL) are formed during inflammatory processes, and they are known to induce cellular stress and apoptosis. The role OxPL play in lung inflammation is not known. OxPL are recognized by the IgM natural antibody $(\mathrm{Ab})$ E06, which can bind to and block many of the pro-inflammatory properties of OxPL.To investigate the role of OxPL in hyperoxia-induced acute lung injury (HALI) and whether neutralizing OxPL using E06 would ameliorate or prevent hyperoxia induced lung injury.

Methods Used C57BL/6J (B6) sensitive and DBA/2J (DBA) resistant mice were exposed to hyperoxia for $48 \mathrm{~h}$ to induce lung injury. We examined the content of OxPL by immunochemistry with E06 and examined inflammatory responses by measuring changes in immune cell composition in the lung by fluorescence-activated flow cytometry and by immunohistochemistry. We measured gene expression changes in whole lung by RNA-seq. Data were analyzed with FlowJo and HOMER. To examine the pathogenic role of OxPL, we also exposed E06-scFv transgenic mice to hyperoxia. These mice generate a high plasma level of functional E06-scFv (singlechain variable fragment of E06).

Summary of Results Using immunohistochemistry, we observed an accumulation of OxPL in the lungs of sensitive B6 mice after hyperoxia. OxPL were more abundant in lungs of B6 mice compared to resistant DBA mice. To further explore the
A

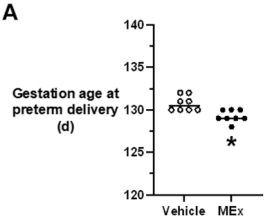

D

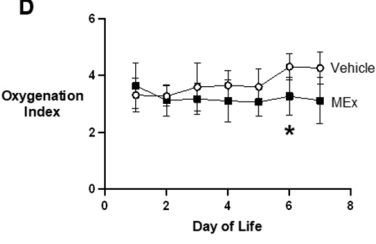

B
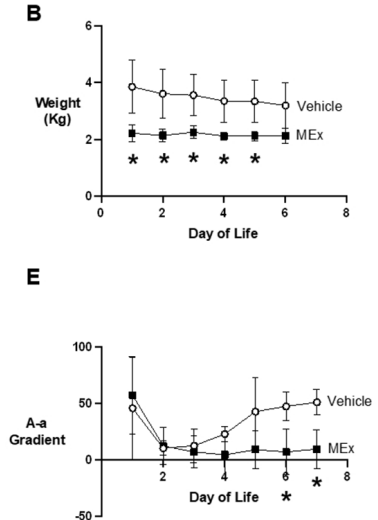

C

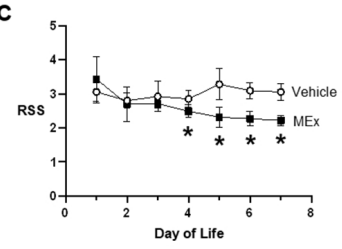

$\mathbf{F}$

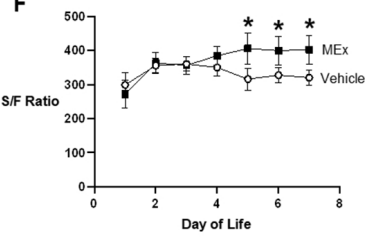

Abstract \#66 Figure 1 Respiratory system physiological outcomes for preterm lambs treated with MEx (black circles) or vehicle (white circles) 
molecular determinants of interstrain susceptibility to oxygen, we performed transcriptomic analysis of the whole lung. Transcripts that most distinguished B6 from DBA mice were associated with apoptotic and cell death pathways. To test whether OxPL have a pathogenic role, we exposed E06-scFv mice-on B6 background-to same hyperoxic conditions. Unlike B6 mice, E06-scFv mice did not show activation of apoptosis and cell death related gene pathways.

Conclusions We observed significant increases in OxPL accumulation following acute hyperoxia exposure in the lungs of injury sensitive compared to resistant mice. OxPL accumulation in the lungs of B6 mice was associated with upregulation of apoptosis and cell death related genes. Blocking of OxPL by the secreted $\mathrm{E} 06-\mathrm{scFv} \mathrm{Ab}$ resulted in a significant reduction of apoptosis protecting the lung from HALI. These data suggest that OxPL are not only a useful biomarker for hyperoxia induced lung injury but that an OxPL neutralizing antibody could be used to ameliorate or prevent HALI. Furthermore, the magnitude of interstrain variability in lung gene expression could form the basis for understanding human interindividual variability in susceptibility to oxygen induced injury.

\section{\#68 ASYNCHRONOUS CHEST COMPRESSIONS WITH HIGH FREQUENCY VENTILATION IMPROVES GAS EXCHANGE DELIVERY IN ASPHYXIATED CARDIAC ARRESTED PRETERM LAMBS}

${ }^{1}$ E Giusto*, ${ }^{2} \mathrm{~A}$ Lesneski, ${ }^{2} \mathrm{H}$ Joudi, ${ }^{2} \mathrm{M}$ Hardie, ${ }^{2} \mathrm{LI}$ Zeinali, ${ }^{2} \mathrm{D}$ Sankaran, ${ }^{2} \mathrm{~S}$ Lakshminrusimha, ${ }^{2} \mathrm{P}$ Vali. ' University of California Davis, Sacramento, CA; ${ }^{2}$ University of California Davis, Davis, CA

\subsection{6/jim-2022-WRMC.67}

Purpose of Study Gas exchange is severely impaired during cardiopulmonary resuscitation (CPR) in the cardiac arrested lamb model despite ventilation with $100 \% \quad \mathrm{O}_{2}$. Optimizing gas exchange during neonatal CPR may improve cerebral oxygen delivery $\left(\mathrm{cDO}_{2}\right)$, prevent rapid fluctuations in $\mathrm{PaCO}_{2}$, and stabilize cerebral blood flow. We hypothesize that asynchronous continuous chest compressions with high frequency percussive ventilation (HFPV) in preterm asphyxiated cardiac arrested lambs will result in improved gas exchange and $\mathrm{cDO}_{2} \mathrm{com}$ pared to 3:1 compression-to-ventilation $(\mathrm{C}: \mathrm{V})$ resuscitation.

Methods Used Time-dated preterm ( $\sim 125 \mathrm{~d}$ gestation; equivalent human $\sim 25$ weeks) fetal lambs were intubated, instrumented to measure cerebral blood flow and arterial blood pressure, and catheterized to collect venous and arterial blood. After instrumentation, lambs were asphyxiated by umbilical
Abstract \#68 Table 1 Comparison of blood gases at fixed timepoints

\begin{tabular}{|c|c|c|c|c|c|}
\hline \multicolumn{6}{|c|}{ Control Group (3:1 C:V resuscitation) } \\
\hline Time Point & $\begin{array}{l}\mathrm{PaCO} 2 \\
(\mathrm{~mm} \mathrm{Hg})\end{array}$ & $\begin{array}{l}\mathrm{PaO2} \\
(\mathrm{mm} \mathrm{Hg})\end{array}$ & $\begin{array}{l}\mathrm{CaCO} 2(\mathrm{~mL} \\
02 / \mathrm{dL})\end{array}$ & $\begin{array}{l}\text { Brain DO2 (mL } \\
02 / \mathrm{kg} / \mathrm{dL})^{*}\end{array}$ & $\mathrm{FiO2}$ \\
\hline $\begin{array}{l}\text { During Chest } \\
\text { Compressions }\end{array}$ & $153(11)$ & $13(15)$ & $2.38(3.2)$ & $0.05(0.06)$ & 1.0 \\
\hline At ROSC & $153(11)$ & $26(24)$ & $4.58(4.8)$ & $0.62(0.59)$ & 1.0 \\
\hline $\begin{array}{l}15 \text { minutes post- } \\
\text { ROSC }\end{array}$ & $158(24)$ & $47(42)$ & $7.05(6.6)$ & $1.97(2.21)$ & 1.0 \\
\hline Intervention Group & continuous & ynchronous & hest compres & ons with HFPV) & \\
\hline $\begin{array}{l}\text { During Chest } \\
\text { Compressions }\end{array}$ & $110(5)$ & $22(3.1)$ & $5.04(1.8)$ & $0.12(0.11)$ & 1.0 \\
\hline At ROSC & $85(20)$ & $57(19)$ & $13(1.4)$ & $3.8(0.14)$ & 1 \\
\hline $\begin{array}{l}15 \text { minutes post- } \\
\text { ROSC }\end{array}$ & $50(11)$ & $60(24)$ & $13.7(1.5)$ & $2.42(0.71)$ & $\begin{array}{l}0.48 \\
(0.21)\end{array}$ \\
\hline
\end{tabular}

Data are mean $(S D){ }^{*} n=2$ for intervention group

cord occlusion until asystole and delivered. Lambs were randomized to (1) 3:1 C:V resuscitation using a T-piece resuscitator following the neonatal resuscitation program (NRP) algorithm (control), or (2) asynchronous continuous chest compressions (120 compressions/min) with HFPV using a TXP-5 ventilator (intervention). First dose of epinephrine $(0.03 \mathrm{mg} / \mathrm{kg})$ was given at three minutes and repeated $\mathrm{q} 3 \mathrm{~min}$ until return of spontaneous circulation (ROSC). Lambs in the control group that achieved ROSC were managed on conventional ventilation and lambs in the intervention group were maintained on HFPV. Ventilation parameters and $\mathrm{O}_{2}$ were adjusted to maintain $\mathrm{SpO}_{2}$ at $90-95 \%$ and $\mathrm{PaCO}_{2}$ between 45-60 mmHg.

Summary of Results Eight lambs were studied and all achieved ROSC. Baseline characteristics, time to ROSC, and epinephrine doses were similar between groups (figure 1). Mean (SD) $\mathrm{PaCO}_{2}$ was 158 (24) $\mathrm{mmHg}$ and the mean (SD) $\mathrm{PaO}_{2}$ was 47 (42) $\mathrm{mmHg} 15$ minutes post-ROSC despite maximum ventilation support and $100 \% \mathrm{O}_{2}$ in the control group compared to a mean (SD) $\mathrm{PaCO}_{2}$ of 50 (11) $\mathrm{mmHg}$ and $\mathrm{a}_{2} \mathrm{PaO}_{2}$ of 60 (24) $\mathrm{mmHg}$ in the intervention group (table 1).

Conclusions Resuscitation using asynchronous continuous chest compressions during HFVP is feasible with similar success of ROSC and improved gas exchange in an asphyxiated cardiac arrested neonatal lamb model. Further studies are required to validate our results and to assess lung injury by immunohistochemistry and biomarkers.

\begin{tabular}{|c|c|c|c|}
\hline Parameters & Control 3:1 (N = 4) & Intervention HFPV (N = 4) & P-Value \\
\hline Weight & $2.89(0.21)$ & $2.69(0.46)$ & 0.47 \\
\hline Time to Asystole (min) & $28(8.5)$ & $18(1.3)$ & 0.09 \\
\hline Aphsyxia pH & $6.80(0.08)$ & $6.83(0.04)$ & 0.59 \\
\hline Asphyxia $\mathrm{PaO}_{2}$ & Undectable & Undectable & N/A \\
\hline Asphyxia $\mathrm{PaCO}_{2}$ & $145(14)$ & $137(12)$ & 0.40 \\
\hline Asphyxia Lactate & $9(1.3)$ & $11(3.9)$ & 0.40 \\
\hline ROSC success & $100 \%$ & $100 \%$ & N/A \\
\hline Time to ROSC (min) & $4.4(1.8)$ & $4.1(0.6)$ & 0.61 \\
\hline \# of Epi Doses & 1 & $1.25(0.5)$ & 0.39 \\
\hline
\end{tabular}

Abstract \#68 Figure 1 Comparison of baseline characteristics between groups. There was no significant difference of characteristics between groups $(p>0.05)$ 


\section{MECHANICAL FUNCTION IN NEONATAL RATS}

E Bye*, T Gonzalez, G Seedorf, B Smith, N Galambos, SH Abman, E Mandell. University of Colorado Denver School of Medicine, Aurora, CO

\subsection{6/jim-2022-WRMC.68}

Purpose of Study Continuous positive airway pressure (CPAP) is an increasingly common method of non-invasive respiratory support for premature infants to avoid more invasive and potentially injurious ventilation strategies. However, the longterm effects of CPAP on the developing lung are poorly understood. Therefore, we seek to understand the effect of daily CPAP on the structure and mechanical function of the developing lung in neonatal rats.

Methods Used Control dams were kept in room air and allowed to give birth spontaneously. At day 1, pups were divided into three groups: pups that were secured to CPAP device and receiving daily positive pressure of $6 \mathrm{~cm} \mathrm{H}_{2} \mathrm{O}$ (CPAP-6); pups that were secured to CPAP device and receiving daily airflow but no pressure (CTL-0); and pups that were simply removed from their cage without being secured to the CPAP device for the duration of daily experiments (CTL). Daily CPAP lasted for 2 hours on days $1-2$, and 3 hours on days 3-13. At day 14, we measured lung mechanics by flexiVent (total respiratory system resistance (Rrs) and compliance (Crs)). Lung structure was determined by mean linear intercepts (MLI), radial alveolar counts (RAC), and pulmonary vessel density (PVD).

Summary of Results There were no differences in body weights between groups. CPAP-6 rats demonstrated decreased Rrs $(\mathrm{p}<0.05)$ compared to CTL and CTL-0, and increased Crs $(\mathrm{p}<0.01)$ when compared to CTL. Lungs from CPAP-6 rats showed impaired alveolarization compared to CTL as assessed by decreased RAC $(p<0.05)$ and increased MLI $(p<0.001)$. Pulmonary vessel density was reduced in CPAP-6 vs CTL rats $(p<0.01)$. There were no significant differences in lung structure between CPAP-0 rats with CTL or CPAP-6 rats.

Conclusions We found that daily CPAP decreased alveolar and vascular growth and altered lung mechanics in infant rats. We speculate that although less invasive than other ventilation strategies, non-invasive positive pressure respiratory support can potentially have negative effects on the normal developing lung, but its net benefits or harm in the setting of lung disease remains uncertain.
Surgery I

\section{Concurrent session}

\section{2:45 PM}

\section{Thursday, January 20, 2022}

\section{$\# 70$ HEMOGLOBIN A1C AS A PROGNOSTIC INDICATOR OF POSTOPERATIVE INFECTION FOLLOWING IMMEDIATE BREAST CANCER RECONSTRUCTION}

CA Czaykowsky*, S Gupta. Loma Linda University School of Medicine, Loma Linda, CA

\subsection{6/jim-2022-WRMC.69}

Purpose of Study Hemoglobin A1c (HgbA1c) is a marker of an individual's glycemic exposure over a preceding 2-3 month period. Minimal evidence currently exists to support increased infection risk following mastectomy. We aimed to evaluate the association of HgbA1c with the incidence of surgical site infection (SSI) in patients undergoing mastectomy and immediate breast reconstruction.

Methods Used An institutional database was queried for patients with CPT code for reconstruction AND diagnosis code for breast malignancy for patients from January 1, 2014 to June 20, 2021. We defined SSI incidence by diagnosis or procedure for SSI within 90 days following mastectomy. A one sample t-test was performed to determine if there is a significance difference in the average HgbA1c of the standard patient population and the sample SSI group. A chi-square test was used to analyze data for correlations between SSI rates in diabetics and non-diabetics. The patients were analyzed using a standard chi-square based on a $2 \times 2$ contingency table.

Summary of Results A total of 1386 patients were included in the query; 268 of which having received a pre-op HgbA1c and with 136 having received a pre-op HgbA1c and a DM diagnosis. Only 22 patients fit our defined SSI sample group criteria. The average population HgbA1c was $6.74(\mathrm{~N}=268)$, the average HgbA1c of diabetics was $7.33(\mathrm{~N}=136)$, the average HgbA1c of non-diabetics was $6.13(\mathrm{~N}=132)$. The average sample HgbA1c was $6.94(\mathrm{~N}=22)$, the average HgbA1c of diabetics was $7.34(\mathrm{~N}=17)$, the average HgbA1c of non-diabetics was $5.58(\mathrm{~N}=5)$. The one sample t-test of the average HgbA1c value in patients with DM vs nondiabetics in the

\begin{tabular}{|c|c|c|c|}
\hline $\begin{array}{l}\text { Mastectomy Patients with Post Surgical Breast Malignancy } \\
\text { (January 1, } 2014 \text { to June 30, 2021) }\end{array}$ & & $\begin{array}{l}\text { Sampling of Patients with Surgical Site Infection } \\
\text { (January 1, } 2014 \text { to June } 30,2021 \text { ) }\end{array}$ & \\
\hline CPT code for reconstruction AND Diagnosis code for breast malignancy & $N=1386$ & CPT code for reconstruction AND Diagnosis code for breast malignancy & $N=22$ \\
\hline Patients with 'Diabetes' diagnosis & $N=181$ & Patients with 'Diabetes' diagnosis & $N=17$ \\
\hline Patients with pre-op HgbA1C & $N=268$ & Patients with pre-op HgbA1C & $N=22$ \\
\hline Patients with Diabetes diagnosis AND pre-op HgbA1C & $N=136$ & Patients with Diabetes diagnosis AND pre-op HgbA1C & $\mathrm{N}=17$ \\
\hline Average value of $\mathrm{HgbA1C}(\%)$ & $\begin{array}{l}6.74 \\
(N=268)\end{array}$ & Average value of $\mathrm{HgbA1C}(\%)$ & $\begin{array}{l}6.94 \\
(N=22)\end{array}$ \\
\hline Average value of $\mathrm{HgbA1C}$ in patients with Diabetes diagnosis (\%) & $\begin{array}{l}7.33 \\
(N=136)\end{array}$ & Average value of $\mathrm{HgbA1C}$ in patients with Diabetes diagnosis (\%) & $\begin{array}{l}7.34 \\
(N=17)\end{array}$ \\
\hline Average value of HgbA1c in non-diabetic patients (\%) & $\begin{array}{l}6.13 \\
(N=132)\end{array}$ & Average value of HgbA1c in non-diabetic patients (\%) & $\begin{array}{l}5.58 \\
(\mathrm{~N}=5)\end{array}$ \\
\hline
\end{tabular}

Data are presented as mean values or as column percentage. 
sample group was not significant, $t(21)=-4.210, p=3.94 \mathrm{E}-$ 4. The chi-square test revealed diabetics more likely than nondiabetics to develop SSI following mastectomy, $X^{2}(1, N=$ 1254) $=76.43, p<.001$.

Conclusions The association of HgbA1c with the incidence of surgical site infection (SSI) following undergoing mastectomy is evident. We found the presence of a DM diagnosis as a better prognostic tool for SSI than HgbA1c level alone. Chisquare analysis determined the relative risk of SSI following mastectomy in diabetic patients at $2.28 \mathrm{x}$ that of a nondiabetic. Considering the SSI sample group, a HgbA1c threshold of 7.34 is what we propose as presenting great additional postsurgical complication risk.

\section{$\# 71$ THE IMPACT OF THE COVID-19 PANDEMIC ON SPINE SURGERY PRACTICE AND OUTCOMES IN AN URBAN HEALTHCARE SYSTEM}

J Parekh, B Attaripour, SY Xiang*, M Siow, B Mitchell, B Shahidi. University of California San Diego, University of California San Diego, La Jolla, CA, San Diego, CA

\subsection{6/jim-2022-WRMC.70}

Purpose of Study To elucidate changes due to COVID-19 on patient demographics, surgical care, logistics, and patient outcomes in spine patients.

Methods Used This is a retrospective study of patients who had spine surgery at UCSD from $3 / 1 / 19$ to $5 / 31 / 19$ (preCOVID-19) and $3 / 1 / 20$ to $5 / 31 / 20$ (first COVID-19 surge). 331 subjects met the study criteria. Demographic and surgical data were collected from medical records. Pain levels at preoperative, discharge, short- (3-6 month) and long-term(9-15 month) timepoints were extracted.

Summary of Results There were no significant differences in patient demographics including age, BMI, gender, race, ethnicity, ASA rating, smoking status, or diabetes status between groups $(\mathrm{p}>0.14)$. The diagnostic indications for surgery (spondylolisthesis, tumor/infection, spondylosis, fracture) were not different between groups ( $p>0.13$ ). There were no differences in operating room duration and skin-to-skin time $(\mathrm{p}>0.64)$, however length of stay was 4.7 days shorter during the COVID-19 pandemic $(p=0.03)$ and more cases were classified as 'urgent' $(p=0.04)$. Preoperative pain scores did not differ

\begin{tabular}{lllll} 
Abstract \#71 Table 1 & & & \\
\hline Length of Stay & & Pre-Covid-19 & During Covid-19 & P-value \\
\cline { 2 - 4 } & & $10.23(28.3)$ & $5.57(6.8)$ & 0.032 \\
\hline Estimated Blood Loss & & $125.7(174.6)$ & $173.5(264.2)$ & 0.064 \\
Minutes in OR & & $317.9(158.5)$ & $309.45(176.1)$ & 0.678 \\
Minutes skin-to-skin & & $223.7(137.2)$ & $216.3(144.6)$ & 0.641 \\
Discharge Disposition N(\%) & Home & $131(70.8 \%)$ & $110(75.3 \%)$ & 0.400 \\
& Nursing Facility & $34(18.4 \%)$ & $27(18.5 \%)$ & \\
Type of Case N(\%) & Other & $14(7.6 \%)$ & $6(4.1 \%)$ & \\
Preoperative Pain & Non-Urgent & $150(81.1 \%)$ & $104(71.2 \%)$ & 0.035 \\
Discharge Pain & Urgent & $35(18.9 \%)$ & $42(28.8 \%)$ & \\
Short Term Pain & & $5.9(2.9)$ & $6.0(2.8)$ & 0.583 \\
\hline
\end{tabular}

between groups $(p=0.58)$, however pain levels at discharge were significantly higher in patients operated upon during COVID $(p=0.04)$ and trended towards remaining higher in the short- $(p=0.06)$ but not long-term $(p=0.21)$ after surgery (table 1).

Conclusions The pandemic resulted in a greater proportion of 'urgent' spine surgery cases and shorter hospital length of stay. Pain levels upon discharge and at short-term timepoints were higher following surgery, however these differences did not persist in the long term.

\section{\#72 MEASURING THE CLINICAL VALUE OF SECONDARY HAND SURGERY IN PATIENTS WITH COMPLEX HAND INJURIES}

${ }^{1}$ BC Goodlin*, ${ }^{1}$ CA Czaykowsky, ${ }^{2}$ S Gupta. 'Loma Linda University School of Medicine, Loma Linda, CA; ${ }^{2}$ Loma Linda University, Loma Linda, CA

\subsection{6/jim-2022-WRMC.71}

Purpose of Study The objective of this study is to assess the value of performing secondary hand surgery in a population of complex hand procedures at a quaternary referral site academic medical center.

Methods Used This was done by obtaining electronic medical records (EMR) of 166 patients over a five year period using specific keywords relating to the types of surgeries generally performed as secondary procedures such as tenolysis, contracture release, and capsulotomies. Of those patients, 50 were found to fit within the parameters of the study. For these 50 remaining patients, hand therapy data was obtained and the percentage of motion that each patient had before the secondary surgery and following the secondary surgery were calculated and this data was used to calculate the overall change in motion. A paired sample t-test was performed to determine if there is a significant difference in the average motion data in the measurements taken before and after secondary hand surgeries. One sample t-tests were performed to determine if there is a significance difference in the average change in the range of motion between common comorbidities (asthma, diabetes mellitus, hypertension, obesity, age over 45 years) or gender between the patient population and each subgroup.

Summary of Results During a median follow-up period of 8.5 months and an average follow-up period of 18.87 months, a total of 75 complications in 50 patients were recorded. The average percentage of full motion before surgery was $49.43 \%$ and the average percentage of full motion after surgery was $66 \%$ to give an overall change in motion to be $+16.58 \%$. Hand motion measurements following secondary hand surgery $(M=0.491, S D=0.188)$ compared to the hand motion measurements preceding secondary hand surgery $(M=0.660$, $S D=0.259$ ) demonstrated a significantly better change in motion percent change, $t(50)=24.50, p=4.177 \mathrm{E}-5$.

Conclusions Although we speculate that we would have seen an even greater impact from secondary hand surgeries if there had been greater adherence to post-surgical hand therapy, our $\mathrm{p}$ value indicates results that are statistically significant. Therefore, we conclude that secondary hand surgery performed on patients with complex hand injuries has a significant measurable impact and we believe that a similar study in a larger population would yield similar results. 
\#73 ASSOCIATION OF THE GERIATRIC NUTRITION RISK INDEX AND POSTOPERATIVE COMPLICATIONS IN HEAD AND NECK CANCER

J Chen*, M Abouyared. University of California Davis, Sacramento, CA

10.1136/jim-2022-WRMC.72

Purpose of Study Malnutrition is associated with increased morbidity and mortality in patients with head and neck cancer (HNC) undergoing surgery. Despite the profound impact malnutrition has on this patient population, objective screening tools are still lacking in a clinical setting. Without a clear approach to identify malnutrition, there is currently a barrier to capturing patients with inadequate nutrition, delaying interventions that could otherwise be implemented to optimize their nutritional status. Therefore, recognizing the need for a tool, the aim of this study is to assess the ability to use the geriatric nutrition risk index (GNRI) to screen for malnutrition among HNC patients and determine if there is an association between GNRI scores and postoperative complications.

Methods Used A retrospective review of medical records was conducted for patients undergoing surgical resection at a tertiary academic hospital from June 2012 to June 2021. Patients were included if surgical excision was the primary treatment modality and if a serum albumin was obtained 6 months prior to their surgery. A total of $44 \mathrm{HNC}$ patients were included in the study and analysis. Preoperative body weight and serum

$\begin{aligned} & \text { Abstract \#73 Table } 1 \\
& \text { high GNRI scores }\end{aligned}$
\begin{tabular}{llll} 
& CNRI $<97.5$ & GNRI $\geq 97.5$ & P Value \\
& $\mathrm{N}=12$ & $\mathrm{~N}=32$ & \\
\hline Number of patients with complications (\%) & $8(67)$ & $6(19)$ & 0.016 \\
Return to the OR (\%) & $5(42)$ & $4(13)$ & 0.087 \\
Total LOS, day (mean \pm SD) & $18 \pm 23$ & $7 \pm 7$ & 0.119 \\
Discharge to SNF & $4(33)$ & $2(6)$ & 0.039 \\
\hline GNRI, geriatric nutrition risk index. & & &
\end{tabular}

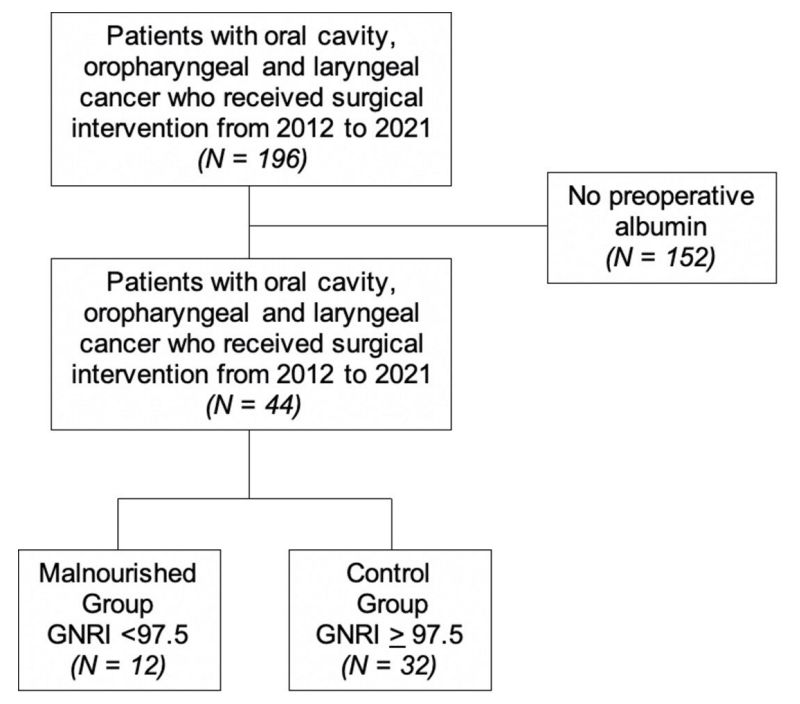

Abstract \#73 Figure 1 albumin were abstracted from medical records to calculate the GNRI.

Summary of Results Of the 44 patients included in the study, there were 30 men (68\%) and 14 women (32\%), with a total mean age of $62 \pm 12$ years. Malnutrition was defined by a GNRI score of $<97.5$ and was present in $27 \%$ of patients $(n=12)$. Malnourished patients had significantly higher rates of postoperative complications and required discharge to a skilled nursing facility (SNF) more often compared to the control group.

Conclusions A low GNRI score appears to be a predictor of increased complications after head and neck surgery. The GNRI is a simple tool that utilizes serum albumin and body weight to objectively assess nutritional status. Results from this study suggest that, in the future, the GNRI may be a clinically useful approach to screen for malnutrition and identify patients who are at high risk for complications during the postoperative course.

\section{\#74 DEMOGRAPHICS, FRACTURE CHARACTERISTICS, AND TREATMENT STRATEGIES FOR PERIPROSTHETIC DISTAL FEMUR FRACTURES COMPARED TO NATIVE DISTAL FEMUR FRACTURES}

${ }^{1} S$ Kong*, ${ }^{1}$ B Bautista, ${ }^{2,3}$ A Saiz, ${ }^{2}$ MR Haffner, ${ }^{2} \mathrm{~J}$ Kelley, ${ }^{2}$ MA Lee. ${ }^{1}$ University of California Davis, Sacramento, CA; ${ }^{2}$ University of California Davis Health System Department of Orthopaedic Surgery, Sacramento, CA; ${ }^{3}$ The University of Texas Health Science Center at Houston, Houston, TX

\subsection{6/jim-2022-WRMC.73}

Purpose of Study The incidence of periprosthetic distal femur fractures is increasing due to the increasing number of knee arthroplasties being performed in the aging population. The purpose of this study was to analyze the demographics, fracture characteristics, and treatment strategies associated with periprosthetic distal femur fractures (PDFF) compared to native distal femur fractures (NDFF) in order to identify important clinical differences between these groups that might help guide management.

Methods Used A retrospective study was conducted of 209 patients $>18$ years old who underwent surgical treatment for either a native distal femur fracture (NDFF) or a periprosthetic distal femur fracture (PDFF) about a total knee arthroplasty (TKA) from January, 2006 to December, 2020. Fracture classification of CT images by the Association for Osteosynthesis/Orthopedic Trauma Association (AO/OTA) was reported. Demographics, fracture characteristics, fixation constructs, and surgical outcomes were compared between subjects with PDFF vs. NDFF.

Summary of Results Out of 70 patients with PDFF, 81.1\% were female and $18.6 \%$ were male, with an average age of 80 years old (range $=49-102$ yrs). PDFFs were most often isolated $(80 \%)$ or comminuted $(85 \%)$ injuries with AO classification 33A.3 (71.4\%). Out of 139 patients with NDFF, $53.2 \%$ were female and $46.8 \%$ were male with an average age of 57 years old (range $=18-96$ yrs). NDFFs were commonly comminuted (92.1\%) injuries with AO classification 33C.2 (28.1\%) or 33A.3 (25.2\%). NDFFs were extra-articular $(54.0 \%)$ or intra-articular $(46.0 \%)$. Nearly half of subjects with NDFF (48.2\%) experienced concomitant fracture of the ipsilateral knee $(14.4 \%)$ or tibial plateau (15.1\%). Intramedullary nailing was the most common fixation construct for both fracture groups $(42.6 \%$ PDFF;36.7\% NDFF). The second 
most common fixation construct for PDFF was combined nail/ plate $(17.3 \%)$ and lateral locking plate (20.9\%) for NDFF. Patients with PDFF experienced shorter length-of-stays (6.36 days vs. 11.4 days) but had higher complication rates compared to NDFF $(5.7 \%$ vs $4.4 \%)$. Incidence of low bone density (osteopenia or osteoporosis) was higher in those with PDFF compared to NDFF (55.7\% vs. $19.4 \%$ ).

Conclusions PDFFs frequently occur as isolated comminuted injuries with greater complication rates compared to NDFF. Though intramedullary nailing remains the most common fixation construct for both NDFF and PDFF, stabilization via combined plate/nail is increasingly being used for PDFFs. Elderly women with TKA and poor bone quality are a high risk group for PDFF. Further research should entail how physicians can improve their surgical and clinical approach for this type of fracture in the affected population.

\section{$\# 75$ EFFECT OF ANTICOAGULANT AND ANTIPLATELET USE ON DERMATOLOGIC AND COSMETIC PROCEDURE INTRAOPERATIVE OUTCOMES}

M Gruber, E Foltz*. Washington State University Elson S Floyd College of Medicine, Pullman, WA

\subsection{6/jim-2022-WRMC.74}

Purpose of Study The use of antithrombotic medications in patients is widely utilized for numerous medical conditions. Research has demonstrated that antiplatelet and anticoagulant use can influence surgical outcomes as well as prolong intraoperative times. However, sparse literature exists examining the effects of antithrombotic use on dermatologic surgery outcomes, specifically in Mohs and cosmetic flap procedures. The purpose of this study is to elucidate the relationship of anticoagulant and antiplatelet therapy on intraoperative time and closure size in dermatologic and cosmetic surgery patients. We hypothesize that those who use daily antithrombotics will have resultantly longer intraoperative times and larger closure sizes in dermatologic flap procedures.

Methods Used A retrospective medical record review was conducted of all patients who underwent Mohs or cosmetic flap surgery at Chesnut Institute of Cosmetic \& Reconstructive Surgery in Spokane, Washington between March 5, 2019 and December 14, 2020. Procedures of 40 minutes duration or less were included. This yielded a total of 243 surgeries with complete information about intraoperative outcomes. Patients were stratified into 5 cohorts based on medication usage (table 1) with documentation of skin closure size and total procedure length.

\begin{tabular}{lll} 
Abstract \#75 Table 1 & \\
\hline Cohort & Medications & $\begin{array}{l}\text { Number of } \\
\text { Patients }\end{array}$ \\
\hline Cohort 1 & Supplements (Fish oil, krill oil, garlic, turmeric) & 12 \\
Cohort 2 & COX inhibitors & 104 \\
Cohort 3 & ADP inhibitors, phosphodiesterase inhibitors, glycoprotein Ilb/ & 11 \\
& IIla inhibitors & \\
Cohort 4 & Vitamin K antagonists, direct thrombin inhibitors, direct Xa & 25 \\
& inhibitors, indirect thrombin inhibitors & 91 \\
Cohort 5 & No antithrombotic medications & \\
\hline
\end{tabular}

Summary of Results A statistically significant reduction in intraoperative times (p-value 0.03) was observed in patients who were not taking any form of antithrombotic medication (cohort 5), as compared to patients in cohort 4 who were actively taking anticoagulant medications. Other classes of antithrombotic medication (cohorts 1-3) were associated with higher average intraoperative times relative to cohort 5, however the difference was not statistically significant. There was no statistically significant difference in closure size across the cohorts.

Conclusions The use of oral anticoagulants in patients undergoing Mohs and cosmetic flap surgeries results in significantly longer intraoperative times. Further investigation of this relationship and consideration of this finding may influence management of dermatologic and cosmetic procedures.

\section{\#76 MULTIMODAL PAIN CONTROL UTILIZING BUPRENORPHINE FOR ROBOTIC ASSISTED LAPAROSCOPIC PROSTATECTOMY: A QUALITY IMPROVEMENT COMPARISON TO CONVENTIONAL OPIOID MANAGEMENT}

${ }^{1} \mathrm{M}$ Hajiha, ${ }^{1} \mathrm{D}$ Lien*, ${ }^{1} \mathrm{G}$ Stier, ${ }^{1} \mathrm{~L}$ Soloniuk, ${ }^{1} \mathrm{H}$ Ruckle, ${ }^{1} \mathrm{~A}$ Amasyali, ${ }^{1} \mathrm{~K}$ Shete, ${ }^{1} \mathrm{M}$ Douglas, ${ }^{1} \mathrm{~J}$ Calvert, ${ }^{1} \mathrm{M}$ Chang, ${ }^{1} \mathrm{R}$ Tone, ${ }^{2} \mathrm{~J}$ Johnson, ${ }^{2}$ I Muchiutti, 'S Wraich. 'Loma Linda University Adventist Health Sciences Center, Loma Linda, CA; ${ }^{2}$ Loma Linda University School of Medicine, Loma Linda, CA

\subsection{6/jim-2022-WRMC.75}

Purpose of Study New persistent opioid use is recognized as a complication in both major and minor surgeries. Prolonged postoperative pain is often the impetus for patients seeking renewal of opioid prescriptions that can lead to persistent use and substance abuse. Clinicians have responsibilities to provide adequate pain relief and limit harmful unnecessary opioid use. By developing alternative analgesic pathways that are as effective as the opioid-inclusive analgesic protocol in managing post-operative pain levels, surgeons can decrease surgical patient's need and access to opioids in an uncontrolled home environment. Buprenorphine, a mu receptor agonist, has been used for acute and chronic pain management since U.S FDA approval in 1981. However, few studies have tested its efficacy in perioperative administration.

Methods Used Patients with localized prostate cancer scheduled for RALP were recruited to receive either of two pathways. Forty patients received the standard opioid pathway, and forty-one patients received the buprenorphine-inclusive pathway. In this novel pathway, intravenous buprenorphine was administered intraoperatively and as needed postoperatively. Post-operative analgesic management was as standard, while avoiding non-buprenorphine opiates. Patients were administered a questionnaire regarding their post-operative complications, pain level at discharge, and at-home analgesics used at five days post-op to monitor pain control. Our primary endpoint was adequate pain control, and our secondary endpoints were analgesic consumption at home, opioid-related side effects, and patient satisfaction.

Summary of Results There was no difference between the buprenorphine group and the conventional group in length of stay (1.1 vs 1.3 days, $p=0.18)$, pain control $(0-10$ scale) at the time of discharge $(5.2$ vs $5.7, \mathrm{p}=0.4)$ and overall patient satisfaction $(p=0.1)$. Our study demonstrates buprenorphine's analgesic capabilities to maintain non-inferior levels of pain control, length of stay, and patient satisfaction comparable to 
patients on opioid-inclusive analgesia during RALP while decreasing post-surgical and home opioid use.

Conclusions By markedly decreasing post-surgical opioids prescriptions, we can reduce the risk opioid addiction and the associated harm to the patient. This study is a proof of principle that buprenorphine use for perioperative analgesia during RALP is an alternative to traditional opioid-inclusive analgesic pathways. We believe such a strategy will decrease the incidence of opioid use disorder and have benefits including less associated healthcare spending, improved patient health and reduced social harm.

\section{\#77 RETROSPECTIVE COMPARISON OF LAMINOPLASTY VERSUS LAMINECTOMY WITH FUSION FOR TREATING CERVICAL SPONDYLOTIC MYELOPATHY}

${ }^{1} \mathrm{O}$ Bakr*, ${ }^{1} \mathrm{~B}$ Van, ${ }^{1} \mathrm{~B}$ Bautista, ${ }^{1} \mathrm{Z}$ Booze, ${ }^{2}$ RF Roberto. ' University of California Davis School of Medicine, Sacramento, CA; ${ }^{2}$ University of California Davis Health System, Sacramento, CA

\subsection{6/jim-2022-WRMC.76}

Purpose of Study This study compares the outcomes, features, and costs of laminectomy and fusion (LEF) versus laminoplasty (LP) as surgical treatments for patients with cervical spondylotic myelopathy (CSM).

Methods Used Elective LEF and LP procedures performed at a single institution between 2014 and 2020 were identified. Included patients had no prior cervical spine surgery. All patients received pre- and postoperative outpatient evaluations in the outpatient clinic. Only procedures involving three or more spinal levels were included. Clinical data was collected from electronic medical records. SPSS 27 was used for statistical analysis. Hospital costs were obtained from hospital billing for a subgroup of patients for whom this information was available.

Summary of Results 135 patients were included: 76 underwent LP and 59 underwent LEF. Mean follow-up time was 14 months. Compared to LEF, LP procedures involved fewer levels $(4.2$ vs 4.8 levels, $\mathrm{p}<.001)$ and trended shorter operative time per level (47 vs 62 minutes, $p<.001$ ). Intraoperative blood loss and fluid replacement were similar between groups $(\mathrm{p}=.79$ and $\mathrm{p}=.08)$. Patients in the LP group were discharged an average of 1.1 days earlier $(\mathrm{p}=.001)$. LP was not associated with higher rates of C5 palsy $(p=.28)$. Patients who underwent LEF were five times more likely to develop wound infection or dehiscence (risk ratio $=5.2,95 \%$ CI:1.1 to 23.4). Postoperative ground-level falls requiring an emergency department (ED) visit occurred more frequently in the LEF group $(11.9 \%$ vs $2.6 \%, p=.04)$. The frequency of ED visits for postoperative neck pain did not differ between groups $(\mathrm{p}=.42)$. Likewise, rates of new-onset neck pain were similar $(p=.45)$. Both groups reported improved VAS neck pain over the course of follow-up $(p=.001)$. Surgery type, involvement of the C7 level, and the number of levels involved were not predictive of differences in postoperative neck pain $(\mathrm{p}=.66, \mathrm{p}=.31$, and $\mathrm{p}=.87)$. Opioid analgesic needs in the year before and the year after surgery were similar between groups $(\mathrm{p}=.41$ and $\mathrm{p}=.33)$. The LP cohort had greater preoperative cervical lordosis (C2-C7 cobb angle: 11.69 vs $6.59, \mathrm{p}=.01)$ and lost more lordosis postoperatively $(-7.9$ vs $-1.8, \mathrm{p}=.004)$. LEF cases at this hospital incurred $18 \%$ and $34 \%$ greater fixed and variable costs $(\mathrm{p}=$ .03 and $\mathrm{p}<.001)$
Conclusions When used to treat patients with multilevel CSM, LP does not seem to be associated with new or worsening axial neck pain compared to LEF. Neck pain may be expected to improve similarly with either surgery. When cervical deformity is not prohibitive, LP could be offered as a less morbid and more cost-efficient alternative to LEF. Modern patient-reported outcomes and randomized controlled trials are still needed to optimize the utility of both procedures.

\section{\#78 LOWER LIMB AMPUTATIONS IN PATIENTS WITH DIABETES HOSPITALIZED WITH FOOT BURNS - A NATIONAL TRAUMA DATABANK ANALYSIS}

${ }^{1} \mathrm{D}$ Perrault, ${ }^{2} \mathrm{~J}$ Cobert, ${ }^{3} \mathrm{~V}$ Gadiraju*, ${ }^{1} \mathrm{G}$ Gurtner, ${ }^{4} \mathrm{~T}$ Pham, ${ }^{1} \mathrm{C}$ Sheckter. ${ }^{1}$ Stanford Medicine, Stanford, CA; ${ }^{2}$ University of Rochester, Rochester, NY; ${ }^{3}$ University of Washington School of Medicine, Seattle, WA; ${ }^{4}$ University of Washington Medical Center, Seattle, WA

\subsection{6/jim-2022-WRMC.77}

Purpose of Study Diabetes Mellitus (DM) has a significant burden in the United States and results in worsening health outcomes. Patients are at risk of peripheral neuropathy, which increases the risk of lower extremity burns, delay in burn presentation, and more complications that translate to more amputations. However, there are limited reports regarding the incidence and outcomes of DM foot burns. We aim to better understand DM health outcomes, specifically lower limb amputations, in DM foot burns at national Level 1 and 2 trauma centers.

Methods Used Implementing a retrospective cohort study design, we reviewed de-identified data on 116,796 adult admissions from 2007-2015 from the National Trauma Databank (NTDB) for patient age, DM, foot burn status, sex, race/ethnicity, region, burn size, and comorbidities. An exploratory logistic regression of factors associated with lower limb amputations was performed.

Summary of Results Of the 7,963 (7\%) foot burn patients, $1,338(17 \%)$ had DM (median age 56 years [17]) and 378 $(28 \%)$ were male. Common comorbidities included alcohol use, smoking, and chronic kidney disease (table 1). Using an exploratory logistic regression analysis, when all other variables were kept the same, factors (OR, CI) linked with total lower limb amputations are DM (3.70, [2.98, 4.59]), alcohol

Abstract \#78 Table 1 NTDB DM foot burn cohort characteristics (2007-2015)

\begin{tabular}{ll}
\hline & Cohort Characteristics \\
\hline Age (median, IQR) & $56[17]$ \\
Diabetes Mellitus & $1,338(100)$ \\
Alcohol & $61(5)$ \\
Smoking & $247(18)$ \\
Chronic Kidney Disease & $73(5)$ \\
$20-29 \%$ TBSA & $45(3)$ \\
$39-39 \%$ TBSA & $13(1)$ \\
$>40 \%$ TBSA & $25(2)$ \\
African-American/Black & $340(26)$ \\
Male & $378(28)$ \\
\hline
\end{tabular}

All values are denoted as $n, \%$ unless otherwise specified. Cohort is patients with DM and foot burns of $n=1,338$ ( $1 \%$ of NTDB adult admissions). Only covariates of significance are included in this table.\%TBSA is the percentage of total body surface area affected by a burn and represents burn size. 


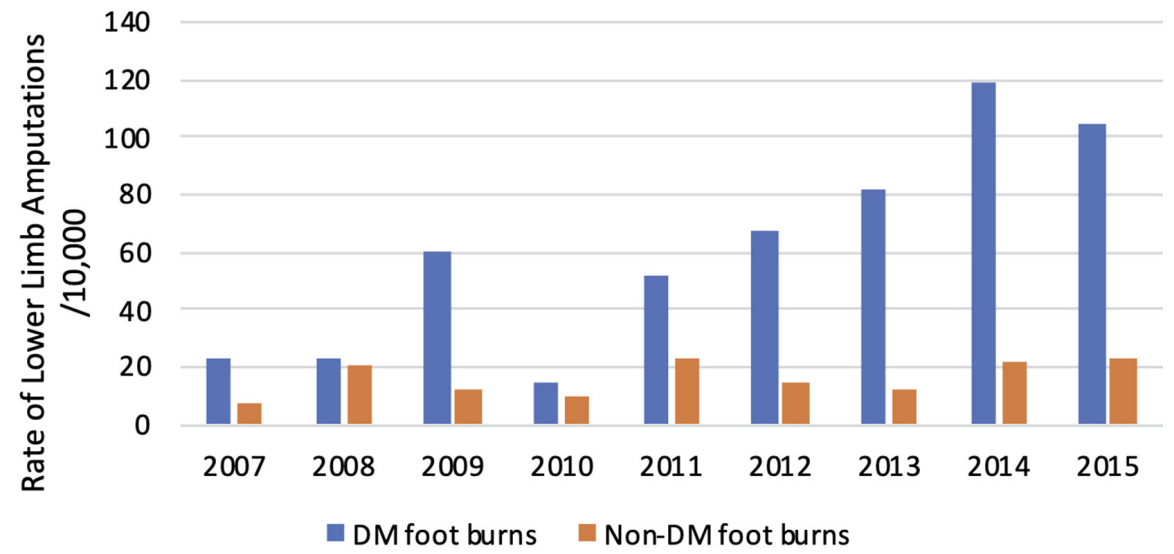

Abstract \#78 Figure 1 Rate per 10,000 of total lower limb amputations increases in DM foot burn vs. non-DM foot burn populations from 20072015.

use $(2.78,[2.13,3.61])$, smoking $(0.78,[0.62,1.00])$, chronic kidney disease $(2.90,[1.72,4.88])$, burn size $>20 \% \quad(4.12$, [2.96, 5.73]), African-American/Black race (1.61, [1.29, 2.01], male sex $(1.61,[1.28,2.02])$, and age $>40$ years. There was a higher rate of lower limb amputations in patients with DM foot burns.

Conclusions There is a higher rate of total amputations in DM foot burn patients, which indicates the need for increased patient education and treatment protocols that address the incidence of complications in this group. Future steps include confirmatory research to assess the risk of lower limb amputations in DM foot burn patients.

\section{Cardiovascular II}

\section{Concurrent session}

\section{3:15 PM}

\section{Thursday, January 20, 2022}

\section{\#79 OUTCOME OF HOSPITALIZED HEART TRANSPLANT PATIENTS WITH COVID INFECTION AT A LARGE WEST COAST CENTER}

${ }^{1} \mathrm{~A}$ Mohanty ${ }^{*},{ }^{2} \mathrm{~S}$ Kim, ${ }^{2} \mathrm{~N}$ Patel, ${ }^{2} \mathrm{~T}$ Singer-Englar, ${ }^{2} \mathrm{M}$ Hamilton, ${ }^{2} \mathrm{~J}$ Kobashigawa. ${ }^{1}$ Santa Clara University, Santa Clara, $C A ;{ }^{2}$ Cedars-Sinai Smidt Heart Institute, Los Angeles, CA

\subsection{6/jim-2022-WRMC.78}

Purpose of Study The COVID-19 pandemic infected large portions of the US community and infected many heart transplant (HTx) patients, but in distinct geographical patterns. HTx programs have reported mortality in the range of $23-29 \%$ and in non-transplant patients in the range of $15-17 \%$. The impact of hospitalized HTx patients with COVID infection in a large West Coast heart transplant program has not been reported. We now report our outcomes for hospitalized patients with COVID.

Methods Used Between March 2020 and March 2021, we assessed 22 HTx patients who were admitted to the CedarsSinai Medical Center (CSMC) for COVID infections. COVID is known to affect many systems within the body, and we report the effects on lungs, heart, and kidney. Morbidity and mortality, including risk of death, were included within 90 days post-infection.

Summary of Results Of the $22 \mathrm{HTx}$ patients hospitalized at the CSMC, 7 patients died (31.8\%). All patients had COVID pneumonia requiring supplemental oxygen and 5 patients required ventilatory support. The mean peak $\mathrm{FiO} 2$ of the patients was $79.7 \%$. 16 of these patients also were noted to have an increase in serum creatinine, with 6 patients requiring kidney dialysis. Cardiac function was maintained in all patients with COVID-19 and no myocarditis or cardiac dysfunction was observed. 9 patients received remdesivir and 19 patients received corticosteroids. 4 patients received tocilizumab antiinflammatory therapy.

Conclusions COVID-19 resulted in significant morbidity and mortality in hospitalized HTx patients. The immunosuppressed state appears to be a risk factor for poor outcome and is higher compared to non-transplant hospitalized patients.

\section{\#80 IS SACUBITRIL/VALSARTAN A RISK FACTOR FOR VASOPLEGIA/PRIMARY GRAFT DYSFUNCTION AFTER HEART TRANSPLANTATION?}

${ }^{1} \mathrm{M}$ Oda*, ${ }^{2} \mathrm{~T}$ Singer-Englar, ${ }^{2} \mathrm{~N}$ Patel, ${ }^{2} \mathrm{~S}$ Kim, ${ }^{2} \mathrm{M}$ Hamilton, ${ }^{2} \mathrm{~J}$ Kobashigawa. ${ }^{1}$ University of California Los Angeles, Los Angeles, CA; ${ }^{2}$ Cedars-Sinai Smidt Heart Institute, Los Angeles, CA

10.1136/jim-2022-WRMC.79

Purpose of Study Primary graft dysfunction (PGD) is seen in approximately $7-29 \%$ of heart transplant ( $\mathrm{HTx})$ patients. Many of these patients with PGD also develop significant vasoplegia which requires high doses of intravenous vasoconstrictors. Outcomes of these patients with severe PGD is compromised within 30 days after HTx. Risk factors for the development of severe PGD have included angiotensin-converting enzyme inhibitors (ACEi). There may be a connection between ACEi and the kallikrein-kinin system whereby bradykinin is increased, thus resulting in more vasoplegia and PGD. It is not known whether the new drug sacubitril/valsartan (S/ $\mathrm{V})$ is also a risk factor for the development of vasoplegia/ severe PGD as bradykinin is also increased with sacubitril. Therefore, we reviewed our large HTx program to see if there is a correlation of $\mathrm{S} / \mathrm{V}$ as a risk factor for this complication. 
Abstract \#80 Table 1 Comparison of Entresto vs. ACEi/ARB

\begin{tabular}{llll}
\hline Endpoint & $\begin{array}{l}\text { Entresto } \\
(\mathbf{n}=65)\end{array}$ & $\begin{array}{l}\text { ACEi/ARB control } \\
(\mathbf{n}=65)\end{array}$ & p-value \\
\hline 1-year survival & $89.2 \%$ & $90.8 \%$ & 0.716 \\
1-year freedom from cardiac dysfunction & $78.5 \%$ & $72.3 \%$ & 0.432 \\
1-year freedom from NF-MACE & $95.3 \%$ & $87.7 \%$ & 0.139 \\
Development of severe PGD & $4.6 \%$ & $6.2 \%$ & 1.000 \\
Development of vasoplegia & $29.2 \%$ & $33.9 \%$ & 0.706 \\
\hline
\end{tabular}

Methods Used Between 2015 and 2020, we assessed 65 HTx patients who were on $\mathrm{S} / \mathrm{V}$ at the time of transplantation. Vasoplegia was defined as requiring more than 2 vasoconstricting drugs with BP systolic $<90 \mathrm{mmHg}$, and PGD was defined as per the ISHLT classification scheme (within 24 hours posttransplant). These patients on $\mathrm{S} / \mathrm{V}$ were compared to patients on ACEi/ARB (1:1 control group for age, sex, transplant year). Outcomes included death, cardiac dysfunction, and nonfatal major adverse cardiac events (NF-MACE: MI, new CHF, PCI, ICD/pacemaker, or stroke) in the first year after HTx.

Summary of Results Compared to ACEi/ARB, S/V had similar risk for the development of vasoplegia or severe PGD. Furthermore, 1-year survival, and 1-year freedom from cardiac dysfunction and NF-MACE were not significantly different between groups.

Conclusions Patients undergoing HTx on S/V do not appear to be at risk for vasoplegia or severe PGD.

\section{\#81 THE EFFECT OF HYPOMAGNESEMIA POST HEART TRANSPLANTATION}

${ }^{1} \mathrm{~K}$ Ravellette ${ }^{*},{ }^{2} \mathrm{~N}$ Patel, ${ }^{2} \mathrm{~S}$ Kim, ${ }^{2} \mathrm{~T}$ Singer-Englar, ${ }^{2} \mathrm{M}$ Hamilton, ${ }^{2} \mathrm{~J}$ Kobashigawa. ${ }^{1}$ University of California Los Angeles David Geffen School of Medicine, Los Angeles, CA; ${ }^{2}$ Cedars-Sinai Smidt Heart Institute, Los Angeles, CA

\subsection{6/jim-2022-WRMC.80}

Purpose of Study The calcineurin inhibitors (CNIs), including tacrolimus and cyclosporine, have revolutionized heart transplantation (HTx) in terms of maintaining low rejection rates. However, CNIs have significant side effects, such as nephropathy, hypertension, malignancy, and hypomagnesemia. It is this hypomagnesemia that has not been addressed as to whether this has an impact on outcome after HTx. Hypomagnesemia has been involved in muscle cramping and cardiac arrhythmias. Therefore, we reviewed our HTx patients and assessed magnesium $(\mathrm{Mg})$ levels to assess outcome in the first 6 months after HTx.

Methods Used Between 2010 and 2020, we assessed 956 HTx patients and recorded their $\mathrm{Mg}$ levels in the first 6 months after HTx. Patients with low Mg levels less than or equal to $1.8 \mathrm{mg} / \mathrm{dL}$ were assessed for complications including muscle cramping, cardiac arrhythmias, rehospitalization, rejection episodes, and death. Patients with low Mg levels were grouped into mildly low $\mathrm{Mg}$ levels $(1.7-1.8 \mathrm{mg} / \mathrm{dL}$ ) and moderately low $\mathrm{Mg}$ levels (1.4-1.7 mg/dL). Patients were compared to control patients who had normal $\mathrm{Mg}$ levels $(>1.8 \mathrm{mg} / \mathrm{dL})$ during this period of time.

Summary of Results Patients with mildly or moderately low $\mathrm{Mg}$ levels compared to patients with normal $\mathrm{Mg}$ levels had no difference in muscle cramping, rejection episodes, cardiac arrhythmias, and use of antihypertensive medications. Kidney

\begin{tabular}{|c|c|c|c|c|}
\hline & $\begin{array}{l}\text { Moderate } \\
\text { Low } \\
\text { Magnesium } \\
{[1.4-1.7 \mathrm{mg} /} \\
\mathrm{dL}] \\
(\mathrm{n}=173)\end{array}$ & $\begin{array}{l}\text { Mild Low } \\
\text { Magnesium } \\
\text { [1.7-1.8 } \\
\mathrm{mg} / \mathrm{dL}] \\
(\mathrm{n}=158)\end{array}$ & $\begin{array}{l}\text { Normal } \\
\text { Magnesium } \\
{[>1.8 \mathrm{mg} /} \\
d \mathrm{~L}] \\
(\mathrm{n}=625)\end{array}$ & P-Value \\
\hline 6-Month Average Magnesium & $1.66 \pm 0.06$ & $1.79 \pm 0.02$ & $2.05 \pm 0.15$ & $<0.001$ \\
\hline $\begin{array}{l}\text { Incidence of Muscle Cramping } \\
\text { within 6-Months Post-Transplant }\end{array}$ & $12.1 \%$ & $8.2 \%$ & $11.5 \%$ & 0.444 \\
\hline $\begin{array}{l}\text { Incidence of Cardiac Arrhythmias } \\
\text { within 6-Months Post-Transplant }\end{array}$ & $5.2 \%$ & $10.8 \%$ & $21.9 \%$ & $<0.001$ \\
\hline $\begin{array}{l}\text { Rehospitalization within 6- } \\
\text { Months Post-Transplant }\end{array}$ & $17.3 \%$ & $22.8 \%$ & $25.1 \%$ & 0.099 \\
\hline $\begin{array}{l}\text { 6-Month Freedom from Any } \\
\text { Treated Rejection }\end{array}$ & $89.0 \%$ & $88.6 \%$ & $87.4 \%$ & 0.634 \\
\hline 6-Month Survival & $100.0 \%$ & $99.4 \%$ & $93.6 \%$ & $<0.001$ \\
\hline 6-Month Average Creatinine & $1.25 \pm 0.46$ & $1.28 \pm 0.48$ & $1.74 \pm 0.78$ & $<0.001$ \\
\hline
\end{tabular}

function was abnormal in those patients with normal magnesium levels.

Conclusions Mild-moderate hypomagnesemia did not have significant adverse effects in heart transplant patients in terms of muscle cramping, cardiac arrhythmias, cardiac rejection, or cardiac function.

\section{\#82 ACUTE ABDOMINAL COMPLICATIONS IMMEDIATELY FOLLOWING HEART TRANSPLANTATION}

${ }^{1} \mathrm{~K}$ Duggin ${ }^{*},{ }^{2} \mathrm{~S} \mathrm{Kim},{ }^{2} \mathrm{~T}$ Singer-Englar, ${ }^{2} \mathrm{~N}$ Patel, ${ }^{2} \mathrm{M}$ Hamilton, ${ }^{2} \mathrm{~J}$ Kobashigawa. ${ }^{1}$ University of California Santa Barbara, Santa Barbara, CA; ${ }^{2}$ Cedars-Sinai Smidt Heart Institute, Los Angeles, CA

\subsection{6/jim-2022-WRMC.81}

Purpose of Study It is not uncommon to have an acute abdomen following heart transplantation. Patients who have atherosclerotic vascular disease (coronary artery disease) as the need for heart transplant may also have risk for ischemic bowel associated with their surgeries. In addition, patients who have had gallstones are at increased risk for having cholecystitis immediately following cardiac surgery. It has not been well established as to the frequency of these abdominal complications that warrant urgent abdominal surgery. Furthermore, the presence of increased inflammation in abdominal surgery may trigger an immune response and thereby cause a rejection episode. We sought to evaluate these complications in our large, single center experience.

Methods Used Between 2010 and 2021, we assessed 1154 patients who underwent heart transplantation and reviewed the frequency of acute abdomen requiring surgical intervention (11 patients) in the first month following heart transplant surgery. These patients were assessed for 30-day and 1-year survival, episodes of cardiac rejection, and infectious complications requiring intravenous antibiotics and/or readmission in the ensuing 3 months. The acute abdomen patients were compared to a case-controlled group, matched by age, sex, and time of transplant.

Summary of Results $1.0 \%$ (11/1154 patients) of our patient population developed an acute abdomen and required surgical intervention within the 30 days following heart transplant 


\begin{tabular}{|c|c|c|c|}
\hline & $\begin{array}{l}\text { Acute Abdomen within } 30 \text { Days } \\
\text { Following Heart Transplantation } \\
(\mathrm{n}=11)\end{array}$ & $\begin{array}{l}\text { No Acute } \\
\text { Abdomen } \\
(n=22)\end{array}$ & $\begin{array}{l}\text { P- } \\
\text { Value }\end{array}$ \\
\hline 30-Day Survival & $72.7 \%$ & $100.0 \%$ & 0.010 \\
\hline 1-Year Survival & $45.5 \%$ & $90.9 \%$ & 0.002 \\
\hline $\begin{array}{l}\text { Infectious } \\
\text { Complications }\end{array}$ & $36.4 \%(4)$ & $40.9 \%(9)$ & 0.801 \\
\hline $\begin{array}{l}\text { 3-Month Freedom } \\
\text { from Infections }\end{array}$ & $72.7 \%$ & $77.3 \%$ & 0.442 \\
\hline Rejection Episodes & $18.2 \%(2)$ & $4.5 \%(1)$ & 0.199 \\
\hline $\begin{array}{l}\text { 3-Month Freedom } \\
\text { from ATR }\end{array}$ & $90.9 \%$ & $100.0 \%$ & 0.083 \\
\hline $\begin{array}{l}\text { 3-Month Freedom } \\
\text { from ACR }\end{array}$ & $90.9 \%$ & $100.0 \%$ & 0.083 \\
\hline $\begin{array}{l}\text { 3-Month Freedom } \\
\text { from AMR }\end{array}$ & $100.0 \%$ & $100.0 \%$ & 1.000 \\
\hline
\end{tabular}

surgery. Types of surgical interventions included hemicolectomy, cholecystectomy, and exploratory laparoscopy. Compared to the control group, the acute abdomen group had significantly worse 30-day survival and 1-year survival. In the study group, infectious complications occurred in an additional $36.4 \%$ of these patients who required rehospitalization with administration of intravenous antibiotics. Rejection episodes following these events was not different from the control population.

Conclusions Acute abdomen immediately post-heart transplant resulting in urgent abdominal surgery requiring hemicolectomy and/or cholecystectomy has significant morbidity/mortality. For patients awaiting heart transplant with gallstones, prophylactic laparoscopic cholecystectomy might be considered.

\section{\#83 CLINICAL MANIFESTATION AND PROGNOSIS OF DIFFERENT CARDIOMYOPATHY TYPES ON THE BASIS OF GENETIC BACKGROUND}

${ }^{1} \mathrm{~A}$ Paldino, ${ }^{2} \mathrm{~K}$ Medo*, ${ }^{2} \mathrm{~L}$ Mestroni, ${ }^{1} \mathrm{M}$ Merlo, ${ }^{2} \mathrm{M}$ Taylor. ' Universita degli Studi di Trieste, Trieste, Italy; ${ }^{2}$ University of Colorado Denver School of Medicine, Aurora, CO

\subsection{6/jim-2022-WRMC.82}

Purpose of Study Cardiomyopathies (CMP) are a heterogeneous group of heart disease characterized by structural and electrical abnormalities lacking secondary causative etiology and frequently related to mutations in CMP genes. Recent studies in this field have showed important phenotype overlaps between Dilated Cardiomyopathy (DCM) and Arrhythmogenic Cardiomyopathy (ACM), making the diagnosis a challenging task. The aim of this study is to assess whether a classification of CMP patients (not hypertrophic) based on genetic characterization outperforms in diagnostic and prognostic accuracy the classical, phenotype-driven, diagnostic approach.

Methods Used We analyzed a population of patients affected by genetically determined DCM and ACM, including carriers of 'pathogenic' or 'likely pathogenic' (P/LP) variants, registered into the Heart Disease Centers of Trieste and Denver hospitals. We described the phenotype distribution in our population with a clinical and echocardiographic evaluation based on the different disease-related mutated genes. Then, we examined the prognostic impact of the single gene/genetic cluster in determining these outcomes: 1) all-cause mortality and heart transplant; 2) heart failure-related death, heart transplant or destination left ventricular assist device implantation (DHF/HTx/VAD); and 3) sudden cardiac death, sustained ventricular tachycardia/ventricular fibrillation or appropriate defibrillator shock (SCD/VT/VF/shock).

Summary of Results 281 patients carrying P/LP variants $(82 \%$ DCM) were included in the study. Titin (TTN) and sarcomeric genes (SARC) variants were the most prevalent (TTN: 95 patients, 34\% of total population; SARC: 63 patients, $22 \%$ of total population) and almost completely related to DCM phenotype (TTN: 100\% DCM, SARC: 95\% DCM); lamin (LMNA) patients (29 patients, 10\% of total population, 96\% DCM). A more heterogeneous phenotypic distribution between DCM and ACM were noted for desmoplakin (DSP), plakoglobin (PKP2) and filamin (FLNC) variants. Patients with uncategorized DCM phenotype and carriers of DSP, PKP2, FLNC and LMNA variants (arrhythmic genes) experienced more frequent SCD/VT/VF/shock events ( $\mathrm{p}$ value $=0.002$ and $\mathrm{p}=0.023$ ), compared to patients with DCM phenotype, during follow-up (median=132 months). The analysis shows only $\mathrm{P} /$ LP variants of arrhythmic genes, early age of onset and male gender, were associated with an increased risk of SCD/VT/VF/ shock events during follow-up. Additionally, there were no differences in terms of $\mathrm{HF}$ events was significantly related to genotype.

Conclusions In a large DCM and ACM population with a positive genetic test for $\mathrm{P} / \mathrm{LP}$ variants, the classification based on specific genotypes is a useful tool in arrhythmic prognostication. These findings support the need of extensive genetic testing to support CMP diagnosis and prognosis.

\section{\#84 SEX DIFFERENCES IN DESENSITIZATION FOR PATIENTS AWAITING HEART TRANSPLANTATION: IS THERE A DIFFERENCE?}

${ }^{1} \mathrm{~N}$ Yamamoto*, ${ }^{2} \mathrm{~N}$ Patel, ${ }^{2} \mathrm{~T}$ Singer-Englar, ${ }^{2} \mathrm{~S}$ Kim, ${ }^{2} \mathrm{M}$ Hamilton, ${ }^{2} \mathrm{~J}$ Kobashigawa. ${ }^{1}$ Western University of Health Sciences College of Osteopathic Medicine of the Pacific, Pomona, CA; ${ }^{2}$ Cedars-Sinai Smidt Heart Institute, Los Angeles, CA

\subsection{6/jim-2022-WRMC.83}

Purpose of Study For patients awaiting heart transplantation (HTx) who have high levels of circulating antibodies (greater than 70\%), desensitization therapy may be indicated. This will allow expansion of the donor pool for a compatible donor. As women appear to be more highly sensitized (due to multiple pregnancies), it is not clear as to whether women can benefit from desensitization therapy. We sought to answer this question with review of our large, single center database.

Methods Used Between 2008 and 2020, we assessed 49 patients awaiting HTx who underwent desensitization therapy. These patients were divided into groups by sex for their response to desensitization therapy. Our desensitization protocols consist of regimens including intravenous immune globulin, anti-CD20 monoclonal antibody, plasmapheresis, and/or proteosome inhibitors. A response to desensitization therapy was assessed by the decline of the dominant circulating antibody determined by mean fluorescence intensity (MFI). PostHTx data was assessed for 1-year survival and freedom from rejections (acute cellular rejection [ACR], antibody-mediated rejection $[\mathrm{AMR}])$. Rejection episodes were compared to a 


\begin{tabular}{llll} 
Abstract \#84 Table 1 & & & \\
\hline & $\begin{array}{l}\text { Male } \\
(\mathbf{n}=\mathbf{1 1})\end{array}$ & $\begin{array}{l}\text { Female } \\
(\mathbf{n}=\mathbf{3 8})\end{array}$ & $\begin{array}{l}\text { P- } \\
\text { Value }\end{array}$ \\
\hline Immunodominant Antibody MFI Pre-Treatment & $\begin{array}{l}12613 \pm \\
3642\end{array}$ & $\begin{array}{l}12779 \pm \\
\text { Immunodominant Antibody MFI Post-Treatment }\end{array}$ & 0.891 \\
& $\begin{array}{l}12045 \pm \\
12055 \pm\end{array}$ & 0.994 \\
Change in Immunodominant Antibody MFI & 3514 & 3925 & \\
& $-568 \pm$ & $-723 \pm 1782$ & 0.798 \\
Average Time on Waitlist (years) & 1687 & & \\
1-Year Survival & $0.61 \pm 0.62$ & $0.65 \pm 0.82$ & 0.896 \\
1-Year Freedom from Acute Cellular Rejection & $100.0 \%$ & $89.5 \%$ & 0.299 \\
(ACR) & $81.8 \%$ & $94.7 \%$ & 0.106 \\
1-Year Freedom from Antibody-Mediated & $72.7 \%$ & $78.9 \%$ & 0.691 \\
Rejection (AMR) & & & \\
\hline
\end{tabular}

control group of non-sensitized patients transplanted during the same period $(n=771)$.

Summary of Results Desensitization therapy in women appeared to be comparable to men, considering similar desensitization protocols. There were no significant differences in waitlist mortality, time on the waitlist, 1-year post-transplant survival, or 1-year freedom from ACR or AMR between the two groups. Compared to non-sensitized patients, freedom from AMR was significantly lower in both sensitized men and women $(72.7 \%$ men vs. $78.9 \%$ women vs. $96.5 \%$ control group, $\mathrm{p} \leq 0.001)$.

Conclusions Sensitized women awaiting HTx compared to men appear to have similar response to various desensitization regimens. Post-HTx, there was more AMR in both groups, suggesting memory B-cells may be responsible.

\section{\#85 A CASE OF POST-COVID MYOCARDITIS IN A 39 YEAR OLD FEMALE WITH PCOS AND INCIDENTAL FINDING OF CAD}

S Ratnayake*, A Mesfin, L Moosavi, F Joolhar, A Ghandforoush. Kern Medical Center, Bakersfield, CA

\subsection{6/jim-2022-WRMC.84}

Purpose of Study Reports of cardiovascular manifestations in the setting of COVID-19 have included arrhythmia, pericarditis, heart failure, acute coronary syndrome, and myocarditis. Myocarditis is defined as inflammation of the heart muscle and is commonly associated with viral infection. Common symptoms of myocarditis can include chest pain, shortness of breath, as well as arrhythmia and fatigue. While endomyocardial biopsy remains the gold standard for diagnosis, clinically suspected myocarditis in low-risk patients can be established through presentation and non-invasive diagnostic findings. Here, we aim to highlight the association between coronavirus disease of 2019 infection (COVID-19) and cardiovascular complications such as myocarditis in this case report. In our case, heart catheterization demonstrated $60 \%$ stenosis of the proximal left anterior descending artery; however, this lesion was not suspected to be the culprit lesion causing myocardial injury. Etiology of injury was thought to be caused by global ischemia in the setting of post-COVID-19 infection.

Methods Used Retrospective chart review after IRB approval.
Summary of Results This is a 39 -year-old Hispanic female with history of PCOS, hyperlipidemia, hypertension, Oral Contraceptive Pill, provoked Deep Vein Thrombosis and Pulmonary Embolism on Rivaroxaban, who presented to emergency department with 4 days of new onset intermittent severe substernal chest pain radiating down to her left arm. She had SARS-CoV-2 pneumonia the month prior to this presentation significant for cough, anosmia, and myalgias, which resolved without hospitalization. On arrival, she was hypertensive, tachycardic, and afebrile. Coagulation panel was normal, troponin-I was elevated at 6.25 with a peak of 9.27. Toxicology was negative for stimulants. She tested positive for SARS-CoV2 but remained asymptomatic. Patient was started on dual anti-platelet therapy and anti-coagulation therapy. Repeat ECG showed no new changes. A second episode of chest pain revealed lateral ST-elevations and $\mathrm{Q}$-waves in inferior leads. Troponin continued to downtrend. Left heart catheterization was performed with incidental finding of $60 \%$ stenosis of the proximal LAD with a smooth plaque. This patient clinically improved without further chest pain and was discharged with dual-antiplatelet therapy.

Conclusions Evaluation and tracking of clinically suspected myocarditis in the setting of COVID-19 infection may give insight into the pathophysiology of infection in cardiomyocytes due to SARS-CoV-2. This case report aims to illustrate the possible association between COVID-19 and myocarditis in the hopes of decreasing morbidity and mortality.

\section{\#86 APICAL HYPERTROPHIC CARDIOMYOPATHY MIMICKING AS MYOCARDIAL INFARCTION}

${ }^{1}$ VK Narang ${ }^{*},{ }^{2} \mathrm{P}$ Chan, ${ }^{1} \mathrm{~F}$ Joolhar, ${ }^{1} \mathrm{~T}$ Win. ${ }^{1}$ UCLA-Kern Medical, Bakersfield, CA; ${ }^{2}$ Ross University School of Medicine, Miramar, FL

\subsection{6/jim-2022-WRMC.85}

\section{Case Report}

Purpose Hypertrophic cardiomyopathy (HCM) is known to have a wide spectrum of patterns. This case highlights an uncommon form of HCM called apical hypertrophic cardiomyopathy (ApHCM) which was seen to mimic myocardial infarction.

Methods Retrospective Study.

Summary A 46-year-old Punjabi male with hypertension presented to an outside hospital with chest pain and was to have elevated troponin levels of $0.31 \mathrm{ng} / \mathrm{mL}$. Nuclear Lexiscan stress test at that time showed 'reversible defect of the cardiac apex suggestive of ischemia', cardiac catheterization was negative, and transthoracic echocardiogram (TTE) showed preserved left ventricular function and mild mitral regurgitation. Troponin trended down to $0.23 \mathrm{ng} / \mathrm{mL}$ and the patient was discharged.

Patient then comes to the medicine clinic to establish care and was complaining of palpitations that are intermittent and last about 2-3 minutes per episode. Patient reports that the episodes are initiated by physical activity such as walking about 100 feet and alleviated with rest. Patient denied any chest pain or shortness of breath. Positive history for heavy alcohol use, drinks 6-8 alcoholic beverages 2-3 times a week. Electrocardiogram (ECG) done in the clinic showed left ventricular hypertrophy and abnormal $\mathrm{T}$ waves in inferior leads. Repeat TTE showed left ventricular ejection fraction is estimated at $>65 \%$ and apical to mild LV is unusually thickened 
which is consistent with ApHCM. Patient was then referred to the cardiology clinic for further management. The patient will be treated with appropriate beta-blocker and cardiac monitoring for further risk stratification.

Conclusion There are many different spectrums to hypertrophic cardiomyopathy with the most common form being asymmetric septal hypertrophy (ASH). There is a more rare form called ApHCM which is more prevalent in the Asian population $(25 \%)$ than in non-Asians (1\% to $10 \%)$. Compared to the ASH, it is more sporadic and associated with more atrial fibrillation (AF) and different risk factors for sudden cardiac death (SCD). There are no current guideline recommendations for diagnosis, screening, or patient risk stratification available for ApHCM.

This case illustrates the importance of understanding and diagnosing patients with ApHCM since patient symptoms mimicked a myocardial infarction. The accurate and timely diagnosis may highly improve the clinical outcome and overall well-being of the patient.

\section{Diversity, equity, inclusion I}

\section{Concurrent session}

\section{3:15 PM}

\section{Thursday, January 20, 2022}

\section{\#87 STAKEHOLDER ENGAGEMENT EFFORTS TO IMPROVE DIVERSITY IN RARE DISEASE RESEARCH: INSPIRING NEW SCIENCE IN GUIDING HEALTHCARE IN TURNER SYNDROME (INSIGHTS) REGISTRY}

\begin{abstract}
1,2,3 S Davis*, 1,4V Bamba, 1,5W Brickman, 1,2,3 A Carl, 1,6 ${ }^{1,6}$ Dowlut-McElroy, 1,7 M Good, ${ }^{1,2,3}$ S Howell, ${ }^{1,8} \mathrm{R}$ Kanakatti Shankar, ${ }^{1,9} \mathrm{~J}$ Law, ${ }^{1,10} \mathrm{~S}$ Prakash, ${ }^{1,7} \mathrm{~K}$ Ranallo. ${ }^{1}$ INSIGHTS Consortium, Overland Park, KS; ${ }^{2}$ Children's Hospital of Colorado, Aurora, CO; ${ }^{3}$ University of Colorado - Anschutz Medical Campus, Aurora, $\mathrm{CO}^{4}{ }^{4}$ The Children's Hospital of Philadelphia Division of Endocrinology and Diabetes, Philadelphia, PA; ${ }^{5}$ Ann Robert H Lurie Children's Hospital of Chicago, Chicago, IL; ${ }^{6}$ National Institute of Child Health and Human Development, Bethesda, MD; ${ }^{7}$ Turner Syndrome Global Alliance, Overland Park, KS; ${ }^{8}$ Children's National Hospital, Washington, DC; ${ }^{9}$ University of North Carolina at Chapel Hill School of Medicine, Chapel Hill, NC; ${ }^{10}$ The University of Texas Health Science Center at Houston, Houston, TX
\end{abstract}

\subsection{6/im-2022-WRMC.86}

Purpose of Study Turner syndrome (TS) occurs in $\sim 1$ in 2,000 females who are born with partial or complete absence of the second sex chromosome. Like many rare disease conditions, most research in TS has been focused on specific features (particularly growth), limited to single centers, included minimal diversity, and lacked community engagement. The Inspiring New Science in Guiding Healthcare in Turner Syndrome (InsighTS) Registry was developed to address these limitations. Methods Used A Steering Committee with stakeholders comprised of researchers, multidisciplinary clinicians, and patient advocates was formed to develop the goals, infrastructure, data collection tools, protocols and engagement strategies for a national, collaborative clinic-based longitudinal registry for individuals with TS. Six institutions with multidisciplinary TS clinics across geographical regions were onboarded as recruitment sites with the goal of $>80 \%$ of eligible patients enrolling with diversity in age, race, ethnicity, payor status, and timing of diagnosis. The team identified patient-centered multidisciplinary outcomes obtainable through medical records and optional additional study procedures.

Summary of Results To date, 154 participants representing all regional centers have enrolled in InsighTS with an average enrollment rate of 15 per month. The average age at enrollment of $11.9 \pm 11$ years (range $0-67,16.9 \% \geq 18$ at enrollment). $18.5 \%$ identify as Hispanic/Latinx ethnicity and racial distribution includes 6.2\% Asian, 13.7\% Black, 71.9\% White, and $11.0 \%$ Other Race. TS was identified prenatally in $30.3 \%$ of participants. The majority of participants agreed to be contacted for future studies (89\%), complete annual surveys $(83 \%)$ and contribute to the biobank (61\%).

Conclusions Stakeholder engagement for the development of a national clinic-based registry for the rare genetic condition of TS has successfully led to a diverse cohort representative of the US population. Additional engagement strategies to increase enrollment while prioritizing diversity are underway.

\section{\#88 PARENTAL PERSPECTIVES TOWARDS COVID-19 VACCINES AND RETURN TO SCHOOL: FOCUSING ON HEALTH DISPARITIES}

${ }^{1} \mathrm{~K} \mathrm{He}^{*},{ }^{2,3} \mathrm{M}$ Berra, ${ }^{2,3} \mathrm{M}$ Kung, ${ }^{2,3} \mathrm{~B}$ Afghani. ${ }^{1}$ Children's Hospital of Los Angeles, Los Angeles, CA; ${ }^{2}$ UC Irvine School of Medicine, Orange, CA; ${ }^{3}$ Children's Hospital of Orange County, Orange, $C A$

10.1136/jim-2022-WRMC.87

Purpose of Study The COVID-19 pandemic has disproportionately impacted children from low socioeconomic and minority groups. Parents encounter new decisions regarding vaccinating their child against COVID-19 and return to school in fall of 2021. Prior studies show COVID-19 vaccine hesitancy is associated with income, race, and marital status. However, few studies examine the demographics behind COVID-19 vaccine hesitancy in relation to return to school in vulnerable communities. Understanding both are crucial to addressing challenges for children with healthcare inequities.

Methods Used A cross-sectional survey was conducted at inpatient and outpatient settings at an academic center and its affiliated site between September 2020 - September 2021. Parents were recruited to complete an anonymous mobilephone based survey using REDCap regarding perspectives on COVID-19 vaccines and factors affecting children's return to school during the pandemic. Statistical analyses were performed to examine the association between demographic factors (gender, marital status, education, ethnicity, and household income), COVID-19 vaccine hesitancy, and healthcare inequities affecting return to school.

Summary of Results Of 189 respondents, 65.5\% were married, $41.9 \%$ had less than college education, and $37.0 \%$ had households of $>2$ people. $64.6 \%$ were minorities and $53.9 \%$ were from low income families. COVID-19 vaccine acceptance was positively associated with marital status and number of household members: 60.9\% of married individuals reported they would vaccinate their child compared to $30.4 \%$ of unmarried individuals $(\mathrm{p}=0.001) .62 .1 \%$ of households of $>2$ people would vaccinate compared to $43.1 \%$ with households 2 or less $(p=0.015$, table 1$)$. Those who accepted or rejected COVID-19 vaccines were more likely to prefer onsite school compared to those who were unsure $(p=0.020)$. Education, ethnicity, and income were not associated with COVID-19 vaccine acceptance (table 1) or parental decisions in having their child return to school. Those with less than college 


\begin{tabular}{|c|c|c|c|c|}
\hline Demographic Factor (N) & Yes $(\%)$ & $\begin{array}{l}\text { Unsure } \\
(\%)\end{array}$ & No $(\%)$ & $\begin{array}{l}\mathrm{p} \text { - } \\
\text { value }\end{array}$ \\
\hline \multicolumn{5}{|l|}{ Gender (171) } \\
\hline Mother (143) & $\begin{array}{l}68 \\
(47.6 \%)\end{array}$ & $\begin{array}{l}51 \\
(35.7 \%)\end{array}$ & $\begin{array}{l}24 \\
(16.8 \%)\end{array}$ & 0.119 \\
\hline Father (28) & $\begin{array}{l}19 \\
(67.9 \%)\end{array}$ & $5(17.9 \%)$ & $4(14.3 \%)$ & \\
\hline \multicolumn{5}{|l|}{ Marital Status (171) } \\
\hline Unmarried (56) & $\begin{array}{l}17 \\
(30.4 \%)\end{array}$ & $\begin{array}{l}25 \\
(44.6 \%)\end{array}$ & $\begin{array}{l}14 \\
(25.0 \%)\end{array}$ & 0.001 \\
\hline Married (115) & $\begin{array}{l}70 \\
(60.9 \%)\end{array}$ & $\begin{array}{l}31 \\
(27.0 \%)\end{array}$ & $\begin{array}{l}14 \\
(12.2 \%)\end{array}$ & \\
\hline \multicolumn{5}{|l|}{ Educational Level (171) } \\
\hline College and Up (100) & $\begin{array}{l}52 \\
(52.0 \%)\end{array}$ & $\begin{array}{l}30 \\
(30.0 \%)\end{array}$ & $\begin{array}{l}18 \\
(18.0 \%)\end{array}$ & 0.605 \\
\hline Grade and High School (71) & $\begin{array}{l}35 \\
(49.3 \%)\end{array}$ & $\begin{array}{l}26 \\
(36.6 \%)\end{array}$ & $\begin{array}{l}10 \\
(14.1 \%)\end{array}$ & \\
\hline \multicolumn{5}{|l|}{ Number of Household Members (168) } \\
\hline 1-2 people (102) & $\begin{array}{l}44 \\
(43.1 \%)\end{array}$ & $\begin{array}{l}35 \\
(34.3 \%)\end{array}$ & $\begin{array}{l}23 \\
(22.5 \%)\end{array}$ & 0.015 \\
\hline 3-7 people (66) & $\begin{array}{l}51 \\
(62.1 \%)\end{array}$ & $\begin{array}{l}20 \\
(30.3 \%)\end{array}$ & $5(7.6 \%)$ & \\
\hline \multicolumn{5}{|l|}{ Ethnicity (171) } \\
\hline Non-Minority (White, Asian) (61) & $\begin{array}{l}32 \\
(52.5 \%)\end{array}$ & $\begin{array}{l}19 \\
(31.1 \%)\end{array}$ & $\begin{array}{l}10 \\
(16.4 \%)\end{array}$ & 0.941 \\
\hline $\begin{array}{l}\text { Minority (Hispanic, Black, Pacific Islander) } \\
\text { (110) }\end{array}$ & $\begin{array}{l}55 \\
(50.0 \%)\end{array}$ & $\begin{array}{l}37 \\
(33.6 \%)\end{array}$ & $\begin{array}{l}18 \\
(16.4 \%)\end{array}$ & \\
\hline \multicolumn{5}{|l|}{ Income (171) } \\
\hline High $(>50 \mathrm{~K})(78)$ & $\begin{array}{l}46 \\
(59.0 \%)\end{array}$ & $\begin{array}{l}21 \\
(26.9 \%)\end{array}$ & $\begin{array}{l}11 \\
(14.1 \%)\end{array}$ & 0.151 \\
\hline Low (<50K) (93) & $\begin{array}{l}41 \\
(44.1 \%)\end{array}$ & $\begin{array}{l}35 \\
(37.6 \%)\end{array}$ & $\begin{array}{l}17 \\
(18.3 \%)\end{array}$ & \\
\hline
\end{tabular}

education, low income families, and minority groups favored returning to school because of school-provided lunches and availability of internet.

Conclusions Our study shows that parents from all ethnicities and incomes may experience hesitancy towards COVID-19 vaccines. COVID-19 vaccine acceptance is positively associated with marital status and number of people in the household. Parents from vulnerable communities experience barriers influencing their decision of sending children back to school. Larger studies are needed to examine the underlying demographic factors behind COVID-19 vaccine hesitancy and return to school. Unique interventions are needed to target children experiencing healthcare inequities in order to increase COVID-19 vaccine confidence and promote safe return to school.

\section{\#89 BLACK INDIVIDUALS, HEALTHCARE UTILIZATION, AND COVID-19: PREFERENCES IN ACCESSING CARE}

${ }^{1}$ I Pasumarthi*, ${ }^{1} \mathrm{~T}$ Hollar, ${ }^{1} \mathrm{~N}$ Alety, ${ }^{2} \mathrm{E}$ Solano, ${ }^{2} \mathrm{M}$ Edman, ${ }^{2} \mathrm{G}$ Ochoa. ${ }^{1}$ Nova Southeastern University, Fort Lauderdale, $\mathrm{FL}_{i}{ }^{2} Y M C A$ of South Florida, Fort Lauderdale, FL

\subsection{6/jim-2022-WRMC.88}

Purpose of Study Black individuals in the United States have historically faced barriers to accessing healthcare, only exacerbated by the COVID-19 pandemic. The purpose of this study was to examine the self-reported likelihood of utilizing care within this population in the midst of the COVID-19 pandemic.

Methods Used Housing authority residents in Broward County, Florida were asked about their likelihood of visiting their doctor during the COVID-19 pandemic as part of a COVID-19 testing and education initiative conducted by the YMCA of South Florida, in partnership with the Housing Authority of the City of Fort Lauderdale and the Broward County Housing Authority. Secondary data analysis of program data was conducted, including descriptive statistics for describing respondents, Chi-square and t-tests for detecting significant differences around likelihood of seeking care between groups, and logistic regression to determine the odds of particular groups' likelihood of seeking care.

Summary of Results Significant differences were found between respondents $(n=147)$ reporting they were more likely to visit their doctors in terms of race/ethnicity (X2 [1,n=147] = $8.15, \mathrm{p}<.01)$. Black respondents had three times the odds of claiming to be more likely to visit their doctor (aOR 2.76, 95\% CI 1.36-5.60) than other groups. However, Black respondents reported being significantly more afraid of contracting the virus that causes COVID-19 on the way to the doctor's office than non-Black respondents (X2 [1,n=147] $=$ 4.23, $\mathrm{p}<.05)$. Black respondents also reported being more concerned of contracting the virus that causes COVID-19 at the doctor's office than non-Black respondents $(\mathrm{X} 2[1, \mathrm{n}=147]$ $=5.29 \mathrm{p}<.05)$.

Conclusions Black Housing Authority residents seemed to have a high likelihood of utilizing care if needed. However, this high utilization is coupled with a fear of contracting the virus that causes COVID-19 in the process of utilizing care. Areas for further research include determine the rationale behind this positive attitude toward healthcare utilization and figuring out specific areas of concern for contracting COVID-19 in the process of utilizing care (ex. fears of contracting the virus through the use of public transportation on the way to the clinic, etc.).

\section{\#90 THE EFFICACY OF EDUCATIONAL WORKSHOPS TO IMPROVE HUMAN PAPILLOMAVIRUS LITERACY IN HISPANIC POPULATIONS}

${ }^{1} Y$ Hwang ${ }^{*}{ }^{2,3} \mathrm{C}$ Aristizabal, ${ }^{4,5}$ L Baezconde-Garbanati. ' Eastern Virginia Medical School, Norfolk, VA; ${ }^{2}$ University of Southern California Norris Comprehensive Cancer Center, Los Angeles, $\mathrm{CA} ;{ }^{3} \mathrm{NCl}$ Center to Reduce Cancer Health Disparities, Bethesda, MD; ${ }^{4}$ University of Southern California Keck School of Medicine, Los Angeles, CA; ${ }^{5} \mathrm{NCl}$ Center to Reduce Cancer Health Disparities, Bethesda, MD

\subsection{6/jim-2022-WRMC.89}

Purpose of Study Hispanic populations experience disparities with regards to human papillomavirus (HPV) vaccine uptake despite ranking highest among racial groups for rates of cervical cancer. It is well-established that HPV vaccination confers a high degree of protection against HPV-related cancers. Yet barriers to HPV vaccination contribute to low rates of vaccine initiation and series completion in Hispanic populations with only $35-46 \%$ of adolescents fully vaccinated against HPV. Notably, literature suggests low health literacy as a common deterrent to vaccine uptake. The purpose of this study is to assess the utility of educational workshops in the improvement 
of HPV literacy in Hispanic populations in the Los Angeles area.

Methods Used Educational interventions, consisting of a onehour slideshow presentation delivered via Zoom video platform, were conducted from August 2020 to April 2021 and addressed the clinical significance of HPV and the HPV vaccine. Study participants $(n=92)$ were recruited through community partners and by word-of-mouth in a snowball fashion. A pre- and post-test design was used to investigate study participants' knowledge of HPV and the HPV vaccine before and after educational intervention. Lecture presentations and pre-/ post-questionnaires were offered in English and Spanish, depending on the participant's language preference. A paired t-test was used to compare HPV and HPV vaccine literacy scores pre- and post-lecture. Analysis was completed using SPSS Statistics Version 28.0.

Summary of Results Of the 92 study participants who attended the lectures, $58(63.0 \%)$ completed both the pre- and postquestionnaires. Prior to educational intervention, HPV and HPV vaccine literacy scores for study participants were $70.4 \%$ and $70.7 \%$ accuracy, respectively. Post-lecture, HPV and HPV vaccine literacy scores increased to $83.1 \%$ and $77.8 \%$ accuracy, respectively, demonstrating a statistically significant improvement $(p<0.001)$ in HPV literacy with educational intervention.

Conclusions The implementation of educational lectures was effective in improving HPV and HPV vaccine literacy in a Hispanic population. These findings demonstrate the need for increased emphasis on HPV education to close the knowledge gap disproportionately affecting Hispanic populations. Future research should focus on vaccine acceptability post-educational intervention in Hispanic populations to identify possible strategies to improve HPV vaccination rates. A potential explanation behind the diminished response rate may be attributed to hesitancy among undocumented study participants to reveal personally identifiable information.

\section{\#91 UNDERREPRESENTED IN MEDICINE MINORITY PHYSICIANS IN EMERGENCY MEDICINE RESIDENCY LEADERSHIP}

${ }^{1}$ J Smittick, ${ }^{2}$ ET Reibling, ${ }^{2} \mathrm{~B}$ Jones, ${ }^{2} \mathrm{~L}$ Fierro, ${ }^{2} \mathrm{M}$ Kiemeney*. 'Loma Linda University School of Medicine, Loma Linda, CA; ${ }^{2}$ Loma Linda University, Loma Linda, CA

\subsection{6/jim-2022-WRMC.90}

Purpose of Study It's evident in academic literature that representation of underrepresented in medicine (URiM) minorities in Emergency Medicine (EM) is sparse. This disparity is more drastic amongst EM leadership. Faculty and residents are directly involved in recruiting, interviewing, and ranking potential incoming residents. The lack of URiM participation in that process impacts the potential for future URiM physicians to be appointed to EM residency leadership positions. Our study sought to identify potential areas for increased representation in the future and factors that may increase URiM involvement.

Methods Used We administered a survey to the U.S. Emergency Medicine Residency Program Directors (PDs) listed on FREIDA, the American Medical Association (AMA) residency and fellowship database. We drafted and piloted the online survey instrument before sending it to participants via Qualtrics. Survey items focused on ethnic identity in program leadership, career preparation such as mentors and previously held roles, and strategies used to encourage URiM recruitment. Participants received one announcement email and three reminder emails following the survey distribution. We used Microsoft Excel for primary data analysis.

Summary of Results We received 57 completed surveys. 22\% of the respondents identified as URiM, of which 9\% identified as Black and $7 \%$ identified as Latinx. The median percentage of Residents identifying as URiM was 13\% (IQR 1\%-32\%). Eight programs (14\%) reported having at least one Chief Resident identifying as URiM. $72 \%$ of respondents reported that a mentor was instrumental in their ascension to PD. $11 \%$ reported that their mentor identified as URiM. We asked PDs to confirm which strategies they've implemented to encourage URiM participation (Boatright 2008). The most commonly implemented strategies were, 'Know the institution's local and community demographics, and address those needs' (51\%), followed by 'Broaden selection criteria beyond USMLE scores to include intangibles such as leadership, community service, and other life experiences' (49\%), and 'Develop curricula to address topics on diversity, cultural competence, and implicit bias' (47\%).

Conclusions The disparity of URiM PDs in EM may be a result of a lack of URiM mentorship. 29\% of respondents were URiM but only $11 \%$ reported having a URiM mentor. This lack of mentor-mentee concordance may be an area of further study and improvement. More intentional utilization of URiM recruitment strategies could also drastically improve representation. Increased URiM participation in EM leadership has great potential to improve diversity, equity, and inclusion in EM overall.

*Boatright N, et al. The Impact of the 2008 Council of Emergency Residency Directors (CORD) Panel on Emergency Medicine Resident Diversity. DOI: 10.1016/j. jemermed.2016.06.003.

\section{\#92 NOVEL HEALTH EQUITY ADVOCACY CURRICULUM TO INCITE ENGAGEMENT IN MEDICAL TRAINEES}

${ }^{1} \mathrm{C}$ Conklin*, ${ }^{1} \mathrm{~N}$ Rodman, ${ }^{1} \mathrm{D}$ Smith, ${ }^{1,2} \mathrm{~W}$ Joshi. ${ }^{1}$ University of California San Diego School of Medicine, La Jolla, CA; ${ }^{2}$ Rady Children's Hospital San Diego, San Diego, CA

\subsection{6/jim-2022-WRMC.91}

Purpose of Study Medical education health equity curriculums rarely emphasize advocacy and community engagement, further exploiting the minority tax in pursuing health equity work. Health equity curriculums must include three components: history, outcomes and interventions. The Journal Club and Advocacy Lab (JC-AL) schema was added to the Health Equity Thread (HET) preclinical curriculum at UC San Diego (UCSD) School of Medicine to teach and support interventions to health disparities.

Methods Used Preclinical students receive HET credit by attending JC-ALs. JC-AL workflow is depicted in figure 1; the JC and AL are held 1-2 weeks apart. Participants took a survey, approved by the UCSD Institutional Review Board, before the JC and after the AL. Survey responses from November 2020-June 2021 were gathered and summarized for each timepoint using R.

Summary of Results Of participants surveyed, 141 (28.5\%) identified as underrepresented in medicine. About a quarter of participants saw an increase in $\operatorname{mood}(25 \%)$, resilience $(27 \%)$, 


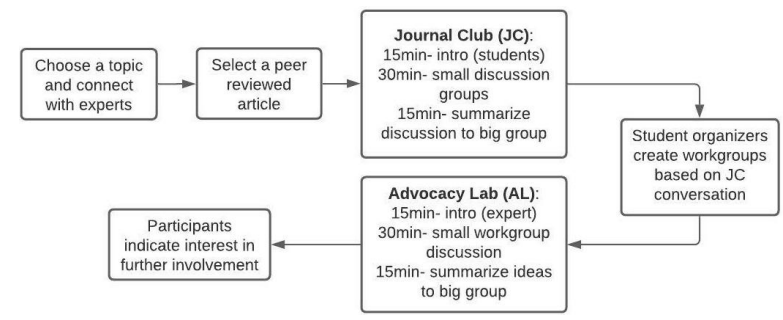

Abstract \#92 Figure 1 Workflow for JC-AL

sense of community (24\%) and/or motivation (29\%) regarding health equity work after the intervention. 158 participants $(67.2 \%)$ reported being somewhat or very likely to stay involved in the advocacy project, and 93 participants (39.6\%) reported being likely to lead a session in the future. Almost all of the JC-ALs have manifested long term projects including:

- Educational material for healthcare providers and preclinical students regarding removal of race from eGFR and adoption of cystatin $\mathrm{C}$ and addition of cystatin $\mathrm{C}$ in UCSD Health Laboratory Medicine Formula

- Learning modules for preclinical students on gender affirming and trauma informed care

- Elective on obtaining a health equity history in the emergency department

Conclusions The JC-AL schema is a feasible approach to engage trainees in the community and institution to enact change. It is a well-received component of the HET ranging from 30-100 participants at each event.

\section{\#93 USING VALIDATED INSTRUMENTS TO ASSESS LONELINESS, SOCIAL SUPPORT, AND BURNOUT AMONGST RACIAL AND LGBTQ+ RESIDENTS}

DA Mesa*. University of Colorado, Denver, CO

\subsection{6/jim-2022-WRMC.92}

Purpose of Study There is very little data published exploring the impact that racial or sexual minority identity has on a resident's training experience. Given that a high percentage of internal medicine training programs are predominantly white it's important to understand the emotional and supportive barriers minority residents face. We began the important work looking into these barriers with a survey-based needs study.

Methods Used 174 residents enrolled in the University of Colorado Internal Medicine Residency Program were asked to participate in an online survey. This survey consisted of several validated instruments including: the PHQ-4, the MOS Social Support Survey, and the UCLA Loneliness scale. The survey included a demographics section and each respondent utilized a unique PID to maintain anonymity. 65 out of 174 residents responded to the survey. The answers to the survey were coded and scored per the original publications. Analysis of the data was done using two tailed T-Tests in the SAS software.

Summary of Results The average MOS total support score was significantly lower in LGBTQ+ residents compared to NonLGBTQ+ residents (Mean 65.80 vs. 79.16; $\mathrm{P}=0.035$ ). LGBTQ+ residents also trended towards having higher amounts of burnout, though this wasn't statistically significant (Mean 0.38 vs $0.17 ; \mathrm{P}=0.19$ ). Notably 3 out of 9 LGBTQ
+ residents reported feeling burnt out compared to 9 out of 56 Non-LGBTQ+ residents (33\% vs 16\%). Notably most of the significant findings were amongst single vs non-single residents with significance in: UCLA loneliness scale $(\mathrm{P}=0.03)$, MOS total support score $(\mathrm{P}<0.0001)$, MOS emotional support score $(\mathrm{P}=0.008)$, MOS affectionate support score $(\mathrm{P}$ $<0.0001)$, MOS tangible support score $(\mathrm{P}<0.0001)$, MOS positive interactions score $(\mathrm{P}=0.001)$ and PHQ-Depression sub-domain $(P=0.025)$. Racial minority residents had lower average levels of burnout compared to non-minority residents (Mean 0.12 vs $0.23 ; \mathrm{P}=0.32$ ). However, racial minority residents had lower average levels of overall social support compared to non-minority residents (Mean 73.16 vs 78.74; $\mathrm{P}=$ 0.270) with the tangible support subdomain score being the closest for significance (Mean 13.55 vs 15.93; P $=0.120$ ).

Conclusions The sample size for the survey-based study was smaller than anticipated. However, it was large enough to find significance for LGBTQ+ residents having less social support, and also revealed higher levels of burnout. It's also important to note that while it didn't reach significance minority residents experienced lower average levels of overall social support. Surprisingly minorities had fewer burnout numbers which may be an indicator of increased resilience or utilization of protective mechanisms. Further reasearch needs to be conducted to better understand the needs of LGBTQ+ and racial minority residents. Future directions include expansion nationwide to gather a larger sample size and assess for geographic differences.

\section{\#94 IDIOPATHIC SUBGLOTTIC STENOSIS IN NON-CAUCASIAN WOMEN}

${ }^{1} \mathrm{~A}$ Suk ${ }^{*},{ }^{2} \mathrm{~L}$ Reder, ${ }^{3} \mathrm{~K}$ O'Dell, ${ }^{4} \mathrm{~S}$ Verma, ${ }^{5} \mathrm{M}$ Harmon, ${ }^{5} \mathrm{P}$ Weissbrod, ${ }^{6} \mathrm{P}$ Krishna, ${ }^{6} \mathrm{~B}$ Crawley. ${ }^{1}$ Loma Linda University School of Medicine, Loma Linda, CA; ${ }^{2}$ Kaiser Permanente Baldwin Hills, Los Angeles, CA; ${ }^{3}$ University of Southern California, Los Angeles, CA; ${ }^{4}$ University of California Irvine, Irvine, CA; ${ }^{5}$ University of California San Diego, La Jolla, CA; ${ }^{6}$ Loma Linda University School of Medicine, Loma Linda, CA

\subsection{6/jim-2022-WRMC.93}

Purpose of Study To analyze the presentation, disease course, and treatment of idiopathic subglottic stenosis of non-Caucasian women.

Methods Used In this multi-institutional retrospective study, information extracted included date of birth, age at symptom onset, age and date of diagnosis, race, Cotton Meyer grade, stenosis length and distance from glottis, BMI, comorbidities, medication to manage iSGS, age at first surgery, additional treatment with serial intralesional steroid injections, the date of each surgery, occupation, autoimmune labs, and family history of autoimmune diseases.

Summary of Results 35 non-Caucasian women with idiopathic subglottic stenosis were identified. Of the 35 women, 31 were Hispanic while one was African-American, two were Asian, and one was non-Hispanic mixed race. Their average BMI was $31.8 \pm 2.19 \mathrm{~kg} / \mathrm{m}^{2}$ and $51.4 \%$ of the patients were obese (BMI>30). 31.4\% had hypertension. Their average age of onset was 45.8 years old (95\% CI, 42.2-49.3) with a range of 26-69 years old. The average age at diagnosis was 47.8 years $(95 \% \mathrm{CI}, 44.3-51.3)$ with a Charlson comorbidity index of 0.85 (95\% CI, 0.42-1.28). At diagnosis, $13.4 \%$ were CM I, $43.3 \%$ were CM II and $43.3 \%$ were CM III $(n=30)$. The average age at their first surgery was 46.8 (95\% CI, 43.250.4) years and 17 received SILSI. While treatment type 
varied given this was a retrospective surgery, none of the 35 women received open reconstruction. 62.9\% experienced disease recurrence after their first surgery with a median of 11 months between their first and second surgery and received an average of 2.5 surgeries.

Conclusions Our results show that the non-Caucasian population does not differ from the majority Caucasian population published elsewhere in current literature on idiopathic subglottic stenosis, which calls into question the homogeneity of the disease and the need to adjust recruitment methods to include more people of color and provide a more accurate representation of the patient population.

\section{\#95 SCOPING REVIEW OF SOCIOECONOMIC FACTORS AND HIDRADENITIS SUPPURATIVA}

${ }^{1}$ OS Cherepakhin*, ${ }^{2} \mathrm{~K}$ DeNiro. ${ }^{1}$ University of Washington School of Medicine, Seattle, WA; ${ }^{2}$ University of Washington, Seattle, WA

\subsection{6/jim-2022-WRMC.94}

Purpose of Study Hidradenitis suppurativa (HS) is an autoinflammatory disease characterized by painful boils beneath the skin. While socioeconomic factors have been linked to HS individually, there has been no scoping review that synthesizes these correlations. Our objective was to assess the published data on the associations between HS and the factors of income, education, and work.

Methods Used A search limited to English publications was conducted in PubMed, Embase, Web of Science and Cochrane from database origin to $07 / 26 / 21$. The terms used were 'hidradenitis' combined with 'socioeconomic,' 'insurance, 'class, 'disparities,' 'disparity,' 'education,' 'income,' 'work,' 'employment,' 'job,' 'insurer,' 'medicaid,' or 'professional activity.' Eligible publications were peer-reviewed and examined the association between HS and income level, educational attainment, occupation class, employment status, work impairment, or insurance status. Records were evaluated by O.C. and K.D. In the event of a disagreement, another reviewer was available to resolve the discrepancy.

Summary of Results After duplicate removal, 413 records were screened by title/abstract. 79 full-text records were then assessed for eligibility and 33 articles met inclusion criteria. By manually searching article references, an additional 3 papers were included. 29 research articles, 6 reviews, and 1 case report from 13 different countries were qualitatively synthesized according to the defined categories of associations.

3 articles found that HS patients had lower income levels but one of those studies, after adjusting for age/sex, found that this was not significant. 6 articles elucidated an association between HS and lower educational attainment. An association between HS and lower class of occupation was found by 1 study, and 7 publications (6 articles, 1 review) demonstrated a higher probability of being unemployed as an HS patient. 16 articles, 1 case report, and 5 reviews discussed the association between HS and work impairment. A higher likelihood for HS patients to have government-funded insurance was found by 3 studies. 4 articles utilized a combination of the factors as measures of SES. 3 of them found associations between low SES and HS, while one Israeli study found the opposite.

Conclusions Our qualitative synthesis demonstrates that HS globally is linked with lower income levels, reduced educational attainment, unemployment, work impairment, and government-funded insurance coverage. Though one study found that higher SES is associated with HS, this can be explained by their usage of dermatologist-diagnosed HS patients and the fact that in Israel, dermatology encounters require co-payments unlike primary care visits. Though the directionality between HS and lower SES cannot be determined from the current research, our work shows the importance of considering SES when treating HS patients.

\section{\#96 AMERICAN OSTEOPATHIC ASSOCIATION OTOLARYNGOLOGY AND OPHTHALMOLOGY PROGRAM CLOSURES AS A MODEL TO HIGHLIGHT CHALLENGES OF MAINTAINING GRADUATE MEDICAL EDUCATION IN HIGH NEED AREAS}

${ }^{1} \mathrm{~K}$ Vo*${ }^{*}{ }^{2} \mathrm{H}$ Ahmed, ${ }^{3} \mathrm{~W}$ Robbins. ${ }^{1}$ Western University of Health Sciences, Pomona, $C A_{i}$ ${ }^{2}$ Loma Linda University, Loma Linda, $\mathrm{CA} ;{ }^{3}$ OhioHealth, Columbus, $\mathrm{OH}$

\subsection{6/jim-2022-WRMC.95}

Purpose of Study While 90\% of former American Osteopathic Association (AOA) residency programs transitioned to Accreditation Council for Graduate Medical Education (ACGME) accreditation, surgical subspecialty programs such as otolaryngology (ENT) (62\%) and ophthalmology (47\%) struggled to gain accreditation. DOs have actively participated in serving underserved communities, and losing AOA surgical specialty programs may decrease access to surgical care in rural and non-metropolitan areas.

Methods Used A directory of former AOA ENT and ophthalmology programs was obtained from the American Osteopathic Colleges of Ophthalmology and Otolaryngology-Head and Neck Surgery (AOCOO-HNS). A secured survey was sent out to16 eligible ENT and ophthalmology program directors. The survey contained both quantitative and qualitative aspects to help assess why these programs did not pursue or failed to receive ACGME accreditation.

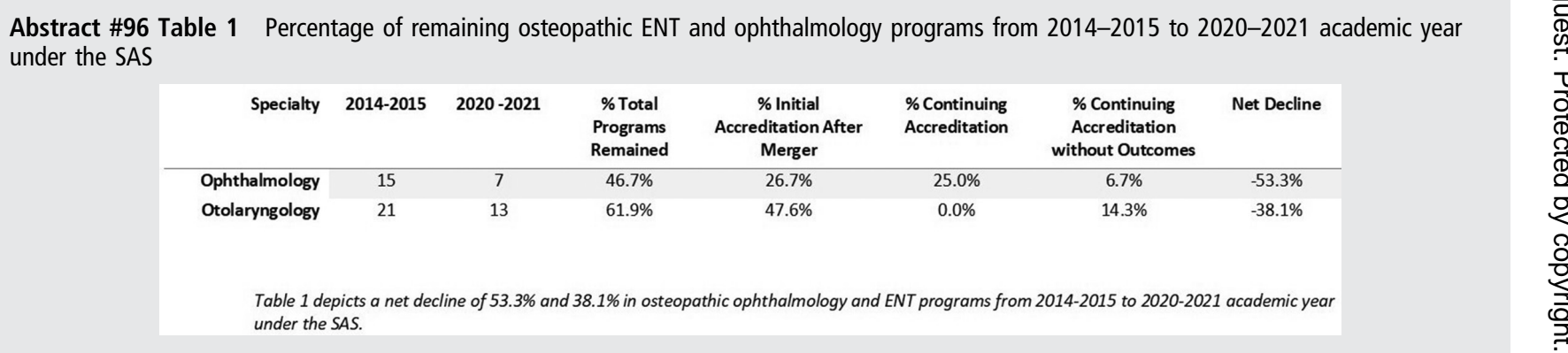


Overall reasons for not pursuing or obtaining ACGME accreditation

(Combined ENT and Ophthalmology Program Director Responses)

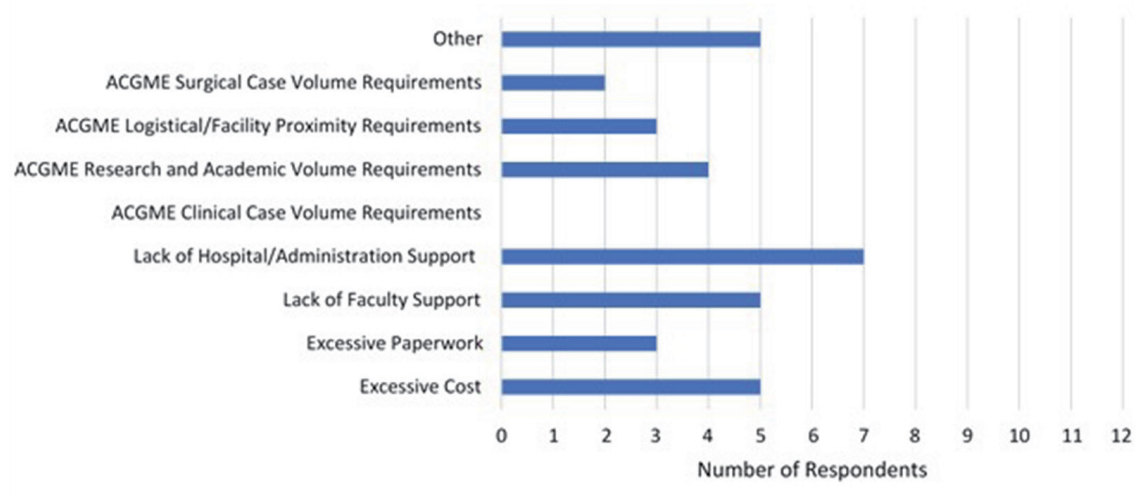

\section{Abstract \#96 Figure 1}

Summary of Results 12 of 16 eligible programs responded: 6 ophthalmology and 6 ENT program directors. $83 \%$ of respondents did not pursue accreditation (6 ophthalmology and 4 ENT programs), and $17 \%$ (2) were unsuccessful in achieving accreditation despite pursuing accreditation. Across 12 respondents, $58 \%$ (7) cited lack of hospital/administrative support and 42\% (5) cited excessive costs and lack of faculty support as reasons for not pursuing or obtaining ACGME accreditation.

Conclusions The survey results reflect financial issues associated with rural hospitals. Lack of hospital/administrative support and excessive costs to transition to the ACGME were key drivers in the closures of AOA surgical specialty programs. Considering these results, we have 4 recommendations for various stakeholders, including program directors, designated institutional officials, hospital chief medical officers, and health policy experts. These recommendations include expanding Teaching Health Center Graduate Medical Education to surgical subspecialties, identifying and learning from surgical fields such as urology that fared well during the transition to ACGME, addressing the lack of institutional commitment and prohibitive costs of maintaining ACGME accredited subspecialty programs in under-resourced settings, and reconsidering Centers for Medicare \& Medicaid Services (CMS) pool approach to physician reimbursement.

\section{Hematology and oncology I}

\section{Concurrent session}

\section{3:15 PM}

\section{Thursday, January 20, 2022}

\section{\#97 NOVEL PERINEPHRIC NEOVASCULARITY SCORING SYSTEM IN RENAL CELL CARCINOMA TUMOR STAGING}

C Fateri* , A Peta, N Kar, T Bui, B Roth, J Glavis-Bloom, L Limfueco, J Landman, R Houshyar. University of California Irvine, Irvine, CA

10.1136/jim-2022-WRMC.96
Purpose of Study Renal cell carcinoma (RCC) is the most common type of kidney cancer worldwide. Angiogenesis plays a major role in providing adequate blood flow and nutrients to promote tumor growth and RCC progression. While radiologists assess enhancement patterns of renal tumors to predict tumor pathology, to our knowledge, no formal scoring system has been created and validated to assess the level of neovascularity in RCC, despite its critical role in cancer metastases. In this study, we characterized and analyzed the level of angiogenesis in tumor-burdened kidneys and their benign counterparts. We then created and validated a scoring scale for neovascularity that can help predict tumor staging for RCC.

Methods Used After Institutional Review Board approval, the charts of patients who had undergone surgery for RCC between January 13, 2014 and February 4, 2020 were retrospectively reviewed for inclusion in this study. Inclusion criteria were a diagnosis of RCC, simple/radical nephrectomy, pre-operative contrast enhanced computed tomography (CT) scans, and complete pathology reports. Neovascularity was scored on a scale of 0 to 4 where $0=$ no neovascularity detected, $1=$ a single vessel $<3 \mathrm{~mm}$ wide, $2=$ a single vessel $\geq 3 \mathrm{~mm}$ wide, $3=$ multiple vessels $<3 \mathrm{~mm}$ wide, and $4=$ multiple vessels $\geq 3 \mathrm{~mm}$ wide. Each patient was scored by a senior medical student and then validated by a board-certified abdominal radiologist. Statistical analysis was performed using RStudio ${ }^{\circledR}$ Version 3.5.1. Demographics and tumor characteristics were compared using a Kruskall-Wallis ANOVA or Chi-squared test; neovascular score was compared using a Wilcoxon Rank-Sum test. Statistical significance was considered as $\mathrm{p}<0.05$.

Summary of Results A total of 217 patients were included in this study. There was no significant difference in patient demographics between tumor stages. Additionally, the majority of tumor pathology was clear cell carcinoma, regardless of tumor staging. The average neovascularity score was 1.07 for $\mathrm{pT} 1 \mathrm{x}$ tumors, 2.83 for pT2x tumors, and 3.04 for pT3x tumors. The average neovascularity score for the benign counterparts was $0.124,0.385$, and 0.458 , respectively. There was a significant difference in neovascularity score between $\mathrm{pT} 1 \mathrm{x}$ and pT2 $\mathrm{x}$ tumors $(\mathrm{p}=0.0046), \mathrm{pT} 1 \mathrm{x}$ and $\mathrm{pT} 3 \mathrm{x}$ tumors $(\mathrm{p}<$ 
0.0001), and benign kidneys and kidneys with RCC ( $\mathrm{p}=$ $<0.0001)$.

Conclusions Our novel vascular scoring system for renal cell carcinoma demonstrates a significant correlation with RCC pathological tumor staging. This scoring system may be utilized as part of a comprehensive radiological assessment of renal tumors, potentially improving tumor characterization and clinical decision making.

\section{\#98 STRESS-INDUCED DIFFERENTIAL MIR-4633-5P EXPRESSION IN THYROID CANCER HEALTH DISPARITIES}

'J Mancao*, 'S Khan, 'J Lee, ${ }^{2} \mathrm{~K}$ Rood, ${ }^{1} \mathrm{R}$ Davis, ${ }^{1} \mathrm{M}$ Perez, ${ }^{3} \mathrm{~A}$ Simental, ${ }^{4} \mathrm{~S}$ Roy. 'Loma Linda University School of Medicine, Loma Linda, CA; ${ }^{2}$ Loma Linda University Department of Basic Sciences, Loma Linda, CA; ${ }^{3}$ Loma Linda University Medical Center, Loma Linda, CA; ${ }^{4}$ The University of Texas at El Paso, El Paso, TX

\subsection{6/jim-2022-WRMC.97}

Purpose of Study Filipino Americans (FA) are known to have higher rates of thyroid cancer incidence and disease recurrence compared to European Americans (EA). FA are also known to be two times more likely to die of thyroid cancer compared to EA. Epidemiological studies in California have shown that thyroid cancer is the second most common cancer among FA women. Currently, there are no studies that demonstrate the mechanism behind these discrepancies. Evidence shows a strong correlation between obesity and more aggressive forms of thyroid cancer; obesity has an increased frequency in FA populations. The exact connection between the mechanisms of obesity and cancer is poorly understood. This epigenetic phenomenon may be due to microRNAs (miRNAs), which post-transcriptionally regulate gene expression. Dysregulated miRNA profiles have been associated with various diseases including obesity and cancer. MiRNAs are linked to different types of cancer; tumor suppressor genes and oncogenes are subject to modulation by dysregulated miRNAs. No study elucidates the association of miRNAs to tumor staging or prognosis in thyroid cancer health disparities.

Methods Used In this study, we determined miRNA expression profiles and found significant differences in the miRNA profiles between FA and EA thyroid cancer patients. Our pilot study showed several dysregulated miRNAs, from which we chose to assay dysregulated miR-4633-5p segments that are known to be associated with thyroid cancer signaling. We used QIAGEN's miRNA extraction kit to obtain high-quality miRNA from paraffin-embedded thyroid tissues. We performed next-generation miRNA sequencing using equal number of FA and EA samples and identified the top ten significantly upand down-regulated miRNAs from the pool of differentially expressed miRNAs by qPCR assays.

Summary of Results Our investigation demonstrated a 1.5-2fold higher expression of an upregulated miR-4633-5p in FA versus EA miRNA samples $(n=70)$ after normalized to controls. In contrast, miR-323b-3p showed no difference between FA and EA after normalized to controls.

Conclusions For our future work, we plan to analyze multiple up- and down-regulated miRNAs by qPCR, determine whether the miRNA signatures are consistent between samples from FA versus EA, and explore the use of these miRNA signature differentials for affordable and rapid thyroid cancer screening and prognosis.
\#99 MELANOMA OF UNKNOWN PRIMARY: A SINGLEINSTITUTION EVALUATION OF CLINICAL OUTCOMES

${ }^{1,2}{ }^{S}$ Dwabe ${ }^{*}{ }^{1,2} \mathrm{G}$ In. ${ }^{1}$ Los Angeles County University of Southern California Medical Center, Los Angeles, CA; ${ }^{2}$ Keck Hospital of USC, Los Angeles, CA

\subsection{6/jim-2022-WRMC.98}

Purpose of Study Melanoma of unknown primary (MUP) is clinically uncommon and is understudied as a disease. There have been studies evaluating the utility of local resection with radiation therapy for treatment of MUP. However, it has been only within the last few years that MUP has been routinely treated with targeted or immunotherapy.

Methods Used We conducted a retrospective review of patients with MUP treated at LAC-USC Medical Center and Norris

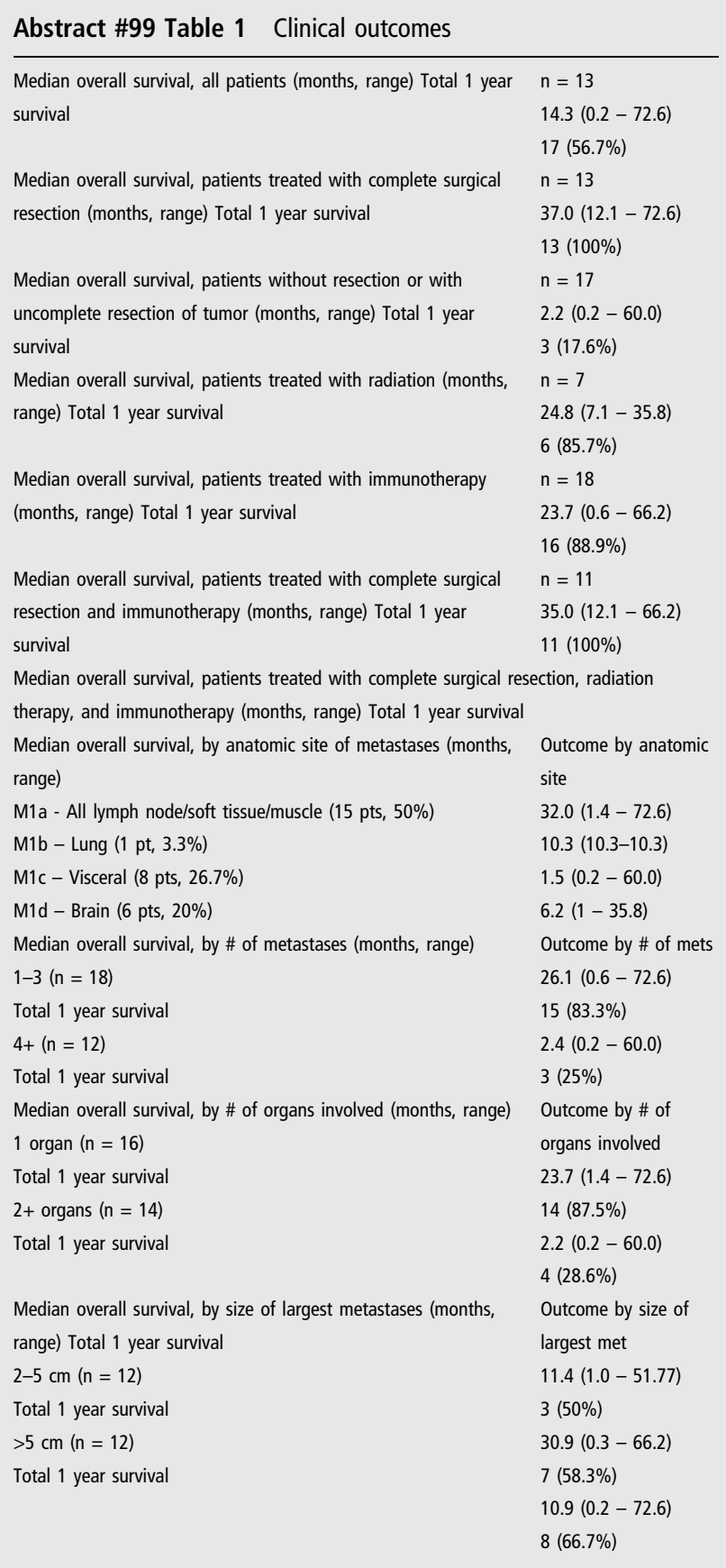


Cancer Center from 2008 to December 1st, 2020. We recorded the presentation, treatment course, and outcomes of each patient within our database. Data points collected include demographic information, clinical staging, size of largest metastases, location and number of metastatic sites. Treatment modalities, including metastatectomy, and systemic therapy were reviewed. The primary outcomes studied were median overall survival and 1-year overall survival.

Summary of Results Data was collected from 32 patients identified as having MUP. Sites of melanoma metastases included lymph node, soft tissue/muscle, lung, liver, brain/leptomeningeal disease, and bone. Thirteen patients (40.6\%) were found to have one metastatic tumor, $6(18.8 \%)$ were found to have 2-3 metastases, and $13(40.6 \%)$ were found to have $4+$ metastatic tumors on presentation. Two patients were lost to follow up shortly after their diagnosis. The 30 remaining patients had a 14.3-month median survival with 17 (56.7\%) surviving past one year.

In total, 15 patients underwent surgical metastatectomy, and 18 patients were treated with immunotherapy. With all patients surviving after one year, the 13 patients who had a complete resection of their tumor burden were noted to have a median survival time of 37.0 months following their diagnosis compared to a 2.2-month median survival among the 17 patients who did not have surgery or who had incomplete resection of tumor. The 18 patients treated with immunotherapy (PD-1 \pm CTLA-4 inhibition) were found to have a median survival time of 23.7 months with 16 (88.9\%) surviving past one year. Eleven patients who were treated both with complete surgical resection and adjuvant immunotherapy, were found to have a median survival time of 35.0 months with $11(100 \%)$ surviving past one year. When analyzing outcomes of patients with MUP based on the number of metastases, number of organs involved, and largest size of metastases, survival was correlated with less than 4 metastases and less than 2 organs involved. Size of largest metastases had no effect on survival outcomes.

Conclusions Outcomes among patients with MUP may vary, depending on treatment modality, and tumor burden. Based on our data, patients who have MUP with low burden of disease may benefit from multi-modality therapy, including both surgical metastatectomy, and immune checkpoint blockade. Further validation using larger cohorts is warranted to help confirm these findings.

\section{$\# 100$ SECOND HITS IN NF1-TUMORS REVEAL PREVALENCE OF COPY NEUTRAL LOSS-OF-HETEROZYGOSITY IN INDIVIDUALS WITH NEUROFIBROMATOSIS TYPE 1}

${ }^{1} S$ Tong*, ${ }^{2} \mathrm{~J}$ Shieh, ${ }^{2} \mathrm{P}$ Devine. ' UCSF Benioff Children's Hospital Oakland, Oakland, CA;

${ }^{2}$ University of California San Francisco, San Francisco, CA

\subsection{6/jim-2022-WRMC.99}

Purpose of Study Multiple mechanisms may give rise to biallelic variants in NF1-related tumors. Deletion and copy-neutral loss of heterozygosity (LOH) are potential mechanisms of somatic NF1 loss, distinct from point mutations. Tumor multigene sequencing demonstrates co-mutations in genes in addition to NF1, which may be tumor dependent and which may help molecularly classify tumors seen in NF1. This study asks whether excised tumors from individuals with NF1 demonstrate additional gene variants and differentiates first and second hits in NF1 using paired germline and somatic sequencing.

Methods Used The hypothesis is NF1 second hits and comutational patterns may be found by analyzing cancer driver genes. To test this hypothesis, data from 6381 tumors previously sequenced on a 529- cancer gene panel were analyzed to yield 391 NF1-mutated tumors. LOH analysis over NF1 was done for all cases.

Summary of Results NF1 LOH was common, seen in 133/391 tumor samples. There were 40 tumors from individuals with constitution NF1. Tumors from individuals with constitutional NF1 had more prevalent copy neutral LOH (p-value $<0.0001$, two proportion z-test), suggesting somatic intrachromosomal recombination. Osteosarcoma was noted in association with NF1 with copy-neutral LOH, adding to accumulating reports of this rare tumor in NF1. NF1-associated MPNST versus non-NF1-associated MPNST, harbored comutations in TP53 as well as CDKN2A/2B deletion. Additionally, NF1 second-hit data from tumors were informative for annotating missense variants that were conflicting in ClinVar, potentially helping to improve NF1 annotation. The results provide an additional 162 deleterious NF1 variants to add to current gene annotation efforts.

Conclusions Sequencing of paired tumor and normal samples in NF1-associated tumors uncovers a spectrum of second hits to the NF1 locus. Future work will be aimed at a mechanistic understanding of these distinct patterns of mutation and strategies aimed at mitigating tumor risk.

\section{\#101 IMPACT OF SPONSOR ON ADVANCED NON-SMALL CELL LUNG CANCER CLINICAL TRIAL ENROLLMENT CRITERIA}

${ }^{1}$ RA Cooper * , ${ }^{2} Y$ Chai, ${ }^{1} \mathrm{~J}$ Nieva. ' University of Southern California Keck School of Medicine, Los Angeles, CA; ${ }^{2}$ Children's Hospital of Los Angeles Saban Research Institute, Los Angeles, $C A$

\subsection{6/jim-2022-WRMC.100}

Purpose of Study Clinical trials use inclusion and exclusion criteria to control for confounding variables in patient populations. Largely inspired by the ASCO-Friends of Cancer Research recommendation documents (2017 and 2021), there has been a recent drive to loosen clinical trial enrollment criteria to improve generalizability in trial outcomes. We sought to determine if the sponsor of a clinical trial impacted the transparency and selection of inclusion and exclusion criteria.



Abstract \#101 Figure 1 Percentage of studies with strict, loose, and no restrictions on performance status. Actual number of studies within each group are included as data labels 
Methods Used Using clinicaltrials.gov, phase 2 and 3 non-small cell lung cancer (NSCLC) drug trials were sorted into one of three sponsor categories: Industry, government/cooperative group, and academic. Fisher Exact tests were used to assess variability in strictness of specific criteria and level of transparency in listing organ function requirements. Independent sample $t$ tests were used to analyze differences in total number of criteria.

Summary of Results Industry sponsored NSCLC drug trials more often omit from clinicaltrials.gov complete organ function requirements compared to government/cooperative group $\left(\mathrm{p}=2.3 \times 10-^{10}, \alpha=0.01\right)$ and academic $\left(\mathrm{p}=1.8 \times 10-^{4}\right.$, $\alpha=0.01$ ) sponsored trials. Industry sponsored trials are also more likely to have stricter performance status requirements compared to government/cooperative group sponsored studies $\left(\mathrm{p}=5.7 \times 10-^{6}, \alpha=0.01\right)$.

Conclusions Industry funded NSCLC clinical trials are more rigorous in excluding patients with worse performance status and are less transparent in listing all study requirements on clinicaltrials.gov.

\section{\#102 ASSESSING ADULT PATIENTS' UNDERSTANDING OF TERMS IN CONSENT RELATED TO SECONDARY MALIGNANCY RISK IN RADIATION THERAPY}

N Vartanian*, M Wilson, R Ermoian. University of Washington School of Medicine, Seattle, WA

\subsection{6/jim-2022-WRMC. 101}

Purpose of Study Informed consent entails that healthcare providers effectively describe adverse effects associated with medical treatments to patients. In radiation oncology, the terms 'second tumors', 'secondary malignancies', or 'secondary tumors' are used in patient consents to describe the appearance of new and different tumors caused by radiation treatment. Furthermore, these incidences are sometimes described in consents as 'rare', although the incidence varies greatly from nearly negligible in patients treated with palliative intent, to $20 \%$ in patients undergoing myeloablative total body irradiation for stem cell transplant. We evaluated whether non-cancer patients without prior knowledge of or exposure to radiation therapy interpret the terms 'secondary malignancy', 'rare', and 'small chance' in a way consistent with physician intent.

Methods Used We screened 164 adult subjects who did not require medical interpreters at a university affiliated family medicine clinic, excluding cancer patients and those with any prior knowledge of or experience with radiation treatment. One hundred subjects were eligible for and completed our 12question multiple choice questionnaire, which assessed their understanding of the term 'secondary tumor', and how they would interpret the terms 'small chance' or 'rare' in the context of a 'bad side effect' arising from medical treatment.

Summary of Results Twenty-nine percent of subjects correctly identified that 'secondary tumors' referred to new and different tumors caused by treatment. Forty-nine percent thought the term referred to their original tumor coming back, and twenty-two percent thought the term referred to new and different tumors not caused by radiation therapy. In the context of a 'bad side effect' occurring 'rarely', $2 \%$ of subjects attributed 'rare' to a $1 / 10$ chance; $16 \%$ to a $1 / 100$ chance; $33 \%$ to a $1 / 1000$ chance; and $49 \%$ to a $1 / 100,000$ chance.
In the context of a 'bad side effect' having a 'small chance' of occurrence, $8 \%$ of subjects attributed 'small chance' to odds of $1 / 10 ; 33 \%$ to $1 / 100 ; 41 \%$ to $1 / 1000$; and $18 \%$ to $1 /$ 100,000 .

Conclusions Patients without prior radiation therapy exposure have a demonstrably different understanding than radiation oncologists of the terms 'secondary malignancy', 'second tumor', or 'secondary tumor'. Additionally, there is great variability in patient understanding of the terms 'rare' or 'small chance'. Radiation oncologists must use different and more descriptive terms for secondary malignancies and their incidence, to ensure patients are truly informed when undergoing treatment. The results of this study may have implications for all medical fields in which patients are consented for procedures associated with a risk for consequential side effects.

\section{$\# 103$ REGULATION OF CHEMORESISTANCE BY CHRM1 IN NON-SMALL CELL LUNG CANCER}

S Simpson*, T Bland. WWAMI, Seattle, WA

\subsection{6/jim-2022-WRMC.102}

Purpose of Study Chemotherapy is a mainstay treatment for late-stage non-small cell lung cancer (NSCLC), yet most tumors develop resistance to these agents. Studies in our lab have shown that chemoresistant NSCLC cells overexpress the muscarinic acetylcholine receptor 1 (CHRM1). We hypothesize that CHRM1 regulates chemoresistance in NSCLC cells, and that the combination of a repurposed CHRM1 antagonist dicyclomine, clinically used to treat IBS, and a chemotherapeutic agent has the potential to sensitize and kill chemoresistant NSCLC cells.

Methods Used Chemosensitive (A549) and chemoresistant $\left(\mathrm{A} 549^{\mathrm{R}}\right)$ NSCLC cells were utilized in this study. Cell survival and colony formation assays were utilized to measure DTX sensitivity by pretreating with designated drug (24 hr) before addition of DTX (48 hr). Western blot and phosphokinase array were utilized to measure protein expression and intracellular pathway activation. The designed receptors exclusively activated by designer drugs (DREADD) system was utilized to isolate CHRM1 signaling. All data are expressed as the mean \pm SEM. Multiple comparisons were analyzed using one-way ANOVA with post-hoc Tukey's analysis and single comparisons were analyzed using a two-tailed, unpaired Student's t test.

Summary of Results CHRM1 expression is enhanced in A549 ${ }^{\mathrm{R}}$ cells, suggesting that CHRM1 may play a role in chemoresistance. This was supported by the ability of a CHRM1 agonist, dicyclomine (Dic), to sensitize $\mathrm{A} 549^{\mathrm{R}}$ cells to the chemotherapeutic agent docetaxel (DTX) measured by cell survival (IC50: DTX, not reached > 1M; DTX + Dic $(10 \mu \mathrm{M}), 49.91 \mu \mathrm{M}$; DTX + Dic $(25 \mu \mathrm{M}), 12.11 \mu \mathrm{M})$. Furthermore, these results were duplicated by colony formation assay. However, activation of a CHRM1 in A549 cells by the acetylcholine mimetic carbachol did not protect cells from DTX-induced cell death, suggesting that CHRM1 expression is necessary for chemoresistance in the A549 ${ }^{\mathrm{R}}$ cells, but not sufficient. A phospho-kinase array was used to determine the intracellular signaling pathway activated by CHRM1, which showed increased phosphorylation of multiple kinases including CREB, EGFR, STAT3, and ERK1/2. Increased CREB phosphorylation was 
validated by western blot with carbachol stimulation in $\mathrm{A} 549^{\mathrm{R}}$ and M1D samples suggesting these as possible targeting pathways downstream of CHRM1.

Conclusions Chemoresistant NSCLC shows increased CHRM1 expression, which when antagonized, resensitizes these cells to DTX-induced cell death. While CHRM1 expression is not sufficient to instill resistance, it is necessary in the A549 ${ }^{\mathrm{R}}$ cells, and may play a role in enhancing EGFR signaling. This provides a potential promising new therapy for lethal chemoresistant NSCLC which utilizes the repurposed IBS drug dicyclomine.

\section{\#104 TISSUE IS THE ISSUE: CHEMOTHERAPY RESPONSE SCORE (CRS) IS MOST PREDICTIVE OF RESPONSE TO NEOADJUVANT CHEMOTHERAPY IN ADVANCED, HIGH GRADE SEROUS OVARIAN CANCER}

${ }^{1} \mathrm{~A}$ Knickerbocker*, ${ }^{2} \mathrm{~K}$ Kuchta, ${ }^{2} \mathrm{C}$ Donaldson, ${ }^{2} \mathrm{E}$ Diaz Moore, ${ }^{2} \mathrm{M}$ Lippitt, ${ }^{2} \mathrm{G}$ Rodriguez, ${ }^{2} \mathrm{~W}$ Watkin, ${ }^{2} \mathrm{~T}$ Jenkins Vogel. ${ }^{1}$ Washington State University, Spokane, WA; ${ }^{2}$ NorthShore University HealthSystem, Evanston, IL

\subsection{6/jim-2022-WRMC.103}

Purpose of Study Favorable chemotherapy response score (CRS) has prognostic value and correlates with progression free and overall survival in advanced ovarian cancer. CRS has not been compared to other clinical measures used to gauge response to neoadjuvant chemotherapy (NACT). We sought to examine whether CRS is a better predictor of outcome compared to traditional clinical and radiographic response measures.

Methods Used Clinical data from 2003-2020 was obtained through retrospective chart review. Radiographic review preand post-NACT was performed via RECIST 1.1 with responses characterized as complete/near-complete (CR/NCR), partial with $>50 \%$ reduction in tumor $(\mathrm{PR}>50)$, partial with $<50 \%$ reduction in tumor $(\mathrm{PR}<50)$, stable disease $(\mathrm{SD})$ and progressive disease (PD). Histologic response in surgical specimens was characterized using CRS 1-3. Survival was assessed using the Kaplan-Meier method with log-rank tests, and Cox regression with hazard ratios (HR).

Summary of Results 128 patients who underwent NACT for high grade serous ovarian cancer (HGSOC) were included. Increasing CRS was associated with improved recurrence free (RFS) and overall survival (OS). OS at 5 years for CRS 1,2 and 3 was $24.7 \%, 57 \%$ and $73.7 \%(p<0.0001)$. More favorable radiographic response was predictive of decreased recurrence risk with $\mathrm{RFS}$ at 3 years for $\mathrm{PR}<50$ and $\mathrm{CR} /$ NCR $(16.3 \%$ and $54.8 \%, \mathrm{p}=0.0005)$ but not predictive of OS. Patients with CR/NCR more commonly had CRS 3 vs CRS 1 (47.1 vs $17.7 \%, p=0.022)$. Among radiographic response groups, increasing CRS was associated with decreased risk of recurrence and death. For example, in patients with CR/NCR risk of recurrence HR 5.38 $(p=0.0243)$ and risk of death $8.24(p=0.006)$ with CRS 1 vs 3 . Number of NACT cycles prior to surgery did not differ among patients regardless of CRS. Rate of R0 resection was similar among all three CRS subgroups, and for the entire cohort was $85.2 \%$. Recurrence rates were significantly higher with CRS $1(89.1 \%)$ and 2 (73.9\%) compared to CRS $3(38.9 \%)(p<0.0001)$. Median CA 125 prior to surgery was lower with CRS 3 compared to CRS 1 (28 vs 81, $\mathrm{p}=0.0017)$. Of the 12 germline BRCA2+ patients in the study, $7(58.3 \%)$ had a pathologic CRS 3.

Conclusions Our data confirms that favorable CRS is associated with improved overall and recurrence free survival in HGSOC. While radiographic response appears predictive of recurrence, it was not associated with overall survival in our study. Among patients with similar radiographic response, CRS remained predictive of outcome and is associated with other clinical factors traditionally felt to confer favorable prognosis. Pathologic CRS is an important predictive factor in determining response to neoadjuvant chemotherapy in HGSOC and may provide the best means to characterize prognosis.

\section{\#105 THE IMPACT OF AN EDUCATIONAL TRAINING PROGRAM ON COVID-19 AND CANCER: ENHANCING COMMUNITY UNDERSTANDING, FOR DISEASE PREVENTION, BETTER TREATMENT, AND OPTIMAL OUTCOMES}

J Miller*, E McGhee. Charles Drew University of Medicine and Science, Los Angeles, CA

\subsection{6/jim-2022-WRMC.104}

Purpose of Study The CDC reported cancer patients as at-risk for severe illness from severe acute respiratory syndromerelated coronavirus 2 (COVID-19). Cancer patients were 2 times more likely than non-cancer patients to exhibit cellular sequelae due to COVID-19. Those with hematological malignancies exhibited a case-fatality rate 2 times more than those with solid tumors. This research aims to educate and enhance community understanding of factors that lead to increased mortality rates in COVID-19 cancer patients by using a community training program in SPA 6 of Los Angeles, California.

Methods Used Data were obtained from post-training surveys of SPA 6 community members which included cancer diagnosis, demographics, and knowledge of COVID-19 with cancer. Impact assessments utilizing Likert-scale response options to analyze and measure the data. Fisher's exact test was utilized to measure and evaluate participant understanding of the community training program in regards to increased mortality rates of COVID-19 cancer patients. Data analyses were performed using statistical tests SPSS. P-values of $<0.05$ were considered significant.

Summary of Results There is a significant need for COVID-19 educational training programs for cancer patients in African American and Latino underserved communities. Impact assessments distributed to 100 participants demonstrated positive change in social behavior and willingness to be vaccinated. Post lecture reviews, quizzes, and feedback surveys were distributed to 100 participants. The information received showed a notable change in participants' overall knowledge of COVID-19 regarding the increased risk in cancer patients.

Conclusions The data exhibit educational training programs in underserved communities that were hardest hit by COVID-19 increase the understanding of COVID-19 cancer knowledge. The educational training program indicated an association with a greater increase in willingness to participate in COVID-19 prevention practices and willingness to be vaccinated. This research demonstrated the positive 
change from educational training programs to be utilized to make a significant impact on health outcomes and cancer mortality rates.

\section{\#106 PREDICTIVE VALUE OF THE MINUTE WALK TEST IN ONCOLOGY AND PRE-REHABILITATION: A LITERATURE REVIEW}

${ }^{1} \mathrm{RC}$ Ellis*, ${ }^{2} \mathrm{~A}$ Blough, ${ }^{2} \mathrm{M}$ Clark. 'Western University of Health Sciences College of Osteopathic Medicine of the Pacific, Pomona, CA; ${ }^{2}$ University of Kansas Medical Center, Kansas City, KS

\subsection{6/jim-2022-WRMC.105}

Purpose of Study To review the predictive value of the minute walk physical function test in hematologic malignancy.

Methods Used A literature review of PubMed using the terms and synonyms of 'hematologic cancers' and 'functional evaluation' on June 3, 2021 elicited 1,256 manuscripts. After reviewing each abstract for clinical outcomes in relation to minute walk physical function tests in hematologic malignancy, and with the exclusion criteria of confounding intervention or lack of original research, we included 3 published studies.

Summary of Results Increased frailty before and during cancer treatments has been demonstrated to predict mortality, disability, and hospitalization for cancer patients. The minute walk test is an objective measurement of frailty that measures the distance walked in a set amount of time, with decreased distance walked indicative of increased frailty. While there are multiple published manuscripts documenting the association between the minute walk test and clinical outcomes in cancer patients, few studies validate this test in hematologic cancer patients. Our review found 3 studies using the minute walk test as a functional correlate for rates of mortality. Only 1 of the reviewed manuscripts reported significant increase in mortality with decreased physical function measured by the minute walk test, while the other 2 studies showed no significant change. The study that showed a significant change used a follow up period of 2 months, while the studies with nonsignificant results used a 1 to 2 year follow up.

Conclusions The frequency of nonsignificant results and the shorter follow up period of the significant results suggest that the minute walk test may be an unreliable predictor of mortality in hematologic cancers. This affects oncology and physiatry alike. The minute walk is one of multiple frailty assessments that oncologists use to determine the intensity and type of treatment a patient should receive. This would also impact physiatry, as there is a growing practice of 'prerehabilitation', of improving physical function before and during cancer treatment to improve clinical outcomes. If the minute walk test is an inaccurate predictor of mortality, then pre-rehabilitation may focus less on walking mobility. It is possible that the minute walk test may be an accurate predictor of other outcomes in this patient population, such as patient satisfaction or unplanned hospitalizations. Further research, including a meta-analysis, is necessary to determine the predictive value of the minute walking test in hematologic malignancies. As more rehabilitation and oncology practices embrace pre-rehabilitation, the need for validated and standardized methods of objectively assessing physical mobility increases.
Neonatology general II

\section{Concurrent session}

\section{3:15 PM}

\section{Thursday, January 20, 2022}

\section{\#107 PREVENTING GROWTH FAILURE AND CHARACTERIZING MICROBIOME IN NEONATES WITH GASTROSCHISIS}

${ }^{1} \mathrm{~K}$ Strobel ${ }^{*},{ }^{2} \mathrm{~K}$ Kramer, ${ }^{3} \mathrm{E}$ Fernandez, ${ }^{4} \mathrm{C}$ Rottkamp, ${ }^{5} \mathrm{C}$ Uy, ${ }^{3} \mathrm{~L}$ Moyer, ${ }^{3} \mathrm{SL}$ Leibel, ${ }^{5} \mathrm{M}$ Aslam, ${ }^{4} \mathrm{~F}$ Poulain, ${ }^{1} \mathrm{KL}$ Calkins. ${ }^{1}$ University of California Los Angeles, Los Angeles, CA; ${ }^{2}$ University of California San Francisco, San Francisco, CA; ${ }^{3}$ University of California San Diego, La Jolla, $C A ;{ }^{4}$ University of California Davis, Davis, $C A ;{ }^{5}$ University of California Irvine, Irvine, $C A$

\subsection{6/jim-2022-WRMC.106}

Purpose of Study 55\% percent of infants with gastroschisis in the University of California Fetal Consortium (UCFC) have growth failure (GF). The etiology of GF is multifactorial and associated with caloric/nutrient deficiencies. Intestinal dysbiosis may play a role. In this prospective study of infants with gastroschisis, we aimed to investigate 1) if a nutritional pathway would decrease GF, and 2) the relationship between the microbiome and GF.

Methods Used The UCFC implemented a pathway to decrease GF by standardizing parenteral nutrition dosing, human milk feedings, and GF detection and treatment. Adherence was monitored, and a contemporary cohort $(n=45)$ was compared to a historical cohort $(2015-2019, n=125)$. GF was defined as a decline in weight or length $\mathrm{z}$-score $\geq 0.8$. Shotgun next generation sequencing of the fecal microbiome was performed in a subset of gastroschisis $(n=7)$ and late preterm infants $(\mathrm{n}=7)$.

Summary of Results Good adherence to the pathway was noted. Demographics were similar for the cohorts except

\begin{tabular}{lll} 
Abstract \#107 Table 1 & & \\
\hline & Historic & Prospective \\
\hline Gestational Age (weeks) & $37(35-37)$ & $36(35-37)$ \\
Birth Weight (grams) & $2482(2168-2881)$ & $2330(1948-2644)^{*}$ \\
Days Until Full Feeds & $23(18-41)$ & $24(17-33)$ \\
Days of Antibiotics & $4(2-7)$ & $3(1-6)$ \\
Length of Stay & $32(23-60)$ & $34(26-47)$ \\
\hline
\end{tabular}

Cohort characteristics. Continuous variables, median (IQR). * $\mathrm{p}<0.03$


Abstract \#107 Figure 1 A) Heat map showing associations of bacteria with gastroschisis, delivery mode, and antibiotics; B) Dot plots showing relative abundances (log10 transformed) of species 
birth weight (table 1). Historical controls exhibited a decline in weight and length $\mathrm{z}$-scores at 30 days $(-0.10 \mathrm{z}$ score units/week and -0.11 z-score units/week, respectively, $p<0.001$ for all). In the prospective cohort, weight and length $\mathrm{z}$-scores remained stable. When the cohorts were compared, the prospective cohort demonstrated a decrease in length GF at 14 days $(p=0.002), 30$ days $(p=0.03)$, and discharge $(p=0.002)$. However, weight GF was similar at all time points. When compared to preterm infants, gastroschisis infants had a higher abundance of Bacteroides thetaiotaomicon ( $\mathrm{q}=0.003), \quad$ Bacillus coagulans $\quad(\mathrm{q}=0.061)$, Lactobacillus animalis $(\mathrm{q}=0.13)$, and Akkermansia municphilla $(\mathrm{q}=0.13)$, and less Bifidobacterium bifidum $(\mathrm{q}=0.19)$ and longum $(\mathrm{q}=0.19)$ even after adjustment for delivery mode and antibiotic days (figure 1).

Conclusions This study suggests that a multi-institutional nutritional pathway is feasible and may decrease linear GF in infants with gastroschisis. Research is needed to determine how the microbiome contributes to GF in this population.

\section{\#108 ARACHIDONIC AND DOCOSAHEXAENOIC ACID AND RETINOPATHY OF PREMATURITY}

${ }^{1} \mathrm{~T}$ Gillespie ${ }^{*},{ }^{2} \mathrm{E}$ Kim, ${ }^{2} \mathrm{H}$ Kim, ${ }^{2} \mathrm{~T}$ Tsui, ${ }^{2} \mathrm{~A}$ Chu, ${ }^{2} \mathrm{KL}$ Calkins. ${ }^{1}$ University of California Los Angeles David Geffen School of Medicine, Los Angeles, CA; ${ }^{2}$ University of California Los Angeles, Los Angeles, CA

\subsection{6/jim-2022-WRMC.107}

Purpose of Study Worldwide, 20,000 infants each year are legally blind from retinopathy of prematurity (ROP). We have demonstrated that preterm infants develop docosahexaenoic (DHA) and arachidonic acid (ARA) deficits after birth. These polyunsaturated fatty acids play an important role in regulating inflammation and angiogenesis. The aim of this research is to investigate DHA and ARA status in infants at risk for ROP.

Methods Used Inclusion criteria for this single site retrospective study: $\leq 30$ weeks gestational age (GA) or $\leq$ birthweight $(\mathrm{BW})<1.5 \mathrm{~kg}$, and ROP screenings until ROP development, complete vascularization, or 42 weeks postmenstrual age. DHA and ARA in the red blood cell membrane were quantified with gas chromatography-mass spectrometry. DHA, ARA, and ARA:DHA were compared throughout the first month of life, stratified by either severity of (Type 1 ROP, low grade ROP, no ROP) or treatment for ROP.

Summary of Results table 1 depicts subject demographics. At week 1, ARA was lower in the Type 1 ROP group vs. the no ROP group $(17.9 \pm 2.2 \%$ vs. $20.5 \pm 1.7 \%, \mathrm{p}<0.01)$. At week 2, significant differences were noted in DHA and ARA

\section{Abstract \#108 Table 1}

\begin{tabular}{|c|c|c|c|c|c|}
\hline & $\begin{array}{l}\text { No ROP } \\
(n=23)\end{array}$ & $\begin{array}{l}\text { Type } 1 \text { ROP } \\
(n=11)\end{array}$ & $\begin{array}{l}\text { Low Grade } \\
\text { ROP }(n=17)\end{array}$ & $\begin{array}{l}\text { Not Treated } \\
(n=37)\end{array}$ & $\begin{array}{l}\text { Treated } \\
(n=14)\end{array}$ \\
\hline GA (weeks) & $29.7(2.2)$ & $24.3(1.5)^{*}$ & $26.8(2.1)^{*}$ & $28.5(2.5)$ & 25 (2.5)\# \\
\hline BW Z Scores & $\begin{array}{l}0.17 \\
(1.86)\end{array}$ & $-0.78(1.16)$ & $-0.39(1.28)$ & $-0.16(1.53)$ & $\begin{array}{l}-0.89 \\
(1.45)\end{array}$ \\
\hline $\begin{array}{l}\text { Birth Length Z } \\
\text { Scores }\end{array}$ & $\begin{array}{l}-0.75 \\
(1.67)\end{array}$ & $-0.64(1.44)$ & $-0.39(1.28)$ & $-0.62(1.35)$ & $\begin{array}{l}-0.80 \\
(1.71)\end{array}$ \\
\hline Male & 12 (55\%) & $6(55 \%)$ & $9(53 \%)$ & $18(49 \%)$ & $8(57 \%)$ \\
\hline $\begin{array}{l}\text { Day of Life of } \\
\text { Full Feed }\end{array}$ & $22(17)$ & $125(37)^{*}$ & 27 (14) & $24(16)$ & 44 (23)\# \\
\hline $\begin{array}{l}\text { Chronic Lung } \\
\text { Disease }\end{array}$ & $5(22 \%)$ & $8(73 \%)^{*}$ & $14(82 \%)^{*}$ & $5(36 \%)$ & 18 (49\%)\# \\
\hline $\begin{array}{l}\text { Length of Stay } \\
\text { (days) }\end{array}$ & $76(32)$ & $125(37)^{*}$ & $96(23)^{*}$ & $82(30)$ & 121 (34)\# \\
\hline
\end{tabular}

Data is represented as mean (SD). * $p$

(figure 1) but not ARA:DHA. No significant differences in DHA, ARA, and ARA:DHA were observed in weeks 3-4.

Conclusions This study demonstrates that preterm infants with more severe ROP, either Type 1 or ROP requiring treatment, have lower ARA and DHA levels than infants without ROP. It remains unclear if DHA and ARA supplementation shortly after birth will improve ROP outcomes.

\section{$\# 109$ TYPE OF INTRAVENOUS LIPID EMULSION AND CLINICAL OUTCOMES IN INFANTS WITH GASTROINTESTINAL DISEASE}

U Lee*, E Kim, T Romero, KL Calkins. University of California Los Angeles David Geffen School of Medicine, Los Angeles, CA

\subsection{6/jim-2022-WRMC.108}

Purpose of Study Intravenous lipid emulsions (ILEs) are an important component of parenteral nutrition (PN) for neonates with gastrointestinal disorders (GD). Neonates with GD are at high risk for parenteral nutrition associated cholestasis (PNAC) and associated complications, including liver failure. $100 \%$ soybean oil (SO) contains a high concentration of hepatotoxic phytosterols and omega- 6 fatty acids, which contribute to PNAC. A composite oil (CO) containing 15\% fish oil has high amounts of a-tocopherol and omega-3 fatty acids, and less phytosterols. This study aims to compare PNAC and clinical outcomes in infants with GD who received SO or CO. Methods Used Inclusion criteria for this observational study included: 1) born between 2014 and 2019, 2) GD

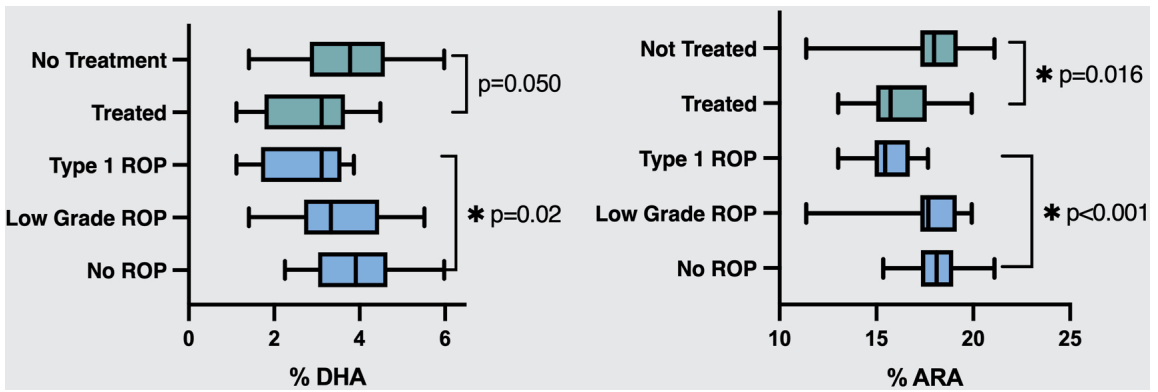

\section{Abstract \#108 Figure 1}




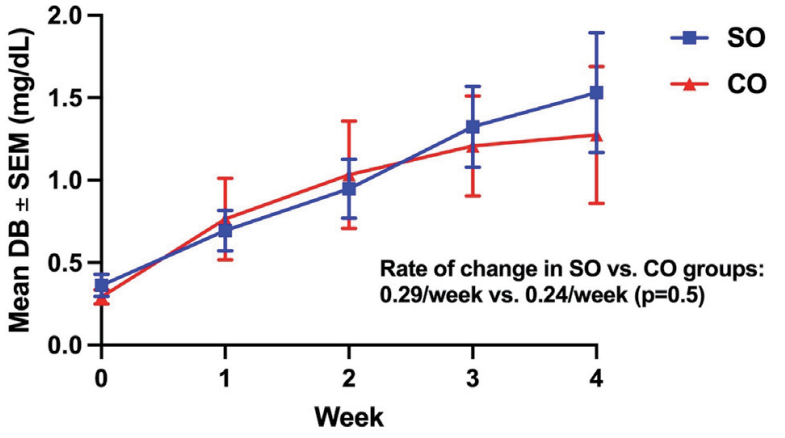

Abstract \#109 Figure 1

(gastroschisis, omphalocele, intestinal atresia, motility disorder, volvulus, necrotizing enterocolitis, or intestinal perforation), 3) exposure to $\mathrm{SO}$ or $\mathrm{CO}>7$ days, and 4) survival to discharge. The primary outcome was cholestasis (conjugated bilirubin (CB) $>1 \mathrm{mg} / \mathrm{dL}$ ). Gas chromatography/mass spectrometry was used to measure fatty acids in the red blood cell membrane in a subset of infants.

Summary of Results The mean $( \pm$ SD) gestational age was 37 \pm 3 and $36 \pm 3$ weeks for the SO $(n=29)$ and CO $(n=21)$ groups, respectively $(\mathrm{p}=0.47)$. The two groups were well matched for GD diagnosis $(\mathrm{p}=0.5)$ and number of GI surgeries $(1.8 \pm 0.8$ for both groups, $\mathrm{p}=0.90)$. Nutrition delivery was similar for the SO and CO groups, including days to full enteral feeds $(33 \pm 32$ vs. $30 \pm 25$ days, $\mathrm{p}=0.85)$ and ILE days $(25 \pm 21$ vs. $30 \pm 27$ days, $\mathrm{p}=0.77)$. Weight $\mathrm{z}$-score declined from birth to discharge $(-1.0 \pm 0.9$ vs. $-0.8 \pm 1.0, p<0.01$ for both), but there was no difference between groups $(p=0.52)$. There was no difference in PNAC incidence $(48 \%$ vs $48 \%$, $\mathrm{p}=0.99)$ and maximum $\mathrm{CB}(2.0 \pm 1.8$ vs. $1.9 \pm 1.6 \mathrm{mg} / \mathrm{dL}$, $\mathrm{p}=0.79$ ) when the SO group was compared to the CO group (figure 1). Fatty acid profiles were similar between the two groups.

Conclusions In this study of infants with GD, when compared to infants who received SO, infants who received $\mathrm{CO}$ had similar fatty acid trajectories, growth, and clinical outcomes, including PNAC. Further investigation is needed to determine the optimal ILE to decrease PNAC incidence in this population.

\section{\#110 NEONATAL ENCEPHALOPATHY FOLLOWING SELECTIVE SEROTONIN REUPTAKE INHIBITOR EXPOSURE IN THE THIRD TRIMESTER OF PREGNANCY: A POPULATION- BASED STUDY}

${ }^{1} \mathrm{M}$ Cornet ${ }^{\star},{ }^{2} \mathrm{H}$ Forquer, ${ }^{1} \mathrm{~A}$ Scheffler, ${ }^{1} \mathrm{~A}$ Yeaton-Massey, ${ }^{1} \mathrm{~T}$ Newman, ${ }^{2} \mathrm{M}$ Kuzniewicz, ${ }^{1} Y$ Wu. ${ }^{1}$ University of California San Francisco, San Francisco, CA; ${ }^{2}$ Kaiser Permanente, Oakland, CA

\subsection{6/jim-2022-WRMC.109}

Purpose of Study About 4-8\% of pregnant women are treated with selective serotonin reuptake inhibitors (SSRI). SSRI exposure in the third trimester may cause poor neonatal adaptation and abnormal movement in neonates, both potential signs of encephalopathy. We assessed whether exposure to SSRI during the third trimester of pregnancy, and dose of SSRI, are associated with neonatal encephalopathy (NE).

Methods Used In a cohort study comprising all Kaiser Permanente Northern California births $\geq 35$ weeks from 2011 to
Abstract \#110 Table 1 Neurologic outcomes of neonates exposed and unexposed to SSRI

\begin{tabular}{|c|c|c|c|c|c|}
\hline & $\begin{array}{l}\text { No SSRI } \\
\text { exposure in 3rd } \\
\text { trimester } \\
(\mathrm{N}=297,403)\end{array}$ & $\begin{array}{l}\text { SSRI } \\
\text { exposure in } \\
\text { 3rd trimester } \\
(\mathrm{N}=8,024)\end{array}$ & $\begin{array}{l}\text { Risk } \\
\text { ratio } \\
(95 \% \\
\mathrm{Cl})\end{array}$ & $\begin{array}{l}\text { Risk } \\
\text { difference } \\
(95 \% \mathrm{Cl})\end{array}$ & $\begin{array}{l}\text { NNH } \\
\text { (assuming } \\
\text { causality) }\end{array}$ \\
\hline Encephalopathy & $1.6 / 1,000$ & $4.4 / 1,000$ & $\begin{array}{l}2.7 \\
(1.9- \\
3.8)\end{array}$ & $2.7 / 1,000$ & 370 \\
\hline $\begin{array}{l}\text { Metabolic } \\
\text { acidosis }(\mathrm{BE}<-16)\end{array}$ & $16 / 1,000$ & $21 / 1,000$ & $\begin{array}{l}1.32 \\
(0.81- \\
2.1)\end{array}$ & NS & NS \\
\hline Apgar $5 \mathrm{~min}<5$ & $5 / 1,000$ & $15 / 1,000$ & $\begin{array}{l}3.3 \\
(2.7- \\
3.9)\end{array}$ & $10.5 / 1,000$ & 95 \\
\hline$P P V$ in $D R$ & $30 / 1,000$ & $92 / 1000$ & $\begin{array}{l}3.0 \\
(2.8- \\
3.2)\end{array}$ & $61 / 1,000$ & 16 \\
\hline $\begin{array}{l}\text { Admission to } \\
\text { neonatal unit }\end{array}$ & $71 / 1,000$ & $123 / 1,000$ & $\begin{array}{l}1.7 \\
(1.6- \\
1.8)\end{array}$ & $52 / 1,000$ & 19 \\
\hline
\end{tabular}

BE: Base excess; PPV: Positive pressure ventilation; DR: Delivery room; NNH: Number needed to harm.

2019, we defined NE as 5-minute APGAR score $<7$ and abnormal level of consciousness, activity, tone, or reflexes. We used logistic regression to adjust for potential confounders.

Summary of Results Of 305,426 infants, 8,024 (2.6\%) were exposed to SSRI in the third trimester, and $510(0.17 \%)$ had NE. After adjusting for maternal depression or anxiety, maternal age, race, and hospital, exposed neonates had 2.7 times higher odds of NE (95\% CI 1.9-3.8). The average risk difference between SSRI-exposed and unexposed mothers was 2.7/ 1000 (95\%CI 1.8-4.1/1000). This relationship was dosedependent. Each $25 \mathrm{mg} / \mathrm{d}$ increase in the sertraline equivalent dose was associated with a 31\% (95\% CI: 23-39\%) increase in the odds of developing NE.

Conclusions Exposure to SSRI in the third trimester is associated with increased risk of neonatal encephalopathy. Given that $\mathrm{NE}$ is rare, if this association is causal the number needed to cause is high $(\mathrm{N} \sim 370)$ and should be balanced with the potential maternal and neonatal benefits of treatment. Future directions include EEG and MRI analyses to correlate SSRI exposure with severity of $\mathrm{NE}$ and brain abnormalities.

\section{$\# 111$ BREASTFEEDING RATE REMAINS LOW DURING COVID- 19 PANDEMIC IN INFANTS WITH NEONATAL OPIOID WITHDRAWAL SYNDROME (NOWS)}

A Fisher*, A McDougal, M McLaren, C Fung. University of Utah Health, Salt Lake City, UT

\subsection{6/jim-2022-WRMC.110}

Purpose of Study Breastfeeding is a well-established non-pharmacological way to improve severity of NOWS in infants with in-utero drug exposure. Given the barriers imposed on mothers during their inpatient stay in the COVID-19 pandemic, the purpose of this study was to assess breastfeeding rate among infants with NOWS during that time. This study also 
compared the rate of breastfeeding at discharge and the trends in substance use to our published cohort at the University of Utah prior to the COVID-19 pandemic. $^{1}$

Methods Used This was a retrospective chart review of a single academic center at University of Utah. Infants born at $\geq 34$ weeks gestational age, between January 1-December 31, 2020, who received Neonatal Withdrawal Inventory (NWI) scoring were reviewed. Infants who received NWI for non-intrauterine drug exposure were excluded. We calculated the percentages of breastfeeding rates of eligibility, initiation, and continuation at discharge. Eligibility for breastfeeding was determined by the provider permitting such use. We additionally noted infant and maternal demographic data, modes of delivery, and drug exposures per cord toxicology screens.

Summary of Results Of the 125 infants reviewed, 102 infants met eligibility. Table 1 summarizes the data. Mothers of $77 \%$ infants received medication-assisted therapy (MAT) compared to only $61 \%$ in our prior study. Similar to our prior study, $21 \%$ infants had isolated opioid exposure compared to $79 \%$ with polysubstance exposure which included opioid and nonopioid substances. Sixty-five (64\%) of the infants were deemed eligible to breastfeed or to receive expressed maternal milk. Fifty-seven $(56 \%$ of total, $88 \%$ of breastfeeding eligible) infants received maternal milk at least once during hospitalization. However, only 37 (36\% of total, $57 \%$ of breastfeeding eligible) infants were receiving maternal milk at discharge compared to $48 \%$ in our prior study. 18 (18\%) infants were discharged to adoptive family or state custody and three of

\begin{tabular}{ll} 
Abstract \#111 Table 1 & \\
\hline Infant Demographics & $\mathrm{n}(\%)$ or median (Q1, Q3) \\
\hline Gestational age at delivery (weeks) & $38(37,39)$ \\
Female sex & $41(40.2)$ \\
Male sex & $61(59.8)$ \\
Birth weight (grams) & $2958(2724,3240)$ \\
SGA (BW $\leq 10 \%)$ & $10(9.8)$ \\
Inborn & $99(97)$ \\
Infants discharged to non-maternal guardianship & $18(17.6)$ \\
Maternal Demographics & $\mathrm{n}(\%)$ or median (Q1, Q3) \\
Maternal age (years) & $31(28,34)$ \\
COVID-19 tested & $54(52.9)$ \\
COVID-19 positive & $1(1)$ \\
Caucasian/White & $69(67.6)$ \\
African American/Black & $3(2.9)$ \\
American Indian/Alaskan Native & $2(2)$ \\
Other/Unknown & $26(25.5)$ \\
Primigravida & $10(9.8)$ \\
Mothers receiving medication-assisted treatment & $79(77.5)$ \\
Mode of Delivery & $\mathrm{n}(\%)$ \\
Vaginal & $65(63.7)$ \\
Cesarean section & $37(36.3)$ \\
Breastfeeding & $\mathrm{n}(\%)$ \\
Maternal milk eligible & $65(63.7)$ \\
Any maternal milk provided & $57(55.9)$ \\
Breastfeeding attempted & $46(45.1)$ \\
Breastfeeding at discharge & $37(36.3)$ \\
Prenatal Exposures & $\mathrm{n}(\%)$ \\
Opioids alone & $21(20.6)$ \\
Polysubstance (opioids + & $81(79.4)$ \\
\hline & \\
& \\
\hline
\end{tabular}

them were eligible to receive maternal milk but did not due to social limitations.

Conclusions Despite a higher rate of maternal MAT with no change in the substance exposure rates, infants with NOWS during COVID-19 suffered from the loss of benefits of breastfeeding/breastmilk feeding. The provision of maternal milk when medically safe in infants with NOWS is vital to optimizing short- and long-term outcomes. However, in this population of vulnerable mother-infant dyads, establishing and sustaining breastfeeding remains a complex challenge particularly during the COVID-19 pandemic when additional psychosocial factors and unanticipated barriers may dominate.

\section{REFERENCE}

1. Morris $\mathrm{E}$, et al. Am J Perinatol 2020.

\section{\#112 IDENTIFYING BARRIERS TO MOTHER'S MILK FEEDING IN EARLY PRETERM BLACK INFANTS IN AN URBAN NEONATAL INTENSIVE CARE UNIT}

${ }^{1}$ MA Chang *, ${ }^{2} S$ Curtis, ${ }^{2}$ NL Davis. ' University of Southern California, Los Angeles, CA; ${ }^{2}$ University of Maryland School of Medicine, Baltimore, MD

\subsection{6/jim-2022-WRMC.111}

Purpose of Study Greater use of mother's milk (MM) is associated with improved outcomes for preterm infants admitted to neonatal intensive care units (NICUs). Healthcare disparities exist in MM provision to preterm infants and further research is needed to better identify barriers to providing $\mathrm{MM}$ in highrisk populations. Our urban Level IV NICU serves a patient population who are $60 \%$ non-Hispanic Black, allowing us to better study this important demographic. The study objective was to evaluate incidence and predictors of provision of $\mathrm{MM}$ to early preterm non-Hispanic Black infants in Baltimore, Maryland.

Methods Used We performed a retrospective medical record review of non-Hispanic Black infants (as identified by their mother) born $<34$ weeks gestational age (GA), between 9/ 2014 - 12/2020 in an urban Level IV NICU. We performed bivariate analyses comparing: 1) maternal and neonatal characteristics of infants who received $\mathrm{MM}$ at any point during NICU admission vs. those who did not, and 2) neonatal outcomes based on exposure to any MM vs. none.

Summary of Results We identified 422 early preterm, nonHispanic Black infants during the study period, of whom 332 (79\%) received some MM during their NICU admission. Maternal factors associated with receiving no MM during admission included higher maternal gravidity $(p=0.0011)$, increased parity of term deliveries $(p<.0001)$ and mothers with increased number of living children $(\mathrm{p}<.0001)$. Maternal age and medical comorbidities such as pre-eclampsia, chronic hypertension, and diabetes did not have a significant impact on provision of MM. Infants of mothers with bipolar disorder were less likely to receive MM ( $p=0.0068)$ while those of mothers with anxiety were more likely to receive $\mathrm{MM}(\mathrm{p}=0.0245)$. There was no difference in $\mathrm{MM}$ provision for those whose mothers had preexisting depression or who screened positive for postpartum depression. Mothers of infants who did receive MM were significantly more likely to have had documented lactation consultation during admission (74\% vs. 20\%, $\mathrm{p}<0.001)$. Infants who received no $\mathrm{MM}$ had higher birth weights $(p<0.0001)$, were born less prematurely $(p=0.0002)$, and 
were more likely to have been on a ventilator $(p=0.0219)$ during their admission, though there was no difference in rates of intraventricular hemorrhages.

Conclusions Identifying barriers to $\mathrm{MM}$ provision for nonHispanic Black infants will allow clinicians to focus supportive and educational interventions. Interestingly, although medical comorbidities such as diabetes, hypertension, and depression did not lower likelihood of providing MM, having more living children did decrease incidence of MM provision. Inpatient lactation consultation had one of the strongest associations, so enhancing access to lactation consultation may significantly increase MM provision in early preterm neonates.

\section{\#113 IMPROVING ENTERAL FEEDING PRACTICES AND HUMAN MILK CONSUMPTION IN MULTIPLE NICUS: A QUALITY IMPROVEMENT PROJECT}

1J Parker*, ${ }^{2} \mathrm{~L}$ Ulm, ${ }^{2} \mathrm{~S}$ Kaprolet, ${ }^{2} \mathrm{C}$ Wieweck, ${ }^{2} \mathrm{M}$ Shea, ${ }^{2} \mathrm{~S}$ Akey, ${ }^{2} \mathrm{~J}$ Wickland, ${ }^{2} \mathrm{~W}$ Ruben, ${ }^{2} \mathrm{O}$ Kudin, ${ }^{2} \mathrm{~J}$ Weber, ${ }^{2} \mathrm{M}$ Ellsworth, ${ }^{2} \mathrm{~K}$ Ellsworth, ${ }^{2} \mathrm{P}$ Griffiths, ${ }^{2} \mathrm{~K}$ Allred, ${ }^{2} \mathrm{~K}$ Marshall, ${ }^{2} \mathrm{~N}$ Whittington, ${ }^{2} \mathrm{M}$ Elkhwad, ${ }^{2} \mathrm{G}$ Martin, ${ }^{2} \mathrm{NS}$ Bhopal. ${ }^{1}$ The University of Arizona College of Medicine Phoenix, Phoenix, AZ; ${ }^{2}$ Division of Neonatology, Phoenix Children's Hospital, Phoenix, AZ

\subsection{6/jim-2022-WRMC. 112}

Purpose of Study Optimal nutrition is essential to overcome common disease processes in preterm and high-risk term newborns; however, introduction of enteral feedings creates a possible risk of developing necrotizing enterocolitis (NEC). NEC is a potentially devastating inflammatory disease of the gastrointestinal tract, which can result in intestinal perforation and possibly death. Simple interventions such as prioritizing human milk over formula feeds and following a standardized feeding protocol for initiating and advancing feeds are well established practices for improving outcomes and reducing NEC. Phoenix Children's Hospital Division of Neonatology was established in 2020, providing medical services to a level 4 NICU and to two level 2 NICUs. We introduced a standardized feeding protocol and used quality improvement methodology to measure compliance with our non-surgical infants $<37$ weeks, with a goal to increase compliance by $>10 \%$ and measured human milk use during hospitalization and at discharge in infants of all gestational ages with a goal to increase human milk consumption by $>10 \%$.

Methods Used We collected data on our feeding practices at all 3 NICUs to measure compliance with the feeding protocol from December 2020 to July 2021. Outcomes were compared in 2 distinct epochs: Epoch 1 from December 2020 to March 2021 and Epoch 2 from April to July 2021. To increase compliance with our feeding protocol, we educated providers, and nurses about the protocol upon its roll-out. Awareness was increased by posting copies at medical provider work stations (February 2021), reviewing interim compliance data with the medical team (April 2021) and placing copies in bedside charts (May 2021).

Summary of Results Feeding data was tracked on 265 infants. The mean gestational age and birth weight were 36 weeks ( \pm 3 weeks) and $2700 \mathrm{~g}( \pm 100 \mathrm{~g})$. In both epochs, breast milk was used for the initial feed in $58 \%$ of all babies admitted to the NICU. The mean time to full feeds was 4 days \pm 2 days in preterm infants $<34$ weeks. Compliance with protocol improved with time from $72 \%$ in epoch 1 to $77 \%$ in epoch 2 in babies $<37$ weeks, and from $66 \%$ to $75 \%$ in babies <
34 weeks. Babies discharging home exclusively on breast milk increased from $16.9 \%$ in epoch 1 to $43.5 \%$ in epoch 2 . There was 1 case of medical NEC in both epochs and no cases of surgical NEC.

Conclusions In this quality improvement project, we improved compliance with a feeding protocol and increased exclusive human milk usage through hospital discharge. While there was 1 case of medical NEC in both epochs, there were no cases of surgical NEC in our data set.

\section{$\# 114$ CLINICAL AND ECONOMIC IMPACT OF USING EXCLUSIVE HUMAN MILK IN VERY LOW BIRTH WEIGHT INFANTS}

M Tetarbe*, M Chang, L Barton, E Toushin, R Ramanathan, R Cayabyab. University of Southern California, Los Angeles, CA

\subsection{6/jim-2022-WRMC.113}

Purpose of Study The AAP recommends use of expressed breast milk (EBM) or donor human milk (DHM) in preterm infants fortified with proteins, minerals, and vitamins to ensure optimum nutrient intake. Unfortunately, the implementation of EBM/DHM fortified with human milk-based fortifiers (EHM) can place an economic burden on individual institutions raising concerns on the economic feasibility of such products. The objective of this study is to assess the clinical impact of using EHM in very low birth weight infants (VLBW) infants, and to perform a cost-benefit analysis of its use.

Methods Used Retrospective study of all VLBW infants admitted to neonatal intensive unit before and after the implementation on the use of EHM. Neonatal demographics and clinical outcomes such as necrotizing enterocolitis (NEC), severe retinopathy of prematurity (ROP), bronchopulmonary dysplasia (BPD), late-onset sepsis (LOS), and average length-ofstay (ALOS) were collected from January - December 2016 (before implementation) and January - December 2020 (after

\begin{tabular}{|c|c|c|c|c|c|}
\hline Clinical Outcome & $\begin{array}{l}\text { Pre- } \\
\text { EHM } \\
2016 \\
\mathrm{~N}=45\end{array}$ & $\begin{array}{l}\text { Post- } \\
\text { EHM } \\
2020 \\
\mathrm{~N}=27\end{array}$ & $\begin{array}{l}\text { Change } \\
\text { Post vs Pre }\end{array}$ & $\begin{array}{l}\text { Estimated } \\
\text { cost per case }\end{array}$ & $\begin{array}{l}\text { Cost } \\
\text { Avoidance }\end{array}$ \\
\hline $\begin{array}{l}\text { Medical necrotizing } \\
\text { enterocolitis, } n\end{array}$ & 2 & 1 & 1 & $\$ 74,004$ & $\$ 74,004$ \\
\hline Late-onset sepsis, n & 4 & 5 & -1 & $\$ 10,055$ & $-\$ 10,055$ \\
\hline $\begin{array}{l}\text { Bronchopulmonary } \\
\text { dysplasia, } n\end{array}$ & 9 & 5 & 4 & $\$ 31,565$ & $\$ 126,260$ \\
\hline $\begin{array}{l}\text { Severe retinopathy } \\
\text { of prematurity, } n\end{array}$ & 2 & 3 & -1 & $\$ 35,749$ & $-\$ 35,749$ \\
\hline $\begin{array}{l}\text { Total parenteral } \\
\text { nutrition, days* }\end{array}$ & 34 & 27 & 7 & $\$ 1,436$ & $\$ 271,404$ \\
\hline $\begin{array}{l}\text { Length-of-stay, } \\
\text { days* }^{*}\end{array}$ & 87.2 & 74.3 & 12.9 & $\$ 3,500$ & $\$ 1,219,050$ \\
\hline Cost Avoidance & & & & & $\$ 1,644,914$ \\
\hline $\begin{array}{l}\text { Product Acquisition } \\
2020\end{array}$ & & & & & - $\$ 313,784$ \\
\hline Net Financial Impact & & & & & $\$ 1,331,130$ \\
\hline
\end{tabular}


implementation). The net cost to the institution was estimated using published data for each outcome measure.

Summary of Results After excluding deceased infants in both time periods, 45 infants were included in the pre-EHM analysis period, (mean birth weight (BW): $1034 \mathrm{~g}$, mean gestational age (GA): 27.9 weeks), and 27 infants were included in the post-EHM analysis period (mean BW: $1070 \mathrm{~g}$, mean GA: 28.8 weeks). Our institution's product acquisition cost in 2020 was estimated to be $\$ 313,784$. The implementation of the EHM protocol saw a reduction in the ALOS by 12.9 days and average total parenteral nutrition (TPN) use by 7 days per infant in the post-EHM group equating to a net savings of $\$ 1,176,670$. While there was a small difference in the number of morbidities between the two time periods, when combining the cost avoidance to include medical NEC, and BPD, the estimated financial impact excluding insurance reimbursement rose to $\$ 1,331,130$ (table 1 ).

Conclusions Our preliminary findings suggest that implementation of exclusive human-milk feeding in VLBW infants is a cost-effective option for NICUs that can result in decrease in NEC, BPD, TPN use and length of stay for these infants at nominal cost.

\section{Neonatology pulmonary II}

\section{Concurrent session}

\section{3:15 PM}

\section{Thursday, January 20, 2022}

\section{\#115 PERINATAL ELECTRONIC CIGARETTE-EXPOSURE INDUCES ASTHMA IN RAT OFFSPRING}

A Harb, C Yu, J Liu, R Sakurai, Y Wang, V Rehan. The Lundquist Institute, Torrance, CA

\subsection{6/jim-2022-WRMC.114}

Purpose of Study An exponential increase in the use of electronic cigarettes (e-cig), including by pregnant women, exposes an increasing number of fetuses to potentially harmful e-cig chemicals with little knowledge of its repercussions. Perinatal nicotine exposure-induced asthma is associated with downregulated PPAR $\gamma$ signaling and upregulated Wnt signaling in the developing lung. However, the impact of maternal nicotine vaping on the developing lung is unknown. Here, we use an established rat model to determine the effect of perinatal maternal e-cig vaping on offspring pulmonary function and markers of airway contractility.

Methods Used Pair-fed pregnant rat dams received saline, vehicle (e-cig without nicotine), or e-cig with nicotine daily from embryonic day 6 until postnatal day (PND) 21. Using an established e-cig delivery system and mimicking real-life puffing topography, dams were exposed to four-sec puffs, one puff (puff volume $35 \mathrm{ml}$ ) every 30s, 3h/day, and 7 days/week. Average maternal plasma nicotine level $(7 \pm 4 \mathrm{ng} / \mathrm{ml})$ using this vaping regimen is well within the range observed in moderate cig smokers. Pups delivered spontaneously at term and breastfed ad-lib, but not directly exposed to e-cig aerosols at any time. At PND21, lung resistance and compliance were determined following the methacholine challenge. At sacrifice, the lungs were collected to determine the expression of airway contractility markers, i.e., $\alpha$-SMA, Calponin, Fibronectin, Collagen I/III, and key Wnt/PPAR $\gamma$ signaling intermediates by qRT-PCR, immunoblotting, and immunostaining.

Summary of Results Compared to controls, perinatal e-cig exposure resulted in a significant increase in airway resistance and decreased airway compliance following the methacholine challenge. mRNA levels of Collagen III and LEF-1 increased, and those of PPAR $\gamma$ and ADRP decreased in the e-cig group. Immunoblotting showed that in the e-cig group, airway contractility markers ( $\alpha$-SMA, Calponin, Fibronectin, Collagen I and III), Wnt signaling intermediates ( $\beta$-catenin and LEF-1), and nicotinic acetylcholine receptors $\alpha 3$ and $\alpha 7$ levels increased. In contrast, compared to controls, PPAR $\gamma$, which interacts directly with Wnt signaling intermediates, levels decreased. Immunostaining of whole lung sections confirmed immunoblotting data.

Conclusions For the first time, we unequivocally demonstrate offspring asthma following perinatal maternal e-cig vaping and explain likely molecular mechanisms involved. Our data add to the accumulating evidence contradicting the idea that e-cigs are'safe.'

Grant Support NIH (HL151769, HD127237, HD071731, and HL152915)) and TRDRP (23RT-0018, 27IP-0050, and T29IR0737).

\section{\#116 COMBINED PRE- AND POSTNATAL GROWTH RESTRICTION INCREASE EXPRESSION OF A NOVEL DOMINANT NEGATIVE PPAR $\gamma$ SPLICE VARIANT IN THE RAT LUNG}

A Cohen*, R Gage, T Benally, H Wang, J Zhao, L Joss-Moore. University of Utah Health, Salt Lake City, UT

10.1136/jim-2022-WRMC.115

Purpose of Study Preterm infants frequently suffer growth restriction, increasing the risk and severity of neonatal lung disease, characterized by impaired alveolar development and worse outcomes in male infants. We showed that growth restriction in the prenatal (IUGR) or postnatal (PGR) period reduces rat lung PPAR $\gamma$ gene expression, which results in impaired alveolar development. PPAR $\gamma$ variants, including the novel delta 5 splice variant $(\operatorname{PPAR} \gamma \Delta 5)$, can impact the downstream effects of PPAR $\gamma$ activation. As PPAR $\gamma \Delta 5$ is a dominant negative variant, the effect of increasing PPAR $\gamma \Delta 5$ is a reduction in PPAR $\gamma$ signaling. Whether PPAR $\gamma \Delta 5$ is expressed in the rat lung, and the effect by growth restriction on expression is unknown. We hypothesize that $\operatorname{PPAR} \gamma \Delta 5$ will be expressed in rat lung, and that the combination of IUGR and PGR will increase expression of PPAR $\gamma \Delta 5$.

Methods Used IUGR and PGR were generated in Sprague Dawley rat pups by bilateral uterine artery ligation and variation in litter size respectively. Lungs were collected at postnatal day 12 from Control, IUGR only, PGR only, and PGR +IUGR rat pups. Male and female rats were treated as sperate groups. PCR, gel electrophoresis, and sequencing were used to confirm the presence of PPAR $\gamma \Delta 5$ in the rat lung. Full length and PPAR $\gamma \Delta 5 \mathrm{mRNA}$ and protein were assessed using real-time RT PCR and western blotting. Differences were assessed by one-way ANOVA and fishers post hoc test. Summary of Results Results are IUGR as $\%$ of control $\pm S D$, ${ }^{*} P<.05$. Sequence confirmed PPAR $\gamma \Delta 5 \mathrm{mRNA}$ is expressed in 
rat lung at postnatal day 12 . PGR model resulted in significantly lower weights on D12 $(66.9 \pm 3 \%$ for PGR only, 64.8 $\pm 3.2 \%$ in IUGR+PGR). In male rat lung, PPAR $\gamma \Delta 5$ mRNA was increased $(325 \pm 79 \% *)$ by IUGR+PGR. Similarly, in the male rat lung, PPAR $\gamma \Delta 5$ protein was increased by IUGR+PGR $(163 \pm 14 \% *)$. In female rat lung, neither PPAR $\gamma$ transcript was affected. However, lung protein levels of PPAR $\gamma \Delta 5$ were increased in female IUGR+PGR $(146 \pm 24 \% *)$.

Conclusions We conclude that the PPAR $\gamma \Delta 5$ is expressed in rat lung, and that IUGR+PGR increase expression. We speculate that increased PPAR $\gamma \Delta 5$ expression in male IUGR+PGR rat lungs may further impair $\operatorname{PPAR} \gamma \Delta 5$ signaling, leading to impaired alveolar development.

\section{\#117 EVIDENCE FOR THE INTERGENERATIONAL PULMONARY EXPRESSION OF GENES DIFFERENTIALLY METHYLATED IN SPERM CELLS OF PERINATALLY NICOTINE EXPOSED RATS}

L Afrose*, Y Wang, J Liu, C Yu, T Rho, T Dao, D Hatai, V Rehan. The Lundquist Institute, Torrance, $C A$

\subsection{6/jim-2022-WRMC.116}

Purpose of Study Nicotine exposure to the developing fetus results in asthma that can be transmitted across generations. However, the underlying mechanism remains unknown. We recently demonstrated differential DNA methylation in the proximity of nicotine-response genes in sperms of the perinatally nicotine exposed F1 animals. Gene ontology and pathway enrichment analysis suggested a possible link between the spermatozoal differential DNA methylation and the offspring asthma phenotype. We hypothesize that nicotine-induced spermatozoal epigenetic changes drive the intergenerational transmission of nicotine-induced asthma. The expression of genes in F2 lungs differentially methylated in the spermatozoa of nicotine exposed F1 males was determined to test this hypothesis.

Methods Used Sprague Dawley rat dams (F0) received nicotine $(1 \mathrm{mg} / \mathrm{kg}, \mathrm{sc})$ or saline from embryonic day 6 (E6) until postnatal day 21 (PND21). Pups (F1) were weaned at PND21 and used as breeders to generate F2 without any subsequent exposure to nicotine in the F1 progeny. F2 pups were weaned at PND21. At PND60, F2 males ( $\mathrm{n}=20 ; 10$ control, 10 nicotine) were sacrificed, and their lungs were collected and flash frozen for performing qRT-PCR for the top 11 differentially methylated genes (AABR07051515.1, Dio1, Gabra4, Htr6, Map4k2, Men1, Mnu, Orai2, Rars, Sec1415, and Slc7a11) in sperm cells of the nicotine exposed F1 males.

Summary of Results In line with data from F1 lungs, the expression of the top 2 differentially hypermethylated genes $A A B R 07051515.1$, a lincRNA, known to modulate lung function, and Dio1 (iodothyronine deiodinase 1) in the nicotine exposed F1 sperm cells was upregulated or downregulated, respectively ( $p \leq 0.05$ ), in F2 lungs. In addition, similar to the F1 progeny, Mnu and Sec1415 genes' expression was downregulated $(\mathrm{p} \leq 0.05)$ in F2 lungs of the nicotine-exposed group. In contrast, the expression of the other 7 differentially methylated genes in F1 spermatozoa did not change significantly.

Conclusions Our data further support the concept that perinatal nicotine exposure-induced spermatozoal epigenetic reprogramming, specifically DNA methylation alterations in nicotine response- and lung development-related genes, likely drive the intergenerational transmission of perinatal nicotine-induced asthma.

Grant Support NIH (HL151769, HD127237, HD071731, and HL152915) and TRDRP (23RT-0018; 27IP-0050; and T29IR0737).

\section{\#118 THE EFFECT OF SEROTONIN DEPLETION ON HYPOXIA INDUCED NEONATAL MURINE BRONCHOPULMONARY DYSPLASIA AND PULMONARY HYPERTENSION}

D Roberts*, JN Posey, J Archambault, E Nozik, C Delaney. University of Colorado Anschutz Medical Campus, Aurora, CO

10.1136/jim-2022-WRMC.117

Purpose of Study Pulmonary hypertension (PH) associated with bronchopulmonary dysplasia (BPD) leads to worse outcomes in former preterm neonates. Serotonin (5-hydroxytryptamine, 5 -HT) is a potent pulmonary vasoconstrictor, smooth muscle mitogen, and is increased in the lungs of infants who died with severe BDP. Tryptophan hydroxylase 1 (TPH1), the rate limiting enzyme in 5-HT synthesis, is increased in adult patients and animals with experimental $\mathrm{PH}$. Serotonin signaling blockade decreases pulmonary vascular resistance and prevents pulmonary vascular remodeling in preclinical models. We hypothesized that TPH1 knock-out (KO) neonatal mice would be protected from hypoxia induced BPD associated with $\mathrm{PH}$.

Methods Used Neonatal wild-type (WT) and TPH1 KO offspring were placed in hypoxia or remained in normoxia at Denver altitude for 2 weeks. To assess alveolar development, inflation fixed lungs were analyzed for surface area (SA) and mean linear intercept (MLI). To identify total number of small vessels $(<30 \mu \mathrm{m})$, lung sections were immunostained with Factor VIII. PH was assessed by Fulton's index and right ventricular systolic pressures (RVSP). Platelet poor plasma (PPP), platelet, and lung homogenate 5-HT levels were measured by ELISA. Data were analyzed by Prism with unpaired t-test or 2-way ANOVA with Bonferroni post-hoc analysis. Significance level $\mathrm{p}<0.05$.

Summary of Results At baseline, WT mice have more platelet poor plasma, platelet, and lung $5-\mathrm{HT}$ than $\mathrm{KO}$ mice $(53 \pm 6$ - $9 \pm 1, \mathrm{p}<0.0001 ; 275 \pm 11-56 \pm 12, \mathrm{p}<0.0001 ; 18 \pm 2$ $10 \pm 1, \mathrm{p}<0.004$; respectively, $\mathrm{ng} / \mathrm{mL})$. TPH1 KO mice were not protected from hypoxia-induced alveolar simplification, shown by no difference compared to WT mice MLI and SA, nor were they protected against hypoxia-induced pulmonary vascular simplification, shown by no difference compared to WT mice vessel density. TPH1 KO mice were attenuated to hypoxia-induced pulmonary vasoconstriction, shown by reduction in RVSP $(32 \pm 0.66-29 \pm 0.55, \mathrm{p}<0.006, \mathrm{mmHg})$. There was less PPP and platelet 5-HT in hypoxia-exposed WT mice compared to WT mice at baseline $(20 \pm 2-53 \pm 6, \mathrm{p}<0.0001$ and $117 \pm 27-275 \pm 11, \mathrm{p}<0.0001$, respectively, $\mathrm{ng} / \mathrm{mL}$ ). There was less lung 5-HT in hypoxia-exposed $\mathrm{KO}$ mice than in $\mathrm{KO}$ mice at baseline $(2 \pm 1-10 \pm 1, \mathrm{p}<0.001, \mathrm{ng} / \mathrm{mL})$.

Conclusions Neonatal TPH1 KO mice are not protected against hypoxia-induced lung injury. Surprisingly, this study contradicts the current understanding of the role of 5-HT in adults with $\mathrm{PH}$ and in adult models of hypoxia-induced $\mathrm{PH}$. We found decreased plasma and platelet 5-HT following hypoxia exposure. We speculate that decreased 5-HT observed in hypoxia may contribute to neonatal hypoxia-induced 
alveolar simplification and impaired vascular development. Further studies are needed to elucidate the role of $5-\mathrm{HT}$ in the developing lung.

\section{$\# 119$ THE EFFECT OF PLATELET ALPHA GRANULE DEFICIENCY ON HYPOXIA INDUCED NEONATAL PULMONARY HYPERTENSION}

D Roberts*, JN Posey, E Nozik, C Delaney. University of Colorado - Anschutz Medical Campus, Aurora, $\mathrm{CO}$

\subsection{6/jim-2022-WRMC.118}

Purpose of Study Pulmonary hypertension $(\mathrm{PH})$ associated with bronchopulmonary dysplasia (BPD) leads to worse outcomes in former preterm neonates. Elevated platelets at birth are an independent predictor of BPD, increased platelet derived protein after birth is associated with higher rates of neonatal pulmonary vascular disease, and perinatal platelet transfusions are associated with higher rates of mortality and BPD. Circulating platelets from neonatal mice with experimental $\mathrm{PH}$ are increased and express a higher percentage of active $\alpha \operatorname{IIb} \beta 3$, a marker of platelet activation. NBEAL 2 knock-out (KO) mice lack platelet alpha granules, have low platelet counts, and have decreased platelet function in vitro and in vivo. We hypothesized that NBEAL $2 \mathrm{KO}$ neonatal mice would be protected from hypoxia-induced $\mathrm{PH}$.

Methods Used Neonatal wild-type (WT) and NBEAL $2 \mathrm{KO}$ offspring were placed in hypobaric hypoxia (18,000 feet) or remained in normoxia at Denver altitude for 2 weeks. $\mathrm{PH}$ was assessed by Fulton's index as a marker of right ventricular hypertrophy (RVH) and right ventricular systolic pressures (RVSP). Data were analyzed by Prism with unpaired t-test or 2-way ANOVA with Bonferroni post-hoc analysis. Significance level $\mathrm{p}<0.05$.

Summary of Results Right ventricular systolic pressure is higher in NBEAL2 KO mice than in WT mice at baseline $(24.2 \pm 0.5$ - 21.5 $\pm 0.3, \mathrm{p}<0.0001, \mathrm{mmHg}$ ). There is no difference between baseline right ventricular hypertrophy between NBEAL2 KO mice and WT mice $(0.27 \pm 0.01-0.31 \pm 0.01$, ns). NBEAL2 KO mice display comparable hypoxia-induced increase in RVSP compared to WT mice $(29 \pm 1-30 \pm 1$, ns, $\mathrm{mmHg}$ ). NBEAL2 KO mice display comparable hypoxiainduced increase in RVH compared to WT mice $(0.3 \pm 0.03$ $-0.4 \pm 0.03$, ns).

Conclusions Platelet alpha granule deficiency is a risk factor for neonatal pulmonary vasoconstriction at baseline. Further studies are needed to elucidate the role of platelets in neonatal PH associated with BPD.

\section{\#120 METABOLITES IMPORTANT IN THE DIFFERENTIATION OF HUMAN PLURIPOTENT STEM CELLS TO LUNG PROGENITOR CELLS}

${ }^{1}$ SL Leibel ${ }^{*},{ }^{2}$ I Tseu, ${ }^{3} \mathrm{~A}$ Zhou, ${ }^{4} \mathrm{~A}$ Hodges, ${ }^{4} \mathrm{~J}$ Yin, ${ }^{2} \mathrm{C}$ Bilodeau, ${ }^{2} \mathrm{O}$ Goltsis, ${ }^{2} \mathrm{M}$ Post. ${ }^{1}$ University of California San Diego, La Jolla, CA; ${ }^{2}$ SickKids Research Institute, Toronto, ON, Canada; ${ }^{3}$ Johns Hopkins University, Baltimore, MD; ${ }^{4}$ Sanford Burnham Prebys Medical Discovery Institute, La Jolla, CA

\subsection{6/jim-2022-WRMC.119}

Purpose of Study Metabolism is vital to cellular function and tissue homeostasis during human lung development. In utero, embryonic stem cells undergo endodermal differentiation towards a lung progenitor cell (LPC) fate that can be modeled in vitro using pluripotent stem cells (hPSCs). We previously showed differences in lung cell composition and gene expression between wild type and surfactant protein B (SP-B) deficient lung organoids. These differences may be impacted by changes in metabolites during early lung development. We hypothesize that SP-B deficient cells will express a different metabolomic profile compared to wt cells during the differentiation to lung progenitor cells.

Methods Used To examine metabolites that differ during endodermal differentiation, we used an untargeted metabolomics approach to evaluate the changes in metabolites at the stem cell (hPSC), definitive endoderm (DE), anterior foregut endoderm (AFE) and lung progenitor (LPC) stage between wt and SP-B deficient cell lines. At each differentiation step, the cells were sorted for surface markers specific to their differentiation stage in quadruplicate. The homogeneous cell lysates were analyzed using a Biocrates p180 metabolite kit including hexoses, amino acids, phosphatidylcholines, lysophosphatidylcholines, sphingolipids, acylcarnitines, and biogenic amines. The metabolomic multivariate data analysis was performed using XLSTAT.2016 software (Addinsoft) and MetaboAnalyst.

Summary of Results We found that the largest metabolic changes during endodermal differentiation occurred from hPSC to DE with a change from glycolytic respiration to oxidative phosphorylation. The metabolites most enriched during the differentiation from hPSC to LPC, independent of cell line, were sphingomyelin and lecithin. In the wt cell lines, metabolites for oxidation of fatty acids and tryptophan metabolism were up-regulated, while metabolites for ammonia recycling and aspartate metabolism were down-regulated. In the SP-B deficient cells, metabolites in fatty acid oxidation and carnitine synthesis were up-regulated and metabolites for amino acid metabolism, the urea cycle, and multiple energybased pathways were down-regulated.

Conclusions Differentiation to lung progenitor cells from pluripotent stem cells resulted in increased fatty acid metabolism and decreased urea cycle and aspartate metabolism in both wt and SP-B deficient cell lines. Therefore, metabolite composition in early lung development is not influenced by the loss of SP-B expression.

\section{$\# 121$ EFFECTS OF VITAMIN A AND VITAMIN D TREATMENT ON LUNG GROWTH AND FUNCTION IN OFFSPRING FROM MATERNAL VITAMIN D DEFICIENT RATS}

${ }^{1} \mathrm{~N}$ Galambos*, ${ }^{1} \mathrm{E}$ Bye, ${ }^{1} \mathrm{~T}$ Gonzalez, ${ }^{1} \mathrm{G}$ Seedorf, ${ }^{1} \mathrm{~B}$ Smith, ${ }^{2} \mathrm{JC}$ Fleet, ${ }^{1} \mathrm{SH}$ Abman, ${ }^{1} \mathrm{E}$ Mandell. ${ }^{1}$ University of Colorado - Anschutz Medical Campus, Aurora, CO; ${ }^{2}$ The University of Texas at Austin College of Natural Sciences, Austin, TX

\subsection{6/jim-2022-WRMC.120}

Purpose of Study Maternal vitamin D deficiency (M-VDD) is associated with perinatal pulmonary morbidities. We have demonstrated that offspring of rodent maternal VDD dams have sustained abnormalities of distal lung structure, increased airway hyperreactivity and abnormal lung mechanics. In pulmonary endothelial cells, vitamin A (VA) and vitamin $\mathrm{D}$ (VD) co-dimerize on retinoid $\mathrm{x}$ receptor. VA therapy has been shown to improve lung development in pre-clinical and clinical studies, but whether combined postnatal (PN) treatment with VA and VD further enhances lung development in offspring of M-VDD dams is unknown. Therefore, 
we seek to determine if $\mathrm{PN}$ VA and VD supplementation improves lung development and function in offspring of $\mathrm{M}$ VDD dams.

Methods Used Newborn rats from control (CTL) and M-VDD dams received daily treatment of retinoic acid (VA) alone, VA and 1,25-OHD (VD) (VA-VD) or saline (SAL) for 14 days. On DOL 14 lung structure was assessed by mean linear intercept (MLI), radial alveolar count (RAC) and pulmonary vessel density (PVD). Lung mechanics were measured using flexiVent. Summary of Results Lungs from VDD-SAL rats had increased MLI $(p<0.001)$ and decreased pulmonary vessel density $(p<0.05)$ as compared to CTL-SAL. VDD rats that received VA had increased RAC compared to VDD-SAL $(p<0.05)$. VDD-SAL rats had increased resistance $(\mathrm{p}<0.01)$ and decreased compliance $(p<0.01)$ as compared to CTL-SAL. VDD-VA rats had decreased elastance as compared to VDDSAL pups $(p<0.05)$.

Conclusions M-VDD decreases distal lung and vascular development and impairs lung function in infant rats. PN VA therapy improved RAC and decreased elastance in VDD pups. These findings suggest that abnormal lung development after PN VA therapy may improve alveolarization and lung mechanics of M-VDD pups. We speculate that M-VDD leads to persistent abnormalities in infant lung growth that may be responsive to $\mathrm{PN}$ VA.

\section{\#122 MATERNAL VITAMIN D DEFICIENCY ALTERS PULMONARY ENDOTHELIAL CELL GROWTH AND MRNA EXPRESSION IN NEWBORN RATS}

${ }^{1} \mathrm{~T}$ Gonzalez*, ${ }^{1} \mathrm{E}$ Bye, ${ }^{1} \mathrm{~N}$ Galambos, ${ }^{1} \mathrm{G}$ Seedorf, ${ }^{2} \mathrm{JC}$ Fleet, ${ }^{1} \mathrm{SH}$ Abman, ${ }^{1} \mathrm{E}$ Mandell. ${ }^{1}$ University of Colorado - Anschutz Medical Campus, Aurora, $\mathrm{CO}^{2}{ }^{2}$ The University of Texas at Austin College of Natural Sciences, Austin, TX

\subsection{6/jim-2022-WRMC.121}

Purpose of Study Vitamin D deficiency (VDD) during pregnancy is associated with chronic lung disease in preterm infants, and the underlying mechanisms are not understood. We have shown that vitamin D (VD) preserves lung structure and prevents pulmonary hypertension $(\mathrm{PH})$ in an experimental model of bronchopulmonary dysplasia, and that VD treatment increases pulmonary artery endothelial cell growth and function. However, the direct effects of maternal VDD on pulmonary endothelial cell (PEC) growth and function are unknown. Thus, we seek to determine whether PEC from newborn rats exhibit altered growth and mRNA expression at birth after exposure to maternal VDD and whether these changes persist during infancy.

Methods Used Female rats were fed VDD chow and shielded from UV-B light to achieve 25-OHD levels less than $10 \mathrm{ng} / \mathrm{ml}$ before mating. PEC were isolated from offspring of maternal VDD (VDD) or control (CTL) dams at postnatal days 0 and 14. PECs were used for proliferation assays and response to exogenous VEGF and 1,25-OHD. PEC lysates were also collected for RT-qPCR analysis.

Summary of Results PEC isolated from VDD pups at both D0 and D14 demonstrate decreased growth compared to CTL D0 and D14 $(\mathrm{p}<0.01)$. VEGF or 1,25-OHD treatment increased CTL PEC growth from both D0 and D14 when compared to untreated CTL D0 and D14 PEC $(p<0.01)$. In contrast, neither VEGF nor 1,25-OH treatment increased D0 VDD PEC growth. D14 VDD PEC showed an increased growth with VEGF treatment compared to untreated D14 VDD PEC $(\mathrm{p}<0.01)$. RNA isolated from D0 VDD PEC demonstrate decreased expression of KDR and eNOS and increased VEGF expression compared to D0 CTL PEC $(p<0.01)$, no expression changes seen at D14.

Conclusions We found that D0 PEC from newborn offspring of maternal VDD dams demonstrate decreased baseline PEC growth and no responsiveness to angiogenic stimuli. At D14 VDD PEC grew poorly at baseline, and were responsive to VEGF but not 1,25-OHD treatment. We speculate that maternal VDD disrupts normal PEC function, which persists into postnatal life and may contribute to high risk for late cardiopulmonary disease.

\section{$\# 123$ THE EFFECT OF PERINATAL NICOTINE EXPOSURE ON THE LUNG CIRCADIAN MOLECULAR CLOCK}

D Hatai*, Y Wang, R Sakurai, J Liu, L Afrose, T Dao, T Rho, C Yu, V Rehan. The Lundquist Institute, Torrance, CA

\subsection{6/jim-2022-WRMC.122}

Purpose of Study Dysregulated peripheral circadian rhythm is associated with enhanced inflammatory response and cellular senescence. Recent studies have demonstrated an association of exposure to cigarette smoke and dysregulated peripheral molecular clock in Chronic Obstructive Pulmonary Disease (COPD) and asthma patients. This has also been confirmed in rodent models. Although developmental smoke/nicotine exposure predisposes to asthma and COPD, its impact on circadian clock genes is unknown. Here, we test the hypothesis that developmental nicotine exposure alters the molecular clock, which lasts well into adult life.

Methods Used Pair-fed pregnant Sprague-Dawley rat dams received once-daily $1 \mathrm{mg} / \mathrm{kg}$ nicotine or saline diluent from embryonic day 6 (E6) to postnatal day 21 (PND21). Lungs from pups were collected on E21, PND21, or PND60 and flash-frozen for later mRNA and protein analysis. The expression of core clock genes (Bmal1, Clock, Cry1, Cry2, Per1, Per2, Rev-erba, Rev-erbb, Rora, and Sirt1) was determined by qRT-PCR on mRNA isolated from lungs. Protein levels of key clock genes Bmal1, Clock, and Rev-erba were determined using western analysis on proteins extracted from PND 21 lungs.

Summary of Results Overall, the mRNA expression of Bmal1, Clock, Cry1, Cry2, Per1, Per2 , Rev-erba, Rev-erbb, Rora, and Sirt 1 was significantly decreased $(\mathrm{p}<0.05)$ in the nicotine treated group vs. the control group at E21 and PND21. Perinatal nicotine exposure-induced downregulation of key clock genes Bmal1 and Rev-erba was also confirmed by their down-regulated protein levels by western analysis at PND 21. Interestingly, the expression of several downregulated clock genes at E21 and PND21 in the nicotinetreated group was either not different or upregulated versus the control group at PND60, suggesting a dynamic response of perinatal nicotine exposure on the peripheral molecular clock.

Conclusions Perinatal nicotine exposure leads to peripheral clock dysregulation in the lung that lasts at least through adolescence. These results suggest a new mechanism that underlies the effects of perinatal nicotine-induced lung injury. Further studies are needed to determine the impact of perinatal nicotine exposure-induced dysregulated peripheral clock on lung health, gender specificity, and how long these effects last. 
Grant Support NIH (HL151769, HD127237, HD071731, and HL152915) and TRDRP (23RT-0018; 27IP-0050; and T29IR0737).

\section{$\# 124$ ASSOCIATION OF SYSTEMIC SEROTONIN WITH PERSISTENT PULMONARY HYPERTENSION OF THE NEWBORN}

${ }^{1} \mathrm{~J}$ Archambault*, ${ }^{1} \mathrm{C}$ Palmer, ${ }^{1} \mathrm{E}$ Nozik, ${ }^{2} \mathrm{CC}$ Galambos, ${ }^{1} \mathrm{C}$ Delaney. ${ }^{1}$ University of Colorado, Aurora, $\mathrm{CO} ;{ }^{2}$ University of Colorado Denver - Anschutz Medical, Aurora, CO

\subsection{6/jim-2022-WRMC.123}

Purpose of Study Pulmonary hypertension (PH) is a life-threatening condition that affects infants, children, and adults. However, treatment strategies are limited, and morbidity and mortality remain significant. We have previously demonstrated in robust animal models that serotonin $(5-\mathrm{HT})$ contributes to the pathogenesis of experimental neonatal $\mathrm{PH}$ and know that infants who died due to severe lung disease have a 34-fold increase in lung 5-HT. We designed an exploratory pilot study to test the hypothesis that systemic 5-HT is increased in infants with persistent pulmonary hypertension of the newborn (PPHN).

Methods Used Near term and term infants ( $\geq 36$ weeks) were recruited from the NICUs at Children's Hospital Colorado and University of Colorado Hospital beginning in March 2021. Infants with culture proven sepsis, metabolic/genetic abnormality, major cardiac defect, renal failure, or antenatal exposure to SSRIs were excluded. $\mathrm{PH}$ was defined on echocardiogram by an estimated systolic pulmonary artery pressure $\geq 40 \mathrm{~mm} \mathrm{Hg}$, end-systolic eccentricity index $\geq 1.16$, or presence of a right-to-left shunt. 5-HT is an unstable neurotransmitter that degrades quickly; thus, we measured its more stable metabolite 5-hydroxyindolacetic acid (5-HIAA). Urine samples were collected on DOL 1 and DOL 3, and 5-HIAA was analyzed via mass spectrometry. Monthly follow up samples were collected if $\mathrm{PH}$ persisted. Demographics, clinical characteristics, and interventions were obtained through chart review and summarized for the patient cohort. 5-HIAA levels were summarized using medians and ranges.

Summary of Results To date, 6 infants with PPHN and 7 agematched controls have been enrolled. 54\% were male and $46 \%$ female. The mean gestational age was 38.2 weeks. All infants with PPHN were classified as having severe $\mathrm{PH}$ on initial echo. $83 \%$ were born with congenital diaphragmatic hernia $(\mathrm{CDH})$ and $100 \%$ had a patent ductus arteriosus (PDA). $83 \%$ required vasopressors, with $60 \%$ initiated in the delivery room. $100 \%$ required steroids for blood pressure and/or respiratory support. $83 \%$ required pulmonary vasodilators with inhaled nitric oxide and sildenafil being the most common. $50 \%$ of infants with PPHN were followed for refractory $\mathrm{PH}$ for a mean of 2.67 months. At DOL 1, the median 5-HIAA level was 14.89 (min, max: 14.17, 19.1) in the PPHN group and $14.17(11.56,16.63)$ in the control group. At DOL 3, the median was $18(8.89,40.5)$ in the PPHN group and 15 $(10.95,17.14)$ in the control group.

Conclusions This study investigated the association of PPHN with systemic alterations in 5-HIAA. Our current results offer a preliminary description; however, enrollment is ongoing. With additional data we will test our hypothesis that 5-HIAA is significantly associated with $\mathrm{PH}$ severity. Ultimately, we aim to establish it as a noninvasive biomarker to follow treatment response and predict the later development of $\mathrm{PH}$ in high-risk infants.

\section{Neuroscience I}

\section{Concurrent session}

\section{3:15 PM}

\section{Thursday, January 20, 2022}

\section{\#125 COMBINED SUPEROTEMPORAL RETINAL AMYLOID AND RETINAL VENULAR TORTUOSITY INDEX PREDICTS VERBAL MEMORY PERFORMANCE IN COGNITIVELY IMPAIRED SUBJECTS}

1,2 $\mathrm{T}_{\text {Torbati }}{ }^{*}{ }^{2}$ J Sheyn, ${ }^{2} \mathrm{DS}$ Sherman, ${ }^{3} \mathrm{MM}$ Khansari, ${ }^{2} \mathrm{KL}$ Black, ${ }^{2,3} \mathrm{PD}$ Lyden, ${ }^{2} \mathrm{Y}$ Koronyo, ${ }^{2} \mathrm{M}$ Koronyo-Hamaoui, ${ }^{4} \mathrm{OM}$ Dumitrascu. ${ }^{1}$ Western University of Health Sciences, Pomona, $C A ;{ }^{2}$ Cedars-Sinai Medical Center, Los Angeles, $C A ;{ }^{3}$ University of Southern California Keck School of Medicine, Los Angeles, $C A ;{ }^{4}$ Mayo Clinic Arizona, Scottsdale, AZ

\subsection{6/jim-2022-WRMC.124}

Purpose of Study Alzheimer's disease (AD) is commonly characterized by pathognomonic amyloid-beta $(A \beta)$ burden in the brain, and recent reports demonstrate the vital role of cerebral vascular pathology in $\mathrm{AD}$ development. Given that the retina is a CNS organ amenable to noninvasive imaging, our team previously pioneered retinal curcumin-fluorescence imaging (RFI) and identified a significant correlation between retinal amyloid burden in the proximal mid-periphery (PMP) of the superotemporal retina with cognitive performance and hippocampal volume. The rising hypothesis of vascular neuropathology in $\mathrm{AD}$, coupled with RFI clinical feasibility targeting both vasculature and $A \beta$, warrants the implementation of both neurovascular and retinal $\mathrm{A} \beta$ for early $\mathrm{AD}$ detection. Considering the crucial yet unmet need for such multimodal detection models, we used RFI to examine retinal vascular parameters in relation to retinal $A \beta$ in patients with varying neurocognitive status.

Methods Used 29 subjects underwent neuropsychometric cognitive evaluations and quantitative RFI to measure retinal amyloid burden. We also quantified vessel tortuosity index (VTI), inflection index and branching angle from segmented retinal blood vessels. Using linear regression models, we conducted correlation analyses between retinal vascular and amyloid measures in relation to various cognitive domain $\mathrm{Z}$-scores.

Summary of Results Total and PMP retinal amyloid count were markedly increased in patients with cognitive impairment (CI) as compared to those with normal cognition (NC, $\mathrm{p}=$ 0.0012). Venous VTI was significantly different across levels of Clinical Dementia Rating (CDR) cognitive scores $(\mathrm{p}=$ 0.026). Patients with CI displayed considerably higher combined PMP amyloid-venous VTI index in comparison to NC subjects $(p=0.0068)$. Increased combined PMP amyloidvenous VTI index significantly correlated with decreased WMS-IV Z-scores ( $\mathrm{r}=-0.537, \mathrm{p}=0.001)$ as well as with reduced SF-MCS-36 Z-scores $(r=-0.338, p=0.039)$.

Conclusions This study reveals that combined PMP amyloid count-venous VTI index may predict verbal memory loss and cognitive-related quality of life performance. Future larger investigations are needed to further refine the practical utility of RFI in a clinical setting. 
\#126 THYROID HORMONE TREATMENT REVEALS GENE EXPRESSION PLASTICITY IN CONE PHOTORECEPTORS OF ADULT ZEBRAFISH

1,2P Thomas", 'A Farre, 'D Stenkamp. ${ }^{1}$ University of Idaho, Moscow, ID; ${ }^{2}$ University of Washington School of Medicine, Seattle, WA

\subsection{6/jim-2022-WRMC.125}

Purpose of Study In humans, as well as other vertebrates, color vision requires the differential expression of specific cone opsins in photoreceptor cone cells. One model for the regulation of the human long and medium wavelength sensitive (LWS/MWS) opsin tandem array suggests an upstream regulatory region interacts with replicated opsin genes at random, resulting in mutually exclusive expression of a specific opsin. A similar orthologous long wavelength sensitive (lws 1/lws 2) array in zebrafish provides a good model for study of this regulation. However, our prior investigations into this array suggest that thyroid hormone $(\mathrm{TH})$ and retinoic acid serve as trans regulators in larvae/juveniles (Mitchell et al., 2015, PLOS Genetics; Mackin et al., 2019, PNAS). This study investigates whether cone opsin expression remains plastic to $\mathrm{TH}$ treatment in adult zebrafish, where cone distribution is considered stable.

Methods Used Adult zebrafish (6-18 months old) were treated with $\mathrm{NaOH}(0.01 \%$, control) or $\mathrm{TH}(386 \mathrm{nM})$ for 1 or 5 days. qRT-PCR was performed on homogenized eyes. Whole retinas were treated by hybridization chain reaction in-situ and then analyzed by confocal imaging for mRNA expression.

Summary of Results In adult zebrafish, exogenous $\mathrm{TH}$ drastically increased lws 1 expression in both 1 and 5 day-treated groups ( $\mathrm{p}<1 \mathrm{e}-7,0.01$, respectively) while decreasing lws 2 expression $(\mathrm{p}<0.001,0.001)$. Other phototransduction-related transcripts (gngt2b, rh2-1) also demonstrated expression changes following $\mathrm{TH}$ treatment. Exogenous $\mathrm{TH}$ induced a drastic shift from lws 2 to lws 1 in adult zebrafish, consistent with previous studies of larvae and juveniles.

Conclusions This shift from lws2 expression to lws1 expression occurs as rapidly as 1 day when exposed to $\mathrm{TH}$, which shows that cones remain highly plastic even into adulthood. Plasticity in spectral sensitivity (to be sensitive to higher wavelengths) in response to $\mathrm{TH}$ suggest a role in visual system function well into adulthood. These results oppose earlier models suggesting that regulation between tandemly replicated opsin genes is stochastic and fixed.

\section{$\# 127$ EFFECTS OF METHAMPHETAMINE ON FENFLURAMINE- INDUCED HEAD-TWITCH RESPONSE AND C-FOS EXPRESSION IN MICE PREFRONTAL CORTEX}

Y Sun, S Chebolu, S Skegrud, S Kamali*, N Darmani. Western University of Health Sciences College of Osteopathic Medicine of the Pacific, Pomona, CA

\subsection{6/jim-2022-WRMC.126}

Purpose of Study The head-twitch response (HTR) is evoked following stimulation of postsynaptic serotonin $2 \mathrm{~A}\left(5-\mathrm{HT}_{2 \mathrm{~A}}\right)$ receptors in the prefrontal cortex (PFC). D-Fenfluramine (FF) is a selective 5-HT releaser, it produces the HTR via release of serotonin from nerve terminals through the 5-HT uptake carrier working in reverse. Methamphetamine (MA) is a nonselective releaser of monoamines 5-HT, norepinephrine (NE) and dopamine (DA). We investigate whether pretreatment with either MA (1-5 mg/kg, i.p.) or the $5-\mathrm{HT}_{2 \mathrm{~A}}$ receptor selective antagonist EMD $281014(0.001,0.005,0.01,0.05 \mathrm{mg} / \mathrm{kg}$, i. p.) can alter: 1) the mean frequency of FF-induced HTR at different ages (20-, 30- and 60-day old), and 2) the expression of $\mathrm{c}$-fos evoked by $\mathrm{FF}$ in different regions of the PFC. We also explored whether blockade of serotonergic $5-\mathrm{HT}_{1 \mathrm{~A}^{-}}$ or adrenergic $\alpha_{2}$-receptors can alter the effect of MA on FFinduced HTR across the above ages.

Methods Used The HTR was observed for 30 min following the injection of $\mathrm{FF}$ in each mouse. We use immunohistochemistry study to evaluate the changes of c-fos expression in the PFC.

Summary of Results Pretreatment with MA (1-5 mg/kg, i.p.) dose-dependently suppressed the FF-induced HTR across different ages. $\mathrm{MA}$ at $1 \mathrm{mg} / \mathrm{kg}$ in 20- and 30-day old mice, and at $5 \mathrm{mg} / \mathrm{kg}$ in 60-day old mice significantly suppressed the FF-induced HTR. Pretreatment with EMD 281014 (0.001, $0.005,0.01,0.05 \mathrm{mg} / \mathrm{kg}$, i.p.) also blocked the FF-induced HTR in an age- and dose-dependent manner. The selective 5$\mathrm{HT}_{1 \mathrm{~A}}$ receptor antagonist WAY 100635 (0.25 mg/kg, i.p.) and the adrenergic $\alpha_{2}$-receptor antagonist RS $79948(0.1 \mathrm{mg} / \mathrm{kg}$,i. p.) significantly reversed the inhibitory effect of MA on the mean frequency of HTR in 20-day old mice, but not in 30and 60- day old mice. Moreover, FF significantly increased cfos expressions in several PFC regions in 30-day old mice. Despite the inhibitory effect of MA or EMD 281014 on FFinduced HTR, pretreatment with either MA (1 mg/kg, i.p.) or EMD 281014 (0.05 mg/kg, i.p.) significantly increased c-fos expression in different regions of the PFC in 30-day old mice.

Conclusions The inhibitory effect of MA on the FF-evoked HTR appears to be mainly due to functional interactions between the stimulatory $5-\mathrm{HT}_{2 \mathrm{~A}}$ - and the inhibitory $5-\mathrm{HT}_{1 \mathrm{~A}}$ and/or adrenergic $\alpha_{2}$-receptors. The MA-induced increase in cfos expression in different PFC regions is probably due MAevoked increases in synaptic concentrations of 5-HT, NE and/ or DA. EMD 281014 failed to prevent the increase in c-fos expression induced by FF, which may be due to the increased 5 -HT synaptic concentration that activates other serotonergic receptors, such as, $5-\mathrm{HT}_{1 \mathrm{~A}}$.

\section{\#128 PROGRESSION OF GEOGRAPHIC ATROPHY IN AGE- RELATED MACULAR DEGENERATION PATIENTS TREATED WITH LEVODOPA}

${ }^{1} \mathrm{G}$ Muigai* ${ }^{*}{ }^{2} \mathrm{RW}$ Snyder, ${ }^{2,3} \mathrm{C}$ Christensen, ${ }^{4} \mathrm{~S}$ Purewal. ${ }^{1}$ Vanderbilt University, Nashville, $T N ;{ }^{2}$ University of Arizona, Tucson, AZ; ${ }^{3}$ Des Moines University College of Osteopathic Medicine, Des Moines, IA; ${ }^{4}$ Banner University Medical Center Tucson, Tucson, AZ

\subsection{6/jim-2022-WRMC. 127}

Purpose of Study Geographic atrophy (GA) is a severe and poorly understood progression of dry age-related macular degeneration (AMD). Patients with GA are also more likely to develop choroidal neovascularization. Carbidopa-levodopa treatment has demonstrated successful reduction in neovascular AMD. In this study, we investigate the effects of carbidopalevodopa treatment on progression of GA.

Methods Used A retrospective analysis of patients with already existing GA who participated in our proof-of-concept study was performed. Fundus autofluorescence (FAF) and optical coherence tomography (OCT) were utilized to confirm the presence of geographic atrophy. This study followed the 2018 retina consensus meeting requirements to measure geographic atrophy markers in patients. The primary outcomes measures 
were complete retinal pigment epithelium and outer retinal atrophy (cRORA), hypertransmission through Bruch's membrane, and $\mathrm{mm} /$ year change from initiation of study drug.

Summary of Results We included 5 patients with already existing geographic atrophy in 6 eyes. This cohort consented to carbidopa-levodopa treatment and was predominantly male (3 patients) with a median (IQR) age of 82 (5). The mean GA change in cRORA 1 year before and after treatment initiation was $-0.000433 \mathrm{~mm} /$ year and $0.0061 \mathrm{~mm} /$ year. The mean GA change in hypertransmission 1 year before and after treatment initiation was $0.0085 \mathrm{~mm} /$ year and $0.135 \mathrm{~mm} /$ year.

Conclusions Geographic atrophy progressed in all eyes except one. According to a 2021 Ophthalmic Research meta analysis, the average GA growth rate seen across 23 studies was 0.33 $\mathrm{mm} / \mathrm{year}$. Thus, our results indicate that the carbidopa-levodopa treatment provides benefit in slowing progression rates of GA. Further studies are indicated into the pathogenesis of GA and the role that carbidopa-levodopa might play in its treatment.

\section{\#129 HOW DO MEDICAL AND PHYSICAL THERAPY STUDENTS LEARN ABOUT CONCUSSIONS?}

1,2W Cheung*, 1,2EA Tangog, ${ }^{1,2}$ L McKay, ${ }^{3} T$ Alsky, ${ }^{1} D$ Baron. ${ }^{1}$ Western University of Health Sciences, Pomona, CA; ${ }^{2}$ Western University of Health Sciences College of Osteopathic Medicine of the Pacific, Pomona, CA; ${ }^{3}$ The University of Arizona College of Medicine Tucson, Tucson, $A Z$

\subsection{6/jim-2022-WRMC. 128}

Purpose of Study This study assesses medical and physical therapy students' knowledge level in concussion symptoms, diagnosis, and treatment. Parameters we evaluated included how education level, sports background, and concussion history influenced students' concussion knowledge. The study assessed how these students learn about concussions and whether gaps in knowledge exist. The ultimate goal is to use the survey results to help educators better prepare medical and physical therapy students for patient care.

Methods Used The first phase of our study involved sending a 14-question electronic survey to osteopathic medical schools across the United States, which assessed demographics, concussion knowledge level, source of concussion education, and interest in curriculum-based learning. The second phase consists of sending a similar electronic survey that expanded to allopathic and physical therapy schools. This survey consisted of 16 questions, with 2 additional demographic questions inquiring about gender and type of pursued degree.

Summary of Results Preliminary collection of over 800 responses and analysis of the data show that $60.2 \%$ of $\mathrm{MD}$, DO, and physical therapy students played sports in either high school, college, or professionally. In addition, $42.7 \%$ of participants reported sustaining at least one concussion throughout their lives. $26.9 \%$ of our participants reported learning about concussions through non-academic means, while $70 \%$ reported learning via academic means such as through lectures, literature reviews, or clinical rotations. Our results showed that $80 \%$ of our participants agreed they would like more formal education on concussions.

Conclusions While data collection is still ongoing, the preliminary results of our study indicate that having a sports background or personal experience with concussion may influence their knowledge in concussion diagnosis and treatment. A large percentage of our participants learned about concussions through non-academic methods. While data is forthcoming, this may indicate that an alternative means to learning about concussions is through a sports background and/or concussion history. Participants agree that in order to solidify or supplement concussion knowledge, more education is needed to best prepare rising health care professionals in clinical settings.

\section{\#130 NEW THROMBUS FORMATION IMMEDIATELY AFTER ANDEXANET ALFA INFUSION: A CASE REPORT AND LITERATURE REVIEW}

${ }^{1,2} \mathrm{~T}$ Torbati, ${ }^{3} \mathrm{OA}$ Elshaigi ${ }^{*},{ }^{2} \mathrm{M}$ Kayyali, ${ }^{3} \mathrm{OM}$ Dumitrascu. ${ }^{1}$ Western University of Health Sciences, Pomona, CA; ${ }^{2}$ Cedars-Sinai Medical Center, Los Angeles, CA; ${ }^{3}$ Mayo Clinic Arizona, Scottsdale, $A Z$

\subsection{6/jim-2022-WRMC.129}

Case Report Andexanet alfa was FDA approved in May 2018 to reverse anticoagulant effects of Factor Xa inhibitors like Apixaban and Rivaroxaban, thereby generating pro-thrombotic mechanisms. Thromboembolic complications within 30 days of Andexanet alfa administration have been reported. Here, we present for the first time a thrombotic cerebral event that appeared immediately after Andexanet alfa infusion in a patient with acute intraventricular hemorrhage (IVH).

A 73-year-old man presented to our emergency department with sudden onset of a severe headache. Head CT demonstrated $2.4 \mathrm{~mL}$ of IVH. CT angiogram showed 60\% stenosis of the left supraclinoid internal carotid artery (ICA). The patient had been taking $5 \mathrm{mg}$ Apixaban twice daily for atrial fibrillation, with his last dose 5.5 hours prior to presentation. $\mathrm{IVH}$ indicated the patient may benefit from anticoagulation reversal via Andexanet alfa.

A $400 \mathrm{mg}$ bolus of Andexanet alfa was administered followed 30 minutes later by a 2-hour infusion of an additional $480 \mathrm{mg}$, immediately upon which the patient exhibited global aphasia, temporarily alleviated by head-of-the-bed flattening. A left ICA territory mismatch $(342 \mathrm{~mL})$ and $76 \mathrm{~mL}$ core infarct were observed on CT perfusion. Shortly afterwards, the patient developed a persistent and severe left middle cerebral artery (MCA) stroke syndrome with NIH stroke scale (NIHSS) score of 23. Emergent cerebral angiogram was then performed, revealing a new sizeable thrombus in the left cervical ICA. Successful thrombectomy yielded a resulting TICI score of 2B. However, neurologic status remained poor due to development of a large left MCA territory infarct, and the patient's family chose to withdraw supportive care.

Our observation of a thrombotic event induced immediately after Andexanet alfa challenges current administration guidelines. The ANNEXA-4 phase 3 trial reported thrombotic events within 7 days of Andexanet alfa administration in 4\% of subjects and within 30 days in 10\%. The earliest event in literature was noted 1 day following treatment. Also, Andexanet alfa appears to have a higher thrombotic risk than other reversal agents like four-factor prothrombin complex concentrate by as much as $7 \%$. Further elucidation of its effects on the coagulation cascade are warranted to improve safe clinical practices. Interestingly, no thrombotic events were reported in ANNEXA-4 patients who restarted anticoagulation protocols. Thus, it may be advisable to monitor patients closely and broadly for thrombotic events until future studies update protocols for resuming anticoagulation therapy after reversal treatment. 
novel biomarker for inflammatory lung injury in ARDS resulting from multiple etiologies and strategies targeting NET formation may improve outcomes in ARDS.

\section{Pulmonary and critical care}

\section{Concurrent session}

\section{3:15 PM}

Thursday, January 20, 2022

\section{\#132 PLASMA NEUTROPHIL EXTRACELLULAR TRAP LEVELS CORRELATE WITH ACUTE RESPIRATORY DISTRESS SYNDROME SEVERITY}

J Aoki* , F Denorme, J Rustad, D Perry, M Cody, E Harris, EA Middleton, CC Yost. University of Utah Health, Salt Lake City, UT

\subsection{6/jim-2022-WRMC.130}

Purpose of Study Acute Respiratory Distress Syndrome (ARDS) is characterized by hypoxic respiratory failure, multi-organ dysfunction, and mortality. ARDS results from inflammatory alveolar injury precipitated by direct and indirect lung injury. Neutrophils play a central role in the pathology of ARDS and release neutrophil extracellular traps (NETs) to trap and kill pathogens. Dysregulated NET formation, however, can cause inflammatory tissue damage and exacerbate acute lung injury as in COVID-19 associated ARDS. Whether NETs participate pathogenically in non-COVID-19 associated ARDs remains unknown. We hypothesized that plasma NET levels correlate directly with disease severity and mortality in non-COVID-19 ARDS patients.

Methods Used We obtained previously collected plasma samples from patients $(n=200)$ with moderate to severe ARDS enrolled in the Re-evaluation of Systemic Early Neuromuscular Blockade (ROSE) trial at three different time points (admission, 24 hours, and 48 hours after admission) complete with clinical outcome data through 28 days after admission. We also examined age- and gender-matched healthy donor plasma $(n=20)$. We assayed cell-free DNA levels via fluorescence as a surrogate for NETs in each plasma sample. Clinical outcomes from ROSE trial participants were correlated with the quantification of NETs. We also assessed NET formation by neutrophils isolated from healthy adults following incubation with ARDS patient and healthy donor plasma samples using live cell imaging and confocal microscopy.

Summary of Results We demonstrated elevated cell-free DNA in ARDS plasma compared to healthy donor plasma. Deceased study participants demonstrated higher plasma cell-free DNA levels on admission and at 48 hours as compared to ARDS survivors (admission: $p=0.0045$ and 48 hours: $p=0.0050$ ). Increased cell-free DNA on admission, at 24 hours, and 48 hours also correlated with illness severity. Furthermore, ARDS plasma samples induced NET formation in vitro in neutrophils isolated from healthy donors while control plasma did not.

Conclusions NET formation is increased in plasma from patients with ARDS compared to healthy donor plasma, consistent with the inflammatory alveolar injury seen in ARDS. Additionally, plasma from ARDS patients induces NET formation in vitro in PMNs isolated from healthy adult donors. We speculate that exaggerated NET formation may serve as a

\section{\#133 PLATELET ALPHA GRANULE DEFICIENT MICE ARE PROTECTED AGAINST THE DEVELOPMENT OF HYPOXIA- INDUCED RIGHT VENTRICULAR HYPERTROPHY}

${ }^{1} \mathrm{JN}$ Posey*, ${ }^{2} \mathrm{E}$ Nozik, ${ }^{1} \mathrm{C}$ Delaney. 'University of Colorado Denver School of Medicine, Aurora, CO; ${ }^{2}$ University of Colorado Denver Department of Medicine, Aurora, CO

\subsection{6/jim-2022-WRMC.131}

Purpose of Study Pulmonary Hypertension (PH) is a life-threatening disorder characterized by increased pulmonary vascular resistance, right ventricular systolic pressures (RVSPs) and right ventricular hypertrophy (RVH), driven in part by inflammation. Our previous studies have demonstrated that platelets are activated in mice with hypoxia exposure, leading to the release of the proinflammatory chemokines Platelet Factor 4 (PF4) and CCL5, contributing to hypoxia induced lung inflammation. Nbeal2 KO mice are platelet alpha-granule deficient. Alpha granules contain numerous chemokines, including PF4 and CCL5. We hypothesized that Nbeal2 $\mathrm{KO}$ mice would be protected from hypoxia-induced $\mathrm{PH}$.

Methods Used Male and female C57BL/6 and Nbeal2 KO mice were exposed at $8-9$ weeks of age to $10 \%$ hypobaric hypoxia or remained in normoxia for 21 days. Whole blood was collected via RV cardiac puncture using heparin coated syringes and analyzed immediately. RVSPs were obtained by closed-chest RV puncture. Hearts were dissected to obtain the weights of the RV and septum + left ventricle (LV). Fulton's index, $(\mathrm{RV} / \mathrm{LV}+\mathrm{S})$ was used to determine $\mathrm{RV}$ hypertrophy as an indicator of the development of $\mathrm{PH}$.

Summary of Results Nbeal2 $\mathrm{KO}$ mice have lower platelet numbers $\left(434 \pm 43.5-780 \pm 39.6\left[\mathrm{x} 10^{3} / \mathrm{uL}\right], \mathrm{p}<0.001\right)$ and larger platelets, demonstrated by increased mean platelet volume (MPV) $(4.7 \pm 0.05-5.3 \pm 0.04[\mathrm{fL}], \mathrm{p}<0.0001)$ compared to WT controls. RVSP under control conditions was similar in Nbeal2 $\mathrm{KO}$ and WT mice $(28.14 \pm 0.91$ - 31.04 $\pm 1.01[\mathrm{mmHg}])$. There was a significantly greater hypoxiainduced increase in RVSP in Nbeal2 KOs compared to WT mice $(34.5 \pm 1.05-37.8 \pm 0.66[\mathrm{mmHg}], \mathrm{p}<0.05)$. Though, we saw statistically higher Nbeal2 KO RVSP compared to WT, they both showed a $22 \%$ increase in RVSP with hypoxia exposure (WT CO vs. HPX $28.1 \pm 0.91-34.5 \pm 1.1$ $[\mathrm{mmHg}], \mathrm{p}<0.001 ; \mathrm{KO} \mathrm{CO}$ vs. HPX $31.0 \pm 1.01-37.8 \pm$ $0.66[\mathrm{mmHg}], \mathrm{p}<0.0001)$. Fulton's index under control conditions was similar between Nbeal2 $\mathrm{KO}$ and WT mice. (0.29 $\pm 0.014-0.29 \pm 0.001)$. As expected, WT mice show the development of $\mathrm{RVH}$ when exposed to prolonged hypoxia $(0.29 \pm 0.01-0.39 \pm 0.02, \mathrm{p}<0.001)$. Nbeal2 KO mice did not develop hypoxia-induced RVH $(0.29 \pm 0.001-0.32$ $\pm 0.02)$.

Conclusions Mice deficient in alpha granules (Nbeal2 KO) have similar hypoxia-induced pulmonary vasoconstriction, but are protected against the development of RVH. Our future studies will address whether Nbeal2 $\mathrm{KO}$ mice demonstrate impaired platelet activation and/or decreased recruitment to the pulmonary circulation conferring protection from inflammatory mediated pulmonary vascular remodeling and $\mathrm{PH}$. 
\#134 EVALUATION OF THE ACCURACY OF MINIMALLYINVASIVE CARDIAC OUTPUT MONITORS BEFORE AND AFTER CARDIOPULMONARY BYPASS

K Cheung ${ }^{*}$, N Fleming. University of California Davis, Sacramento, CA

\subsection{6/jim-2022-WRMC.132}

Purpose of Study Cardiac output (CO) monitoring is an important tool for hemodynamic optimization. Bolus thermodilution (iCO) with a pulmonary artery catheter (PAC) remains the gold standard for $\mathrm{CO}$ measurement, but is invasive and has been associated with complications. This study evaluates the level of agreement of $\mathrm{CO}$ values measured from multiple minimally-invasive $\mathrm{CO}$ monitor systems before and after cardiopulmonary bypass (CPB). CCO uses a modified thermodilution technology. Cheetah is based on thoracic bioreactance. ClearSight reconstructs the brachial arterial pressure waveform from the finger arterial pressure. CNAP CO is based on continuous non-invasive arterial pressure from the finger. LiDCO is based on the radial arterial blood pressure waveform. FloTrac calculates $\mathrm{CO}$ from the radial arterial pulse contour.

Methods Used The IRB reviewed and approved this quality improvement study. Sixty patients were enrolled. 8 patients were excluded due to missing iCO measurements. $\mathrm{CO}$ measurements from 52 patients were evaluated using Bland-Altman analysis. CO values were measured simultaneously by bolus thermodilution with a PAC and the CO monitors listed above. Summary of Results All values were not available at all time points. The Bland-Altman plots are presented in figure 1 and the corresponding values are summarized in table 1 .

Conclusions Based upon percentage errors, the relative accuracy of the minimally-invasive $\mathrm{CO}$ monitors when compared to iCO were: $\mathrm{CCO}>$ Cheetah $>$ ClearSight $>$ FloTrac $>$ CNAP $>$ LiDCO. Measurements after $\mathrm{CPB}$ have slightly smaller percentage of
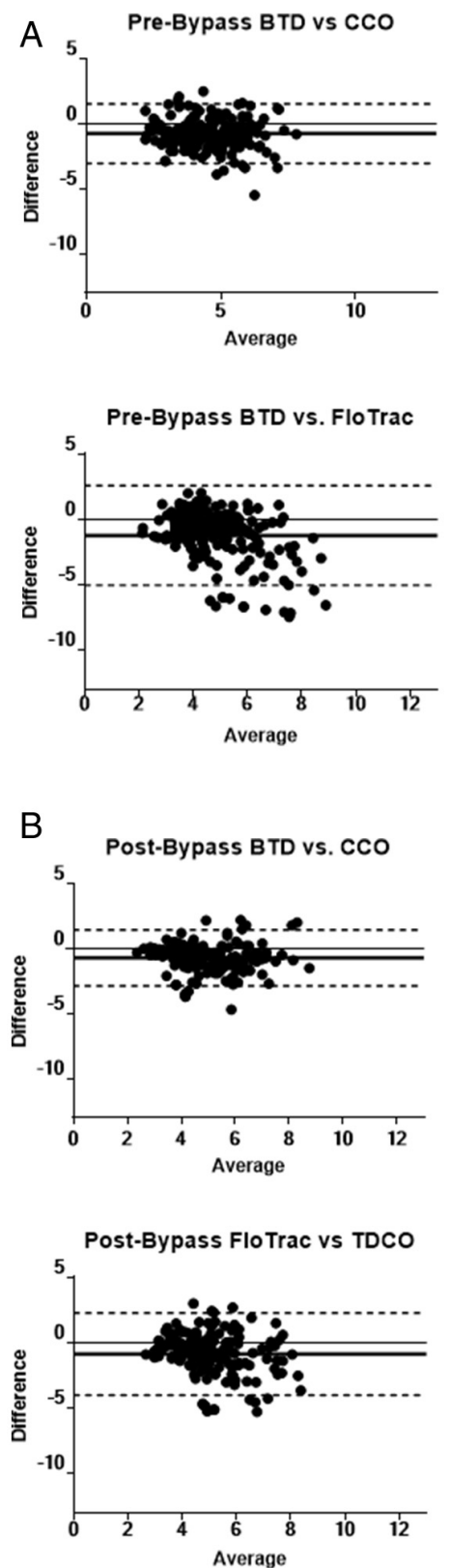
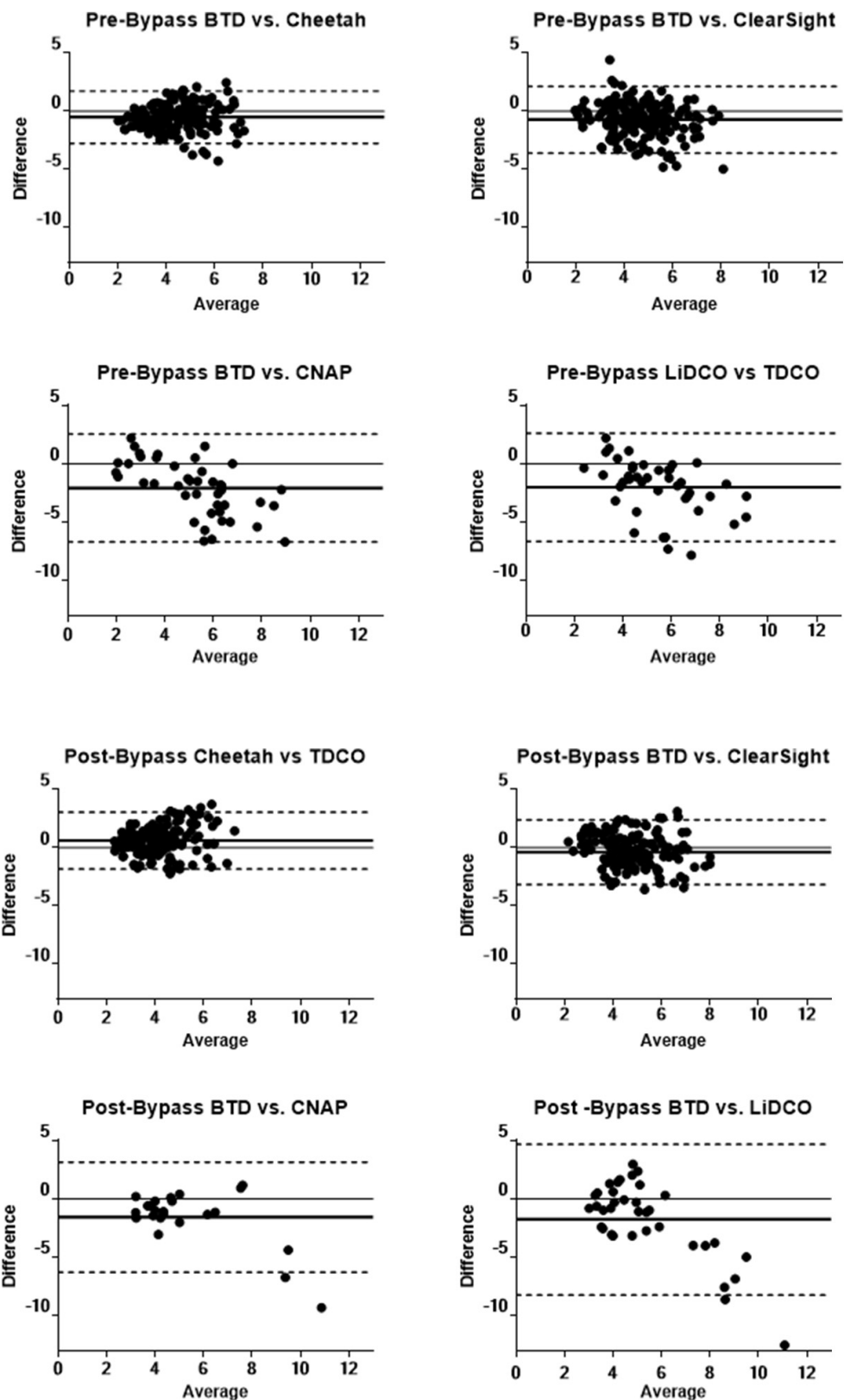

Abstract \#134 Figure 1 Bland-altman analysis of minimally-invasive CO monitors pre-bypass and post-bypass 


\begin{tabular}{lllllll}
$\begin{array}{l}\text { Abstract \#134 Table } 1 \\
\text { invasive CO monitors }\end{array}$ & \multicolumn{6}{l}{ Bland-altman analysis of minimally- } \\
\hline PRE-BYPASS: & CCO & Cheetah & ClearSight & FloTrac & CNAP & LiDCO \\
\hline Number of values & 192 & 178 & 183 & 204 & 43 & 42 \\
Percentage errors & $54.5 \%$ & $53.3 \%$ & $68.1 \%$ & $90.8 \%$ & $110.2 \%$ & $110.6 \%$ \\
Bias & -0.75 & -0.51 & -0.73 & -1.2 & -2.0 & -2.0 \\
SD of bias & 1.1 & 1.1 & 1.4 & 1.9 & 2.3 & 2.3 \\
95\% Limits of Agreement & & & & & & \\
From & -3.0 & -2.7 & -3.5 & -5.0 & -6.7 & -6.6 \\
To & 1.5 & 1.7 & 2.1 & 2.5 & 2.5 & 2.6 \\
& & & & & & \\
POST-BYPASS: & CCO & Cheetah & Cheetah & FloTrac & CNAP & LiDCO \\
Number of values & 176 & 155 & 169 & 178 & 23 & 36 \\
Percentage errors & $46.8 \%$ & $53.3 \%$ & $60.2 \%$ & $68.5 \%$ & $102.7 \%$ & $140.7 \%$ \\
Bias & -0.71 & 0.60 & -0.39 & -0.87 & -1.57 & -1.79 \\
SD of bias & 1.10 & 1.24 & 1.41 & 1.6 & 2.4 & 3.3 \\
95\% Limits of Agreement & & & & & & \\
From & -2.8 & -1.8 & -3.1 & -4.0 & -6.3 & -8.2 \\
To & 1.4 & 3.0 & 2.3 & 2.2 & 3.1 & 4.6 \\
\hline
\end{tabular}

errors. Percentage error $<30 \%$ is considered acceptable (Critchely et al., 1999). On this basis, the minimally-invasive CO monitors cannot replace the PAC for accurate CO measurement in cardiopulmonary bypass surgery.

\section{\#135 A CASE OF DABBING-INDUCED LIPOID PNEUMONIA}

LW Boucher*, GA Loh. Dwight David Eisenhower Army Medical Center, Fort Gordon, GA

\subsection{6/jim-2022-WRMC.133}

\section{Case Report}

Introduction Dabbing is an emerging form of cannabis consumption. Similar to vape-associated lung injury, it can result in acute respiratory distress syndrome (ARDS), and diagnosis is often obscured by a broad infectious differential.

Case Description The patient is a 36 year old male with recurrent admissions for various infections in the setting of a splenectomy who was admitted for an undifferentiated inflammatory syndrome. He presented with fever, leukocytosis with bandemia, several episodes of emesis, and fatigue. $\mathrm{He}$ denied any cough, dyspnea, sputum production, or chest pain. Aside from fever, his vital signs were otherwise normal and he was admitted to the general medical ward. Given his immunocompromised state and lack of clear infectious source he was started on vancomycin, azithromycin, and meropenem. Despite this regimen his fever persisted, leukocytosis worsened, and hypoxia developed; the latter rapidly progressed requiring intubation for hypoxemic respiratory failure. CT scan of the chest revealed bilateral consolidation and ground glass opacities. He met Berlin criteria for ARDS and he was started on glucocorticoids. Cytology from bronchoscopy revealed abundant alveolar macrophages with oil red $\mathrm{O}$ staining and mixed inflammatory cells. Comprehensive infectious workup including Covid-19 PCR x2, 3 sets of blood cultures, urine culture, and bronchioaveolar lavage for bacteria, acid fast organisms, and fungus was negative. This negative infectious workup coupled with cytology findings indicates lipoid pneumonia as the etiology of his illness. His clinical condition improved after several days and he was successfully extubated and eventually discharged on room air. It was discovered that the patient had been vaping with THC products including dabs. Dabs, a wax-like THC product, are likely the mechanism by which lipids were introduced into his lungs resulting in pneumonia.

Discussion This case represents a presentation of lipoid pneumonia secondary to 'dabbing,' a relatively novel form of ingesting cannabis. There have been few reported cases of respiratory failure secondary to 'dabbing,' and this case identifies lipoid pneumonia as the cause of lung injury. This case highlights the need for physicians to be aware of specific forms of recreational drugs and routes of delivery.

\section{$\# 136$ LUNG EPITHELIAL CELL ENVIRONMENT MODULATES TEMPORAL KINASE SIGNALING DYNAMICS}

N DeCuzzi*, D Murphy, A Ram, M Pargett, K Chmiel, AA Zeki, J Albeck. University of California Davis, Davis, CA

\subsection{6/jim-2022-WRMC.134}

Purpose of Study Signaling in lung epithelial cells plays a role in respiratory disease pathogenesis. ERK, NFkB, and AMPK are key kinases regulating cell growth and proliferation that are implicated in airway inflammation and disease. Importantly, ERK and AMPK display heterogenous and temporally dynamic signaling activity that can be linked to cell behavior but has yet to be investigated in the context of airway disease. We hypothesize that unique signatures of short-term oscillatory signaling activity (minutes) differentially regulate long-term (>24 hour) inflammatory responses in part via regulation of the transcription factor STAT3 at the cellular level.

Methods Used Using fluorescent biosensors and live-cell imaging, we track single-cell kinase signaling activity in our Human Bronchial Epithelial (HBE1) cell line, and primary human bronchial epithelial cells (pHBE), continuously at 6-minute intervals, both in submerged and Air-Liquid Interface (ALI) culture conditions. Computational image analysis extracts kinase signaling activity profiles in response to growth factors, and inflammatory cytokines. After 24 hours of ligand exposure, cells are fixed and immunofluorescent stain for nuclear pSTAT3 is performed to measure cellular inflammatory response.

Summary of Results Comparison of HBE1's ERK signaling activity from control and ligand stimulated cells, reveals heterogeneous and dynamic single-cell responses to inflammatory ligands relevant to both subtypes of asthma and COPD (IL-6, IL-1 $\beta, \&$ TNF $\alpha$ ), that are both ligand- and concentrationdependent. Intriguingly, pHBE cells in submerged culture display dynamic and heterogeneous ERK signaling activity, that is similarly dependent on ligand and concentration, but are unique from those seen in HBE1 cells. Notably, both HBE1 and pHBE cells displayed unique ERK responses to inflammatory ligands than those of EGF treated cells. We note a similar heterogeneity in STAT3 activation dependent on inflammatory ligand, that is attenuated in the presence of metabolic perturbation and AMPK activation.

Conclusions These results support our central hypothesis and demonstrate the importance of this novel and unique approach using both airway epithelial cell lines and primary bronchial epithelial cells in ALI. Ongoing work: 1) Finishing data collection for ERK and NFKB in pHBE cells cultured in ALI, and AMPK activity in HBE1 cells, 2) Using statistical modeling to determine ERK, AMPK, and NFKB's contribution to 
modulating the STAT3 response to inflammatory ligands, and 3) Assessing how pharmacological agents alter activity profiles to modulate long-term inflammatory responses in these model systems. Our technique will reveal deeper knowledge about airway epithelial kinase signaling mechanisms relevant to asthma and COPD, and potentially, other lung diseases.

\section{\#137 THE EFFECTS OF ACUTE AND CHRONIC KIDNEY INJURY ON MORTALITY IN OLDER PATIENTS CRITICALLY ILL WITH COVID-19}

MD Rockstrom*, S Windham, KM Erlandson, J Hippensteel. University of Colorado, Denver, $\mathrm{CO}$

\subsection{6/jim-2022-WRMC. 135}

Purpose of Study Older age is a well-recognized risk factor for increased mortality due to COVID-19. Similarly, older age is associated with high rates of critical illness and admission to intensive care units. Limited data suggests that frailty, as measured by the presence of comorbidities prior to admission, may predispose to severe illness. There is currently an inadequate description of what factors can be attributed to the increase in mortality in aged, critically ill patients with COVID-19.

Methods Used In this retrospective, cohort study, we examined 200 patients admitted to a quaternary referral center intensive care unit (ICU) with COVID-19 from March to June of 2020. Data collected included demographics, number of comorbidities, residence in assisted living or skilled nursing facilities prior to admission, vital signs, and laboratory findings at time of admission to the hospital and the ICU.

Bivariate analysis was performed with age, comorbidities, and place of residence prior to admission as independent variables and mortality, length of stay, rates, and severity of acute respiratory distress syndrome (ARDS), rates of acute kidney injury (AKI) and hemodialysis, and rates and severity of shock as dependent variables. Multivariate analysis evaluated the relationship of age, comorbidities, end organ dysfunction, and disease severity.

Summary of Results ICU mortality was positively correlated with age $(\mathrm{p}<0.001)$ and number of comorbidities $(\mathrm{p}<$ 0.001). Furthermore, age and number of comorbidities were directly correlated with development of AKI ( $p=0.012)$; age was inversely correlated with severity of lung disease as measured by P:F ratio $(\mathrm{p}=0.0092)$. Pre-existing chronic kidney disease (CKD) was highly predictive of development of AKI (OR 6.93, $\mathrm{p}=0.003$ ) Multivariable analysis demonstrated that the odds of mortality were higher with increasing age $(\mathrm{OR}=1.06[95 \%$ CI $1.02,1.11]$ per year, $\mathrm{p}=0.005)$, decreased with increasing P:F ratio (OR 0.336 [95\% CI 0.16, 0.61] for every increase in P:F of 50, p $<0.001)$, and increased with AKI requiring hemodialysis (OR $=4.61[95 \%$ CI 1.185, 19.7], $\mathrm{p}=0.031$ ).

Conclusions Recognition of CKD in older adults critically ill with COVID-19 is critical in identifying those who are high risk of severe kidney dysfunction and death. CKD has been shown previously to be associated with mortality in COVID19 and this study offers a mechanistic understanding of this relationship. This is especially important given that older patients do not seem to have the same propensity for severe lung disease seen in younger patients. The role of kidney disease in the mortality of older patients critically ill with COVID-19 needs to be better defined prospectively.

\section{\#138 EFFECT OF POSITIVE END EXPIRATORY PRESSURE IN PREVENTION OF POSTOPERATIVE PULMONARY COMPLICATIONS IN OBESE PATIENTS UNDER GENERAL ENDOTRACHEAL ANESTHESIA: SYSTEMATIC REVIEW AND META-ANALYSIS OF RANDOMIZED TRIALS}

JY Choi*, MA Al-Saedy, BJ Carlson. Washington State University, Spokane, WA

\subsection{6/jim-2022-WRMC.136}

Purpose of Study In non-obese patients, low positive endexpiratory pressure (PEEP) of $<5 \mathrm{cmH}_{2} \mathrm{O}$ is a widely accepted practice. Although the safety of low tidal volume plus low PEEP has been established in non-obese patients, this strategy may induce atelectasis and post-operative pulmonary complications in obese patients. In obese patients, use of PEEP $>10 \mathrm{cmH}_{2} \mathrm{O}$ has shown to prevent intraoperative atelectasis but there is no conclusive data for the postoperative complications from high PEEP. Therefore, this meta-analysis aims to compare the rate of postoperative complications (PPCs) associated with high PEEP and low PEEP in obese patients who received surgery under endotracheal general anesthesia.

Methods Used The protocol for this meta-analysis was published on PROSPERO (CRD42021224041). We searched MEDLINE, EMBASE, CENTRAL, and CINAHL databases using pre-specified search strategies. We included only RCTs that compared the effect of low PEEP and high PEEP in obese patients $\left(\mathrm{BMI}>30 \mathrm{~kg} / \mathrm{m}^{2}\right)$ who received open or laparoscopic surgeries under endotracheal general anesthesia. All patients received the tidal volume of $6-8 \mathrm{ml}$ per $\mathrm{kg}$ of predicted body weight. Risk of bias was assessed using the Covidence review manager. Statistical analysis was conducted using Cochrane RevMan software (Mantel-Haenszel, Fixed Effects, Risk Ratio). Primary outcome was the number of PPCs. Secondary outcomes included extrapulmonary complications, intraoperative complications, and mortality during hospital stay.

Summary of Results There was no significant difference in the rate of postoperative complications between low PEEP and high PEEP groups $(\mathrm{RR}=0.93 ; 95 \% \mathrm{CI} 0.79$ to $1.10 ; \mathrm{P}=0.41)$. We identified 4 randomized controlled trials involving 2,116 participants. Two trials had a low risk of bias, and the other two trials had an immediate and substantial risk of bias. We observed significant heterogeneity within included trials $\left(\mathrm{I}^{2}=53 \%\right)$. Secondary outcomes were not reported by all trials. Incidence of intraoperative hypotension was reported in 3 trials and was significantly lower in the low PEEP group $(\mathrm{RR}=1.84 ; \mathrm{P}<0.01)$.

Conclusions Overall, the evidence is not robust enough to determine the protective effect of high PEEP and low PEEP in obese patients. Out of 2,116 total participants, 1,976 participants were from 1 trial that included both laparoscopic and open abdominal surgery. This may explain the heterogeneity observed since the other three trials only included patients who received laparoscopic abdominal surgeries. Although exclusion of the large study significantly lowered the incidence of PPCs in the low PEEP group with relative risk of $(\mathrm{RR}=8.0 \mathrm{~m} \mathrm{P}=0.05)$, the other trials were smaller studies and had higher risk of publication bias. More multi-center RCTs are warranted to compare the rate of PPCs between low PEEP and high PEEP in obese patients under endotracheal general anesthesia. 
\#139 ALVEOLAR HEMORRHAGE DUE TO SECONDARY PULMONARY VASCULITIS IN A PATIENT WITH NEW FEATURES OF AUTOIMMUNE DISEASE

${ }^{1} \mathrm{~A}$ Garcia*, ${ }^{2} \mathrm{~T}$ Issa, ${ }^{2} \mathrm{R}$ Garcia-Pacheco, ${ }^{1} \mathrm{~J}$ Oberndorf. ${ }^{1}$ Ross University School of Medicine, Miramar, FL: ${ }^{2}$ Kern Medical Center, Bakersfield, CA

\subsection{6/jim-2022-WRMC.137}

Case Report Pulmonary Vasculitis is a manifestation of a specific set of disorders pathologically defined by inflammatory destruction of blood vessels within the lungs. Secondary immune mediated vasculitis, as in autoimmune processes like rheumatoid arthritis (RA), is one subset of these disorders. We present a rare case of alveolar hemorrhage caused by pulmonary vasculitis in a patient positive for anti-CCP antibody but no laboratory or imaging to meet diagnostic criteria for RA. CASE A 50-year-old female with history of pulmonary embolism (PE) presented to the ED with three episodes of hemoptysis. She also complained of chest pain, arthralgias and fatigue. She was not on anticoagulation therapy. Family history was positive for lupus and RA in two sisters. Vital signs were stable. Labs showed ESR 36, CRP 1.53, and D-dimer 749. CT chest angiogram revealed scattered ground glass and patchy alveolar densities throughout lung fields; no evidence of PE. Flexible bronchoscopy with bronchoalveolar lavage (BAL) was diagnostic for alveolar hemorrhage. Cytology from BAL yielded numerous red blood cells and macrophages with hemosiderin granules. Autoimmune panel was significant for elevated ANA 1:80 and CCP Ab IgG >250. Patient was started on Prednisone $60 \mathrm{mg} /$ day for diagnosis of alveolar hemorrhage secondary to immune mediated capillaritis and discharged home. As an outpatient, Xrays of wrists and hands revealed no inflammatory or crystalline arthropathy. Labs showed normal rheumatoid factor (RF), but persisting elevation in CRP and ESR. Methotrexate was started to prevent recurrent alveolar hemorrhage and Prednisone was continued pending rheumatology referral.

Discussion Although there have been some rare cases of diffuse alveolar hemorrhage (DAH) preceding the diagnosis of RA, DAH commonly arrives as a manifestation of long standing RA. Anti-CCP antibody is known to not only be as sensitive as RF but significantly more specific when diagnosing RA. Studies have shown a subgroup of patients testing positive for anti-CCP antibodies with no evidence of RA, who subsequently developed RA within short followup.

Conclusion The unique disease course of DAH in patients with underlying features of autoimmune disease is not well documented. Implications of positive anti-CCP antibody but no definitive diagnosis of RA in patients with lung disease still requires further investigation. Anti-CCP antibody has been shown to be highly predictive of future development of RA. Patients that present with pulmonary symptoms like hemoptysis due to autoimmune vasculitis and capillaritis should be closely monitored. Whether to initiate early treatment for RA should also be strongly considered. As such, clinicians need to remain vigilant when suspecting immune-mediated $\mathrm{DAH}$ in patients with unclear autoimmune disease.

\section{$\# 140$ USE OF STRICT SPINAL PRECAUTIONS FOR SPINAL CORD INJURIES BY SKI PATROLS: DO THEY WORK?}

C O' Driscoll, D Deng* ${ }^{*}$ E Guenther. Western University of Health Sciences College of Osteopathic Medicine of the Pacific-Northwest, Lebanon, OR

10.1136/jim-2022-WRMC.138
Purpose of Study For decades, hard backboards have been the standard of care for emergency medical personnel and ski patrollers treating known or suspected spinal cord injuries in the field. Recent studies have shown that prolonged immobilization on hard backboards can cause injuries to patients and are no longer recommended unless specific criteria are met. In 2018, the National Ski Patrol implemented guidelines consistent with the Position Statement of the National Association of EMS Physicians and the American College of Surgeons Committee on Trauma. The effectiveness of those guidelines for ski patrollers in the field are quantified in this survey study.

Methods Used This study was approved by the Western University of Health Sciences Institutional Review Board. An anonymous online survey was distributed electronically to 168 ski patrols across the United States using contact information obtained from the National Ski Patrol website. The survey was optional, and participants voluntarily completed the survey between April 2 and April 27, 2021. A total of 19 ski patrols responded to the survey and 19 are represented in the data. They were asked questions regarding how many backboards they used per season before and after the new guidelines were implemented, frequency of backboard-specific training, whether the mountain also has a paid patrol division, as well as confidence of their patrol utilizing the new spinal protection guidelines.

Summary of Results The majority of respondents (14 out of 19) reported a decrease of at least 5 backboards used in the season following implementation of the new guidelines. Patrols that conducted more than two backboard-specific trainings per year reported a larger decrease in backboards (6-10 per season) in the season following the new guidelines than patrols that conducted 1-2 yearly trainings (up to 5 per season). The average reduction in backboards in both patrols that have a paid division and those that do not was approximately 5 backboards per year. 10 out of 19 patrols reported that they were 'very confident' their patrols could implement the new guidelines and 9 out of 19 reported they were 'somewhat confident'. No respondents reported that they were 'not very confident'.

Conclusions The new guidelines and training from the National Ski Patrol resulted in a decrease in hard backboard use by the majority of surveyed patrols while still allowing ski patrols to utilize potentially life-saving devices when necessary.

\section{Surgery II}

\section{Concurrent session}

\section{3:15 PM}

\section{Thursday, January 20, 2022}

\section{\#141 DISPLACED FEMORAL NECK FRACTURES IN ELDERLY PATIENTS SHOULD NOT BE FIXED WITH CLOSED REDUCTION AND PERCUTANEOUS PINNING}

${ }^{1} \mathrm{D}$ Skerrett*, ${ }^{2} \mathrm{M}$ Coale, ${ }^{2} \mathrm{~W}$ Lack. ' University of Washington School of Medicine, Seattle, WA; ${ }^{2}$ University of Washington Department of Medicine, Seattle, WA

10.1136/jim-2022-WRMC.139

Purpose of Study The Garden classification has been used to grade femoral neck fractures since 1961. Nondisplaced 
fractures on AP radiographs are described as Garden I or II, while displaced fractures are designated Garden III or IV. These distinctions are useful when selecting an operative treatment. In elderly patients, nondisplaced fractures are often treated with internal fixation (i.e. screws) while displaced fractures are often treated with arthroplasty. Poor bone quality in the elderly limits screw purchase and impairs healing, particularly in the setting of Garden III/IV fractures. Nonetheless, many displaced fractures in elderly patients are treated with internal fixation operations, such as closed reduction and percutaneous pinning (CRPP). This observational study compared rates of failure between displaced and nondisplaced femoral neck fractures treated with closed reduction and percutaneous pinning in Seattle.

Methods Used We identified 374 patients who suffered femoral neck fractures and were treated with internal fixation between 2010 and 2020 at 3 hospitals in Seattle. Patients older than 50 and fixed with CRPP met the inclusion criteria. Pathologic fractures and fractures fixed with dynamic hip screw, dynamic helical hip screw, arthroplasty, or open approach/visualized reduction were excluded. Treatment failure was defined as avascular necrosis, nonunion, or conversion to arthroplasty. 267 records met these criteria. For each patient, data was collected on displacement, age, sex, dementia status, ASA, baseline ambulation, independence, and injury mechanism. Chi-squared tests were used to assess correlation between these variables and treatment failure.

Summary of Results Displaced fractures treated with CRPP were significantly more likely to fail than nondisplaced fractures treated with CRPP $(\mathrm{p}<0.00001)$. Absence of dementia was also associated with CRPP failure ( $p=0.0197)$. Age, sex, ASA, baseline ambulation, independence, and injury mechanism were not found to be associated with treatment failure.

Conclusions In elderly patients, displaced femoral neck fractures are significantly more likely than nondisplaced fractures to fail treatment with CRPP. Initial treatment with hemiarthroplasty or total arthroplasty should therefore be considered in this demographic to reduce the risk of pain and reoperation. Absence of dementia was also correlated with treatment failure; however, dementia patients' differing goals of care and impaired communication with caretakers confound this result. Future studies could compare outcomes between displaced fractures treated with CRPP versus arthroplasty. In addition, future work may further examine the association between absence of dementia and CRPP failure.

\section{\#142 ALTERATIONS IN SHOULDER TENDON STRUCTURAL PROTEINS IN ATHEROSCLEROSIS}

WH Fang*, S Sekhon, FG Thankam, DK Agrawal. Western University of Health Sciences, Pomona, $C A$

\subsection{6/jim-2022-WRMC. 140}

Purpose of Study Hyperlipidemia is a hallmark of the atherosclerotic process and can impact every system of the body, including the musculoskeletal system as evident from the increased comorbidity of tendinopathies in atherosclerotic patients. Indeed, lipid deposits within the extracellular matrix (ECM) have been found in tendon tissues with changes in the biomechanical properties of the tendon. However, there is limited information on the development and progression of tendon pathology in atherosclerotic patients. Here, we examined the expression status and molecular crosstalk of the ECM proteins in atherosclerosis using hyperlipidemic microswine model.

Methods Used Shoulder tendons tissues $(\mathrm{N}=12)$ from hyperlipidemic Yucatan microswine were harvested, fixed, embedded in paraffin and longitudinal thin sections were used for tissue morphology with hematoxylin and eosin (H\&E), Masson trichrome, and Pentachrome staining.

Immunofluorescence staining (IF) was performed for collagen types I, III, IV, V, VI, and XVII, MMP2, and MMP9 following standard protocols. The fluorescence intensity (MFI) of each protein was quantified using Image J software. The variation with respect to control (tendon tissue harvested from normal swine) was calculated from the average $\mathrm{MFI} /$ nuclei and the results are presented as $\log _{2}$ fold-change (FC).

Summary of Results H\&E staining showed disorganized ECM in atherosclerotic tissue with greater deposition of adipocytes. Trichrome staining revealed disorganization in collagen fibers with poorly defined vasculature and increased infiltration of adipocytes in atherosclerotic tendons compared to the control. The pentachrome staining highlights decreased collagen expression and increased mucin deposition in the atherosclerotic tissues. Also, the atherosclerotic shoulder tendons demonstrated decreased expression of COL III $(\mathrm{FC}=-0.38 \pm 1.49)$, COL IV $(\mathrm{FC}=-0.61 \pm 1.75)$, and a pronounced decrease in COL XVII $(\mathrm{FC}=-1.6 \pm 1.85)$ and $\mathrm{COL} \mathrm{I}(\mathrm{FC}=-2.24 \pm 0.41)$. However, there was an increased expression of $\mathrm{COL} V$ $(\mathrm{FC}=1.02 \pm 2.13)$, MMP-9 $(\mathrm{FC}=0.9 \pm 0.60)$, and a marked increase in MMP-2 $(\mathrm{FC}=2.05 \pm 1.53)$.

Conclusions The findings demonstrated that there was considerable structural alteration in ECM composition and components in hyperlipidemic tendon when compared with normal shoulder tendons. There was a decrease in collagen proteins and an upregulation of the MMP class of proteolytic enzymes. The decreased collagen and increased MMP expression are correlated with increased tendon injury and rupture. Such pathological alterations support the existence of increased comorbidity of tendinopathies in hyperlipidemic patients.

\section{$\# 143$ AN INVESTIGATION IN THE EVIDENCE BASE FOR ADAPTOGENS IN WOUND HEALING AND SKIN REJUVENATION}

D Sokolov*, S Gupta. Loma Linda University, Loma Linda, CA

10.1136/jim-2022-WRMC.141

Purpose of Study Despite having a long history of use in traditional and herbal medicine, adaptogens have recently reentered the spotlight due to their potential to augment the body's response to stress. Originally mentioned in 1974 in a Soviet literature review as 'New substances of plant origin that Increase non-specific resistance', the term 'adaptogen' has grown to include most any compound that increases the body's resilience against a variety of mechanisms of cellular stress. Considering that the skin is highly affected by many of these pathways, this implies a potential application for adaptogens in the context of specialties concerned with aesthetic outcomes. This study identified adaptogens with an evidencebased clinical application in the areas of skin health and wound healing.

Methods Used A literature search was conducted using the term 'adaptogen' in the article databases of Google Scholar, 


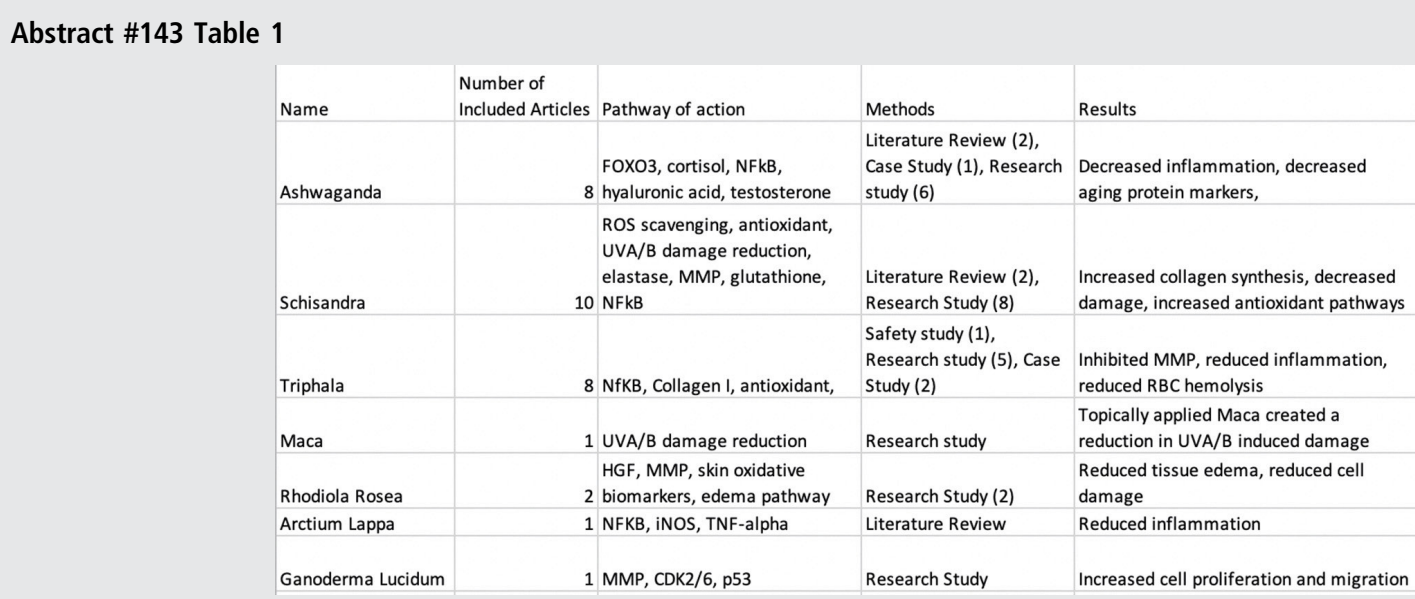

JSTOR, NCBI and PubMed. Upon finding mention of specific compounds, separate searches were then performed for each compound. Inclusion criteria consisted of articles pertaining to skin improvement and anti-inflammatory properties. Additionally, special attention was given to studies that used human keratinocyte cell lines. Exclusion criteria included general information articles, and articles with described benefits outside the realm of skin and regenerative capacity.

Summary of Results The number of relevant articles found spoke to a large need for further research. While there are numerous articles and studies done on the systemic benefits of various adaptogens, research specific to skin and wound healing is very limited. However, the research completed consistently shows agreement in the nature of the benefits of various adaptogens. A common theme of inflammation reduction quickly emerged through the course of the search, with the most prevalent pathway affected being NFkB. While Ashwagandha, Schisandra and Triphala held the most promise in terms of an evidence base, the number of applicable articles often corresponded with the total amount of available literature on a certain compound.

Conclusions This serves to highlight how much potential there is in this area for further research. If the benefits proposed in the current literature stood the test of clinical studies, adaptogens could become part of standard perioperative and post traumatic care.

\section{\#144 HOW DOES DIMETHYL SULFOXIDE AFFECT IN-VITRO FAT GRAFT VIABILITY?}

NM Sarli*, S Gupta. Loma Linda University Adventist Health Sciences Center, Loma Linda, CA

\subsection{6/jim-2022-WRMC. 142}

Purpose of Study Since the popularization of fat grafting in the 1980s, surgeons have sought to improve the viability of the grafts. Research demonstrates that the majority of harvested cells die within hours to days of transplantation. One of the most promising interventions which may improve survival during this time are pharmacologic agents added to the graft or recipient site. Several additives have been trialed. One such compound is dimethyl sulfoxide (DMSO). In one study, rats treated with DMSO showed improved fat graft survival. Due to the challenge of studying new treatments in humans we sought to observe the impact of DMSO on viability of adipocytes in vitro.

Methods Used DMSO was added to samples of human lipoaspirate to produce three concentrations: 500:1, 2000:1, and 8000:1 and a control sans DMSO. The samples were centrifuged to isolate the fat from the aqueous fraction. Each sample was then divided into several $900 \mu \mathrm{L}$ portions and incubated with $100 \mu \mathrm{L}$ of AlamarBlue at $37 \mathrm{C}$ for 4 hours. Each portion was then diluted with $3 \mathrm{~mL}$ of saline and centrifuged to suspend the dye. Absorbance was measured at $570 \mathrm{~nm}$ and $600 \mathrm{~nm}$. The experiment was repeated thrice. The patients were females aged 41, 61, and 48. Normalized absorbances were calculated by subtracting the $600 \mathrm{~nm}$ absorbance from the $570 \mathrm{~nm}$ absorbance.

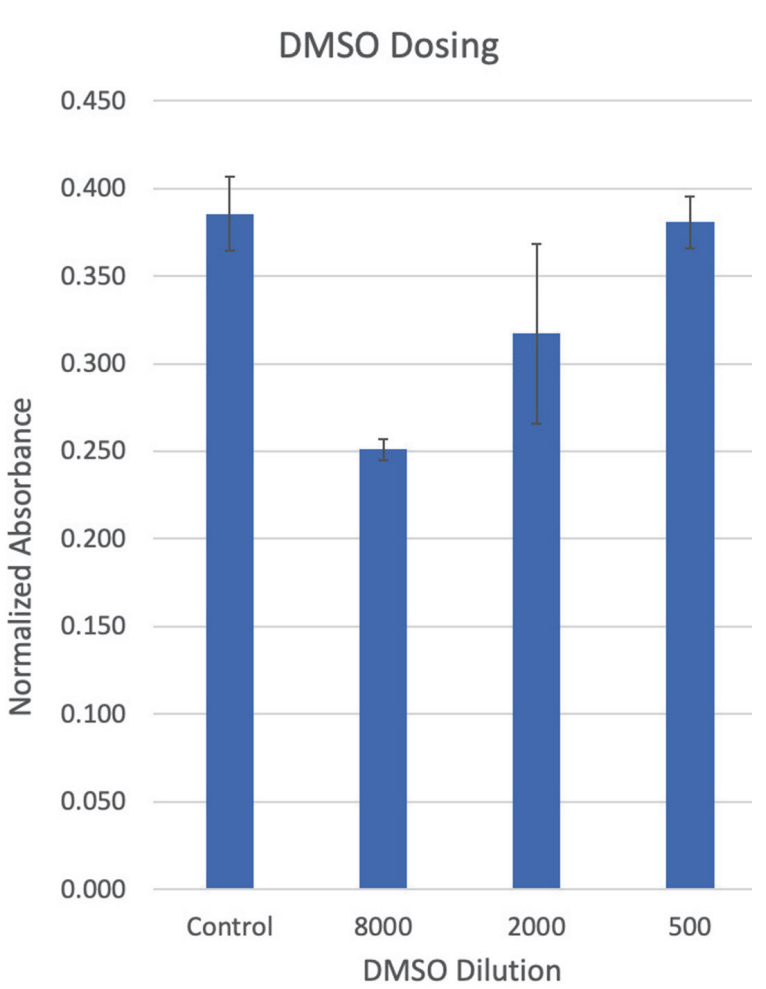

Abstract \#144 Figure 1 
Summary of Results Trial \#1, (figure 1), showed a decrease in absorbance across all doses of DMSO tested [ Control (0.386), 1:8000 (0.251 p = 0.004), 1:2000 (0.317), and 1:500 (0.381)]. Trial \#2 (figure 2), demonstrated a dose-

\section{DMSO Dosing}

0.250



Abstract \#144 Figure 2

\section{DMSO Dosing}

0.250

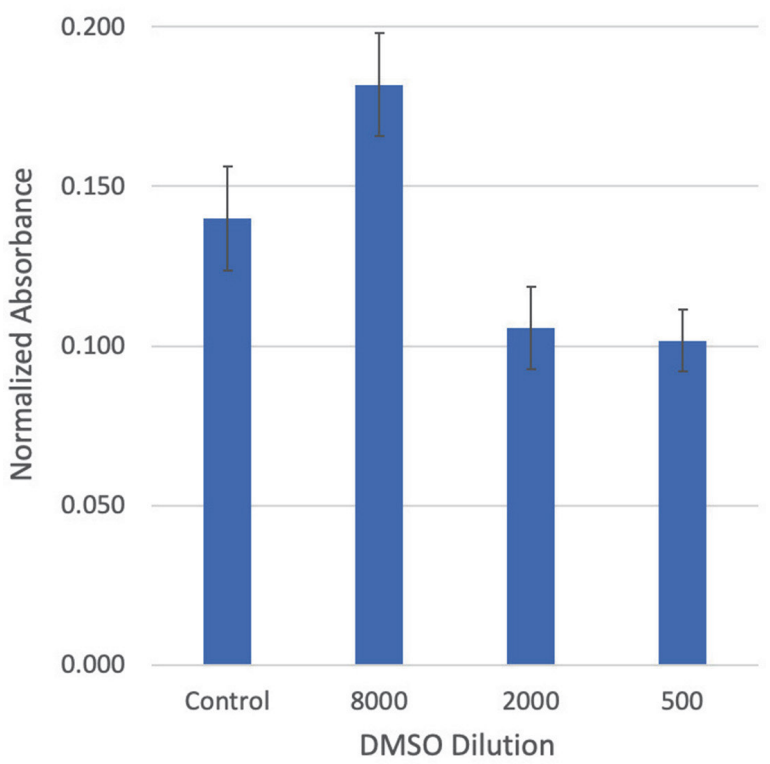

Abstract \#144 Figure 3 dependent decrease in absorbance across the three concentrations [Control (0.201) 1:8000 (0.186, p = .03), 1:2000 $(0.183, \mathrm{p}=0.2), 1: 500(0.145, \mathrm{p}=0.02)]$. Finally, trial \#3, (figure 3) yielded an increase in absorbance at a low (1:8000) concentration of DMSO (0.182, $\mathrm{p}=0.02)$ vs Control (0.140). 1:2000 and 1:500 were found to have a negative effect on absorbance $(0.106 \mathrm{p}=0.1$ and $0.102 \mathrm{p}=0.07$, respectively).

Conclusions Our results are, thus far, inconclusive. However, we believe that there are confounds which can be eliminated. The researcher noted an inconsistent mixing of assay with fat and also noted that small variations in pipetting technique introduced bubbles in the cuvette that could affect readings. If minor technique changes rectify these errors, more consistent results are possible.

If future in vitro trials find DMSO to offer a viability benefit to graft cells, further research could be done on topical applications in humans as DMSO has been safely used topically for years.

\section{\#145 COMPARISON OF COMPLICATIONS IN PATIENTS WITH NORMAL AND COMPROMISED RENAL FUNCTION UNDERGOING ADJUVANT RADIATION THERAPY FOLLOWING ROBOTIC-ASSISTED LAPAROSCOPIC RADICAL PROSTATECTOMY}

${ }^{1} \mathrm{R}$ Chen ${ }^{*},{ }^{2} \mathrm{~S}$ Song, ${ }^{2} \mathrm{~A}$ Amasyali, ${ }^{2} \mathrm{C}$ Ritchie, ${ }^{1} \mathrm{C}$ Baas, ${ }^{2} \mathrm{D}$ Baldwin. ${ }^{1}$ Loma Linda University, Loma Linda, CA; ${ }^{2}$ Loma Linda University Adventist Health Sciences Center, Loma Linda, CA

\subsection{6/jim-2022-WRMC.143}

Purpose of Study Chronic kidney disease (CKD) and end-stage renal disease (ESRD) are associated with high morbidity and mortality. Kidney transplantation may be a life-saving therapy for these patients. However, ESRD patients with untreated prostate cancer may be excluded from transplantation due to risk for cancer progression, while patients treated with robotic-assisted laparoscopic radical prostatectomy (RALP) who have undetectable PSA's may be cleared for immediate transplantation. Patients with detectable PSA's may require adjuvant radiation, which may result in radiation cystitis, a clinical diagnosis ranging from mild dysuria to severe hematuria. In patients with prior RALP, adjuvant radiation therapy (XRT) may result in a higher mean radiation dose to the bladder due to its low volume and new post-surgical location, which may increase the risk for hemorrhagic cystitis. The purpose of this study was to compare the complications between patients with normal and compromised renal function undergoing adjuvant radiation therapy following RALP.

Methods Used A retrospective review was conducted of all patients in a single academic institution undergoing RALP followed by XRT between December 2006 and July 2020. Demographic variables, surgical parameters, cancer stage, outcomes and complications were compared between patients with CKD $0-2$ and CKD 3-5. The primary outcome was radiation related bladder complications including hematuria, catheter insertion, blood transfusions, continuous bladder irrigation, surgical clot evacuation, and bladder fulguration. Statistical analysis was performed by Mann Whitney $U$ and Independent $\mathrm{T}$ test with $\mathrm{p}<0.05$ considered significant.

Summary of Results When comparing the 101 patients with CKD 0-2 who underwent RALP followed by XRT, to the 9 patients with CKD 3-5, there was no difference in age $(67.7$ 
vs. 63.7 yrs.), length of follow-up (39.6 vs. 38.2 mos) or cancer stage ( $p>0.05$ for all). However, patients in the CKD 3-5 group were significantly more likely to require Foley placement for clot evacuation $(33.3 \%$ vs $1.1 \%$; $<<0.01)$, hospitalization for continuous bladder irrigation $(33.3 \%$ vs $1.1 \%$; $\mathrm{p}<0.01)$, blood transfusion $(33.3 \%$ vs $1.1 \%$; $<<0.01)$, hyperbaric oxygen treatment $(33.3 \%$ vs $0 \% ; \mathrm{p}<0.01)$ and surgical fulguration $(22.2 \%$ vs $1.1 \%$; $<<0.01)$.

Conclusions Individuals with CKD 3-5 are at significantly greater risk for severe radiation-induced hemorrhagic cystitis. Lower volume bladder combined with increased risk of coagulopathy may partly explain this finding. Adjuvant radiation should be used with caution in patients with significant renal dysfunction.

\section{\#146 FACTORS ASSOCIATED WITH SPONTANEOUS LATERAL SKULL BASE CEREBROSPINAL FLUID}

JS De Armas*, E Miles, A Miller, Y Liu, N Wycliffe. Loma Linda University Medical Center, Loma Linda, CA

\subsection{6/jim-2022-WRMC. 144}

Purpose of Study Most lateral skull base CSF leaks have an identifiable cause, however, spontaneous cerebrospinal fluid (CSF) leaks do not. We aimed to determine risk factors for spontaneous lateral skull base CSF leaks.

Methods Used Retrospective chart review of all patients seen at a tertiary referral center over the last 10 years for spontaneous lateral skull base CSF leak who underwent temporal bone CT scan. Patients with spontaneous CSF otorrhea were included. Patients with lateral skull base CSF leaks secondary to known causes such as trauma, cholesteatoma, or iatrogenic were excluded. Sex and age-matched controls were randomly selected from all patients seen at the same center with temporal bone CT scan and without major otologic pathology. Demographics collected and main outcomes analyzed included age, sex, ethnicity, body mass index (BMI), smoking status, diagnosis of obstructive sleep apnea, and other comorbid medical conditions. Statistical analysis included analysis of variance, student t-test, and chi-squared between CSF leak patients and controls.

Summary of Results Fourteen patients were identified with spontaneous lateral skull base CSF leak. 32 sex and agematched controls with CT temporal bone imaging were randomly selected. $93 \%$ of patients in the CSF leak group were female with an average age of 66.9 years. Patients in the CSF leak group had higher BMI $(33.4$ vs $27.4, \mathrm{p}<0.01)$ with higher rates of cardiovascular disease (93\% vs. $63 \%, \mathrm{p}=0.04)$ and sleep apnea or snoring (43\% vs. $3 \%, \mathrm{p}<0.01)$.

Conclusions Risk factors associated with spontaneous lateral skull base CSF leaks include female gender, elevated BMI, OSA, and cardiovascular disease. Further research is warranted to identify potential associations between lateral skull base thickness and spontaneous lateral skull base CSF leaks.

\section{\#147 UTILITY OF PEDIATRIC VESICOSTOMY IN PROTECTING THE URINARY TRACT}

${ }^{1} \mathrm{~J}$ Byer* ${ }^{2}{ }^{2} \mathrm{C}$ Ritchie, ${ }^{2} \mathrm{~J}$ Chamberlin. 'Loma Linda University School of Medicine, Loma Linda, $C A ;{ }^{2}$ Loma Linda University, Loma Linda, CA

10.1136/jim-2022-WRMC. 145
Purpose of Study In pediatric patients, a vesicostomy is a surgical option for temporary or permanent urinary diversion as a protective measure for the integrity of the patient's urinary tract and renal system. The objective of this study is to quantify the indications for the pediatric patient population undergoing vesicostomies at an academic children's hospital.

Methods Used We performed a retrospective chart review of all pediatric patients (0-18 years) who underwent cutaneous vesicostomy from a single tertiary children's hospital from 2002 to 2021. Demographic information, pathological indications for vesicostomy, medical management, urinary tract infections, urodynamics, renal function, complications, and reversal of vesicostomy were evaluated. The primary outcome was improvement in hydronephrosis and vesicoureteral reflux, comparing before and after vesicostomy. Two-tailed, Student- $t$ tests were calculated, with $\mathrm{p}$ values less than 0.05 considered significant.

Summary of Results At our institution, 33 pediatric patients (20 males and 13 females) underwent vesicostomy. The age at time of surgery ranged from 0 to 179 months (mean 39.2 months) with median follow-up 70.5 months (range 2-210). The indication for vesicostomy included neurogenic bladder (14), chromosomal anomalies (8), anatomical malformation (7), prune belly syndrome (4), secondary vesicoureteral reflux (3), posterior urethral valves (3), and solitary kidney (3). Two patients underwent vesicostomy prior to kidney transplant clearance and one had a vesicostomy concurrently with kidney transplant. Prior to surgery, $21(63.6 \%)$ were started on clean intermittent catheterization and $20(60.6 \%)$ were on an anticholinergic. Fifteen $(45.5 \%)$ had $\geq 2$ confirmed UTIs before surgery (range 0 to 11). Either unilateral or bilateral SFU Grade $\geq 2$ Hydronephrosis was present in 25 patients $(75.8 \%)$ prior to surgery and present in 12 (36.4\%) after surgery $(\mathrm{p}<0.01)$. Vesicoureteral reflux Grade $\geq 2$ was present in 15 patients $(40.5 \%)$ prior to surgery and persistent in $5(15.2 \%)$ after surgery $(p=0.036)$. The median percentage of expected bladder capacity was $83.3 \%$. Complications after vesicostomy included vesicostomy site fungal rash (23), sepsis due to a UTI (10), and renal or bladder calculi (4). Additionally, 4 developed stricture, 4 developed stomal stenosis, 8 had to catheterize the stoma to maintain patency, and 5 had bladder prolapse through the vesicostomy site with 2 requiring revisional surgery. Six underwent excision and closure of their vesicostomy. One had their cutaneous vesicostomy modified to an ileovesicostomy. Two underwent repeat vesicostomy.

Conclusions Cutaneous vesicostomy are useful and effective forms of urinary diversion in pediatric patients with unsafe urinary tracts that have otherwise been refractory to medical treatment.

\section{\#148 DOES A NEEDLE HOLDER REDUCE FLUOROSCOPIC RADIATION EXPOSURE TO THE SURGEON'S HAND COMPARED TO LEAD GLOVES AND CONVENTIONAL GLOVES?}

${ }^{1} \mathrm{E} J 00^{*},{ }^{1} \mathrm{C}$ Baas, ${ }^{1} \mathrm{R}$ Chen, ${ }^{1} \mathrm{JC}$ Hartman, ${ }^{2} \mathrm{JD}$ Belle, ${ }^{2} \mathrm{~A}$ Amasyali, ${ }^{2} \mathrm{D}$ Baldwin. ${ }^{1}$ Loma Linda University School of Medicine, Loma Linda, CA; ${ }^{2}$ Loma Linda University Adventist Health Sciences Center, Loma Linda, CA

10.1136/jim-2022-WRMC. 146

Purpose of Study Fluoroscopy is commonly employed while gaining needle renal access prior to removal of kidney stones during percutaneous nephrolithotomy (PCNL). Excess radiation 
exposure has been linked to possible deleterious outcomes for both patients and surgeons. Some of the radiation the surgeon receives is due to scatter radiation, but the surgeon's hand may be exposed to the highest radiation dose when placed directly in the fluoroscopy beam. To reduce this exposure, some surgeons wear lead gloves, and more recently, a specialized needle holder has been developed. The purpose of this study is to compare direct radiation dose to the surgeon's hand while holding the needle with a conventional surgical glove, a lead glove, or a novel needle holder.

Methods Used A PCNL procedure was simulated using a cadaver to represent a patient and a separate cadaver arm to mimic a surgeon's hand. Three different techniques for holding the needle were tested: holding the needle directly in the fluoroscopy beam while wearing a conventional surgical glove, holding the needle while wearing a lead glove, and using a novel needle holder designed to distance the surgeon's hand from the direct beam. Thermoluminescent dosimeters were attached to the thumb, middle finger, hypothenar eminence, wrist of the cadaver hand, and skin of the cadaver patient, dorsal to the kidney. Five trials were performed for each treatment arm. In each trial, five minutes of fluoroscopy was delivered using automatic exposure control. Radiation doses between treatment arms were compared using ANOVA with $\mathrm{p}<0.05$ considered significant.

Summary of Results Using a lead glove resulted in a significant reduction of radiation to the surgeon's hand compared to when the surgeon directly held the needle wearing a conventional glove (mean dose 248.4 vs. 391.9 mRad; p<0.001). However, the greatest radiation reduction was seen when using the novel needle holder $(137.3 \mathrm{mRad})$ compared to a lead glove (248.4 mRad) and a conventional glove (391.9 mRad; $\mathrm{p}<0.001)$. An unexpected finding was a significant reduction in the cadaver patient's radiation dose when the novel needle holder was used, compared to the use of a lead glove and a conventional glove (703 vs 842.90 and 816.65 mRad, respectively; $\mathrm{p}=0.024)$.

Conclusions This study showed that using a needle holder during PCNL reduced radiation exposure to the surgeon's hand compared to the use of a lead glove and a conventional glove. In addition, by removing dense objects from the radiation field, the dose to the patient was significantly less when a needle holder was used. These findings demonstrate that keeping the surgeon's hand out of the beam will reduce radiation exposure, not only to the surgeon, but also to the patient, and may thereby decrease the risks of radiation exposure, including cancer.

\section{\#149 RISK FACTORS FOR URINARY TRACT INFECTIONS IN CHILDREN WITH PRENATAL HYDRONEPHROSIS}

${ }^{1}$ A Fisher ${ }^{*},{ }^{1} \mathrm{C}$ McKinney, ${ }^{2} \mathrm{C}$ Ritchie, ${ }^{2} \mathrm{~J}$ Chamberlin. 'Loma Linda University School of Medicine, Loma Linda, CA; ${ }^{2}$ Loma Linda University Medical Center, Loma Linda, CA

10.1136/jim-2022-WRMC. 147

Purpose of Study Prenatal hydronephrosis is common in pregnancy and is detected in up to $5 \%$ of pregnancies. Children with PNH are at risk of developing a UTI in childhood. The aim of this study was to determine the incidence and risk factors for urinary tract infections (UTIs) in children diagnosed with prenatal hydronephrosis.
Methods Used We performed a retrospective review of children who presented to a tertiary children's hospital with confirmed prenatal hydronephrosis between 2012-2021. Longitudinal data was collected in demographics, imaging studies, sex, grade of hydronephrosis, presence or absence of vesicoureteral reflux, and presence or absence of dilated ureter. The primary outcome was the development of a clinically diagnosed UTI treated with antibiotics. Statistical analysis was completed using a Chi-square test, where a p $<0.05$ is significant.

Summary of Results 259 children with prenatal hydronephrosis were included in the study. Of these, 51 developed UTIs. 175 of the patients underwent a voiding cystourethrogram (VCUG) to determine if vesicoureteral reflux was present. Of these, 38 patients had vesicoureteral reflux. $39.5 \%$ these patients with reflux developed UTIs. $17.3 \%$ of patients who did not have reflux $17.3 \%$ of patients developed UTIs. $(p=0.004)$. Ureteral dilation was also examined. 79 patients had ureteral dilation diagnosed on ultrasound. $29.9 \%$ of these patients developed UTIs, vs $15.7 \%$ of patients without ureteral dilation developed UTIs $(p=0.011)$.

Conclusions In children with prenatal hydronephrosis, a dilated ureter and vesicoureteral reflux increase the risk of UTI.

\section{\#150 COMPARISON OF SMARTPHONE TO WEARABLE SENSORS FOR ASSESSMENT OF GAIT IN AN ORTHOPAEDIC CLINIC}

${ }^{1} \mathrm{D}$ Ahmadian*, ${ }^{2} \mathrm{G}$ Coyle, ${ }^{1} \mathrm{M}$ Dohm. ${ }^{1}$ The University of Arizona College of Medicine Tucson, Tucson, $A Z_{i}^{2}$ The University of Arizona, Tucson, $A Z$

\subsection{6/jim-2022-WRMC.148}

Purpose of Study Recently, a host of smartphone-based applications have been developed that advertise the ability to effectively measure gait kinematics compared to traditional methods. These applications, if proven to have clinical utility, would provide a more accessible and affordable alternative for measuring gait on a long-term, continual basis. Thus, the aim of this study is to compare the utility of a smartphone-based gait analysis platform to that of an inertial motion capture (IMC) wearable sensor in a clinical setting.

Methods Used Gait data was collected from 7 patients presenting to the Orthopaedic clinic over a 1-month period. During each appointment, the patient performed a Self-Paced Walking Test (SPWT) with 5 LEGSys wearable sensors attached to their thighs, shanks, and waist. After this test, the most recent

\section{Abstract \#150 Table 1}

Smartphone vs. LEGSys Comparison of Mean Stride Length and Walking Speed

\begin{tabular}{|c|c|c|c|}
\hline Mmartphone Stride Length (m) & 1.13 & t-test & p-value \\
LEGSys Stride Length (m) & 1.17 & -0.555 & 0.6 \\
\hline Smartphone Walking Speed (m/s) & 0.906 & -1.04 & 0.3 \\
LEGSys Walking Speed (m/s) & 0.963 & & \\
\hline
\end{tabular}


values for the patient's Step Length and Walking Speed were obtained from the Health application found in their iPhone and recorded in RedCap, a secure web application for managing online databases. A paired t-test was performed to compare the means of the values of Stride Length (m) and Walking Speed $(\mathrm{m} / \mathrm{s})$ recorded from the patient's iPhone compared to that of the LEGSys sensors. This quality study was granted an IRB exemption, and patient data was de-identified before analysis.

Summary of Results Overall, the means for the iPhone and LEGSys Stride Length were 1.13 (m) and $1.17(\mathrm{~m})$, respectively, with a p-value of 0.6. The means for the iPhone and LEGSys Walking Speed were $0.906(\mathrm{~m} / \mathrm{s})$ and $0.963(\mathrm{~m} / \mathrm{s})$, respectively, with a p-value of 0.3 . (Table 1 ).

Conclusions In this study of 7 participants, it was shown that there is not a notable difference between the mean measurements of Stride Length $(\mathrm{m})$ and Walking Speed $(\mathrm{m} / \mathrm{s})$ as measured by iPhone and LEGSys wearable sensors, thus providing evidence that smartphone sensors may be useful in measuring these gait parameters. Smartphone-based gait sensors have the potential to serve as a useful clinical alternative to traditional wearable sensors in measuring gait. They are cost-effective and easily accessible in a clinical setting, saving time for the clinician during a patient encounter. Future research should focus on collecting data on more patients comparing these two technologies to increase the sample size and power of the study.

\section{Poster session}

\section{Adolescent medicine and general pediatrics}

\section{6:00 PM}

\section{Thursday, January 20, 2022}

\section{\#151 ABSTRACT WITHDRAWN}

\section{\#152 IMPLEMENTING TELEHEALTH INTO PEDIATRIC TRAUMA CONSULTATIONS}

${ }^{1} \mathrm{~S}$ Garrison*, 'JP Marcin, ${ }^{1} \mathrm{~J}$ Galante, ${ }^{2} \mathrm{JL}$ Rosenthal, ${ }^{1} \mathrm{~T}$ Rinderknechkt, ${ }^{2} \mathrm{~K}$ Grether-Jones, ${ }^{2} \mathrm{MY}$ Hamline, ${ }^{1} \mathrm{M}$ Zwienenberg, ${ }^{3} \mathrm{~B}$ Haus, ${ }^{1} \mathrm{~K}$ Matthews, ${ }^{1} \mathrm{~K}$ Rominger, ${ }^{1} \mathrm{~A}$ Sanders, ${ }^{1} \mathrm{R}$ Dizon, ${ }^{1} \mathrm{~N}$ Kuppermann. ${ }^{1} U \mathrm{C}$ Davis Health, Sacramento, CA; ${ }^{2} U C$ Davis Children's Hospital, Sacramento, CA; ${ }^{3}$ University of California Davis Health System Department of Orthopaedic Surgery, Sacramento, CA

\subsection{6/jim-2022-WRMC. 149}

Purpose of Study We are evaluating the implementation of telehealth and image sharing services to connect pediatric trauma specialists to rural and community hospital emergency departments to create a Virtual Pediatric Trauma Center (VPTC). The goal of the study is to compare the current standard of care to the VPTC model of care with regards to the parent/ family experience of care, distress, healthcare utilization, and out-of-pocket cost burden.

Methods Used We are comparing the current standard of care to the VPTC model of care with regards to: 1) the parent/ family experience of care and distress at 3, 30, 60 and 90 days following a childhood injury; 2) the 30-day healthcare utilization following the injury event; and 3 ) the out-of- pocket costs and financial burdens experienced by parents/ families 3-days and 30-days following the injury. We've implemented a stepped wedge trial among a stratified selection of 10 hospital EDs in Northern California, with a goal of enrolling 380 patients by November 2022. We've deployed pole mounted videoconferencing units with highdefinition monitors, omnidirectional microphones, and remote-controlled pan-tilt-zoom cameras. Pediatric trauma providers were provided access to workstations as well as video capable mobile devices.

Summary of Results To date, 122 pediatric trauma patients have been enrolled. Protocol adherence for videoconferencing has occurred in 18 of 43 eligible patients; adherence for image sharing has occurred in 16 of 38 eligible patients. Collection rates of surveys of parent/family experience of care, distress, and financial burden surveys has been $65 \%$. The ability of the specialty providers to connect using telemedicine has been limited by other clinical responsibilities, and protocol relies heavily on the NPs to administer telehealth communications and recommendations. Changes in implementation strategies and the workflow were made to increase the reliability and fidelity of the intervention, which will be shared in the presentation.

Conclusions Implementing telehealth into acute pediatric trauma care is challenging. Challenges include incorporating videoconferencing by busy providers and implementing project-specific platforms at partner sites. However, with this commitment, acutely injured children can receive regionalized pediatric trauma expertise at the bedside in a receiving hospital ED. Current findings illustrate the need for qualitative data to improve the family experience.

\section{Poster Session}

\section{Behavior and Development}

\section{6:00 PM}

\section{Thursday, January 20, 2022}

\section{\#153 A SURVEY OF STUDENT ATTITUDES ABOUT ONLINE LEARNING VIA THE ZOOM PLATFORM}

C Bauer*, A Craft. Western University of Health Sciences, Pomona, CA

\subsection{6/jim-2022-WRMC.150}

Purpose of Study Despite decreasing attendance at live in-person lectures, this teaching method has remained a mainstay in medical education. Prior surveys of medical students have found that M1 students attend live lectures more than M2 students, while both years utilize the lecture recordings to supplement their education. With the COVID-19 pandemic, more lecture content has moved online, with some delivered synchronously and some recorded for asynchronous review.

A study of dental students' perception found that $44 \%$ of students felt learning 'somewhat worsened' and 26\% thought learning 'significantly worsened' with the move to online platforms during COVID-1. The same study found that students preferred synchronous learning experiences despite often choosing not to attend live lectures prior to the pandemic. This study seeks to evaluate similar issues in osteopathic medical students to determine a preferred mode of 
online learning, perception of their learning, and signs of burnout.

Methods Used An anonymous survey was created and distributed via email to the students in the Classes of 2022, 2023, and 2024 at the College of Osteopathic Medicine of the Pacific. The survey consisted of 8 questions, three were yes or no responses, two were categorical responses, and three questions were based on a 10-point Likert scale. A goal of a $20 \%$ return of surveys was pre-established.

Summary of Results Of the 995 students who received the survey, $292(29.3 \%)$ completed the anonymous online survey. $54.1 \%$ of respondents were in the Class of $2024,34.2 \%$ were in the Class of 2023, and $11.6 \%$ were in the Class of 2022.

On a 10-point scale, with 1 being highly dissatisfied, 55 (18.8\%) students selected a score between 1 and 4 when evaluating satisfaction with a synchronous lecture via Zoom video conference technology compared to a live-in person lecture. $231(79.1 \%)$ students found the video conference lecture as or more helpful than an in-person lecture. 165 (56.5\%) respondents noted they were MORE likely to ask a question via the video conference lecture platform, compared to an inperson lecture.

When asked how likely they were to attend live in person lectures in the future, the responses demonstrated that students were evenly distributed, with 41 students (14.1\%) responding absolutely not and 45 students (15.4\%) responding definitely. Overall, 171 (58.6\%) students stated that synchronous video conference-based lectures could fully replace live in person lectures.

Conclusions Overall, students at this single medical school appear to have no significant preference for synchronous video conference-based lectures compared to live in-person lecture. Some students even suggested a greater willingness to participate and ask questions, whether verbally or via the chat function, during video, conference-based lectures. This preliminary data may allow for further investigation into how technology may impact the pedagogy for future generations of medical students.

\section{\#154 HOW DO MEDICAL STUDENTS RATE RESILIENCY IN A PANDEMIC?}

S Dulani*, E Salimi, AL Nelson, M Hudson. Western University of Health Sciences College of Osteopathic Medicine of the Pacific-Northwest, Lebanon, OR

10.1136/jim-2022-WRMC. 151

Purpose of Study To assess the perceptions of medical students about the importance of resiliency in general and their own resiliency in a time of pandemic and to assess what external factors contribute to resilience, especially in the midst of a pandemic.

Methods Used This IRB approved a survey for osteopathic medical students. The student survey questions were beta tested 3 times by students at other universities as well as examined by a professional in the field. All enrolled students at $\mathrm{COMP} / \mathrm{COMP}-\mathrm{NW}$ were sent an invitation to participate ( 1,300 students) ages 18 and up. The invitation letter asked them to self-exclude if they were pregnant or believed that participation in the study could cause them to feel uncomfortable. We based our questions on the academic resilience scale to see if there are any associations between self-perception of stress, relationship style, negative affect and resilience levels in students. The survey consisted of 9 Likert scale questions, 4 multiple choice questions, and 1 open ended question. Multiple questions are derived from previous resilience scales.

Summary of Results We obtained 130 responses (10\% response rate). $46 \%$ were first year, $24 \%$ second year, $14 \%$ third year and $16 \%$ fourth year students. 51\% were female. $40 \%$ said they had above average self-esteem, while $30 \%$ were neutral and $30 \%$ had below average self- esteem. 96\% admitted to having anxiety in personal or professional relationships. $52 \%$ were likely to reflect on a stressful day, while $54 \%$ were likely to reflect on a successful day. This may show that students can be optimistic amid ongoing stress. $85 \%$ of students felt confident in their ability to persevere through challenging times. $65 \%$ of students were willing to ask for help in times of need while $16 \%$ were not willing to, and $19 \%$ were unsure. Students are most likely to turn to their spouse, friends, and themselves for support. $75 \%$ of respondents who had an opinion said that medical school had been the hardest time of their life. Only $40 \%$ of students were satisfied with their academic performance, and only $42 \%$ were satisfied with their general wellbeing. Financial hardship contributes to stress levels in $40 \%$ of students. The financial hardship does not seem to correlate with those that are unhappy in their academic performance and general well-being.

Conclusions The students surveyed are optimistic; more of them were likely to reflect on a successful day compared to a stressful one. The high rate of anxiety in personal as well as professional relationships may impede students' ability to seek personal or professional support when they need it. Students who do not have access to friends or family during times of hardship may have a harder time coping with stress.

\section{\#155 IMPACT OF COVID-19 ON ROCKY VISTA UNIVERSITY MEDICAL STUDENTS' MENTAL HEALTH: A CROSS- SECTIONAL SURVEY}

D Paz*, V Kuo, M Bains, V Bandi, M Zueger, M Payton, R Ryznar. Rocky Vista University, Parker, CO

\subsection{6/jim-2022-WRMC.152}

Purpose of Study The high prevalence of anxiety and depression among medical students has been previously linked to the rigor of medical school curriculums. Since late 2019, the COVID-19 pandemic has forced schools to make unprecedented yet unavoidable changes to their educational curriculums. This study is a quantitative analysis of the educational and psychological impacts of COVID-19 on first and secondyear osteopathic medical students at Rocky Vista University.

Methods Used A cross-sectional survey was administered to students at Rocky Vista University during the COVID-19 pandemic. Anxiety and depression levels were measured using validated questionnaires GAD-7 and PHQ-9. The remainder of the survey was a self-designed questionnaire that investigated potential associated factors, including sociodemographic characteristics, educational changes, and COVID-19 related concerns. The questionnaire also gauged the impact of COVID-19 on behavioral changes and strategies used to cope with the pandemic.

Summary of Results One hundred fifty-two Rocky Vista University OMS-I and OMS-II students across both campuses participated. Overall, depression and anxiety levels due to the COVID-19 pandemic were increased from previously reported 
prevalence rates. Results showed that while the transition to an online based-curriculum was met with mixed feelings, most students found ways to effectively adapt their study habits amidst the pandemic. Notably, students reported feeling concerned that the transition in the curriculum will negatively impact their preparedness for clinical rotations. Overall, COVID-19 was found to have a negative impact on students' mental health according to nearly all measures. However, the majority of students also reported finding new ways to cope with their stress and anxiety levels and nearly all students reported a willingness to assist with the pandemic.

Conclusions This study demonstrates the significant detrimental effects of the pandemic on medical student mental health, which could impact the quality of care these future physicians provide. Thus, it is imperative to establish effective interventions to mitigate the negative repercussions of the pandemic.

* This work was presented in part at Rocky Vista University Appreciation Day (virtual) on October 15, 2021.

\section{\#156 SURVEYS OF THE PERCEPTIONS OF FACULTY ABOUT STRESS AND RESILIENCE}

E Salimi*, S Dulani, AL Nelson, M Hudson. Western University of Health Sciences College of Osteopathic Medicine of the Pacific, Pomona, CA

\subsection{6/jim-2022-WRMC.153}

Purpose of Study To find out what the faculty's perceptions are about resilience and its relationship to academic success in osteopathic medical school. Can resilience be acquired or is it an innate trait? If it can be acquired, then can it be formally taught? Who is responsible for this formal training and when could it be provided? Should resilience be a part of the DO curriculum?

Methods Used This is an IRB-approved survey for all COMP faculty who interact within students in the pre-clinical years. The questionnaire was beta tested by 10 outside physicians and researchers. It consisted of 13 multiple choice questions and 2 open ended questions. 4 of the 13 questions used Likert scales. Letters of invitation that outlined the study purpose and its voluntary and anonymous nature were sent with the link to the survey, conducted via Qualtrics. Faculty were asked not to participate if they were pregnant or if they anticipated that the topic might cause them discomfort. We also sent out a reminder $\sim 3$ weeks after the initial letter that thanked those who had already responded and ask them not to do so again. At the same time, select faculty were approached to see if they would be willing to encourage their colleagues to participate in the study. We calculated the response rate and percentage distribution of the answers.

Summary of Results The response rate is $21 \%$. 55\% of the respondents were clinical faculty, $30 \%$ basic science faculty, $5 \%$ both. $60 \%$ thought that resilience is more learned than intrinsic. 34\% believed that resilience can be learned through life experiences and another $34 \%$ believed that it can be learned through informal teaching in context of individual challenge. $35 \%$ believed that there should be a more formal screening for resilience in applicants during the admissions process while $30 \%$ disagreed. $100 \%$ believed that resilience is either very important or extremely important to a medical student's success. $75 \%$ believed that financial burdens are either very important or extremely important to students' stress levels to their growth as a physician. 90\% believed that resilience improves student's academic performance by a lot or a great deal. All respondents reported that they work with students in some way to increase their resilience. $65 \%$ believe that formal resilience training should be a part of the DO curriculum for students and $85 \%$ believe that faculty would benefit from formal training about how to teach resilience.

Conclusions Although osteopathic medicine places importance on holistic approaches to health, there are no requirements in the COCA curriculum to explicitly provide resilience training. The majority of the faculty thought that resilience is more learned than intrinsic. Therefore, it could be worthwhile to explore the possibilities of providing resilience training for both students and faculty.

\section{Poster session}

\section{Cardiology}

\section{6:00 PM}

\section{Thursday, January 20, 2022}

\section{$\# 157$ NOVEL CHOLESTEROL LOWERING AGENTS IN SETTING OF STATIN INTOLERANCE}

${ }^{1} \mathrm{~S}$ Noh*, ${ }^{1} \mathrm{~K}$ Mai, ${ }^{1} \mathrm{M}$ Shaver, ${ }^{1} \mathrm{~S}$ Yong, ${ }^{1} \mathrm{M}$ Mostaghimi, ${ }^{2} \mathrm{G}$ Oh, ${ }^{1} \mathrm{MM}$ Radwan. ${ }^{1}$ Western University of Health Sciences, Pomona, CA; ${ }^{2}$ University of the Pacific Thomas J Long School of Pharmacy, Stockton, CA

\subsection{6/jim-2022-WRMC.154}

Purpose of Study Statins are the gold standard lipid-lowering therapy for atherosclerotic diseases. However, there is increasing evidence in support of combination therapies providing greater benefits to certain patient populations, particularly those intolerant to statins. Here, we discuss the efficacy, safety, and practical use of novel alternative and adjuvant agents that target low-density lipoprotein cholesterol (LDL-C) or lipoprotein(a) (Lp(a)).

Methods Used A literature review was conducted on PubMed and Google Scholar based on research and clinical trials of cholesterol modulators primarily within the past decade.

Summary of Results PCSK9 antibodies have been effective as adjuvant therapies in further reducing LDL-C levels and coronary atherosclerosis progression when added to statins. Importantly, the FOURIER and ODYSSEY OUTCOMES trials demonstrated that the addition of evolocumab and alirocumab remarkably reduced the risk of major cardiovascular events, respectively. Yet, expensive cost and administrative burden are impediments to practical use. PCSK9 siRNA inclisiran is an appealing alternative because of the marked reduction of LDL$\mathrm{C}$ without significant adverse events as well as its lower cost and administrative burden. Bempedoic Acid (BemA) is another promising LDL-C lowering agent highlighted for its efficacy as both monotherapy and add-on to statins. BemA exhibits a favorable tolerability profile due to its exclusive activation in the liver, suggesting its potential as an alternative to statinrelated myopathies. Future studies on its impact on cardiovascular outcomes may provide a greater appreciation of where BemA stands among LDL-C therapies. Antisense oligonucleotide therapies IONIS-APO(a)rx, IONIS-APO(a)Lrx, and AKCEA-APO(a)-Lrx have emerged as encouraging alternatives due to their efficacy and safety as $\mathrm{Lpa}(\mathrm{a})$-lowering agents. 
Continued investigation as phase 3 trials may establish the potential of $\mathrm{Lp}(\mathrm{a})$ therapies as treatments for atherosclerotic disease.

Conclusions Further investigation for all these novel approaches is needed to better appreciate the long-term efficacy, safety, and effects on cardiovascular outcomes. Overall, these cholesterol targeting therapies provide a promising outlook as alternatives for atherosclerotic patients for whom statins have been ineffective or intolerable.

\section{Poster session}

\section{Diversity, equity, inclusion research}

\section{6:00 PM}

\section{Thursday, January 20, 2022}

\section{\#158 IMPACT OF IMPLEMENTATION OF SMART GOALS FOR RADIOLOGY TRAINEE RESEARCH AND MENTORSHIP}

${ }^{1} \mathrm{~T}$ Bui*, ${ }^{1} \mathrm{C}$ Fateri, 'D Kwan, ${ }^{1} \mathrm{~J}$ Glavis-Bloom, ${ }^{2} \mathrm{~A}$ Ushinsky, ${ }^{1} \mathrm{M}$ Helmy, ${ }^{1} \mathrm{D}$ Floriolli, ${ }^{1} \mathrm{R}$ Houshyar. ${ }^{1}$ University of California Irvine, Orange, $C A ;{ }^{2}$ Washington University in St Louis School of Medicine Mallinckrodt Institute of Radiology, Saint Louis, MO

\subsection{6/jim-2022-WRMC. 155}

Purpose of Study Active research programs are particularly crucial to the field of radiology because the development of imaging technologies and subsequent translation of them into clinical practice drives the field forward. The SMART (Specific, Measurable, Attainable, Realistic, Time-bound) Goals framework is a validated goal-setting tool that has been successfully applied to medical education. In this prospective cohort study, we evaluated the implementation of the SMART Goals framework in radiology research. We hypothesized that engaging research trainees in formalized goal-setting would result in higher engagement in research and greater productivity.

Methods Used After Institutional Review Board approval, medical students and radiology residents from a radiology research $\mathrm{lab}$ at a major academic center between the academic year of August 1, 2020 to July 31, 2021 were invited to participate. Inclusion criteria were those who filled out a SMART Goals form and participated in a formal review meeting. The comparison group was comprised of lab members from previous academic years (August 1, 2016 to July 31, 2020). Productivity was assessed by the number of publications, abstracts, and grants. For purposes of streamlining the data, the acceptance year was defined as the same as the submission year to best match the timeframe during which most of the work was completed. Additionally, participants' beliefs about their knowledge and abilities were assessed using five-point Likert scale pre- and post-surveys. Descriptive statistics and two-tailed ttests were calculated. Statistical significance was considered to be $p<0.05$.

Summary of Results There were 19 individuals in the postSMART group and 28 individuals in the pre-SMART group. The mean number of publications was 0.79 and 1.54 per individual in the pre-SMART and post-SMART groups, respectively $(p=0.02)$. The mean number of abstracts was 1.04 and 2.37 per individual in the pre-SMART and post-SMART groups, respectively $(p=0.02)$. The mean number of successful grants was 0.05 and 0.347 per individual in the pre-SMART and post-SMART groups, respectively $(p=0.01)$. Based on survey results, participants' knowledge and comfort with research goals increased 1.54 points $(p<0.01)$; with manuscript writing increased $1.21(p<0.01)$; and with abstract submission increased 0.60 points $(p=0.01)$.

Conclusions Dedicated learning goal creation through the SMART Goals framework has the potential to significantly increase the number of radiology trainees producing high-quality research, improve medical student and resident knowledge in research, and increase trainees' confidence in their research skills.

\section{\#159 COVID-19 IN RAVALLI COUNTY, MONTANA: CAPTURING THE VOICES OF HEALTHCARE LEADERS}

N Johnson*. University of Washington School of Medicine, Seattle, WA

\subsection{6/jim-2022-WRMC.156}

Purpose of Study COVID-19 has strained rural Montana healthcare resources. Ravalli County, MT, is a rural county, and has dealt with the consequences of COVID-19 in its small towns. The National Humanities Center (NHC) in partnership with the University of Washington School of Medicine gave medical students the opportunity to perform qualitative research by gathering oral histories at rural sites. The interviewer for this project worked to record and preserve the stories of healthcare leaders in Ravalli County. The interviewer's goal was to remain unbiased, listen openly to all stories, limit interruptions, and learn about the effect COVID-19 had on the county.

Methods Used Interviewees for the project were recruited through email, phone call, and text message. Connections were made with the help of Ravalli Family Medicine. Four total interviews were done with folks in healthcare positions including: two Directors, an Administrator, and a Technical Supervisor. All were conducted in person at private locations, each being audio recorded and lasting about an hour. The same set of questions was used with all interviewees. Interview skills training was led by Jacqueline Kellish, Ph.D., Director of the COVID-19 Oral History Initiative at the NHC. Training emphasized skills in active listening and communication, focusing on building trust with interviewees.

Summary of Results Information gathered on the community response to COVID-19, how folks worked to survive during the pandemic, and how vaccination roll-out happened in the county created a story of the pandemic in rural Montana. These oral histories and their take-aways will be used to educate others about rural experiences during the pandemic and contribute to the larger NHC collective database. In the county, these interviews sparked discussion about vaccines and education.

Conclusions Ravalli County was hit hard by COVID-19 and serves as a window into the rural experience of the pandemic. Leaders emphasized the importance of advocacy, education, and preparedness and have brought these lessons to their communities. Strategies for vaccine education and confidence would benefit rural communities and reduce a strain on resources. This individual project is limited by its small sample set of four interviews. Sharing these stories and their lessons will contribute to a meaningful positive discourse on living through a pandemic. 
\#160 JOURNAL CLUB IMPLEMENTATION AS AN EDUCATIONAL TOOL FOR MEDICAL TRAINEE RESEARCH AND MENTORSHIP IN RADIOLOGY, A PILOT STUDY

D Kwan*, T Bui, C Fateri, J Glavis-Bloom, R Houshyar. University of California Invine, Orange, $C A$

\subsection{6/jim-2022-WRMC.157}

Purpose of Study Interpreting medical literature is an essential skill set for physicians to acquire as physicians must be able to determine the validity of research that may influence their practice. A baseline competency in the basic components of research (i.e., identifying bias, data interpretation, etc.) is imperative for effective clinical practice and clinical research. The purpose of this study is to assess the impact of a journal club on trainee's confidence and knowledge base in fundamental components of research literature.

Methods Used After Institutional Review Board approval, members of the Computational Abdominal Radiology research lab, predominantly medical students and radiology residents, at a major academic center were invited to participate in this study. Journal club sessions were conducted from April to September 2021. Articles from leading peer-reviewed radiology journals were selected to highlight a specific component of research. Pre and post journal club session surveys were distributed among lab members via Microsoft Forms using Likert scales to assess trainee confidence and multiple-choice questions to assess knowledge base. Survey results were compared using a paired two-tail t-test with statistical significance set as $p<0.05$.

Summary of Results On average, participants displayed an increase in confidence in topic knowledge (pre-test mean = $4.29 \pm 0.31$ versus post-test mean $=5.91 \pm 0.33, p=$ 0.004). However, the difference in percent correct answers between the pre-test (mean $=0.77 \pm 0.25$ ) and post-test (mean $=0.85 \pm 0.13$ ) indicated that there was not a significant increase in correct answers after the journal club sessions $(p=0.42)$.

Conclusions The results of this pilot study indicate that a journal club can be effective in increasing participants' confidence in the basic fundamentals of research, although its limited application has not yet been shown to increase aptitude. Additional journal club sessions, repeated exposure to key topics, and longer follow up beyond this pilot study are needed to better assess the efficacy of the impact of journal clubs in the setting of research and clinical mentorship in radiology.

\section{\begin{tabular}{|l|l}
$\# 161$ & INDOOR WALKING TRACK AS OPPORTUNITY FOR
\end{tabular} OLDER ADULTS TO EXERCISE DURING WINTER IN VALDEZ, ALASKA}

M Weyhrauch*. University of Washington School of Medicine, Seattle, WA

\subsection{6/jim-2022-WRMC.158}

Purpose of Study The two most recent community health need assessments performed in Valdez, Alaska identified 'lack of physical activity/overweight' as a top health need. $66 \%$ of Valdez adult residents are reportedly overweight or obese and about $10 \%$ report they do not engage in any form of physical activity. Rural residents are generally less physically active than their urban counterparts and disproportionately affected by chronic diseases associated with insufficient activity. Getting the amount of recommended exercise in rural areas is a challenge where lack of infrastructure, weather dependency, and safety are common barriers.

Methods Used Information was gathered in the context of a clinical immersion in Valdez, Alaska. Patient and provider interviews were conducted at the Valdez Medical Clinic to discover community health concerns and local organizations that are working to counter concerns. An interview at the Department of Parks, Recreation, and Cultural Services (DPRCS) for the City of Valdez detailed available programs, resources, and future proposals. A literature review was then conducted to examine interventions that could build on Valdez's existing assets.

Summary of Results A literature review identifies that increasing steps per day is an easy way to start and maintain a healthy lifestyle, however this is a challenge during the extended winter months. Step count drops precipitously as snow level increases, daylight decreases, and temperatures drop. Fear of falling is a major environmental barrier for older adults in rural areas. DPRCS has an ongoing feasibility study for a new recreation facility that could include an indoor track if community members show interest. This research highlighting the potential benefits of an indoor walking track has been sent to a manager at the DPRCS. This could potentially be used to advance the current feasibility study.

Conclusions An indoor track, as part of a new recreation facility, could provide a safe, affordable, and accessible place for older adults to exercise year-round. Annual property taxes paid to Valdez by the Trans-Alaska Pipeline could provide an avenue to pay for the infrastructure. This indoor track could combat the tough environmental conditions that make exercise particularly challenging during the Valdez winter.

\section{Poster session}

\section{Genetics}

\section{6:00 PM}

\section{Thursday, January 20, 2022}

\section{\#162 VARIABLE EXPRESSIVITY IN NTRK1-CONGENITAL INSENSITIVITY TO PAIN WITH ANHIDROSIS (CIPA)}

AD Niehaus*, CG Tise, M Manning, D Stevenson. Stanford University, Stanford, CA

\subsection{6/jim-2022-WRMC.159}

Purpose of Study Congenital insensitivity to pain with anhidrosis (CIPA) is a rare disorder caused by biallelic loss-offunction (LOF) pathogenic variants in NTRK1. NTRK1 encodes TrkA, a receptor tyrosine kinase for nerve growth factor essential for survival of autonomic sympathetic postganglionic neurons. The disorder is characterized by decreased pain perception and inability to perspire effectively, as well as varying degrees of intellectual disability for which the underlying mechanism is not well understood. Insensitivity to pain often leads to repeated injuries, oral self-mutilation, skin infections, and fractures; anhidrosis predisposes individuals to hyperthermia and seizures. Here we describe two individuals with CIPA secondary to different homozygous LOF pathogenetic variants in NTRK1 and provide further evidence of the extreme phenotypic variability of the disorder. 
Methods Used Chart review, physical examination, and literature review.

Summary of Results The first individual was a male evaluated at 20-months of age with severe global developmental delay, diffuse hypotonia, and extreme self-mutilation habits including tongue-biting requiring tooth extraction and finger-biting requiring restraints/bandages. He was found to be homozygous for a novel intragenic deletion including exons 4 and 5. The second individual was a typically-developing girl evaluated at 5 years of age with a history of irritability as an infant secondary to anhidrosis, successfully recognized and since treated by her parents with cold, wet towels. She had no history of autonomic or temperature crises, severe self-mutilation, fractures, nor prior hospitalizations, but was found to be homozygous for the founder variant NTRK1 (c.851-33T>A) after parents were identified as carriers by preconception carrier testing prior to their second child.

Conclusions While the individuals described here were found to have different variants in NTRK1, the profound differences in their outcomes highlights the phenotypic variability of this disorder. These cases support a previously published hypothesis that individuals homozygous for the founder variant (c.851-33C $>\mathrm{T}$ ) may present with a milder phenotype, despite this variant having a deleterious effect on protein structure (Wang et al., 2018). Lack of a clear genotype-phenotype correlation in the literature indicates genetic or environmental modifiers may be at play. Lastly, the value of early diagnosis should be further explored, especially if there is evidence suggesting earlier intervention improves outcomes.

\section{\#163 NOVEL HETEROZYGOUS P3H2 VARIANTS IN A CHILD WITH EARLY-ONSET NON-SYNDROMIC DEGENERATIVE HIGH MYOPIA}

${ }^{1}$ VD Tang*, 'A Egense, ' $\mathrm{M}$ Estrada, 1,2SP Shankar. 'University of California Davis, Sacramento, CA; ${ }^{2}$ University of California Davis, Davis, CA

\subsection{6/jim-2022-WRMC. 160}

Purpose of Study To report on $\mathrm{P} 3 \mathrm{H} 2$ variants causing non-syndromic high degenerative myopia guiding precision medical care.

Methods Used Chart review, Next-generation sequencing (NGS) panel, and exome trio genetic testing.

Summary of Results A 3-year-old male with bilateral degenerative high myopia (-12.75 diopter spherical (DS)) and refractive amblyopia diagnosed at 2 years of age, was referred to genomic medicine. He was born full-term with an unremarkable prenatal and birth history. His developmental milestones were normal other than mild articulation difficulty. Family history was significant for myopia of -3.00 DS. Physical exam was normal other than midfacial hypoplasia and depressed nasal bridge. Testing in the connective tissue panel, including Stickler syndrome genes and FBN1 for Marfan's syndrome, was negative. Several rare non-syndromic myopia genes have been reported; however, given the lack of NGS panel, an exome trio was ordered. This identified two pathogenic variants in the $\mathrm{P} 3 \mathrm{H} 2$ gene, c.1372 C>T, p.Q458* and c.1328del, p.G443Vfs* in trans, confirmed by parental testing. P3H2, also called LEPREL1, encodes the Prolyl 3-hydroxylase2 enzyme, which performs the 3-hydroxylation of proline residues in collagens. Tissues from the eye, including the sclera, cornea (type 1), and lens capsule (type 4), are composed of collagen. The abnormal collagen molecule processing may cause weakened collagen in eye tissues. Mutations in the $\mathrm{P} 3 \mathrm{H} 2$ gene have been associated with non-syndromic earlyonset severe myopia with cataracts, lens instability, vitreoretinal degeneration, and risk of retinal detachment mostly in consanguineous families as homozygous variants. There are no reported skeletal issues or syndromic features. The variants in our proband have not been published in the past, but they have been reported once in ClinVar with different variants considered pathogenic.

Conclusions The degenerative high myopia in our proband is due to pathogenic heterozygous variants in the $\mathrm{P} 3 \mathrm{H} 2$ gene, consistent with non-syndromic severe myopia and refractive amblyopia. This molecular diagnosis guides management of potential ocular risks and prevents unnecessary systemic evaluation, such as echocardiograms. It will influence the patient's future lifestyle choices, such as avoiding contact sports and choosing suitable careers. It also eased parental anxiety and offered more accurate inheritance and recurrence risk counseling for parents and extended family.

\section{\#164 IMPROVED ACCESS TO CLINICAL GENETICS SERVICES VIA GENETIC COUNSELOR LED HEARING LOSS CLINIC}

M Dutra-Clarke*, S Glover, B Russell. University of California Los Angeles, Los Angeles, CA

\subsection{6/jim-2022-WRMC.161}

Purpose of Study There are over 200 genes associated with non-syndromic and syndromic hearing loss (HL). Knowing the underlying genetic etiology helps to determine prognosis including whether it is expected to be stable vs progressive, isolated, or associated with other organ systems requiring surveillance (e.g. eyes, heart, kidneys), and when planning for cochlear implant. It guides medical management such as early access to developmental services, inform recurrence risks for the family, eligibility for future gene therapy, and avoidance of invasive imaging (e.g. CT scan) and aminoglycosides. Early genetic diagnosis is critical. Due to long wait times to see a clinical geneticist, we developed a genetic counseling (GC) clinic where patients with non-syndromic HL receive genetic counseling and testing by a certified genetic counselor. The purpose of the study was to compare wait times for a consult with a GC vs clinical geneticist and determine the diagnostic yield on genetic testing for apparently non-syndromic HL. The intent was to track productivity, measure the effort allocated for this clinic, and plan adjustments and future needs.

Methods Used We conducted a retrospective chart review of patients with apparently non-syndromic HL seen in the Genetics Division at UCLA between Jan 2020-Sept 2021 (ages 2 mos-58 yrs). We separated patients into two groups: (1) seen by a clinical geneticist with a genetic counselor $(M D+G C)$ or (2) seen only by a genetic counselor (GC-only). We compared time from referral to initial visit to return of results. Panelbased genetic testing of 150-224 genes was done in a clinical setting.

Summary of Results There were 25 patients seen by MD+GC and 17 patients seen by GC-only. The average time from referral to initial visit was 80 days for $\mathrm{MD}+\mathrm{GC}(\mathrm{n}=22)$ and 16 days for GC-only $(n=17)$. The average time for testing was 48 days in the MD+GC group $(n=25)$ and 79 days in the GC-only group $(n=6)$, which varied between labs. The average time from referral to return of results was 207 days 
for MD+GC $(n=21)$ and 126 days for GC-only $(n=6)$. The diagnostic yield on genetic testing was 53\%, and up to $69 \%$ when filtered for prelingual bilateral sensorineural hearing loss. In total, 17 were positive, 5 had variants of uncertain significance, and 10 were negative $(n=32)$. Four patients with postlingual onset HL had pathogenic variants in GJB2, MYO7A, SLC26A4, CEACAM16. Two patients with unilateral prelingual onset HL had pathogenic variants in MITF and MTTK.

Conclusions A genetic counseling (GC) clinic for non-syndromic HL was effective as measured by improved turnaround time for results, patient satisfaction, feedback from referring providers, and reimbursement by insurance. GC clinics allow genetic counselors to practice at the top of their scope and improves patient care.

\section{\#165 UNCOMMON NEUROIMAGING FINDINGS IN INBORN ERRORS OF METABOLISM}

${ }^{1} \mathrm{JA}$ Morales*, ${ }^{2} \mathrm{FP}$ Velez-Bartolomei, ${ }^{1} \mathrm{M}$ Ruzhnikov, ${ }^{1} \mathrm{GM}$ Enns. 'Stanford University, Stanford, $C A_{;}{ }^{2}$ San Jorge Children and Women's Hospital, San Juan

\subsection{6/jim-2022-WRMC. 162}

Purpose of Study Inborn errors of metabolism (IEM) are often associated with neurodevelopmental/neurological features such as intellectual disability, autism, epilepsy, hearing loss, hypotonia and movement disorders.

Underlying structural brain abnormalities, like disorders of neuronal migration and/or primarily symmetric signal abnormalities of basal ganglia or white matter, are also common features for certain IEMs.

In this study, we describe two individuals with distinct IEMs: Glutaric acidemia type 1 (GA1) and Congenital disorder of glycosylation type IIs, (CDGIIs), who presented with intracranial abnormalities not previously or rarely described.

Methods Used Literature \& retrospective chart review, clinical evaluations.

Summary of Results 1. An 18-year-old male previously diagnosed with GA1, presented with a first-time generalized tonicclonic seizure. Comorbidities include spastic quadriparetic cerebral palsy and lack of verbal speech, likely secondary to past metabolic crises.

Brain MRI showed diffuse cerebral white matter, basal ganglia and brainstem abnormal diffusion. Multiple subependymal nodules along the ventricular margins were also detected.

A comprehensive brain malformations gene panel was not clinically relevant.

2. A 21-year-old male with an underlying diagnosis of CDGIIs, presented with an episode of left face and arm numbness, and left facial droop at age 15 years. His comorbidities include complex partial epilepsy, hypogammaglobulinemia, beta-thalassemia intermedia, tethered cord, kyphosis, cirrhosis, and portal hypertension. Brain MRI demonstrated severe narrowing of the supraclinoid internal carotid arteries concerning for Moyamoya disease. This was confirmed through cerebral angiogram, and corrected by bilateral cerebral artery bypass grafts.

Conclusions These specific brain imaging findings have rarely been reported in IEMs. Subependymal nodules have been found in four other patients affected by GA1, three of whom were adults. Moyamoya disease is thought to arise from either tunica intima proliferation or abnormal angiogenesis and can be associated with genetic disorders such as Down syndrome or RNF213. While the number of known patients with CDGIIs is small, none have been identified with Moyamoya disease and we are unaware of other CDG subtypes associated with this.

Given the low incidence of these rare conditions, it is not possible to determine a pathophysiologic link or direct correlation. However, in the absence of a secondary condition that could explain these findings, it is worth considering them as part of the broad phenotypic spectrum for these particular IEMs. Neuroimaging should be part of the diagnostic work-up upon presence of suggestive neurologic symptoms.

\section{\#166 PEDIATRICS RESIDENTS AND FUNDAMENTALS OF GENETIC TESTING: SELF-PERCEIVED KNOWLEDGE AND CONFIDENCE}

R Gates*, L Hudgins, LC Huffman. Stanford University, Stanford, CA

\subsection{6/jim-2022-WRMC.163}

Purpose of Study Genetic testing is becoming ubiquitous in the field of medicine and is often ordered or requested by primary care providers, non-genetics subspecialists, and patients themselves. Previous studies have demonstrated that primary care providers are often not comfortable ordering genetic testing and counseling families about genetic testing results. There have been initiatives to teach these concepts to practicing physicians via continuing medical education, however, there is no standardized training to teach resident physicians about genetic testing.

Methods Used This was an IRB-exempt study. In SeptemberOctober 2020, we recruited all active Stanford pediatrics residents via email $(n=102)$. Participant residents completed an investigator-developed Qualtrics electronic survey that addressed self-perceived current level of knowledge about fundamentals of genetic testing, as well as confidence discussing these fundamental issues with families. The survey included 19 items, with Likert scale and open-text response formats. Likert scale responses (Not at All/Slightly and Moderately/Very/Extremely) were combined into two response categories (Insufficiently and Adequately). Chi-Square test of independence was performed using IBM SPSS Statistics software.

Summary of Results Response rate was 46/102 (45\%); all training levels were represented (PGY1 33\%, PGY2 24\%, PGY3+ $42 \%)$. Proportions of respondents who were 'Adequately' knowledgeable about fundamentals of genetic testing ranged from $20 \%$ to $72 \%$. Smaller proportions of respondents were 'Adequately' confident discussing these concepts with families, ranging from $7 \%$ to $65 \%$. Self-reported knowledge and confidence were highest across items for the PGY1 group, with statistically significant differences in proportions of PGY1, PGY2, and PGY3 + describing themselves as 'Adequately' knowledgeable of basic concepts (93\%, 73\%, and 53\%, respectively; $\mathrm{p}=0.034)$ and in proportions describing themselves as 'Adequately' confident during discussions of genetic testing limitations $(64 \%, 9 \%$, and 27\%, respectively; $\mathrm{p}=0.045)$. The majority $(89 \%)$ of pediatrics residents agreed that a curriculum teaching basics of genetic testing would be helpful to them. Desired curricular topics included: 
indications/limitations of genetic testing, procedure for testing, and counseling families.

Conclusions Despite its increasing importance in medicine, medical genetics education is lacking in pediatrics residency programs. This single-institution educational needs assessment suggests that more advanced pediatrics residents have a greater awareness of deficits in genetics-related knowledge and confidence. Residents agree that they would benefit from a curriculum that teaches fundamentals of genetic testing during training. Future studies should evaluate these themes across multiple institutions and implement a curriculum that assesses pre- and post- intervention knowledge and confidence.

\section{\#167 NEONATAL COVID-19 ENCEPHALITIS WITH AN INCIDENTAL PTEN PATHOGENIC VARIANT}

A Sosa*1, J Woods ${ }^{1,2}$. 'Valley Children's Healthcare, Madera, CA; ${ }^{2}$ Stanford Medicine, Stanford, $C A$

\subsection{6/jim-2022-WRMC. 164}

Purpose of Study Neonatal COVID-19 encephalitis is a disease that has been rarely reported during the COVID-19 pandemic. Given the rarity of the disease the signs associated with neonatal COVID-19 encephalitis may easily be confused with other encephalopathies. Here we describe a case of neonatal COVID-19 encephalitis that prompted genome sequencing due to findings on a brain MRI. Genome sequencing discovered a pathogenic variant in PTEN, which is likely unrelated to the COVID-19 encephalitis but has serious lifelong health implications.

Methods Used Chart review.

Summary of Results The patient was a 3 week-old female who was admitted to the PICU for seizures and upper respiratory tract infection signs in the setting of COVID-19 positive contacts at home. An MRI of the head demonstrated patchy white matter degeneration interpreted by radiology as consistent with viral encephalitis or a metabolic disorder. The genetics service was then consulted and determined that the patient likely had COVID-19 encephalitis. However, out of an abundance of caution rapid trio genome sequencing was sent. The genome demonstrated a de novo pathogenic missense variant in PTEN (chr10-89720679 C>T). This variant is well known to be pathogenic of PTEN hamartoma tumor syndrome (PHTS). This variant was determined to be unlikely relevant to her inpatient care and the baby discharged home on Keppra five days later.

Conclusions Rapid genetic testing is a powerful tool for clinical decision support, though incidental findings may complicate genetic counseling and clinical care. There is no evidence supporting pathogenic variants in PTEN increasing the susceptibility to COVID-19 encephalitis. Neonatal onset PHTS would also be unusual and macrocephaly would be expected and the patient was actually microcephalic. The parents opted not to learn about incidental findings, which further complicated the disclosure of results as the PTEN variant was reported by the lab despite the fact that it was an incidental finding unrelated to the patient's current illness. However, the parents were thankful to learn about the PTEN variant given the risks for neurodevelopmental delay and neoplasms associated with PHTS. PHTS is associated with a variety of neoplasms, most of which are adult onset. However, follicular or papillary thyroid cancer can occur as young as seven years of age in PHTS. Thus the patient will follow up with genetics for evaluation of neurodevelopment and thyroid cancer screening.

\section{Poster session}

\section{Healthcare delivery research}

\section{6:00 PM}

\section{Thursday, January 20, 2022}

\section{$\# 168$ TRUST IN OREGON HEALTH AUTHORITY POLICIES DURING COVID-19}

H Graham*, M Gentry, M Jette, E Kim, J Spaan. Western University of Health Sciences College of Osteopathic Medicine of the Pacific-Northwest, Lebanon, OR

\subsection{6/jim-2022-WRMC.165}

Purpose of Study The purpose of this project was to discern trust in the healthcare system during the COVID-19 pandemic in Oregon communities. Our hypothesis was that if a person trusted the health care system, then they would agree with the statements and follow the COVID-19 policies put forth by the Oregon Health Authority (OHA).

Methods Used We measured trust by analyzing when behavior matched opinion in accordance with the research by Lazarus et al, 2019 that said higher trust in a healthcare system can be demonstrated by higher compliance with policies. To assess this, we conducted a survey that asked people about their behavior during the timeframe of April 2020 through November 2020 with a yes or no question. We then asked them to answer if a specific OHA policy would help prevent the spread of COVID-19 on a Likert scale. Our survey was distributed via email and over social media from the timeframe of April 4th, 2021, through April 7th, 2021. We closed our survey on April 7th, 2021, after we realized we had aberrant data most likely due to a computer-generated survey hack that began to occur on April 8th, 2021. After closing the survey, we were left with a total sample size of 124 responses from 12 different Oregon counties aged 18 years and older.

Summary of Results In order to analyze the data, we gave a numerical value to each question response so that the higher values would be awarded to the responses that aligned with the OHA policies. In the policy agreement section, the scores were: strongly disagree $=1$, disagree $=2$, agree $=3$, strongly agree $=4$. If they answered with a 'neutral' regarding their feeling of OHA policies protecting against COVID, they were assigned a score of 0 . The lowest scores correlated with people that never followed OHA recommended behavior and who strongly disagreed that the OHA policies would prevent infections. The higest possible score was 38 which represented the most trust in OHA. When analyzing the results, we found our respondents demonstrated a spectrum of trust in OHA with the total lowest score being 13 and our highest score 
being 38 with an average score of 31.4. This average score correlates with people having COVID conscious behaviors and agreeing or strongly agreeing with most of the OHA statements.

Conclusions In conclusion, these data points align with our hypothesis because they illustrate an agreement with OHA policies and a behavior that acts in accordance with that policy. Further research should include a larger sample size to better represent the population of Oregon, as well as measures to prevent survey hacking. Results could also be collected at multiple time points as the status of the pandemic is constantly in flux. When applied on a larger scale, this data could help public health officials gauge the trust of the populations they serve and lead to better health outcomes.

\section{\#169 DOES INSURANCE STATUS INFLUENCE MATERNAL MORTALITY: A SCOPING REVIEW}

1J Hannan*, 1,2A Bertotti Metoyer. 'University of Washington, Seattle, WA; ${ }^{2}$ Gonzaga University, Spokane, WA

\subsection{6/jim-2022-WRMC. 166}

Purpose of Study Maternal mortality in the US is the highest of all developed countries. Change in health insurance policy, such as extension of postpartum Medicaid, is frequently proposed as a possible solution to reduce maternal deaths. The goal of this review is to identify and summarize literature describing association of health insurance status on maternal mortality.

Methods Used We performed a scoping search of the databases PubMed, Web of Science Core Collection, Embase, and CINAHL using keywords related to maternal mortality and health insurance. Inclusion criteria required studies to be within the US and use empirical data from 1990 to present. Studies were excluded if they did not compare more than one insurance type or did not examine mortality separately from morbidity. Any numerical data related to mortality was extracted. Search results and articles were screened by two researchers.

Summary of Results Our database search yielded 894 results, of which 38 proceeded to full text screening and 17 were included in this review. Our study found that literature directly examining the association of insurance on maternal mortality is not very prevalent. Among the literature that is available, study design is varied. Units of analysis are either state aggregate data or individual mortality data. Additionally, some studies focus only on in-hospital mortality, while others include postpartum deaths within six weeks to one year of delivery. Three included studies examine state level policy, and within aggregated data, generally show increased insurance coverage rates are correlated with lower maternal mortality rates. The remaining 14 included studies use individual mortality data. Only two specifically examine insurance type as a causal factor. The other 12 examine insurance as one of many factors related to mortality and within a subsection of larger analyses, such as investigations of specific causes of maternal deaths or regional mortality case reviews. Two case control studies show a non-statistically significant effect of insurance, and one showed a higher proportion of private insurance among women who died. The remaining nine individual data studies show varying degrees of mostly beneficial correlation between being insured and reduced maternal mortality, with private insurance being more protective than Medicaid when examined. Effect size varies significantly by racial group both in state and individual data.

Conclusions Despite significant commentary on the topic, literature examining the effect of health insurance status on maternal mortality is scant. However, state-level research suggests that health insurance access may reduce probability of maternal mortality. This trend requires further examination at the individual level to determine mechanism and degree of effect. Understanding this effect would aid development of future health insurance policy accordingly.

\section{$\# 170$ INTERVENTIONS TO ADDRESS HEALTH DISPARITIES IN COLORECTAL CANCER SCREENING AMONG THE MINORITY POPULATIONS IN THE UNITED STATES}

${ }^{1} S$ Hosseinian*, 'S Afzal, 'E Bolt, ${ }^{1} \mathrm{~J}$ Gu, ${ }^{1} \mathrm{D}$ Lee, ${ }^{1} \mathrm{P}$ Pragash, 'S Sacchetto, ${ }^{1,2} \mathrm{~B}$ Afghani. ${ }^{1}$ UC Irvine school of medicine, Orange, CA; ${ }^{2}$ Children's Hospital of Orange County, Orange, $C A$

\subsection{6/jim-2022-WRMC. 167}

Purpose of Study Colorectal cancer in underserved or minority populations is associated with increased morbidity and mortality. Factors related to this disparity are complex and multifactorial but one of the reasons is due to lower participation rates in colorectal cancer (CRC) screening. The purpose of this study is to review the interventional studies and compile the best practices to increase CRC in minority populations.

Methods Used A literature review was conducted through PubMed, Google Scholar, and Sci-Hub databases using keywords: 'colorectal cancer' 'screening' 'minority' African-American' 'Hispanic' and 'intervention.' Inclusion criteria included interventional studies: that included a control group, consisted of a population of at least $50 \%$ minority, participants over the age of 50, and published after 2005 .

Summary of Results A total of 8 studies satisfied our inclusion criteria (see table 1). The majority of the studies showed an improvement in CRC screening rates but the outcomes measured varied, and included fecal occult blood test, sigmoidoscopy, or colonoscopy. Interventions also varied, and included patient navigation in most of the studies and/or physician-level education in a few studies. Therefore, it was difficult to compare the interventions in different studies because of different study designs and outcomes. After intervention, the CRC screening rate ranged from $27 \%$ to $94 \%$ in different studies. It appears that interventions that were more personalized and required repetitive reminders or contact seemed to be more effective.

Conclusions Our literature review suggests interventional strategies are useful in increasing colorectal cancer screening in the minority population. However, even after intervention, CRC screening remained suboptimal. Larger studies are needed to identify barriers at the individual, health provider and community level and measure the impact of targeted intervention to overcome those barriers. 
Abstract \#170 Table 1 Interventional studies to improve colorectal cancer (CRC) screening among the minority population

\begin{tabular}{|c|c|c|c|c|c|}
\hline $\begin{array}{l}\text { First author, } \\
\text { year } \\
\text { published }\end{array}$ & Control and intervention definition & $\begin{array}{l}\text { Total number of } \\
\text { subjects } \\
\text { (intervention group) }\end{array}$ & $\begin{array}{l}\text { Total number of } \\
\text { controls (standard or no } \\
\text { intervention) }\end{array}$ & $\begin{array}{l}\text { \% Minority: African- } \\
\text { Americans and } \\
\text { Hispanics }\end{array}$ & $\begin{array}{l}\text { CRC screening completion rate: } \\
\text { Controls vs. Intervention, p- } \\
\text { value }\end{array}$ \\
\hline \multirow[t]{4}{*}{ Myers, 2007} & Control: No intervention & Group 1: $N=387$ & \multirow[t]{4}{*}{$\mathrm{N}=387$} & \multirow[t]{4}{*}{$58 \%$} & Controls: $32.56 \%$ \\
\hline & Group 1: Standard letter & Group 2: $N=386$ & & & Group 1: $45.74 \%$ \\
\hline & Group 2: Standard letter and tailored messages & Group 3: $N=386$ & & & Group 2: $43.78 \%$ \\
\hline & Group 3: Standard, tailored message and phone call & & & & Group 3: $48.45 \%, P<0.05$ \\
\hline \multirow[t]{2}{*}{ Horne, 2014} & Control: Educational Material & $\mathrm{N}=578$ & \multirow[t]{2}{*}{$\mathrm{N}=642$} & \multirow[t]{2}{*}{$>50 \%$} & Controls vs intervention: $91 \%$ vs \\
\hline & Intervention: Patient Navigator & & & & $94 \%, P=0.04$ \\
\hline \multirow[t]{3}{*}{ Cole, 2017} & Control: Patient Motivational Interview for Blood Pressure & $\mathrm{N} 1=234$ & \multirow[t]{3}{*}{$N=238$} & \multirow[t]{3}{*}{$100 \%$} & Control vs intervention 1 vs \\
\hline & Intervention1: Patient Navigation & $\mathrm{N} 2=254$ & & & intervention $2: 8.4 \%$ vs $17.5 \%$ vs. \\
\hline & Intervention 2: Patient Navigation and Motivational Interview & & & & $17.8, P<0.01$ \\
\hline DeGroff, & Control: Standard Care & $\mathrm{N}=429$ & $\mathrm{~N}=427$ & $>50 \%$ & Control vs Intervention: $53.2 \%$ vs \\
\hline 2017 & Intervention: Patient Navigation & & & & $61.1 \%, P=0.02$ \\
\hline Ford, 2006 & $\begin{array}{l}\text { Control: Patients did not receive monthly communication from } \\
\text { case managers } \\
\text { Intervention: Patients received monthly communications from } \\
\text { case managers }\end{array}$ & $N=352$ & $N=351$ & $100 \%$ & $\begin{array}{l}\text { Control vs Intervention: } 51.3 \% \text { vs } \\
68.9 \%, P=.10\end{array}$ \\
\hline Basch, 2006 & $\begin{array}{l}\text { Control: patients were mailed printed materials } \\
\text { Intervention: Patients received a tailored telephone outreach }\end{array}$ & $N=226$ & $N=230$ & $>50 \%$ & $\begin{array}{l}\text { Control vs Intervention: } 6.1 \% \text { vs } \\
27.0 \%, 95 \% \mathrm{Cl}: 2.6,7.7\end{array}$ \\
\hline $\begin{array}{l}\text { Khankari, } \\
2007\end{array}$ & $\begin{array}{l}\text { Control: baseline screening rate } \\
\text { Intervention: Mailing screening-eligible patients a physician } \\
\text { letter. Physicians were also trained to review health literacy } \\
\text { and 'best practices." }\end{array}$ & $N=154$ & $N=174$ & $>95 \%$ & $\begin{array}{l}\text { Control vs Intervention: } 11.5 \% \text { vs } \\
27.9 \%, P<.001\end{array}$ \\
\hline $\begin{array}{l}\text { Friedman, } \\
2007\end{array}$ & $\begin{array}{l}\text { Medical residents received educational intervention } \\
\text { Control = Rate of CRC screening by residents } 6 \text { months prior } \\
\text { to education } \\
\text { Intervention = Rate of CRC screening by residents } 6 \text { months } \\
\text { post education }\end{array}$ & $N=132$ & $N=116$ & $100 \%$ & $\begin{array}{l}\text { Control vs Intervention: } 26.7 \% \text { vs } \\
59.1 \%, P<0.001\end{array}$ \\
\hline
\end{tabular}

\section{\#171 A COMPREHENSIVE REVIEW OF WOMEN'S QUESTIONS FOLLOWING MISCARRIAGE ON DIFFERENT SOCIAL MEDIA PLATFORMS}

${ }^{1} \mathrm{EG}$ Ong*, 'L Davis, ${ }^{1} \mathrm{~A}$ Sanchez, ${ }^{1} \mathrm{R}$ Curiel, ${ }^{2} \mathrm{H}$ Stohl, ${ }^{1} \mathrm{~A}$ Nelson, ${ }^{2} \mathrm{~N}$ Robinson. ${ }^{1}$ Western University of Health Sciences College of Osteopathic Medicine of the Pacific, Pomona, CA; ${ }^{2}$ Harbor-UCLA Medical Center, Torrance, CA

\subsection{6/jim-2022-WRMC.168}

Purpose of Study The purpose of this study was to investigate common questions and the accuracy of advice related to miscarriage that women post on social media to identify any gaps in miscarriage care, counseling and give learners insight into the experience of miscarriage.

Methods Used Public social media posts from January 1st 2019 to June 30th 2021 were searched using the keywords of 'miscarriage', 'preterm birth', 'pregnancy loss', 'early pregnancy loss', 'early fetal loss', 'spontaneous abortion', 'spontaneous pregnancy loss' and '\#IHadAMiscarriage'. Only public posts in English and originating from the United States were included. No private identifiable information was collected. The inclusion criteria for posts varied by site: all posts in public Facebook Miscarriage groups, YouTube videos with $\geq 1000$ views, Reddit r/Miscarriage with $\geq 175$ upvotes, Instagram photos with $\geq 50$ likes and Tweets with $\geq 1$ interaction were analyzed for mentions of questions, advice, and conflicts of interest. Each theme in a post was counted. Advice was classified into categories of 'accurate' if it was supported by current professional association guidelines, including the American College of Obstetricians and Gynecologists (ACOG). 'Insufficient evidence' was advice only supported by published reports, and 'inaccurate' if any part was against clinical guidelines or was unsupported.

Summary of Results For women and families experiencing miscarriage, social media has become a popular outlet and resource for support. 103 posts were identified for common themes. The most common themes identified included: questions on grief $(20.4 \%)$, blame $(19.4 \%)$, quality of post-partum counseling (14.6\%), and lack of medical support/follow-up (13.6\%). $82.9 \%$ of advice mentioned the emotional consequences of loss. $17.1 \%$ of posts offered medical advice eligible for evaluation, with the most accurate platforms being YouTube (66.6\%), Instagram (100\%) and Reddit (100\%). The least accurate platform was Facebook (33.3\%). Twitter yielded the fewest results that met the inclusion criteria.

Conclusions Many women who post on social media struggle with grief, blame and look for better support from the medical community. The majority of the online community of women who have had miscarriages offer advice related to grief and coping with loss rather than questions regarding medical information. Thus, clinicians may advise women to use social media as a resource to connect with others in their loss and grief. Social media may also be used as an invaluable resource for learners to empathize with women and their families after miscarriage. The ability to better understand the experience and impact of miscarriage will hopefully improve the quality of miscarriage care. 


\section{\#172 MEDICAL LEARNERS VIEWS ON LEARNER FEEDBACK TO} BUILD PROGRAM CAMARADERIE

${ }^{1} \mathrm{R}$ Buller, ${ }^{2} \mathrm{AG}$ Raja*, ${ }^{1} \mathrm{P}$ Poysophon, ${ }^{1} \mathrm{~T}$ Allison-Aipa. ${ }^{1}$ Riverside University Health System, Riverside, $C A ;{ }^{2}$ Western University of Health Sciences, Pomona, CA

\subsection{6/jim-2022-WRMC.169}

Purpose of Study There is little focus on how residents and fellows can give feedback to program faculty in residencies and fellowships in the United States. This study aims to determine if learners feel comfortable giving feedback, the preferred method of feedback, and whether this feedback correlates with a culture of camaraderie between residents and faculty. It has been shown that building camaraderie improves health outcomes for patients and improves physician wellness by creating an overall positive atmosphere.

Methods Used A questionnaire using both multiple choice and open response questions was sent to all 125 residents and fellows at the Riverside University Health System medical program. The survey was composed of questions regarding honesty in giving feedback, preferred feedback method, and if learners thought increased feedback helped build camaraderie. Cronbach's alpha was calculated to determine internal validity. Descriptive statistics and a correlation coefficient were calculated from survey responses.

Summary of Results From the 26 responses, results showed that $54 \%$ of learners felt comfortable giving feedback to their program and $50 \%$ of learners preferred individual, anonymous, written feedback as their method of choice. There were strong correlations between the use of feedback and feelings of camaraderie. Learners who were able to provide feedback had a strong correlation with learners who felt camaraderie with their faculty $(\mathrm{p}<.001)$. $75 \%$ of learners who preferred one-to-one verbal feedback in general also preferred this method to improve camaraderie, compared to $14 \%$ of learners who chose group written feedback. When they differed, students chose group verbal feedback to improve camaraderie.

Conclusions Our study shows that allowing residents to give feedback to their mentors can increase camaraderie, which is known to improve outcomes: patient health outcomes and physician wellness. Furthermore, this study provides a look into learners perspective and leads the way for future studies to provide more causal links between learner feedback to physician wellness and patient health.

\section{\#173 OPTIONS TO EXPAND HOME HEALTHCARE IN CORDOVA, AK}

1,2JT Bramante*. 'University of Washington, Seattle, WA; ${ }^{2}$ University of Alaska Anchorage, Anchorage, $A K$

\subsection{6/jim-2022-WRMC. 170}

Purpose of Study Patients in Cordova, Alaska lack access to standard home healthcare services such as home health agencies (HHAs) or assisted living facilities (ALFs). Patients with complicated medical issues who are homebound and often do not have robust social support in the community must rely on physician home visits or be admitted to the hospital's long term care facility. In order for either of the primary care clinics to bill medicare for home visits, a physician must be present, which significantly limits availability of home visits.

Methods Used Patient and provider interviews were conducted at the Cordova Community Medical Center and Ilanka
Community Health Center (Ilanka). An asset-based research approach was used to identify other community entities that could potentially impact health for homebound individuals. An interview was conducted with the Cordova Police Department, which provides wellness checks for individuals in the community. A literature review was conducted to evaluate the efficacy of home health intervention programs in addressing needs present in the community such as hospice and wound care. State and national regulatory institutions were then contacted to ascertain the options for expanding Centers for Medicare \& Medicaid Service (CMS) coverage of the local primary care centers to include visiting nurse services.

Summary of Results It was determined that Ilanka, which is registered as a Federally Qualified Healthcare Center with CMS, would be eligible to be compensated for visiting nurse services given that there is a shortage of HHAs in the area. However, since they are not located in an area that has been officially designated as having a shortage by the state, and there is no official path to achieve this in Alaska, they must petition their CMS Regional Office.

Conclusions This asset-based research approach identified a strong community need in Cordova for expanded home healthcare. A CMS regulation was identified that would allow Ilanka to be compensated for visiting nurse services. However, information on the approval process was very difficult to find. This need is likely shared by other rural communities in the state that are large enough to have a primary care center but not necessarily support an independent HHA or ALF, but there are no governmental processes to support approval. Recent efforts in the state of Washington might provide a template for centralized support and streamlining of the approval process.

\section{\#174 THE IMPACTS OF COVID-19 ON THE SOCIAL PERCEPTIONS OF BREASTFEEDING}

${ }^{1} \mathrm{~A}$ Mehta*, ${ }^{1}$ EA Wright, ${ }^{1} \mathrm{AL}$ Nelson, ${ }^{2} \mathrm{H}$ Stohl, ${ }^{2} \mathrm{M}$ Economidis. ${ }^{1}$ Western University of Health Sciences College of Osteopathic Medicine of the Pacific, Pomona, CA; ${ }^{2}$ University of California Los Angeles David Geffen School of Medicine, Los Angeles, CA

\subsection{6/jim-2022-WRMC.171}

Purpose of Study There is a growing concern amongst physicians that the increased stress of COVID-19 has significantly impacted the number of women choosing to breastfeed. However, not many qualitative studies address whether COVID-19 has impacted breastfeeding perceptions. This study aims to identify hidden challenges women face while breastfeeding and to determine if those changed with the onset of the pandemic.

Methods Used This research was conducted manually across two social media sites, Reddit and TikTok, using the search terms 'breastfeeding,' 'breastisbest,' and 'fedisbest.' Posts with the most comments from pre-pandemic times [Jan 1, 2018 to March 15, 2020] were compared to the posts with the most comments from [March 15, 2020 to June 15, 2020]. Each post and comment was categorized into a theme, and thematic saturation was achieved after three comments mentioned the same theme. In order to establish a theme, there must be at least five posts speaking about that topic.

Summary of Results 248 posts were analyzed with a total of 433 mentions of the selected themes. Fifteen themes were noted across both Reddit and TikTok, and the top 2 themes across both platforms were 'providing medical advice' and 
'supporting women's breastfeeding decisions.' On Reddit, 'supporting women's breastfeeding decisions' was the most mentioned theme before the onset of the pandemic (37.2\%), but 'providing medical advice' became the most mentioned theme after COVID-19 (42.4\%). $60.0 \%$ of the posts on TikTok: After COVID19 included 'providing medical advice' while only $42.4 \%$ of the posts on Reddit: After COVID19 provided medical advice. Despite data showing reduction in breastfeeding support on the maternity ward and a shortage of breastfeeding support equipment, $10.6 \%$ of posts on Reddit noted COVID-19 positively affected breastfeeding. Some comments included the fact that not allowing visitors in hospitals and in their homes initially allowed them to successfully connect with their babies and initiate breastfeeding. Two women mentioned that they could choose to stay away from their in-laws who discouraged breastfeeding, using the excuse of the pandemic. Other comments mentioned a more flexible online work schedule allowed them to plan their meetings around scheduled breastfeeding sessions.

Conclusions Social media platforms provide a niche community for breastfeeding women to communicate with and support each other. Despite the stress of COVID-19, posts on Reddit illustrated that the pandemic positively impacted women's abilities to breastfeed. We hope that this study encourages physicians to engage in deeper conversations with their breastfeeding patients to help them better critically analyze information online but still receive the positive community support they need.

\section{\#175 FUNCTION OF SOCIAL MEDIA ON PREMENSTRUAL DYSPHORIC DISORDER}

N Poladian*, A Maron, T Ghazarian, AL Nelson, Y Fernandez-Sweeny. Western University of Health Sciences College of Osteopathic Medicine of the Pacific, Pomona, CA

\subsection{6/jim-2022-WRMC.172}

Purpose of Study Premenstrual Dysphoric Disorder (PMDD) is a common and debilitating disorder that affects reproductive age women. With society's growing reliance on social media and the emergence of various social platforms, many patients turn to social media outlets for information. This practice also holds true for women seeking information surrounding PMDD, and those investigating their symptoms. This study was designed to provide clinicians with a better understanding of the content, support, as well as wealth and accuracy of information surrounding PMDD found in a popular social media site, Reddit, often used to investigate medical topics.

Methods Used In this IRB-approved study, we analyzed the content of posts on the subreddit titled 'PMDD,' in public domain Reddit, from January 2020 through May 2021. Posts needed to include greater than five upvotes and at least three comments. Posts with only photos were excluded. These posts and their respective comments were manually analyzed for qualitative data, and were categorized by prevalent themes that all authors confirmed.

Summary of Results We analyzed a total of 232 posts that fulfilled the inclusion criteria. The most prevalent theme in posts regarding PMDD was expression of feelings $(n=116)$, centered around the topics of depression, anxiety, identity crisis, rejection, and frustration about the lack of support and understanding from both their community and healthcare providers. The second most common theme was sharing of experience- based insight $(n=56)$, which contained advice suggesting varying therapeutics and coping strategies to decrease PMDD symptom severity. While therapeutic suggestions of SSRI's, NSAID's, and lifestyle modifications have clinical relevance, user misconceptions were noted regarding contraceptive use and hysterectomy for PMDD symptom relief. The third most common theme was relationship complications due to PMDD $(n=31)$. Health-related questions $(n=28)$, such as whether a new or recurring symptom was associated with PMDD, were the least frequent. Analysis of commentary on user posts led to further identification of four themes: offering support, sharing stories/advice, validating one another's feelings, and showing appreciation for one another's insights.

Conclusions This study provides clinicians with insight on the interactions between patients, not observed in clinic. Reddit primarily serves for PMDD as a positive space for promoting belonging, and in doing so, may be beneficial to patients. Furthermore, identification of user frustration and discussion pertaining to failed therapies may set realistic expectations of treatment efficacy. Clinicians may inform patients about specific therapeutic misinformation seen online, promoting further open discussion between patient and physician.

\section{\#176 INTERNAL MEDICINE EDUCATION FOR MEDICAL TRAINEES IN NAIVASHA, KENYA}

${ }^{1} \mathrm{LV}$ Savochka*, ${ }^{1} \mathrm{D}$ Li, ${ }^{1,2} \mathrm{~L}$ Onofrey, ${ }^{3} \mathrm{D}$ Bosibori, ${ }^{1,2} \mathrm{~J}$ Beste, ${ }^{1} \mathrm{C}$ Farquhar. ${ }^{1}$ University of Washington School of Medicine, Seattle, WA; ${ }^{2}$ Harborview Medical Center, Seattle, WA; ${ }^{3}$ Naivasha County Referral Hospital, Naivasha, Kenya

10.1136/jim-2022-WRMC.173

Purpose of Study In Kenya, there has been a decrease in government funding for medical education which has led to outdated educational guidelines to treat common diseases. In this project, the Clinical Education Partnership Initiative (CEPI) between the Naivasha County Referral Hospital (NCRH) and University of Washington (UoW) was used to strengthen the medical education curriculum for internal medicine trainees and pharmacy students from the University of Nairobi (UoN) who are obtaining their medical training at NCRH.

Methods Used The NCRH internal medicine trainees and pharmacy students were surveyed via Zoom on what internal medicine curriculum topics they would like updated over the course of this 8 -week long project. The most popular topics were updated using current medical guidelines and recommendations from World Health Organization, UptoDate and Kenya Ministry of Health. The community CEPI partners at the NCRH helped tailor the guidelines to their resource availability. The updated curriculum was then presented at weekly seminars to the medical trainees and was also shared with the medical community to be used as a reference in the future.

Summary of Results A total of six internal medical education curriculums were updated and six seminars were delivered on topics such as asthma, chronic obstructive pulmonary disease, COVID-19, meningitis, liver failure, acute coronary syndrome and acute kidney injury. The attendance of each seminar ranged from 5-10 students and included internal medicine residents, as well as pharmacy students. Throughout the project, positive feedback regarding the sessions was given by the site coordinator, as well as the attendees. In the end-of-project survey, $83 \%$ of the attendees strongly agreed that they felt more up-to-date with the topics presented and have been using the information presented in clinical settings. Additionally, 63\% of 
the attendees strongly agreed that they felt more confident in diagnosing and $83 \%$ felt more confident treating based on those topics. Participant feedback also revealed that case-based questions and Zoom polling were an effective way to engage attendees.

Conclusions This project successfully updated six outdated internal medicine curriculum didactic sessions for internal medicine trainees and pharmacy students at NCRH. Participants reported to have an improved confidence in diagnosis and treatment on the presented topics. The plan is for future lectures to continue incorporating case-based questions and polling to emphasize teaching points using Zoom. The presentations were also sent to the site coordinator for future use and reference by NCRH trainees.

\section{$\# 177$ DEVELOPMENT OF A WHATSAPP-BASED PILOT PROGRAM TO IMPROVE STROKE EDUCATION AND RISK FACTOR REDUCTION IN PATIENTS WITH ARTERIAL HYPERTENSION IN HUARAL, PERU}

${ }^{1} \mathrm{~K}$ Turk ${ }^{*},{ }^{1} \mathrm{~J}$ Zunt, ${ }^{2} \mathrm{C}$ Abanto, ${ }^{3} \mathrm{~A}$ Sanchez. ${ }^{1}$ University of Washington School of Medicine, Seattle, WA; ${ }^{2}$ Instituto Nacional De Ciencias Neurologicas, Lima, Peru; ${ }^{3}$ Universidad Peruana Cayetano Heredia, Lima, Peru

\subsection{6/jim-2022-WRMC.174}

Purpose of Study Cerebrovascular disease and stroke are the second-leading cause of death in Peru. Despite the high burden of stroke, Peru lacks a national stroke program, and there is a shortage of neurologists, stroke units, and thrombolytic therapy, particularly in rural areas. It is imperative to reduce patients' risk factors for stroke, especially hypertension since it is the most significant modifiable risk factor in preventing stroke. WhatsApp, one of the most popular communication platforms in Peru, provides an exciting opportunity to disseminate health information to patients. The aim of this project was to develop materials for a WhatsApp-based pilot program to improve stroke education and risk factor reduction in patients with hypertension living in rural areas of Huaral, a province in the Lima Region of Peru.

Methods Used A stakeholder analysis was conducted to identify key partners whose input was essential in creating this project. Guidelines from the National Institute of Neurologic Sciences (INCN) in Peru and the American Heart Association/ American Stroke Association were used to create infographics addressing stroke awareness and risk reduction. An informative outline of the pilot program was created for Huaral Hospital partners. A cohort of patients with hypertension was identified by the Huaral Hospital Teaching and Training Unit Director. Surveys addressing patients' stroke knowledge and comfort using WhatsApp were generated. To ensure content was medically accurate and culturally appropriate, all materials were first edited by a medical student in Peru and subsequently by Peru's Chief of the Center of Research in Cerebrovascular Disease at the INCN.

Summary of Results Materials created for the pilot program include a detailed program outline, an informed consent adapted to WhatsApp, pre- and post-program surveys, and five educational infographics. These materials are ready to be sent to patients with hypertension in Huaral via WhatsApp. There is enthusiasm and optimism from hospital staff about the potential impact of the pilot program.

Conclusions This project will increase access to accurate stroke education for patients with hypertension living in
Huaral. Now that the materials have been created, the next step is to pilot their use in the selected cohort of patients. The program's success will be determined by comparing participants' pre- and post-program survey responses. By using WhatsApp, this program could provide a sustainable means to improve stroke education while limiting travel and financial burdens for patients living in rural areas. If successful, this program could be replicated in other rural areas in Peru.

\section{Poster session}

\section{Infectious diseases}

\section{6:00 PM}

\section{Thursday, January 20, 2022}

\section{\#178 A CASE OF PSEUDOMONAS AERUGINOSA ASSOCIATED DIARRHEA IN A LONG-TERM HOSPITALIZED PATIENT}

C Besmanos*, N Raza, H Lai, S Mishra, S Ragland, A Heidari. Kern Medical Center, Bakersfield, CA

\subsection{6/jim-2022-WRMC.175}

Purpose of Study Pseudomonas aeruginosa associated diarrheal disease is not common in adults and if seen, is mostly reported in pediatric population. It has been classified into Shanghai fever, enterocolitis and antibiotics associated diarrhea in pediatric literature. In adults, immunocompromising conditions such as malignancies, neutropenia, and admission to long-term care and intensive care unit (ICU) are known risk factors. Here we describe a case of Pseudomonas aeruginosa associated diarrhea in a long-term hospitalized patient who had rectal tube.

Methods Used Retrospective case review

Summary of Results A 53-year-old Caucasian man with a history of alcohol use disorder, hypertension, and hypothyroidism presented with myxedema coma with TSH 148 requiring intubation. He had a complicated hospital course with ventilatorassociated pneumonia with MRSA, sepsis with candida, and abdominal compartment syndrome requiring decompressive laparotomy. The patient slowly recovered despite 43 days of hospitalization. Before leaving his 31 days of admission to ICU, a flexi-seal rectal tube was placed due to fecal incontinence. Five days later when he was already transferred out of the ICU, he started having loose watery stools with leukocytosis and left shift. Clostridioides difficile (C.diff.) colitis was suspected and he was placed on oral vancomycin empirically. His stool test for C.diff. came back negative, and his rectal tube was subsequently removed. Imaging did not show any abscess, perforated viscus, or fistula formations. His stool culture grew heavy colony numbers of Pseudomonas aeruginosa. Vancomycin was stopped and he was started on oral ciprofloxacin 750 mg twice a day with complete resolution of his diarrhea and leukocytosis.

Conclusions Pseudomonas aeruginosa associated diarrheal disease is not common in adults. Complicated prolonged hospitalization, administration of broad-spectrum antibiotics, and immunocompromising conditions perhaps play role in colonization and eventually infection in the right setting. The role of rectal tube is unknown. 
\#179 HHV-8 ASSOCIATED MULTICENTRIC CASTLEMAN DISEASE: A DIAGNOSTIC CHALLENGE IN A PATIENT WITH ACQUIRED IMMUNODEFICIENCY SYNDROME AND FEVER

R Dunn*, F Venter, R Jariwal, S Mishra, J Bhandohal, E Cobos, A Heidari. Kern Medical Center, Bakersfield, CA

\subsection{6/jim-2022-WRMC.176}

Purpose of Study HHV-8 associated Multicentric Castleman Disease (MCD) is an angiofollicular lymphoproliferative disorder that affects multiple regions of lymph nodes simultaneously. Incidence of Castleman Disease is about 6500 to 7700 in the United States, $75 \%$ of them being associated with HHV-8. All cases of MCD in HIV patientd are HHV-8 associated. Common symptoms include fever, lymphadenopathy, Hepatosplenomegaly, pulmonary involvement, edema, and ascites. Here we describe a case of HIV with fever of unknown orgin in that after extensive work up diagnosed with MCD.

Methods Used This is a single case review after IRB approval. Summary of Results A 28 year old male with history of HIV, non-aherence with antiretroviral therapy (ART) presents with constant abdominal pain, vomiting, and fevers over 5 days. CBC showed pancytopenia with a CD4 count of 27 cells/ $\mathrm{mcL}$ and an HIV viral load of 95,200 copies/mL. Work up for Syphilis, Gonorrhea, Chlamydia, Hepatitis A/B/C, Cocci, Cryptococcus, TB, Toxoplasma, Histoplasmosis, Bartonella, Giardia, Brucella, CMV PCR, Parvo B19 PCR, Coxiella brunetii, Cryptosporidium and all of his cultures were negative. Bone marrow biopsy and culture were negative. Imaging showed diffuse lymphadenopathy in the mediastinum, hilar, axillary, retroperitoneum, iliac and inguinal lymph nodes. IR Lymph node excision and HHV-8 staining confirmed HHV-8 associated MCD with serum HHV-8 levels of 2,288,277 copies $/ \mathrm{ml}$. Patient was given Rituximab infusions and started on ART.

Conclusions Diagnosis of HHV-8 Multicentric Castleman Disease in HIV host could be challenging and usually is delayed. Starting antiretroviral and Rituximab is the most agreed upon therapeutic modality. This approach almost doubles the survival rate at 2 years from $42 \%$ to $90 \%$.

\section{\#180 ELEVATED ADENOSINE DEAMINASE LEVELS IN A CASE OF COCCIDIOMYCOSIS}

C Salib*, L Moosavi, K Radicic. Kern Medical Center, Bakersfield, CA

\subsection{6/jim-2022-WRMC. 177}

Purpose of Study Elevated adenosine deaminase (ADA) levels have been a useful diagnostic tool associated with tuberculosis (TB). Diagnostic testing for Coccidioidomycosis (Cocci) involves serologic testing of IgM and IgG antibodies. Though there are a variety of diagnostic testing options for $\mathrm{TB}$, in a case of high clinical suspicion, pleural fluid analysis for ADA is often completed as an additional confirmatory test, due to its high sensitivity and specificity. This case demonstrates a patient who presented with elevated ADA levels despite having negative sputum cultures for $\mathrm{TB}$ and with elevated serological titers for Cocci.

Methods Used A single patient case report was conducted after IRB approval.
Case Presentation A 26-year-old male with history of uncontrolled Type 1 diabetes presented to the ED with shortness of breath and productive cough of two day duration. The patient denied history of travel and reported a nonspecific amount of weight loss over the past few months. He presented febrile, tachycardic, and hypoxic. CT scan found a lung abscess and empyema. He was seen by Interventional Radiology for placement of a pigtail catheter for drainage of pleural fluid. $\mathrm{He}$ was initially treated with Fluconazole but then transitioned to Ambisome.

During his admission, patient remained consistently tachycardic and tachypneic with worsening hypoxia. Requiring ICU admission, he was intubated three times and was subsequently extubated within a few days. The patient underwent bronchoscopy and was found to have pleural ADA level of 468 . Despite having negative QuantiFERON results, the patient was started on RIPE therapy due to suspected TB infection.

Results from Mycobacterial sputum culture returned negative weeks after patient was discharged from the hospital, confirming that the patient did not have an active TB infection. However, serologic test was confirmatory for Cocci.

Conclusions ADA levels have been known to have both a high sensitivity and specificity for diagnosing $\mathrm{TB}$, which makes it an ideal diagnostic tool. In this instance, there was the unusual presentation of increased levels of ADA along with a negative AFP culture. The role and expected actions of the ADA enzyme can be indicative of T-lymphocyte activity. When thought of more generally, this diagnostic tool can be used to diagnose disease conditions other than $\mathrm{TB}$, such as Cocci.

\section{\#181 HYDROXYCHLOROQUINE PRE-EXPOSURE PROPHYLAXIS FOR COVID-19 IN HEALTHCARE WORKERS FROM INDIA: A META-ANALYSIS}

RB Stricker*, MC Fesler. Union Square Medical Associates, San Francisco, CA

\subsection{6/jim-2022-WRMC.178}

Purpose of Study While vaccines have taken center stage in the battle against COVID-19, alternate approaches for prevention of SARS-CoV-2 infection have been ignored. Hydroxychloroquine pre-exposure prophylaxis (HCQ PrEP) has been used to prevent COVID-19 in high-risk healthcare workers (HCWs) in a number of studies from India. We have performed a meta-analysis to evaluate the safety and efficacy of a standard HCQ PrEP regimen in HCWs enrolled in these studies.

Methods Used We performed a search of the medical literature based on the PRISMA checklist (http://www.prisma-statement. org/) using PubMed, Google Scholar, medRxiv and ResearchGate to obtain all relevant publications and preprints. Using this method we obtained eleven nonrandomized controlled trials of weekly HCQ PrEP involving 7,616 HCWs in India (3,489 treated, 4,127 controls). The HCQ PrEP regimen consisted of an $800 \mathrm{mg}$ loading dose in the first week, then $400 \mathrm{mg}$ weekly thereafter according to the guidelines of the Indian Council of Medical Research. SARS-CoV-2 infection was documented by seroconversion or positive polymerase chain reaction (PCR) testing. We used random-effects metaanalysis to summarize the risk ratio (RR) of infection across the studies.

Summary of Results Sex distribution was available for nine studies and age distribution was available for eight studies. 
The sex distribution was 58\% male and $42 \%$ female, and the mean age was $33.1 \pm 7.7$ years. In eight studies that reported HCW roles, 63\% were involved in direct patient care (doctors and nurses) while $37 \%$ provided support services (laboratory technicians, housekeeping staff and other hospital workers). In the 11 studies that included HCWs who used any HCQ PrEP, the infection rate was significantly decreased (RR $0.56,95 \%$ CI $0.37-0.83, p=0.0040$ ). In the five studies that included HCWs who took at least six doses of weekly HCQ PrEP, the infection rate was reduced even further (RR 0.25, 95\% CI 0.13-0.50, p $<0.0001$ ). No deaths were recorded in either the HCQ PrEP or control group. Three studies involving $667 \mathrm{HCWs}$ reported adverse events (AEs). The most common AEs were headache (8\%) followed by nausea (7\%) and dyspepsia (6\%). No arrhythmias were reported by the HCWs. AEs were generally mild and well tolerated, as shown in one study where the HCQ discontinuation rate due to AEs was $4 \%$.

Conclusions Weekly HCQ PrEP appeared to be safe and effective for prevention of COVID-19 in high-risk HCWs from India. Further studies of HCQ PrEP are warranted to supplement vaccines in the prevention of COVID-19.

\section{\#182 A UNIQUE CASE OF COVID-19 PRESENTED AS FOCAL SEIZURES WITH IMPAIRED AWARENESS}

R Sharma*, S Ratnayake, H Lai, S Mishra, A Heidari. Kern Medical Center, Bakersfield, CA

\subsection{6/jim-2022-WRMC.179}

Purpose of Study Severe acute respiratory syndrome coronavirus 2 (SARS-CoV2) has rapidly become a global pandemic with millions of confirmed cases worldwide. Encephalitis and seizure associated with COVID-19 has been seen and reported. Here described is a unique case of SARS-CoV2 infection presented with focal seizure with impaired awareness.

Methods Used A retrospective review following IRB approval. Summary of Results A 54-year-old man unvaccinated for COVID-19 with no known past medical history presented to the emergency department with altered mental status. Three days prior to presentation he complained of frontal headaches and blurry vision and one day prior his son noticed he was unable to speak. Minutes upon arrival he suffered a focal seizure for which levetiracetam was administered. He was found to be oriented to self and age with difficulty finding words answering only with 'yes'. EEG was performed and he was diagnosed with focal seizure with impaired awareness. His chest $\mathrm{x}$-ray showed multifocal bilateral hazy infiltrations. SARS-Cov2 PCR test came back positive. His brain MRI revealed 2 acute subcortical superior right frontal lobe lacunar infarcts. His lumbar puncture was negative. He suffered nine seizures of left occipital origin despite being treated with levetiracetam and valproic acid. Phenytoin was added which eventually controlled his seizure and he became fully oriented. Overcoming a tumultuous hospital course, he was also found to have newly diagnosed poorly controlled diabetes and pulmonary cavitary coccidioidomycosis. He was discharged stably on hospital day 16.

Conclusions Focal seizure with impaired awareness associated with SARS-CoV2 has not been reported. Further studies are warranted to understand the pathophysiology and definitive treatment.

\section{\#183 METHICILLIN-RESISTANT STAPHYLOCOCCUS EPIDERMIDIS ENDOCARDITIS, FROM TUNNEL TO SPINE}

${ }^{1}$ VK Narang ${ }^{*},{ }^{2} \mathrm{H}$ Sidhu, ${ }^{1} \mathrm{C}$ D'Assumpcao, ${ }^{1} \mathrm{~L}$ Moosavi, ${ }^{1} \mathrm{TT}$ Win, ${ }^{1} \mathrm{~A}$ Heidari. ${ }^{1}$ Kern MedicalUCLA, Bakersfield, CA; ${ }^{2}$ Ross University School of Medicine, Miramar, FL

\subsection{6/jim-2022-WRMC. 180}

Case Report Staphylococcus epidermidis is a common clinically encountered species of coagulase-negative staphylococci. Its ability to produce biofilm particularly in the presence of central lines can lead into serious infections including endocarditis. Here we demonstrate a case of a 68 -year-old female on hemodialysis with exposed tunnel part of her dialysis catheter resulting in infective endocarditis and spinal osteomyelitis.

Methods Approval was obtained from IRB. A single patient case report was conducted.

Case Presentation Patient is a 68-year-old female with End Stage Renal Disease on hemodialysis who presented with 4 weeks of worsening lumbar spine pain. Upon presentation she was afebrile. Her examination was significant for lumbar spine point tenderness and left jugular tunneled catheter entry site dehiscence exposing the catheter. Patient stated the skin over the tunnel opened five months prior. Her laboratory studies showed ESR of 100 and CRP of 25. Her blood culture grew Methicillin-resistant S. epidermidis (MRSE). Her tunneled catheter was removed, and a new catheter was place in same area due to lack of access and stenosis of central venous on the other side. MRI lumbar spine showed near complete loss of the intervertebral disc at L4-L5 with severe erosive endplate changes.

An Interventional radiology (IR) guided bone biopsy of lumbar spine also grew MRSE. The patient persistently remained bacteremic with MRSE despite IV antibiotics. Transesophageal echocardiogram revealed sub-aortic $0.6 \times 0.9 \mathrm{~cm}$ with no signs of abscess or valvular dysfunction. Despite lack of alternative access for dialysis due to stenosis there was no choice but to remove the tunneled catheter again and temporary catheter was placed at an alternate site. IR performed balloon angioplasty for central venous stenosis and insertion of left, IJ tunneled dialysis catheter after blood cultures remained negative.

Conclusion Persistent bacteremia with Staphylococcus epidermidis in the presence of central line can lead into serious and metastatic infections. This requires successful source control in addition to antibiotic therapy.

\section{\#184 PULMONARY GIANT CAVITARY COCCIDIOIDES WITH FUNGAL BALL AND HEMOPTYSIS}

${ }^{1}$ VK Narang*, ${ }^{2} \mathrm{~K}$ Dao, ${ }^{2} \mathrm{~S}$ Jaratanian, ${ }^{1} \mathrm{C}$ D'Assumpcao, ${ }^{1} \mathrm{R}$ Kuran, ${ }^{1} \mathrm{~A}$ Munoz, ${ }^{1} \mathrm{~A}$ Heidari. ${ }^{1}$ Kern Medical-UCLA, Bakersfield, CA; ${ }^{2}$ Ross University School of Medicine, Miramar, FL

10.1136/jim-2022-WRMC. 181

Case Report Coccidioidomycosis is a fungal pneumonia with risk of cavitation in select populations, such as diabetics. Cavitary lesions can hemorrhage and have superimposed infections. We discuss a case of giant cavitary coccidioidomycosis in an 
uncontrolled diabetic presenting with hemoptysis and mycetoma.

Methods A single patient case report was conducted after approval from IRB.

Case Presentation A 48-year-old Hispanic male with diabetes, untreated pulmonary coccidioidomycosis, and history of COVID-19 infection one year prior presented with sudden hemoptysis, night sweats, and a $45-\mathrm{lb}$ weight loss. He was diagnosed with coccidioidomycosis seven years prior but did not start treatment. Three years prior he was admitted elsewhere with shortness of breath. Serum coccidioidal complement fixation titer was 1:32. Imaging found a right lower lobe cavity measuring $4 \times 3 \mathrm{~cm}$ with right sided pneumothorax and bronchopulmonary fistula. He underwent videoassisted thoracotomy with pleurodesis and treated with fluconazole for 4 months, improving his titers to $1: 4$. He was then lost to follow.

Upon presentation to our facility with worsening hemoptysis, he had a larger cavity measuring $10 \times 8 \times 7 \mathrm{~cm}$ in the right lower lobe with central filling mass. He hemoptysized daily while admitted. Bronchoscopy confirmed coccidioidal mycetoma by direct stain and fungal cultures. Interventional radiology performed arterial embolization of right tracheobronchial and intercostal bronchial arteries to control hemoptysis. He was restarted on fluconazole and discharged home. One week later he returned in respiratory distress with fever, shortness of breath, and hypoxemia. Imaging revealed new left lower lobe and lingular consolidations. He was thought to have aspiration of right sided cavitary material to the left lung. His oxygen requirements increased significantly, concerning for severe pulmonary coccidioidomycosis and he was placed on steroids and liposomal amphotericin B. His symptoms improved and steroid was stopped. His antifungal was switched to Posaconazole and was able to go home on room air.

Discussion Management of giant pulmonary cavitary coccidioidomycosis with mycetoma and bleeding is challenging. Poorly controlled diabetics are already at increased risk for cavitation. In a patient presenting with hemoptysis and a cavitary lesion, a multidisciplinary team consisting of pulmonology, infectious diseases, thoracic surgery and interventional radiology is essential.

\section{$\# 185$ A CASE OF PSEUDOTERRANOVA, HAVING CEVICHE WITH SPECIAL FLAVOR}

${ }^{1,2}{ }^{2}$ Prasad ${ }^{*},{ }^{2} C$ D'Assumpcao, ${ }^{2} \mathrm{R}$ McPheeters, ${ }^{2} \mathrm{~W}$ Stull, ${ }^{2} \mathrm{~A}$ Heidari. ${ }^{1}$ Adtalem Global Education Inc, Downers Grove, IL; ${ }^{2}$ Kern Medical Center, Bakersfield, CA

\subsection{6/jim-2022-WRMC. 182}

Case Report Anisakiasis is a parasitic disease of the gastrointestinal tract caused by Anisakis species or Pseudoterranova species. Humans acquire this disease by ingestion of raw or undercooked fish that are infected with larvae of these parasites. We describe a 17-year-old male who had consumed ceviche one week prior and presented after he coughed up a worm. The worm was identified as Pseudoterranova species. Epidemiology of food sources in Southwestern United States is discussed.

Methods Used Retrospective case study.
Summary of Results A 17-year-old male with no significant medical history presented to our hospital after he coughed up a worm earlier that morning. He also complained of ongoing rhinorrhea and sore throat for the past four days. He denied nausea, vomiting, rash, diarrhea, fever, chills, night sweats, hematochezia, hematemesis, abdominal pain, abdominal bloating, headache, weight loss, or change in appetite. Patient stated that he was from Mexico but had been living in the United States for the past two years. A dietary history revealed that one week ago he had eaten his favorite ceviche made from fresh fish brought by a family member visiting from Ensenada, Mexico. Patient's physical examination, lab values, and imaging were all unremarkable. He had brought the worm, which he had coughed up, to the hospital. The worm was sent to the pathology lab and identified as Pseudoterranova species. Patient was discharged with instructions to return if symptomatic. He was referred for follow-up in the outpatient setting.

Conclusion Anisakiasis is rare with current United States food handling regulations. The clinical suspicion is raised when raw fish from alternative sources of fresh seafood is consumed. Removal of the worm via endoscopy or even surgery might be necessary and is considered therapeutic. Diagnosis is made by direct visualization of the nematode.

\section{$\# 186$ MANDIBULAR OSTEOMYELITIS DUE TO AGGREGATIBACTER ACTINOMYCETECOMITANS}

${ }^{1} S$ Ratnayake* ${ }^{1,2} \mathrm{H}$ Sidhu, ${ }^{1} \mathrm{C}$ D'Assumpcao, ${ }^{1} \mathrm{G}$ Petersen, ${ }^{1} \mathrm{~A}$ Heidari. ${ }^{1}$ Kern Medical Center, Bakersfield, CA; ${ }^{2}$ Ross University School of Medicine, Miramar, FL

\subsection{6/jim-2022-WRMC.183}

Case Report Aggregatibacter actinomycetemcomitans is frequently associated with localized aggressive periodontitis. A. actinomycetemcomitans is a Gram-negative facultative anaerobe that is a member of the HACEK group of fastidious Gramnegative bacteria that can rarely cause endocarditis. We report a 21 year-old Hispanic male with osteonecrosis of the mandible from a tooth infected with $A$. actinomycetecomitans requiring antibiotics and surgical intervention.

Case Description A 21-year-old Hispanic male with no known past medical history presented to the emergency department with a purulent right jaw and neck abscess. Two weeks prior he began having right lower tooth pain. Over the next 10 days his pain and swelling progressed, limiting his ability to open his mouth and to eat and drink. He then noticed an enlarging mass over the right jaw and neck with purulent discharge. Intolerable pain brought him to the ED. Admission CT of the soft tissue of the neck found right mandibular angle and ramus osteomyelitis, adjacent masticator and sternocleidomastoid infectious myositis, reactive right parotiditis, and severe right cervical cellulitis. There were also small periapical abscess of the right mandibular first molar, and small pockets of localized edema in the right suprahyoid neck without organization and right upper cervical adenopathy. $\mathrm{He}$ was started on vancomycin and pipericillin-tazobactam. Ear nose and throat surgeon aspirated the abscess. Aspirated abscess culture grew A. actinomycetecomitans. Antibiotics were narrowed to ceftriaxone and metronidazole. ENT then performed incision and drainage of the right jaw and neck abscess and extracted two infected teeth. He was 
successfully discharged on post-operative day one on moxifloxacin with goal of 6 weeks of therapy with close ENT follow up.

Conclusion Early recognition and treatment of periodontal infections is important to prevent complications such as abscess formation and osteonecrosis from osteomyelitis. We report a prototypical example of an acute progression of a simple tooth ache developing into severe osteonecrosis by a rare HACEK organism not commonly encountered requiring aggressive antibiotics and surgical management.

\section{\#187 MALLET FINGER COMPLICATED BY DISTAL PHALANX OSTEOMYELITIS}

${ }^{1} \mathrm{D}$ Deng*, ${ }^{2} \mathrm{~N}$ Hatchard, ${ }^{3} \mathrm{R}$ Patel. ${ }^{1}$ Western University of Health Sciences College of Osteopathic Medicine of the Pacific-Northwest, Lebanon, OR; ${ }^{2}$ Philadelphia College of Osteopathic Medicine, Philadelphia, PA; ${ }^{3}$ Inspira Health Network, Vineland, NJ

\subsection{6/jim-2022-WRMC. 184}

Case Report A 24-year-old male presented with a right index finger injury which occurred during a soccer game. The mechanism of injury was the ball hitting the top of the second digit and lacerating the most distal portion of the digit. A diagnosis of mallet finger was made, and later on osteomyelitis of the distal phalanx.

The patient was initially evaluated in the emergency room where he was diagnosed with mallet finger and placed in a distal interphalangeal (DIP) extension QuickCast splint. A follow-up visit revealed five degree DIP flexion posture and ten degree proximal interphalangeal extension posture. DIP active range of motion flexion was noted at 30 degrees and only 45 degrees and painful for PIP active and passive range of motion. As X-ray of the right index finger revealed no evidence of fracture, dislocation, or degenerative changes, surgical intervention was not indicated at the time. However, patient was showing persistent edema, erythema, and pain 8 weeks after the initial accident. Follow-up MRI imaging identified fluid collection in the DIP joint. With suspicion for infectious process, surgical debridement of the finger was performed which revealed necrotizing tissue at the mid dorsal metaphysis of the distal phalanx spreading through a crevice to the germinal matrix. This impacted the patient's recovery and rehabilitation of the joint along with the structural and functional outcome. The patient was ultimately diagnosed through MRI and bone biopsy and found to have Enterobacter kobei osteomyelitis of the distal interphalangeal joint. The patient was treated with oral ciprofloxacin post-surgical debridement and was able to fully recover from the osteomyelitis infection. However, continued and permanent droop of the DIP with inability to regain full ROM, strength, and utility in the right index phalanx was seen during the patient's follow-up appointment.

Our aim with this case study is to demonstrate the possible complications of open mallet finger injury and its rehabilitation considerations. In this case, the patient's lacerated mallet finger led to osteomyelitis of the digit. While mallet finger itself is a very common injury, its recovery is rarely complicated by infection in previously healthy individuals.

This case highlights the importance of considering bone infection in non-healing joint injuries such as mallet finger and subsequently appropriately adjusting therapies to include prophylactic antibiotics and splinting to facilitate the structural and functional rehabilitation.
Poster session

Morphogenesis and malformations

\section{6:00 PM}

\section{Thursday, January 20, 2022}

\section{\#188 PREAXIAL POLYDACTYLY: CHARACTERIZING SONIC HEDGEHOG REGULATION}

${ }^{1} \mathrm{M}$ Malone* ${ }^{2} \mathrm{~K}$ Ball, ${ }^{3} \mathrm{C}$ Pira, ${ }^{3} \mathrm{~K}$ Oberg. ' Loma Linda University School of Medicine, Loma Linda, CA; ${ }^{2}$ Loma Linda University Department of Basic Sciences, Loma Linda, CA; ${ }^{3}$ Loma Linda University, Loma Linda, CA

\subsection{6/jim-2022-WRMC.185}

Purpose of Study Preaxial polydactyly is associated with ectopic Sonic hedgehog $(S h h)$ expression in the presumptive thumb. Shh is secreted from the zone of polarizing activity (ZPA), the signaling center that directs limb development along the radialulnar (anterior-posterior) axis. Shh is necessary for development of the ulna and posterior four digits, while the thumb develops in the absence of Shh. A limb-specific enhancer, the ZPA regulatory sequence (ZRS), is necessary for $S h b$ expression, although little is known about how the ZRS restricts Shb expression to the ZPA. Single nucleotide variations (SNVs) within the ZRS cause ectopic $S h h$ expression resulting in preaxial polydactyly. These SNVs are spread across three highly conserved regions: peaks 1,2 , and 3 . We hypothesize that one or more of these peaks is necessary for ZRS activity.

Methods Used Plasmids containing full-length ZRS, or each peak respectively were electroporated into Hamburger-Hamilton stage 14 chicken embryo presumptive limb buds. The embryos were incubated for 48 hours then observed by fluorescence microscopy. Fluorescent activity of each peak was then compared to that of full-length ZRS.

Summary of Results Constructs containing peak 1 and peak 2 have no detectable activity in the limb. Peak 3, however, maintains some activity, but markedly less than full-length ZRS.

Conclusions Peak 3 is necessary and sufficient for activity while peaks 1 and 2 are not, suggesting peak 3 contains core sequences necessary for ZRS activity. It is surprising that peaks 1 and 2 lack activity as many clinically relevant SNVs occur in these two regions and the reported Hand 2 binding site is in peak 2. Since peak 3 activity is less than that of full-length ZRS, peaks 1 and 2 likely enhance the activity of peak 3 . Further studies will assess the quantitative difference in ZRS peak activity and determine how the ZRS localizes Sh expression to the ZPA. Mapping the ZRS will clarify the role of critical sequences in $S h h$ regulation that contribute to limb development and malformation.

\section{\#189 VARIATIONS IN THE PERONEUS TERTIUS MUSCLE: EVOLUTIONARY AND CLINICAL PERSPECTIVES}

K Kay*, J Scott, M Wedel. Western University of Health Sciences, Pomona, CA

10.1136/jim-2022-WRMC.186

Purpose of Study Peroneus tertius (PT) is a muscle in the anterior compartment of the leg that functions in dorsiflexion and eversion of the foot at the ankle. PT was long thought to be unique to humans, but it is now known to be variably present 
in many other primates, including bonobos, chimpanzees, gorillas, and several species of monkeys. However, there is still disagreement between anthropologists and comparative anatomists regarding the evolutionary origin of the muscle. PT is highly variable in origin, insertion, size, and number of musculotendinous slips. Previously published studies have also reported PT to be extremely variable in terms of prevalence, ranging between $38 \%$ and $100 \%$ in different populations. Our goals are to summarize the evolutionary origin, anatomical variations, and clinical implications of the muscle.

Methods Used We synthesized information from the literature on the evolution, prevalence, variability, and clinical correlations of the PT using Google Scholar, NCBI, and ResearchGate. For statistical analysis, we measured prevalence using the number of limbs with PT present per number of legs total. In particular, we compared studies that observed the muscle in human cadavers $(n=11)$ versus studies that used palpation on living subjects $(n=11)$.

Summary of Results Numerous variants of PT have been identified, including an origin from the extensor hallucis longus. The muscle is occasionally doubled, in both humans and gorillas. Peroneus tertius is associated with multiple causes of ankle pain, and can be used in surgery for repair of other tendons. We found that the prevalence of PT varies depending on the method of data collection. Studies that use cadaveric dissection consistently report a significantly $(\mathrm{p}<0.0001)$ higher prevalence of PT $(86-100 \%)$ than studies that rely on palpation of the muscle in living subjects (38-84\%).

Conclusions Although the higher prevalence of PT in humans than in other primates has been linked to the evolution of bipedality, PT is not crucial for an erect bipedal gait. Recent studies have found no significant difference in ROM or in the strength of dorsiflexion or eversion among patients with and without PT. PT has been used in tendoplasty, tendon transfer, resection surgeries, transposition to correct ankle laxity, and transplantation surgeries for foot drop. The significant difference between dissection and palpation studies in the reported prevalence of PT suggests that palpation consistently underestimates the true prevalence of the muscle. We suggest that the variability in the size and course of PT, as well as with the potential for a small PT to be difficult to palpate through the extensor retinaculum, combine to make the muscle difficult to diagnose via palpation. Although palpation studies are potentially faster and less expensive than dissection-based studies, we urge caution in interpreting their results.

\section{Poster session}

\section{Neonatology general}

\section{6:00 PM}

\section{Thursday, January 20, 2022}

\section{\#190 GENETIC ASSOCIATIONS WITH PREECLAMPSIA, INTRAUTERINE GROWTH RESTRICTION, AND SPONTANEOUS PRETERM BIRTH}

${ }^{1}$ AL Baranoff*, ${ }^{1,2}$ A Paquette. 'Seattle Children's Research Institute, Seattle, WA; ${ }^{2}$ University of Washington School of Medicine, Seattle, WA

10.1136/jim-2022-WRMC. 187
Purpose of Study Pregnancy-related conditions are attributable to a combination of factors and may increase one's risk for later disease. Three of the most common conditions are preeclampsia (PE), intrauterine growth restriction (IUGR), and spontaneous preterm birth/delivery (SPTB/SPTD). The genetic basis of these conditions is illustrated through the findings that women who experience SPTB/D are at increased risk of subsequent SPTB/D of the same gestational age, and women who experienced PE or IUGR are also more likely to experience these same complications in subsequent pregnancies. We investigated the genetic profiles of each outcome and analyzed which maternal and fetal genomic variants are most associated with multiple pregnancy complications. This knowledge will increase our ability to better treat and/or prevent adverse pregnancy outcomes by identifying persons who may be at higher risk of complications prior to their manifestation in disease.

Methods Used We conducted a literature review of studies that assessed the association between fetally and/or maternallybased single nucleotide polymorphisms (SNPs) and PE, IUGR, and $\mathrm{SPTB} / \mathrm{D}$. We selected genetic variants that were significantly associated with these pregnancy outcomes in an initial candidate gene study, genome-wide association study (GWAS), or summarized in a meta-analysis (MA). The significance of association between SNP and pregnancy outcome was reported at either a study-wide significance level (for GWAS and MA studies) or $\mathrm{p}<0.05$ for candidate gene studies.

Summary of Results Our search yielded 103 articles, of which 59 investigated genetic associations in PE, 27 in IUGR, and 18 in SPTB. We identified five variants found in both PE and IUGR: rs1918975, rs10774624, rs3184504, rs4769612, rs1884082 which were in regulatory regions of four genes: FLT1, SERPINA3, MECOM, and SH2B3. We identified no variants found in both IUGR and SPTB. We identified five genes that contained SNPs in both IUGR and SPTB (ADCY5, WNT4, IGF1R, EBF1, IGF1), although none of the SNPs overlapped. No genetic variations or gene profiles were found to be shared amongst all adverse pregnancy outcomes.

Conclusions We identified five genetic variants associated with both PE and IUGR which correlated to four genes as well as five genes associated with both IUGR and SPTB. Several of these gene variants are also risk factors for the development of diseases that impact health throughout life, such as cardiovascular and kidney disease as well as neurological delay, revealing that adverse pregnancy outcomes and adult disease have shared and complex genetic risk factors. The contribution of the identified shared genetic variants in the pathogenesis of PE, IUGR, and SPTB/D should be the focus of future studies.

\section{$\# 191$ FACTORS ASSOCIATED WITH HYPOTHERMIA AND HYPOGLYCEMIA ON ADMISSION IN VERY LOW BIRTH WEIGHT INFANTS}

C Marquez*, K Ramm, Y Shao, NS Nanduri, A Hisey, L Barton, R Ramanathan, M Biniwale. LAC + USC Medical Center, Los Angeles, CA

\subsection{6/jim-2022-WRMC.188}

Purpose of Study Maintaining temperature and glucose in preterm infants are vital as these abnormalities can predispose them to many undesirable complications in early neonatal period. The present study was conducted to identify the 


\section{Abstract \#191 Table 1}

\begin{tabular}{llll}
\hline & $\begin{array}{l}\text { Hypothermia on } \\
\text { admission }\end{array}$ & $\begin{array}{l}\text { Normothermia } \\
\text { on admission }\end{array}$ & P value \\
\hline Birth weight (g) & 942 & 991 & 0.116 \\
Gestational age (wks) & 28 & 28 & 0.483 \\
Resuscitation in delivery room (\%) & 42.8 & 31 & 0.008 \\
Chest compressions (\%) & 19.6 & 10.3 & 0.006 \\
Epinephrine in delivery room (\%) & 15.1 & 8.1 & 0.018 \\
Median 5 min apgar score & 6 & 7 & 0.013 \\
Metabolic acidosis on first gas (\%) & 11.6 & 3.4 & 0.001 \\
Lowest pH & 7.21 & 7.25 & 0.015 \\
\hline
\end{tabular}

factors associated with hypothermia and hypoglycemia in very low birth weight (VLBW) preterm infants.

Methods Used The data consisting of VLBW infants born at our hospital was collected retrospectively from 2009 through 2021. Maternal factors, delivery room events and early neonatal morbidities were analyzed against infant's first temperature and glucose done on admission to NICU. Hypothermia was defined as temperature $<36.5$ C. Hypoglycemia was defined as blood glucose $<45 \mathrm{mg} / \mathrm{dl}$ on admission checked by point of care testing. IRB approval was obtained to review the data from electronic medical records. SPSS version 28 statistical software was used to analyze the data.

Summary of Results From all VLBW infants born during this period 152/642 (23.7\%) had temperature below $36.5 \mathrm{C}$ on admission to NICU while $104 / 652(15.9 \%)$ were diagnosed to have hypoglycemia. Birth weight or gestational age had no impact on either hypothermia or hypoglycemia on NICU admission. Infants needing resuscitation including chest compressions and epinephrine administration were at highest risk for hypothermia. These infants were also noted to have metabolic acidosis and low $5 \mathrm{~min}$ apgar scores. Infants presented with hypoglycemia were small for gestational age $(37 \%$ vs $24 \% \mathrm{p}=0.009)$. Maternal medical conditions including diabetes did not put these infants at additional risk for hypoglycemia. Hypoglycemia on admission was also associated with additional risk of requiring higher ventilation as well as oxygen requirement in the first 24 hours of NICU stay.

Conclusions VLBW Infants needing resuscitation in the delivery room are at risk for hypothermia. These infants may present with metabolic acidosis on admission to NICU. Hypoglycemia on admission may predispose VLBW infants for more respiratory support.

\section{\#192 THE EFFECT OF SARS-COV-2 ON THE RATES OF BREASTFEEDING IN THE NEWBORN NURSERY}

1J Wang*, ${ }^{2} \mathrm{AF}$ Ahmed, ${ }^{2} \mathrm{R}$ Ramanathan, ${ }^{2} \mathrm{~A}$ Yeh. ${ }^{1} \mathrm{LAC}+$ USC Medical Center/USC, LoS Angeles, CA; ${ }^{2}$ LAC+USC Medical Center, Keck School of Medicine of USC, LA, CA

\subsection{6/jim-2022-WRMC.189}

Purpose of Study Exclusive breastfeeding for the first six months of life is recommended by the American Academy of Pediatrics and the Centers for Disease Control for its benefits to infant immunity, maternal-child bonding, and long-term health. While these benefits are well studied, the SARS-CoV-2 pandemic raises questions about the safety of breastfeeding among SARS-CoV-2-positive mothers. In addition, the pandemic's effects on hospital staffing, patient-provider facetime, and healthcare access may impact breastfeeding rates. This study aims to explore the effect of the SARS-CoV-2 pandemic on breastfeeding in the newborn nursery.

Methods Used This is a retrospective cohort study comparing breastfeeding rates between neonates at LAC+USC Medical Center Newborn Nursery from January 2019 to April 2021. We defined the pre-SARS-CoV-2 group as all neonates born prior to April 2020, and the during-SARS-CoV-2 group as those born from April 2020 to April 2021. Maternal data gathered included gravidity and parity, ethnicity, age, mode of delivery, and pregnancy complications. Infant data gathered included gestational age, birth weight, sex, and hyperbilirubinemia requiring intensive phototherapy. Newborns with maternal contraindications to breastfeeding, such as positive toxicology screen, positive HIV status, incarceration, and placement in foster care were excluded. Rates of exclusive breastfeeding and any breastfeeding were calculated for each month within this time period and compared using T-test. Pvalue less than 0.05 was considered significant.

Summary of Results Of the 964 newborns screened in the preSARS-CoV-2 cohort, 913 were included. Of the 800 screened during-SARS-CoV-2 cohort, 763 newborns were included. There were no significant differences in the demographics between the two cohorts (table 1). We found a 11\% decrease in the rate of exclusive breastfeeding $(p<0.05)$ and a $4 \%$ decrease in any breastfeeding $(p<0.05)$ during the SARSCoV-2 period (Image 1).

Conclusions The SARS-CoV-2 pandemic had a negative impact on the rates of both exclusive breastfeeding and any

Abstract \#192 Table 1 Maternal and neonatal demographics and characteristics

\begin{tabular}{|c|c|c|}
\hline & Pre-SARS-CoV2 & During-SARS-CoV2 \\
\hline \multicolumn{3}{|l|}{ Study Subjects } \\
\hline Total Newborns Born & 964 & 800 \\
\hline Total Newborn Included In This Study & 913 & 763 \\
\hline \multicolumn{3}{|l|}{ Exclusion Criteria } \\
\hline Drug Screen Positive\% & 1.5 & 2.0 \\
\hline Maternal Incarceration \% & 2.4 & 1.9 \\
\hline DCFS Case $\%$ & 1.7 & 0.8 \\
\hline \multicolumn{3}{|l|}{ Baby Demographics } \\
\hline Hispanic or Latino\% & 81.6 & 83.2 \\
\hline African American $\%$ & 8.8 & 7.3 \\
\hline Others\% & 9.6 & 9.4 \\
\hline \multicolumn{3}{|l|}{ Maternal Characteristics } \\
\hline Average Maternal Age & 28.3 & 29.0 \\
\hline Average Maternal Gravida & 2.7 & 2.9 \\
\hline Average Maternal Para & 2.2 & 2.3 \\
\hline Maternal Preeclampsia\% & 4.4 & 6.0 \\
\hline Infant of Diabetic Mother\% & 10.5 & 13.1 \\
\hline \multicolumn{3}{|l|}{ Baby Characteristics } \\
\hline Average Baby Gestational Age & 38.9 & 38.9 \\
\hline Preterm Baby\% & 8 & 8.4 \\
\hline Birthweight $<2.5 \mathrm{~kg} \%$ & 4.1 & 4.1 \\
\hline Birthweight $>4 \mathrm{~kg} \%$ & 7.1 & 6.2 \\
\hline Baby Underwent Intensive Phototherapy\% & 10.3 & 10.5 \\
\hline \multicolumn{3}{|l|}{ Breastfeeding Rate } \\
\hline Exclusive Breastfeeding Rate\% & 73.2 & 62.4 \\
\hline Any Breastfeeding Rate\% & 94.6 & 91.2 \\
\hline
\end{tabular}



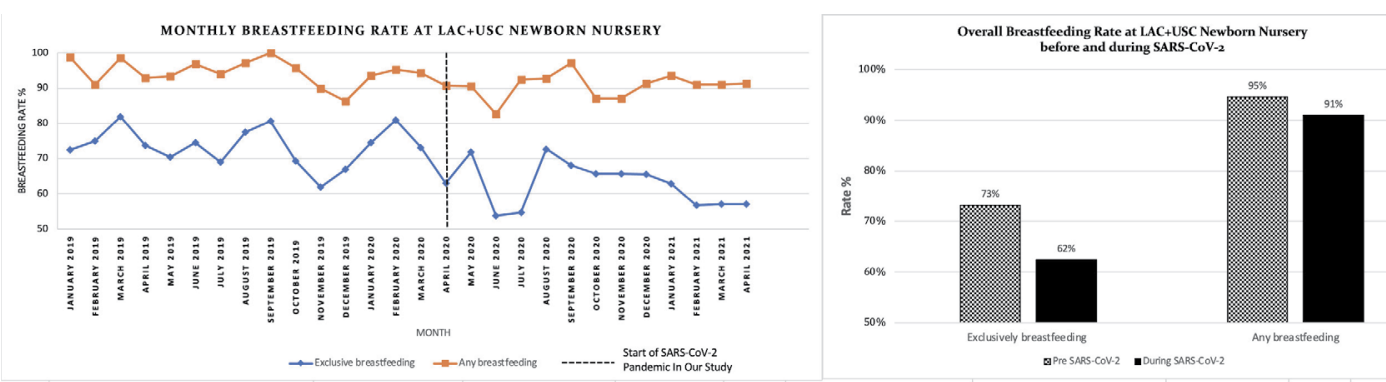

Abstract \#192 Figure 1 Breastfeeding rate at LAC+USC medical center before and during SARS-CoV-2

breastfeeding among newborns in the normal nursery from a single center in Los Angeles. These results prompted the creation of a specific task force to counter the detrimental effect of the pandemic on breastfeeding. Prospective studies would be useful in assessing the long-term effects of the SARS-CoV-2 pandemic on breastfeeding rates and associated effects on infant immunity, maternal-child bonding, and long-term health.

\section{\#193 EXPECTED GROWTH TRENDS IN A LARGE COHORT OF ALMOST 7000 PRETERM INFANTS FROM BIRTH TO EIGHTEEN YEARS}

1,2J Barnard*, ${ }^{2}$ A Defante, ${ }^{1,2}$ J Ryu. ${ }^{1}$ University of California San Diego, La Jolla, CA; ${ }^{2}$ Rady Children's Hospital San Diego, San Diego, CA

\subsection{6/jim-2022-WRMC. 190}

Purpose of Study Despite numerous studies about the growth of preterm infants (PI) postnatally, there is still no consensus on expected growth rates for PI through childhood. The standard of care is to correct for gestational age (GA) until age two years during which time PI are expected to 'catch up.' In addition to correcting for GA, there may be a need to account for growth restriction. There are conflicting studies on whether infants born small for gestational age (SGA) 'catch up' by age two years. However, to the best of our knowledge, there are no studies with this cohort size following the growth of SGA PI over 18 years.

Methods Used This retrospective cohort study of 6916 followed infants born between 23-32 weeks over 18 years. Data was pulled from Rady Children's Hospital electronic medical record system which includes specialists as well as over 30 general pediatrician offices. Being the main institution for follow up of premature infants in a very large catchment area allowed for longitudinal follow up of a large cohort. Infants were categorized as SGA if their birthweight was $\leq$ tenth percentile birthweight for their GA, AGA if tenth to ninetieth percentile and LGA if $\geq 90$ th percentile based on the WHO Fetal Growth Charts. Their weights and BMIs at ages two through 18 years were categorized as below the tenth percentile, between the tenth and ninetieth percentiles and above the ninetieth percentile by CDC standards.

Summary of Results Using a Chi Squared test, preliminary results show statistically significant differences ( $\mathrm{p}$-values all $<$ 0.001) in the counts of PI who are below the tenth percentile, between the tenth and ninetieth percentiles and above the ninetieth percentile by CDC standards at ages two through 18 years based on whether they were born SGA, AGA, or LGA. Those born SGA had more infants remain 'small' than those born AGA or LGA. But for all groups, we observed significantly more PI stay 'small,' less than the tenth percentile for weight, at ages two through 18 years than expected. This held true for BMI as well for ages two through 18 years for PI born SGA, AGA, or LGA with p-values all $<0.001$.

Conclusions As more extremely premature and very low birth weight infants are surviving, there is a need for further assessment of this subpopulation's expected postnatal growth. The observed distribution of PI over two through 18 years differed significantly by their size at birth. Infants born SGA may continue below the tenth percentile for weight for several years and this may not be 'abnormal' growth for them. These infants may be seen by various specialists for failure to thrive but might just need different standards. This study validates the need for different expectations of growth for infants born growth-restricted and very premature.

\section{\#194 GENERAL MOVEMENT ASSESSMENTS IN THE SURGICAL GASTROINTESTINAL NEONATAL POPULATION}

S Bell, S Espinosa*, K Kesavan, KL Calkins. University of California LoS Angeles, LoS Angeles, CA

\subsection{6/jim-2022-WRMC.191}

Purpose of Study Infants with gastrointestinal disorders (GD) have increased survivorship with advances in neonatal medicine and surgery. GD infants are at risk for neurodevelopmental impairment; they require surgery and are at high risk for sepsis, growth failure, and prolonged hospital stays. There is evidence that the General Movement Assessment (GMA) is an early biomarker of motor delays, including cerebral palsy. Most studies have focused on extremely low birth weight infants (ELBWs) and have neglected GD infants.

Methods Used In this retrospective single-site study, GD infants (i.e, gastroschisis, omphalocele, atresias) who underwent surgery within the first 90 days of life were compared to ELBWs (2/20/18 - 5/01/2021). The primary outcome was GMA results during the writhing stage at 36-49 weeks corrected gestational age (normal or abnormal (poor repertoire (PR), cramped synchronous (CS), or chaotic)) and fidgety stage at 3-4 months corrected gestational age (normal or abnormal). Abnormal fidgety was defined as fidgety movements that were not observed or movements that have exaggerated amplitude, speed, or jerkiness.

Summary of Results There were 55 GD infants; 31 (56\%) had at least one GMA. There were 33 ELBWs; 28 (85\%) with one GMA. Gestational age and birth weight were significantly different when the cohorts were compared. However, the

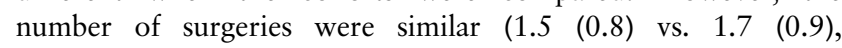
$\mathrm{p}=0.3)$ for the GD an ELBW cohort, respectively (table 1). Of the GD infants, $60 \%$ had an abnormal writhing stage GMA (0\% CS, 60\% PR) and 20\% had an abnormal fidgety 
Abstract \#194 Table 1 Characteristics of GD and ELBW infants

\begin{tabular}{lll}
\hline & GD (n=31) & ELBW (n=28) \\
\hline Maternal age (years) & $30(7)$ & Mean (SD), $\%$ \\
Cesarean\% & 48 & $34(6)^{*}$ \\
Maternal gravida & $2(2)$ & $89^{*}$ \\
Maternal parity & $2(1)$ & $2(2)$ \\
Chorioamnionitis\% & 10 & $1.6(0.8)$ \\
Female\% & 39 & 11 \\
Gestational age (weeks) & $36(4)$ & 50 \\
Birth weight (kg) & $2.5(0.9)$ & $26(2)^{*}$ \\
Birth length (cm) & $45(7)$ & $0.7(0.2)^{*}$ \\
Birth head circumference (cm) & $32(4)$ & $32(3)^{*}$ \\
Small for gestational age\% & 29 & $23(2)^{*}$ \\
Maternal tobacco use & 7 & 36 \\
Maternal illicit drug use & 17 & 11 \\
Length of stay (days) & $44(42)$ & $0^{*}$ \\
Necrotizing enterocolitis & 3 & $106(29)^{*}$ \\
Late onset sepsis & 3 & 11 \\
Age of first feed (days) & $11(12)$ & 19 \\
Total number of surgeries & $1.5(0.8)$ & $5(8)^{*}$ \\
\hline * $p$ & & $1.7(0.9)$ \\
\hline
\end{tabular}

stage GMA. Of the ELBW infants, 35\% had abnormal writhing stage GMA (15\% CS, 20\% PR) and 22\% had an abnormal fidgety stage GMA. Rates for abnormal GMAs were similar when the groups were compared $(p=0.1,0.9)$.

Conclusions In this study, GD and ELBW infants were at high risk for abnormal GMAs. GMAs maybe a helpful tool to ensure that GD infants receive long-term follow-up and resources required to reach their developmental potential. Further longitudinal research is required to determine the accuracy of GMAs in the GD population.

\section{\#195 ABNORMAL BLOOD GAS AND OXYGEN REQUIREMENTS IN FIRST 24 HOURS OF LIFE AS INDICATORS OF MORBIDITY IN VERY LOW BIRTH WEIGHT INFANTS}

${ }^{1,2} \mathrm{NS}$ Nanduri ${ }^{*},{ }^{1,3} \mathrm{~A}$ Hisey, ${ }^{1} \mathrm{Y}$ Shao, ${ }^{1} \mathrm{~K}$ Ramm, ${ }^{1} \mathrm{~L}$ Barton, ${ }^{1} \mathrm{R}$ Ramanathan, ${ }^{1} \mathrm{M}$ Biniwale. ${ }^{1}$ Los Angeles County University of Southern California Medical Center, Los Angeles, CA; ${ }^{2}$ Drexel University College of Medicine, Philadelphia, PA; ${ }^{3}$ Loyola University Chicago Stritch School of Medicine, Maywood, IL

10.1136/jim-2022-WRMC.192
Purpose of Study Oxygen requirement as well as abnormal blood gas values have often been used as indicators of morbidity in the premature neonate, however, there is mixed evidence regarding its utility to predict short and long term morbidity. This study aims to assess the impact of abnormal pCO2, pH, and $\mathrm{FiO} 2$ values in the first 24 hours of life of very low birth weight (VLBW) infants on short and long term outcomes.

Methods Used Data on all VLBW infants was retrospectively gathered from the electronic medical record between the years of 2009 and 2021. IRB approval was obtained prior. For blood gas levels, significance was calculated based on $\mathrm{pH}$ of 7.15 and $\mathrm{CO} 2$ of $50 \mathrm{~mm} \mathrm{Hg}$. $\mathrm{FiO} 2$ was considered significant if the infant required $50 \%$ in the first 24 hours of life. Each category was statistically analyzed against common neonatal outcomes including intraventricular hemorrhage (IVH), bronchopulmonary dysplasia (BPD), and retinopathy of prematurity (ROP).

Summary of Results Of 564 VLBW infants studied $46.6 \%$ had an abnormal pCO2 and 53.4\% had a normal pCO2. $78.7 \%$ of these infants had an abnormal pH and $19.5 \%$ had a normal pH. $87.2 \%$ had a normal $\mathrm{FiO} 2$ and $12.8 \%$ had an abnormal FiO2. Infants with elevated $\mathrm{pCO} 2$, decreased $\mathrm{pH}$, and high FiO2 were significantly more likely to need intubation in the delivery room, emergently, remained intubated at 24 hours and had significantly higher days of invasive mechanical ventilation (table 1). These infants were more likely to need surfactant and had higher mortality (table 1). Lastly, these infants were at increased risk of IVH and ROP (table 1). BPD was correlated with higher $\mathrm{FiO} 2$ as well as low $\mathrm{pH}$. Neonates with abnormal $\mathrm{FiO} 2$ also showed an increased risk for having abnormal MRI before discharge $(51.6 \%$ vs $69.0 \% \mathrm{p}=0.034)$.

Conclusions Abnormal blood gas and higher $\mathrm{FiO} 2$ in the first 24 hours of life is associated with increased need for intubation as well as invasive mechanical ventilation in the delivery room and NICU. VLBW infants showed increased likelihood of developing long term complications including BPD, severe $\mathrm{IVH}$, and severe ROP.

\section{\#196 INTUBATION IN THE FIRST 24 HOURS AS AN INDICATOR OF NEONATAL MORBIDITY}

${ }^{1} \mathrm{~N}$ Nanduri*, ${ }^{1,2} \mathrm{~A}$ Hisey, ${ }^{1} \mathrm{Y}$ Shao, ${ }^{1} \mathrm{~K}$ Ramm, ${ }^{1} \mathrm{C}$ Marquez, ${ }^{1} \mathrm{~L}$ Barton, ${ }^{1} \mathrm{R}$ Ramanathan, ${ }^{1} \mathrm{M}$ Biniwale. 'Los Angeles County University of Southern California Medical Center, Los Angeles, CA; ${ }^{2}$ Loyola University Chicago Stritch School of Medicine, Maywood, IL

10.1136/jim-2022-WRMC.193

\section{Abstract \#195 Table 1}

\begin{tabular}{|l|ccc|ccc|ccc|}
\hline & \multicolumn{3}{|c|}{$\mathrm{pCO} 2$} & \multicolumn{3}{c|}{$\mathrm{pH}$} & \multicolumn{3}{c|}{ FiO2 } \\
\hline & $<50$ & $>50$ & $\mathrm{p}$-value & $>7.15$ & $<7.15$ & $\mathrm{p}$-value & $<50$ & $>50$ & $\mathrm{p}$-value \\
\hline Death & $3.0 \%$ & $13.7 \%$ & $<0.001$ & $3.1 \%$ & $29.8 \%$ & $<0.001$ & $4.1 \%$ & $29.0 \%$ & $<0.001$ \\
Duration of invasive ventilation & 8.8 & 26.4 & $<0.001$ & 13.9 & 32.8 & 0.003 & 27.0 & 29.3 & 0.010 \\
Intubation in delivery room & $37.0 \%$ & $54.9 \%$ & $<0.001$ & $40.4 \%$ & $65.9 \%$ & $<0.001$ & $35.6 \%$ & $72.5 \%$ & $<0.001$ \\
Emergency intubation & $32.1 \%$ & $55.0 \%$ & $<0.001$ & $37.5 \%$ & $65.5 \%$ & $<0.001$ & $36.9 \%$ & $59.0 \%$ & 0.008 \\
Intubated at 24 hours & $26.6 \%$ & $67.9 \%$ & $<0.001$ & $37.7 \%$ & $82.8 \%$ & $<0.001$ & $36.9 \%$ & $88.6 \%$ & $<0.001$ \\
Surfactant & $49.8 \%$ & $64.3 \%$ & 0.002 & $53.4 \%$ & $69.8 \%$ & 0.006 & $48.4 \%$ & $59.2 \%$ & 0.089 \\
Severe IVH & $2.3 \%$ & $9.8 \%$ & 0.01 & $2.4 \%$ & $20.4 \%$ & $<0.001$ & $3.6 \%$ & $20.6 \%$ & 0.001 \\
Severe ROP & $5.5 \%$ & $21.9 \%$ & $<0.001$ & $8.5 \%$ & $36.6 \%$ & $<0.001$ & $10.3 \%$ & $30.4 \%$ & 0.011 \\
BPD & $48.4 \%$ & $59.4 \%$ & 0.055 & $50.8 \%$ & $65.5 \%$ & 0.043 & $4.1 \%$ & $29.0 \%$ & $<0.001$ \\
\hline
\end{tabular}




\begin{tabular}{|c|c|c|c|}
\hline & $\begin{array}{l}\text { Invasive ventilation in } \\
\text { the first } 24 \text { hours (\%) }\end{array}$ & $\begin{array}{l}\text { Non invasive ventilation in } \\
\text { the first } 24 \text { hours (\%) }\end{array}$ & $P$ value \\
\hline Birth Weight & $749.7 \pm 279 \mathrm{~g}$ & $1100 \pm 253 \mathrm{~g}$ & $<0.001$ \\
\hline Gestational Age & $25.9 \pm 2.3$ weeks & $29.1 \pm 2.3$ weeks & $<0.001$ \\
\hline $\begin{array}{l}\text { Histologic } \\
\text { Chorioamnionitis }\end{array}$ & 18.7 & 6.8 & 0.002 \\
\hline $\begin{array}{l}\text { Intubation in the } \\
\text { delivery room }\end{array}$ & 76.5 & 24.3 & $<0.001$ \\
\hline $\begin{array}{l}\text { Chest } \\
\text { compressions }\end{array}$ & 33.8 & 4.5 & $<0.001$ \\
\hline Severe IVH & 10.2 & 1.1 & $<0.001$ \\
\hline PDA & 75.2 & 31.4 & $<0.001$ \\
\hline $\begin{array}{l}\text { PDA requiring } \\
\text { surgery }\end{array}$ & 26.5 & 2.9 & $<0.001$ \\
\hline $\begin{array}{l}\text { Abnormal brain } \\
\text { MRI }\end{array}$ & 61.0 & 33.7 & $<0.001$ \\
\hline BPD & 63.3 & 25.1 & $<0.001$ \\
\hline Severe ROP & 82.9 & 17.1 & $<0.001$ \\
\hline
\end{tabular}

Purpose of Study Delivery room intubation in the premature neonate has been previously correlated with an increased risk for neonatal morbidities such as bronchopulmonary dysplasia (BPD). Invasive ventilation for longer duration also puts these infants at similar risk. This study further assessed relationships between infants needing invasive ventilation at 24 hours of life and short term neonatal outcomes.

Methods Used Retrospective data of preterm VLBW infants born between 2009 and 2021 at LAC + USC Medical Center was evaluated for invasive ventilation at 24 hours of life and common neonatal morbidities. Institutional IRB approval was obtained. Maternal factors, delivery room interventions and standard neonatal outcomes were analyzed.

Summary of Results Out of the 313 infants meeting inclusion criteria, $136(43.5 \%)$ required invasive ventilation beyond 24 hours of life. Infants born to mothers with histologic chorioamnionitis were more likely to need invasive respiratory support at 24 hours. These infants had lower birth weight as well as lower gestational age. Resuscitation in the delivery room including intubation and chest compressions were also strongly correlated. These infants had a higher incidence of patent ductus arteriosus (PDA) as well as severe intraventricular hemorrhage (IVH). Bronchopulmonary dysplasia (BPD), severe retinopathy of prematurity (ROP) and MRI abnormalities by term gestation were also more often seen.

Conclusions VLBW infants who had invasive ventilation in the first 24 hours of life were more likely to have BPD, PDA, abnormal brain MRIs, and severe ROP during their NICU stay.

\section{\#197 CHARACTERIZING RISK FACTORS AND INVESTIGATING TESTING AT BIRTH FOR CONGENITAL HEPATITIS C VIRUS INFECTION}

${ }^{1} \mathrm{H} \mathrm{Ko}{ }^{*},{ }^{2} \mathrm{M}$ Dodd, ${ }^{2} \mathrm{~T}$ Borunda, ${ }^{1} \mathrm{~K}$ Page, ${ }^{3} \mathrm{I}$ Cervantes, ${ }^{1} \mathrm{~J}$ Maxwell, ${ }^{1} \mathrm{RO}$ Castillo. ${ }^{1}$ University of New Mexico Health Sciences Center, Albuquerque, NM; ${ }^{2}$ Rhodes Group, Albuquerque, $N M{ }^{3}$ University of New Mexico School of Medicine, Albuquerque, NM

10.1136/jim-2022-WRMC.194
Purpose of Study Hepatitis C virus (HCV) is the leading cause of blood-borne infection globally with an associated increase from the ongoing opioid epidemic. Current recommendations call for antibody screening of HCV-exposed infants after 18 months of age or RNA testing after 2 months of age, however, studies have shown low compliance. We hypothesize that many women with HCV do not receive appropriate screening during pregnancy resulting in gaps in infant care. We seek to identify factors associated with suboptimal pediatric HCV screening that could improve screening and subsequent treatment in perinatally exposed and chronically infected children.

Methods Used A retrospective chart review was completed using data obtained by Tricore Laboratories. The data assessed the yearly proportion of $\mathrm{HCV}$ in pregnant women who were tested from 2014-2019 at our institution, characterize their demographic and health information, and identify their infants and HCV testing status. Demographics of mothers and infants with HCV testing were compared to those without testing to determine if certain demographics portend a greater probability of follow up care for infants with possible congenital HCV infection.

Summary of Results From 2014-2019, a total of 14,709 women delivered at our institution with $63 \% \quad(n=9,310)$ receiving $\mathrm{HCV}$ testing. Of these women, 139 (1.5\%) were antibody positive; 107 mother-infant pairs were included in our analysis. Only 29 infants (27\%) had antibody testing and 4 infants (3.7\%) received viral load testing. One child was found to be antibody and viral load positive. The majority of these infants $(n=81)$ were discharged to their birth parent from the nursery or neonatal ICU regardless of testing status. Mean infant gestational age, mothers' gravida/parity, liver enzyme levels, time between initial positivity of the mother and birth of infant, and maternal viral load at prenatal care onset did not differ significantly between the infant groups. However, urine positivity for opioid replacement therapy (ORT; methadone or buprenorphine) appeared to approach significance $(p=0.08)$ for mothers whose infants were tested. Maternal and infant ALT levels showed a 0.34 correlation.

Conclusions Mothers receiving ORT were more likely to have infant testing completed. This could be partly due to involvement of these mothers in programs which subsequently screen their infants. Additionally, a correlation between maternal and infant ALT levels seemed to exist however it is known that LFTs can fluctuate in those with HCV and in newborns. Thus, we are unable to conclude that this finding was clinically significant especially given the small number of infants who had LFT testing.

\section{\#198 PAID FAMILY LEAVE AND VERY LOW BIRTHWEIGHT INFANT HEALTH OUTCOMES}

1J Feister*, ${ }^{1} \mathrm{H}$ Lee, ${ }^{2} \mathrm{~L}$ Greenberg, ${ }^{3} \mathrm{M}$ Parker, ${ }^{4} \mathrm{M}$ Rossin-Slater, ${ }^{2} \mathrm{E}$ Edwards. ' $L$ ucile Salter Packard Children's Hospital at Stanford, Palo Alto, CA; ${ }^{2}$ Vermont Oxford Network, Burlington, VT; ${ }^{3}$ Boston University School of Medicine, Boston, MA; ${ }^{4}$ Stanford University, Stanford, CA

\subsection{6/jim-2022-WRMC.195}

Purpose of Study Paid family leave (PFL) is associated with improved infant health, potentially through increased breastfeeding. Little is known regarding the effects of PFL on very low birthweight (VLBW) infants, a population in which human breast milk (HBM) is critical. California (CA) was the first state to implement PFL in 2004. The primary aim of this 
study was to determine the impact of California's PFL program on use of $\mathrm{HBM}$ at discharge, necrotizing enterocolitis (NEC), and in-hospital mortality in VLBW infants.

Methods Used We conducted a quasi-experimental study by employing a difference-in-differences design using data from Vermont Oxford Network. PFL was defined as the intervention with CA as the exposed group and the rest of the Western U.S. (WUS) as the unexposed group. Singleton infants with birthweight $<1500 \mathrm{~g}$ cared for at VON participant hospitals in CA \& rest of the WUS from 2001-2010 were included. Infants with early mortality or congenital anomalies were excluded. Relative risk (RR) and adjusted RR (aRR) of each outcome for infants born pre 2004 and post 2004 (the year of PFL implementation) were calculated using multivariate regression models, controlling for maternal race/ethnicity, birthweight, mode of delivery, and antenatal steroids. Trends pre/post PFL in CA vs. the WUS were compared to identify the effect of PFL on the outcomes of interest.

Summary of Results Of 41,633 infants who met inclusion criteria, both CA and WUS infants were more likely to receive HBM at discharge post vs pre PFL enactment in CA (CA RR 1.19 [95\% CI 1.15-1.23], aRR 1.17 [1.11-1.24]); WUS RR 1.03 [0.99-1.07], aRR 1.04 [0.98-1.10]). Both groups had higher incidence of NEC post vs pre PFL (CA RR 1.32 [1.18-1.47], aRR 1.29 [1.15-1.45]); WUS RR 1.43 [1.251.64], aRR 1.43 [1.24-1.64]). Incidence of NEC was lower in CA compared to WUS throughout the study period. There were no significant differences in mortality in either group pre vs post PFL. There was a trend of increasing use of HBM at discharge in CA but not WUS both pre and post PFL. Trends in NEC and mortality did not differ between CA and WUS. Overall, no statistically significant effect of PFL on the outcomes of interest was found when comparing difference in differences.

Conclusions Implementation of PFL legislation in CA did not have a clear, significant impact on use of HBM at discharge, NEC, and mortality in VLBW infants. Inadequate duration or utilization of PFL may account for the lack of observed impact. PFL may also not be sufficient for families who spend long periods for NICU hospitalizations. Further research investigating individual level effects of PFL on VLBW infants and patterns of PFL utilization by families with infants in the NICU is warranted.

\section{\#199 TELEMEDICINE EXPOSURE AND TRAINING IN NEONATAL-PERINATAL MEDICINE FELLOWSHIP PROGRAMS: A NATIONAL SURVEY OF FELLOWSHIP DIRECTORS}

1J Rajkumar*, ${ }^{2} \mathrm{~K}$ Lund, ${ }^{1} \mathrm{~T}$ Hyunh, ${ }^{1} \mathrm{~A}$ Hoffman, ${ }^{1} \mathrm{~W}$ Lapcharoensap. ${ }^{1}$ Oregon Health and Science University, Portland, OR; ${ }^{2}$ University of Utah Health, Salt Lake City, UT

\subsection{6/jim-2022-WRMC.196}

Purpose of Study To describe the prevalence of Neonatal-Perinatal Medicine (NPM) fellow exposure to telemedicine, and the amount and type of telemedicine training fellows receive.

Methods Used This study is a cross-sectional national survey of NPM fellowship training program directors. The survey was distributed electronically via the Organization of Neonatal-Perinatal Medicine Training Program Directors (ONTPD) listserv and answers were collected using Qualtrics.
Abstract \#199 Table 1 Perception of telemedicine and fellow telemedicine training

\begin{tabular}{llll}
\hline & Disagree & Neutral & Agree \\
\hline $\begin{array}{l}\text { Telemedicine is an important aspect of NICU } \\
\text { practice }\end{array}$ & $2(11.1 \%)$ & $7(38.8 \%)$ & $9(50 \%)$ \\
$\begin{array}{l}\text { Fellows will encounter telemedicine in their future } \\
\text { career }\end{array}$ & $2(11.1 \%)$ & $4(22.2 \%)$ & $12(66.7 \%)$ \\
$\begin{array}{l}\text { Fellows should learn about telemedicine during } \\
\text { training }\end{array}$ & $2(11.1 \%)$ & $5(27.8 \%)$ & $12(66.7 \%)$ \\
$\begin{array}{l}\text { Fellows should participate in telemedicine during } \\
\text { training }\end{array}$ & $1(5.6 \%)$ & $6(33.3 \%)$ & $11(61.1 \%)$ \\
\hline
\end{tabular}

Summary of Results 21 individuals responded to the survey with 18 total completed surveys. $8(47.37 \%)$ programs had a neonatal telemedicine program. Of these, the types of consultation offered (number of programs in parentheses) included general neonatal consults (7), resuscitation guidance (3), counseling for fetal conditions or anomalies (4), perinatal viability counseling (3), remote rounding (1), and NICU follow-up (2). One program noted during the COVID pandemic, intrahospital consultations, rounds and parental visitation were being conducted via telemedicine. NPM fellows conducted the telemedicine consultations in 3 programs $(37.5 \%)$. One program started fellow consultations during the first year, and two started in the second year. One program provided real-time fellow oversight by an attending during the first year of training and the other 2 indicated none was required. No programs had specific training or curriculum for fellows conducting telemedicine consultations. The overall perception of telemedicine and fellowship telemedicine training is described in table 1. Programs were generally in agreement that telemedicine is important in modern NICU practice, that fellows would likely encounter it in their careers, and that telemedicine training should be provided during fellowship.

Conclusions Telemedicine has a rapidly expanding presence in neonatology. There appears to be minimal involvement of fellows throughout NPM fellowship programs. Further studies describing fellowship telemedicine training (including platform capabilities, demonstration of proficiency, communication techniques, documentation, medicolegal aspects, and simulated encounters) as well as the impact of such training on telemedicine program effectiveness are needed. Furthermore, development of expectations and curricula for telemedicine education in NPM fellowship should be standardized and widely adopted.

\section{\#200 INFANT AND MATERNAL FACTORS ASSOCIATED WITH DEVELOPING NECROTIZING ENTEROCOLITIS IN VERY LOW BIRTH WEIGHT INFANTS}

${ }^{1}$ MA Sacks*, ${ }^{1}$ YS Mendez, ${ }^{1} \mathrm{FA}$ Khan, ${ }^{2} \mathrm{G}$ Gollin, ${ }^{1} \mathrm{~A}$ Radulescu. ${ }^{1}$ Loma Linda University Adventist Health Sciences Center, Loma Linda, CA; ${ }^{2}$ Rady Children's Hospital San Diego, San Diego, CA

\subsection{6/jim-2022-WRMC.197}

Purpose of Study The purpose of this study was to understand the relationship between maternal and infant risk factors associated with developing necrotizing enterocolitis in premature infants. 


\begin{tabular}{|c|c|c|c|}
\hline \multicolumn{4}{|c|}{ Comparing groups characteristics } \\
\hline & $\begin{array}{c}\text { Without NEC } \\
(\mathrm{N}=58)\end{array}$ & $\begin{array}{c}\text { With NEC } \\
(\mathrm{N}=12)\end{array}$ & p-value \\
\hline \multicolumn{4}{|l|}{ Neonate factors $(\mathrm{N}, \%)$} \\
\hline Male & $31(53.4)$ & $6(50)$ & 0.920 \\
\hline Gestational age in weeks, mean (range) & $29.0(23.4-34.7)$ & $25.9(23.0-30.6)$ & $0.002 *$ \\
\hline Apgar score at 1 minute (mean) & 5.6 & 5.2 & 0.544 \\
\hline Apgar score at 5 minutes (mean) & 7.8 & 6.7 & 0.136 \\
\hline Singleton birth & $44(75.9)$ & $10(83.3)$ & 0.697 \\
\hline \multicolumn{4}{|l|}{ Maternal factors $(\mathrm{N}, \%)$} \\
\hline Income less than $\$ 30,000$ (USD)/year & $20(36.4)$ & $4(40)$ & 0.891 \\
\hline Conception age in years (mean, range) & $30.4(18-44)$ & $31.8(25-41)$ & 0.446 \\
\hline Medical conditions, during pregnancy & $23(41.8)$ & $5(41.7)$ & 0.754 \\
\hline Prenatal care started first trimester & $47(81.0)$ & $10(83.3)$ & 0.595 \\
\hline First child & $30(56.6)$ & $8(66.7)$ & 0.747 \\
\hline \multicolumn{4}{|l|}{ Maternal substance usage $(\mathrm{N}, \%)$} \\
\hline Smoking, former/during pregnancy & $8(13.7)$ & $2(16.7)$ & 0.680 \\
\hline Alcohol, during pregnancy & $3(5.2)$ & $1(8.3)$ & 0.537 \\
\hline Drugs, during pregnancy & $3(5.2)$ & $2(16.7)$ & 0.201 \\
\hline \multicolumn{4}{|l|}{ Maternal stressors (mean) } \\
\hline Number of pregnancy complications & 0.9 & 1.1 & 0.508 \\
\hline History of premature child & 0.3 & 0.1 & 0.161 \\
\hline Emotional stressor (N, \%) & $20(35.7)$ & $10(83.3)$ & $0.007 *$ \\
\hline Mean number of stressors & 0.5 & 1.5 & $0.027 *$ \\
\hline
\end{tabular}

Methods Used Following Institutional Review Board (IRB) approval (\#5190190), this prospective study was performed at two large academic Neonatal Intensive Care Units (NICU) in southern California. Our recruitment targeted mothers of very low birth weight infants $(<1500$ grams). After informed consent, they completed a questionnaire including demographics, health, substance usage and socioeconomic status. The infant data was collected from birth until NICU discharge and monitored if the development of NEC.

Descriptive statistics and quatitative analysis were performed as appropiate. $\mathrm{P}$ values $<0.05$ were considered statistically significant.

Summary of Results Seventy infants were enrolled: 37(57.9\%) male and 33(47.1\%) female. Only 12(17.1\%) developed NEC $\geq$ Bell stage 2. NEC infants had a lower gestational age than infants without NEC 25.9 vs. 29.0 weeks ("p<0.05), and lower birth weight at 752.8 vs. 1082.3 grams $(" \mathrm{p}<0.05)$. There were no significant differences in mechanical ventilation, vasopressors, intracranial hemorrhage, infection, or feed initiation.

Mothers of NEC infants reported, on average, more overall stressors 1.5 vs 0.5 and specifically more emotional stressors $10 / 12(83.3 \%)$ vs. $20 / 58(35.7 \%)$ during pregnancy (*p<0.05) (table 1). Other maternal factors were not significantly associated with NEC: age at conception, pregnancy complications, smoking history, alcohol and drug usage, household income, education level, first born child and family history of prematurity.

Conclusions Very low birth weight infants ( $<1500$ grams) with necrotizing enterocolitis (NEC) were smaller and born earlier. Our findings suggest that specifically emotional stressors and overall number of maternal stressors during pregnancy may be risk factors for developing necrotizing enterocolitis.

\section{\#201 EVALUATING THE EFFECTS OF BREASTFEEDING VERSUS BOTTLE FEEDING ON THE PRETERM INFANTS' MICROBIOME AND METABOLOME}

${ }^{1,2} \mathrm{~K}$ Schulkers Escalante*, ${ }^{1,2} \mathrm{SS}$ Bai-Tong, ${ }^{1} \mathrm{M}$ Thoemmes, ${ }^{1} \mathrm{~K}$ Weldon, ${ }^{1} \mathrm{~S}$ Hansen,

${ }^{1,2} \mathrm{D}$ Motazavi, ${ }^{1,2} \mathrm{~J}$ Kitsen, ${ }^{1} \mathrm{~S}$ Jin Song, ${ }^{1} \mathrm{~J}$ Gilbert, ${ }^{1} \mathrm{P}$ Dorrestein, ${ }^{1} \mathrm{R}$ Knight, ${ }^{1,2} \mathrm{~S}$ Leibel,

${ }^{1,2} \mathrm{SL}$ Leibel. 'University of California San Diego, La Jolla, CA; ${ }^{2}$ Rady Children's Hospital San Diego, San Diego, CA

\subsection{6/jim-2022-WRMC. 198}

Purpose of Study Breast milk provides numerous benefits to preterm infants including decreasing the risk of necrotizing enterocolitis and sepsis. Providing breast milk via direct breastfeeding versus a bottle has been shown to improve long term outcomes in term infants. As preterm infants transition from tube to oral feeds, the impact of the route of oral feeds on the infants' microbiome and metabolome is unknown. The purpose of this study is to determine if direct breastfeeding changes the preterm infants' oral and gut microbiome and metabolome versus exclusive bottle feeding.

Methods Used This study proposes using stool, saliva and milk samples collected from a cohort of preterm infants from the study: 'The Association Between Milk Feedings, the Microbiome and Risk of Atopic Disease in the Preterm Population (MAP) Study' (NCT04835935). This study recruited 46 babies $<34$ weeks gestational age. Their clinical data was collected as well as weekly samples of their milk feeds, saliva and stool until discharge. Stool samples were analyzed for microbiome and metabolomic profiles in a subcohort of 18 infants. For 
each subject, 3 longitudinal stool samples were analyzed (at birth, 2 weeks of age, and 4-6 weeks of age). Fifty-four stool samples, including 18 meconium samples, were analyzed. Metabolites were analyzed by untargeted gas chromatographymass spectrometry and Kruskal-Wallis $\mathrm{H}$ test was used for statistical analysis. Bacterial compositions were analyzed by shotgun metogenomic. Differences in bacterial community composition were compared using a permutational multivariate analysis of variance (PERMANOVA).

Summary of Results Forty-two (91\%) infants experienced at least one episode of breastfeeding during their NICU stay. Only 17 infants (40\%) were discharged home primarily receiving maternal breast milk. Analysis of the 54 stool samples showed there was a strong differentiation in bacterial community composition after the initiation of bottle $(p=0.014)$ and breast feeding $(p=0.014)$. This indicated changes to the stool microbiome at the onset of oral feeding following full enteral feeds via a nasogastric tube based on shotgun analysis. Metabolomic analysis showed a trend toward differentiation in the stool after initiation of bottle feeds $(p=0.07)$ but did not show significant difference after the initiation of breastfeeding $(\mathrm{p}=0.31)$.

Conclusions While analysis of stool samples has demonstrated microbiome and metabolomic changes after the initiation of breast versus bottle feeds in a subcohort of preterm infants, the future direction is to analyze all of the stool, saliva and breast milk samples for distinct microbiome and metabolome signatures of preterm infants who were exclusively bottle fed versus breastfed.

\section{\#202 VARIATIONS IN PARENT PARTICIPATION IN NURSING CARE SESSIONS IN THE NICU BY MEDICAID STATUS}

S Takamatsu*, AM Cunningham, J Dempsey, J Kelleher, AG Dempsey. University of Colorado - Anschutz Medical Campus, Aurora, CO

\subsection{6/jim-2022-WRMC. 199}

Purpose of Study Parents are faced with the challenge of navigating other external responsibilities (e.g., parenting of other children, work) while their infant is hospitalized in the NICU. Families may have financial barriers impacting stability of housing, childcare, and transportation. As a result, increasing and stabilizing engagement is a common research interest, as it is influential in skill development and caregiver efficacy. Cares sessions with nursing occur in the NICU throughout the day, offering a structured time to observe and partake in the care of the infant. Participation in cares lends itself to demonstration of skills, explanation of care, and can be helpful for parents to feel competent interacting and caring for their baby. The present study collected data on the frequency of parents' attendance of cares sessions in the NICU. To begin to understand differences in potential social barriers to engagement, differences were compared based on mother's Medicaid status.

Methods Used Our sample included 122 premature infants in the NICU who were part of a larger quality improvement study to enhance family engagement. Attendance of four or more cares sessions with nursing per seven days was set as the target goal. Bedside nurses entered data into the infant's medical record. A $X^{2}$ test was performed to detect a difference in goal attainment by Medicaid status.

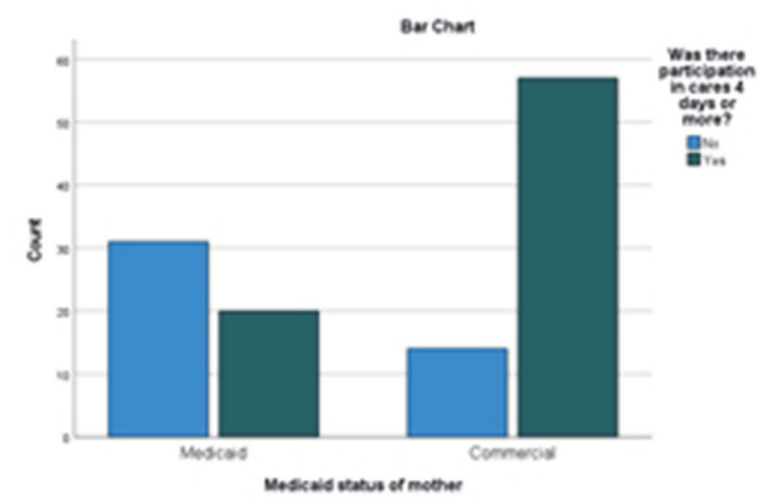

Abstract \#202 Figure 1 Difference in goal attainment by mediciad status

Summary of Results A $X^{2}$ test of independence showed the relation between these variables (e.g., goal attainment and Medicaid status) was significant, $X^{2}(1, N=122)=21.5, p$ $=.000$. Infants with mothers with commercial insurance were more likely than infants with mothers with Medicaid to attend 4 or more cares sessions per week.

Conclusions The data show a difference in participation in cares sessions at least 4 times per week or more when compared by insurance status of the mother, a proxy of socioeconomic status. Further exploration is needed to fully understand barriers to parental engagement. Assessment of potentially related factors to Medicaid status (e.g., other family demands, distance from hospital, transportation difficulties) is recommended. The findings highlight the need for NICU providers to be cognizant of social determinants to bedside engagement and consider virtual options for parents to engage in care.

\section{\#203 PREVALENCE OF NEONATAL INTESNIVE CARE UNIT ADMISISON AMONG PATIENTS WITH GENETIC TESTING}

SB Zoucha*, J Jensen, JL Bonkowsky. University of Utah Health, Salt Lake City, UT

10.1136/jim-2022-WRMC.200

Purpose of Study Genetic disease is estimated to affect many critically ill neonates, but an unbiased determination of genetic disease prevalence in Neonatal Intensive Care Units (NICU) has been unclear. Since rapid and extensive genetic testing is increasingly available and practical and can impact outcomes, there is a need for identifying best clinical practices for genetic testing use. We hypothesized that a history of NICU admission is more common in patients with known or presumed genetic disease; and that neonatal NICU characteristics can guide best use of genetic testing.

Methods Used We performed a retrospective population-based cohort analysis of children on whom genetic testing was performed at a tertiary children's hospital. The hospital and specialists are the only providers of pediatric sub-specialty care in a 500-mile radius. We analyzed the cohort for a history of NICU admission, and clinical characteristics of the admission. We identified 3894 patients with a history of genetic testing with birthdates between $1 / 1 / 09$ and $6 / 1 / 21$ to identify a final cohort of 1611 patients. 
Summary of Results Of this cohort, 132 patients with a history of NICU admissions were identified (8.2\% of the cohort). Of patients with a history of NICU admission; 36\% had a positive (diagnostic) genetic test result, $30 \%$ had a negative test, and $34 \%$ had an uncertain (VUS) result. Compared to children without a history of NICU admission; $38 \%$ had a positive test result, $26 \%$ negative, and $36 \%$ uncertain; differences between these groups was not significant ( $p$ 0.34). The age at testing was lower in those with a history of NICU admission (3.4 years vs 4.6 years; $p<0.001$ ).

Conclusions Our study suggests that a genetic condition is present in a minority of children with a history of NICU admission. Further work into clinical characteristics of NICU children in whom genetic diagnoses are considered will help prioritize use of genetic testing.

\section{\#204 WHAT IS THE EVIDENCE FOR AN ASSOCIATION BETWEEN FIRST TRIMESTER VAGINAL MICROBIOTA ABNORMALITIES AND PRETERM BIRTH?}

${ }^{1} \mathrm{~N}$ Holden*, ${ }^{2} \mathrm{~N}$ Field. ${ }^{1}$ Western University of Health Sciences, Pomona, CA; ${ }^{2}$ University College London, London, UK

\subsection{6/jim-2022-WRMC.201}

Purpose of Study Contemporary research has increasingly explored the possibility that preterm births (PTBs), the livebirth of babies prior to 37 weeks of completed pregnancy, are associated with first trimester vaginal microbiota abnormalities; yet the association between the two remains unclear.

Methods Used A systematic literature review was conducted to assess current evidence for the role of first trimester vaginal microbiota abnormalities driving PTBs. Following the PRISMA (Preferred Reporting Items for Systematic Reviews and MetaAnalyses) 2009 guidelines, scientific databases, including Medline, Embase, and the Maternity \& Infant Care Database (MIDIRS), were searched from January 2009 to March 2019. The search terms used were (vagina* microb* OR vagina* bacteria OR vagina* flora OR vagina* microflora* OR vaginal dysbiosis OR bacterial vaginosis) AND (preterm OR pre term
OR premature OR early term OR early birth). Details on population/sample, study design, method of microbiota determination, measures of microbiota associations with PTBs, and Lactobacillus prevalence were extracted.

Summary of Results Thirteen studies (nine cohort, four randomized control trial) were included in the review. The results provided strong evidence for an association between abnormal first trimester vaginal microbiota and PTBs. Specifically, low-Lactobacillus, high-diversity microbial econiches were found to be associated with an increased likelihood of PTB outcomes. Notably, two studies reported contradictory findings showing a negative association between first trimester AVF and PTBs, and three studies reported no significant association. Possible explanations for the negative association reported in Farr et al., 2015 include regional bias and inclusion of women with chronic conditions. Selection bias was also of concern in Koumans et al., 2010, which also reported a negative association, as participant recruitment was not randomized, possibly shifting the baseline health demographic of recruited women. No definite taxa-specific trends associated with PTB were identified; however, different studies reported Mycoplasma and Ureaplasma parvum increase the odds of PTB manifestation.

Conclusions Associations were largely consistent and strong, suggesting vaginal flora measurements might hold the potential to enable early prediction of PTBs. It is important to note that causality and a biological mechanism for AVF-associated PTBs is not yet proven, with more research being recommended.

\section{\#205 IMPLEMENTING SCREENING FOR NEONATAL DELIRIUM IN THE NICU AT RADY CHILDREN'S HOSPITAL}

${ }^{1,2} \mathrm{M}$ Karmarkar*, ${ }^{2} \mathrm{M}$ Speziale, ${ }^{2} \mathrm{~W}$ Jenkins, ${ }^{2} \mathrm{D}$ Heath, ${ }^{2} \mathrm{~J}$ Kang, ${ }^{2} \mathrm{~J}$ Suvak, ${ }^{2} \mathrm{P}$ Grimm, ${ }^{2} \mathrm{~L}$ Moyer. 'University of California San Diego, La Jolla, CA; ${ }^{2}$ Rady Children's Hospital San Diego, San Diego, CA

10.1136/jim-2022-WRMC.202

Purpose of Study Delirium is defined as fluctuating changes in awareness and cognition occurring in the setting of a medical

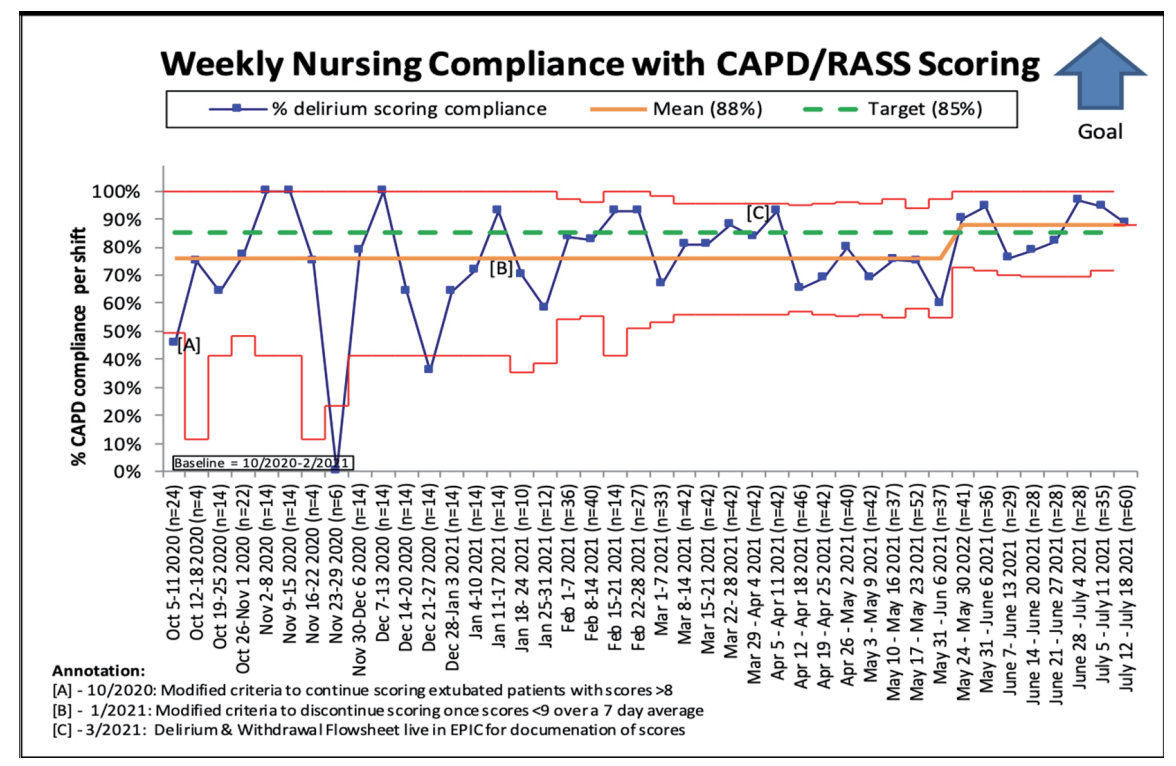

\section{Abstract \#205 Figure 1}


illness, and is associated with adverse neurodevelopmental outcomes. Although delirium is recognized in pediatric ICUs, it is not commonly diagnosed in the neonatal population. The NICU at Rady Children's Hospital is comprised of medically complex patients that are often on multiple medications for pain and sedation. Early recognition and treatment of delirium in the NICU may be helpful in improving clinical outcomes. We conducted a QI project to implement screening for neonatal delirium in high-risk patients. Our objective is to increase delirium screening (RASS/CAPD scores) from $0 \%$ to $85 \%$ in eligible NICU patients by 10/2021. Inclusion criteria are defined as NICU patients $>$ or $=38$ weeks corrected gestational age who are mechanically ventilated $>7$ days and who are receiving any sedatives or opiates.

Methods Used Multiple interdisciplinary meetings were initiated with key stakeholders to develop an algorithm for the evaluation of neonatal delirium. Completion of the RASS (Richmond Agitation and Sedation Scale) and age-adjusted CAPD (Cornell Assessment of Pediatric Delirium) scores were used as the objective tool for delirium screening. Weekly nursing compliance with RASS/CAPD score documentation (figure 1 ) is the primary process measure. Outcome measures include child psychiatry consultations and a diagnosis of delirium.

Summary of Results Implementation of screening and data collection began in October 2020. After implementation, data from 10/2020 through 2/2021 showed an average weekly screening compliance of $76 \%$. Our data shows a sustained shift with an average compliance of $88 \%$, placing us above our goal of $85 \%$ compliance. Targeted future interventions to sustain our goal include: creation of an order set in the medical record and required documentation.

Conclusions Through this QI project, we have increased awareness of neonatal delirium as a diagnosis in our NICU. Our expectation is that early recognition of delirium in our chronic patients will lead to more timely management of symptoms and decreased use of narcotic and sedative medications. This early recognition will be important to these patients' overall recovery.

\section{\#206 IMPROVING ELECTROLYTE AND MINERAL HOMESTASIS IN EXTREMELY PREMATURE INFANTS}

S Markee, J Fuller, A Yaroslaski* ${ }^{*}$ E Shenk, J Maxwell. University of New Mexico Hospital, Albuquerque, NM

\subsection{6/jim-2022-WRMC.203}

Purpose of Study Infants $\leq 28$ weeks gestational age (GA) are at increased risk for developing electrolyte and mineral abnormalities due to reduced baseline bone mineral content. $\sim 80 \%$ of fetal calcium stores are obtained in the third trimester and $\sim 54 \%$ of extremely preterm infants have metabolic bone disease. These infants rely heavily on early parenteral nutrition (PN) to provide appropriate electrolytes, but there is limited evidence on how to optimize calcium and phosphorus. Our primary outcome is to improve calcium and phosphorus by day of life (DOL) 7 in infants $\leq 28$ weeks GA.

Methods Used This Quality Improvement project is currently in the fourth Plan-Do-Study-Act (PDSA) cycle. We reviewed electrolyte and PN data in infants $\leq 28$ weeks GA in 2019 (cohort $0 ; n=16$ ). In March 2020, we obtained daily serum calcium and phosphorus levels in infants $\leq 28$ weeks GA during the first postnatal week $(n=13)$. Upon review, a custom
PN form was designed for the next cohort $(n=9)$, introducing calcium and phosphorus in PN earlier. In our third PDSA cycle we implemented new stock fluids, which included calcium gluconate $0.5 \mathrm{mEq} / \mathrm{dL}$, dextrose, and amino acids for immediate use following birth for those born $\leq 28$ weeks GA $(n=10)$. A comparison was made between all three prospective cohorts. Additionally, serum creatinine and ionized calcium (ical) levels were compared. A Grubb's analysis was used followed by a student's t-test.

Summary of Results Mean serum calcium on DOL1 between cohort 1 and 2 were similar $(6.57 \pm 0.25 \mathrm{mg} / \mathrm{dL}$ and $6.74 \pm$ $0.12 \mathrm{mg} / \mathrm{dL}$, respectively $(p=0.6))$. In cohort 3 , the calcium level was noted to be lower on DOL3 compared to cohort 0 , $(9.6 \pm 0.24 \mathrm{mg} / \mathrm{dL}$ and $9.03 \pm 0.09 \mathrm{mg} / \mathrm{dL}$, respectively $(p=0.08))$. Interestingly, mean serum creatinine on DOL1 nearly significantly decreased in cohort $2(0.76 \mathrm{mg} / \mathrm{dL} \pm 0.05$ $\mathrm{mg} / \mathrm{dL})$, compared to cohort $1(0.90 \mathrm{mg} / \mathrm{dL} \pm 0.05 \mathrm{mg} / \mathrm{dL})$, $p=0.07$. Mean serum creatinine remained $<1 \mathrm{mg} / \mathrm{dL}$ throughout the first week of life for infants in cohorts 2 and 3 , while it trended higher in cohorts 0 and 1 over the first week of life.

Conclusions The new PN allowed a more consistent and gradual increase in serum calcium levels, while remaining in normal limits, during the first postnatal week. Additionally, creatinine levels were lower and ical levels remained in goal range, requiring less therapeutic intervention. Addition of calcium gluconate to stock fluids for infants $\leq 28$ weeks GA on DOL0, as seen in cohort 3 , augmented the improvement and stabilization of serum calcium and creatinine levels. Surprisingly, we have found that although we have been unable to show an improvement in our serum phosphorus for this patient population, we have seen improvement in calcium and creatinine levels in the first postnatal week.

\section{\#207 IMPLEMENTATION OF A NEONATAL MASSIVE TRANSFUSION PROTOCOL}

${ }^{1} \mathrm{H}$ Ko*,${ }^{1} \mathrm{JR}$ Griggs, ${ }^{1} \mathrm{~J}$ Raval, ${ }^{2} \mathrm{~T}$ Zamora. 'University of New Mexico Health Sciences Center, Albuquerque, $N M_{;}{ }^{2}$ Regents of the University of Minnesota, Minneapolis, MN

\subsection{6/jim-2022-WRMC.204}

Purpose of Study A Massive Transfusion Protocol (MTP) is an institutional plan created to facilitate communication, ensure timely lab monitoring and reduce delays/errors when ordering multiple blood products. Though adult and pediatric (MTPs) exist, they are ill-suited for use in the neonatal population. Given the unique nature of neonates, this population could benefit from a neonatal-specific MTP.

Methods Used A pre-implementation survey was sent out to physicians, nurse practitioners, physician assistants, and nurses in the NICU. A multi-disciplinary team was then put together, involving clinical staff from the NICU, transfusion medicine, and the transfusion committee in order to develop a neonatalspecific MTP.

Summary of Results Fifty responses were recorded to 5 questions. 64\% were aware of MTPs in general. Almost all participants (96\%) correctly identified that MTPs were generally used for any patients requiring large blood volume replacements and/or multiple blood products. Forty-six percent responded that they had previously experienced a clinical situation in which there was difficulty obtaining blood products. Of the $46 \%$ who experienced difficulty getting blood 
products, $67 \%$ identified that the delay was mostly attributed to waiting for blood products to arrive.

Conclusions Our multi-disciplinary group collectively developed a neonatal-specific MTP to allow for a safer, timelier, and standardized approach to administering multiple blood products. Massive transfusions are rare but high-risk events in the NICU setting thus this study will hopefully improve patient outcomes related to transfusions. Our pre-implementation survey shows educational and practical barriers exist when attempting to order multiple blood products and further work will need to be done to address these barriers.

\section{\#208 HOSPITAL VARIATION IN EXTREMELY PRETERM BIRTH}

GP Goldstein*, P Kan, C Phibbs, E Main, GM Shaw, H Lee. Stanford University, Stanford, $C A$

\subsection{6/jim-2022-WRMC.205}

Purpose of Study To assess between-hospital variation in extremely preterm birth (EPTB) frequency when stratifying by

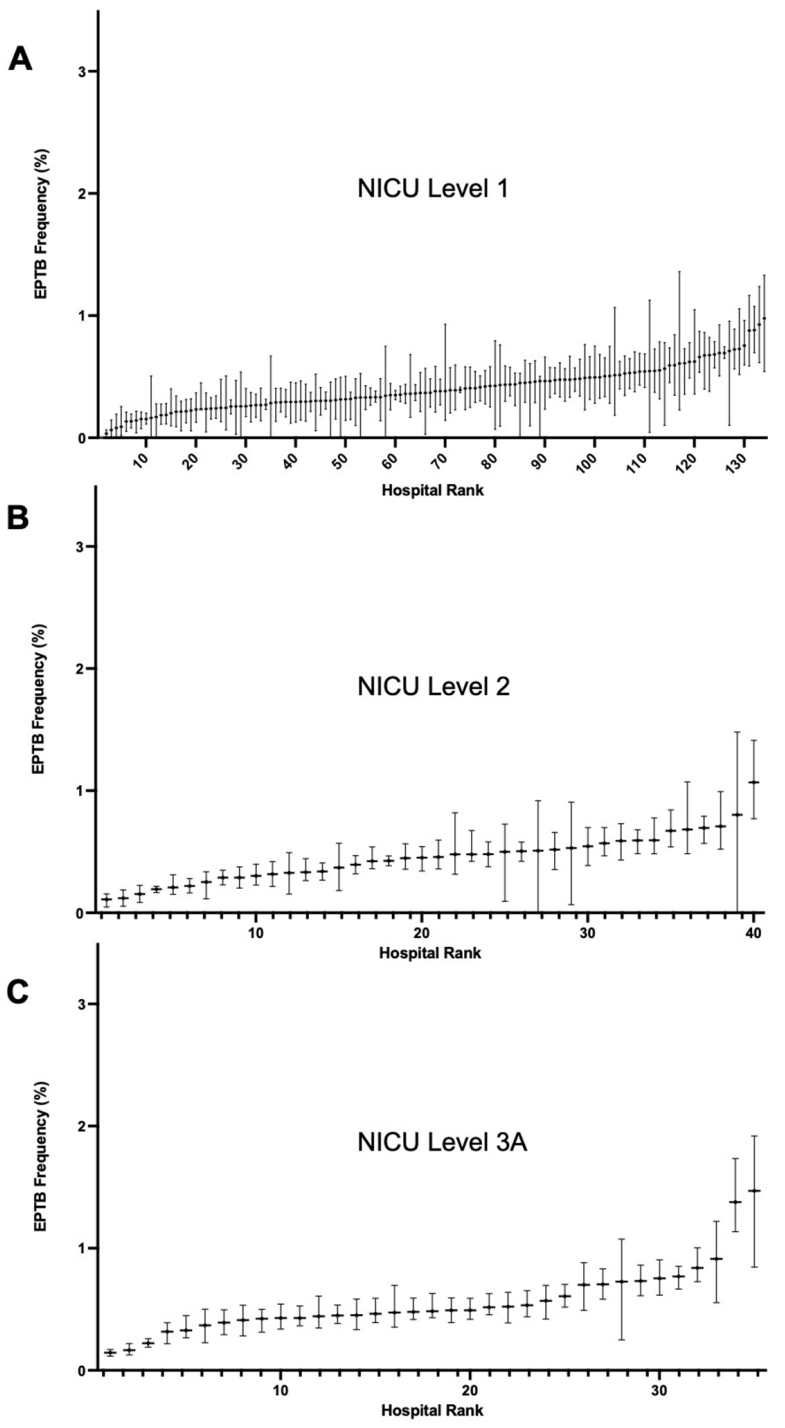

Abstract \#208 Figure 1 Hospital frequencies of extremely preterm birth (EPTB) among hospitals with level 1, 2 and 3A NICUs and by hospital rank hospital level of care, and determine the proportion of variance explained by differences in maternal and hospital factors.

Methods Used We assessed 7,072,562 births in California from 1997 to 2011, using hospital discharge, birth and death certificate data. We estimated the association between maternal and hospital factors and EPTB using multivariable regression, calculated hospital-specific EPTB frequencies and estimated between-hospital variances, intra-class coefficients, and median odds ratios stratified by hospital level of care.

Summary of Results Hospital frequencies of EPTB ranged from $0.01 \%$ to $3.0 \%$. Between-hospital EPTB frequencies varied substantially, despite stratifying by hospital level of care and accounting for confounding factors. This variation appeared to be related to differences in a collection of hospital, maternal sociodemographic and medical factors, and other factors not accounted for in our study, such as barriers to maternal transfer prior to delivery.

Conclusions Our results demonstrate differences in EPTB frequency among hospitals when stratifying by hospital level of care. Proportion of EPTBs at level 1 and 2 NICUs should be further investigated and considered as a hospital quality measure.

\section{\#209 PERCEIVED MOTIVATIONS AND BARRIERS OF NEONATAL NURSE PRACTITIONERS IN FELLOW EVALUATION: A PILOT CASE STUDY}

N Dyess*. University of Colorado Denver School of Medicine, Aurora, CO

\subsection{6/jim-2022-WRMC.206}

Purpose of Study Fellowship programs must provide objective performance evaluations of trainees that are formative and come from multiple evaluators including nonphysicians. The utility of multisource feedback has been well demonstrated in the literature; however, it is difficult to ensure nonphysician staff fill out evaluations. The purpose of this study is to elucidate the neonatal nurse practitioner's (NNP's) perceived motivations and barriers to completing fellow evaluations. As a pilot study, an additional purpose was to assess feasibility and refine methods.

The research questions guiding this study are: how do NNPs view their role in the trainee evaluation process, what motivates NNPs to fill out evaluations, what are the self-perceived barriers to evaluations, and how can we increase NNP evaluation of trainees.

Methods Used I performed a pilot study of a phenomenological, qualitative case study of NNPs at the University of Colorado. A convenience sample of $3 \mathrm{NNPs}$, selected via purposive sampling, participated in semi-structured, one-on-one interviews to explore the perceived motivations and barriers to evaluating fellows. A constructivist epistemological framework guided the study. The data was viewed through a theoretical framework inspired by Maslow's motivation theory. Interview transcripts were coded inductively via the constant comparative method and then clustered into emergent themes using phenomenological reduction, horizontalization, imaginative variation, and thematic analysis.

Summary of Results A conceptual framework emerged from the data, consisting of five themes of driving and restraining forces to completing evaluations which interact in a process akin to a neuronal cell's action potential. The 
Abstract \#209 Table 1 Themes of driving and restraining forces and representative quotes

\begin{tabular}{|c|c|c|}
\hline Themes & Driving Forces & Restraining Forces \\
\hline $\begin{array}{l}\text { Fellow Characteristics } \\
\text { * extremes of behavior; } \\
\text { pattern/repetitive behavior } \\
\text { * values feedback }\end{array}$ & $\begin{array}{l}\text { 'if they go like above and beyond' 'if somethings like stuck out' 'just knowing } \\
\text { that that's helpful for them or that they would appreciate the critique...just } \\
\text { sensing someone's openness or reception to wanting to learn and improve in } \\
\text { different ways impacts my willingness' 'if you have a really positive experience or } \\
\text { a really negative experience then you may think to fill it out' }\end{array}$ & $\begin{array}{l}\text { 'I don't think I've known that people want feedback necessarily' 'most } \\
\text { experiences are kind of like somewhere in the middle... doesn't stick out to you } \\
\text { in the same way' 'what if they're having a bad week, what if they're having a } \\
\text { bad service, what if they're having a bad day' }\end{array}$ \\
\hline $\begin{array}{l}\text { NNP-Fellow Relationship } \\
\text { * continuity/exposure } \\
\text { * perceived hierarch } \\
\text { * fear of fellow reaction or } \\
\text { repercussion }\end{array}$ & $\begin{array}{l}\text { 'if I worked more closely with one of you, then I try to share' 'your relationship } \\
\text { with the fellow, whether or not you have the same communication skills' }\end{array}$ & $\begin{array}{l}\text { 'I feel like there's still a sense of hierarchy...it can feel like intimidating or } \\
\text { strange to be critical of someone that you maybe see as your superior in some } \\
\text { way or leader' 'it's very difficult to know where your boundaries are and where } \\
\text { the line is in as far as giving negative feedback' }\end{array}$ \\
\hline $\begin{array}{l}\text { Evaluation Characteristics } \\
\text { * perceived feasibility } \\
\text { * confidentiality/anonymity } \\
\text { * type of feedback } \\
\text { * degree of specificity } \\
\text { * degree of timeliness }\end{array}$ & $\begin{array}{l}\text { 'if you're giving positive feedback...you hope that the person that you are } \\
\text { writing about actually sees it because you want them to know that they're } \\
\text { appreciated' }\end{array}$ & $\begin{array}{l}\text { 'questions where it's like give me an example...then I have to rack through my } \\
\text { memory of the past month that you've been on service' 'I would be hesitant to } \\
\text { give that exact situation because then they would know who gave that } \\
\text { feedback' 'you're thinking of one specific scenario but I don't know where that } \\
\text { fits in these like } 3 \text { questions that they ask about...it's like you're trying to fit } \\
\text { your feedback into a mold that didn't really go together' 'you are given a } \\
\text { random evaluation and you haven't worked with that person in like a month' }\end{array}$ \\
\hline $\begin{array}{l}\text { NNP-Evaluation } \\
\text { Relationship } \\
\text { * knowledge of evaluator } \\
\text { role and value (to trainee } \\
\text { and to program) } \\
\text { * knowledge of evaluation } \\
\text { process and outcomes } \\
\text { * time constraints, } \\
\text { interruptions, unprotected } \\
\text { time }\end{array}$ & $\begin{array}{l}\text { 'I just think that our feedback because we are in a different role is also } \\
\text { invaluable because you will be working with other nurse practitioners in } \\
\text { community hospitals, other nurses in community hospitals, rather than other } \\
\text { attendings } 24-7 . \text {.' 'it's very important to have the bedside nurse and NNP...join } \\
\text { in on that conversation because you're not just communicating with consultants } \\
\text { and you're not just communicating with other attendings...you speak to an } \\
\text { attending differently than how you speak to a NNP or bedside RN...so I feel like } \\
\text { having a bedside RN and NNP weigh in on what you're doing is important' }\end{array}$ & $\begin{array}{l}\text { 'I guess I've never been explicitly...told about it or that we do have a role in } \\
\text { that' 'in the case of constructive criticism, it's helpful if it's very explicit in where } \\
\text { it goes and who's name is on what so you at least do that in full awareness of } \\
\text { what maybe could be the implications of the criticism you are giving' 'the } \\
\text { simplest answer is no, I do not know the process for giving feedback' 'we get } \\
\text { bombarded with evaluations all the time' 'we are inundated with a bunch of } \\
\text { evaluations with absolutely everything we do' 'everybody thinks like if I give this } \\
\text { negative feedback, something bad is going to happen' }\end{array}$ \\
\hline $\begin{array}{l}\text { NNP Characteristics } \\
{ }^{*} \text { preferred evaluation and } \\
\text { feedback strategies } \\
{ }^{*} \text { new to role, knowledge } \\
\text { of own role, knowledge of } \\
\text { culture/system }\end{array}$ & $\begin{array}{l}\text { 'if it were simplified and we can just... have a place where there is eval } \\
\text { forms... like ok I really have something to say about this person, I don't want to } \\
\text { wait for an evaluation to come out, I can go online and I can click on this tab } \\
\text { and I can fill [it] out' 'more years into the role, like I can see the differences in } \\
\text { where a fellow stands and where they are in their career and how well they do } \\
\text { their job' }\end{array}$ & $\begin{array}{l}\text { 'I prefer to do it verbally, in person.' 'I feel like verbal feedback is way easier, } \\
\text { quicker, gets the point across.' 'I... haven't found a way to give...constructive } \\
\text { criticism, partly because I am trying to figure things out myself' 'it's because I } \\
\text { was a newer NNP...so I wasn't comfortable, and didn't really know the ropes, I } \\
\text { guess, as far as what should be done... what should be expected' }\end{array}$ \\
\hline
\end{tabular}

framework describes the implicit weighing of these forces to determine if a threshold for activation is reached to complete an evaluation. Themes are supported by Maslow's motivation theory, with each of Maslow's levels of need equating to a NNP's level of need for completion of fellow evaluations.

The Fellow Characteristics theme describes how extremes of behavior, repetitive behavior, and a fellow's value of feedback affect evaluation completion. The NNP-Fellow Relationship theme describes how increased exposure and knowledge of fellow repercussion affect evaluation completion. The Evaluation Characteristics theme illustrates how anonymity, specificity, feasibility, and timeliness affect evaluation completion. The NNP-Evaluation Relationship theme describes how knowledge of evaluator role/value and the evaluation process is critical to evaluation completion. The NNP Characteristics theme describes how alignment with preferred feedback strategies and seniority affect evaluation completion.

Conclusions The conceptual framework provides insights into the motivations and barriers to completion of fellow evaluations by NNPs that can inform measures to increase completion rates of trainee evaluations by nonphysicians.

\section{\#210 EVALUATING A NEONATAL OPIOID WITHDRAWAL SYNDROME CURRRICULUM TO IMPROVE CARE IN RURAL HOSPITALS}

${ }^{1} \mathrm{~J}$ Patel ${ }^{*},{ }^{2} \mathrm{~S}$ Sanders, ${ }^{2} \mathrm{H}$ Brakey, ${ }^{2} \mathrm{~T}$ Ozechowski, ${ }^{2} \mathrm{~A}$ Sussman, ${ }^{2} \mathrm{~A}$ Kong, ${ }^{2} \mathrm{H}$ Pratt-Chavez. ${ }^{1}$ University of New Mexico School of Medicine, Albuquerque, NM; ${ }^{2}$ University of New Mexico Health Sciences Center, Albuquerque, NM

\subsection{6/jim-2022-WRMC.207}

Purpose of Study The incidence of neonatal opiate withdrawal syndrome (NOWS) in the US has grown dramatically over the past two decades. Many rural hospitals are not equipped with resources and materials to manage best practices of these patients resulting in transfers to hospitals in bigger cities. The purpose of this study is to evaluate a curriculum we created to support rural hospitals to keep healthy infants with NOWS for observation instead of transferring them.

Methods Used The curriculum was used for quality improvement at a rural hospital and shared with providers in another state that expressed interest. To evaluate the curriculum, we conducted pre- and post-surveys of NOWS knowledge, attitudes, and care practices, plus post-curriculum interviews and focus groups. 
Summary of Results Fourteen participants completed both preand post-curriculum surveys. They indicated an increase in knowledge and care practices, and a decrease in belief that infants with NOWS should be cared for in a critical care environment. Most respondents agreed with positively worded attitude items pre-test and post-test. Although few respondents expressed negative attitudes about mothers of infants with NOWS at pre-test, the training curriculum appeared to have no impact on such attitudes at post-test. Sixteen participants participated in focus groups or interviews. Qualitative data reinforced quantitative results, plus the need to reduce stigma and improve provider/staff interactions with patients.

Conclusions This curriculum has strong positive impacts on NOWS knowledge and care practices. Incorporating focus on core concepts of trauma-informed care and self-regulation in future iterations of the curriculum may strengthen the opportunity to change attitudes and address the needs expressed by participants and improve care and well-being of families and babies with NOWS.

\section{\#211 EFFECTS OF POSTNATAL GLUCOCORTICOIDS ON BRAIN STRUCTURE IN PRETERM INFANTS, A SCOPING REVIEW}

I Robles*, MA Eidsness, HM Feldman, SE Dubner. Stanford University School of Medicine, Stanford, $C A$

\subsection{6/jim-2022-WRMC.208}

Purpose of Study Postnatal GCs (GC) are given for many indications in infants, including for the reduction in incidence and severity of bronchopulmonary dysplasia, a major risk factor for morbidity, mortality, and neurodevelopmental disability in children born preterm. Variation exists in medication, dosing, timing, and reported outcomes. Clinical neurodevelopmental outcomes after GC administration may be due, in part, to GC induced alterations in neonatal brain development. The objective of this scoping review is to identify what is known about the effects of GC treatment on brain structural development in preterm human infants in order to identify potential mechanisms by which GCs may

\begin{tabular}{|c|c|}
\hline Inclusion Criteria & Exclusion Criteria \\
\hline 1. Papers from 1990 and later & 1. Conference abstracts, case studies/ \\
\hline 2. English language & reports if less than 10 subjects, \\
\hline 3. Published and peer reviewed & dissertations; review papers; preprints; \\
\hline 4. Contains empirical data & theoretical papers \\
\hline 5. Human subjects study & 2. Endogenous GC exposure \\
\hline 6. Infants born before 37 weeks gestation & 3. GCs only administered antenatally \\
\hline 7. Postnatal GC exposure (exogenous) & 4. No structural brain outcomes reported \\
\hline 8. GCs administered systemically - enteral, & 6. Animal study with no human participants \\
\hline intravenous, intramuscular, inhaled, & 7. Topical GC application only \\
\hline nebulized, intranasally administered, & 8. GCs only after birth hospitalization \\
\hline sublingual, or subcutaneous & discharge \\
\hline \multicolumn{2}{|l|}{ 9. GCs administered to infants during birth } \\
\hline \multicolumn{2}{|l|}{ hospitalization } \\
\hline \multicolumn{2}{|l|}{ 10. Reports on at least one structural brain } \\
\hline \multicolumn{2}{|l|}{ outcome measured after postnatal GC } \\
\hline exposure & \\
\hline
\end{tabular}

affect later clinical neurodevelopmental outcomes and to identify gaps in the literature.

Methods Used A search query was developed to search online databases for original research on human infants, GCs, and brain structure. Potential article titles and abstracts were screened by two reviewers to identify papers for full text review.

Summary of Results 6565 titles were identified based on the search query for title and abstract review. Inclusion and Exclusion criteria are shown in the table. 70 were included for full text review. Multiple imaging modalities and outcomes were reported.

Conclusions GC effects on brain are of interest to a wide audience of researchers across the lifespan and across many clinical conditions. Relatively few human studies have directly assessed the effect of this intervention on early brain structural development. This study highlights the need for additional research on neonatal GCs and their potential effects on brain development.

\section{\#212 DOES MATERNAL AGE IMPACT FEEDING OUTCOMES IN PRETERM INFANTS?}

${ }^{1} \mathrm{~A}$ Patel ${ }^{*},{ }^{2} \mathrm{C}$ Bradley. ${ }^{1}$ University of California Irvine School of Medicine, Irvine, CA; ${ }^{2}$ University of California Irvine Department of Pediatrics, Irvine, CA

\subsection{6/jim-2022-WRMC.209}

Purpose of Study For preterm infants in the Neonatal Intensive Care Unit (NICU), successful and safe oral feeding is a requirement for discharge from the hospital. There are a variety of factors that contribute to feeding outcomes as neonates learn to coordinate breathing during oral feeding. While studies have detailed the effects of advanced maternal age on various neonatal morbidities, there is a dearth of literature exploring the association between maternal age and feeding outcomes in neonates. This analysis sought to examine whether a correlation exists between maternal age at delivery and feeding outcomes in preterm infants.

Methods Used A retrospective chart review was conducted for 12 healthy infants born at less than 37 weeks gestational age who were admitted to the NICU and were transitioning from gavage to bottle feeding. Data was collected on maternal age at delivery, rate of milk transfer (in milliliters per minute), oral feeding performance (in percentage of milliliters taken during the feeding per milliliters prescribed), and feeding proficiency (in percentage of milliliters taken during the first five minutes of the feeding per milliliters prescribed). A correlation matrix was then developed via a linear regression model using these, and other, maternal and infant health factors to assess for possible correlations.

Summary of Results Maternal age showed a moderately positive correlation with two of the three feeding outcomes studied. The Pearson correlation coefficient (R) for the association between maternal age and feeding performance was +0.66 . The $\mathrm{R}$ for the association between maternal age and feeding proficiency was +0.48 . Maternal age was not associated with rate of milk transfer, with an $\mathrm{R}$ of +0.04 .

Conclusions This preliminary analysis shows that increased maternal age may serve as a protective factor for the complex oral feeding process that is challenging for preterm infants due to immature neurodevelopment. Maternal age at delivery may be valuable to consider in studying progression to full 
oral feeding, and ultimately discharge from the NICU, in this population. While the power of this exploratory study is limited due to small sample size and potential for confounding, this warrants further research that examines the association of maternal age at delivery on various feeding outcome measures.

\section{\#213 WEE NUZZLE: A QUALITY INITIATIVE TO PROMOTE NON-NUTRITIVE BREAST FEEDING IN ORDER TO INCREASE BREASTMILK AT DISCHARGE FOR PRETERM INFANTS IN THE NEONATAL INTENSIVE CARE UNIT}

\author{
${ }^{1,2} \mathrm{~K}$ Schulkers Escalante* ${ }^{*}{ }^{1,2} \mathrm{~J}$ Barnard, ${ }^{1} \mathrm{E}$ Clemens, ${ }^{1} \mathrm{R}$ Hammer, ${ }^{1} \mathrm{C}$ Ritter, ${ }^{1} \mathrm{~K}$ Ko, ${ }^{1} \mathrm{~J}$ Wood, \\ ${ }^{1} \mathrm{~S}$ Freeman, ${ }^{1 J} \mathrm{C}$ Cook, ${ }^{1,2} \mathrm{~K}$ Weiss, ${ }^{1}{ }^{1} \mathrm{SL}$ Leibel. ${ }^{1}$ University of California San Diego, La Jolla, \\ CA; ${ }^{2}$ Rady Children's Hospital San Diego, San Diego, CA

\subsection{6/jim-2022-WRMC.210}

Purpose of Study Reduced opportunities for preterm infants to practice oral feeding can contribute to issues such as delayed hospital discharge and oral aversion. Furthermore, a lack of opportunity for direct latching may discourage mothers and reduce breastmilk feeding at discharge. The NICU at Jacobs Medical Center, University of California, San Diego has a low rate of non-nutritive breast feeding (NNBF) in preterm infants due to: 1) varying practices and comfort levels, especially for preterm infants on non-invasive respiratory support (NRS), 2) lack of formal guidelines, and 3) NNBF not frequently integrated into medical team's daily discussions or documented by bedside staff. The goals of this QI project are to support early introduction to the breast to promote latching and ultimately milk transfer, promote maternal-infant bonding, and facilitate early positive oral experiences. Our SMART AIM is to increase eligible preterm infants attempting at least one session of $\mathrm{NNBF}$ at least once weekly from a baseline of $0 \%$ to $\geq$ $50 \%$ by July 2022 .

Methods Used To support the development of oral feeding skills, called Wee Feeds, in premature infants, a multidisciplinary team was developed that included providers, occupational and respiratory therapists, lactation consultants, nurses, and parents. A new pathway called 'Wee Nuzzle' was created as a pre-feeding developmental pathway primarily focusing on promoting breast feeding by encouraging Skin-to-Skin, Milk Drops, and NNBF. Premature infants' $\geq 30$ weeks are included if they are receiving enteral breast milk, are on NRS or room air, and the parent desires to do NNBF. Infants are excluded if they were intubated or within a week of extubation, have an umbilical arterial catheter, and/or have significant congenital or neurological abnormalities making it unsafe to attempt NNBF. Education for NICU staff has occurred at department meetings, nurse staff meetings and huddles, nursing fair skills day, and via multiple emails over the past 6 months. The primary process measure is percentage of eligible infants attempting at least one NNBF session per week. Outcome measures include the number of preterm infants discharged home on breastmilk and time to discharge from start of Wee Nuzzle. Balancing measures include an increase in respiratory support within 24 hours of NNBF without another explanation.

Summary of Results Implementation of our project is currently ongoing. We plan to measure data monthly and implement PDSA cycles as needed.

Conclusions The oral feeding pathway, Wee Feeds, has been widely accepted and supported by all levels of staff in our NICU and we hope to gain the same support for Wee Nuzzle.
We will continue education, data collection and analysis with the hope to achieve our measures and aims.

\section{Adolescent medicine and general pediatrics II}

\section{Concurrent session}

\section{8:00 AM}

\section{Friday, January 21, 2022}

\section{\#214 IMPACT OF AGE, SEX, AND RACE ON VELOPHARYNGEAL ANATOMY WITHIN THE FIRST TWO YEARS OF LIFE}

${ }^{1} \mathrm{~S}$ Levene*, ${ }^{2} \mathrm{I}$ Neuberger, ${ }^{2} \mathrm{~K}$ Barhaghi, ${ }^{3} \mathrm{~A}$ Piccorelli, ${ }^{3} \mathrm{~K}$ Kotlarek. ${ }^{1}$ University of Washington School of Medicine, Seattle, WA; ${ }^{2}$ Children's Hospital Colorado, Aurora, CO; ${ }^{3}$ University of Wyoming, Laramie, WY

\subsection{6/jim-2022-WRMC.211}

Purpose of Study Children born with cleft palate typically undergo primary palatoplasty within the first 2 years of life with the goal of anatomic restoration of the palate to allow for the development of normal speech and swallowing patterns. To the best of our knowledge, no large-scale quantitative data exists regarding typical velopharyngeal (VP) structures for children within this age range. This retrospective study aimed to (1) quantify the impact of age, sex, and race on the size and orientation of VP structures during the first 2 years of life and (2) provide normative data for future comparison to infants with cleft palate. Based on existing literature, it was hypothesized that VP dimensions would not display sexual dimorphism but would display a significant racial effect.

Methods Used An a priori power analysis was completed. A retrospective chart review was completed for all patients under 24 months of age that underwent an MRI of the head for medical necessity using a 3D FLAIR sequence at a large pediatric hospital within the past 18 months. After excluding patients based on scan quality and medical diagnoses or structural conditions affecting the region of interest (e.g. cleft palate), VP measurements were obtained for 184 patients using ThermoFisher ${ }^{\mathrm{TM}}$ Amira $^{\mathrm{TM}}$ software. Participants were divided into 5 groups based on corrected age. A multivariate analysis of covariance was used to assess differences in VP variables by age group while controlling for sex and race. Inter- and intra-rater reliability was completed for all variables based on $20 \%$ of participants using a Pearson productmoment correlation.

Summary of Results Inter- and intra-rater reliability were excellent $(r=.90+)$. There was a statistically significant $(p<.0001)$ difference between age groups based on overall combination of dependent variables after controlling for sex and race. Regarding corrected age, follow-up analyses revealed significant differences in adenoid depth $(p=.0001)$, effective velar length $(p=.0079)$, levator length $(p<.0001)$, origin to origin distance $(p<.0001)$, pharyngeal depth $(p=.0021)$, sagittal angle $(p=.0016)$, velar insertion distance $(p<.0001)$, velar length $(p<.0001)$, and velar thickness $(p<.0001)$. Regarding sex, follow-up analyses revealed significant differences for effective velar length $(p=.0225)$, levator length $(p<.0001)$, origin to origin distance 
$(p=.0019)$, velar insertion distance $(p<.0283)$, and velar length $(p=.0166)$. No significant differences were observed between race groups.

Conclusions Variations in VP dimensions based on both age and sex were observed among children under 2 years of age. Data from the present study provide a normative database for future comparison to children born with atypical VP anatomy (e.g. cleft palate).

\section{\#215 LIMPING THROUGH A DIFFERENTIAL: AN UNCOMMON PRESENTATION TO A COMMON PEDIATRIC DIAGNOSIS}

J Smith*, J Gardner, K Dreher, S Sanders. University of Arkansas for Medical Sciences, Little Rock, $A R$

\subsection{6/jim-2022-WRMC.212}

Case Report A 4-year-old previously healthy boy presented to the pediatric emergency department (ED) with a onemonth history of a progressively worsening limp with concerns for osteomyelitis. He was recently evaluated by an orthopedic surgeon with x-rays of the ankle showing medial metaphyseal lucency and joint effusion concerning for infection as well as distal fibular sclerosis concerning for a healing fracture. His limp progressed to the inability to bear weight due to worsening left lower extremity pain. There was no history of trauma to the area. He complained of no other bone pain.

Physical Exam Exam was notable for left ankle swelling with tenderness to palpation and pain with passive movement. The left lower extremity had normal sensation and perfusion. The liver edge was palpated several centimeters below the costal margin. No lymphadenopathy was appreciated. No rashes or bruising noted.

Diagnostic Evaluation Laboratory evaluation was significant for elevated inflammatory markers. A complete blood count revealed a borderline microcytic anemia, but was otherwise normal. Magnetic resonance image (MRI) of the ankle showed diffuse abnormal bone marrow enhancement consistent with an infiltrative process. Further questioning revealed a history of night sweats, increasing fatigue, and intermittent fevers over the past month. Lactate dehydrogenase and uric acid were elevated and a peripheral smear showed atypical lymphocytes. The patient underwent bone biopsy. No drainable fluid collection was found. Cultures did not grow bacteria. Bone marrow aspirate with flow cytometry showed 60\% B-lymphoblasts consistent with B-cell acute lymphoblastic leukemia (ALL).

Discussion Acute onset limping and acute lymphoblastic leukemia are commonly encountered problems in the ED. Extremity pain with limping carries a wide differential diagnosis in a child. Studies have shown that the initial diagnosis in a patient with limp is only correct $42 \%$ of the time. ALL often presents with diffuse bone pain; however, presentation with limp as the primary problem is rare. This case highlights the importance of a thorough history and physical exam. It was not until they were asked specifically about night sweats, weight loss, and fevers that the patient's guardians responded in the affirmative. In the workup of the limping child, it is imperative to have malignancy on the differential even for localized bone pain.

\section{REFERENCES}

1. Herman MJ, Martinek M. The limping child. Pediatr Rev 2015;36(5):184-197. doi:10.1542/pir.36-5-184

2. Lefèvre $Y$, Ceroni $D$, Läedermann $A$, et al. Pediatric leukemia revealed by a limping episode: A report of four cases. Orthop Traumatol Surg Res 2009;95(1):7781. doi:10.1016/j.otsr.2008.09.004

\section{\#216 PROTEIN CONCENTRATION OF HUMAN MILK VARIES WITHIN A FEED AND OVER A 24-HOUR PERIOD}

${ }^{1} \mathrm{KA} \mathrm{Bull}{ }^{*},{ }^{2} \mathrm{AC}$ Gogel, ${ }^{2} \mathrm{JE}$ Williams, ${ }^{2} \mathrm{Y}$ Bonney, ${ }^{2} \mathrm{MA}$ McGuire, ${ }^{2} \mathrm{MK}$ McGuire. ${ }^{1}$ University of Washington School of Medicine, Moscow, ID; ${ }^{2}$ University of Idaho College of Agricultural and Life Sciences, Moscow, ID

\subsection{6/jim-2022-WRMC.213}

Purpose of Study Fundamental to understanding human milk composition is determining what is a representative milk sample. Many factors can influence the concentration of some milk components, and controlling for these variables is essential to documenting 'typical' milk composition. While variation in lipid content has been widely studied, there is less research on macronutrients. The purpose of this project was to determine if total protein concentration varies with time of day and during a feed. We hypothesized that we would detect no differences in protein based on these factors.

Methods Used This was an epidemiologic, repeated-measures trial involving 17 healthy women from Moscow, ID and surrounding areas who were $\geq 18$ y of age, not taking antibiotics, and nursing healthy infants. Participants were $31.4 \pm 3.6 \mathrm{y}$ old and $223 \pm 175 \mathrm{~d}$ postpartum. Milk collection kits were delivered to each mother's home with detailed sampling instructions, and study personnel were available (via phone) during most of the sample collection times. During $d$, participants collected milk representing the foremilk, mid-milk, and hindmilk of a feed during a single breast expression. On d 2, milk was collected 4 times (T1-T4), $6 \pm 1.5 \mathrm{~h}$ apart, the first being between 0500-0900 hr. Protein concentrations in milk were measured using a colorimetric method (Pierce BCA Protein Assay Kit) with human serum albumin (Sigma-Aldrich) as the standard. Data were analyzed using linear mixed models with participant as a random variable while assuming an autoregressive correlation structure for the repeated measures within participant as implemented in SAS (v 9.4).

Summary of Results Contrary to our hypotheses, protein concentration varied within a feed $(p=0.0058)$ and over a 24hr period $(p=0.0046)$. Protein concentrations of foremilk and mid-milk were lower than that of hindmilk (17.66 \pm $0.46,17.80 \pm 0.46$, and $18.56 \pm 0.57 \mathrm{~g} / \mathrm{L}$, respectively; $\mathrm{p}$ $=0.0210$ and $\mathrm{p}=0.0015$, respectively). Protein concentrations increased in a stepwise fashion over the 24-hr period (17.31 $\pm 0.51,17.83 \pm 0.44,18.37 \pm 0.45$, and $19.15 \pm 0.44 \mathrm{~g} / \mathrm{L}$ at $\mathrm{T} 1, \mathrm{~T} 2, \mathrm{~T} 3$, and $\mathrm{T} 4$, respectively).

Conclusions Our results demonstrate that human milk protein concentration increases from the beginning to the end of a feed and over a 24-hr period. These findings contradict the majority of published studies and bring into question current published values of 'typical' human milk protein concentration. Our data suggest that time within a feed and time of day must be considered when collecting a representative milk sample for protein analysis. 
\#217 A CASE REPORT OF THE ASSOCIATION OF KLINEFELTER SYNDROME AND AUTOIMMUNE SCLERITIS

S Michalsky, C Klinhom, M Malwane* , K Eckert. University of Nevada Reno, Reno, NV

\subsection{6/jim-2022-WRMC.214}

Case Report Autoimmune (AI) diseases have been shown to be more common in patients with Klinefelter Syndrome (KS) than the general population. To our knowledge, there are no known records of autoimmune scleritis in Klinefelter Syndrome. We report a unique case of a 13-year old male with $\mathrm{KS}$ and AI scleritis.

Methods The medical records of a patient with KS and autoimmune scleritis were reviewed over a 3-year period.

Results A 13-year old male with KS presented 1-2 week of right eye edema, erythema, decrease in vision, and mild ophthalmalgia and ophthalmodynia, worsened with eye movement. Patient reported concurrent pain in knees and shoulders. Patient was noted to have 'red eyes' as a child and diagnosed with keratitis. Family history was positive for arthritis, unexplained deep vein thrombosis in his mother, and antiphospholipid syndrome in grandfather. Physical exam was notable for the right eye with red, injected sclera and conjunctiva.

Further studies included MR of the right orbit showing mild right proptosis, as well as right periorbital, intraorbital and extraconal intraorbital edema and enhancement. Right uveal thickening and enhancement was seen and the extraocular muscles were normal in caliber and symmetry. The globes were otherwise unremarkable and the optic nerve/sheath complexes were normal in configuration. Final MRI impression was significant for right orbital and periorbital cellulitis with retinitis and mild proptosis. Patient had a mildly elevated white blood cell count at $12.7 \mathrm{~K} / \mathrm{uL}$ and was started on IV Vancomycin and Unasyn. There was no clinical response after 2 days of treatment. Ophthalmology determined that presentation was most consistent with a likely autoimmune etiology, after which prednisone treatment was started at $80 \mathrm{mg}$ daily. The patient showed immediate improvement in symptoms. Primary immune screen included: Antinuclear antibodies (ANA) was positive, but titers $<1: 40$ (titers in the range of $1: 40$ to $1: 60$ are considered low), anti-neutrophilic cytoplasmic autoantibody (ANCA), rheumatoid factor (RF), Immunoglobulin (Ig) G subclasses, IgM, and IgA were all normal, SSA/SSB and HLA-B27 were negative. Initial erythrocyte sedimentation rate (ESR) was 31 and normalized to 2 (normal ESR in males : $\leq 15 \mathrm{~mm} / \mathrm{hr}$ ) after being on prednisone treatment. There are no specific tests available to confirm autoimmune scleritis; however, patient was unresponsive to appropriate antibiotic therapy and highly responsive to prednisone treatment, implying an autoimmune etiology.

At the age of 14 , gonadal failure and delayed puberty were diagnosed.

Conclusions Appropriate autoimmune screening should be part of the medical management of patients with KS. As presented in this case report, it is also important to be aware of eye symptoms, particularly scleritis, as the first symptom of autoimmunity in KS patients.

\section{\#218 \\ PERCEPTION AND CONTRACEPTION: ATTITUDES TOWARDS HORMONAL BIRTH CONTROL ON TIKTOK}

H Egnew, V Yu*, AL Nelson, E Guenther. Western University of Health Sciences College of Osteopathic Medicine of the Pacific, Pomona, CA
Purpose of Study Social media sites, such as Twitter, have been used to sample informal attitudes and messages shared about birth control between users. TikTok is a newer platform that appeals to younger users, that has not been used to study this topic. On TikTok, content creators openly share their experiences with birth control through dialogue and humorous interpretations. TikTok's largest age group is 18-24, while Twitter's is $30-49$. Identification of sentiment about hormonal birth control in the previously untapped TikTok population could provide insight into younger patients' attitudes towards birth control.

Methods Used In this qualitative IRB-approved study, 100 videos per hormonal birth control method (oral contraceptive pills [OCP], injections, intrauterine devices [IUD] and implant) were identified through hashtags. Given interest in user experience, we excluded videos posted by self-identified medical providers or commercial agents. Videos were then analyzed for sentiment, factual accuracy, and theme saturation.

Summary of Results $60 \%$ of the content we surveyed on TikTok was negative, while $8 \%$ was positive. A previous study on Twitter had found a majority of birth control content was neutral or positive.

TikTok content most commonly highlighted side effects (59.5\%), experiences with providers (19.5\%), and humor (18.5\%). OCP videos discussed side effects the least $(37 \%)$ and were more likely to include humor (37\%). Implant (70\%), IUD (67\%) and injection (64\%) videos focused on side effects much more than OCP videos. IUD (18\%) and OCP $(13 \%)$ videos were most likely to contain misinformation. IUD contained the most videos mentioning complications (17\%).

We found that videos discussing short-acting methods were over twice likely to be positive (12\%) compared to long-acting methods (5\%). A previous study found that Tweets mentioning long-acting methods were more likely to be positive than short-acting methods.

Humor was utilized most in OCP (37\%) and IUD (16\%). Many of the humorous videos joked about the onset and severity of side effects, or used humor to make fun of children's misbehavior as their motivation for using birth control. One content creator stated, 'I take the pill so my boyfriend doesn't have to be a teen dad'. Much of the humorous content on TikTok relied on trending audio tracks, self-deprecating jokes, and facial expressions.

Conclusions Attitudes and themes towards hormonal birth control options presented on TikTok differ from previous studies on public platforms, and introduce humor and use video formats that both resonate with the application's younger users. Recognition of different perceptions of birth control by younger TikTok users in comparison to the older Twitter users can be used to strategically target misinformation and potentially identify generational differences in perception of birth control.

\section{\#219 KIDZ MEDED: EMPOWERING YOUTH TO CREATE ANIMATED MEDICAL EDUCATION VIDEOS TO FOSTER STEM IDENTITY IN UNDERREPRESENTED YOUTH}

${ }^{1} \mathrm{M}$ Jayakumar*, ${ }^{2} \mathrm{~A}$ Balasubramaniam, ${ }^{3} \mathrm{R}$ Alyssa, ${ }^{4} \mathrm{~N}$ Garza, ${ }^{5} \mathrm{~K}$ Piri, ${ }^{6} \mathrm{R}$ Kinman. ${ }^{1}$ Oakland University, Rochester, MI; ${ }^{2}$ University of Leicester, Leicester, UK; ${ }^{3}$ University of California San Francisco, San Francisco, CA; ${ }^{4}$ University of California Merced, Merced, CA; ${ }^{5}$ California State University Fresno, Fresno, CA; ${ }^{6}$ University of California San Francisco Fresno, Fresno, CA 
Purpose of Study Inequalities in educational opportunities and lack of STEM identity have contributed to underrepresentation of various minority backgrounds in medical professions. We developed Kidz MedEd, an innovative pipeline program to increase STEM identity in impoverished minority youth interested in health careers through the creation of animated medical education videos and medical infographics.

Methods Used High school students were recruited from the UCSF Fresno Doctors Academy Summer Program while premedical college students volunteered to provide mentorship to the high school students. Learners were divided into 3 groups containing medical, college, and high school student(s). Each group was headed by a pediatric subspecialist and met weekly via Zoom for mentorship and medical education. Animated medical education videos were created with Powtoon, with Canva used to create visually appealing medical infographics.

Summary of Results Students learned digital literacy skills and improved their fund of medical knowledge while collaborating with both peers and near-peers to make informative infographics and videos on a variety of topics. An emphasis was placed on copyright literacy, gender and racial neutrality, and provision of medically accurate information to those with low literacy levels. Students were able to make informative infographics and videos on a variety of topics including asthma, constipation, diabetes, hepatic steatosis, hormones, obesity, and thyroid disease.

Conclusions The Kidz MedEd project has provided underrepresented minority high school and college students from California's impoverished Central Valley with an opportunity to gain insight into the medical field, learn valuable digital literacy skills, while simultaneously receiving mentorship from healthcare professionals. It demonstrates that educational technology can be used to create innovative medical learning experiences for high school and college students interested in health careers. Collaborations such as this can benefit both disadvantaged students who wish to gain greater exposure to the medical field and physicians who lack the time to create educational tools such as animated medical videos, infographics, and other social media content.

\section{Cardiovascular III}

\section{Concurrent session}

\section{8:00 AM}

\section{Friday, January 21, 2022}

\section{\#220 SMIDT HEART INSTITUTE TAKOTSUBO REGISTRY: ADJUDICATION METHODS}

\footnotetext{
1J Maughan*, ${ }^{1} \mathrm{O}$ Obrutu, ${ }^{1} \mathrm{~B}$ Tjoe, ${ }^{2} \mathrm{R}$ Herscovici, ${ }^{1} \mathrm{P}$ Moy, ${ }^{1} \mathrm{~N}$ Rojas, ${ }^{1} \mathrm{P}$ Marano, ${ }^{1} \mathrm{~J}$ Wei, ${ }^{1} \mathrm{C}$ Shufelt, ${ }^{1} \mathrm{C}$ Bairey Merz. ${ }^{1} \mathrm{Cedars}$-Sinai Medical Center, Los Angeles, CA; ${ }^{2}$ Sheba Medical Center, Tel Hashomer, Israel
}

\subsection{6/jim-2022-WRMC.217}

Purpose of Study Takotsubo syndrome (TTS) is an acute transient left ventricular dysfunction often triggered by emotional or physical stressors and seen predominantly in post-menopausal women. The Smidt Heart Institute Takotsubo Registry at Cedars-Sinai Medical Center aims to understand the prevalence, recurrence and prospective status of TTS. Rigorous medical record review is needed to adjudicate the diagnosis of TTS according to the International Takotsubo (InterTAK) Diagnostic Criteria.

Methods Used Once enrolled into the Takotsubo Registry, research staff obtain medical records of all prior TTS events from the enrollees directly (upload via HIPAA secure BOX), through medical record request using a signed medical Release of Information Authorization form, or via the electronic health record EPIC and Care Everywhere system. Once medical records are obtained, study staff detail the completion of the records and note the TTS clinical picture, hospitalization labs, TTS triggers, electrocardiograms (ECG), echocardiograms (ECHO) during admission and recover, coronary angiography and ventriculogram findings, past medical history, and past and present medications. The minimum records needed for adjudication are troponin levels with ECGs during event, ECHOs, angiography and hospitalization summary. Once medical records are complete, they are sent to be adjudicated by two board-certified cardiologists in consensus using the following InterTAK Diagnostic Criteria: 1) transient left ventricular dysfunction with or without right ventricular dysfunction presenting as apical ballooning or midventricular, basal, or focal wall motion abnormalities, typically extending beyond a single epicardial vascular distribution, 2) absence of culprit atherosclerotic coronary artery disease 3) new and reversible ECG abnormalities, 4) positive elevation in cardiac troponin, 5) absence of infectious myocarditis. Events are adjudicated on REDCap as definite TTS, probable TTS, probable not TTS and definite not TTS based on the medical record review. Enrollees receive written adjudication results.

Summary of Results From January 2019 to July 2021, 104 of the 131 participants enrolled in the registry have been adjudicated. Of the baseline events, 101 events were adjudicated as 61 definite TTS, 22 probable TTS, 10 probable not TTS and 8 definite not TTS. Furthermore, 32 enrollees reported a total of 55 recurrent events (between 2-5 events per enrollee) which include 14 definite TTS, 11 probable TTS, 7 probable not TTS and 23 definite not TTS events.

Conclusions The Smidt Heart Institute Takotsubo Registry aims to investigate TTS pathophysiology and gain an accurate estimation of TTS reoccurrence. Limitations of the adjudication process include time intensive collection of medical records from institutions which is a focus for improvement.

\section{\#221 RISK FACTORS FOR SEIZURES FOLLOWING HEART TRANSPLANTATION}

${ }^{1} \mathrm{~L}$ Mishalani ${ }^{*},{ }^{2} \mathrm{~N}$ Patel, ${ }^{2} \mathrm{~T}$ Singer-Englar, ${ }^{2} \mathrm{~S}$ Kim, ${ }^{2} \mathrm{M}$ Hamilton, ${ }^{2} \mathrm{~J}$ Kobashigawa. ${ }^{1} \mathrm{Columbia}$ University, New York, NY; ${ }^{2}$ Cedars-Sinai Smidt Heart Institute, Los Angeles, CA

\subsection{6/jim-2022-WRMC.218}

Purpose of Study Seizures following heart transplantation is not uncommon. Patients who have had previous strokes have a higher propensity to develop seizures after open heart surgery. Patients who undergo heart transplant may also have underlying atherosclerotic vascular disease and have a history of stroke or cerebral microvascular disease. Both of these entities may be a risk for developing seizures after heart transplant. It has not been well established what the frequency of seizures is and whether or not we can identify risk factors as to who will develop these seizures. 


\begin{tabular}{llll} 
Abstract \#221 Table $\mathbf{1}$ & \multicolumn{3}{l}{ Univariate analysis } \\
\hline & $\begin{array}{l}\text { Seizures Post-HTx } \\
(\mathbf{n}=\mathbf{4 0 )}\end{array}$ & $\begin{array}{l}\text { No Seizures Post-HTx } \\
(\mathbf{n}=\mathbf{5 2 0})\end{array}$ & $\begin{array}{l}\text { P-Value } \\
\end{array}$ \\
\hline Male Gender & $62.5 \%$ & $70.8 \%$ & 0.271 \\
History of Diabetes & $37.5 \%$ & $35.0 \%$ & 0.750 \\
History of Hypertension & $55.0 \%$ & $35.2 \%$ & 0.012 \\
History of Stroke & $22.5 \%$ & $17.1 \%$ & 0.388 \\
History of Atherosclerosis & $67.5 \%$ & $50.2 \%$ & 0.035 \\
History of Smoking & $40.0 \%$ & $39.6 \%$ & 0.962 \\
History of Previous Seizures & $10.0 \%$ & $2.9 \%$ & 0.017 \\
\hline
\end{tabular}

Abstract \#221 Table 2
\begin{tabular}{|l|c|c|}
\hline Multivariate Analysis \\
\hline & Odds Ratio $(95 \% \mathrm{Cl})$ & P-Value \\
\hline $\begin{array}{l}\text { History of } \\
\text { Hypertension }\end{array}$ & $2.193(1.130-4.259)$ & 0.020 \\
\hline $\begin{array}{l}\text { History of } \\
\text { Atherosclerosis }\end{array}$ & $1.786(0.891-3.581)$ & 0.102 \\
\hline $\begin{array}{l}\text { History of Previous } \\
\text { Seizures }\end{array}$ & $3.924(1.204-12.791)$ & 0.023 \\
\hline
\end{tabular}

Methods Used Between 2015 and 2020, we assessed 560 patients undergoing heart transplantation and found that 40 patients developed seizures within the first month postoperatively. We identified risk factors for these patients such as previous stroke, atherosclerotic vascular disease, previous history of seizures, history of diabetes, history of smoking, and male gender. The main endpoint was to establish the frequency of seizures following heart transplant surgery and to assess risk factors to develop seizures. These patients with seizures were compared to patients without seizures.

Summary of Results The incidence of seizure was 7.1\% following heart transplant surgery. Univariate analysis found that hypertension, history of atherosclerosis, and history of seizures were significant risk factors to developing seizures immediately postoperative. Multivariate analysis found that only hypertension and history of previous seizures were significant for the development of seizures post-heart transplant. Patients who were on tacrolimus appear to have a lower threshold to experience seizures and these patients were subsequently switched to cyclosporine.

Conclusions Patients undergoing heart transplant with hypertension and history of seizures are at risk to develop seizures postoperatively. These patients may be considered for prophylactic anti-seizure therapy for the first 30 days following heart transplant surgery.

\section{\#222 MYOCARDITIS AS AN EARLY MANFIESTATION OF SYSTEMIC LUPUS ERYTHROMATOSUS IN A YOUNG FEMALE}

${ }^{1} \mathrm{P} C$ Chan ${ }^{*},{ }^{2} \mathrm{VK}$ Narang, ${ }^{2} \mathrm{~F}$ Joolhar, ${ }^{2} \mathrm{~T}$ Win. ${ }^{1}$ Ross University School of Medicine, Miramar, $\mathrm{FL}^{2}{ }^{2}$ UCLA-Kern Medical, Bakersfield, CA

10.1136/jim-2022-WRMC.219
Case Report Systemic lupus erythematosus (SLE) is an autoimmune disease where the immune system can attack its own tissue and causes damage to many organs, even the heart. This case report shows a rare presentation of SLE myocarditis in an 18 year old female.

Methods Approval was obtained from IRB. A single patient case report was conducted.

Case Presentation A 18-year-old Filipino female with no past medical history presented to the emergency department with bilateral extremities and periorbital swelling for 10 days with recent development of oral mucosal ulcers. Patient was found to have hyponatremia and nephrotic range proteinuria; in addition to a positive family history of autoimmune disorder, an autoimmune workup was ordered. The work up showed high antinuclear antibodies titer of 1:1280 and high doublestranded DNA antibody of $6 \mathrm{IU} / \mathrm{mL}$ which was highly suspicious of SLE related cause of patient's symptoms. Further workup with ultrasound-guided renal biopsy resulted in lupus podocytopathy. Chest $\mathrm{x}$-ray showed cardiomegaly so transthoracic echocardiogram (TTE) was ordered to rule out pericarditis and pericardial effusion. The TTE showed left ventricular ejection fraction (LVEF) 60\%. Eight days later, the patient was found to be tachycardic to 130-140s with oxygen saturation of $88 \%$ so computerized tomography angiogram of the chest was obtained. It showed no evidence of pulmonary emboli but there was right lower lobe pneumonia, right sided small pleural effusion, and borderline cardiomegaly. Electrocardiogram showed no abnormalities but BNP was elevated to $3608 \mathrm{pg} / \mathrm{mL}$ and repeat TTE showed concentric left ventricular hypertrophy with LVEF $20 \%$ and small circumferential pericardial effusion. Guideline-directed medical therapy (GDMT) with lisinopril and carvedilol initiated. The patient was discharged with outpatient follow up.

Conclusion SLE can affect virtually all organs by the immune system attacking its own tissue and causing widespread inflammation and tissue damage. The heart is a common affected organ usually presenting with pericarditis or premature coronary artery disease in patients with long standing disease while myocarditis is less common.

This case shows a rare presentation of SLE myocarditis in an acute setting seen with a huge decline in cardiac function over 8 days. One can argue that this could be a case of stress myocarditis due to the acute presentation. A better understanding of patients with SLE and the risk for specific cardiac manifestations of SLE should be researched for improvement of clinical outcome for future patients.

\section{\#223 SMIDT HEART INSTITUTE TAKOTSUBO REGISTRY - STUDY DESIGN}

'O Obrutu*, ' J Maughan, 'B Tjoe, ${ }^{2} \mathrm{R}$ Herscovici, ${ }^{1} \mathrm{P}$ Moy, ${ }^{1} \mathrm{~N}$ Rojas, ${ }^{1} \mathrm{P}$ Marano, ${ }^{1} \mathrm{~J}$ Wei, ${ }^{1} \mathrm{C}$ Shufelt, ${ }^{3,4} \mathrm{~T}$ Rutledge, ${ }^{1} \mathrm{C}$ Bairey Merz. ${ }^{1} \mathrm{C}$ edars-Sinai Smidt Heart Institute, Los Angeles, $C A ;{ }^{2}$ Chaim Sheba Medical Center, Tel-Hashomer, Israel; ${ }^{3}$ VA San Diego Healthcare System, San Diego, $C A ;{ }^{4}$ University of California, San Diego, CA

\subsection{6/jim-2022-WRMC.220}

Purpose of Study Takotsubo syndrome (TTS) is an acute form of transient systolic heart failure that occurs predominantly in women, often triggered by emotional or physical stressors. The Smidt Heart Institute Takotsubo Registry at Cedars-Sinai Medical Center aims to establish a database for deep phenotyping of this syndrome. 




Abstract \#223 Figure 1 Recruitment and enrollment flowchart

Methods Used Registry participants are recruited from multiple sources: Deep 6-based medical records review, physician referrals, peer and self referrals (Abstract \#223 figure 1). Deep 6 is an artificial intelligence software that analyzes clinical data to identify patients that match complex clinical trial criteria. Peer and self referrals are sourced through social media advertisements on a Facebook support group for TTS survivors. Recruited participants remotely sign consents and provide detailed information via questionnaires on REDCap. Medical records of TTS events are adjudicated by cardiologists using the International Takotsubo Diagnostic Criteria. MITRA kits are used to remotely collect blood samples for further analysis.

Summary of Results From January 2019 to July 2021, 131 participants (99\% female, mean age: $61.2 \pm 9.8$ years) enrolled in the registry across 25 US states and 3 other countries. About $41 \%$ of enrollees are self or peer referrals. Overall, $89 \%$ completed all baseline questionnaires and 78\% returned filled MITRA kits. Completed questionnaires provide information about participants' general health status, physical function, psychosocial history, and TTS events.

Conclusions The Takotsubo Registry will reach a large and diverse participant base by using a multifaceted approach to recruitment as well as tools to facilitate remote enrollment and study participation. Study questionnaires, blood samples and medical record review will allow for deep phenotyping of participants in order to deepen our understanding of TTS pathophysiology.

\section{\#224 CARDIAC TRANSCRIPTOME ANALYSIS OF THE ELECTRONIC CIGARETTE-INDUCED CARDIAC DYSFUNCTION MOUSE MODEL TREATED WITH ACIPIMOX}

${ }^{1} \mathrm{JL}$ Arambulo*, 'J Espinoza-Derout, ${ }^{1} \mathrm{~K}$ Hasan, ${ }^{2} \mathrm{MC}$ Jordan, ${ }^{1} \mathrm{CJ}$ Lao, ${ }^{1} \mathrm{~J}$ Wilson, ${ }^{2} \mathrm{~K}$ Luna, ${ }^{1} \mathrm{~A}$ Sinha-Hikim, ${ }^{2} \mathrm{~K}$ Roos, ${ }^{1} \mathrm{~T}$ Friedman. ${ }^{1}$ Charles Drew University of Medicine and Science, Los Angeles, CA; ${ }^{2}$ University of California Los Angeles David Geffen School of Medicine, Los Angeles, $C A$

10.1136/jim-2022-WRMC.221
Purpose of Study Electronic cigarettes (e-cigarettes) have increased in popularity and pose a public health crisis, demanding further research on their cardiovascular health effects to better inform future interventions. We have shown that mice treated with e-cigarettes containing nicotine develop increased serum free fatty acid levels (FFAs) and systolic dysfunction associated with inflammation. As such, we aim to study the differentially expressed genes between the hearts of e-cigarette and saline-treated mice and to define the genes controlling the physiological normalization produced by acipimox (a lipolysis inhibitor). We hypothesize that acipimox will reverse the transcriptomic changes associated with e-cigaretteinduced cardiomyopathy.

Methods Used C57BL/6J wild type mice were exposed to saline, e-cigarettes, and e-cigarettes plus acipimox for 12 weeks. Left ventricular RNA was sequenced for differential gene expression and analyzed using Ingenuity Pathway Analysis (IPA) and Gene Set Enrichment Analysis (GSEA).

Summary of Results Our preliminary echocardiographic data showed that acipimox abrogates the e-cigarette-induced increases in serum FFAs and cardiomyopathy. RNA-sequencing analysis showed that 79 genes were dysregulated by e-cigarette treatment, and IPA showed that these changes are associated with inflammation. 30 of these genes were normalized by acipimox treatment and are implicated in inflammation, atherogenesis, and cardiac function. GSEA revealed that acute myocardial infarction, circadian rhythm, and G2/M DNA damage checkpoint genes are enriched in e-cigarette-treated mice compared to e-cigarette plus acipimox and saline-treated mice. Conclusions Our RNA-sequencing analysis offers a mechanistic insight into how acipimox prevents cardiomyopathy in e-cigarette-treated mice. These findings provide gene expression evidence suggesting that lipolysis is necessary for e-cigaretteinduced cardiac dysfunction. This study and future studies will not only illuminate the harms involved with e-cigarettes, but will also aid in the identification of targets for intervention to address complications associated with e-cigarette usage.

\section{\#225 LONG-TERM CARDIAC OUTCOMES OF MULTI-SYSTEM INFLAMMATORY SYNDROME IN CHILDREN (MIS-C)}

${ }^{1} \mathrm{~K}$ Ghasemian* , 'J Chuang, ' $\mathrm{S}$ Datta, ${ }^{1} \mathrm{~A}$ Koka, ${ }^{1} \mathrm{~F}$ Shaik, ${ }^{1} \mathrm{D}$ Sun, ${ }^{1} \mathrm{M}$ Sunkara, ${ }^{1,2} \mathrm{~B}$ Afghani. ${ }^{1}$ University of California Irvine School of Medicine, Irvine, $C A ;{ }^{2}$ Children's Hospital of Orange County, Orange, $C A$

\subsection{6/jim-2022-WRMC.222}

Purpose of Study Due to the novelty and severity of the COVID-19 pandemic, the long-term cardiac outcomes of children diagnosed with multi-system inflammatory syndrome (MIS-C) have not been extensively studied. The purpose of this study was to evaluate cardiac outcomes, comparing echocardiogram and lab results at admission and follow-up periods.

Methods Used A literature review using Google Scholar and Pubmed was conducted, utilizing keywords such as 'long-term cardiac outcomes', 'multi-system inflammatory syndrome in children', and 'Coronarivus Disease 2019'. We included studies of MIS-C patients that reported echocardiographic data and outcomes, troponin levels, and a minimum follow-up period of two weeks. Studies only evaluating electrocardiogram results were excluded.

Summary of Results Left ventricular (LV) dysfunction or decreased LV ejection fraction (LVEF) were the most common 
Abstract \#225 Table 1 Cardiac characteristics and long-term outcomes in patients with multisystem inflammatory syndrome in children (MIS-C)

\begin{tabular}{|c|c|c|c|c|c|c|c|c|c|c|}
\hline $\begin{array}{l}\text { Author, } \\
\text { Location, Year }\end{array}$ & $\begin{array}{l}\text { Mean/ } \\
\text { Median Age } \\
\text { (years) }\end{array}$ & $\begin{array}{l}\text { Initial LV } \\
\text { Dysfunction/ } \\
\text { Decreased LVEF (\%) }\end{array}$ & $\begin{array}{l}\text { Initial } \\
\text { Dilations } \\
(\%)\end{array}$ & $\begin{array}{l}\text { Initial } \\
\text { Aneurysms } \\
(\%)\end{array}$ & $\begin{array}{l}\text { Elevated } \\
\text { Troponin } \\
(\%)\end{array}$ & $\begin{array}{l}\text { Myocarditis, MR, } \\
\text { PCE (\%) }\end{array}$ & $\begin{array}{l}\text { Follow-up LV } \\
\text { Dysfunction/Decreased } \\
\text { LVEF (\%) }\end{array}$ & $\begin{array}{l}\text { Follow-up } \\
\text { Dilations } \\
(\%)\end{array}$ & $\begin{array}{l}\text { Follow-up } \\
\text { Aneurysms } \\
(\%)\end{array}$ & $\begin{array}{l}\text { Follow- } \\
\text { up } \\
\text { Period }\end{array}$ \\
\hline $\begin{array}{l}\text { Minocha et al., } \\
\text { NYC, } 2021\end{array}$ & 2.8 & $4 / 33(13 \%)$ & $2 / 33(7 \%)$ & $0 / 33(0 \%)$ & $7 / 32(22 \%)$ & $\begin{array}{l}1 / 33(5 \%), 5 / 33 \\
(15 \%), 1 / 33(3 \%)\end{array}$ & $0 / 33(0 \%)$ & $0 / 33(0 \%)$ & $0 / 33(0 \%)$ & 14 days \\
\hline $\begin{array}{l}\text { Gaitonde et al., } \\
\text { Georgia, } 2020\end{array}$ & 8 & $8 / 12(67 \%)$ & $1 / 12(8 \%)$ & $0 / 12(0 \%)$ & - & $\begin{array}{l}\text { MR: } 12 / 12 \\
(100 \%), \text { PCE: } 5 / 12 \\
(42 \%)\end{array}$ & $1 / 12(8 \%)$ & $2 / 12(17 \%)$ & $0 / 12(0 \%)$ & $\begin{array}{l}\text { Median = } \\
45 \text { days }\end{array}$ \\
\hline $\begin{array}{l}\text { Clouser et al., } \\
\text { USA, } 2021\end{array}$ & 7.3 & $7 / 18(39 \%)$ & $0 / 18(0 \%)$ & $0 / 18(0 \%)$ & - & PCE: $2 / 18$ (11\%) & $1 / 11(9 \%)$ & $1 / 11(9 \%)$ & $\begin{array}{l}0 / 11 \\
(0 \%)\end{array}$ & $\begin{array}{l}\text { Median = } \\
29 \text { days }\end{array}$ \\
\hline $\begin{array}{l}\text { Dionne et al., } \\
\text { USA, } 2020\end{array}$ & 9.7 & $15 / 25(60 \%)$ & $\begin{array}{l}3 / 25 \\
(12 \%)\end{array}$ & $2 / 25(8 \%)$ & $2 / 25(8 \%)$ & - & $2 / 15(13 \%)$ & - & - & $\begin{array}{l}\text { Median = } \\
51 \text { days }\end{array}$ \\
\hline $\begin{array}{l}\text { Penner et al., } \\
\text { UK, } 2021\end{array}$ & 10.2 & $15 / 46(33 \%)$ & & & $38 / 45$ (84\%) & PCE: $1 / 46(2 \%)$ & $0 / 46(0 \%)$ & $1 / 46(2 \%)$ & $1 / 46(2 \%)$ & 6 months \\
\hline $\begin{array}{l}\text { Kelly et al., } \\
\text { Boston, } 2020\end{array}$ & 3.5 & $7 / 12$ (58\%) & $1 / 12(8 \%)$ & $1 / 12(8 \%)$ & $9 / 12(75 \%)$ & $\begin{array}{l}\text { MR: } 3 / 12(25 \%), \\
\text { PCE: } 4 / 12(33 \%)\end{array}$ & $1 / 8(13 \%)$ & $2 / 8(25 \%)$ & $1 / 8(13 \%)$ & $\begin{array}{l}\text { Median }= \\
16.5 \text { days }\end{array}$ \\
\hline $\begin{array}{l}\text { Jhaveri et al., } \\
\text { NYC, } 2021\end{array}$ & 11.5 & $8 / 15(53 \%)$ & - & $4 / 15(33 \%)$ & - & $\begin{array}{l}\text { MR: } 8 / 15(53 \%), \\
\text { PCE: } 2 / 15(13 \%)\end{array}$ & $3 / 13(23 \%)$ & - & $1 / 13(8 \%)$ & $\begin{array}{l}\text { Median }= \\
28.1 \text { days }\end{array}$ \\
\hline $\begin{array}{l}\text { Feldstein et al., } \\
\text { US, } 2021\end{array}$ & 9.7 & $172 / 539(34 \%)$ & - & $\begin{array}{l}57 / 424 \\
(13 \%)\end{array}$ & - & $\begin{array}{l}\text { PCE: } 125 / 539 \\
(25 \%)\end{array}$ & $1 / 172(0.58 \%)$ & - & $12 / 172(7 \%)$ & $\begin{array}{l}2-20 \\
\text { weeks }\end{array}$ \\
\hline $\begin{array}{l}\text { Kobayashi et } \\
\text { al., Canada, } \\
2021\end{array}$ & 11.4 & $9 / 25(36 \%)$ & $2 / 25(8 \%)$ & $4 / 26(16 \%)$ & 9/23 (39\%) & $\begin{array}{l}\text { MR: } 1 / 25(4 \%) \\
\text { PCE: } 0 / 25(0 \%)\end{array}$ & $2 / 9(22 \%)$ & - & - & $\begin{array}{l}1-2 \\
\text { months }\end{array}$ \\
\hline Totals & $2.8-11.5$ & $13-60 \%$ & $0-12 \%$ & $0-33 \%$ & $8-84 \%$ & - & $0-23 \%$ & $0-25 \%$ & $0-13 \%$ & $\begin{array}{l}2 \text { weeks- } \\
6 \text { months }\end{array}$ \\
\hline
\end{tabular}

initial finding and seen in up to $60 \%$ of patients with MIS-C. Upon follow-up, these abnormalities had resolved in the vast majority of patients, with residual dysfunction seen in a few patients. Coronary artery dilations and aneurysms were seen in up to $33 \%$ of patients initially, and resolved in almost all patients, especially when follow-up period was longer. Elevated troponin levels had normalized at follow-up. Other common MIS-C-associated cardiac abnormalities included myocarditis, mitral valve regurgitation, and pericardial effusion, which were often resolved by the follow-up period.

Conclusions Our review suggests that patients with MIS-C have favorable long-term cardiac outcomes. In general, the initial cardiac complications and findings substantially improve or resolve by follow-up. Persistent cardiac abnormalities are rare, and often mild in severity. Further research with larger sample sizes and longer follow-up periods must be evaluated to better understand the long-term cardiac outcomes of MIS-C.

\section{\#226 WET BERIBERI IN A PATIENT WITH ISCHEMIC CARDIOMYOPATHY AND GASTRIC BYPASS: A CASE REPORT}

T Azenkot* ${ }^{*}$ OM Campa. University of California Davis Health System, Sacramento, CA

\subsection{6/jim-2022-WRMC.223}

Case Report A 49-year-old woman with hypertension and Roux-en-Y gastric bypass 9 years prior presented for subacute exertional dyspnea and chest pain. Her home medications were multivitamins. On admission, blood pressure was $163 / 123 \mathrm{mmHg}$ and heart rate $96 \mathrm{bpm}$. Hemoglobin was $12.9 \mathrm{~g} / \mathrm{dL}$. Troponin was 22 and unchanged on repeat. Btype natriuretic protein was $276 \mathrm{pg} / \mathrm{mL}$. An electrocardiogram was without changes concerning for acute coronary syndrome. An echocardiogram showed global hypokinesis with an estimated left ventricular ejection fraction (LVEF) of $25 \%$. Coronary angiography demonstrated complete total occlusion of the right coronary artery, though her global akinesis was out of proportion to this finding. She was discharged on guideline directed medical therapy for heart failure (HF).

Four months later, the patient returned to the emergency department for dizziness. Her blood pressure was 53/43, with improvement to $91 / 53$ after fluids. Creatinine was 2.29 and granular casts seen on urinalysis. Her alkaline phosphatase and aspartate and alanine transaminases were $1,213 \mathrm{U} / \mathrm{L}, 615 \mathrm{U} / \mathrm{L}$, and $338 \mathrm{U} / \mathrm{L}$, respectively, a pattern consistent ischemic hepatopathy. An echocardiogram showed improved LVEF to $32 \%$ with no focal wall motion abnormalities. Thiamine level was 51 (normal 70-180) nmol/L, concerning for component of thiamine deficiency-induced cardiomyopathy, or wet beriberi. Supplementation was initiated. HF medications were held. Her dizziness and lab abnormalities resolved with fluids.

The prevalence of thiamine deficiency in patients admitted for HF is estimated to be 30\% (Hanninen et al., 2006). This may be in part due to the association of diuretics, including furosemide, with increased renal excretion and decreased intestinal absorption of thiamine. Post gastric bypass patients also have a prevalence of thiamine deficiency up to $30 \%$ due to disruption of thiamine absorption in the duodenum and jejunum (Wilson, 2020). This renders patients with HF and gastric bypass, such as the present case, dually vulnerable to thiamine deficiency. Several small studies have demonstrated improved HF outcomes (i.e., improved LVEF and decreased hospital admission rates) with thiamine supplementation, though further studies are needed (Jain et al., 2015). 
MALIGNANT EFFUSIONS: A RARE CASE OF SUBACUTE, SEVERE REGIONAL CARDIAC TAMPONADE

N Shamapant*, C Duarte. University of Colorado, Denver, CO

\subsection{6/jim-2022-WRMC.224}

Case Report Identify clinical manifestations of regional cardiac tamponade

Case Presentation The patient is a 31 year old female with stage IV ER+, PR+, Her2- breast cancer complicated by recurrent malignant pleural effusions who presented with worsening dyspnea. Vitals on admission included BP 121/91, HR 124, RR 32 and oxygen saturation of 99\%. Shortly after admission, blood pressure dropped to $92 / 68$ and oxygen saturation was $97 \%$ on $2 \mathrm{~L}$ nasal cannula. On exam, patient had increased work of breathing with elevated neck veins. EKG showed low voltage QRS complexes with electrical alternans. CTA chest revealed a large pericardial effusion with no evidence of pulmonary embolus (PE). Given the pericardial effusion, a manual pulsus was checked and was negative. TTE showed a large pericardial effusion with moderate right ventricular (RV) collapse, dilated inferior vena cava and normal left ventricular systolic function. Based on the echocardiographic evidence of pericardial effusion with RV collapse in the context of worsening hypotension and tachycardia, the diagnosis of regional cardiac tamponade was made. Pericardiocentesis was performed with an opening pericardial pressure of $23 \mathrm{mmHg}$ and $680 \mathrm{~mL}$ of fluid was drained with cytology confirming malignant pericardial effusion. Following the pericardiocentesis, the patient's dyspnea and tachycardia resolved. Significant post-procedure pericardial drainage prompted placement of a pericardial window and the patient was discharged to hospice.

Discussion This patient's presentation of dyspnea, tachycardia, progressive hypotension and distended neck veins fits multiple illness scripts including PE and cardiac tamponade. When the CTA was negative for PE, the team pursued tamponade as a possible diagnosis. Normally the lack of a pulsus paradoxus would argue against clinical tamponade physiology. However, this case is particularly unique for the echocardiographic finding of isolated RV collapse, leading to clinically significant regional tamponade. Collapse of a cardiac chamber occurs when pericardial pressure is greater than chamber pressure. Regional tamponade is often seen after procedures (pericardiotomy, cardiac surgery) or myocardial infarction where a loculated effusion or pericardial hematoma forms in a particular region ${ }^{1}$. Regional cardiac tamponade fails to produce pulsus physiology because the increased pericardial pressure is localized to the $\mathrm{RV}^{2}$. The RV's inability to expand prevents interventricular septum from bowing into the left ventricle (LV) on inspiration. As a result, there is no compression of the $\mathrm{LV}$, a phenomenon which is required for the clinical manifestation of pulsus paradoxus. This patient's presentation of dyspnea, hypotension, and tachycardia with negative pulsus paradoxus but echocardiographic findings of pericardial effusion merited a high level of suspicion for regional cardiac tamponade.

\section{Case reports I}

\section{Concurrent session}

8:00 AM

\section{Friday, January 21, 2022}

\section{$\# 228$ EXTENSIVE LEFT VENTRICULAR AND MITRAL VALVE THROMBUS IN A GASTRIC CANCER PATIENT ON DIRECT ORAL ANTICOAGULANTS}

${ }^{1} \mathrm{P}$ Chan*, ${ }^{2} \mathrm{VK}$ Narang, ${ }^{2} \mathrm{~F}$ Joolhar, ${ }^{2} \mathrm{~T}$ Win. ${ }^{1}$ Ross University School of Medicine, Miramar, FL; ${ }^{2}$ UCLA-Kern Medical, Bakersfield, CA

\subsection{6/jim-2022-WRMC.225}

Case Report Thrombotic events are a common complication of cancer but it is rare to have arterial thrombosis. This is an unusual case of left ventricular thrombus and multiple thrombi on the mitral valve while taking direct oral anticoagulants (DOAC) in a patient with stage IV gastroesophageal signet ring adenocarcinoma.

Methods Approval was obtained from the IRB at Kern Medical. A single patient chart review was conducted.

Summary A 45-year-old female with no past medical history presented to the emergency department (ED) with decreased appetite, fatigue, dysphagia, abdominal pain, and unintentional weight loss for 4 months. Initial workup with esophagogastroduodenoscopy (EGD) showed an epigastric mass that was biopsied. The pathology came back showing stage IV gastroesophageal signet ring adenocarcinoma. During this hospitalization, the patient also had a computerized tomography (CT) of the chest done which showed pulmonary emboli (PE), and was started on apixaban for PE therapy.

Four weeks later, a CT of the chest, abdomen, and pelvis was ordered for evaluation of port-a-cath placement evaluation which demonstrated a filling defect within the left ventricular apex measuring $19 \times 17 \mathrm{~mm}$. The patient was referred for a transthoracic echocardiogram (TTE) which revealed a large left ventricular thrombus and multiple thrombi on the mitral valve. The patient was then admitted to the hospital for initiation of anticoagulation with therapeutic Lovenox. The patient was then discharged home with therapeutic Lovenox and instructions to follow up with cardiology outpatient.

Conclusion It is well known that there is a link between thromboembolism and cancer but the underlying mechanism is poorly understood. It is believed that there are many ways the cancer cells activate the coagulation system such as having the ability to produce and secrete procoagulant/fibrinolytic substances and inflammatory cytokines. Deep vein thrombosis (DVT) and pulmonary embolism (PE) are common complications in patients with cancer but arterial thrombosis secondary to malignancy is rare. The case highlights a rare presentation of a large left ventricular thrombus and multiple thrombi in the mitral valve in a patient with stage IV gastroesophageal signet ring adenocarcinoma that was already on a DOAC for a known PE. 


\section{\#229 CARDIOVASCULAR MANIFESTATIONS IN ADOLESCENT MALES AFTER THE PFIZER COVID-19 VACCINE}

${ }^{1} \mathrm{M}$ Arhin* ${ }^{2}{ }^{2} \mathrm{H}$ Hamad, ${ }^{3} \mathrm{C}$ Iheagwara, ${ }^{4} \mathrm{~J}$ Hales. ${ }^{1}$ University of North Carolina at Chapel Hill School of Medicine, Chapel Hill, NC; ${ }^{2}$ Geisinger Commonwealth School of Medicine, Scranton, PA; ${ }^{3}$ North Carolina State University, Raleigh, NC; ${ }^{4} H C A$ Holdings Inc, Nashville, TN

\subsection{6/jim-2022-WRMC.226}

Case Report The Food and Drug Administration (FDA) granted Emergency Use Authorization (EUA) for the Pfizer BioNTech mRNA (BNT162b2) and Moderna COVID-19 vaccines in December 2020 to address rising COVID-19 infections across the nation. Clinical trials showed the vaccines to be effective and safe. Once these vaccines became publicly available, some minor adverse events were reported to the Vaccine Adverse Event Reporting System (VAERS); they included fever, fatigue, and joint pain. Additionally, there have been cases of post-immunization myocarditis and/or pericarditis reported to VAERS.

This case series describes the clinical course of the first three adolescent patients in our hospital that presented with myocarditis or pericarditis within two to four days after administration of their first or second dose of the Pfizer mRNA covid-19 vaccine. The COVID-19 status of the patients was assessed by reverse transcription-polymerase chain reaction (RT-PCR) of nasopharyngeal swabs. Laboratory tests for all patients included a routine complete blood count, basic metabolic panel, troponin, and inflammatory markers. Two patients had testing for SARS-CoV-2/Covid-19 and other viral causes of myocarditis and pericarditis, including the Epstein-Barr virus, cytomegalovirus, parvovirus B19, respiratory syncytial virus, influenza A virus, influenza B virus, Roseola, and human herpesvirus 6 . These tests were all negative.

\begin{tabular}{|c|c|c|c|c|c|c|}
\hline $\begin{array}{l}\text { Case } \\
\text { No. }\end{array}$ & $\begin{array}{l}\text { Age/ } \\
\text { Sex }\end{array}$ & $\begin{array}{l}\text { Peak } \\
\text { CRP* } \\
\text { Peak } \\
\text { Troponin- } \\
\text { I* Peak } \\
\text { BNP** }\end{array}$ & Admission ECG & $\begin{array}{l}\text { RT-PCR } \\
\text { testing for } \\
\text { COVID19 }\end{array}$ & $\begin{array}{l}\text { Admission } \\
\text { CRP and } \\
\text { troponin }\end{array}$ & $\begin{array}{l}\text { Discharge } \\
\text { CRP and } \\
\text { troponin }\end{array}$ \\
\hline 1 & $\begin{array}{l}16 / \\
\text { Male }\end{array}$ & $\begin{array}{l}\text { CRP: } 4.06 \\
\text { mg/dL; } \\
\text { Troponin I: } \\
4.76 \mathrm{ng} / \mathrm{dL} ; \\
\text { BNP: } 53 \\
\mathrm{pg} / \mathrm{mL}\end{array}$ & $\begin{array}{l}\text { Nonspecific ST } \\
\text { and T wave } \\
\text { abnormality and } \\
\text { minimal ST } \\
\text { elevation in the } \\
\text { inferior leads V5 } \\
\text { and V6 }\end{array}$ & $\begin{array}{l}\text { Not } \\
\text { performed }\end{array}$ & $\begin{array}{l}\text { CRP: } 4.06 \\
\mathrm{mg} / \mathrm{dL} \\
\text { Troponin: } \\
4.07 \mathrm{ng} / \mathrm{mL}\end{array}$ & $\begin{array}{l}\text { CRP: } 1.45 \\
\text { mg/dL } \\
\text { Troponin: } \\
1.45 \mathrm{ng} / \mathrm{mL}\end{array}$ \\
\hline 2 & $\begin{array}{l}17 / \\
\text { Male }\end{array}$ & $\begin{array}{l}\text { CRP: } 6.10 \\
\text { mg/dL; } \\
\text { Troponin I: } \\
0.26\end{array}$ & $\begin{array}{l}\text { Right bundle } \\
\text { branch block }\end{array}$ & Negative & $\begin{array}{l}\text { CRP: } 5.0 \\
\mathrm{mg} / \mathrm{dL} \\
\text { Troponin: } \\
1.05 \mathrm{ng} / \mathrm{mL}\end{array}$ & $\begin{array}{l}\text { CRP: } 4.06 \\
\text { mg/dL } \\
\text { Troponin: } \\
0.45 \mathrm{ng} / \mathrm{mL}\end{array}$ \\
\hline 3 & $\begin{array}{l}14 / \\
\text { Male }\end{array}$ & $\begin{array}{l}\text { CRP: } 5.03 \\
\text { mg/dL; } \\
\text { Troponin I: } \\
7.61 ; \text { BNP: } \\
37 \text { pg/mL }\end{array}$ & $\begin{array}{l}\text { T wave inversion } \\
\text { in lateral leads } \\
\text { and ST segment } \\
\text { elevation in V2-V6 }\end{array}$ & Negative & $\begin{array}{l}\text { CRP: } 5.03 \\
\text { mg/dL } \\
\text { Troponin: } \\
5.52 \mathrm{ng} / \mathrm{mL}\end{array}$ & $\begin{array}{l}\text { CRP: } 4.04 \\
\text { mg/dL } \\
\text { Troponin: } \\
3.68 \mathrm{ng} / \mathrm{mL}\end{array}$ \\
\hline
\end{tabular}

*Serum CRP (normal range
The three cases at our hospital consisted of previously healthy male adolescents between the ages of 14 and 17 that presented with chest pain within 4 days of receiving their first or second mRNA covid-19 vaccine. On admission, they all had an abnormal electrocardiogram (ECG), elevated C-reactive protein (CRP), and elevated troponin I levels (table 1). Brain natriuretic peptide (BNP) levels were tested in two patients and found to be within normal limits. All patients had a normal echocardiogram, except for a residual patent foramen ovale in one patient. All patients showed down-trending troponin levels and inflammatory markers as well as complete resolution of symptoms before discharge on hospital day 4 . All patients had normal troponin and CRP levels at post-discharge follow-up with an outpatient pediatric cardiologist visit. No causal relationship has been proven between the vaccine and myocarditis or pericarditis. Currently, the Centers for Disease Control and Prevention (CDC) continues to recommend the COVID-19 vaccination for anyone older than twelve years old as the known risks of illness and possible severe complications far outweigh having a possible rare adverse reaction. Suspected cases of myocarditis and pericarditis after COVID19 vaccination should continue to be reported to VAERS

\section{\#230 METASTATIC AND PERSISTENT BACTEREMIA WITH METHICILLIN-RESISTANT STAPHYLOCOCCUS AUREUS, ASSOCIATED WITH COVID-19 PNEUMONIA, A MANAGEMENT NIGHTMARE}

${ }^{1,2} \mathrm{C}$ Besmanos ${ }^{*},{ }^{2} \mathrm{C}$ D'Assumpcao, ${ }^{2} \mathrm{I}$ Fong, ${ }^{2} \mathrm{R}$ Kuran, ${ }^{2} \mathrm{~A}$ Heidari. ${ }^{1}$ American University of the Caribbean School of Medicine BV, Cupecoy, Sint Maarten (Dutch part); ${ }^{2}$ Kern Medical Center, Bakersfield, CA

\subsection{6/jim-2022-WRMC.227}

Case Report Telavancin is a vancomycin-derivative semisynthetic lipoglycopeptide that has antimicrobial activity against resistant gram-positive organisms, namely methicillin-resistant Staphylococcus aureus (MRSA). The purpose of this study is to describe a case in which telavancin clinically failed in treating persistent MRSA bacteremia in a patient.

Methods Used Retrospective case review.

Summary of Results A 61-year-old man with nasal MRSA colonization and history of previous abscesses was initially admitted for severe COVID-19 pneumonia requiring high flow nasal cannula oxygen support and dexamethasone protocol as per current guidelines. He developed MRSA pneumonia and persistent polyclonal resistant MRSA bacteremia with hematogenous seeding causing lumbar vertebral osteomyelitis despite treatment with vancomycin for 5 days follow with telavancin for 11 days. MRSA susceptibility to the glycopeptide antibiotics as well as ceftaroline and daptomycin were evaluated using E-test to interpret minimum inhibitory concentration (MIC) according to the manufacturer's instructions. Telavancin MIC was initially 0.064 but then on retest was 0.125 . Since MRSA continued to grow in repeated blood cultures after 11 days, telavancin was considered to have clinically failed. He was switched to combination of ceftaroline and daptomycin and subsequently developed daptomycin associated eosinophilic pneumonitis. He was started on prolonged course of prednisone. He was switched to ceftaroline and rifampin to complete 6 weeks total of antibiotics after blood sterilization. Outpatient MRSA decolonization protocol was also started just prior to discharge. 
Conclusion Secondary bacterial infection associated with COVID-19 is on the rise particularly after adoption of dexamethasone as standard of care in severe cases. Persistent bacteremia with MRSA complicated with metastatic seeding in this setting is not described and perhaps is due to hostpathogen mediated mechanisms. Clinical failure of telavancin in deep seeded MRSA infections has not yet been reported.

\section{\#231 A SEVERE CASE OF RECURRENT HERPES SIMPLEX VIRUS-1 ENCEPHALITIS WITH AUTOIMMUNE COMPONENT}

${ }^{1}$ VF Civelli*, ${ }^{2} \mathrm{C} D$ 'Assumpcao, ${ }^{3} \mathrm{M}$ Kaur, ${ }^{2} \mathrm{R}$ Kuran, ${ }^{2} \mathrm{~K}$ Sabetian, ${ }^{2} \mathrm{~A}$ Heidari. ${ }^{1}$ Clinica Sierra Vista, Bakersfield, CA; ${ }^{2}$ Kern Medical Center, Bakersfield, CA; ${ }^{3}$ Ross University School of Medicine, Miramar, $F L$

\subsection{6/jim-2022-WRMC.228}

Case Report The incident of Human Herpes Simplex Virus1 (HSV-1) encephalitis is 4 in one million cases worldwide. From that $5-27 \%$ can become recurrent a rare phenomenon with serious sequela. Differentials include induced autoimmune-mediated sequelae or paraneoplastic encephalitis. Knowing that $69-89 \%$ of first HSV-1 encephalitis will result in permanent neurological deficits, the degree of damage in recurrent cases is estimated to be higher. The pathophysiology of recurrent HSV-1 encephalitis and its aftermath are poorly understood. We report a case of recurrent HSV encephalitis with evidence of autoimmune dysfunction.

Methods Used Retrospective Study

Summary of Results A 58-year-old Hispanic female presented elsewhere for acute onset of confusion. She was diagnosed with HSV-1 encephalitis by PCR testing of the CSF and received 28 days on intravenous (IV) Acyclovir and recovered. She presented to our facility with new onset of seizures, expressive aphasia, short-term memory loss, bowel/bladder incontinence, and unsteady gait. Her mental status declined, and diagnosis of autoimmune encephalitis was made due to negative HSV-1 PCR and brain MRI findings. A 5-day course of IV Immuglobulin and high dose methylprednisolone resulted in transient reliefe of her symptoms. She was discharged home with prednisone for 5 weeks. Autoimmune CSF panel showed elevated acetylcholine receptor ganglionic (alpha 3) $\mathrm{Ab}$ at 172, and elevated VGCC Type P/Q Ab at $>30$, and VGCC Type $\mathrm{N} \mathrm{Ab}$ at 69. She had two episodes of recurrent neurological symptoms resulted in courses of IV high dose steroid with transient relief each time CSF only was positive for mild pleocytosis with negative HSV-1 PCR. Her brain MRI continued to show progressive disease now spreading to both sides. On the third recurrence episode of rapid decline in mentation CSF came back positive for HSV-1 PCR. She was started on a 3-week course of IV Acyclovir. Follow up CSF was negative for HSV-1 PCR and she is placed on prolong course of oral valacyclovir with unknown duration perhaps lifelong.

Conclusion Differential diagnosis of recurrent encephalitis after the first episode of HSV-1 infection includes immune-mediated HSV-1 viral encephalitis vs recurrence of HSV-1 itself. Residual irreversible neurocognitive deficits are expected and may result in protracted disease. The diagnosis is challenging and prognosis is poor.

\section{\#232 HUMAN HERPESVIRUS-6 MENINGOENCEPHALITIS IN AN IMMUNOCOMPETENT MALE}

${ }^{1}$ VK Narang ${ }^{*},{ }^{2} \mathrm{C}$ D'Assumpcao, ${ }^{1} \mathrm{M}$ Valdez, ${ }^{1} \mathrm{~K}$ Radicic, ${ }^{1} \mathrm{~L}$ Moosavi, ${ }^{2} \mathrm{R}$ Kuran, ${ }^{2} \mathrm{~A}$ Heidari. ${ }^{1}$ UCLA-Kern Medical, Bakersfield, CA; ${ }^{2}$ Kern Medical-UCLA, Bakersfield, CA

\subsection{6/jim-2022-WRMC.229}

Case Report Meningoencephalitis with Human herpesvirus 6 (HHV-6) in adults is rare. It is known to occur as a result of reactivation of infected dormant brain cells during childhood, when host becomes immunocompromised in conditions such as organ or bone marrow transplantations. New primary infection in adults has also been described. Diagnosis is difficult and challenging particularly in immunocompetent adults due to low level of suspicious and undefined clinical, central spinal fluid (CSF) and neuroimaging findings. The duration and choice of antiviral also have not been well reported. We present a case of HHV-6 meningoencephalitis in an immunocompetent elderly male who presented with fever and altered mental status.

Methods A single patient case report was conducted after IRB approval.

Case Presentation Patient is a 79-year-old male with a history of dementia and diabetes mellitus who presented after being found down by a bystander. Patient arrived lethargic, tachypneic, and febrile to $39.4{ }^{\circ} \mathrm{C}$. Physical exam was remarkable for rigid extremities with laboratory studies demonstrating a leukocyte count of $14.6 \times 10^{\wedge} 3$ cells $/ \mathrm{mm} 3$ without left shift and hyperglycemia to $550 \mathrm{mg} / \mathrm{dL}$. He was empirically placed on antibiotics to cover bacterial etiologies plus acyclovir. CT of the head demonstrated moderate to severe global volume loss and periventricular leukomalacia. CSF showed WBC count of 17 with 76\% monocytes and 22\% lymphocytes. Extensive initial work up and all cultures came back negative. MRI brain with gadolinium found atrophic and chronic microangiopathic changes without enhancement. CSF Meningoencephalitis Panel (BioFire, BioMerieux, Salt Lake City, Utah) found HHV6 positivity. HHV-6 serology testing found negative IgM and positive IgG. Serum HHV-6 PCR confirmed the diagnosis with $>2$ million copies $/ \mathrm{mL}$. Patient was started on ganciclovir which resulted in significant improvement in symptoms. He was discharged to rehabilitation facility to complete a 30 day course of ganciclovir.

Conclusion HVV-6 meningoencephalitis is a serious but rare condition particularly in otherwise immunocompetent adults. Clinicians should be aware of this infection when initial work up is not diagnostic.

\section{\#233 A CASE OF CONCURRENT DISSEMINATED COCCIDIOIDOMYCOSIS AND EMBRYONAL CARCINOMA WHEN LICE AND FLEAS COEXIST}

M Ke, A Heidari, M Valdez*, A Tsiyer, R Kuran, R Johnson. Kern Medical Center, Bakersfield, CA

\subsection{6/jim-2022-WRMC.230}

Case Report Coccidioidomycosis (CM) is a fungal infection endemic to the southwestern United Stated with a wide range of clinical presentations depending on the infected organ systems. CM causes a primary pulmonary infection. 1 percent of cases disseminate, via hematogenous or lymphatic spread. It is in these cases, that more severe symptoms may present and potentially overlap with those characteristics of other systemic 
illnesses. We report a case of CM disseminated to lymph nodes in a 24-year-old man with concomitant metastatic embryonal carcinoma. It is difficult to identify the primary etiology for many components of his presentation and the relationship between these concurrent disease processes is not entirely clear. Factors that may contribute include locus minoris resistentiae or a shared immune response between infectious organisms and malignant cells.

Case Description 24-year-old man from the central valley of California presented with a new rash. Examination showed periorbital edema and diffuse raised, erythematous, hyperpigmented skin lesions. Differential included psoriasis and CM. $\mathrm{CM}$ serology showed nonreactive IgM, very weakly reactive IgG, and complement fixation $(\mathrm{CF})$ titer $<1: 2$. The patient was lost to follow-up but returned 6 weeks later with 17pound weight loss, progression of the rash, and proximal muscle weakness. He had a heliotrope rash and elevated CK. He was prescribed prednisone $60 \mathrm{mg}$ daily for dermatomyositis. 2 weeks later, he developed fevers and CM CF titer was 1:16. He was started on fluconazole $800 \mathrm{mg}$ daily. 5 weeks later, he developed diffuse lymphadenopathy and imaging showed multiple pulmonary nodules, destructive lesion in the iliac bone, and retroperitoneal and pelvic lymphadenopathy. CM CF titers were now 1:64 and he was started on liposomal amphotericin B. New retroperitoneal and right testicular masses were then identified. Histopathology from orchiectomy and retroperitoneal mass biopsy revealed embryonal carcinoma while inguinal lymph node excision showed granulomatous inflammation with endosporulating spherules diagnostic for CM. He completed 9 weeks of amphotericin and bone scan showed no foci of increased uptake. He is on track to complete 4-6 cycles of chemotherapy. On subsequent clinic visits, weight was up-trending and improvement in both rash and lymphadenopathy were noted.

Conclusion As CM and several types of malignancies may have similar or overlapping presentations, a thorough examination and tissue sampling are generally necessary to distinguish. In rare cases, coexistence may occur. 'Läuse und Flöhe haben.' A German phrase, which translates to 'Having lice and fleas,' refers to having two reasons for a problem. Understanding the etiology or identifying the relationship between the concomitant conditions is essential to formulate the most appropriate treatment plan.

\section{$\# 234$ \\ A RARE CASE OF NEISSERIA MENINGITIDIS PNEUMONIA IN THE ABSENCE OF MENINGITIS}

J Johal, M Valdez*, HK Sandhu, S Mishra, A Heidari. Kern Medical Center, Bakersfield, CA

10.1136/jim-2022-WRMC.231

Case Report Neisseria meningitidis (NM) is a gram-negative diplococcus that typically colonizes the nasopharynx after inhalation of aerosolized particles containing meningococci. NM may also be transmitted via direct contact with respiratory secretions. Once local tissues are colonized, NM can invade the bloodstream, causing numerous forms of meningococcal diseases. The most common manifestations are meningitis and septicemia. Meningococcal pneumonia (MP) is a rare manifestation of meningococcal disease. The incidence of MP is estimated at $5 \%-15 \%$ in patients with invasive meningococcal disease. Only 344 MP cases have been documented worldwide between 1906 and 2015. At least 13 serogroups of meningococci have been identified. Serogroups A, B, C, X, Y, and W-135 are associated with meningococcal disease in humas. Y and W-135 are most associated with pneumonia. The clinical presentation of MP is indistinguishable from pneumonia caused by other infectious organisms. We report a case of an 88-year-old female with concurrent SARS-CoV-2 and NM pneumonia.

Case Presentation 88-year-old woman with diabetes and hypertension presented with 3-day history of dyspnea, productive cough, and subjective fevers. On presentation, she met SIRS criteria and was hypoxic. Initial labs were significant for lactic acid 2.5, procalcitonin 3.62, and testing for SARS-CoV-2 RNA positive. CXR revealed a $7 \mathrm{~cm}$ RUL opacity. CTA chest showed a large RUL consolidation with scattered ground-glass opacities. She was initially started on Ceftriaxone $1 \mathrm{~g} \mathrm{Q} 24 \mathrm{H}$ and Azithromycin $500 \mathrm{mg} \mathrm{Q} 24 \mathrm{H}$ for community acquired pneumonia as well as a 10-day course of dexamethasone for hypoxia associated with SARS-CoV-2. Preliminary blood cultures grew gram-negative diplococci in 1 of 2 bottles and final culture revealed NM. Ceftriaxone was then increased to $2 \mathrm{~g}$ Q24H. Patient remained alert and oriented. She denied headaches and neck stiffness. Neck was supple with no nuchal rigidity or signs of meningismus. Bronchoscopy was considered but was eventually deferred in the setting of concurrent covid pneumonia and given that symptoms improved with appropriate antibiotic therapy. Repeat blood cultures showed no growth and patient was discharged with supplemental oxygen for covid associated hypoxia and oral Amoxicillin/Clavulanic acid to complete a 14 day total course of antibiotics for MP.

Conclusion Neisseria Meningitidis as the underlying etiology for pneumonia should always be considered when blood or sputum cultures identify gram-negative diplococci. Early recognition is critical in order to reduce the risk of transmission to close contacts and health care personnel. Finally, given the high mortality rates associated with untreated meningococcal disease, early initiation of appropriate antibiotic therapy is essential in attempting to improve the outcomes of meningococcal disease.

\section{\#235 GASTRIC MUCORMYCOSIS IN AN ADOLESCENT WITH NEW ONSET DIABETES MELLITUS}

J Timberman*, C Kupelian. Valley Children's Healthcare, Madera, CA

\subsection{6/jim-2022-WRMC.232}

Case Report Mucormycosis is a rare angioinvasive fungal infection associated with a high mortality rate, especially in children and neonates. Recent data suggests the incidence is rising in the United States. Risk factors include diabetes, glucocorticoids, hematological malignancies and iron overload. The most frequent types of mucormycosis include rhinoorbitocerebral, pulmonary, and cutaneous disease.

Here we present a child with newly diagnosed type II diabetes mellitus who developed hematemesis and was found to have gastrointestinal mucormycosis.

Methods Used Case Report

Summary of Results A 17-year old obese male presented to the emergency department with a 1-day history of abdominal pain, vomiting and respiratory distress. Weight loss, polyuria and polydipsia were reported. He had no other significant medical or surgical history. On examination his temperature was 37.8 C, pulse 137, BP 116/77, RR 44, and Pox 96\% in 
room air. He was altered, mucus membranes were dry, and abdomen was distended and diffusely tender. He was subsequently admitted to our pediatric intensive care unit.

The patient was found to be in diabetic ketoacidosis with acute pancreatitis, for which he was treated accordingly. During his hospital course, he acutely developed severe hematemesis and anemia. Esophagogastroduodenoscopy demonstrated the presence of active bleeding and multiple ulcerations in the stomach. Biopsy showed fungal elements with ribbon like hyphae, identified as Rhizopus microsporus.

The patient was diagnosed with hemorrhagic gastritis secondary to mucormycosis. He was started on liposomal amphotericin and posaconazole with resolution of his hematemesis. He received 6 weeks of liposomal amphotericin and was discharged home on a prolonged course of posaconazole.

Conclusions Our case emphasizes the importance of considering gastrointestinal mucormycosis in children with diabetic ketoacidosis and hematemesis.

Gastrointestinal mucormycosis is thought to occur due to ingestion of spores amongst immunocompromised hosts. The angioinvasive nature of the gastrointestinal lesions places children at risk for perforation, bowel infarction, and hemorrhagic shock. Although the prognosis is poor, early initiation of antifungal therapy has been shown to improve outcomes and reduce mortality rates. In this case, biopsy allowed for timely diagnosis and initiation of appropriate therapy, resulting in a favorable outcome.

\section{Endocrinology and metabolism II}

\section{Concurrent session}

\section{8:00 AM}

\section{Friday, January 21, 2022}

\section{\#236 NEURAL CORRELATES OF OBESITY AND INFLAMMATION IN YOUTH WITH CLASSICAL CONGENITAL ADRENAL HYPERPLASIA}

${ }^{1,2} \mathrm{MS} \mathrm{Kim*}{ }^{3} \mathrm{TA}$ Pickering, ${ }^{3} \mathrm{DL}$ Cotter, ${ }^{1} \mathrm{NR}$ Fraga, ${ }^{4} \mathrm{~S}$ Luo, ${ }^{1} \mathrm{C}$ Won, ${ }^{1,2} \mathrm{M}$ Geffner, ${ }^{2} \mathrm{M}$ Herting. ${ }^{1}$ Children's Hospital of Los Angeles, Los Angeles, $C A ;{ }^{2}$ Children's Hospital of Los Angeles Saban Research Institute, Los Angeles, $C A{ }^{3}{ }^{3}$ University of Southern California Keck School of Medicine, Los Angeles, CA; ${ }^{4}$ University of Southern California, Los Angeles, $C A$

\subsection{6/jim-2022-WRMC.233}

Purpose of Study Patients with classical congenital adrenal hyperplasia (CAH) due to 21-hydroxylase deficiency exhibit an increased prevalence of obesity over their lifetime. We have previously shown that $\mathrm{CAH}$ youth exhibit smaller gray matter brain region volumes, including the prefrontal cortex (PFC), amygdala, and hippocampus, as well as white matter microstructure abnormalities. Here, we aim to study the relationship between these patterns of altered brain structure with obesity and inflammation in youth with and without CAH.

Methods Used We studied 27 youth with CAH $(12.6 \pm 3.4$ yr, 16 females) and 35 controls (13.0 $\pm 2.8 \mathrm{yr}, 20$ females) via 3-T MRI, and examined the PFC and its subregions, amygdala, and hippocampus regions of interest (ROIs), as well as white matter tracts including the fornix and stria terminalis (ST). Relaimpo (relative importance for linear regression: $\mathrm{R}^{2}{ }_{\text {lmg }}$ ) analyses identified body composition and inflammatory markers, as well as gray and white matter ROIs most associated with $\mathrm{CAH}$ status. Regression analyses examined associations between brain structure, $\mathrm{CAH}$, and other variables.

Summary of Results Waist-to-height ratio (WHtR; $\mathrm{R}^{2}{ }_{\mathrm{lmg}}=$ 0.12 ) and monocyte chemoattractant protein-1 (MCP-1 R ${ }^{2}{ }_{\text {mg }}$ $=0.21$ ) exhibited the strongest associations with $\mathrm{CAH}$ status amongst body composition variables and inflammatory markers respectively. Analysis of gray matter ROIs showed the PFC was most strongly associated with $\mathrm{CAH}$ status $\left(\mathrm{R}^{2}{ }_{\mathrm{lmg}}=0.13\right)$. Superior frontal (SF) was the only PFC subregion associated with all key variables: $\mathrm{CAH}$ status $(\beta=-0.58, \mathrm{P}=0.001)$, WHtR $(\beta=-0.23, \mathrm{P}=0.009)$, and MCP-1 $(\beta=-0.35, \mathrm{P}<$ $0.001)$. CAH youth exhibited significantly larger WHtR and MCP-1, and smaller SF volume than controls (P $\leq 0.001$ for all). White matter microstructure showed fornix and ST associations with $\mathrm{CAH}$ (fornix $\beta=0.15, \mathrm{P}=0.02$; ST $\beta=0.22$, $\mathrm{P}<0.001$ ) and WHtR (fornix $\beta=0.36, \mathrm{P}=0.01$; ST $\beta=$ $0.35, \mathrm{P}=0.02)$, but only ST associations with MCP-1 $(\beta=$ $0.30, \mathrm{P}=0.02$ ).

Conclusions Youth with $\mathrm{CAH}$ demonstrate an important relationship between altered gray and white matter brain structure, WHtR, and MCP-1 that could have implications for neuroinflammation and obesity in $\mathrm{CAH}$.

\section{\#237 POPULATION-BASED ASSESSMENT OF CARDIOMETABOLIC-RELATED DIAGNOSES IN YOUTH WITH KLINEFELTER SYNDROME: A PEDSNET STUDY}

S Davis*, N Nokoff, A Furniss, A Valentine, L Pyle, A Dempsey. University of Colorado, Denver, $C O$

\subsection{6/jim-2022-WRMC.234}

Purpose of Study Diabetes and cardiovascular diseases are common among men with Klinefelter syndrome (KS) and contribute to higher morbidity and mortality. Cardiometabolic health outcomes have not been evaluated in a large population of youth with KS.

Methods Used Data from electronic health records from six pediatric institutions were used to compared the prevalence of five cardiometabolic-related outcomes among 1,080 youth with KS to 4,497 youth without KS matched for sex, age (mean 13 years at last encounter), year of birth, race, ethnicity, insurance, site, and duration of care (mean 7 years). Odds ratios (OR) and 95\% confidence intervals (CI) both unadjusted and adjusted for potential covariates including obesity, testosterone, and antipsychotic use were computed using generalized estimating equations.

Summary of Results The odds of overweight/obesity (OR 1.6 (95\%CI 1.4-1.8)), dyslipidemia (3.0 (2.2-3.9)), and liver dysfunction (2.0 (1.6-2.5)) were all higher in KS compared to controls. While the adjusted model attenuated the effect of KS on these outcomes, boys with KS still had $45 \%$ greater odds of overweight/obesity (CI 1.2-1.7) and 70\% greater odds of liver dysfunction (1.3-2.2) compared to controls, and both dyslipidemia (1.6 (1.1-2.4)) and dysglycemia (1.8 (1.1-3.2)) were higher in KS but of borderline statistical significance when accounting for multiple comparisons. The odds of 
hypertension were not different between groups in unadjusted or adjusted models.

Conclusions This large, population-based cohort of youth with KS found a higher odds of many cardiometabolic-related diagnoses compared to matched controls. Investigation into the mechanisms that underlie risk for cardiometabolic dysfunction in youth with KS independent of obesity and testosterone is needed.

\section{\#238 REACTIVE HYPOGLYCEMIA FOLLOWING A SUGAR CHALLENGE IS ACCOMPANIED BY HIGHER INSULIN IN ADOLESCENT GIRLS WITH OBESITY}

${ }^{1,2} \mathrm{MA}$ Ware ${ }^{*},{ }^{3} \mathrm{~A}$ Carreau, ${ }^{1} \mathrm{Y}$ Garcia-Reyes, ${ }^{1,2} \mathrm{H}$ Rahat, ${ }^{4} \mathrm{C}$ Diniz Behn, ${ }^{1} \mathrm{M}$ Cree-Green. ${ }^{1}$ University of Colorado - Anschutz Medical Campus, Aurora, CO; ${ }^{2}$ Rocky Vista University, Parker, CO; ${ }^{3}$ Universite Laval Faculte de medecine, Quebec, QC, Canada; ${ }^{4}$ Colorado School of Mines, Golden, $\mathrm{CO}$

\subsection{6/jim-2022-WRMC.235}

Purpose of Study Adolescent girls can present with postprandial, episodic symptoms of dizziness and excess sweating, which are often clinically disregarded. These symptoms can be associated with idiopathic reactive hypoglycemia $(\mathrm{RH})$, reproduced with a glucose challenge, and may relate to increased risk of insulin resistance and type 2 diabetes (T2D). We sought to determine the prevalence of and glycemic measures associated with RH among adolescent girls with obesity following an oral sugar tolerance test (OSTT).

Methods Used Secondary analysis of 112 adolescent girls with obesity (age 12-21 yrs; body mass index (BMI) $\geq 90$ th percentile). Participants completed a 4-hr OSTT (75 g glucose, $25 \mathrm{~g}$ fructose) and were stratified by glucose nadir between 120240 mins: $\square 60 \mathrm{mg} / \mathrm{dL}$ as $\mathrm{RH}, \geq 80 \mathrm{mg} / \mathrm{dL}$ as normoglycemic (NG), and 61-79 mg/dL as indeterminate. Area under the curve (AUC) of glucose, insulin, glucagon, and C-peptide and measures of insulin sensitivity and $\beta$-cell function including oral minimal model, insulinogenic index, and oral disposition index were calculated. Demographic, physical, and metabolic characteristics between RH and NG were compared with Student's t-tests or Mann-Whitney U tests. OSTT curves were compared with repeated measures ANOVA.

Summary of Results $12 \%$ of girls had RH $(n=13)$ and $36 \%$ had NG $(n=40)$. Groups were similar in age, race, ethnicity, and BMI. Glucose concentrations were lower in RH than NG when fasting $(p=0.033)$, at 210 mins $(p=0.001)$, and 240 mins $(\mathrm{p}<0.001)$. In $\mathrm{RH}$, glucose nadir occurred at a median time of $210 \mathrm{~min}$ postprandial. RH had higher glucagon AUC for 90-240 $\mathrm{min}$ of the OSTT $(\mathrm{p}=0.035)$ and a lower insulin at 240 mins $(p=0.007)$ confirming a counterregulatory response. Insulin AUC $(\mathrm{p}=0.074)$ and $\mathrm{C}$-peptide AUC $(p=0.054)$ for $0-120 \mathrm{~min}$ trended to be higher. Groups had similar hemoglobin a1c, family history of T2D, insulin sensitivity, and $\beta$-cell function.

Conclusions RH was relatively common in this cohort of adolescent girls with obesity and occurred at 3-4 hrs. Early higher insulin secretion preceded the counterregulatory $\mathrm{RH}$ responses but was not related to insulin sensitivity. Future studies should aim to further understand the long-term effects of $\mathrm{RH}$ during adolescence and its significance in predicting future hyperglycemia.
\#239 DIETARY QUALITY AND ONE-YEAR PROGRESSION OF GLUCOSE INTOLERANCE IN SUBJECTS WITH OBESITY

A Thatte*, SJ Melhorn, J Rosenbaum, M De Leon, M Webb, E Schur. University of Washington, Seattle, WA

\subsection{6/jim-2022-WRMC.236}

Purpose of Study Previous longitudinal cohort studies have shown that improved dietary quality is correlated with a reduction in the risk of cardiovascular disease and potentially diabetes. There has been limited research into whether a similar relationship holds for the progression of glucose intolerance. The hypothesis for this study is that better dietary quality is correlated with reduced progression in glucose intolerance over the course of one year in people with obesity.

Methods Used This was a prospective cohort study. Participants $(\mathrm{N}=38)$ at baseline completed three 24-hour dietary recalls. The majority $(\mathrm{N}=37)$ were obese and subjects with diabetes were excluded. Each participant's Healthy Eating Index-2015 (HEI) score was calculated from the dietary recalls. The HEI is a measure of dietary quality that assigns weights to meeting adequacy targets (i.e., getting sufficient vegetables) and not exceeding moderation targets (i.e., limiting saturated fat) for a total score between 0 and 100. All participants underwent a 2-hour 75-gram glucose tolerance test at baseline and at 12 months. Glucose tolerance was calculated as the percentage change in the incremental area under the curve (iAUC) between these two time points. Linear regression analysis in STATA was used to determine the relationship between HEI score at baseline (and other dietary measures), and progression of glucose intolerance over one year. One statistically influential outlier with percentage change in glucose iAUC of $>150 \%$ was excluded.

Summary of Results A trend was observed showing that higher dietary quality by HEI score was correlated with less progression of glucose intolerance $\left(\beta=-1.27, \mathrm{R}^{2}=0.102, \mathrm{p}=\right.$ $0.054)$. When age was added as a covariate, the values were $\left(B=-1.21 R^{2}=0.103, p=0.098\right)$. Higher saturated fat intake was correlated with greater progression of glucose intolerance $\left(\beta=5.07, \mathrm{R}^{2}=0.168, \mathrm{p}=0.012\right)$. There were no statistically significant relationships between vegetable, added sugar, or dietary fiber intake and progression of glucose intolerance.

Conclusions In this small study of people with obesity, higher baseline dietary quality as measured by HEI correlated with less progression of glucose tolerance with borderline statistical significance $(p=0.054)$. Higher saturated fat intake correlated with greater progression of glucose intolerance $(\mathrm{p}=$ 0.012). The clinical relevance of this work would be to help optimize dietary recommendations to prevent progression of glucose intolerance which can be seen before manifestation of overt disease. Limitations of this study include the small number of subjects, lack of normal weight subjects, limited follow-up period, and self-reported dietary data. Future directions of research could include increasing sample size, studying normal weight subjects over a longer timeframe, and exploring methods for collecting dietary data that do not rely on self-report. 
\#240 ORAL CONTRACEPTIVE USE IN ADOLESCENTS WITH POLYCYSTIC OVARY SYNDROME AND OBESITY IS ASSOCIATED WITH ALTERED FAT METABOLISM

${ }^{1,2}{ }^{2}$ Finn ${ }^{*},{ }^{1} \mathrm{C}$ Severn, ${ }^{2} \mathrm{Y}$ Garcia-Reyes, ${ }^{2} \mathrm{MA}$ Ware, ${ }^{2} \mathrm{H}$ Rahat, ${ }^{1,2} \mathrm{M}$ Cree-Green. ${ }^{1}$ University of Colorado, Aurora, CO; ${ }^{2}$ Children's Hospital Colorado, Aurora, $\mathrm{CO}$

10.1136/jim-2022-WRMC.237

Purpose of Study Polycystic ovary syndrome (PCOS) is a common endocrine condition characterized by reproductive and metabolic hormone dysregulation and increased risk for metabolic disease. Combined oral contraceptive pills (OCPs) are first-line treatment for management of hyperandrogenic symptoms and menstrual regulation, yet data on the cardiometabolic effects in youth are limited.

Methods Used Participants were enrolled in a cross-sectional trial of girls aged 12-21 years, BMI\% >90th ile with PCOS per NIH 1990 criteria (NCT02157974). Measurements included anthropomorphics, fasting metabolic and hormonal assessments, 6-hour oral sugar tolerance test (OSTT), hepatic fat per MRI, DXA for body composition, and vascular function by EndoPAT and dynapulse. Area under the curve (AUC) for responses to the OSTT were calculated, as were several indices of insulin sensitivity. Participants treated with OCP's for at least 6 months were matched 1:2 with replacement with untreated participants on BMI, race, and ethnicity. The effect of treatment was estimated with paired t-tests.

Summary of Results Data from 10 participants taking OCPs (age $15.7 \pm 1.3$ years, BMI $34.2 \pm 5.6 \mathrm{~kg} / \mathrm{m}^{2}$ ) were compared to

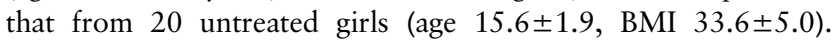
The OCP group had decreased free testosterone $(p \leq 0.001)$, increased sex hormone binding globulin $(p=0.001)$, and decreased free androgen index $(\mathrm{p} \leq 0.001)$. The OCP group had higher total cholesterol $(\mathrm{p}=0.045)$, body fat percentage $(p=0.028)$, and serum leptin $(p=0.038)$. The OCP group also had higher free fatty acids AUC $(p=0.007)$ and glycerol AUC $(p=0.011)$ during the OSTT. No differences between the groups were noted in glucose metabolism including fasting glucose and insulin, HOMA-IR, c-peptide, glucose AUC, or insulin AUC. Vascular function and hepatic fat fraction were similar between groups.

Conclusions Treatment with OCPs was not associated with worsening dysglycemia or markers of vascular dysfunction. However, there was evidence of altered fat metabolism, including a shift towards greater fat mass and adipose insulin resistance with persistent lipolysis. Additionally, hepatic fat was not improved. The overall therapeutic benefit of OCP's in girls with PCOS and obesity who are at high cardiometabolic risk needs to be carefully evaluated.

\section{\#241 ACIPIMOX PREVENTS CARDIAC DYSFUNCTION INDUCED BY ELECTRONIC CIGARETTES IN MICE}

${ }^{1} \mathrm{CJ} L a 0^{*},{ }^{1} \mathrm{~J}$ Espinoza-Derout, ${ }^{1} \mathrm{~K}$ Hasan, ${ }^{2} \mathrm{MC}$ Jordan, ${ }^{1 J}$ Wilson, ${ }^{1} \mathrm{~J}$ Molina, ${ }^{2} \mathrm{~K}$ Luna, ${ }^{1} \mathrm{JL}$ Arambulo, ${ }^{1} \mathrm{~A}$ S Sinha-Hikim, ${ }^{2} \mathrm{~K}$ Roos, ${ }^{1} \mathrm{~T}$ Friedman. ${ }^{1}$ Charles Drew University of Medicine and Science, Los Angeles, CA; ${ }^{2}$ University of California Los Angeles David Geffen School of Medicine, Los Angeles, $C A$

\subsection{6/jim-2022-WRMC.238}

Purpose of Study Tobacco use is a leading cause of preventable death in the United States. In recent years, electronic cigarettes (e-cigarettes) use by youth has augmented at a disturbing rate. Cardiac dysfunction can lead to heart failure, which is responsible for 287,000 deaths each year in the United States. In a mouse model, we will analyze whether acipimox treatment can rescue the cardiac dysfunction phenotype caused by e-cigarettes with $2.4 \%$ nicotine through the inhibition of lipolysis.

Methods Used For 12 weeks, mice were exposed to saline, ecigarettes (2.4\%), and e-cigarettes plus acipimox in 12-hour cycles through specialized chambers. Acipimox (0.05\%) was delivered in the drinking water. Mouse weights and food consumption data were collected weekly. Echocardiograms were used to analyze heart function.

Summary of Results Weight data indicates that acipimox decreases the rate of weight change over time when compared to both saline control and e-cigarette groups. Acipimox treatment rescued e-cigarette induced phenotypes in fractional shortening, ejection fractions and velocity of circumferential fiber shortening. Triglyceride levels were increased in e-cigarette groups but were not fully rescued after acipimox treatment. Acipimox treatment reduced circulating levels of free fatty acids (FFA), Macrophage Colony-Stimulating Factor (MCSF), and Interleukin-6 (IL-6) when compared to e-cigarette treatment alone. Gene set enrichment analysis (GSEA) indicates that genes involved in the G2/M checkpoint were upregulated by e-cigarette treatment and rescued by acipimox treatment. Heme oxygenase 1 (HO1) and Poly(ADP-Ribose) Polymerase 1 (PARP1) protein levels were rescued after acipimox treatment.

Conclusions E-cigarette use led to an increase in free fatty acids which resulted in increased inflammation and reactive oxygen species within cardiac tissue. This resulted in DNA damage in the heart and halting of the cell cycle at the G2/M checkpoint. The DNA repair pathway was activated with increased levels of PARP1, a protein known for cellular recovery. This led to the cardiac dysfunction phenotype after e-cigarette use. Acipimox rescued this phenotype by inhibiting lipolysis and preventing downstream effects of dysfunctional adipose tissue and increased FFAs on cardiac tissue. In conclusion, the data obtained so far shows that lipolysis is necessary for the e-cigarette induced cardiac dysfunction. Lipolysis may become a new therapeutic target to curve the harmful cardiovascular effects produced by e-cigarettes.

\section{\#242 ACCESS TO CARE FOR METABOLIC SYNDROME AND CARDIOVASCULAR DISEASE AMONG IMMIGRANT POPULATIONS IN THE UNITED STATES}

J Beltran*, M Shaheen, D Pan. Charles Drew University of Medicine and Science, Los Angeles, CA

\subsection{6/jim-2022-WRMC.239}

Purpose of Study It is estimated that one-quarter to one-third of adults meet the Metabolic Syndrome (MetS) criteria in the United States. MetS could be responsible for approximately $7 \%$ of total mortality and up to $17 \%$ of cardiovascular disease (CVD). This research aims to determine the relationship between metabolic syndrome, cardiovascular disease, and access to care by immigration status.

Methods Used We analyzed data from the National Health and Nutrition Examination Survey (NHANES) 2017-2018 using bivariate chi-square and multiple logistic regression, considering the design and sample weight. We presented the data as an adjusted odds ratio and 95\% confidence interval, and a p-value of $<0.05$ will be considered statistically significant. 
Summary of Results Of the 5,265 adults, 19.3\% were immigrants, $8.7 \%$ had CVD, and $34.3 \%$ had MetS. Immigrants had lower prevalence of CVD (5.4\%) compared to non-immigrants $(9.5 \%) \quad(\mathrm{p}<0.05)$. A higher percentage of immigrants were uninsured (24\%) compared to non-immigrants (11\%) $(\mathrm{p}<0.05)$. In the multiple logistic models, there was no significant difference in the odds of CVD, MetS, and access to care among immigrants relative to non-immigrants after adjusting for the confounding variables. Though, in four of the five risk factors for MetS, immigrants had higher odds of CVD than non-immigrants $(\mathrm{p}<0.05)$.

Conclusions Though the prevalence of CVD is not statistically different from non-immigrant, immigrants are more susceptible to the risk factors of MetS which increases the likelihood of developing CVD. Resources need to be distributed to the vulnerable immigrant population to decrease the risk factors of MetS, CVD and increase access to healthcare.

\section{\#243 CAN MEDICAL CARE PROVIDERS PREVENT HEART ATTACKS AND STROKES?}

J Giannini* J Padilla, RP Eaton, K Gonzales, DS Schade. University of New Mexico School of Medicine, Albuquerque, NM

\subsection{6/jim-2022-WRMC.240}

Purpose of Study Cardiovascular disease prevention is an important goal of medical care providers. In the clinical setting, we addressed whether myocardial infarctions and strokes can be prevented in asymptomatic patients with proven subclinical cardiovascular disease.

Methods Used Two hundred six asymptomatic self-selected individuals requested a coronary artery calcium scan (CAC). Of these individuals, 125 had positive scans (Agatston score $>1$ ) and were recommended for medical treatment to prevent heart attacks and atherosclerotic strokes. Eighty-one individuals had zero scores and were not treated. Of the 125 individuals with positive scans, 110 followed medical treatment (rosuvastatin $10 \mathrm{mg} / \mathrm{d}$, ezetimibe $10 \mathrm{mg} / \mathrm{d}$, and a low cholesterol diet) and achieved a low density lipoprotein cholesterol (LDL-C) of $\leq 60 \mathrm{mg} / \mathrm{dl}$. The other 15 individuals did not follow recommended treatment and maintained an elevated LDL-C.

Summary of Results All patients were followed for an average of 3.5 years (minimum 1 year - maximum 5 years). No atherosclerotic clinical events were observed in the group that achieved an LDL-C $\leq 60 \mathrm{mg} / \mathrm{dl}$. The expected number of atherosclerotic cardiovascular events based on their calcium scores and published observational data was 12.6. Two patients died of non-atherosclerotic causes (an auto accident and

Abstract \#243 Table 1 Change in clinical parameters in the LDL$\mathrm{C} \leq 60 \mathrm{mg} / \mathrm{dl}$ group with $\mathrm{Rx}$

\begin{tabular}{lllll}
\hline Parameter & $\begin{array}{l}\text { Avg. Prior to } \\
\text { Rx }\end{array}$ & $\begin{array}{l}\text { Avg. Post } \\
\text { Rx }\end{array}$ & Difference & Significance \\
\hline Total-C mg/dl & $187 \pm 4.8$ & $128 \pm 3.1$ & -59 & $P<0.01$ \\
HDL-C mg/dl & $54.8 \pm 1.7$ & $55.3 \pm 1.7$ & +0.5 & $P=0.43$ \\
Triglycerides mg/ & $130 \pm 5.9$ & $100 \pm 5.4$ & -30 & $P<0.01$ \\
dl & & & & \\
LDL-C mg/dl & $113 \pm 3.6$ & $49.0 \pm 1.4$ & -64 & $P<0.01$ \\
BMl kg/m & $30.0 \pm 0.71$ & $29.0 \pm 0.72$ & -1 & $P=0.4$ \\
\hline
\end{tabular}

Alzheimer's disease.) In the non-compliant group, two heart attacks were observed (one patient died, one had a stent). No events were observed in the zero CAC group. No adverse effects of the treatment regimen occurred.

Conclusions Medical care providers can prevent atherosclerotic heart attacks and strokes by treating asymptomatic patients who have positive calcium scores to an LDL-C goal of $\leq 60$ $\mathrm{mg} / \mathrm{dl}$. A simple regimen of rosuvastatin, ezetimibe, and a low cholesterol diet has no major adverse effects, is inexpensive, and is acceptable by almost all patients.

\section{Gastroenterology}

\section{Concurrent session}

\section{8:00 AM}

\section{Friday, January 21, 2022}

\section{\#244 DIABETIC HYPERGLYCEMIA EXACERBATES INFLAMMATORY BOWEL DISEASE IN MICE WITH DIET- INDUCED OBESITY} ${ }^{1,2} \mathrm{~K}$ Francis*, ${ }^{1} \mathrm{MC}$ Pacheco, ${ }^{2} \mathrm{~K}$ Alonge, ${ }^{2} \mathrm{BA}$ Phan, ${ }^{2} \mathrm{~S} \mathrm{Hu},{ }^{2} \mathrm{M}$ Schwartz, ${ }^{1,2} \mathrm{~J}$ Scarlett.
${ }^{1}$ Seattle Children's Hospital, Seattle, WA; ${ }^{2}$ University of Washington School of Medicine,
Seattle, WA

\subsection{6/jim-2022-WRMC.241}

Purpose of Study Inflammatory bowel disease (IBD), obesity, and type 2 diabetes (T2D) are chronic inflammatory conditions that are increasing in prevalence. Obesogenic high-fat diets (HFD) that promote diet-induced obesity (DIO) and T2D have been shown to worsen IBD. Furthermore, comorbid T2D in patients with IBD is a predictor of poor diseaserelated outcomes. To determine the mechanisms whereby T2D worsens IBD pathology, we examined the impact of hyperglycemia on disease activity in a DIO mouse model of IBD, focusing on measures of intestinal barrier integrity.

Methods Used Wild-type C57BL/6J mice were fed HFD for 8 weeks to induce DIO and then given intraperitoneal injections of vehicle or low-dose streptozotocin (STZ) to induce hyperglycemia. At week 12, 2\% dextran sodium sulfate (DSS) or vehicle control was administered in drinking water for 7 days to induce colitis. Outcomes included serial measures of body weights, blood glucose, and disease activity index (DAI) scores, which incorporate weight loss, rectal bleeding, and stool consistency. Animals were euthanized at the end of DSS course and colon tissue was collected and processed for immunohistochemical staining.

Summary of Results As predicted, STZ administration raised mean blood glucose levels significantly compared to vehicletreated controls $(267 \mathrm{mg} / \mathrm{dL}$ vs. $159 \mathrm{mg} / \mathrm{dL}, \mathrm{p}=0.006)$. Hyperglycemic mice treated with DSS (DSS/STZ) manifested clinical signs of colitis 2 days earlier than their normoglycemic counterparts (DSS/Veh), and their DAI scores were significantly higher from Days 2-7 of DSS $(\mathrm{p}<0.0001)$. Colon length and histologic damage scores also demonstrated higher disease burden in DSS $/ S T Z$ mice (colon length $5.93 \mathrm{~cm}$ vs. $6.88 \mathrm{~cm}$, $\mathrm{p}=0.0003$; histologic score 8.0 vs $6.5, \mathrm{p}=0.03$ ). Colonic tissues demonstrated significantly decreased quantity of the tightjunction protein E-cadherin (E-cad) in DSS/STZ mice compared to DSS/Veh (percent area 24.1 vs. $34.9, p=0.004$ ), and 
a similar decrease in colonic mucin barrier was detected by Alcian Blue (AB) staining (percent area 55.3 vs. 71.0, $\mathrm{p}<0.0001)$. Notably, measures of both colonic E-cad and $A B$ were inversely correlated with both DAI scores and blood glucose levels.

Conclusions In DIO mice, clinical and pathological IBD outcomes are exacerbated by moderate hyperglycemia in a DSS model of colitis. We identify two potential mechanisms by which hyperglycemia contributes to intestinal pathology: 1) reduced tight-junction protein quantity in the colonic epithelium and 2) a decrease in the protective colonic mucin barrier. These results support the hypothesis that hyperglycemia induces intestinal barrier dysfunction, which, in combination with an obesogenic diet, increases the risk for IBD progression.

\section{$\# 245$ ACCURACY OF INTRAOPERATIVE CHOLANGIOGRAM FOR THE EVALUATION OF CHOLEDOCHOLITHIASIS}

${ }^{1}$ SK Choi* ${ }^{2} \mathrm{M}$ Syed, ${ }^{2} \mathrm{~J} \mathrm{Kim,}{ }^{2} \mathrm{JJ} \mathrm{Kim.}{ }^{1}$ Loma Linda University School of Medicine, Loma Linda, CA; ${ }^{2}$ Loma Linda University Medical Center, Loma Linda, CA

\subsection{6/jim-2022-WRMC.242}

Purpose of Study Intraoperative cholangiography (IOC) is frequently performed during cholecystectomy and considered definitive testing for evaluation of common bile duct (CBD) stones. Previous studies evaluating the accuracy of IOC for the evaluation of choledocholithiasis are inconsistent, and false-positive testing may lead to increased utilization of diagnostic ERCP. The aim of this study is to evaluate the diagnostic property of IOC and to assess the clinical outcomes of patients who underwent ERCP with or without retained choledocholithiasis.

Methods Used Consecutive hospitalized patients who received cholecystectomy with IOC at Loma Linda University Medical Center (1/2014-6/2021) were identified. Positive IOC was defined by the presence of filling defect, meniscus sign, and/or failure to drain contrast into the duodenum necessitating additional diagnostic evaluations. Definitive diagnosis of choledocholithiasis was defined by the presence of choledocholithiasis and/or sludge on any of the definitive testing for choledocholithiasis (MRCP, EUS, and/or ERCP) performed after IOC. Patients who had prior sphincterotomy or negative IOC without documented $\geq 2$-year follow-up were excluded.

Summary of Results The mean age of 259 patients who underwent same-day cholecystectomy and IOC was 46.0 \pm 19.8 years, 189 (73\%) were female, and 144 (56\%) were of Latino ethnicity/race. On presentation, $22(9 \%)$ patients had bilirubin $\geq 4.0 \mathrm{~g} / \mathrm{dL}, 111(43 \%)$ had a dilated $\mathrm{CBD} \geq 6 \mathrm{~mm}$, and 7 (3\%) had choledocholithiasis detected on abdominal ultrasound. During cholecystectomy, 103 (40\%) patients had abnormal IOC including the presence of filling defect in 94 (36\%), meniscus sign in $2(1 \%)$, and failure of passage of contrast to the duodenum in 7 (3\%). Subsequently $122(46 \%)$ patients had definitive testing including ERCP in 102 (39\%), MRCP in $45(17 \%)$, and EUS in $14(5 \%)$. Diagnostic property of IOC for choledocholithiasis are shown in the table 1. ERCP was performed at a median of 2 days (range, 0-141 days) from IOC. Of 102 patients, 8 (3\%) had adverse events including pancreatitis in 6 (moderate in 3 , mild in 3 ) and perforation in 2; five patients with adverse events received diagnostic ERCP for evaluation of false-positive IOC.
Abstract \#245 Table 1 Diagnostic property of intraoperative cholangiography for choledocholithiasis

\begin{tabular}{lllll}
\hline & $\begin{array}{l}\text { Choledocholithiasis } \\
\text { with or without } \\
\text { Sludge }\end{array}$ & $95 \% \mathrm{Cl}$ & $\begin{array}{l}\text { Choledocholithiasis } \\
\text { Alone }\end{array}$ & $95 \% \mathrm{Cl}$ \\
\hline Prevalence & $79 / 259(30.5 \%)$ & & $67 / 260(25.9 \%)$ & \\
Accuracy & $86.1 \%$ & $81.3-90.1 \%$ & $82.2 \%$ & $77.0-87.0 \%$ \\
Sensitivity & $92.4 \%$ & $84.2-97.2 \%$ & $92.5 \%$ & $83.4-97.5 \%$ \\
Specificity & $83.3 \%$ & $77.1-88.5 \%$ & $78.7 \%$ & $72.2-84.2 \%$ \\
PPV & $70.9 \%$ & $63.6-77.2 \%$ & $60.2 \%$ & $53.3-66.7 \%$ \\
NPV & $96.2 \%$ & $92.0-98.2 \%$ & $96.8 \%$ & $92.8-98.6 \%$ \\
Positive LR & 5.4 & $4.0-7.7$ & 4.3 & $3.3-5.7$ \\
Negative LR & 0.09 & $0.04-0.20$ & 0.09 & $0.04-0.22$ \\
\hline
\end{tabular}

Conclusions Although IOC demonstrated high sensitivity and negative predictive value of $>90 \%$ for ruling out retained CBD stones, nearly a third of the patients received diagnostic ERCP. Given adverse events associated with ERCP, other less invasive definitive tests such as MRCP and EUS should be considered for evaluations of patients with positive IOC prior to ERCP.

\section{\#246 ROLE OF ETHNICITY IN PREDICTING THE SEVERITY OF LIVER FIBROSIS BY THE IMPLEMENTATION OF FOUR CLINICAL LIVER CIRRHOSIS PREDICTION MODELS AMONG HISPANIC AND CAUCASIAN WOMEN: A PILOT STUDY}

${ }^{1} \mathrm{Xu}$ LC*,${ }^{2} \mathrm{JR}$ Fine, ${ }^{3} \mathrm{~A}$ Molfino, ${ }^{1} \mathrm{~V}$ Medici. ' University of California Davis Health System, Sacramento, CA; ${ }^{2}$ University of California Davis, Davis, CA; ${ }^{3}$ Sapienza University of Rome, Rome, Italy

\subsection{6/jim-2022-WRMC.243}

Purpose of Study To determine whether ethnicity and other risk factors can predict the severity of fibrosis or cirrhosis in women with NAFLD applying the four predictor models FIB4, NFS, BARD, and APRI.

Methods Used We performed a retrospective study among Hispanic $(n=567)$ and Caucasian $(n=136)$ women with history of NAFLD (mean age $55.7 \pm 11.4$ SD years) at The University of California Davis Medical Center. Univariate analyses for their FIB-4, NFS, BARD, and APRI scores were conducted by using Chi-Square or Fisher's Exact Test for categorical variables, two-sample t-test for continuous variables, and WilcoxonMann-Whitney test for continuous non-parametric variables. Associations between ethnicity (Hispanic vs. Caucasian) and liver fibrosis severity calculated using the four cirrhosis predictor models were explored using backward selection multinomial logistic regression.

Summary of Results We observed that Hispanic women compared to Caucasian showed lower BMI $(p<0.001)$, higher HDL $(p<0.05)$, higher HbA1c $(p<0.001)$, higher prevalence of stage 4 and 5 CKD $(p=0.005)$, lower prevalence of bariatric surgery $(p=0.002)$, lower likelihood to smoke $(p=0.002)$, and had lower APRI score $(p=0.03)$. The percentage of advanced fibrosis in each models and ethnicity was as follows: Hispanic NFS: 35.1\%, BARD: 82.6\%, FIB-4: 3.5\%, and APRI: $1.3 \%$; in Caucasian NFS: $36.3 \%$, BARD $83.2 \%$, FIB-4: $7.7 \%$, APRI: $6.3 \%$. The odds of having a severe 
fibrosis or cirrhosis was not significantly higher in those who are Hispanic compared to Caucasian in FIB-4, NFS, BARD, and APRI ( $p=0.16,0.78,0.97$, and 0.18 respectively).

Conclusions Based on our preliminary results, we cannot conclude that ethnicity alone can be used to predict the severity of fibrosis or cirrhosis in women with NAFLD in the multiple predictor models.

\section{\#247 EXAMINING CLINICAL PRESENTATION AND WORKUP OF VETERANS WITH IRRITABLE BOWEL SYNDROME IN A SINGLE MEDICAL CENTER}

${ }^{1} \mathrm{PL}$ Claassen*, ${ }^{2} \mathrm{M}$ Riddle. 'Washington State University Elson S Floyd College of Medicine, Spokane, WA; ${ }^{2}$ VA Sierra Nevada Health Care System, Reno, NV

\subsection{6/jim-2022-WRMC.244}

Purpose of Study Irritable Bowel Syndrome (IBS) is a disorder of gut-brain interaction (DGBI) that afflicts about 5\% of the population with significant quality of life and economic impacts. Recent ACG guidelines have been established to improve diagnosis and management of IBS. Within the Veteran population, there is poor understanding of the epidemiology of DGBI despite significant occupation exposures, comorbid mental health problems and risk of deployment-associated diarrhea. Less is known about the management of DGBI in Veterans compared to other populations.

Methods Used As part of a quality improvement initiative, a case series analysis of Veterans seen at a new IBS Clinic was performed to better understand the patient population and their preceding diagnostic workup. Data from the VA Electronic Health Record and a standardized IBS Clinic intake form was retrieved. All charts were comprehensively reviewed to determine the dates of gastrointestinal (GI) symptom onset, first functional GI disorder diagnosis, first IBS diagnosis, as well as basic demographics, current comorbidities and medications. Descriptive analytic methods were applied.

Summary of Results A total of 11 Veterans referred to the IBS Clinic were included with a median age of 43 years, all male, majority Caucasian $(81.8 \%)$ and predominantly from the Persian Gulf war (72.7\%), post-Vietnam (18.2\%) and Vietnam eras (9.1\%). A median of 4 (IQR 2.5-14.5) years delay between time of symptom onset to first functional GI disorder diagnosis was noted. Of those diagnosed with IBS, the phenotypes were diarrhea (57.1\%), mixed (28.6\%) and unclassified (14.3\%). Patients had a median of 10 (IQR 615) comorbidities and took a median of 9 (IQR 5-10) medications (i.e. selective serotonin reuptake inhibitors) which could contribute to diarrhea. Mental health problems were very common, with $55 \%$ having post-traumatic stress disorder. Additionally, $60 \%$ of patients presented with at least one red flag symptom that warranted colonoscopy. With respect to ACG guidelines, less than 50\% received testing for celiac disease and inflammatory bowel disease. Only 20\% received unnecessary stool ova or parasite testing and $10 \%$ an unneeded colonoscopy.

Conclusions IBS is understudied in Veterans. Many of these patients have psychological comorbidities and take medications which may contribute to their symptoms. While some aspects of their workup aligned well with guidelines, others did not. Future efforts are needed to improve diagnosis and management of Veterans with DGBI.
\#248 RISK FACTORS ASSOCIATED WITH INCREASED FIBROSIS AND STEATOSIS IN NON-ALCOHOLIC FATTY LIVER DISEASE UTILIZING TRANSIENT ELASTOGRAPHY (FIBROSCAN) IN A COMMUNITY MULTISPECIALTY PRACTICE

${ }^{1} \mathrm{~B}$ Stratford*, ${ }^{2} \mathrm{C}$ Gonzales. 'Weber State University College of Science, Ogden, UT; ${ }^{2}$ Ogden Clinic, South Ogden, UT

10.1136/jim-2022-WRMC.245

Purpose of Study Non-alcoholic fatty liver disease (NAFLD) is associated with increased risk of fibrosis in patients with obesity, diabetes, and metabolic syndrome. Noninvasive liver assessment using FibroScan is an important part of the evaluation of these patients. The aim of this study is to assess the efficacy of the FibroScan in helping to risk stratify patients with NAFLD in order to improve patient outcomes.

Methods Used Over a 16 month period (3/2020 to 6/2021), 331 patients with suspected NAFLD underwent liver FibroScan assessment. A retrospective chart review was conducted for risk factors of metabolic syndrome (obesity, HTN, diabetes, sleep apnea, hyperlipidemia), FibroScan measurements of liver stiffness (MLS), steatosis, and liver biopsy outcomes.

Summary of Results A total of 331 patients were reviewed with a mean age of 49 years and mean BMI of 35 (range 18-58). Fifty-three percent of patient were obese (BMI > 30) and $20 \%$ were morbidly obese (BMI $>40)$. Metabolic risk factors were common; HTN (45\%), hyperlipidemia (37\%), sleep apnea (31\%) and diabetes (23\%). Greater fibosis risk was seen with increased BMI (normal vs. morbidly obese BMI, mean MLS 5.5 kPa vs. $9.9 \mathrm{kPa}, \mathrm{p}<0.001$ ), metabolic risk factors (0 vs. 5 risk factors, mean MLS $5.7 \mathrm{kPa}$ vs. 13.5 $\mathrm{kPa}, \mathrm{p}<0.0003$ ) and presence of diabetes (no diabetes vs. diabetes, mean MLS $7.3 \mathrm{kPa}$ vs. $10.3 \mathrm{kPa}, \mathrm{p}<0.003$ ). Increased steatosis scores were seen in increased BMI (normal vs. morbidly obese BMI, mean CAP $236 \mathrm{~dB} / \mathrm{m}$ vs. $334 \mathrm{~dB} / \mathrm{m}, \mathrm{P}<$ $0.0001)$, metabolic risk factors (0 vs. 5 risk factors, mean CAP $265 \mathrm{~dB} / \mathrm{m}$ vs. $340 \mathrm{~dB} / \mathrm{m}, \mathrm{p}<0.0001)$ and presence of diabetes (no diabetes vs. diabetes, mean CAP $307 \mathrm{~dB} / \mathrm{m}$ vs. $333 \mathrm{~dB} / \mathrm{m}, \mathrm{p}<0.0005)$. Twenty-five patients underwent liver biopsy evaluation and all patients were diagnosed with nonalcoholic steatohepatitis (NASH). Stage II-III fibrosis was diagnosed in 12 patients (48\%) and stage 4 bridging fibrosis/cirrhosis in 3 patients (12\%).

Conclusions This cohort of patients undergoing Fibroscan testing in a community practice were mostly obese and had $>1$ metabolic syndrome risk factors. Elevated liver stiffness and steatosis were significantly correlated with increasing BMI, presence of diabetes and increased risk factors for metabolic syndrome. These results will help guide clinician utilization of Fibroscan testing in clinical practice.

\section{\#249 ANTIPHOSPHOLIPID ANTIBODY SYNDROME IN A YOUNG MALE WITH BILIARY ADENOCARCINOMA}

${ }^{1,2} \mathrm{H}$ Ipalawatte* ${ }^{1,2} \mathrm{~S}$ Sathian, ${ }^{1} \mathrm{~K}$ Radicic, ${ }^{1} \mathrm{~S}$ Mishra. ${ }^{1}$ Kern Medical Center, Bakersfield, CA; ${ }^{2}$ Ross University School of Medicine - Barbados Campus, Bridgetown, Barbados

10.1136/jim-2022-WRMC.246

Case Report Cholangiocarcinoma(CC) is a rare cancer of the biliary system, common in elderly patients. Risk factors vary including alcoholic liver disease, hepatitis, HIV infection, or parasitic infection. Common associations include ulcerative 
colitis(UC) and primary sclerosing cholangitis (PSC), in which case CC can arise decades sooner (Lowe 2021; Anderson 2021). Here, we present an atypical presentation of a 27 yo male with PSC in the absence of UC symptoms diagnosed with CC and antiphospholipid syndrome(APS), a condition that is more prevalent in women.

Case Presentation A 27yo male with history of alcohol abuse, hypertension and polysubstance abuse presented to the Emergency Department (ED) with jaundice, scleral icterus, and pruritus. He was found to have abnormal liver function tests (LFTs) during a routine evaluation with a month history of exacerbating symptoms.

In the ED, labs showed an albumin level of 3.3, alkaline phosphatase of 972, ALT 219, AST 131, direct bilirubin 5.9, total bilirubin 7, and total protein 8.6. Urine analysis showed urobilinogen of 2 and moderate bilirubin levels. At this time, there was a concern for any autoimmune processes, and further studies, found positive anticardiolipin IgG and IgM in the serum, anti-smooth muscle antibodies. There was high suspicion for APS. Ultrasound of the abdomen showed stigmata of cirrhosis with suboptimal visualization of the right liver lobe with dilated intrahepatic bile ducts. CT scan showed a mass-like bulge in the inferior aspect of the right hepatic lobes with heterogeneous enhancement involving the right hepatic lobe. MRCP showed a 'beaded' appearance of the bile ducts and obstruction at the confluence of the main hepatic ducts. CA 19-9 was elevated. Findings were highly suggestive of malignancy such as CC. He underwent ERCP with sphincterotomy with biopsy confirming biliary adenocarcinoma. He was referred for hepatobiliary surgery and further evaluation with hematology/oncology.

Conclusion CC is a rare condition with a low 5-year survival rate (Nakeed, 1996). The faster a diagnosis is made, the more options that a patient has in terms of therapy. Our patient had PSC leading ho more work up and finding CC, a condition often seen much later in life, and has APS, which is often seen in females rather than males. He did not exhibit any ulcerative colitis symptoms, which are commonly associated with CC. He deviates from many common associations.

In the real world, we may encounter atypical presentations that do not fit into previously learned models. With this case, we wanted to emphasize the importance of being vigilant, have broad differentials and early intervention based on clinical suspicion.

\section{\#250 ILEAL ENDOCRINE TUMOR WITHOUT METASTASIS}

VR Mendiola, J Rahesh*, B Quraishi, S Pathapati. Texas Tech University Health Sciences Center School of Medicine, Amarillo, TX

\subsection{6/jim-2022-WRMC.247}

Case Report A 65-year-old Caucasian male with a past medical history of hypertension and alcohol abuse presented to the ED with 2 months of watery diarrhea consisting of 3-4 episodes a day. He was transferred from an outside facility for acute renal failure, severe metabolic acidosis, diarrhea, dehydration, and hypokalemia.

Initial episodes of watery diarrhea presented with fever, chills, and nausea. Watery diarrhea increased to about 10 episodes a day during this initial course. He also reported generalized weakness and recurrent falls due to syncope. He selfmedicated Ivermectin in high doses meant to be used for horses that he was able to buy from his hometown veterinarian with no prescription meant to treat his diarrhea as well as prevent Covid-19 infections. He used this drug for 10 days and reported a 20 pounds weight loss over 2 months as well as worsening oliguria.

Follow this initial course, his diarrhea continued at a lesser rate of only 3-4 episodes a day. However, three days prior to presenting to the ED he noticed fresh blood in his stool, prompting him to seek medical attention. On admission he denied abdominal pain, flushing, dyspnea, or chest pain. On examination, he had mild diffuse abdominal tenderness. The patient denied any history of hepatitis, pancreatitis, peptic ulcer disease or GERD. He had never had a colonoscopy or endoscopy at this point.

On admission his labs showed acute renal failure with an SCr of 18, a BUN of 161, and bicarb of 6 . After he was started on fluid resuscitation his kidney function improved and urinary output increased, and his acute kidney injury eventually resolved.

His CT abdomen was remarkable for 'misty mesentery' suggestive of panniculitis. There was also an enhancing nodularity of the distal small bowel mesentery in the right mid abdomen with mild to moderate circumferential bowel wall thickening of the underlying small bowel loops. In addition to this there were scattered diverticula of the sigmoid colon with no diverticulitis. The carcinoid tumor was also able to be identified on CT.

Colonoscopy showed a large $3 \mathrm{~cm}$ submucosal polypoid nodular mass at $10 \mathrm{~cm}$ distance from ileocecal junction. Biopsy reported a well differentiated neuroendocrine tumor (carcinoid), spanning at least $2 \mathrm{~mm}$ involving the lamina propria and submucosa. Liver ultrasound showed only hepatic steatosis with no lesions. 24hr urine HIAA was 12.5, chromogranin A $1048 \mathrm{ng} / \mathrm{ml}$ and serum serotonin level of $883 \mathrm{ng} / \mathrm{ml}$. TTE showed no valvular abnormalities.

Right hemicolectomy with small bowel mass was resected, surgical pathology reported well differentiated neuroendocrine tumor, forming multiple (13) mucosal masses extending up to $2.3 \mathrm{~cm}$ with focal angiolymphatic invasion and 3/18 regional lymph nodes involvement. After this, diarrhea improved, and patient followed up with a medical oncologist.

\section{\#251 A CHALLENGING CASE OF COLITIS: INFLAMMATORY OR INFECTIOUS OR BOTH}

${ }^{1} \mathrm{~S}$ Ratnayake*, ${ }^{1} \mathrm{~K}$ Grewal, ${ }^{1} \mathrm{G}$ Singh, ${ }^{1} \mathrm{~S}$ Kaur, ${ }^{2} \mathrm{~N}$ Singh, ${ }^{1} \mathrm{~A}$ Heidari, ${ }^{1} \mathrm{G}$ Petersen. ${ }^{1} \mathrm{Kern}$ Medical Center, Bakersfield, CA; ${ }^{2}$ Ross University School of Medicine, Miramar, FL

\subsection{6/jim-2022-WRMC.248}

Purpose of Study Indeterminate colitis (IC) is a form of Inflammatory Bowel Disease (IBD), when the diagnosis of Ulcerative colitis (UC) or Crohn's disease cannot be made due to mixture of findings. The incidence of Clostridioides difficile (C. diff) infection is $6-9 \%$ in patients with IBD, which can lead to increased risk for colectomy or death. Here we discuss a case of newly diagnosed IC with superimposed C. diff infection.

Methods Used Retrospective chart review after IRB approval. Summary of Results A 26-year-old male with no known medical history presented to our facility with progressive one month history of abdominal cramping pain and bloating. This was associated with watery hematochezia up to 15 episodes 
per day. Upon admission, he was found to have fever as high as 39.4C, WBC 7.5, CRP $23.8 \mathrm{mg} / \mathrm{dL}$, ESR $68 \mathrm{~mm} / \mathrm{hr}$, Hgb $12.7 \mathrm{~g} / \mathrm{dL}$. His work up also showed stool Calprotectin 3520 $\mathrm{mcg} / \mathrm{g}$, positive stool Lactoferrin, and atypical P-ANCA titer of $1: 160$, commonly elevated in UC. Stool studies were also positive for C.diff toxin B PCR and GDH antigen, and oral vancomycin was started. Colonoscopy with biopsies showed mixture of findings, from 2 pathology readings, for UC and $\mathrm{CD}$ with focal active colitis with focal ulcerations (consistent with UC), involvement of the crypts, lamina propria, and submucosa (consistent with CD). The diagnosis of IC was made and he was started on Methylprednisolone $60 \mathrm{mg}$ TID and Mesalamine $1600 \mathrm{mg}$ BID. Patient eventually improved with less frequency of hematochezia. Upon follow up in outpatient clinic his was down to only 5 episodes of diarrhea without hematochezia. Follow up colonoscopy when C.diff infection is resolved is planned for making a definitive diagnosis between UC and CD.

Conclusions Diagnosis of Ulcerative Colitis vs Crohn's Disease can be challenging in the setting of superimposed infection with Clostridioides difficile. Management of this coexistence is difficult, and duration of treatment is also not well studied. Further work up and repeat biopsy might be needed for definitive diagnosis.

\section{Session: genetics I}

\section{Concurrent session}

\section{8:00 AM}

\section{Friday, January 21, 2022}

\section{\#252 DPH5 : A NOVEL GENE CAUSING DIPHTHAMIDE BIOSYNTHESIS DISORDERS}

${ }^{1} \mathrm{SP}$ Shankar*, ${ }^{2} \mathrm{~K}$ Grimsrud, ${ }^{1} \mathrm{~L}$ Lanoue, ${ }^{1} \mathrm{~A}$ Egense, ${ }^{1} \mathrm{~B}$ Willis, ${ }^{1} \mathrm{P}$ Shankar, ${ }^{3} \mathrm{~J}$ Horberg, ${ }^{4} \mathrm{~L}$ Albadi, ${ }^{5} \mathrm{~K}$ Mayer, ${ }^{7} \mathrm{~K}$ Ütkür, ${ }^{6} \mathrm{~K}$ Monaghan, ${ }^{8} \mathrm{~J}$ Krier, ${ }^{8} \mathrm{~J}$ Stoler, ${ }^{7} \mathrm{R}$ Schaffrath, ${ }^{4} \mathrm{~F}$ Alkuraya, ${ }^{5} \mathrm{U}$ Brinkmann, ${ }^{3} \mathrm{~L}$ Eriksson, ${ }^{1} \mathrm{~K}$ Lloyd, ${ }^{1} \mathrm{KA}$ Rauen. ${ }^{1}$ University of California Davis, Sacramento, CA; ${ }^{2}$ University of California Davis, Davis, CA; ${ }^{3}$ Goteborgs universitet, Goteborg, Sweden; ${ }^{4}$ King Faisal Specialist Hospital and Research Center, Riyadh, Saudi Arabia; ${ }^{5}$ F Hoffmann-La Roche AG Research and Development Division, Penzberg, Germany; ${ }^{6} G e n e D x$, Gaithersburg, MA; ${ }^{7}$ Universitat Kassel Institut fur Biologie, Kassel, Germany; ${ }^{8}$ Boston Children's Hospital, Boston, MA

\subsection{6/jim-2022-WRMC.249}

Purpose of Study Neurodevelopmental disorders (NDDs) are genetically heterogeneous lifelong conditions with a known etiology in approximately $50 \%$ of individuals. Here, we report DPH5 (Diphthamide biosynthesis protein 5) as a novel cause of embryonic lethality, multisystem dysfunction and profound NDDs in three unrelated families. DPH5 is critical to the biosynthesis of diphthamide, a post translationally modified histidine on eEF2 (eukaryotic elongation factor 2), essential for ribosomal translocation and protein synthesis in cells.

Methods Used Exome or genome sequencing, transgenic Dph5

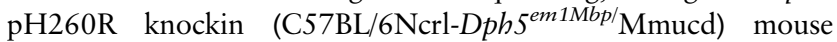
model development, patient recrutiment through GeneMatcher program, ADP-ribosylation assays in DPH5ko cells transfected with pN110S, pN174fs, pR207*, and p260R, site-directed mutagenesis in yeast cells for the missense variants and functional assays, computational modeling to evaluate effect of variants on DPH5 protein structure and assess its interaction with eEF2.

Summary of Results DPH5 variants, homozygous pH260R, compound heterozygous pN110S and pR207* and homozygous pN174fs were found in three unrelated families (F1, 2 $\&$ 3). Similar craniofacial features such as broad forehead, sparse eyebrows, epicanthal folds, short upturned nose, downturned corners of the mouth, profound NDDs, CNS anomalies such as enlarged cisterna magna, cardiac features such as ventricular septal defect, pulmonary stenosis and pericardial effusion, and digital anomalies were noted. Dph5 pH260R targeted knockin mouse model generated only one homozygous (HOM) female exhibiting extremely low birthweight, craniofacial dysmorphism, polydactyly and abnormal behaviors with early death at 24-days of age. Embryonic studies of HOMs revealed abnormal head shape, exencephaly, eye anomalies, edema shortened frontonasal prominence, facial cleft and polydactyly. ADP ribosylation assays showed absent to decreased function in $\mathrm{KO}$ and yeast cells. Insilico modeling showed disruption of interaction of DPH5 with eEF2.

Conclusions We provide clinical and functional evidence that DPH5 variants in three unrelated families are likely pathogenic establishing DPH5 as a novel cause of embryonic lethality or profound NDDs with multisystem involvement expanding the Diphthamide biosynthesis disorders.

\section{\#253 MACROANGIOPATHY, NOT SO COMMON IN MELAS?}

JP Ramos*, JA Morales, M Manning. Stanford Medicine, Stanford, CA

\subsection{6/jim-2022-WRMC.250}

Purpose of Study MELAS (mitochondrial encephalopathy, lactic acidosis, and stroke-like episode) is a multisystem disorder caused by a known mitochondrial DNA mutation most commonly affecting the MT-TL1 gene. The majority of individuals develop symptoms between the ages of 2 to 40 years old.

When MELAS affected patients present with stroke-like symptoms, the expected etiology is the underlying mitochondrial disorder causing a stroke-like episode. Common clinical findings include stroke-like episodes, encephalopathy with seizures or altered mental status, muscle weakness, hearing impairment, peripheral neuropathy amongst others. MELAS is a condition with phenotypic variability due to multiple causes (heteroplasmy, tissue distribution and threshold effect).

There are approximately 13 reported cases of macroangiopathy in patients with MELAS. We report a previously undiagnosed cause of MELAS in an adult patient who presented with right middle cerebral artery (MCA) ischemic stroke and found to have severe intracranial atherosclerosis, a finding not typically reported in MELAS patients.

Methods Used Chart review, physical examination, and literature review

Summary of Results A 44 year-old female was evaluated for a possible mitochondrial condition given a past medical history of sensorineural hearing loss following physiologically demanding circumstances (pregnancy), type 1 diabetes and acute neurologic symptoms concerning for a stroke after removal of a cochlear implant. Molecular testing revealed a mtDNA pathogenic variant: $3243 \mathrm{~A}>\mathrm{G}$ (tRNA Leu gene) with $24.7 \%$ heteroplasmy, a finding highly indicative of MELAS. Head CT angiography found severe intracranial atherosclerosis involving large vessels requiring thrombectomy. Imaging noted a tapering 
occlusion in a large segment of the right MCA, multifocal irregularity and moderate stenosis of the left internal carotid artery and proximal left middle cerebral artery, with diffusely narrowing caliber of the internal carotid arteries.

Conclusions Of the MELAS reported cases, intracranial large vessel involvement (macroangiopathy) is a rare finding in MELAS, described in very few individuals. Macroangiopathy can include stenosis, vasoconstriction, atherosclerosis, and aneurysms, and theoretically could be a lesser-known finding in patients with chronic MELAS. Given the severity of clinical complications in our patient, we call attention to macroangiopathy as a possible complication in patients with MELAS to inform their clinical team when preparing for invasive procedures, especially those involving intracranial vascular system.

\section{\#254 MT-ATP6-ASSOCIATED MITOCHONDRIAL DISEASE CAN PRESENT WITH LOW CITRULLINE BY NEWBORN SCREENING AND MORE: DEFINING THE BIOCHEMICAL PHENOTYPE}

${ }^{1} \mathrm{CG}$ Tise*, ${ }^{1} \mathrm{C}$ Lee, ${ }^{2} \mathrm{~B}$ Mendelsohn, ${ }^{1,3} \mathrm{~J}$ Woods, ${ }^{1} \mathrm{~K}$ Cusmano-Ozog. ${ }^{1}$ Stanford University, Stanford, CA; ${ }^{2}$ Kaiser Permanente, Oakland, $C A ;{ }^{3}$ Valley Children's Healthcare, Madera, CA

\subsection{6/jim-2022-WRMC.251}

Purpose of Study MT-ATP6 encodes a subunit of mitochondrial complex V (ATP synthase). Pathogenic variants are associated with a form of Leigh syndrome exhibiting considerable phenotypic heterogeneity and maternal inheritance. We have identified seven children from five families with MT-ATP6-associated disease who presented with low citrulline by California newborn screening (NBS). Further evaluation revealed abnormalities on plasma amino acids (PAA), urine organic acids (UOA), and acylcarnitine profile (ACP), and in several cases, maternal relatives with a similar phenotype. We report these findings and characterize the biochemical phenotype associated with MT-ATP6-associated disease.

Methods Used Medical literature and chart review, physical examination, and laboratory testing.

Summary of Results Seven probands were found to have a maternally-inherited homoplasmic pathogenic variant in $M T$ ATP6 (m.8993 T>G, p.L156R) after presenting with abnormal NBS for low citrulline. Biochemical evaluation revealed PAA with low citrulline and high alanine, UOA with elevations of lactate and 3-hydroxyisovaleric acid without orotic aciduria, and ACP with elevations of propionylcarnitine (C3) and 3-hydroxyisovalerylcarnitine (C5-OH). Overall findings were suggestive of a mitochondrial disorder, as opposed to the intended primary screening target of a proximal urea cycle disorder, warranting mitochondrial DNA studies. Some maternal relatives were found to have the same biochemical phenotype as the probands $(n=3)$, as well as clinical features of this disorder including ataxia, neuropathy, myopathy, and/or retinitis pigmentosa; others are reportedly asymptomatic.

Conclusions MT-ATP6-associated disease can present with low citrulline by NBS and a unique biochemical phenotype consisting of the triad of low citrulline, elevated C3, and elevated $\mathrm{C} 5-\mathrm{OH}$, in addition to evidence of mitochondrial dysfunction by PAA and UOA. For this reason, we recommend including ACP in the evaluation of individuals who screen positive by NBS for low citrulline; maternal history and laboratory studies should also be considered. Future studies include metabolomic profiling of NBS dried blood spots from individuals with MT-APT6-associated disease and relevant negative and positive controls to further delineate the biochemical phenotype and aptly identify individuals with this mitochondrial disorder.

\section{\#255 ZELLWEGER SPECTRUM DISORDER IN CALIFORNIA'S CENTRAL VALLEY: EVIDENCE OF A FOUNDER MUTATION IN MIXTECO PATIENTS AFFECTED WITH A NOVEL PEX6 VARIANT}

${ }^{1} \mathrm{KR}$ Wong*${ }^{*},{ }^{1,2} \mathrm{~J}$ Woods, ${ }^{1} \mathrm{~J}$ Carmichael, ${ }^{1} \mathrm{Cl}$ Galarreta Aima. 'Valley Children's Hospital, Madera, CA; ${ }^{2}$ Stanford Medicine, Stanford, CA

\subsection{6/jim-2022-WRMC.252}

Purpose of Study Zellweger spectrum disorders (ZSD) are a group of autosomal recessive disorders caused by mutations in the PEX genes essential for peroxisomal biogenesis. Affected patients have defective lipid metabolism and cell detoxification, resulting in hypotonia, neurologic deficits, congenital malformations, adrenocortical dysfunction, and liver disease. Prevalence varies among different regions of the world and founder mutations in distinct populations have previously been reported. California's Central Valley has a large Mixteco population compared to other parts of the United States as many of these families have emigrated from the southern Mexico states of Oaxaca, Guerrero, and Puebla. We have observed an increased frequency of ZSD at our center over the past few years in patients of Mixteco ethnicity. This study reports the spectrum of clinical and genotypic features of ZSD patients at our institution.

Methods Used We performed a retrospective chart review by searching for ZSD patients seen at our center between 2010 and 2020 using ICD-10 codes E71.50, E71.51, E71.511, E71.518, E71.53 and E71.54, and ICD-9 code 277.86. Patients with an alternative diagnosis were excluded.

Summary of Results Seven patients with ZSD were identified, all diagnosed over the past four years, presenting at birth with generalized hypotonia and facial dysmorphisms such as large anterior fontanelles. Two had poor visual response to light, three had lagophthalmos, and four had failed hearing tests. Four patients are now deceased and three died prior to one year of age. Although two were lost to follow-up, they both presented with severe symptoms including worsening hypotonia and poor feeding by three months of age. One patient is currently alive at 12 months of age. Biochemical testing demonstrated characteristic elevations of very long chain fatty acids. Six patients were found to be homozygous for the PEX6 novel variant c.1409G>C (p. Gly470Ala) and were of Mixteco ethnicity. One was homozygous for PEX6 c.2095-21_2095del and was the only patient with parental consanguinity. This patient's ethnicity is unknown.

Conclusions ZSD has a prevalence of $1: 50,000$ to $1: 75,000$ in the United States. In the Central Valley, we found an increased frequency of ZSD per zip code population in which these patients lived, ranging from $1: 4,477$ to $1: 72,280$, with a mean of 1:44,891. This over-representation of the novel PEX6 variant affecting ZSD patients of Mixteco ethnicity suggests a founder mutation within this patient population. 


\section{\#256 CLINICAL FINDINGS IN CRB2-RELATED SYNDROME}

${ }^{1,2}{ }^{\mathrm{M}}$ Adutwum*, ${ }^{3} \mathrm{~A}$ Hurst, ${ }^{4} \mathrm{G}$ Mirzaa, ${ }^{5} \mathrm{~A}$ Slavotinek. ${ }^{1}$ Children's Hospital Oakland Research Institute, Oakland, CA; ${ }^{2}$ UCSF Benioff Children's Hospital Oakland, Oakland, $C A ;{ }^{3}$ University of Alabama at Birmingham, Birmingham, $\mathrm{AL}_{;}{ }^{4}$ Center for Integrative Brain Research, Seattle, $W A ;{ }^{5}$ University of California, San Francisco, San Francisco, CA

\subsection{6/jim-2022-WRMC.253}

Purpose of Study CRB2-related syndrome was first described as a triad of cerebral ventriculomegaly, renal findings including nephrotic syndrome (NS), and greatly elevated alpha-fetoprotein levels. Additional clinical manifestations in CRB2-related syndrome have included congenital heart defects and retinitis pigmentosa (RP). The condition is rare and caused by biallelic, pathogenic variants in the CRB2 gene. Recent reports of CRB2-related syndrome have highlighted NS which can be associated with severe presentations. The objective of this project was to compile a list of the clinical and variant data for CRB2-related syndrome.

Methods Used We conducted a literature review of reported patients with biallelic pathogenic variants in CRB2 and identified additional unreported cases. We compared clinical features, survival, and variant location in CRB2 in patients with manifestations in different body systems. For those with NS, treatments and their effectiveness were recorded.

Summary of Results We ascertained 31 patients with biallelic, pathogenic variants in CRB2; detailed information was available for the majority. 18/29 (62\%) were diagnosed during pregnancy and $9 / 29$ (31\%) from 0 to 10 years of age. Two patients were diagnosed at 46 and 51 years and had RP as the sole clinical finding. Of the 30 patients with recorded outcomes, 20 (66.7\%) were alive, whereas 10 (33.3\%) were deceased, 7 from termination of pregnancy. An analysis of clinical findings showed that renal involvement was the most frequent $(22 / 31 ; 71 \%)$, with $11(35 \%)$ patients having only renal manifestations with NS. 17/31 (55\%) patients had manifestations in multiple body systems, and cardiac findings and abnormal retinae were each found in $3 / 31$ (10\%). Pathogenic variants were mostly located in exons $7,8,10,12$, and 13 , with greatest representation of exon 10 in all patients, whereas exon 7 had the greatest representation in patients with only renal disease. Details of treatments and their effectiveness were inconsistently reported and prevented comparison, but one patient transiently responded to steroids and only one surviving patient required a renal transplant.

Conclusions Most patients with CRB2-related syndrome included in this review survived. The commonest clinical finding in recently reported patients with pathogenic CRB2 variants was NS. Further information is needed to determine optimal treatment and patient care.

\section{\#257 ASYMPTOMATIC THALASSEMIA: A REASON TO REFORM CURRENT SCREENING ALGORITHMS IN REGIONS OF ASIA}

K Mai, K Chen*. Western University of Health Sciences, Pomona, CA

\subsection{6/jim-2022-WRMC.254}

Purpose of Study Many regions in Asia including Taiwan hold a high thalassemia carrier rate. The diagnosis of thalassemia in these regions mainly consists of a screening algorithm relying on mean corpuscular volume (MCV) and mean corpuscular hemoglobin $(\mathrm{MCH})$ values. However, individuals who are silent carriers and who do not phenotypically fit the screening algorithms tend to go undiagnosed, leading to future complications. While genetic screening panels may be useful in this context, a standardized regime is limited in this region. Thus, this study evaluates the clinical significance of implementing a standardized genetic screening panel for $\alpha$-thalassemia. Overall, the study assesses the efficacy of this screening in identifying phenotypically asymptomatic patients who were otherwise undiagnosed, and the risk posed in passing down more severe carrier traits.

Methods Used This is a cross-sectional study utilizing next-generation sequencing (NGS) carrier screening panels to identify $\alpha$-thalassemia silent carriers in a population within Taiwan. Long range PCR is followed to confirm patient's genetic status.

Summary of Results A total of 22 patients were identified as thalassemia silent carriers. 21 out of 22 individuals $(95.5 \%)$ were $\alpha$-thalassemia silent carriers, with 2 of them being couples. Therefore, in this population set there is a $9.5 \%$ chance of passing down more severe alpha thalassemia carrier traits. Furthermore, only 1 out of 22 patients (4.5\%) were found to be $\beta$-thalassemia carriers. These individuals were asymptomatic, and all held a MCV of $>80$. Of these individuals, only 4 were identified with a low hemoglobin level.

Conclusions Our data suggests the implementation of a standardized genetic screening for $\alpha$-thalassemia will be beneficial in many regions within Asia, including Taiwan. Previous screening algorithms focused on measuring $\mathrm{MCV}$ and $\mathrm{MCH}$ levels. However, in this study we demonstrate that MCV and $\mathrm{MCH}$ levels alone may be insufficient in identifying carriers. Maintenance of this screening algorithm would thus result in false negatives within this patient population. Overall, the use of NGS-based carrier screening panels allowed for the diagnosis of individuals who were previously undiagnosed due to them not phenotypically conforming to the current regional screening algorithms. This study also allowed for the identification of individuals at an increased risk of passing down traits such alpha thalassemia carriers and hemoglobin $\mathrm{H}$ $(\mathrm{HbH})$. The utilization of genetic screening for $\alpha$-thalassemia would be cost-efficient and beneficial especially in these patients by allowing for earlier genetic counseling, education, and potential treatment to prevent costly complications later in life.

\section{\#258 LINKAGE DISEQUILIBRIUM SCORE REGRESSION ANALYSES TO DETERMINE THE GENETIC ARCHITECTURE OF THE DIFFERENCES BETWEEN DEPRESSION AND BIPOLAR DISORDER}

S Assaf*. University of California Los Angeles, Los Angeles, CA

\subsection{6/jim-2022-WRMC.255}

Purpose of Study Psychiatric illness is a pressing health concern and there is a greater need to understand genetic differences between influencing traits. This project analyzed genome-wide differences among three genome-wide association studies (GWAS), aiming to understand genetic differences between patients with depression and bipolar disorder. Being influenced by several genes and confounding factors complicates the genetic study of these polygenic illnesses. One tool available to help understand genome wide differences from GWAS is linkage disequilibrium score regression (LDSC) which allows 


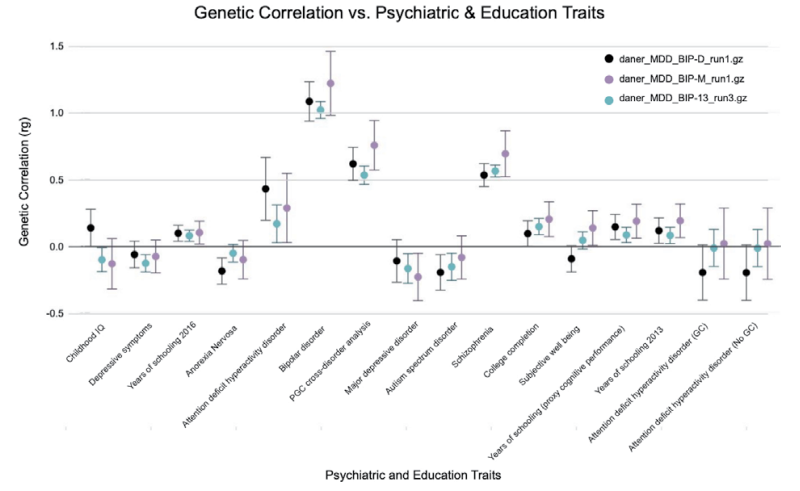

Abstract \#258 Figure 1

for the analysis of differences among psychiatric patient cases who present with bipolar disorder (BP) or major depressive disorder (MDD) with different onsets.

Methods Used Three GWAS files were analyzed through LDSC: daner13 (case-only BP vs. MDD file), danerD (BPonset-MDD vs. MDD file), and danerM (BP-onset-mania vs. MDD file). Unix and Python shell scripting were used to run a series of awk commands and the ldsc command-line tool was used to munge summary statistics. Once the files were munged, LDHub computed genetic correlation for psychiatric and educational traits along with the heritability of traits amongst two cohorts. The three GWAS were then compared using observed heritability values and genetic correlation of the traits.

Summary of Results The genetic correlation of 16 traits for each of the GWAS were plotted with their respective standard errors (figure 1). The heritability values for the daner13, danerD, and danerM files were 0.2184 (SE 0.0209), 0.2079 (SE 0.0478), and 0.1556 (SE 0.057), respectively. Psychiatric traits showed higher genetic correlation than educational traits with BP, PGC cross-disorder analysis and schizophrenia having the strongest correlation $(>0.5)$ across all GWAS. Major depressive disorder and depressive symptoms had weak negative correlations across all GWAS.

Conclusions These heritability results demonstrate that genetic differences exist between MDD and BP. The greater heritability of traits in the danerD file than the danerM file could be attributed to the higher number of BP patients who are first diagnosed with MDD before switching to BP. In addition to genome-wide analyses, individual-level genotype data may also help to predict risk scores for patients who switch from MDD to BP. In the future, these genetic differences may be used to predict the course of illness in depression patients.

\section{\#259 DIFFERENT EXONS, DIFFERENT DISORDERS: ATHELIA AND BRANCHIAL SINUS ANOMALIES ARE FEATURES OF A DISTINCTIVE KMT2D-ASSOCIATED DISORDER}

${ }^{1} \mathrm{R}$ Frankel ${ }^{*},{ }^{1} \mathrm{CG}$ Tise, ${ }^{2} \mathrm{M}$ Sanyoura, ${ }^{1} \mathrm{~B}$ Bernstein, ${ }^{3} \mathrm{LH}$ Philipson. ${ }^{1}$ Stanford University, Stanford, CA; ${ }^{2}$ The University of Chicago, Chicago, IL; ${ }^{3}$ University of Chicago Department of Medicine, Chicago, IL

\subsection{6/jim-2022-WRMC.256}

Purpose of Study Here we describe two individuals with KMT2D-associated disorder to highlight distinct features, helping to define the phenotype associated with variants in this gene. In 2020, Cuvertino et al. described a cohort of nine individuals with heterozygous variants in exons 38 and 39 of KMT2D presenting with a multisystem disorder distinct from Kabuki syndrome. Overlapping clinical features of the nine individuals included typical intelligence, choanal atresia, athelia or hypoplastic nipples, branchial sinus abnormalities, neck pits, lacrimal ducts anomalies, hearing loss, external ear malformations, and thyroid abnormalities.

Methods Used Clinical evaluation, molecular sequencing, chart review, and literature review.

Summary of Results Two unrelated individuals were found to have the same de novo pathogenic variant in exon 39 of KMT2D (c.10784A $>$ G, p.Tyr3595Cys) by exome sequencing after presenting with multiple congenital malformations. The first individual is a 27 year-old female with a coloboma, choanal atresia, congenital deafness, absent ribs, small teeth, bilateral 2nd branchial cleft sinus tracts, aplasia of the posterior semicircular canals, loose anagen hair, amastia/athelia, nonautoimmune diabetes mellitus, and typical cognitive development. The second individual is an 11 year-old female with bilateral microtia, bilateral sensorineural hearing loss, right facial nerve palsy, left sided branchial cleft cyst or pit, bilateral breast hypoplasia, and typical development.

Conclusions Typical intelligence, branchial sinus anomalies, and amastia/athelia in females appear to be distinct features of KMT2D-associated disorder. This syndrome presents with features overlapping with other multiple malformation syndromes including Kabuki, CHARGE, and brachiootorenal syndrome. These individuals exemplify the distinct phenotype of KMT2D-associated disorder and highlight the need for a natural history study of this condition to allow for better characterization and ultimately improved diagnosis and outcomes.

\section{Neonatology general III}

\section{Concurrent session}

\section{8:00 AM}

\section{Friday, January 21, 2022}

\section{\#260 PREDICTIVE VALUE OF PATENT FORAMEN OVALE MEASURED AT BIRTH FOR DEVELOPMENT OF ATRIAL SEPTAL DEFECT IN EXTREMELY LOW BIRTHWEIGHT INFANTS}

S Gaffar*, B Siassi, R Cayabyab, M Ebrahimi, M Uzunyan, R Ramanathan. Los Angeles County University of Southern California Medical Center, Los Angeles, CA

\subsection{6/jim-2022-WRMC.257}

Purpose of Study The foramen ovale (FO) is a fetal channel that allows right-to-left (R-L) shunting throughout fetal development. After birth, some term infants have minor incompetence of the flap valve, allowing a small left to right (L-R) shunt which usually resolves by 18 days of postnatal life. The aim of this study is to evaluate the echocardiographic change in FO size with postnatal growth to determine if progression to atrial septal defect (ASD) can be predicted in extremely low birth weight infants (ELBW).

Methods Used Descriptive study of all inborn ELBW infants admitted to a neonatal intensive care unit between 2015 and 2021 who had echocardiograms (ECHO) performed within 


\section{Abstract \#260 Table 1}

\begin{tabular}{|c|c|c|c|c|c|c|c|c|c|c|c|c|c|}
\hline & & Postnatal age & $\begin{array}{l}\text { Weight } \\
\text { (g) }\end{array}$ & $\begin{array}{l}\text { Foramen oval } \\
(\mathrm{mm})\end{array}$ & $\begin{array}{l}\text { Left } \\
\text { ratio }\end{array}$ & trium:Aort & $\begin{array}{l}\text { Shor } \\
\text { fract }\end{array}$ & $\begin{array}{l}\text { rtening } \\
\text { tion (\%) }\end{array}$ & $\begin{array}{l}\text { Ejec } \\
\text { fract } \\
(\%)\end{array}$ & tion & & & \\
\hline & & $\begin{array}{l}\text { ECHO1 } \\
\text { (days) }\end{array}$ & $\begin{array}{l}\text { ECHO2 } \\
\text { PMA (weeks) }\end{array}$ & $\begin{array}{l}\text { ECHO1 } \\
\text { birth } \\
\text { weight }\end{array}$ & $\begin{array}{l}\mathrm{ECHO2} \\
\text { weight at } \\
\text { discharge }\end{array}$ & ECHO1 & $\mathrm{ECHO2}$ & ECHO1 & $\mathrm{ECHO2}$ & ECHO1 & ECHO2 & ECHO1 & $\mathrm{ECHO2}$ \\
\hline Entire cohort $(n=52)$ & & $\begin{array}{l}2 \\
(1-3)\end{array}$ & $\begin{array}{l}36 \\
(35-40)\end{array}$ & $\begin{array}{l}733 \\
(633-835)\end{array}$ & $\begin{array}{l}2253 \\
(2000- \\
2825)\end{array}$ & $\begin{array}{l}1.9 \\
(1.3-2.5)\end{array}$ & $\begin{array}{l}2.3 \\
(1.5-2.6)\end{array}$ & $\begin{array}{l}1.2 \\
(1.0-1.4)\end{array}$ & $\begin{array}{l}1.3 \\
(1.2-1.4)\end{array}$ & $\begin{array}{l}40 \\
(35-42)\end{array}$ & $\begin{array}{l}41 \\
(36-44)\end{array}$ & $\begin{array}{l}75 \\
(69-77)\end{array}$ & $\begin{array}{l}75 \\
(70-78)\end{array}$ \\
\hline \multirow[t]{2}{*}{$\begin{array}{l}\text { Stratified cohort } \\
(n=52)\end{array}$} & $\begin{array}{l}\text { Small FO } \\
(n=48)\end{array}$ & $\begin{array}{l}2 \\
(1-3)\end{array}$ & $\begin{array}{l}36 \\
(35-40)\end{array}$ & $\begin{array}{l}750 \\
(633-840)\end{array}$ & $\begin{array}{l}2253 \\
(1998- \\
2730)\end{array}$ & $\begin{array}{l}1.9 \\
(1.2-2.3)\end{array}$ & $\begin{array}{l}2.2 \\
(1.3-2.6)\end{array}$ & $\begin{array}{l}1.2 \\
(1.0-1.4)\end{array}$ & $\begin{array}{l}1.3 \\
(1.2-1.4)\end{array}$ & $\begin{array}{l}39 \\
(35-42)\end{array}$ & $\begin{array}{l}41 \\
(37-44)\end{array}$ & $\begin{array}{l}75 \\
(69-77)\end{array}$ & $\begin{array}{l}75 \\
(70-78)\end{array}$ \\
\hline & Large FO $(n=4)$ & $\begin{array}{l}2 \\
(1-2)\end{array}$ & $\begin{array}{l}38 \\
(36-42)\end{array}$ & $\begin{array}{l}700 \\
(615-748)\end{array}$ & $\begin{array}{l}2590 \\
(2118- \\
3645)\end{array}$ & $\begin{array}{l}3.3 \\
(3.1-3.7)\end{array}$ & $\begin{array}{l}6.2 \\
(5.7-7.0)\end{array}$ & $\begin{array}{l}1.2 \\
(1.1-1.2)\end{array}$ & $\begin{array}{l}1.3 \\
(1.1-1.4)\end{array}$ & $\begin{array}{l}42 \\
(36-45)\end{array}$ & $\begin{array}{l}37 \\
(36-38)\end{array}$ & $\begin{array}{l}76 \\
(70-81)\end{array}$ & $\begin{array}{l}71 \\
(69-73)\end{array}$ \\
\hline
\end{tabular}

the first week of postnatal life and before discharge. Size of valve-incompetent $\mathrm{FO}$ was determined by measuring the width of L-R color doppler flow in coronal posterior and sagittal subcostal viewing windows. The largest measurement was taken as the diameter of the septal defect.

Summary of Results Fifty-two infants with median gestational age (GA) of 25 weeks (IQR; 24-26) and median birth weight (BW) of 733 grams (IQR; 633-835) were included in the study. First ECHO was obtained at a median postnatal day of life 2. Forty-eight infants had initial median FO diameter of $1.9 \mathrm{~mm}$ at birth that grew to $2.2 \mathrm{~mm}$ by median postmenstrual age (PMA) of 36 weeks. Majority of infants at birth (37/ $46,80 \%)$ and at discharge $(39 / 44,89 \%)$ had L-R shunt. Only 1 infant (1.9\%) at birth and 4 infants $(7.7 \%)$ at discharge had a closed FO. In contrast, 4 ELBW infants born at median GA of 25 weeks (IQR; 24-26) and median BW of 700 grams (IQR; 615-748) had initial FO diameter of $3.3 \mathrm{~mm}$ that grew into a $6.2 \mathrm{~mm}$ septal defect by 38 weeks PMA. All 4 infants had L-R shunt at FO on discharge. Table 1 shows other echocardiographic parameters measured that were within normal limits.

Conclusions This study demonstrated that in ELBW infants, FO greater than $3 \mathrm{~mm}$ in diameter at birth enlarges with postnatal growth to possibly become a large atrial septal defect that needs echocardiographic follow up as outpatient. If these large defects remain as atrial septal defects on long term follow up, it will indicate a much higher incidence of ASD in ELBW infants compared to full term infants. Further study of a larger population of ELBW infants is needed to confirm this finding.

\section{\#261 SYSTEMIC AND END ORGAN HEMODYNAMIC CHANGES DURING BRADYCARDIC EPISODES IN INFANTS UNDERGOING THERAPEUTIC HYPOTHERMIA}

${ }^{1,2} \mathrm{D}$ Cho* ${ }^{1,2} \mathrm{~T}$ Wu. ${ }^{1}$ Children's Hospital of Los Angeles, Los Angeles, CA; ${ }^{2}$ University of Southern California Keck School of Medicine, Los Angeles, CA

\subsection{6/jim-2022-WRMC.258}

Purpose of Study Therapeutic hypothermia (TH) is the standard therapy for newborn hypoxic-ischemic encephalopathy (HIE). Lower temperature slows firing of sinoatrial node and

\section{Abstract \#261 Table 1}

\begin{tabular}{|c|c|c|c|c|c|c|}
\hline & \multicolumn{2}{|l|}{$H R \leq 100$} & \multicolumn{4}{|l|}{$H R>100$} \\
\hline & Median & IQR & Median & IQR & Difference & p-value \\
\hline $\begin{array}{l}\text { Heart Rate (beats } \\
\text { per min) }\end{array}$ & 91 & $88-94$ & 102 & 100-106 & 11 & $<0.001$ \\
\hline $\begin{array}{l}\text { Mean Blood } \\
\text { Pressure (mmHg) }\end{array}$ & 46 & $43-53$ & 52 & $46-56$ & 6 & $<0.001$ \\
\hline $\begin{array}{l}\text { Cardiac Output } \\
\text { (ml/kg/min) }\end{array}$ & 153 & $140-165$ & 168 & 159-183 & 15 & $<0.001$ \\
\hline $\begin{array}{l}\text { Systemic Vascular } \\
\text { Resistance } \\
\left(\text { dyne }^{*} \mathrm{~s} / \mathrm{cm}^{-5}\right)\end{array}$ & 7.2 & $6.5-8.0$ & 6.5 & $5.9-7.5$ & -0.6 & $<0.001$ \\
\hline CrSO2 (\%) & 83 & $81-88$ & 82 & $80-86$ & -1 & ns \\
\hline RrSO2 (\%) & 76 & $67-79$ & 75 & $69-83$ & -1 & ns \\
\hline
\end{tabular}

Comparison of hemodynamic parameters between bradycardic events and HR $>100$ (pvalue $<0.05$ ). Comparative analysis was made using Mann-Whitney test.

can lead to sinus bradycardia. The heart rate threshold at which end organ perfusion may be compromised is unknown. We aim to investigate systemic and end-organ hemodynamic changes during bradycardic episodes.

Methods Used Continuous hemodynamic data, including heart rate (HR), mean arterial blood pressure (MBP), cardiac output (CO) by impedance cardiometry, and regional brain $\left(\mathrm{CrSO}_{2}\right)$ and renal $\left(\mathrm{RrSO}_{2}\right)$ oxygen saturation were collected prospectively and time-synchronized in infants with HIE undergoing $\mathrm{TH}$ (figure 1). Systemic vascular resistance (SVR) was derived using measured CO and MBP. Bradycardia was defined as sustained $\mathrm{HR} \leq 100$ beats per minute $(<1$ st percentile for age) for 3 minutes. For each bradycardic episode, we selected a corresponding 3-minute period when $\mathrm{HR}>100$, within a 10 minute time frame of the bradycardic event. Hemodynamic parameters were compared between the two groups $(\mathrm{HR} \leq 100$ vs. $H R>100$ ) and reported as medians with IQR (non-normally distributed). Mann-Whitney test was used for non-parametric comparisons.

Summary of Results Twenty infants ( 9 male, 11 female, mean GA $385 / 7$ weeks) were included in the analysis. Cumulatively, 433 bradycardic episodes were noted and occurred in $13 \%$ (21.7 out of 160.7 hours) of the overall monitoring period. 


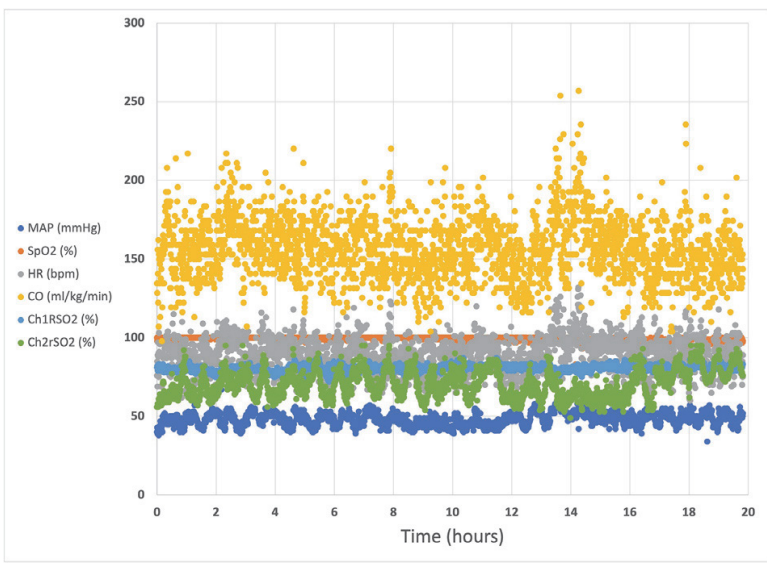

Abstract \#261 Figure 1 Sample data collection for 1 patient to illustrate continuous monitoring of hemodynamic parameters (MAP: mean arterial blood pressure, Sp02: oxygen saturation, HR: heart rate, CO: cardiac output, Ch1RSO2: brain oxygen saturation, Ch2rSO2: renal oxygen saturation).

There was a significant decrease in MBP and CO, and a compensatory increase in SVR during bradycardic episodes (table 1). There was no significant difference in $\mathrm{SaO} 2, \mathrm{CrSO} 2$ or RrSO2.

Conclusions Although there were systemic hemodynamic alterations during bradycardia events, overall end organ perfusion and/or oxygenation remain unchanged.

\section{\#262 DECREASING PERCENT OF INFANTS WITH VERY LOW 5- MINUTE APGAR SCORES AT A DENVER SAFETY-NET LEVEL III NICU}

${ }^{1} \mathrm{E}$ Harding ${ }^{*},{ }^{2} \mathrm{C}$ Stenzel, ${ }^{2} \mathrm{G}$ Roosevelt, ${ }^{1} \mathrm{~T}$ Grover, ${ }^{2} \mathrm{M}$ Hayashi. ${ }^{1}$ University of Colorado, Denver, $\mathrm{CO}^{2}{ }^{2}$ Denver Health, Denver, $\mathrm{CO}$

10.1136/jim-2022-WRMC.259
Purpose of Study Low Apgar scores are a strong predictor of neonatal mortality and may be associated with poor neurologic outcomes. Our hospital's percentage of infants born with very low Apgar scores at 5 minutes was higher than the Vermont Oxford Network national average (2.4\%). We aimed to decrease the percentage of infants with Apgar scores $<4$ at 5 minutes of life from a mean of $5.2 \%$ (range $1-11 \%$ ) to less than $2.5 \%$ and decrease the percentage of infants receiving chest compressions prior intubation from 21\% to less than 5\% by December 2017 and sustain through August 2021.

Methods Used Data was collected from January 2012 to August 2021. Four plan-do-study-act (PDSA) cycles were done from April 2015 through February 2017 including: formulated a multidisciplinary team (PDSA 1), provided 24-

Abstract \#262 Table 1 Short- and long-term outcomes for infants with very low 5-minute Apgar scores

\begin{tabular}{|c|c|c|c|c|c|}
\hline & & $\begin{array}{l}\text { Baseline } \\
(n=66)\end{array}$ & $\begin{array}{l}\text { Intervention } \\
(\mathrm{n}=37)\end{array}$ & $\begin{array}{l}\text { Post } \\
(n=35)\end{array}$ & $\begin{array}{l}P \\
\text { value }\end{array}$ \\
\hline Short-Term & Hypothermia (\%) & $7(11)$ & $6(16)$ & $7(20)$ & 0.42 \\
\hline \multirow[t]{8}{*}{ Outcomes } & HIE (\%) & $16(24)$ & $11(30)$ & $7(20)$ & 0.16 \\
\hline & HIE mild (\%) & $6(9)$ & $1(3)$ & 0 & $* *$ \\
\hline & HIE moderate (\%) & $6(9)$ & $4(11)$ & $3(9)$ & ** \\
\hline & HIE severe (\%) & $4(6)$ & $5(14)$ & $4(11)$ & ** \\
\hline & HIE unknown (\%) & $4(6)$ & $5(14)$ & $4(11)$ & ** \\
\hline & Seizures (\%) & $4(6)$ & $5(14)$ & $7(20)$ & 0.10 \\
\hline & $\begin{array}{l}\text { Gastrostomy tube } \\
\text { (\%) }\end{array}$ & $2(3)$ & $2(5)$ & $3(9)$ & 0.48 \\
\hline & $\begin{array}{l}\text { Conventional } \\
\text { Ventilation (\%) }\end{array}$ & $36(55)$ & $27(73)$ & $22(63)$ & 0.18 \\
\hline Long-term & Cerebral Palsy (\%) & $3(5)$ & $3(8)$ & $1(3)$ & $* *$ \\
\hline \multirow[t]{2}{*}{ Outcomes } & $\begin{array}{l}\text { Developmental } \\
\text { Delay (\%) }\end{array}$ & $11(17)$ & $10(27)$ & $14(40)$ & ** \\
\hline & Speech Delay (\%) & $17(26)$ & $12(32)$ & $10(29)$ & $* *$ \\
\hline
\end{tabular}

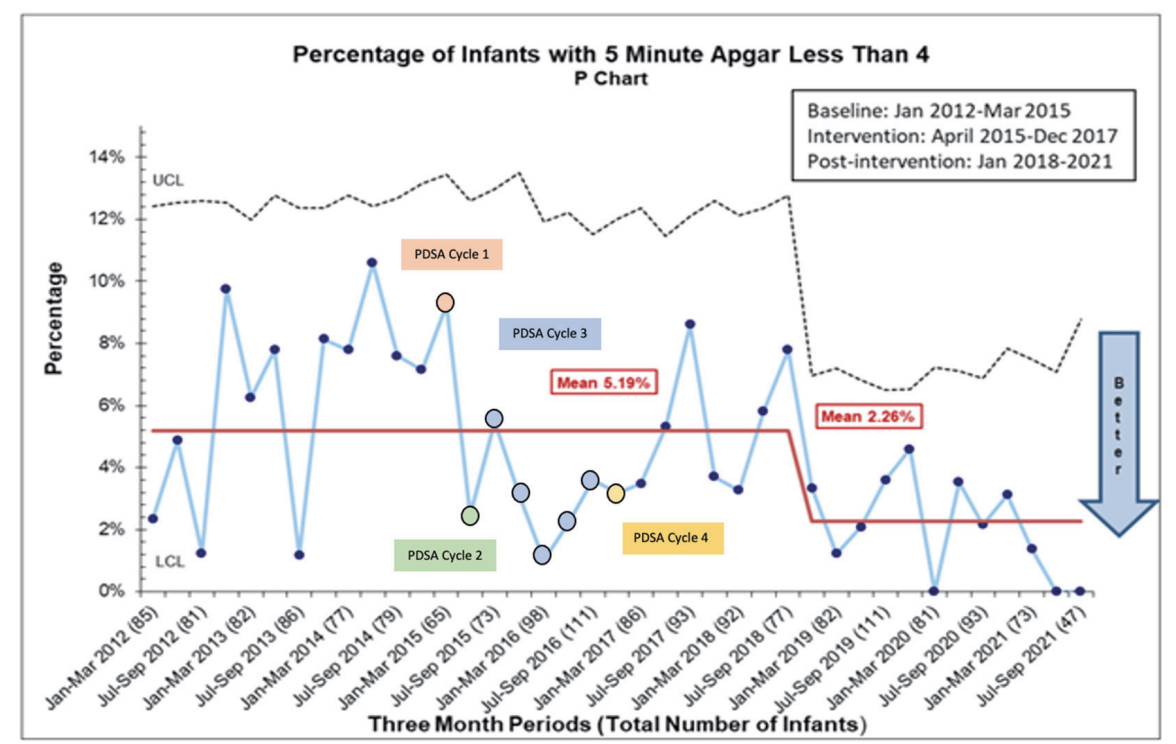

\section{Abstract \#262 Figure 1}


hour Advanced Practice Providers (APP) coverage and education campaign for providers to secure the airway prior starting compressions (PDSA 2), initiated APP-led hands-on scenarios for rotating NICU residents (PDSA 3), and developed 'Go Bags' to be taken to deliveries to ensure adequate supplies. A statistical process control (SPC) p-chart was used to evaluate our primary outcome measure of 5-minute Apgar scores $<4$.

Summary of Results There were no demographic differences across the 3 time periods. The percentage of infants with Apgar scores $<4$ at 5 minutes decreased from $5.2 \%$ in the baseline and intervention period to $2.2 \%$ in the post-invention period as special cause was detected in the SPC chart with 8 points below the center line. The percentage of infants receiving chest compression prior to intubation decreased from $21 \%$ during the baseline period to $3 \%$ in the intervention and post-intervention periods $(\mathrm{p}<0.002)$, with a relative risk of 7.9 for infants born in the baseline period (95\% CI: 1.9, 33.7).

Conclusions We were able to significantly decrease the percentage of infants with 5-minute Apgar scores $<4$ and percentage of infants receiving chest compressions prior to intubation which has been sustained for over 3 years. The addition of APPs as champions with a consistent presence in the NICU involved in all resuscitations and resident education has been critical to improving our care in the delivery room.

\section{\#263 VASOACTIVE THERAPY DURING THERAPEUTIC HYPOTHERMIA IS LINKED TO ADVERSE OUTCOMES AT NEONATAL INTENSIVE CARE UNIT DISCHARGE}

\footnotetext{
${ }^{1} S$ Perugu*, ${ }^{1,2} \mathrm{M}$ Moroze, ${ }^{2} \mathrm{D}$ Patel, ${ }^{2,3} \mathrm{~J}$ Cleary. ${ }^{1}$ Harbor-UCLA Medical Center Department of Pediatrics, Torrance, $C A ;{ }^{2}$ Children's Hospital of Orange County, Orange, $C A ;{ }^{3}$ University of California Irvine, Irvine, $C A$
}

\subsection{6/jim-2022-WRMC.260}

Purpose of Study Evaluate if hemodynamic parameters and vasoactive medication requirements during therapeutic hypothermia predict outcomes such as duration of mechanical ventilator support, clinical parameters, and neurodiagnostics in neonates with moderate-severe Hypoxic Ischemic Encephalopathy (HIE).

Methods Used Retrospective cohort study of thirty four neonates with moderate-severe HIE from 2010 to 2013 at a quaternary neonatal intensive care unit (NICU) with neurodevelopmental assessments until 2016. Data points were extracted from electronic medical records and supplemented by chart review. The data set was analyzed to compare differences between two groups, with vasoactives $(n=18)$ and without vasoactives $(n=16)$ during therapeutic hypothermia. Primary outcomes (heart rate, blood pressures and cerebral NIRS) were analyzed at five time points. Secondary outcomes were duration of mechanical ventilation, clinical and biochemical parameters, brain MRI, EEG, and Bayley III scaled and composite scores between 6-24 months.

Summary of Results There were no statistically significant differences between heart rate, blood pressure or oxygen delivery as measured by cerebral NIRS [6,12, 24, 48 and 72 hours timepoints after birth] in babies with, and without vasoactive therapies. Neonates with vasoactive requirements had more severe hypoxemia, higher blood lactate, lower albumin and hemoglobin, and require prolonged ventilation $(\mathrm{P}=0.027)$.
Additionally, they were $30 \%$ more likely to have abnormal background EEG with low voltage pattern during cooling ( $\mathrm{p}$ $<0.05$ ). Almost $50 \%$ of the patients in the vasoactive group had moderate or severe brain injury on 10-12 day MRI. Despite these important differences at NICU discharge, newborns exposed to vasoactives did not have significantly worse neurodevelopmental delays at six months.

Conclusions Vasoactive medication requirement in babies with moderate-severe HIE predicts or perhaps contributes to adverse outcomes at NICU discharge. However, infants treated with vasoactives during cooling did not have significantly worse early neurodevelopmental delays. It is critical to comprehensively analyze if therapies are meeting their intended goal of oxygen delivery. Prognosticating risks for neurocognitive deficits in childhood thus remains as an important question for pediatric research.

\section{\#264 EFFECTS OF MATERNAL PREECLAMPSIA ON CARDIAC STRUCTURE AND FUNCTION IN VERY LOW-BIRTH- WEIGHT INFANTS}

GA Kohbodi*, R Cayabyab, M Ebrahimi, R Ramanathan, B Siassi. University of Southern California Keck School of Medicine, Los Angeles, CA

\subsection{6/jim-2022-WRMC.261}

Purpose of Study Complications related to maternal preeclampsia (PE) are numerous, such as mortality, growth restriction, prematurity, respiratory distress, and morbidities related to neurodevelopment and cardiovascular system in later life. It has been shown in few small studies that exposure to PE can lead to cardiac remodeling in the fetus and term infants, but, this has not been studied in very low birth weight (VLBW) infants. The purpose of the study is to determine whether exposure to maternal $\mathrm{PE}$ is associated with hypertrophic cardiac changes and cardiac dysfunction in VLBW infants.

Methods Used We retrospectively reviewed the echocardiographic data on VLBW infants born to mothers with PE (cases) and compared them to infants not exposed to PE (controls) between January 2017 to December 2020. Cases were matched by birth weight (BW) and gestational-age (GA). Exclusion criteria were presence of congenital heart disease and no echocardiograms (ECHO) in first 7 days of life. Data was analyzed with Wilcoxon Sign Rank test and McNemar's Test where appropriate.

Summary of Results Our data from 21 cases and controls showed that infants exposed to PE had higher lactate level at birth, significantly higher systolic blood pressure during the first three days of life and a lower rate of vasopressor use within the first 24 hours of life. On ECHO, infants exposed to PE had significantly lower ejection fraction (EF) and fractional shortening (FS) compared to controls. Patent ductus arteriosus (PDA) peak flows were lower in infants with $\mathrm{PE}$ compared to controls though not statistically significant. (Table 1).

Conclusions Our preliminary data show that as a result of exposure to increased resistance to flow through placenta, VLBW infants born to preeclamptic mothers have higher systemic and pulmonary vascular resistance as evidenced by elevated systolic blood pressure after birth and lower peak flow velocity through PDA. Lower EF and SF may also be due to increase systemic vascular resistance. This information may be helpful for choice of inotropic agent for treatment of infants 
Abstract \#264 Table 1 Demographics, clinical outcomes and echocardiographic parameters

\begin{tabular}{|c|c|c|c|}
\hline & $\begin{array}{l}\text { Exposure to } \\
\text { Maternal } \\
\text { Preeclampsia } \\
\text { (Cases) } \\
\mathrm{n}=21\end{array}$ & $\begin{array}{l}\text { Non-Exposure to Maternal } \\
\text { Preeclampsia (Controls) } \\
\mathbf{n}=21\end{array}$ & $\begin{array}{l}P \\
\text { value }\end{array}$ \\
\hline \multicolumn{4}{|l|}{ Demographics } \\
\hline Gestational age (weeks)* & $27.9(26.3-30.9)$ & $26.9(25.4-29)$ & 0.09 \\
\hline Birth weight $(\mathrm{g})^{*}$ & $990(665-1,255)$ & $950(710-1,240)$ & 0.58 \\
\hline $\begin{array}{l}\text { Small for Gestational Age, } \\
\mathrm{n}(\%)\end{array}$ & $10(48)$ & $3(14)$ & 0.04 \\
\hline Male sex, n (\%) & $12(57)$ & $12(57)$ & 1 \\
\hline Cesarean section, n (\%) & $19(90)$ & $15(71)$ & 0.29 \\
\hline $\begin{array}{l}\text { Antenatal steroids, n (\%) } \\
\text { Clinical outcomes }\end{array}$ & $18(85.7)$ & $21(100)$ & 0.25 \\
\hline $\begin{array}{l}\text { Cardiomegaly on } \\
\text { admission Chest X-Ray, } n \\
(\%)\end{array}$ & $6(28.6)$ & $1(4.8)$ & 0.13 \\
\hline $\begin{array}{l}\text { Vasopressor use within } \\
\text { the first } 24 \text { hours, n (\%) }\end{array}$ & $1(5)$ & $7(35)$ & 0.03 \\
\hline Lactate Day $0(\mathrm{mmol} / \mathrm{L}) *$ & $3.3(1.8-9.3)$ & $1.8(1.5-3.3)$ & 0.03 \\
\hline Lactate Day $1(\mathrm{mmol} / \mathrm{L})^{*}$ & $2.9(1.7-3.9)$ & $1.7(1.3-2.3)$ & 0.11 \\
\hline Lactate Day $2(\mathrm{mmol} / \mathrm{L}) *$ & $1.6(1.4-2.2)$ & $1.65(1.4-2.4)$ & 0.47 \\
\hline $\begin{array}{l}\text { Systolic blood Pressure } \\
\text { Day } 0^{*}\end{array}$ & $49(41-59)$ & $42(33-50)$ & 0.04 \\
\hline $\begin{array}{l}\text { Systolic blood Pressure } \\
\text { Day 1* }\end{array}$ & $45(39-51)$ & $41(31-43)$ & 0.03 \\
\hline $\begin{array}{l}\text { Systolic blood Pressure } \\
\text { Day 2* } \\
\text { Echocardiographic Data }\end{array}$ & $49(44-54)$ & $43(39-46)$ & 0.02 \\
\hline $\begin{array}{l}\text { Fractional Shortening }(\%) \\
\text { * }\end{array}$ & $35.7(31.8-41.9)$ & $40.6(36.8-44.6)$ & 0.03 \\
\hline Ejection Fraction $(\%)^{*}$ & $69.1(64.3-77.2)$ & $76.1(71.1-79.6)$ & 0.04 \\
\hline $\begin{array}{l}\text { Patent ductus arteriosus } \\
\text { size }(\mathrm{mm})^{*}\end{array}$ & $2.2(1.7-2.8)$ & $1.57(1.4-2.3)$ & 0.21 \\
\hline $\begin{array}{l}\text { Patent ductus arteriosus } \\
\text { Peak Flow }(\mathrm{m} / \mathrm{s})^{*}\end{array}$ & $1.3(1-2)$ & $1.95(1.3-2.4)$ & 0.17 \\
\hline
\end{tabular}

*median (interquartile range).

with hemodynamic compromise born to mothers with PE. Data collection is ongoing to have a larger sample size to confirm these findings.

\section{\#265 COMBINING PROBABILITY SCORES TO OPTIMIZE CLINICAL USE OF THE ONLINE NEONATAL BRONCHOPULMONARY DYSPLASIA OUTCOME ESTIMATOR}

${ }^{1}$ RM Leigh* ${ }^{2,3} \mathrm{H}$ Yeh, ${ }^{1} \mathrm{~F}$ Chou. ${ }^{1}$ Loma Linda University School of Medicine, Loma Linda, CA; ${ }^{2}$ Children's Mercy Hospitals and Clinics, Kansas City, MO; ${ }^{3}$ University of Missouri Kansas City School of Medicine, Kansas City, MO

\subsection{6/jim-2022-WRMC.262}

Purpose of Study Bronchopulmonary dysplasia (BPD) primarily affects extremely preterm infants and has been associated with high mortality and morbidity. An online estimator for BPD severity was developed by NICHD that may guide interventions. This study aims to investigate the optimal use of the estimator for BPD prediction.

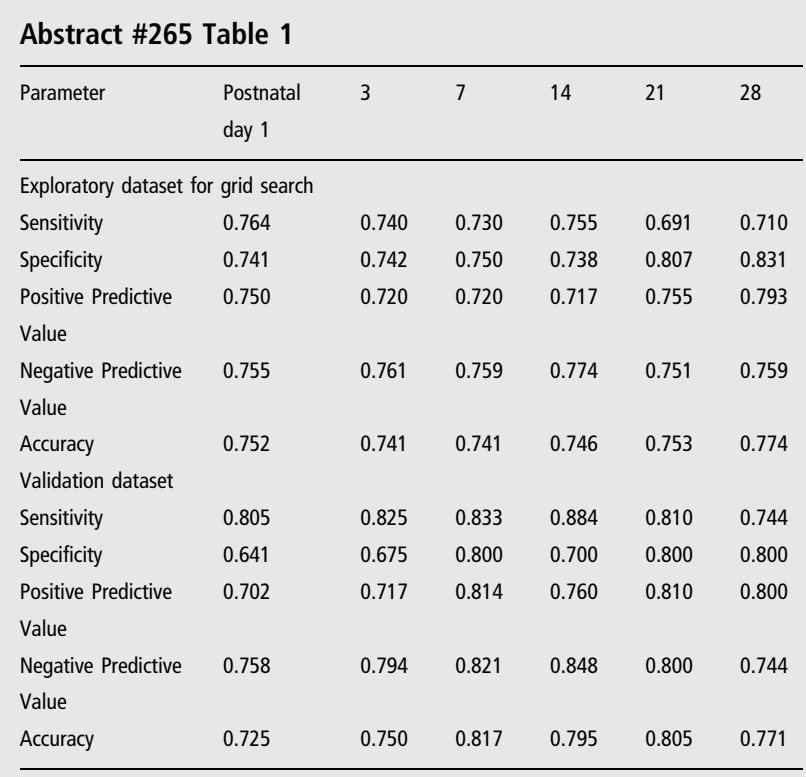

Performance of the NICHD Neonatal BPD Outcome Estimator using combined scores from the severe and death categories with a cutoff number of 21, above which predictive of a severe (positive) disease outcome and below which predictive or a non-severe (negative) disease outcome.

A
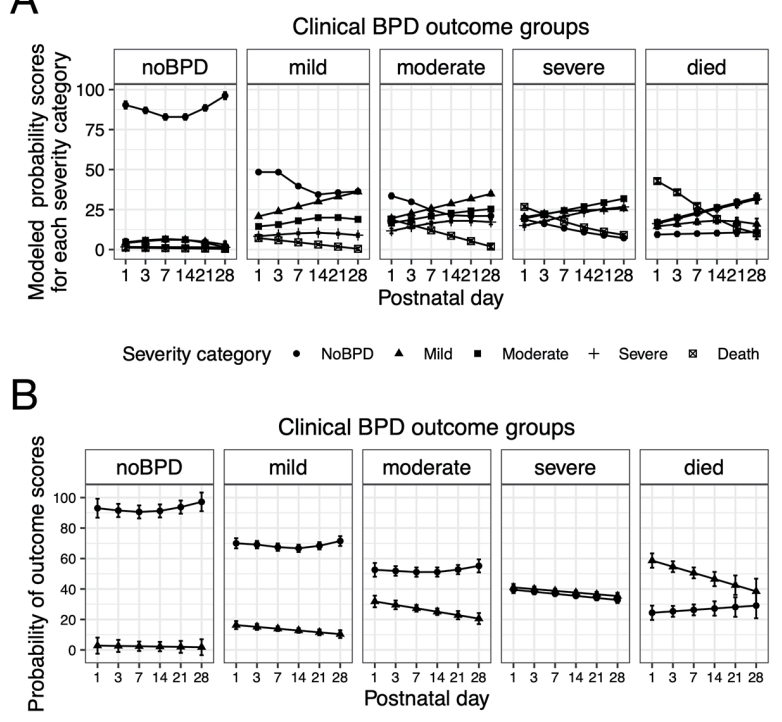

Scores - Combining No BPD and Mild A Combining Severe and Death

C

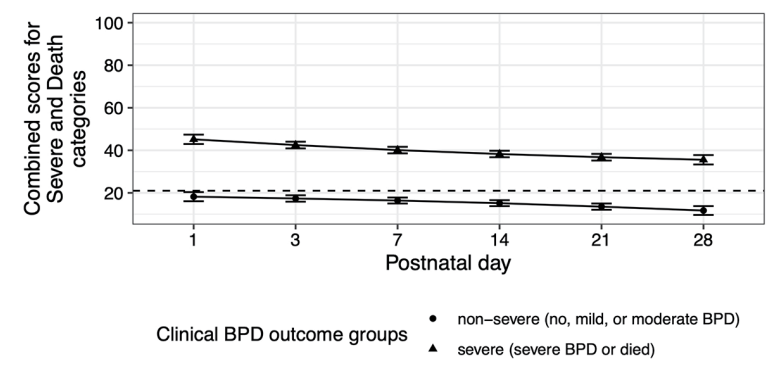

Abstract \#265 Figure 1 
Methods Used This retrospective single-center study included infants of 23-30 weeks gestation weighing $\leq 1,250$ grams. Relevant variables were entered into the estimator which provides severity probability scores for five categories: no BPD, mild, moderate, severe, or death. Generalized additive modeling was used to develop probability score trajectories for each severity category. A receiver operating characteristic curve and the Youden's J statistics, followed by a narrowed grid search, were used to assess predictability of severe BPD or death.

Summary of Results A total of 469 infants were included in the study. Direct use of the highest probability score for BPD prediction yielded 29\% accuracy due to close proximity of probability scores between categories as assessed by probability score trajectories (figure 1A). Using combined severe and death scores as well as combined no BPD and mild BPD scores resulted in improved trajectory separations, which may convey improved predictability (figure 1B). After a grid serach, we found a combined severe and death probability score with a cutoff value of $21 \%$ resulted in an optimal overall accuracy rate of $75 \%$ in predicting a composite outcome of severe BPD or death (table 1 and figure 1C).

Conclusions Combining probability scores of different categories may improve BPD outcome prediction.

\section{Neonatology - perinatal biology I}

\section{Concurrent session}

\section{8:00 AM}

\section{Friday, January 21, 2022}

\section{\#266 GESTATIONAL LONG TERM HYPOXIA AND PHENOTYPIC TRANSFORMATION OF FETAL SHEEP PULMONARY ARTERIES}

${ }^{1} \mathrm{M}$ Lee*, ${ }^{2} \mathrm{E}$ Leslie, ${ }^{1} \mathrm{C}$ Gheorghe, 'L Zhang, ${ }^{3} \mathrm{M}$ La Frano, ${ }^{1} \mathrm{~S}$ Wilson. ' Loma Linda University School of Medicine, Loma Linda, CA; ${ }^{2}$ University of New Mexico Health Sciences Center, Albuquerque, NM; ${ }^{3}$ California Polytechnic State University, San Luis Obispo, CA

\subsection{6/jim-2022-WRMC.263}

Purpose of Study Pulmonary hypertension is a serious medical condition characterized by abnormally high blood pressures in the pulmonary circulation, which can ultimately lead to rightheart failure and other sequelae. Gestational long-term hypoxia (gLTH) is a known risk factor in the development of pulmonary arterial hypertension of the newborn. The pathophysiology of this process is not fully understood, though various processes that cause structural remodeling include enhanced cell growth and proliferation. The purpose of the current study was to test the hypothesis that gLTH would increase markers of cell growth and proliferation and decrease markers of smooth muscle differentiation.

Methods Used Pregnant sheep were divided into low- and high-altitude groups. Low-altitude sheep were transported to LLU $(355 \mathrm{~m})$ for study while high-altitude sheep were transported to the Barcroft Research Station at the University of California White Mountain Research Center (3801 m) and housed there for the latter $\sim 110$ days of gestation to induce gLTH, before being transported to LLU just prior to delivery. Fetal sheep were delivered by c-section near-term at 130-140 days of gestation at LLU. Fetal pulmonary arterial segments were isolated and frozen in LN2 and stored at -80C for subsequent analysis of metabolites as well as proteins using metabolomics and proteomics approaches along with miRNA in plasma exosomes through next-gen sequencing. Based on the results from the proteomics analysis, immunohistochemical analysis was performed as well.

Summary of Results Following gLTH there were increases in metabolomic and proteomic markers of cell proliferation and phenotypic transformation that were associated with changes in exosomal miRNA. There was a decreased expression of a number of structural proteins including myosin heavy chain 11, several collagen isoforms, along with S100 proteins, histones, Caveolin 1, and markers of cellular inflammation. Metabolomic analyses indicate there were increases in oxidative stress, the pentose phosphate shunt, and arachidonic acid metabolism. There were select changes in exosomal miRNA, including upregulation of let-7a-5P, mir-221, mir-103, mir-21 and downregulation of let-7.

Conclusions Our data provide new evidence across multiple platforms regarding the mechanistic underpinnings associated with pulmonary arterial hypertension in newborns due to gLTH. Overall, the results indicate that following gLTH ovine fetal pulmonary arteries are taking on a synthetic phenotype, which likely promotes medial wall thickening along with increases in tissue inflammation and oxidative stress. We are in the process of examining the phenotypic transformation in more depth and working to elucidate the molecular underpinnings to the disease process.

\section{\#267 IUGR DECREASES PHOSPHATIDYLCHOLINE-DHA AND LIPOPHOSPHATIDYLCHOLINE-DHA IN ASSOCIATION WITH INCREASED MFSD2A IN THE RAT LUNG}

A Cohen*, R Gage, T Benally, H Wang, J Cox, A Maschek, L Joss-Moore. University of Utah Health, Salt Lake City, UT

\subsection{6/jim-2022-WRMC.264}

Purpose of Study Human neonates rely on adequate uptake of docosahexaenoic acid (DHA) for appropriate lung development. Low circulating neonatal DHA is associated with the development of bronchopulmonary dysplasia and is exacerbated by intrauterine growth restriction (IUGR). Lung uptake of DHA from the neonatal circulation is primarily in the form of phosphatidylcholine (PC) and lisophosphatidylcholine (LPC) DHA. Cellular uptake of PC-DHA and LPC-DHA is maximized by the omega-3 fatty acid transporter MFSD2a, which in other tissue types increases expression under conditions of low DHA. We previously demonstrated, in a rat model, that IUGR decreases total circulating DHA in male but not female rat pups. However, the effect of IUGR on serum and lung PC-DHA and LPC-DHA, as well as on lung $M f s d 2 a$ mRNA levels is currently unknown. We hypothesize that IUGR causes sex-divergent decreases in serum and lung PC-DHA and LPCDHA in association increased $M f s d 2 a$ in the rat.

Methods Used IUGR was induced via bilateral uterine artery ligation in pregnant Sprague Dawley rats. Control dams received anesthesia only. Rat pups were euthanized at term csection delivery, and serum and lung tissue collected. PC-DHA and LPC-DHA were measured using GC/MS. MFSD2a mRNA was measured using real-time RT PCR. Two-way ANOVA was used to assess interaction effects, t-test was used to assess group differences. 
Summary of Results Results are IUGR as $\%$ of control \pm SD, ${ }^{*} P<.05$. A significant IUGR-sex interaction effect occurred for serum PC-DHA whereby IUGR decreased serum PC-DHA in male rat pups $\left(64 \pm 7 \%^{*}\right)$, with no change in female rat pups. No other interaction effects were observed. IUGR decreased serum LPC-DHA $(65 \pm 30 \% *)$. Within the lung, IUGR decreased PC-DHA and LPC DHA $(85 \pm 8 \% *$ and $79 \pm 23 \% *$ respectively). IUGR also increased lung $M f s d 2 a$ mRNA (159 $\pm 76 \% *)$.

Conclusions IUGR decreases lung PC-DHA and LPC-DHA in rat pups in association with increased $M f s d 2 a$ mRNA expression of both sexes. In contrast, the IUGR-induced decreased serum PC-DHA is restricted to male rat pups. We speculate that upregulation of lung Mfsd2a may be a compensatory mechanism to maximize lung DHA uptake in IUGR.

\section{\#268 ATTENUATED GLUCOSE-STIMULATED INSULIN SECRETION FOLLOWING A 90-MINUTE IGF-1 INFUSION INTO FETAL SHEEP DOES NOT PERSIST IN ISOLATED ISLETS}

A White* I Stremming, LD Brown, P Rozance. University of Colorado, Aurora, CO

\subsection{6/jim-2022-WRMC.265}

Purpose of Study Pregnancy complications such as gestational diabetes and maternal obesity, which can result in elevated insulin-like growth factor-1 (IGF-1) concentrations and fetal overgrowth, are associated with abnormal pancreatic islet development and beta cell failure later in life. We have demonstrated that a 1-week IGF-1 LR3 infusion into fetal sheep results in reduced in vivo and in vitro insulin secretion. However, acute exposure to IGF-1 enhances insulin secretion in adult rodent islets. Therefore, we hypothesized that a 90minute IGF-1 LR3 infusion into fetal sheep would potentiate in vivo and in vitro glucose-stimulated insulin secretion (GSIS).

Methods Used Late gestation fetal sheep $(n=10)$ were infused for 90 minutes at $6.6 \mu \mathrm{g} / \mathrm{kg} / \mathrm{hr}$ with either IGF-1 LR3 (IGF1) or vehicle control (CON). Each animal received both infusions spaced 2-3 days apart. Fetal arterial blood gases and plasma insulin, glucose, and lactate were measured. At minute 90 of infusion, and with infusates still running, GSIS was measured utilizing a hyperglycemic clamp with frequent glucose and insulin measurements. At the end of the second infusion (IGF-1, $\mathrm{n}=5$; CON, $\mathrm{n}=5$ ), fetal islets were isolated and incubated with $1.1,2.7$, or $11 \mathrm{mmol} / \mathrm{L}$ glucose or 30 $\mathrm{mmol} / \mathrm{L} \mathrm{KCl}$ and then pelleted. Insulin concentrations of the media and cell pellet were measured by ELISA. Insulin secretion was calculated as the fraction of total islet insulin secreted into the media.

Summary of Results Plasma insulin concentrations decreased during IGF-1 infusion and were 48\% lower at the end of infusion compared to $\mathrm{CON}(P<0.05)$. Fetal arterial blood gases and plasma glucose and lactate were not different between IGF-1 and CON at the end of infusion. During the GSIS study, glucose concentrations were similar, but insulin concentrations were $66 \%$ lower with IGF-1 infusion compared to $\mathrm{CON}(P<0.0001)$. Insulin secretion in isolated fetal islets was not different based on infusion just prior to necropsy.
Conclusions IGF-1 LR3 infusion for 90 minutes into late gestation fetal sheep lowers plasma insulin concentrations and attenuates fetal GSIS. However, reduced insulin secretion does not persist in isolated fetal islets exposed to IGF-1 in vivo at the time of necropsy. Therefore, while acute increases in fetal IGF-1 may directly suppress insulin secretion, the fetal beta cell in vitro retains the ability to recover GSIS. With more prolonged elevations in fetal IGF-1, beta cells become programmed to secrete less insulin in response to glucose. We speculate that chronically, but not acutely, elevated IGF-1 concentrations during the fetal period in pregnancies complicated by gestational diabetes or maternal obesity may underlie the risk of beta cell failure and diabetes later in life.

\section{\#269 THE EFFECT OF NITRIC OXIDE ON IRON-HANDLING PATHWAYS IN THE PLACENTA}

${ }^{1} \mathrm{P}$ Principe ${ }^{*},{ }^{2} \mathrm{G}$ Mukosera, ${ }^{3} \mathrm{~N}$ Gray-Hutto, ${ }^{3} \mathrm{C}$ Gheorghe, ${ }^{1} \mathrm{~A}$ Blood. ${ }^{1}$ Loma Linda University Department of Basic Sciences, Loma Linda, CA; ${ }^{2}$ Loma Linda University School of Medicine, Loma Linda, CA; ${ }^{3}$ Loma Linda University Medical Center, Loma Linda, CA

\subsection{6/jim-2022-WRMC.266}

Purpose of Study Nitric oxide (NO) is an endogenous gasotransmitter with crucial roles in pregnancy under both physiological and pathophysiological conditions. NO can bind heme and non-heme iron (Fe) with high affinity, forming relatively stable iron nitrosyl compounds. In addition to preserving the bioactivity of NO, these reactions have been shown to affect the function of proteins bound to the iron and alter intracellular iron metabolism. We have recently demonstrated that placentas from preeclamptic pregnancies have significantly elevated levels of iron nitrosyl complexes (FeNOs). Therefore we hypothesized that NO added to cultured placental cells would result in production of FeNOs and altered expression of genes involved in iron homeostasis.

Methods Used This hypothesis was tested using syncytialized BeWo cells, induced with $50 \mu \mathrm{M}$ FSK. BeWo cells were treated for $24 \mathrm{~h}$ with an NO donor (260nM), nitrite control, nitrate control or nothing as naive control. Gene expression was assessed by qPCR. Ozone-based chemiluminescence was used to measure concentrations of $\mathrm{NO}$ and its metabolites in culture media following each experiment.

Summary of Results Our results showed significant 3-fold and 8 -fold $(\mathrm{p}<0.05)$ respective increases in expression of heme oxygenase 1 (HMOX1) and hepcidin antimicrobial peptide (HAMP) treated with NO donor while no significant difference was seen in the other iron homeostasis targets studied, including transferrin, transferrin receptor 1 , ferroportin, and divalent metal transporter.

Conclusions These results could explain in part the mechanism behind previous reports of increased HMOX1 and HAMP levels in preeclamptic placentas and provide insight into the placental cell population mainly responsible for this interplay, the syncytiotrophoblast. Future work aims to corroborate these results in placental explants, measure levels of proteins involved in iron handling, and to investigate the effects of hypoxia on NO-mediated changes in these target genes. 


\section{\#270 ELEVATED TRIGLYCERIDE CONCENTRATIONS IN UMBILICAL CORD PLASMA FROM HUMAN INTRAUTERINE GROWTH RESTRICTED PREGNANCIES CORRELATE WITH ULTRASOUND MARKERS OF POOR FETAL WELLBEING}

SS Chassen*, K Zemski-Berry, S Raymond-Whish, C Driver-Rigdon, J Hobbins, T Powell. University of Colorado, Aurora, CO

\subsection{6/jim-2022-WRMC.267}

Purpose of Study Intrauterine growth restriction (IUGR) is associated with decreased subcutaneous fat depots in the fetus and health consequences including neurodevelopmental impairment for the child. Intrauterine brain development depends on long chain polyunsaturated fatty acids (LCPUFA), but their metabolism in IUGR pregnancies is poorly understood. Circulating fetal LCPUFA levels and their relation to ultrasound (US) markers of fetal growth and wellbeing (estimated fetal weight [EFW], umbilical artery [UA] and middle cerebral artery [MCA] Doppler) in human pregnancies have also not been elucidated. We hypothesized that LCPUFA concentrations are decreased in IUGR cord plasma and correlate with US markers of severe IUGR.

Methods Used Pregnant women with an EFW of $<10$ th\% for gestation in 2 nd or 3 rd trimester were included. Prenatal US was obtained every 1-4 weeks from enrollment, with UA and MCA pulsatility index (PI) assessed by Doppler. Birth weight (BW) was used to classify the infants as pathologically small (BW <5th\%, IUGR), small for gestational age (BW 5-10th\%, SGA), or appropriate for gestational age (BW >10th\%, AGA). Cord plasma samples (umbilical vein) were collected at delivery and targeted lipidomic analyses performed by liquid chromatography mass spectrometry (LCMS) following extraction of fatty acids in triglycerides (TG), phosphatidylcholine (PC), and lysophosphatidylcholine (LPC) lipid classes. Statistical differences and correlations were assessed using student's $t$ test and Pearson's coefficient.

Summary of Results Birth weights were $25 \%$ smaller $(p=0.004)$ and UA PI $122 \%$ higher $(p=0.02)$ in the IUGR group $(n=8)$ compared to combined AGA/SGA group $(n=12)$. Concentrations of all LCPUFA in the TG fraction were greater in IUGR cord plasma compared to combined AGA/SGA group $(\mathrm{p}<0.05)$, and negatively correlated with EFW (avg $\mathrm{r}=$ $0.577, \mathrm{p}<0.05$ ), BW (avg $\mathrm{r}=-0.592, \mathrm{p} \leq 0.01$ ), and MCA PI $(\mathrm{r}=-0.469, \mathrm{p}<0.05)$. LCPUFA concentrations in the PC fraction were similar between groups, though LPC-docosahexaenoic acid (DHA) was decreased in IUGR $(p<0.01)$.

Conclusions We found increased TG-LCPUFA in IUGR cord plasma that correlated with US markers of compromised fetal growth. LPC-DHA (vital for brain development) is believed to be the lipid form for transport across the blood brain barrier, but our data show that LCPUFA packaging into TG (a storage form) may occur at the expense of LPC formation. We speculate that the increased stored TG-LCPUFA represents the fetal response to an adverse in utero environment, such as oxidative stress, and may have detrimental consequences for ultimate LCPUFA transport to the brain for development.
\#271 EFFECTS OF CHRONIC HIGH ALTITUDE HYPOXIA DURING GESTATION ON MITOCHONDRIAL OXYGEN CONSUMPTION RATES IN PULMONARY ARTERIES FROM FETAL AND NEWBORN SHEEP

SF Hanson*, ST Murray, S Wilson, L Zhang, A Blood. Loma Linda University School of Medicine, Loma Linda, CA

\subsection{6/jim-2022-WRMC.268}

Purpose of Study Chronic hypoxia during gestation has been associated with neonatal pulmonary hypertension in newborns of both humans and sheep. MicroRNA-210 levels have been found to be upregulated in response to hypoxia via HIF-1a in nearly every mammalian tissue studied. This, together with evidence that miR210 orchestrates many cellular responses to hypoxia, has led to use of the term 'universal hypoxamir.' One effect of miR210 is downregulation of the expression of iron sulfur cluster assembly proteins that are critical for normal mitochondrial function. We hypothesized that chronic hypoxia during gestation results in decreased mitochondrial oxygen consumption capacity in pulmonary arteries of near-term fetal and 2-week-old newborn lambs.

Methods Used Pregnant ewes were quartered at either low altitude $(330 \mathrm{~m})$ or high altitude $(3801 \mathrm{~m})$ for the last 100 days of gestation (term $=150$ days). Lungs were harvested from fetal lambs following c-section within 12 days before term gestion, and from newborn lambs at 10 to 14 days after birth. Pulmonary arteries were isolated from the 4th to 6 th branching generation, segments of these arteries were studied with a Seahorse XFe 24 analyzer to evaluate oxygen consumption rate (OCR) associated with basal, ATP-linked, maximal, proton leak, and non-mitochondrial respiration. OCR was normalized to vessel weight, and comparisons between normoxic/hypoxic and fetal/newborn groups were made using 2-way ANOVA with xx post hoc analysis (significance at $\mathrm{p}<0.05)$.

Summary of Results Compared to sea level controls, chronic hypoxia resulted in a significant decrease in basal oxygen consumption in both fetal (from $241.23 \pm 32.89$ to $176.74 \pm$ $31.91 \mathrm{ml} / \mathrm{mg}$ protein) and newborn (from $281.74 \pm 26.56$ to $168.03 \pm 43.25 \mathrm{ml} / \mathrm{mg}$ protein) pulmonary arteries. However, contrary to our hypothesis, maximal oxygen consumption rates were not affected by chronic hypoxia. Likewise, hypoxia had no effect on ATP-linked or non-mitochondrial OCR. Unexpectedly, hypoxia resulted in a decrease in oxygen consumption due to mitochondrial proton leak in both fetal (from $55.69 \pm 9.54$ to $13.76 \pm 6.37 \mathrm{ml} / \mathrm{mg}$ protein) and newborn (from $71.25 \pm 12.16$ to $37.62 \pm 4.98 \mathrm{ml} / \mathrm{mg}$ protein) hypoxic arteries. The magnitude of decrease in OCR due to proton leak nearly accounted for the overall decrease in baseline OCR, suggesting that the decrease in baseline OCR is due largely to decreased proton leak.

Conclusions These differences in proton leak when exposed to hypoxic conditions might be explained by downstream effects in the regulation of mitochondrial respiration involving uncoupling proteins and ROS signaling in complex with fatty acid oxidative metabolism and a glycolytic shift. 
Surgery III

\section{Concurrent session}

\section{8:00 AM \\ Friday, January 21, 2022}

\section{\#272 URINE SODIUM TO URINE CREATININE RATIO AS A MARKER OF TOTAL BODY SODIUM IN INFANTS WITH INTESTINAL FAILURE}

${ }^{1} S$ Choi ${ }^{*}$, 1,2L Casey, ${ }^{1,3}$ S Albersheim, ${ }^{1,3} \mathrm{R}$ Van Oerle, $4,5 \mathrm{MA}$ Irvine, ${ }^{1,2} \mathrm{HG}$ Piper. ${ }^{1} T h e$ University of British Columbia Faculty of Medicine, Vancouver, BC, Canada; ${ }^{2} B C$ Children's Hospital, Vancouver, BC, Canada; ${ }^{3}$ British Columbia Women's Hospital and Health Centre, Vancouver, BC, Canada; ${ }^{4} B C$ Centre for Disease Control, Vancouver, BC, Canada; ${ }^{5}$ Simon Fraser University, Burnaby, BC, Canada

\subsection{6/jim-2022-WRMC.269}

Purpose of Study Urine sodium (UNa) is a measure of total body sodium in infants with intestinal failure (IF) but can be misleading as it does not reflect volume status. Urine sodium to urine creatinine ratio (UNa:UCr) may offer a more accurate measure, but is not routinely used. This study compares $\mathrm{UNa}$ : $\mathrm{UCr}$ to $\mathrm{UNa}$ as a maker of sodium status in infants with IF.

Methods Used A retrospective review of infants with IF, from a single centre, from 2018-2020 was conducted (REB H2000816). IF etiology, intestinal anatomy, nutritional intake, urine electrolytes and anthropometrics were collected. Linear mixed effects models adjusting for repeated measures were used to associate $\mathrm{UNa}$ and $\mathrm{UNa}: \mathrm{UCr}$ with weight gain and sodium intake.

Summary of Results Twenty-two infants with a median gestational age of 31 weeks were included. IF etiology included gastroschisis (41\%), necrotizing enterocolitis (23\%), and intestinal perforation (14\%). Infants had an average of 3 paired $\mathrm{UNa}$ and UNa:UCr measures for a total of 74 paired measurements. UNa:UCr more strongly correlated with sodium intake compared to $\mathrm{UNa}(\mathrm{R}=0.25, \mathrm{p}=0.032$ vs. $\mathrm{R}=0.10$, $\mathrm{p}=0.38)$. Overall, neither $\mathrm{UNa}(\mathrm{p}=0.21)$ nor $\mathrm{UNa} \mathrm{UCr}$ $(\mathrm{p}=0.16)$ were significantly correlated with weight gain. However, for infants receiving $\leq 50 \%$ nutrition enterally, weight gain correlated with $\mathrm{UNa}(\mathrm{p}=0.01)$ and $\mathrm{UNa}: \mathrm{UCr}(\mathrm{p}=0.01)$. $\mathrm{UNa}$ UCr $>35$ predicted adequate growth regardless of enteral intake (92\% sensitivity, 59\% specificity).

Conclusions UNa: UCr is a measure of total body sodium that correlates with sodium intake in infants with IF. Our study indicates UNa:UCr $>35$ is associated with adequate growth and can be used to guide further validation studies.

\section{\#273 IDENTIFYING NONADHERENCE IN LIVER TRANSPLANT CANDIDATES USING SELF-REPORTED QUALITY OF LIFE}

\footnotetext{
1,2 J Nichols*, 2,3P Vutien, 2,3S Biggins, ${ }^{2,3,4} \mathrm{~A}$ Dick, ${ }^{3} \mathrm{~S}$ McCandlish, ${ }^{2,3}{ }^{\mathrm{K}}$ Bambha, 2,3,4 Jeyes, $2,3 \mathrm{JD}$ Perkins. 'University of Washington School of Medicine, Seattle, WA; ${ }^{2}$ Clinical and Bio-Analytics Transplant Laboratory, University of Washington, Seattle, WA; ${ }^{3}$ University of Washington Medical Center, Seattle, WA; ${ }^{4}$ Seattle Children's Hospital, Seattle, WA
}

\subsection{6/jim-2022-WRMC.270}

Purpose of Study Nonadherence remains an intractable barrier to undergoing liver transplantation (LT) for some transplant candidates and can result in candidate delisting and poor survival. Currently, there is no objective tool to evaluate pretransplant nonadherence risk. We investigated if the Liver Disease Health-Related Quality of Life Short Form (SF-LDQOL) could help proactively identify LT candidates at risk of nonadherence.

Methods Used We conducted a retrospective cohort study of 720 adults ( $\geq 18$ years old) listed for LT at the University of Washington Medical Center from 9/1/2012 to 8/30/2017, including those who completed the SF-LDQOL prior to listing. Univariable and multivariable competing risk analysis was performed to estimate the risk of delisting due to nonadherence. Results were statistically significant if $\mathrm{P}<0.05$.

Summary of Results $358(49.7 \%)$ LT candidates responded to the SF-LDQOL prior to being listed. Ultimately, 23 (6.4\%) were delisted due to nonadherence, 205 (57.3\%) underwent transplantation, $79(22.1 \%)$ died on the wait list or became too sick for transplantation, $19(5.3 \%)$ were delisted due to cancer progression beyond the Milan Criteria, 19 (5.3\%) were delisted for clinical improvement, and 13 (3.6\%) remained actively listed at the end of follow up. In the multivariable competing risk analysis, SF-LDQOL responses indicating 'poor memory' (SHR: 3.48; 95\% CI: 1.44-8.42; $\mathrm{P}=0.006$ ) and 'poor future outlook' (SHR: 2.96; 95\% CI: 1.01-8.62; $\mathrm{P}=0.047$ ) and being listed for repeat LT (SHR: 16.80; 95\% CI: $1.76-160.30 ; \mathrm{P}=0.01)$ were associated with a higher risk of delisting due to nonadherence. Female sex (SHR: 0.30; 95\% CI: $0.10-0.94 ; \mathrm{P}=0.04)$ and having a history of previous abdominal surgery (SHR: $0.28 ; 95 \%$ CI: $0.10-0.80$; $\mathrm{P}=0.02$ ) were associated with a lower risk of delisting due to nonadherence.

Conclusions Responses in the SF-LDQOL indicating 'poor memory' and 'poor future outlook' may suggest an increased risk of nonadherence in LT candidates. Tools that assess for these specific measures may help identify those candidates in need of additional support to avoid delisting.

\section{\#274 LAMINECTOMY OR FUSION FOR INTRADURAL EXTRAMEDULLARY TUMORS?}

${ }^{1,2} \mathrm{~K} \mathrm{Mo}{ }^{*},{ }^{2} \mathrm{H}$ Al Farii, ${ }^{2} \mathrm{~S}$ Lee. ${ }^{1}$ Western University of Health Sciences, Pomona, CA; ${ }^{2}$ Johns Hopkins University, Baltimore, MD

\subsection{6/jim-2022-WRMC.271}

Purpose of Study Laminectomy and laminectomy with fusion have both been demonstrated as surgical techniques that can treat intradural extramedullary tumors (IDEM). However, we are not aware of any studies comparing outcomes between laminectomy versus laminectomy with fusion. The purpose of this study was to compare the rate of 30-day complications following laminectomy with or without fusion for IDEM.

Methods Used Adult patients undergoing laminectomy for IDEM from 2012 to 2018 were identified in the National Surgical Quality Improvement Program database. Patients undergoing laminectomy for IDEM were substratified into 2 cohorts: those who received fusion and those who did not. In this analysis, pre-operative patient characteristics and demographic variables such as Age, BMI, Gender, ASA Class, Smoking, Functional Status, Diabetes, Chronic Obstructive Pulmonary Disease, Congestive Heart Failure, Hypertension, Renal Failure, Dialysis, Weight Loss, Steroid Use, Bleeding Disorder, Dyspnea at Rest, Dyspnea at Moderate Exertion, and 


\begin{tabular}{|c|c|c|c|c|}
\hline & $\begin{array}{l}\text { Odds } \\
\text { Ratio }\end{array}$ & P-Value & $\begin{array}{l}\text { Lower CI } \\
\text { bound }\end{array}$ & $\begin{array}{l}\text { Upper } \mathrm{Cl} \\
\text { bound }\end{array}$ \\
\hline $\begin{array}{l}\text { Length of Stay > } 5 \\
\text { days }\end{array}$ & 2.73 & $<0.001$ & 1.95 & 3.820 \\
\hline Transfusion & 3.15 & $<0.011$ & 1.78 & 5.57 \\
\hline Myocardial Infarction & 5.62 & 0.241 & 0.31 & 101.00 \\
\hline
\end{tabular}

General Anesthesia were assessed. 30-day wound, sepsis, cardiac, pulmonary, renal, and thromboembolic complications, as well as mortality, postoperative transfusions, extended length of stay, and reoperation were assessed. Bivariate analyses, including chi-squared and t-test, and multivariable logistical regression were performed.

Summary of Results Of 2,027 total patients undergoing laminectomy for IDEM, $181(10 \%)$ also had fusion. There were $72 / 373(24 \%)$ laminectomies with fusion in the cervical region, $67 / 801(8 \%)$ laminectomies with fusion in the thoracic region, and $42 / 776(6 \%)$ laminectomies with fusion in the lumbar region. Following adjustment, patients who received laminectomy with fusion were more likely to have increased length of stay (OR 2.73, $\mathrm{P}<0.001)$ and increased rate of postoperative transfusion (OR 3.15, $\mathrm{P}<0.001)$. Patients undergoing laminectomy in the cervical spine for IDEM had a tendency to receive additional fusion $(\mathrm{P}<0.001)$. Laminectomy with fusion for IDEM is associated with higher anesthesia class $(\mathrm{P}=0.046)$, hypertension $(\mathrm{P}=0.035)$, dialysis $(\mathrm{P}=0.004)$, and steroid use $(\mathrm{P}=0.03)$.

Conclusions Increased length of stay and rate of post-operative transfusion were associated with laminectomy with fusion for IDEM. Patients undergoing laminectomy in the cervical spine for IDEM had a tendency to receive additional fusion. Laminectomy with fusion was also associated increased anesthesia class, hypertension, dialysis, and steroid use.

\section{\#275 TIME EFFICIENCY AND PERFORMANCE OF DISPOSABLE VERSUS REUSABLE CYSTOSCOPES: A PROSPECTIVE, RANDOMIZED BENCHTOP COMPARISON}

${ }^{1} \mathrm{C}$ Baas*, ${ }^{1} \mathrm{R}$ Chen, ${ }^{1} \mathrm{DR}$ Peverini, ${ }^{1} \mathrm{JC}$ Hartman, ${ }^{2} \mathrm{~A}$ Amasyali, ${ }^{2} \mathrm{JD}$ Belle, ${ }^{2} \mathrm{E}$ Baldwin, ${ }^{2} \mathrm{D}$ Baldwin. 'Loma Linda University School of Medicine, Loma Linda, CA; ${ }^{2}$ Loma Linda University Medical Center, Loma Linda, CA

\subsection{6/jim-2022-WRMC.272}

Purpose of Study While reusable cystoscopes are commonly used for urologic procedures, bulky equipment and lengthy sterilization times can impact time efficiency. Disposable cystoscopes may offer advantages including increased availability, greater portability, and faster setup times. The aim of this study is to compare procedure time, cystoscope specifications, and physician satisfaction between disposable and reusable cystoscopes.

Methods Used Ten urologists (5 attendings and 5 residents) performed timed, simulated bedside cystoscopies with target identification using a prospective, randomized, crossover study design. Each subject used both a new disposable Ambu aScope 4 Cysto and a reusable Olympus CYF-5 flexible cystoscope. Afterward, participants completed a satisfaction survey. Time required for supply-gathering, setup, cystoscopy, and cleanup were compared. Image definition, field of view, deflection angle, force required for deflection, irrigation rate, weight, and working length were also compared.

Summary of Results The disposable cystoscope required less time for supply-gathering $(187.5$ vs. $289.4 \mathrm{~s}, \mathrm{p}<.05)$, setup (203.3 vs. 327.5 s, p<.01), and cleanup $(183.7$ vs $356.2 \mathrm{~s}$, $\mathrm{p}<.05)$ compared to the reusable, while cystoscopy times were similar (230.4 vs. $274.1 \mathrm{~s} ; \mathrm{p}=.575)$.

Optical testing showed higher image definition for the disposable cystoscope (6.30 vs 2.00 line pairs $/ \mathrm{mm}, \mathrm{p}<.001)$, but a smaller field of view $\left(66.54^{\circ}\right.$ vs $\left.107.91^{\circ}, \mathrm{p}<.001\right)$ and a lack of user-adjustable optical settings. The disposable cystoscope also had increased deflection $\left(214^{\circ}\right.$ up $/ 182^{\circ}$ down vs $198^{\circ} \mathrm{up} / 109^{\circ}$ down, $\left.\mathrm{p}<.001\right)$, yet required more force to deflect $180^{\circ}$ up $(6.86$ vs $4.46 \mathrm{~N}, \mathrm{p}<.001)$ and $90^{\circ}$ down $(4.66$ vs $3.55 \mathrm{~N}, \mathrm{p}<.01)$. The reusable cystoscope had a greater mass $(325.11$ vs $159.03 \mathrm{~g}, \mathrm{p}<.001)$, shorter working scope length $(37.45 \mathrm{~cm}$ vs $38.97 \mathrm{~cm}, \mathrm{p}<.001)$, and a faster irrigation rate at $200 \mathrm{~cm} \mathrm{H} \mathrm{H}_{2} \mathrm{O}(494.10$ vs $387.40 \mathrm{~mL} / \mathrm{min}$, $\mathrm{p}<.001)$. Post-testing, deflection was reduced for two of the ten disposable cystoscopes.

Survey results showed higher ratings in time-efficiency $(9.5$ vs $6.2 / 10, \mathrm{p}=.000)$ and overall satisfaction (9.3 vs 7.9/10, $\mathrm{p}=.004)$ for the disposable cystoscope. There was no difference in ratings for maneuverability, image quality, confidence in sterility, or cystoscopy time ( $p>.05$ for all).

Conclusions While the disposable cystoscope had better image quality, greater deflection, and was faster to assemble and disassemble, the reusable cystoscope had greater durability, more optical settings, wider field of view, faster irrigation rates, and required less force for deflection. Knowledge of the strengths and weaknesses of each device could assist surgeons in optimizing cystoscope utilization in specific clinical scenarios. Further studies are warranted and should include relative costeffectiveness and infection risks.

\section{\#276 SELF-DETERMINATION OF APTITUDE IN SURGICAL SPECIALITES}

M Dea*. Rocky Vista University College of Osteopathic Medicine, Parker, CO

10.1136/jim-2022-WRMC.273

Purpose of Study The field of surgery remains a highly coveted and competitive specialty. There are currently no standardized assessments used to assess a student's psychomotor skills, innate aptitude and other technical surgical skills. Laparoscopic surgery involves specific skills such as a strong appreciation of depth perception, manual/bi-manual dexterity, reduced tactile feedback all with operating with increased hand tremor. This study investigated two main objectives. Firstly, to evaluate the relationship between motor skills, such as drawing, playing videogames or musical instruments and surgical simulator performance. The second objective was to evaluate how a student's self-perception of their motor skills before and after a simulation assessment related to their actual performance.

Methods Used A cohort of unconditioned medical students defined as having no prior experience with surgical simulators completed a pre-task survey, which included demographic information, previous exposure to playing videogames, painting/drawing, playing a musical instrument, organized sports and knitting/sewing. The students also provided information on their medical specialty of interest and their own predictive 
assessment of their hand-eye coordination. Participants then completed various simulation tasks, which included penalties for mistakes made, and then finished with a post-task survey.

Summary of Results Painting and drawing were found to be significant activities that related to the simulation scores. Additionally, the age in which participants started certain activities played a role. For painting, the effect of age had a positive direction. For playing sports, the effect had a negative direction. Participants who gave ranked their hand-eye coordination highly beforehand significantly increased their predicted score. Additionally, the participants' views of surgical specialties did not change even after completing the tasks as most participants who were interested in pursuing surgery indicated the same view post-simulation.

Conclusions It was found that starting to play sports at an older age was associated with lower scores on the simulation tasks, while starting to draw/paint at an older age was beneficial towards a higher score. Expanding on these factors can enhance surgical residency training by implementing improvement interventions earlier. With implementation of a standardized surgical simulation test, students may be able to assess their own skills, as well as their own future direction into a surgical or non-surgical specialty. By students knowing their competence in these skills, a more tailored learning approach can be created. Patients will also benefit as medical errors may potentially be reduced with increased surgical education and training. Residency program directors may also benefit by having an extra metric in which to assess surgical residency candidates, as the field becomes more competitive each year.

\section{\#277 LEADERSHIP AND COMMUNICATION IMPROVEMENTS IN ORTHOPEDIC SURGERY OPERATING ROOM TIME-OUT HUDDLES}

${ }^{1} \mathrm{~F}$ Qureshi* ${ }^{*},{ }^{1} \mathrm{~S}$ Singh, ${ }^{1} \mathrm{~A}$ Ramprasad, ${ }^{1} \mathrm{P}$ Patel, ${ }^{2} \mathrm{D}$ Derylo, ${ }^{1} \mathrm{M}$ Mardelli, ${ }^{2} \mathrm{R}$ Khan. ${ }^{1}$ University of Missouri Kansas City, Kansas City, MO; ${ }^{2}$ AMITA Health, Chicago, IL

\subsection{6/jim-2022-WRMC.274}

Purpose of Study Time-out huddles, in which all information is confirmed, have significantly reduced adverse outcomes, near misses, and never events. During this critical portion of the procedure, the group leader takes charge and reviews necessary information with the team. The procedure does not continue until each person agrees on all information. This creates an interesting dynamic where everyone shares responsibility for a high-stakes event though most communication and leadership are in the hands of a sole spokesperson. We aim to uncover whether or not members of the team feel valued in the decision-making process as well as suggest means of improvement.

Methods Used We sent out surveys across the United States to active members operating inside the orthopedic surgery operating room and asked them to verify their position. We then asked them to express how valued they feel in terms of the time-out huddles on a scale from 1-10 with 1 being not valued at all and 10 being extremely valued. 30 orthopedic surgeons, 41 anesthesiologists, 66 nurses, 59 medical students, and 79 technicians were interviewed. The response rate remained at $78.57 \%$.

Summary of Results Each team member's average response in alphabetic order is presented. Anesthesiologists reported an average response of 8.44 . Medical students reported an average response of 5.56. Nurses reported an average response of 8.52. Orthopedic surgeons reported an average response of 9.87. Technicians reported an average response of 8.80 .

Conclusions Our study shows significant differences in the reported level of feeling of value in the time-out huddles in orthopedic surgery operating rooms. There are three significantly different groups at a confidence level of 99\%. The high level among orthopedic surgeons can be explained by the fact that these are typically the ones conducting the discussions and therefore they have a lot of control. The middle group includes anesthesiologists, nurses, and technicians. The group with the lowest level was medical students. This is concerning because keeping future health care professionals engaged and responsible is crucial in part of a sustainable future. Many reported that they were passive observers rather than participants. They also reported fear of poor evaluations if they spoke out in any way. It remains true that this is the only group that could be graded.

\section{\#278 ABSTRACT WITHDRAWN}

\section{\#279 COMPARING THE MORPHOMETRIC OUTCOMES OF OPEN AND ENDOSCOPIC SAGITTAL SYNOSTOSIS REPAIR}

${ }^{1}$ LA Chen ${ }^{*},{ }^{2} \mathrm{E}$ Mercan, ${ }^{2,3} \mathrm{~B}$ Massenburg, ${ }^{2,3} \mathrm{~A}$ Lee, ${ }^{2,3} \mathrm{R}$ Ellenbogen, ${ }^{2,3} \mathrm{R}$ Hopper, ${ }^{2,3} \mathrm{C}$ Birgfeld. ${ }^{1}$ University of Washington School of Medicine, Seattle, WA; ${ }^{2}$ Seattle Children's Hospital, Seattle, WA; ${ }^{3}$ University of Washington, Seattle, WA

\subsection{6/jim-2022-WRMC.275}

Purpose of Study Surgical management of sagittal synostosis (SS) aims to improve head shape and relieve growth restriction to prevent increased intracranial pressure. For patients presenting younger than 4 months old, endoscope-assisted suturectomy (ES) coupled with postoperative helmet therapy is offered as a less aggressive alternative to posterior vault remodeling (PVR). The purpose of this study was to compare the skull morphometry of SS patients treated with posterior vault remodeling (PVR cohort) to that of SS patients treated endoscopically (ES cohort), both immediately after surgery and two years after the procedure.

Methods Used Nineteen ES patients and nineteen age-, sexand ethnicity-matched PVR patients were included. Morphometric analysis was performed on 171 computed tomography (CT) scans which included preoperative, postoperative and 2year postoperative CTs for ES and PVR cohorts, and normal control CTs $(\mathrm{N}=57)$ from patients with no craniofacial abnormalities. Data for each patient's procedure was compiled in a chart review.

Summary of Results In immediate postop CTs, PVR cohort had significantly larger posterior vaults and wider skulls compared to the ES cohort $(\mathrm{p}<0.05)$. In 2 -year postoperative CTs, the ES cohort had a significantly lower cephalic index with longer and narrower skulls on average when compared to the PVR cohort. By 2-year follow-up, bossing angles and cranial vault volumes for both PVR and ES cohorts were not different than normal controls. On average, the ES cohort underwent shorter surgeries, with shorter anesthesia durations, and less blood loss compared to the PVR cohort. ES cohort patients were followed for an average of 389 days longer than open cohort patients. 
Conclusions The skull shape differences seen between the two surgical cohorts right after surgery were expected, given the immediate expansion that occurs with PVR. By 2-year followup, both surgical cohorts had morphometric results that were comparable, and both cohorts' cranial vaults had normalized to match control CT scans. Our chart review supports differences in anesthesia exposure, blood loss, and follow-up duration. When choosing a surgical approach for SS, a holistic evaluation that considers other factors beyond the morphometric outcome may be beneficial.

\section{Behavior and development I}

\section{Concurrent session}

\section{0:15 AM}

\section{Friday, January 21, 2022}

\section{\#280 BEHAVIORAL OUTCOMES IN INDIVIDUALS WITH FRAGILE X SYNDROME AND VARYING AUTISM SEVERITY}

${ }^{2} Y$ Tak$^{*},{ }^{1} \mathrm{H}$ Biag, ${ }^{1} \mathrm{M}$ Salcedo-Arellano, ${ }^{1} \mathrm{R}$ Aishworiya, ${ }^{1} \mathrm{~K}$ Kim, ${ }^{1} \mathrm{~S}$ Afzal, ${ }^{1} \mathrm{D}$ Hessl, ${ }^{1} \mathrm{RJ}$ Hagerman. ${ }^{1}$ University of California Davis MIND Institute, Sacramento, $C A ;{ }^{2}$ University of California Davis School of Medicine, Sacramento, CA

\subsection{6/jim-2022-WRMC.276}

Purpose of Study Fragile X Syndrome (FXS) individuals have developmental delay, learning disabilities and social/behavioral deficits. Approximately $60 \%$ of males and $20 \%$ of females with FXS have Autism Spectrum Disorder(ASD). Individuals with both FXS and ASD show more severe behavioral, cognitive and language problems than individuals without ASD, however the behavioral profile varies depending on ASD severity. We aim to evaluate the behavioral profile of individuals with FXS and varying degrees of Autism.

Methods Used Outcome measure data was collected from baseline behavioral questionnaires/assessments of patients $(n=41$, ages 6 to 25) enrolled in the UC Davis MIND Metformin Clinical Trial. Outcomes included five different self-reported questionnaires [Pediatric Quality of Life(PedsQL), Anxiety, Depression and Mood Screening(ADAMs), Aberrant Behavior Checklist(ABC), Child Sleep Habits Questionnaire(CSHQ), Swanson, Nolan and Pelham-IV(SNAP-IV)] and 2 standardized assessments(Leiter and Vineland). Autism severity was measured by the Autism Diagnostic Observation Scale (ADOS-2), which indicated ASD severity [no/mild ASD $(n=8)$, moderate ASD $(n=15)$, severe ASD $(n=18)]$. One-way ANOVA compared mean differences in behavioral outcomes among three ASD severity groups followed by Tukey's post-hoc tests. Significance of differences was determined at p-value $<0.05$.

Summary of Results The Leiter(non-verbal IQ) score of the no/ mild ASD group significantly differed from the moderate ASD $(p=0.0348)$ and severe $\operatorname{ASD}(p=0.0045)$ groups. The Vineland adaptive behavior composite score was significantly different between the no/mild ASD and severe ASD groups $(p=0.0109)$ (table 1). Of the five different self-reported questionnaires, one sub-score from the Peds Quality of Life showed a significant difference between the moderate ASD and severe ASD groups(School Functioning, $p=0.005$ ). The remaining PedsQL scores(Physical Functioning, Emotional Functioning and Social
Abstract \#280 Table 1 Comparisons of behavioral outcome measures among three ASD severity groups

\begin{tabular}{|c|c|c|c|c|}
\hline $\begin{array}{l}\text { Behavioral Outcome } \\
\text { Measure }\end{array}$ & $\begin{array}{l}\text { No/Mild } \\
\text { Autism Avg } \\
(\mathrm{N}=8)\end{array}$ & $\begin{array}{l}\text { Moderate } \\
\text { Autism Avg } \\
(\mathrm{N}=15)\end{array}$ & $\begin{array}{l}\text { Severe } \\
\text { Autism Avg } \\
(\mathrm{N}=18)\end{array}$ & $\begin{array}{l}\text { 1-way } \\
\text { ANOVA, } \\
\text { overall p- } \\
\text { value }\end{array}$ \\
\hline $\begin{array}{l}\text { PedsQL School } \\
\text { Functioning Total }\end{array}$ & $55 \pm 14.392$ & $\begin{array}{l}50.667 \pm \\
11.318\end{array}$ & $\begin{array}{l}67.368 \pm \\
16.446\end{array}$ & 0.005 \\
\hline $\begin{array}{l}\text { Leiter Non-Verbal IQ } \\
\text { Score }\end{array}$ & $\begin{array}{l}65.5 \pm \\
13.512\end{array}$ & $47.8 \pm 15.667$ & $\begin{array}{l}44.167 \pm \\
17.743\end{array}$ & 0.006 \\
\hline $\begin{array}{l}\text { Vineland Adaptive } \\
\text { Behavior Composite } \\
\text { Standard Score }\end{array}$ & $\begin{array}{l}69.5 \pm \\
18.328\end{array}$ & $54.6 \pm 15.810$ & $\begin{array}{l}45.421 \pm \\
17.008\end{array}$ & 0.013 \\
\hline
\end{tabular}

Functioning), Child Sleep Health total, SNAP-IV scores, Aberrant Behavior Checklist total, Anxiety, Depression and Mood Screening scores did not show significant differences between the groups.

Conclusions Our results identified significant differences between individuals with varying autism severity in non-verbal IQ and adaptive behavior. There were no significant differences in other parent-reported behavioral measures and overall pediatric quality of life except for that related to school functioning. Increased sample size may lead to emergence of further significant differences in the other behavioral measures. Nonetheless, facilitating further school accommodations/inclusion for children with ASD can help to improve their schoolrelated quality of life.

\section{\#281 INVESTIGATING RELATIONS BETWEEN MATERNAL MOOD DISORDERS AND RATES OF DEVELOPMENTAL CARE PRACTICES IN THE NEONATAL INTENSIVE CARE UNIT}

M Chan Morales*, E Brignoni-Pérez, VA Marchman, E Armer, R Shaw, KE Travis, M Scala. Stanford University, Stanford, CA

\subsection{6/jim-2022-WRMC.277}

Purpose of Study Developmental care practices in the NICU, such as positive touch and skin-to-skin holding, have led to substantial improvements of clinical and neurodevelopmental outcomes in children born preterm, and represent the largest source of NICU parenting behaviors. Parents of children born preterm are at risk for experiencing mood disorders, such as depression, anxiety, and post-traumatic stress disorder (PTSD). Mood disorders have been shown to have negative impacts on parenting behaviors for healthy term infants. Developmental care activities have been used at other institutions as part of treatment interventions for NICU parents with mood disorders. However, few studies have explored relations between perinatal parental mood disorders and rates of participation in developmental care activities. The purpose of this study is to examine the relationship between maternal mood disorders and rates of mother-delivered developmental care activities.

Methods Used Mothers of infants born preterm $(n=59)$ completed screeners for mood disorders when their infant was approximately 14 days old, deriving rates of maternal mood disorders, specifically Depression, Anxiety, and PTSD. Rates of mother-delivered developmental care (minutes of 
developmental care/days of hospitalization) were documented daily in the electronic medical record throughout the infants' hospital stay and were extracted for analyses.

Summary of Results No significant correlations were observed between mood scores and developmental care rates normalized for the total length of stay (Depression $\mathrm{r}=0.02, \mathrm{p}=0.90$; Anxiety $r=0.00, p=.99$; PTSD $r=0.02 p=0.87$ ). Rates of developmental care were not significantly different in mothers who were classified as qualifying for referral to mental health services $(\mathrm{N}=19)$ compared to those who did not $(\mathrm{N}=40)$ (Depression $\mathrm{t}=0.48, \mathrm{p}=0.63$, Anxiety $\mathrm{t}=-0.34, \mathrm{p}=0.74$; PTSD $\mathrm{t}=-0.20 \mathrm{p}=.85$ ). Results were similar after controlling for factors that may have affected rates of developmental care, including SES, gestational age, and infant health.

Conclusions While perinatal maternal mood disorders remain an important issue in the NICU, perinatal mood may not be an important factor affecting engagement in developmental care. Findings suggest that maternal engagement in developmental care activities may not serve as an appropriate indicator of mental health issues. As such, perinatal mood screeners should be implemented in infant healthcare environments, to gauge maternal mental health. Further research should be conducted to determine the extent to which perinatal screening for mental health can inform ways to improve maternal engagement in developmental care and other parenting activities both during and after infant hospitalization.

\section{\#282 THE INFLUENCE OF MATERNAL AND CHILD INTERACTIVE BEHAVIORS ON EARLY CHILD DEVELOPMENT}

${ }^{1} \mathrm{KN}$ Baillie*, ${ }^{1} \mathrm{~T}$ Emery, ${ }^{2} \mathrm{~J}$ Lowe, ${ }^{2} \mathrm{~S}$ Markee. ${ }^{1}$ University of New Mexico School of Medicine, Albuquerque, NM; ${ }^{2}$ University of New Mexico Health Sciences Center, Albuquerque, NM

\subsection{6/jim-2022-WRMC.278}

Purpose of Study The quality of interactions between children and their caregivers significantly impacts a child's development. Positive maternal behaviors during play are associated with better self-regulation and fewer behavioral problems, while poor parental well-being is associated with increased developmental difficulties. We explored the relationship between positive and negative maternal interactive behaviors during play, parental well-being and cognitive and language development in children aged 18 months old (mo). We hypothesized positive maternal behaviors during play will positively correlate with higher Bayley Scales of Infant Development III (BSID-III) cognitive and language scores at 18 mo and poor parental well-being will lead to more negative directing behaviors and child disengagement.

Methods Used Healthy term infants were recruited in Albuquerque, NM between 2010-2012 as part of a longitudinal study of parenting and child development $(n=40)$. BSID-III was used as an objective measure of cognitive and language development. Parental well-being was measured using a parental stress index (PSI) questionnaire in addition to socioeconomic status (SES). The Maternal Attention Directing Manual (S. Landry 2000) was used to code six minute free-play videos of mother-child dyads, focusing on positive maternal directing behaviors, such as 'maintain' and 'introduce' and negative behaviors, such as 'redirect' or 'zaps'. Intercoder reliability was greater than $80 \%$.
Summary of Results A significant positive correlation was found for BSID-III language scale and maternal frequency of maintaining behavior $(\mathrm{R}=+0.510, \mathrm{p}=0.001)$. A significant negative correlation was found for BSID-III language scale and frequency of maternal redirecting behavior $(\mathrm{R}=-0.383$, $\mathrm{p}=0.018)$ and duration of redirecting $(\mathrm{R}=-0.388, \mathrm{p}=0.018)$. Using partial correlations to control for maternal education level, significant positive associations between BSID-III language score at $18 \mathrm{mo}$ persisted for frequency and duration of maintaining behavior $(\mathrm{R}=+0.446, \mathrm{p}=0.004$ and $\mathrm{R}=+0.522$, $\mathrm{p}=0.001$ respectively). In addition, a significant positive correlation between the PSI parent distress scale and duration for redirecting behavior was found $(\mathrm{R}=0.373, \mathrm{p}=0.042)$ when controlling for maternal education.

Conclusions We demonstrate that early child language development was positively impacted by maternal maintaining behavior and negatively impacted by maternal redirecting behavior during play. Increased maternal stress levels resulted in increased redirecting behavior. Overall, these results suggest higher parental stress may have a negative impact on early child language development.

\section{\#283 THE EASY BABY: EXPLORING TEMPERAMENT PROFILES IN YOUNG CHILDREN WITH PRENATALLY IDENTIFIED SEX CHROMOSOME TRISOMIES}

M Louderman*, TG Thompson, R Wilson, S Davis, S Howell, N Tartaglia. University of Colorado, Denver, CO

\subsection{6/jim-2022-WRMC.279}

Purpose of Study Temperament of very young children with sex chromosome trisomy (SCT) has yet to be described in the literature. Young children with developmental differences may present with variation in temperament profiles. Temperament consists of a child's activity level, rhythmicity, approach or withdrawal tendencies, adaptability, threshold of responsiveness, intensity, mood, distractibility, and persistence; all of which may affect a child's development and have diagnostic and treatment implications. This project aimed to evaluate temperament profiles in infants with prenatally identified SCTs using standardized measures.

Methods Used The eXtraordinarY Babies study is a longitudinal natural history study examining developmental, medical, and psychosocial factors in children with prenatally identified SCT (XXY, XYY, or XXX). Data from the Carey Temperament Scale (CTS) completed by parents at the 12-month-visit were used for this analysis. The CTS is a validated tool designed to assess innate behavioral patterns in children. Caregivers rated the frequency of behaviors on a 6-point scale, yielding z-scores in 9 categories of temperament characteristics, as well as a rating of overall manageability of the child's behavior. Descriptive statistics were used to describe the sample, one-sample t-tests were used to compare the SCT sample to the norming sample, and a one-way ANOVA was used to compare SCT groups.

Summary of Results While results of the CTS suggest that children with SCTs have temperament qualities that fall within the average range, there are statistically significant differences in many domains when compared to the norming sample showing low activity level $(\mathrm{p}<.001)$, high rhythmicity $(p<.001)$, low intensity of reaction $(p<.001)$, low persistence 
$(\mathrm{p}<.001)$, and high threshold of responsiveness $(\mathrm{p}=.006)$. There were no statistically significant differences between the SCT conditions. A significant majority (82\%) of parents rated their child's overall manageability as 'easy' or 'very easy'.

Conclusions Results indicate that 12-month old infants with SCTs have temperamental differences from the general population. These data confirm parent-report of child behavior, and previous studies which suggest similar temperamental differences in older children with SCTs. The temperament profile found reflects what specific characteristics make these children easier to manage in the first year of life and defines a new constellation of behavioral patterns that comprise what is commonly referred to as an 'easy baby' in SCTs. Future analyses will further examine temperamental patterns beyond 12 months of age, the relationship of temperament to development and parent-child relationships, and inform genetic counseling, assessment, and intervention recommendations.

\section{\#284 PERINATAL DISTRESS ASSOCIATED WITH NEONATAL CONDITIONS: ANXIETY AND DEPRESSION}

DL Cooke*, H Nsier, J Kelleher, J Dempsey, AG Dempsey. University of Colorado Denver School of Medicine, Aurora, $\mathrm{CO}$

\subsection{6/jim-2022-WRMC.280}

Purpose of Study The purpose of this study was to examine self-reported perinatal depression and anxiety symptomology associated with discrete fetal medical complexities seen in a Fetal Care Center (FCF) in a Children's Hospital.

Methods Used The Edinburgh Postnatal Depression Scale (EPDS) and General Anxiety Disorder (GAD-7) were administered to all patients at a major FCF as part of a quality improvement project for detection of PMADs in the center. Questionnaires were given to all women presenting for both initial and return appointments, however only data from the initially administered questionnaires were included. The EPDS is a self-report measure of depressive symptoms in antenatal populations, with a cutoff of 11.5 suggested for women with high-risk pregnanices. The GAD-7 is a standardized self-report measure of anxiety symptoms, with a cutoff of 10 . Higher scores on both indicate greater symptomatology. Scores and

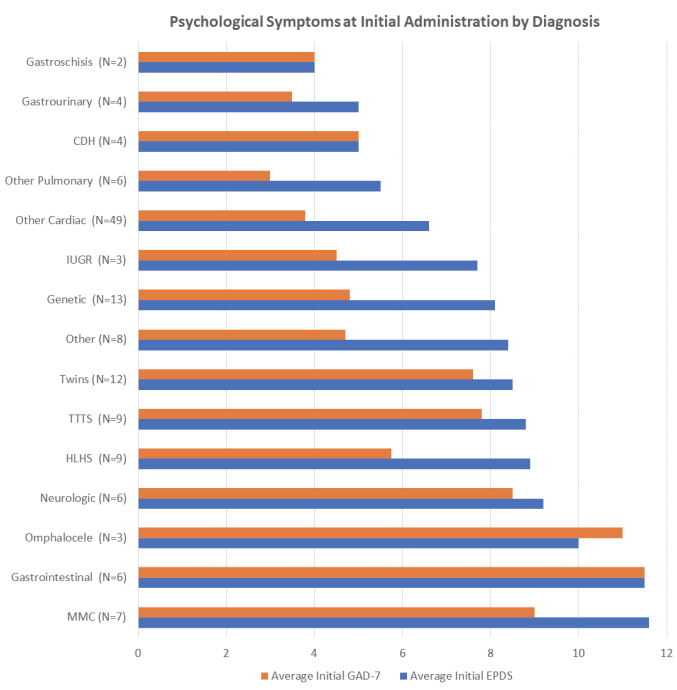

\section{Abstract \#284 Figure 1}

information regarding fetal diagnoses were obtained via retrospective chart review from 141 women and examined descriptively.

Summary of Results The pattern of depressive and anxious symptomatology at first administration varied by medical diagnosis (figure 1). Within the sample 37\% reported an EPDS score of 10 or greater, $21 \%$ reported an EPDS score of 12 or greater, and $20 \%$ reported a GAD-7 score of 10 or greater. Of the observed medical conditions, myelomeningocele, gastrointestinal conditions, and omphalocele were associated with the highest average rates of distress (the average EPDS scores were greater than 10 and GAD-7 scores 9 or greater). Neurologic conditions, hypoplastic left heart syndrome, twin to twin transfusion syndrome, and twins were associated with the second highest average rates of distress (average EPDS scores greater than 8 and GAD-7 scores were greater than 5.75).

Conclusions Certain fetal medical conditions appear to be associated with greater levels of distress in perinatal women. More research is needed to determine if fetal medical conditions increase risk for the development of PMADs. Women with these diagnoses may benefit from increased psychosocial monitoring and support.

\section{\#285 EDUCATIONAL NEEDS OF CHILDREN WITH SEX CHROMOSOME ANEUPLOIDIES}

${ }^{1,2} \mathrm{TG}$ Thompson*, ${ }^{1,2} \mathrm{~J}$ Janusz, ${ }^{1,2} \mathrm{~S}$ Davis, ${ }^{1,2} \mathrm{~N}$ Tartaglia. ${ }^{1}$ University of Colorado Denver School of Medicine, Aurora, CO; ${ }^{2}$ Children's Hospital Colorado, Aurora, CO

\subsection{6/jim-2022-WRMC.281}

Purpose of Study Children with sex chromosome aneuploidies (SCAs) are at an increased risk for neurocognitive and behavioral disorders that may interfere with success in school and ultimately with quality of life, including early developmental delays, learning disabilities, executive function problems, and social communication deficits. The current study aimed to update and extend our understanding of educational supports and outcomes for students with these increasingly common genetic diagnoses.

Methods Used Parents of children with a diagnosed SCA, birth to 21 years, living in the U.S. $(\mathrm{N}=248)$, consented to participant in an electronic survey. Descriptive statistics were used to quantify rates of school support plans, academic accommodations, educational therapies, and school completion. Logistic regression was used to calculate group differences. An inductive qualitative thematic analysis was applied to free-text survey responses.

Summary of Results Results revealed high rates of delayed kindergarten (20\%), grade retention (17\%), and individualized educational programs (IEPs; 71\%). Despite a clear profile of educational need, a majority of respondents with children over age $18(\mathrm{~N}=41)$ reported their child successfully completed high school, and nearly half pursued post-secondary education opportunities. A majority of parents described their child's teachers as having little to no knowledge of how SCAs can impact a child's learning. Analysis of open-ended parent responses emphasized that challenges with reading, executive function, reduced stamina, social skills deficits, and emotional dysregulation acted as barriers to learning, and were frequently triggered by busy or noisy classroom environments. Further, skills hovering in the borderline range are common to the SCA phenotype, and are not often well served by 
special education systems challenged by limited resources and strict cut-offs for qualification. Parents reported the need to advocate strongly for their child to receive adequate school support services.

Conclusions Pediatricians should be aware of the frequent need for accommodations and individualized support plans in children with SCAs, so they can educate families and advocate for early and comprehensive evaluations and intervention plans. Our findings justify a need to train teachers and policy makers in the unique educational needs of children and adolescents with SCAs. We recommend increased collaboration between medical and educational teams and acknowledgement of the significant role the genetic condition plays in the educational experiences of students with SCAs.

\section{\#286 LOW SENSITIVITY OF THE AGES \& STAGES QUESTIONNAIRE IN INFANTS WITH SEX CHROMOSOME TRISOMY AT 6 AND 12 MONTHS OF AGE}

IJ Nevarez, 'TG Thompson, ${ }^{2} \mathrm{~L}$ Pyle, ${ }^{3} \mathrm{~J}$ Ross, ${ }^{1} \mathrm{~N}$ Tartaglia*. ${ }^{1}$ Children's Hospital Colorado, Aurora, CO; ${ }^{2}$ University of Colorado Denver School of Medicine, Aurora, CO; ${ }^{3}$ Alfred I DuPont Hospital for Children, Wilmington, DE

\subsection{6/jim-2022-WRMC.282}

Purpose of Study Sex chromosome trisomy (SCT) conditions are a group of genetic disorders characterized by an extra $\mathrm{X}$ or $\mathrm{Y}$ chromosome, including $\mathrm{XXY} / \mathrm{K}$ linefelter syndrome and $\mathrm{XYY}$ syndrome in males, and Trisomy $\mathrm{X}$ syndrome in females. In early childhood, the most common features of SCT include developmental delays, with previous studies showing that 50$70 \%$ of young children have delays that require early intervention therapies such as speech therapy and/or physical therapy. The diagnosis of SCT in the prenatal period has drastically increased over the past decade, leading to the need for developmental screening guidelines for the large group of SCT infants at risk for delays. The aim of this project is to evaluate a commonly-used standardized developmental screening measure called the Ages and Stages Questionnaire (ASQ) to determine if it is valid and effective in identifying young children with SCT with developmental delays.

Methods Used The eXtraordinarY Babies Study is a longitudinal natural history study examining developmental, medical, and psychosocial factors in children with prenatally identified SCT (XXY, XYY, or XXX). Data from the 6 and 12 month visits were used for this analysis. Parents $(n=121)$ completed the 6 and 12 month ASQ, and results were compared to direct developmental assessment using the Bayley Scales of Infant and Child Development-3 (BSID-III). Sensitivity, specificity, positive and negative predictive values were calculated for domains of communication, fine motor, gross motor, and problem solving, using both a strict ASQ cutoff ( $>2$ SD below the mean) and an expanded cutoff ( $>1 S D$ below the mean).

Summary of Results The sensitivity of all analyses ranged from $0.1-0.6$, all well below the standard accepted sensitivity of $0.7-0.8$, except for the 12-month gross motor analysis. An expanded cut-off improved sensitivity to $0.2-0.9$, but results remained below acceptable levels for a reliable screening tool. Specificity rates were high at a range of $0.7-0.10$ for all analyses and fell within the standard accepted specificity of $0.7-$ 0.9 .

Conclusions Based on findings of low sensitivities and high false negative rates of the ASQ, we conclude that the ASQ is not a good measure to use in the SCT population at 6 or 12 months of age. Future analyses will compare the ASQ to other screening measures to determine if others provide acceptable statistical properties for developmental screening. Based on these results, direct developmental assessment during the first year of life should be part of the care recommendations for all children with SCT until a more rigorous screening measure is identified or developed for this at-risk population.

\section{Diversity, equity and inclusion II}

\section{Concurrent session}

\section{0:15 AM}

\section{Friday, January 21, 2022}

\section{\#287 DISPARITIES IN PARENT-DOCTOR COMMUNICATION DURING THE HOSPITALIZATION OF CHILDREN WITH MEDICAL COMPLEXITY}

1,2S Hussain* ${ }^{1,2} \mathrm{D}$ Chin, ${ }^{1,2} \mathrm{H}$ Nguyen-Tang, 1,2 B Afghani. 'UC Irvine School of Medicine, Orange, $C A ;{ }^{2}$ Children's Hospital of Orange County, Orange, $C A$

\subsection{6/jim-2022-WRMC.283}

Purpose of Study While children with medical complexity (CMC) represent less than $1 \%$ of the pediatric population, they account for one-third of pediatric health care spending. Unfortunately, there is a lack of clinical initiatives and research efforts geared towards improving their care, let alone assessing their health literacy. This population experiences frequent hospitalizations and is often inundated with medical jargon. Clear communication from providers is a key component of health literacy and empowers families to make medical decisions. The objective of this study is to assess communication among physicians and parents prior to discharge.

Methods Used A survey via REDCap was distributed to parents of CMC on the day of discharge from the hospital. The survey was based on the CAHPS Health Literacy survey, a validated tool used to examine the communication barriers that providers can target to improve patient and caretaker's understanding. The survey was modified for the pediatric inpatient population.

Summary of Results Twenty surveys were collected. 20\% said they speak English not well or none at all. About $66 \%$ of the parents had completed high school and some college and the remainder had a college or postgraduate degree. A majority of respondents (72\%) identified themselves as belonging to an

Parent's perspective on physician communication about the care of their hospitalized medically complex child

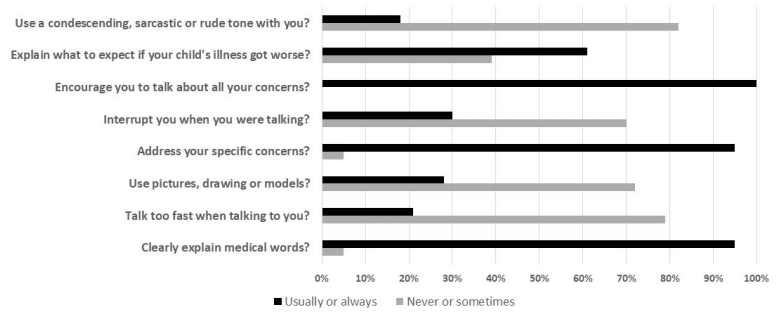

Abstract \#287 Figure 1 
ethnic minority. Overall, parents were satisfied with communication (See figure 1). All parents said that the doctor addressed concerns, but more than $30 \%$ of the respondents felt the doctor did not explain return-to-hospital precautions. $18 \%$ of the respondents endorsed that their doctor spoke to them with some level of condescending, rude, or sarcastic tone, and $30 \%$ of parents said the doctor interrupted them. A third of respondents said that their doctor never used visual aids. Limitations of this study include A) small sample size B) the study does not measure clinical outcomes, and C) as a single-center pilot study, findings may not be generalizable.

Conclusions Although parents were satisfied with communication about their child's care, several areas were identified for improvement, such as decreasing interruptions, increasing anticipatory guidance, and utilizing visual learning methods. Larger studies are underway to address communication gaps in different complex patient populations and measure the impact of interventions on clinical outcomes, such as readmission risk.

\section{\#288 A CALL TO CREATE EVIDENCE-BASED, UPSTREAM PREVENTION PROGRAMS TARGETING YOUTH MENTAL HEALTH THAT ARE EQUITABLE ACROSS ETHNIC/RACIAL SUBGROUPS: ADVOCATES FOR ALL YOUTH (ALLY)}

\footnotetext{
1J Kaar* ${ }^{2} \mathrm{AE}$ Bowen, ${ }^{3} \mathrm{M}$ Pangelinan, ${ }^{3} \mathrm{~A}$ Dadematthews, ${ }^{1} \mathrm{CR}$ Studts, ${ }^{1} \mathrm{~S}$ Simon, ${ }^{4} \mathrm{~L}$ Shomaker. 'University of Colorado Anschutz Medical Campus, Aurora, CO; ${ }^{2}$ Children's Hospital Colorado, Aurora, CO; ${ }^{3}$ Auburn University, Auburn, AL; ${ }^{4}$ Colorado State University, Fort Collins, $\mathrm{CO}$
}

\subsection{6/jim-2022-WRMC.284}

Purpose of Study Youth from disadvantaged racial/ethnic groups have higher rates of anxiety and depression compared to non-Hispanic White youth; however, are less likely to receive mental healthcare. Factors that may underlie these disparities include acculturation pressures and stressors, trauma, peer victimization and microaggressions, and discrimination. With mental health distress exacerbated during and in the wake of the COVID-19 pandemic, there is a need for evidence-based school-based programs that have the potential to reach youth who otherwise may not have access to mental healthcare.

Methods Used ALLY was created based on a review of existing school-based, mental health programs and with input from key stakeholders.

Summary of Results Prevention programs that target the improvement of individual-level protective factors exist, however, tend to not be equitable across ethnic/racial subgroups, especially among low-income Black and Hispanic communities. Schools are viewed as an attractive platform for such programs because they can alter the environment and education of a large number of youths simultaneously. Further, when implemented successfully into the school's structure, have the potential to be integrated and sustained long-term. Despite these advantages, few school-based efforts focused on improving protective factors, such as self-efficacy and resilience, to reduce symptoms of anxiety and depression have been conducted.

Conclusions With continued increases in mental health distress, there is a need for school-based programs that have the potential to reach youth from disadvantaged backgrounds. ALLY is a school-based positive psychology program aimed to increase youth's well-being and reduce symptoms of depression

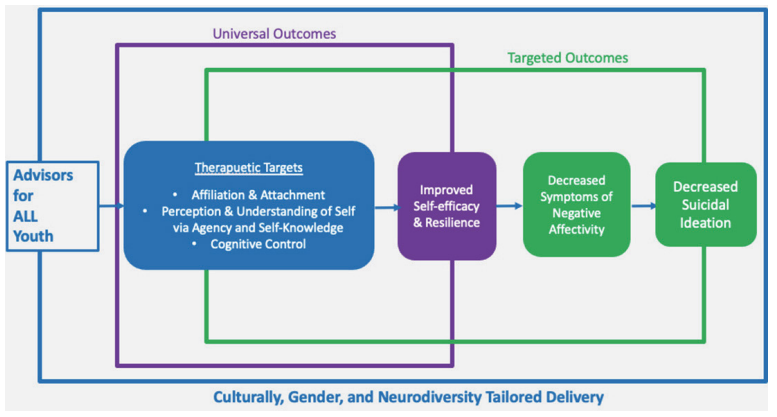

Abstract \#288 Figure 1

and anxiety. Facilitators are selected from the community in which the school is located and receive training in supportive conversations and cultural competency \& humility as well as how to reach youth with varying cultural, gender identity, or neurodiversity differences that may exist. ALLY is examining mental health outcomes among diverse school settings in terms of program participation, efficacy, and implementation. Our findings will inform to what extent the program is efficacious among underrepresented communities.

\section{\#289 SOCIOECONOMIC DIFFERENCES ASSOCIATED WITH CONSUMPTION OF A PLANT-BASED DIET: RESULTS FROM THE NATIONAL HEALTH AND NUTRITION EXAMINATION SURVEY}

M Gonzalgo*, S Nackeeran, A Mouzannar, R Blachman. University of Miami School of Medicine, Miami, FL

\subsection{6/jim-2022-WRMC.285}

Purpose of Study A plant-based diet (PBD) has been associated with potential health benefits, but factors that may affect access to and consumption of a PBD are not well defined. The purpose of this study was to determine the association between plant-based dietary content, racial, ethnic, and socioeconomic status among participants enrolled in the National Health and Nutrition Examination Survey (NHANES).

Methods Used This was a cross-sectional study using data obtained from the NHANES database. The following covariates were assessed: age, sex, race/ethnicity, educational level, marital status, smoking status, physical activity, alcohol use, history of diabetes, and hypertension. Socioeconomic status was categorized according to poverty-income ratio (PIR). Food frequency questionnaires were used to calculate plant-based diet index (PDI) and healthful plant-based diet index (hPDI). Multivariable-adjusted logistic regression was performed to determine the association between PIR, clinical, demographic, and plant-based diet indices.

Summary of Results A total of 5,037 participants were included in the final cohort. Median age of participants was $51 \pm 18.5$ years. Overall PDI and hPDI were 50 (46 - 54) and $52(47-57)$, respectively. Median PDI consumption was significantly different among PIR groups (PDI, $\mathrm{p}=0.018$; hPDI, $\mathrm{p}<0.001)$. On multivariable analysis, participants in the poorest socioeconomic group (PIR $\leq 130 \%$ ) were more likely to have lower consumption of a healthful PBD (hPDI).

Conclusions Lower socioeconomic status (PIR $\leq 130 \%$ ) was associated with decreased consumption of a healthful plantbased diet. These data suggest that socioeconomic disparities 
may limit consumption of healthier food and contribute to the high prevalence of adverse health conditions that exist in certain population groups.

\section{\#290 BILLINGS, MONTANA LGBTQ+ HEALTHCARE ALLIES TRAINING}

M Turner*, T Keys. University of Washington, Seattle, WA

\subsection{6/jim-2022-WRMC.286}

Purpose of Study The aim of the project was to improve LGBTQ+ care in Billings (pop. 125,000 and Montana's largest city) by offering a training to healthcare providers and support staff that educates on how to provide affirming healthcare and reduce barriers to access for the LGBTQ+ population.

Methods Used Bridgercare is a family planning clinic located in Bozeman that offers educational workshops such as the LGBTQ+ Healthcare Allies Training. The 3-hour training educates healthcare providers and support staff and seeks to provide a safe environment for participants to engage with facilitators and a 20-page booklet to follow along with. The evidenced-based booklets are kept by participants and encouraged to be shared after training. The training is led by three facilitators, who either: have experience providing affirming care in a medical setting, belong to an LGBTQ+ community. and/or are allies of LGBTQ+ communities. Bridgercare offered to come to Billings, provide a training, and partner to establish a long-term education class for the Billings area. The training was held at a local museum. Recruitment for attending the training was done through word of mouth and social media. Post-training surveys were given following training.

Summary of Results 30 healthcare professionals attended the first training. Individuals came from 9 different organizations. The training consisted of administration leadership, physicians, PAs, NPs, RDs, nurses, counselors, professors, pharmacists, and care managers. Surveys indicated that 100 percent of individuals felt the training was highly beneficial and learned something. 7 people who were at the training signed up to receive info on how to become a facilitator.

Conclusions Given the positive feedback, next steps will be to partner with another community partner and to train 10 facilitators by December 2021. Trainings with facilitators from Billings will start January 2022. During Spring 2022, training will start to be assessed for how training is impacting LGBTQ + care. Additional metrics to see whether training is an effective intervention may include measuring: the amount of healthcare workers with formal LGBTQ+ education, healthcare worker confidence in treating LGBTQ+ patients, and the amount of healthcare workers who believe their clinic or department adequately addresses the needs of their LGBTQ+ patients.

\section{\#291 PRESENTATION OF ERYTHEMA IN ABSCESSES FOUND IN SKIN OF COLOR}

${ }^{1} \mathrm{~S}$ Bodapati ${ }^{*},{ }^{2} \mathrm{C}$ Wong, ${ }^{3} \mathrm{~S}$ Weis. ${ }^{1}$ Western University of Health Sciences, Pomona, CA; ${ }^{2}$ University of North Texas Health Science Center, Fort Worth, TX; ${ }^{3}$ Medical City Fort Worth, Fort Worth, TX

10.1136/jim-2022-WRMC.287
Purpose of Study Erythema, one of the cardinal signs of inflammation, is one of the hallmark descriptors of a skin abscess. In individuals with lighter skin, such as Caucasians, erythema presents markedly differently from unaffected skin tissue. In individuals with darker skin tone, abscesses may not be as strikingly apparent when discerned based on appearance of erythema alone. The masking of erythema by darker skin tones is a result of differences in skin biology and the overall composition of skin. It highlights the need for dermatologists and medical professionals to rely on other signs of inflammation when diagnosing skin abscesses.

Methods Used Two cases of patients with skin abscesses were selected from University of North Texas Health Science Center and John Peter Smith Hospital. One male and one female case was selected. Photographs were captured in clinic and image analysis of erythema was performed visually.

Summary of Results Variance in the degree of skin pigmentation results from differences in skin composition, and this changes the appearance of erythema. Differences include denser stratum corneum, a greater ratio of eumelanin to pheomelanin, and more oval-shaped melanocytes in darker skinned individuals. Erythema is a result of pathologic increase in inflammation and red blood cells. Hemoglobin is the primary chromophore in red blood cells, and reflects visible red light, causing the typical red appearance. However, as demonstrated in these presentations, appreciating erythema visually as redness is unreliable in darker skinned individuals, because the red hue of oxyhemoglobin often presents as violaceous, brown, or black. While erythema is commonly one of the first described features of an abscess, relying on indicators such as swelling may prevent delays in diagnosis, particularly in people of color.

Conclusions More documentation of the presentation of inflammatory conditions in individuals with skin of color will help build knowledge in diagnosis of dermatologic conditions. Focusing on color-independent markers of inflammation may provide a more equitable approach to patient care.

\section{\#292 DISPARITIES IN USE OF SYRINGE SERVICES PROGRAMS IN KING COUNTY, WA}

${ }^{1} \mathrm{KR}$ Salow*, ${ }^{2} \mathrm{~S}$ Glick. 'University of Washington School of Medicine, Seattle, WA; ${ }^{2}$ University of Washington Department of Medicine, Seattle, WA

\subsection{6/jim-2022-WRMC.288}

Purpose of Study Syringe service programs (SSPs) are an effective harm reduction resource for people who use drugs (PWUD). Recently published cross-sectional studies found that SSPs in two East Coast cities (Miami and New York) were not reaching Black communities. Additionally, qualitative literature showed that women who use drugs experience greater stigma than men and they may feel less inclined to seek harm reduction services. No papers have been published regarding disparities among SSPs in West coast cities, which have different drug use trends and demographics. We used data from the Seattle area to identify disparities in SSP use, and hypothesized that Black PWUD, woman, and gender minorities would be underrepresented in a large Seattle SSP.

Methods Used We compared data from two cross-sectional surveys. One survey was conducted in 2019 at three sites that are part of Public Health - Seattle \& King County's SSP, and evaluated the demographics of 432 SSP clients. The 
comparison survey, the 2018 National HIV Behavioral Surveillance Injection Drug Use (NHBS-IDU) Survey, utilized respondent-driven sampling to create a representative sample of people who inject drugs (PWID) in the region; 555 PWID in the Seattle area completed this survey.

The primary variables of interest were race and gender. Exploratory covariates included age, men who have sex with men (MSM), and housing status. We used chi-squared tests to evaluate differences between people using the SSP and PWID in the Seattle area.

Summary of Results The majority of SSP clients identified as white, which was similar to the demographics of the NHBSIDU survey participants $(p=0.086)$. American Indian/Alaska Native and Black PWUD were underrepresented in the SSP client survey $(p<0.001$ for both). Latinx/Hispanic and Native Hawaiian/Pacific islander PWUD were also underrepresented

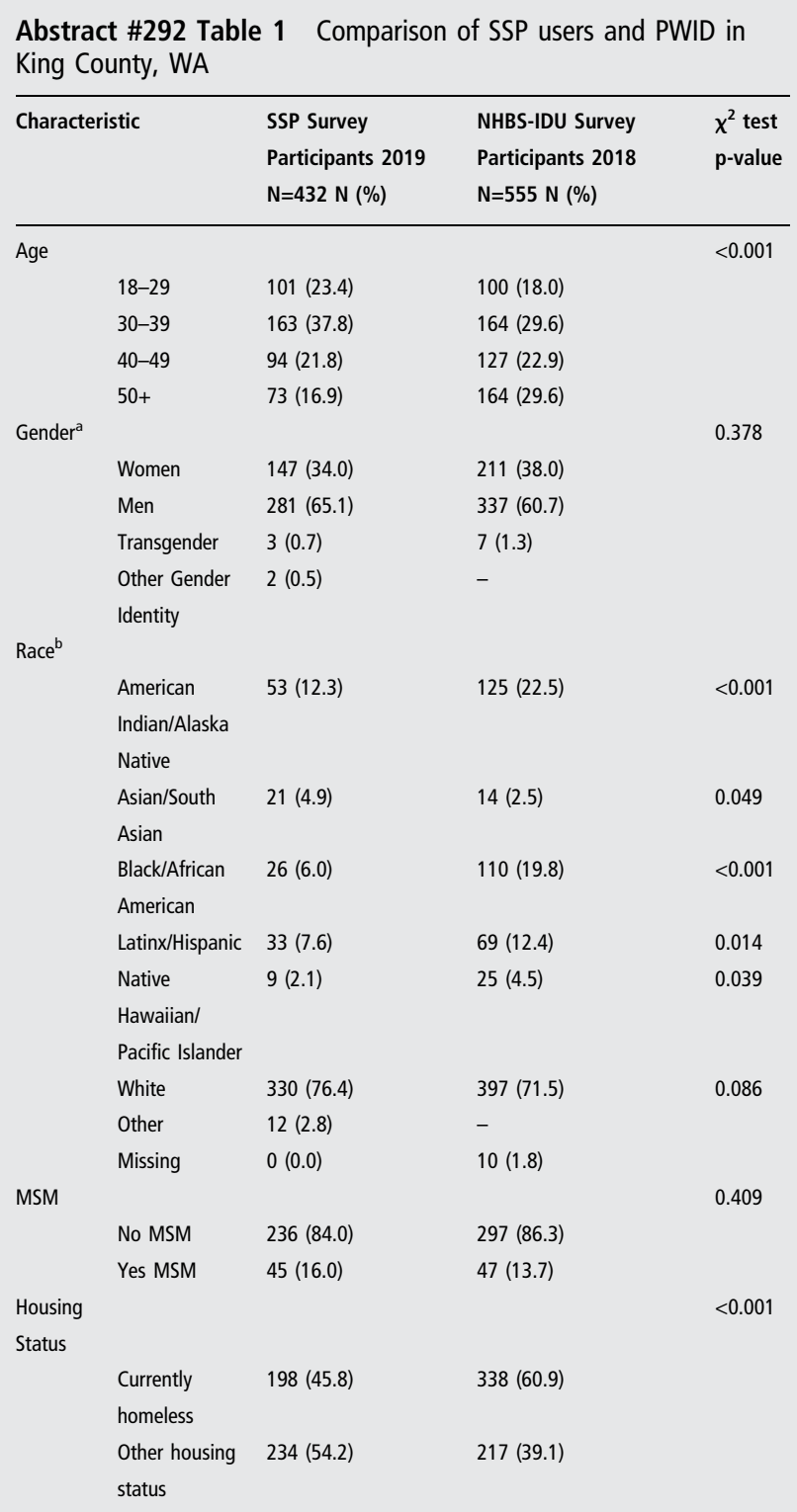

a) SSP participants could select more than one gender, while NHBS-IDU participants could not. b) Both SSP and NHBS-IDU participants could select more than one race. $(\mathrm{p}=0.014$ and $\mathrm{p}=0.039$ respectively). Approximately, twothirds of SSP clients were men and one-third were women, with $<1 \%$ of clients reporting another gender identity and $<1 \%$ reporting being transgender. The gender of SSP clients largely reflects the same distribution seen in a community sample of PWID $(p=0.378)$. Participants in the SSP survey tended to be younger and less likely to be experiencing homelessness than the NHBS-IDU participants $(p<0.001$ for both). The distribution of MSM participants in the two surveys were similar $(\mathrm{p}=0.409)$.

Conclusions This study provides further evidence that Black PWUD are underrepresented in SSPs, consistent with studies in other large US cities. Both nationally and in Seattle, overdose deaths have been increasing among Black PWUD, and harm reduction strategies are vital to reversing this trend. SSPs should explore and test ways to be more accessible to underrepresented populations.

\section{\#293 CHARACTERIZATION OF LEGAL ISSUES IN A NEONATAL INTENSIVE CARE UNIT}

B Haynes*, GM Morrison, L Mutrie, L Gievers. Oregon Health and Science University, Portland, $O R$

\subsection{6/jim-2022-WRMC.289}

Purpose of Study There is a rising standard in pediatric care to screen for social and legal determinants of health (SDH), and to have mechanisms in place to address them. Medicallegal partnerships (MLPs) are innovative collaborations between health and legal organizations that address SDH. Through family-centered legal services, education, training and systemic advocacy, the MLP movement has impacted health outcomes, family well-being, institutional stability and policy reform. Doernbecher Children's Hospital (DCH) introduced the first MLP in the nation to be housed within a neonatal intensive care unit (NICU). The purpose of this study is to characterize the legal needs of families in the NICU.

Methods Used The MLP launched in October 2020 within the NICU at a quaternary, academic medical center located in Portland, Oregon. Sociodemographic, clinical and legal information has been obtained for all MLP-assisted families (including race, ethnicity, county of residence, insurance type, primary language, gestational age at birth, birth weight, reason for admission, length of stay and health outcomes such as number of ED visits and readmissions). Health-harming legal issues were identified by a universally-provided screening tool and with routine social assessments. Descriptive statistics were utilized.

Summary of Results Thirty-one families have received MLP services. Most families identified as non-Hispanic (61.3\%) and Caucasian (83.9\%). English was the most common primary language $(77.4 \%)$. The average gestational age at birth was $32.4 \pm 5.1$ weeks. Long term outcomes are still being collected given limited time since discharge. Fifteen cases (48.4\%) involved family law; custody, child or spousal support, divorce, or paternity. Other legal needs included insurance and disability claims (3), housing instability (2), estate planning (2), employer issues (1), complications of a criminal 
history (1) and complex maternal history (1). Six families were lost to follow up.

Conclusions MLPs are a necessary and important tool to address health-harming legal issues and protect the underserved. There are a wide variety of legal needs within the NICU setting, with family law issues being the most prevalent. By continuing to characterize the legal needs of NICU families, we hope to detect trends in patient needs and develop legislative policy solutions for this unique and deserving community.

\section{\#294 RISK FACTORS FOR MORBIDITY AND MORTALITY IN VERY LOW BIRTH WEIGHT INFANTS FROM RURAL VERSUS URBAN COMMUNITIES}

'DS Ondusko*, ${ }^{2} \mathrm{~J}$ Liu, 'B Hatch, ${ }^{2} \mathrm{~J}$ Profit, ${ }^{1} \mathrm{EH}$ Carter. ${ }^{1} \mathrm{OHSU}$, Oregon Health and Science University, Portland, OR, US, academic/medsch, Portland, OR; ${ }^{2}$ Stanford University, Stanford, $C A$

\subsection{6/jim-2022-WRMC.290}

Purpose of Study People living in rural and frontier areas experience higher morbidity and mortality in part due to limited access to health care. Whether rurality affects care and outcomes for very low birthweight (VLBW) infants is not well understood. This study compares prenatal factors, care patterns, and health outcomes between VLBW infants born to mothers living in rural versus urban zip codes in California.

Methods Used We used data from the California Perinatal Quality Care Collaborative (CPQCC) registry to conduct a population-based retrospective cohort study of VLBW infants born between 2011 and 2018. This descriptive analysis examined associations between the degree of maternal residential rurality and maternal, infant, hospital, and community/county level characteristics based on Rural Urban Commuting Area (RUCA) codes. The associations between all factors and maternal RUCA codes were stratified and compared using Chisquared and ANOVA tests.

Summary of Results Our study included 38,614 VLBW infants. Of those, 36,936 were born to mothers residing in an urban area, 1,113 in a large rural area, and 561 within a small rural or isolated area. Mothers of VLBW infants who live in large rural and small rural/isolated areas were more likely to be younger, publicly insured, have education limited to high school, smoke during pregnancy, and have delayed or no prenatal care.

Infants born to mothers living in large rural and small rural/isolated areas were more likely to have their first hospital within the CPQCC network as a level 4 NICU with greater than 110 annual VLBW admissions but with less than 3500 annual deliveries compared to urban infants.

Birth weight, gestational age, and infant sex were similar across geographic groups. Survival without major morbidities decreased across the rural-urban continuum, with urban maternal residence having the highest percentage of VLBW infants surviving without major morbidities.

Conclusions VLBW infant survival without major morbidity decreased with increasing rurality. This descriptive study highlights various risk and protective factors which may modulate this association, and which suggest the possibility for health policy intervention.
Healthcare delivery research II

\section{Concurrent session}

\author{
10:15 AM
}

Friday, January 21, 2022

\section{\#295 SURVEYS OF CALIFORNIA FAMILY PHYSICIANS AND LOS ANGELES COUNTY PHARMACISTS: PILL PRESCRIBING AND DISPENSING PRACTICES}

I Gedestad*, A Matlack, M Munnangi, A Nelson. Western University of Health Sciences, Pomona, $C A$

\subsection{6/jim-2022-WRMC.291}

Purpose of Study The California Family PACT insurance program provides family planning and reproductive health services at no cost to half a million women ages 15-44 who fall below 200\% of the Federal Poverty Line and who are at risk for unintended pregnancy. As shown in previous studies, women who received 13 packs (a 1 year supply) of oral contraceptives were less likely to become pregnant, conceive an unplanned pregnancy, or receive an abortion when compared to women who received one or three cycles of pills. The purpose of this study was to determine whether women insured by the Family PACT program are able to receive 13 packs of oral contraceptive pills at once from their pharmacies as now required by $\mathrm{CA}$ law.

Methods Used After we obtained a list of all pharmacies in each LA County zip code using Yellow Pages, we organized them by the 8 LA County Service Planning Areas (SPAs). We chose how many pharmacies to contact in each SPA based on the percentage of women living in each SPA. We contacted a proportional number of national and local pharmacies in each SPA based on the distribution of pharmacies in that area. A standardized secret shopper script was used by all researchers when calling pharmacies. We recorded if the pharmacy agreed to dispense 13 packs and if not, how many packs they would dispense and why, among other parameters. Pharmacies were classified as unable to determine if they stated they would need us to come into the pharmacy to determine prescription and insurance eligibility.

Summary of Results In total, we attempted to contact 582 pharmacies. We successfully contacted 432 pharmacies as the remaining 150 pharmacies $(25.3 \%)$ did not answer or had a disconnected number (i.e. were unable to contact). Of the 432 pharmacies that were contacted, only $3.2 \%$ of pharmacies stated 'yes' that they could dispense 13 packs at one time. $47 \%$ stated 'no' they could not dispense 13 packs at one time and $49.7 \%$ did not directly answer yes or no and were classified as 'unable to determine.' Many pharmacies stated Family PACT 'does not work that way' or 'will only cover a 3 month supply so I don't think you can get a year's supply anywhere.' One pharmacy staff member stated 'I've been doing this for 10 years and no insurance covers that amount.' Conclusions While the regulations requiring pharmacies to dispense a one-year supply of oral contraceptive pills to Family PACT patients who have an appropriate prescription was introduced in January 2017, the lack of knowledge of this insurance program and its requirements by pharmacies in this 
study is evident. With only $3.2 \%$ of pharmacies stating they would dispense 13 packs, women on Family PACT insurance face a substantial barrier to accessing the legally allowed amount of oral contraceptives and therefore are at a higher risk for unintended pregnancies.

\section{\#296 CHARACTERIZING OPIOID USER TYPES AND IDENTIFYING PRESCRIBING FACTORS CONTRIBUTING TO LONG TERM USE}

${ }^{1} \mathrm{R} X \mathrm{U}^{*},{ }^{2} \mathrm{~J}$ Bone, ${ }^{2} \mathrm{R}$ Courtemanche, ${ }^{3} \mathrm{~L}$ Yefet, ${ }^{2} \mathrm{M}$ Bucevska, ${ }^{2} \mathrm{M}$ Simmonds, ${ }^{2} \mathrm{E}$ Cattoni, ${ }^{2} \mathrm{G}$ Lauder, ${ }^{2} \mathrm{D}$ Courtemanche. ${ }^{1}$ The University of British Columbia Faculty of Medicine, Vancouver, BC, Canada; ${ }^{2} B C$ Children's Hospital, Vancouver, BC, Canada; ${ }^{3}$ University of Toronto Temerty Faculty of Medicine, Toronto, ON, Canada

\subsection{6/jim-2022-WRMC.292}

Purpose of Study Prescription opioid use is associated with subsequent risk of opioid use disorder and opioid-related death. Variability of prescription practice may further escalate this risk. This is paramount in British Columbia (BC); BC had the highest 2020 Canadian provincial opioid overdose related deaths per capita. Our recent study described BC outpatient opioid prescription patterns (2013-2017). Opioid user types (single, short, intermediate, and long-term) are characterized here with the same dataset to identify prescription factors that increase long-term use.

Methods Used Anonymized data of all BC outpatient opioid prescriptions (2013-2017): patient age and sex, prescriber type, generic drug, dose, and total days of opioid supply, was used. Incident episodes that began within the study timeline were included; those on opioid agonist therapy were excluded. Opioid episode durations: time between first and last day of prescription with none in the following 180 days. Opioid user types: single (one prescription); short-term (<90-day); intermediate ( $\geq 90$-day: $<10$ prescriptions \& $<120$-day supply); and long-term ( $>90$-day: $\geq 10$ prescriptions over $>90$ days or $\geq 120$-day supply).

Opioid user types were described by episode number, median total day supply, median total MME (morphine milligram equivalents) prescribed, first prescribing specialist and first opioid prescribed.

Incidence/prevalence of user types, and prescription trends were described. Hazard ratios for prescription factors associated with long-term use were estimated with cox regression models.

Summary of Results 1,920,073 incident episodes $(8,635,831$ prescriptions) over the 5 years were classified as single (70\%), short (17\%), intermediate (9\%), and long-term (4\%). Median episode length was 5, 13, 15, and 202 days, respectively. Median total MME prescribed was 4.5, 11.3, 13.5, and 79 MME, respectively. General practitioners were the first prescribers for $>50 \%$ of episodes across all user types apart from single (45\%). First opioid prescribed across all user types was codeine (50\%-70\%), tramadol/tapentadol (19\%), and hydromorphone $(5 \%-15 \%)$.

Incidence of long-term users decreased over the 5 years (664 to 537 per 100,000 ) while prevalence remained similar (3000 per 100,000). Factors associated with longer episodes included initial prescription of oxycodone (HR 0.82) or hydromorphone (HR 0.85) relative to codeine; first prescriber speciality: psychiatry (HR 0.79) or diagnostic services (0.89) relative to general practice; and increasing age (HR 0.97 by 5 -year increments).
Conclusions This population-level datal provides a better understanding of the role of prescriber types/prescriptions and factors that contribute to increased likelihood of long-term use.

\section{\#297 BARRIERS TO ACCESSING HEALTHCARE BY THE FEMALE HOMELESS POPULATION: A SURVEY OF SERVICE PROVIDERS}

A Akcam*, AL Nelson, M Fraix. Western University of Health Sciences College of Osteopathic Medicine of the Pacific, Pomona, CA

10.1136/jim-2022-WRMC.293

Purpose of Study This study surveyed service providers to assess perceived barriers that the growing population of homeless, reproductive-age women face accessing health care from the view of those who work with them in San Diego County.

Methods Used Between October 2020 and February 2021, 25 organizations working with unsheltered women were sent links to an anonymous, voluntary survey to distribute to Englishspeaking employees over age 18. It assessed perceived barriers; subgroup analysis was done by participant role in the organization. IRB approval was obtained.

Summary of Results Eighteen of the contacted agencies forwarded the survey to staff; 49 members participated; 38 completed it entirely. Participating organizations included clinics (70.2\%), housing programs (15\%), and outreach and/or school programs $(11 \%)$. Of respondents, $42 \%$ are nurses, $17 \%$ are clinicians, $15 \%$ were members of the management team, and $8.5 \%$ were nursing students.

The top 'major barriers' endorsed by service providers to homeless women accessing care were lack of transportation $(73.8 \%)$, cost of care $(71.4 \%)$, and unfamiliarity with resources $(64.3 \%)$.

Regarding menstrual care, 38 participants reported that women used pads (32.5\%), tampons (28\%) and other products (tee shirts, socks, etc.) (25\%); 60\% provided menstrual supplies. Cost/unfamiliarity with places for low-cost/free products $(60 \%)$, lack of privacy for use $(37.5 \%)$, and lack of storage $(35 \%)$ were endorsed as the top 'major barriers' to care by service providers.

43 participants estimated the number of clients who were sexually active; $21 \%$ estimated $25-50 \% ; 40 \%$ estimated 50 $75 \%$, and $18.4 \%$ estimated $\geq 75 \%$. Nearly $2 / 3$ 's said their organization provided some contraceptive supplies to homeless women; $41 \%$ provided barrier methods, $18 \%$ provided both injectable and oral birth control. Major barriers included cost $(76.3 \%)$ and unfamiliarity with free contraception sites (50\%). Conclusions Unsheltered women face numerous financial and knowledge barriers to care. Based on our results, the best options to increase access to care for homeless women include decreasing costs, and educating women on locations of available products and services. Additionally, organizations like mobile clinics may benefit women more as they eliminate the transportation barrier.

\section{\#298 BARRIERS AND FACILITATORS TO PRIMARY CARE ACCESS FOR REFUGEES IN SPOKANE, WASHINGTON}

K Glover* ${ }^{*}$ A White, AM Cole. University of Washington School of Medicine, Seattle, WA

10.1136/jim-2022-WRMC.294 
Purpose of Study The United States has resettled over three million refugees and Spokane, WA is home to a community of over 10,000 refugees. There is a paucity of research on barriers and facilitators for refugee primary care access (PCA). This study sought to identify barriers and facilitators to PCA amongst resettled refugees in Spokane, WA.

Methods Used This study emphasizes community participation and was developed with local healthcare and refugee assistance organizations (CBO's). Snowball sampling generated key informants from initial referrals from CBOs. Eligible participants were: 1) refugees resettled in Spokane through a formal resettlement agency; 2) healthcare workers who care for refugees; 3) resettled refugees who now provide refugee services. A semi-structured interview guide was developed for each group assessing known barriers and facilitators to PCA, as well as questions developed collaboratively with CBO's to explore refugees' experience of the COVID-19 pandemic. Thematic analysis was performed on the transcribed interview text using an established coding schema of supply- and demandside barriers and facilitators. Demand-side barriers are defined as individual, household or community characteristics that influence the demand for services, and supply-side as "characteristics of the health system that exist beyond the control of potential health service users, such as health facilities, drugs, equipment, finances, human resources, geographic distance, etc.

Summary of Results We interview 24 individuals including refugees $(\mathrm{N}=9)$, care providers $(\mathrm{N}=9)$, and refugees who have become providers $(\mathrm{N}=6)$. Thematic analysis (ongoing at this time) revealed extant supply-side barriers including the complexity of the US healthcare system and inadequate interpretation. Novel supply-side barriers specific to the COVID-19 pandemic emerged such as loss of in-person interpretation services, as well as demand-side barriers including the spread of COVID misinformation. Novel supply-side facilitators include use of communication technologies to share health information. This is one of the first studies of barriers and facilitators to refugee PCA to directly engage refugees resettled on the West Coast. It is also the first such study done in Eastern Washington.

Conclusions These data contribute to the literature on refugee healthcare access in the US in general, as well as in Spokane, WA. This is among the first studies of barriers and facilitators to refugee PCA to assess the impact of the COVID-19 pandemic. These findings offer evidence for CBO's and local and state governments to implement policies that remove barriers and leverage facilitators to refugee PCA.

\section{\#299 AN ANALYSIS OF STREET MEDICINE CHIEF COMPLAINTS AND TREATMENTS FOR THE HOMELESS POPULATION OF SPOKANE, WASHINGTON}

AS Hoppe*, T Kagele, B Hall, BD Messner, A Nichols. Washington State University, Spokane, WA

\subsection{6/jim-2022-WRMC.295}

Purpose of Study The purpose of this study is to evaluate the chief complaints treated by the Spokane Street Medicine team for purposes of resource requirements and allocation.

Methods Used In this analysis, a retrospective chart review of the RANGE Spokane Street Medicine Team was completed on encounters seen between January 2021 and March 2021. To

\begin{tabular}{|c|c|c|}
\hline \multicolumn{3}{|c|}{$\begin{array}{l}\text { TOP } 10 \text { CHIEF COMPLAINTS OF } \\
\text { PERSONS EXPERIENCING HOMELESSNESS IN } \\
\text { SPOKANE, WA BETWEEN JANUARY } 2021 \text { AND } \\
\text { MARCH } 2021\end{array}$} \\
\hline Chief Complaint & Percent & Total $(n=359)$ \\
\hline Wound & $38.2 \%$ & 137 \\
\hline Medication-related & $20.3 \%$ & 73 \\
\hline Pain & $18.4 \%$ & 66 \\
\hline Follow-up & $14.5 \%$ & 52 \\
\hline Cardiovascular & $12.8 \%$ & 46 \\
\hline Infectious & $11.7 \%$ & 42 \\
\hline Respiratory & $10.9 \%$ & 39 \\
\hline Other & $9.7 \%$ & 35 \\
\hline Trauma & $8.4 \%$ & 30 \\
\hline Mental Health & $8.1 \%$ & 29 \\
\hline
\end{tabular}

begin this process, 359 paper charts were transcribed into a Qualtrics format for analysis. Categories included chief complaints, type of chief complaint (acute or chronic), treatment received, and demographic information. These categories were confirmed in meetings with RANGE Street Medicine team members. A qualtrics analysis of basic chief complaint and treatment frequencies was then performed. This is an ongoing analysis.

Summary of Results Primary analysis revealed that the majority of street medicine chief complaints were acute conditions. Of these, the top 5 complaints were Wounds (38.2\%), Medication-Related (20.3\%), Pain (18.4\%), Follow-up (14.5\%), and Cardiovascular (12.8\%) (see table 1.1). Mental health was the 10th most common complaint. In terms of treatment, followups were scheduled with local organizations for $\square$ of patients (33.93\%). Medications were prescribed to 122 patients (39.97\%). No resuscitations were performed or Narcan used.

Conclusions Wound care, medication-related complaints, and pain accounted for the majority of complaints from the Spokane, WA Street Medicine team. Follow-up proved to be another commonly addressed problem, suggesting the difficulties of continuity of care within the homeless population. As such, team resources should be directed towards wound care equipment and assisting with health coordination within the Spokane community. While there is still need for other types of acute care, the team may be best served by pursuing clinic and pharmacy connections for efficient treatment and followup.

\section{\#300 TEXT MESSAGING INTERVENTION TO PROMOTE CARDIAC HEALTH AND COMBAT LONELINESS DURING SOCIAL DISTANCING}

${ }^{1} \mathrm{~K}$ Uehisa*${ }^{2,3} \mathrm{~K}$ Watson, ${ }^{2,3} \mathrm{~T}$ Horwich, ${ }^{2,3} \mathrm{M}$ Calfon-Press. ${ }^{1}$ University of California Los Angeles, Los Angeles, CA; ${ }^{2}$ Ronald Reagan UCLA Medical Center, Los Angeles, CA; ${ }^{3}$ University of California Los Angeles David Geffen School of Medicine, Los Angeles, CA

\subsection{6/jim-2022-WRMC.296}

Purpose of Study The COVID-19 pandemic, with its physical distancing, can lead to loneliness, isolation, and unhealthy behaviors. We aimed to develop an intervention that could address these known cardiovascular risks and hypothesized 
that a health text-based message intervention (HTMI) could mitigate unhealthy behaviors and increase healthy behaviors.

Methods Used We prospectively recruited 101 adults in Southern California. After informed consent, participants received HTMIs for 31 consecutive days. Self-reported health behaviors were assessed by the Health Promoting Lifestyle Profile (HPLP). Subjective loneliness was measured by the UCLA Loneliness Scale 3 (LL), and isolation was measured objectively by the Social Network Index (SNI). Surveys were analyzed via paired $\mathrm{T}$ test and regression analysis. HPLP and LL were scored on a 4-point scale per question.

Summary of Results Participants' median HPLP score increased significantly 2.67 to 2.82 signaling increased health behaviors, and the median LL score decreased signaling decreased loneliness 2.08 or 2.01. SNI showed no significant change in social contacts. $75 \%$ of participants reported increased knowledge of heart health and $81 \%$ reported increased health awareness.

Conclusions HTMIs demonstrated rapid and efficient delivery to a population affected by the COVID-19 pandemic. HTMIs were associated with increased health behaviors and decreased self-reported loneliness.

\section{\#301 RELATIONSHIP BETWEEN PHYSICAL ACTIVITY VITAL SIGN AND HEALTHCARE SYSTEM UTILIZATION}

${ }^{1,2} \mathrm{C}$ Lin, ${ }^{3} \mathrm{~T}$ Ball, ${ }^{4} \mathrm{VF}$ McDonald*, ${ }^{2} \mathrm{~N}$ Gentile, ${ }^{2} \mathrm{~A}$ Humbert. ${ }^{1}$ UW Medicine, Seattle, WA; ${ }^{2}$ University of Washington Medical Center, Seattle, WA; ${ }^{3}$ Northwell Health, New Hyde Park, NY; ${ }^{4}$ University of Washington School of Medicine, Seattle, WA

\subsection{6/jim-2022-WRMC.297}

Purpose of Study Insufficient physical activity (PA) is a leading risk factor for major chronic diseases and all-cause mortality. Engaging in regular PA is associated with improved health, lower risks of chronic disease, and lower all-cause mortality. Insufficient PA has been associated with substantial costs to healthcare systems globally, although reports on these relationships are few, and the association between insufficient PA and healthcare utilization is less known. This study examined the association between self-reported PA with healthcare system utilization for adults in a single academic healthcare system in the United States.

Methods Used The Physical Activity Vital Sign (PAVS) is a standardized and validated tool for collecting self-reported PA from patients into electronic health records. PAVS data from 23,914 patients were collected at outpatient visits between 2018-2020. Association between 6 months of healthcare utilization and the concurrent and prior 6 months (12 months total) of PAVS was evaluated every 6 months for each patient. Generalized estimating equations were used to assess relationships between PAVS and hospital inpatient admissions, emergency department visits, urgent care visits, and primary care outpatient visits respectively.

Summary of Results Sufficient aerobic PA per public health guidelines of $\geq 150 \mathrm{mins} / \mathrm{wk}$ was associated with fewer hospital admissions $(p<0.001)$, emergency department visits $(\mathrm{p}<0.001)$, and primary care visits $(\mathrm{p}<0.001)$ after adjusting for age, sex, body mass index (BMI), and race. PAVS did not show a statistically significant relationship with urgent care visits. Older patients had a larger association between PAVS and fewer hospital admissions and primary care visits $(p=0.002)$. Sex, BMI, and race were not found to modify the association between PAVS and healthcare utilization.
Conclusions We found that higher PAVS is associated with fewer hospital admissions, emergency department visits, and primary care visits. In the public health setting, these findings suggest that PA may be an important modifiable risk factor for health outcomes and healthcare system utilization. Clinically, the results highlight the importance of medical providers assessing PAVS routinely with patients. Implementation of PAVS in the outpatient setting can help identify patients who need support to become more active for their health. Methods and challenges of implementing PAVS as well as increasing PA should be explored. Finally, further research is needed to understand the potential contribution of increasing PA to reductions in healthcare system costs.

\section{\#302 THE IMPACT OF DIRECT-TO-HOME TELEMEDICINE VISITS ON PARENT, PHYSICIAN, AND THERAPIST EXPERIENCE FOR CHILDREN WITH SPECIAL HEALTHCARE NEEDS}

RD Burns* , L Davidson, S Garrison, A Favila, R Dizon, JP Marcin, SC Haynes. University of California Davis School of Medicine, Sacramento, CA

10.1136/jim-2022-WRMC.298

Purpose of Study Pediatric physiatrists provide necessary specialty care to children with physical disabilities. However, pediatric physiatrists are scarce and are concentrated at academic medical centers. To expand access to care for children with special healthcare needs, UC Davis created the SchoolBased Tele-Physiatry Assistance of Rehabilitative and Therapeutic Services (STARS) Program, a novel telemedicine program that connects pediatric physiatrists to patients receiving in-person care from a therapist at a local medical therapy unit (MTU). As a result of the COVID-19 pandemic, this model of care was disrupted, as MTUs moved to direct-to-home video visits with patients. The purpose of this qualitative study was to examine the experience of parents, therapists, and physiatrists on direct-to-home tele-physiatry visits with the goal of informing future use of telemedicine for children with special healthcare needs.

Methods Used Parents were recruited from a list of randomly generated parents of patients who had participated in the STARS program. All therapists and physicians providing care at the participating MTUs were invited to participate. We used a semi-structured guide based on the domains of the Practical Robust Implementation Sustainability Model (PRISM). Following principles of qualitative content analysis, two researchers separately coded each transcript for themes and subthemes using line by line coding in the Dedoose software program. Themes and sub-themes were developed using the constant comparative method and were refined by the larger study team.

Summary of Results Six focus groups (4 parent groups, 1 therapist group, and 1 physician group) were conducted before thematic saturation was reached. Direct-to-home telemedicine visits were more effective when physicians had previously established a therapeutic alliance with patients. Although in-person visits were perceived as higher quality, physicians, therapists, and parents identified aspects of care that improved over telemedicine, including using tools within the patient's home for therapy, understanding the child's environment, and practicing daily tasks. Parents saw direct-to-home video visits as a good option when their child's condition is static, and 
when travel isn't possible due to weather, childcare or work. Effective direct-to-home telemedicine visits are possible when parents are engaged and capable of positioning the child. Using pre-made cards or a doll to show positions to parents helped to improve the quality of visits.

Conclusions Despite inherent limitations, direct-to-home telemedicine visits could benefit children when used appropriately in conjunction with in-person visits. Our findings suggest that there are specific practices that facilitate effective direct-tohome telemedicine visits.

\section{\#303 TELEMEDICINE AS A PREDICTOR OF POSTPARTUM FOLLOW-UP}

${ }^{1} \mathrm{C}$ Archuleta*, ${ }^{2} \mathrm{D}$ Coonrod, ${ }^{2} \mathrm{M}$ Sutton. ${ }^{1}$ The University of Arizona College of Medicine Phoenix, Phoenix, AZ; ${ }^{2}$ Valleywise Health Medical Center, Phoenix, AZ

\subsection{6/jim-2022-WRMC.299}

Purpose of Study This study was part of an ongoing effort at our institution to identify characteristics in our patient population associated with long term (defined as visit between 4-12 weeks) postpartum visit (PPV) attendance and specifically examines whether initiation of telemedicine during the COVID-19 pandemic was a predictor of PPV compliance.

Methods Used A retrospective chart review was performed of patients who delivered between April 10, 2019 - August 31, 2019 and during the same time period in 2020. The main outcome of interest was percentage of deliveries with PPV follow-up in 2020 compared to 2019. Demographics including age, race/ethnicity, parity, total prenatal visits, delivery route, gestational age, birthweight, and length of newborn hospital stay were also reviewed. Statistical analysis was performed using two sample T-test or Mann-Whitney/Wilcoxon Two-Sample Test, or Chi-Squared test as appropriate. Logistic regression was used to identify independent predictors of PPV.

Summary of Results In total, 587 patients were identified in 2019 and 552 in 2020. 13 duplicate charts were removed for a total of 1126 charts in the final analysis. The 2 cohorts were determined to not be significantly different except in terms of parity which was higher in 2019. In 2020, 79\% of patients were compliant with PPV, in 2019 71\% were compliant. This difference was determined to be statistically significant with p-value $=0.001$. 2019 PPV were conducted inoffice only while 2020 PPV were offered as telemedicine or in-person. Approximately 53\% of visits in 2020 were telemedicine.

Other variables predictive of postpartum follow-up included higher age, increased parity, greater total prenatal visits. Cesarean delivery was positively associated with follow-up but it was not significant. Gestational age, race/ethnicity, birthweight, and newborn length of stay were not identified as predictive of follow-up. In the logistic regression delivery year, age, prenatal visits, and parity remained significant and independent predictors of follow-up.

Conclusions This study reviewed 2 cohorts of patients during time periods before and after initiation of telemedicine postpartum visits during the COVID-19 pandemic. Previous research at our institution indicated that telemedicine followup was non-inferior to office follow-up for short term visits in patients with hypertensive disorders. This study expands upon this work by considering long term follow-up in all postpartum patients. Additionally, while demographic characteristics predictive of postpartum attendance have been considered elsewhere in the literature there are fewer studies examining access to telemedicine as a variable. These findings suggest telemedicine may be effective for increasing compliance with postpartum visits.

\section{Hematology and oncology II}

\section{Concurrent session}

\section{0:15 AM}

\section{Friday, January 21, 2022}

\section{\#304 COMPREHENSIVE CARE MODEL FOR SICKLE CELL DISEASE CARE AT KERN MEDICAL}

R Sharma*, L Moosavi, E Cobos. Kern Medical Center, Bakersfield, CA

\subsection{6/jim-2022-WRMC.300}

Purpose of Study Sickle cell disease is the most common genetic hemoglobinopathy in the US. It affects over 100,000 individuals. Sickle cell disease is a blood disorder causing a mutation in the hemoglobin beta chain. It is characterized by acute pain episodes, emergency department visits, hospitalizations, anemia and early mortality. This disease requires a programmatic, multidisciplinary and team-based approach. Currently there are three potential models of care for sickle cell disease patients: classic comprehensive model, embedded care model and specialized medical home model. The model adapted at our institution is a blend of all the three potential models incorporating as per our institutional considerations. This study presents the results following the multidisciplinary care implementation at our institution over the last year.

Methods Used This study was approved by the Institutional Review Board of Kern Medical. A retrospective review of post initiation of a multidisciplinary care intervention at a public academic medical center was reviewed.

Summary of Results This intervention was implemented on July 13th 2020. The multidisciplinary team includes hematologist/ oncologist, pharmacists, primary care physician, pain specialist, sickle cell specialist, behavioral health worker, clinical health worker, medical assistant and registered nurse. In the last six months a total of 170 patients were scheduled to be seen at the sickle cell clinic. 14 patients were admitted inpatient in the last six months which is a decrease in number compared to the data prior to im plementation. 23 additional patients were newly referred from outside institutions. Our findings thus far highlight the importance of gaining patients' trust, providing social support and providing patients with appropriate resources. In the last month we have also established a virtual reality program for pain management for patients. In December of 2020 at the state level, we also gained recognition for standardizing care for sickle cell patients.

Conclusions In conclusion, a multidisciplinary comprehensive care for sickle cell patients is a necessity. At present there are only ten sickle cell clinics in the state of California. Our academic center became one of the founding institutions of the upcoming Networking California for Sickle Cell Model. The model of care for implementation of this disease varies highly upon location, population served, financial and institutional needs. 
\#305 FAMILY-CENTERED CARE: DEFINING THE NEED FOR CHILDCARE SUPPORT FOR CANCER PATIENTS FROM THE HEALTHCARE PROVIDER PERSPECTIVE

${ }^{1} \mathrm{~K}$ Preston*, ${ }^{2} \mathrm{M}$ MacDonald, ${ }^{2} \mathrm{P}$ Ingledew. ${ }^{1}$ The University of British Columbia Faculty of Medicine, Vancouver, BC, Canada; ${ }^{2} B C$ Cancer Agency Vancouver Centre, Vancouver, BC, Canada

\subsection{6/jim-2022-WRMC.301}

Purpose of Study Approximately 20\% of newly diagnosed cancer patients are between the typical parenting ages of 20 and 54 , and so many of these patients are also the primary caregivers of children. Qualitative studies focusing on this demographic indicate that patients who are parents struggle to balance their own care needs with those of their children, but to date, no research has formally evaluated the need for onsite childcare services at cancer centers. This study aims to explore the need for childcare support for cancer patients from the perspective of healthcare professionals providing care at a major Canadian cancer center.

Methods Used Between May and April 2021, healthcare providers at one major Canadian cancer center were invited to partake in semi-structured interviews. The interview guide was developed through consultation with a multidisciplinary team and aimed to elicit the perspectives of healthcare providers on the importance and potential benefits of childcare services for their patients. Specific questions were also designed to explore what might constitute optimal childcare solutions for patients, and how cancer centers and healthcare providers could help to address this need. Interview transcript data was interpreted using thematic analysis.

Summary of Results Semi-structured telephone interviews were conducted with 28 healthcare professionals providing care at a major Canadian cancer center between April and May 2021, including medical, surgical, and radiation oncologists, psychiatrists, general practitioners, registered nurses, radiation therapists, and social workers. Managing childcare responsibilities was described as challenging for patients, and the introduction of childcare services for patients on-site at cancer centers was seen as a way to reduce emotional and financial stress. Other identified benefits of introducing childcare support services for patients included increased system efficiency, improved treatment compliance, increased trust in providers and cancer centers, and additional emotional support for the children of patients.

Conclusions These results indicate that childcare issues are broadly impactful for parents battling cancer, and that providing childcare support for these patients could be highly beneficial from both a medical and social perspective. As such, these findings suggest that cancer centers could consider the implementation of childcare support services to provide a more patient-centered approach to care.

\section{\#306 OUTCOME OF PLANNED CHECKPOINT INHIBITOR DISCONTINUATION IN METASTATIC MELANOMA PATIENTS IN A COMMUNITY ONCOLOGY PRACTICE: A CASE SERIES}

${ }^{1} \mathrm{~L}$ Perez* ${ }^{*}{ }^{1,2} \mathrm{~W}$ Samlowski, ${ }^{1} \mathrm{RD}$ Lopez-Flores. 'University of Nevada Reno School of Medicine, Reno, NV; ${ }^{2}$ University of Nevada Las Vegas, Las Vegas, NV

10.1136/jim-2022-WRMC.302
Purpose of Study Check-point inhibitor (CKI) therapy for melanoma has dramatically changed the prognosis for patients with metastatic melanoma. Twenty to $40 \%$ of patients appear to achieve long-term remissions. It is not clear whether patients require ongoing therapy to maintain remissions or whether treatment can be safely discontinued. We evaluated a potential strategy for elective treatment discontinuation.

Methods Used A retrospective chart review of patients with metastatic melanoma treated with CKI based treatment was performed. Patients who underwent elective treatment discontinuation after 2 negative scans 3 months apart or had biopsies confirming complete remission (CR) were identified for analysis.

Summary of Results Of 139 checkpoint inhibitor treated patients, 53 individuals achieved a CR. The progression free survival was $100 \%$ at 1 year, $95 \%$ at 3 years after treatment discontinuation, with a median duration of follow up of 31 months. Four of 53 individuals with complete remissions (7.5\%) eventually relapsed. The median overall survival of the entire cohort was not reached and was $100 \%$ at 1 year, and was $95 \%$ at 3 years.

Conclusions In this retrospective analysis, we demonstrate an elective treatment discontinuation strategy that is generalizable to a variety of CKI \pm targeted therapy regimens. We have found a high complete response duration after elective treatment discontinuation without a requirement for a specific period of therapy. We hypothesize that this approach can potentially decrease treatment related financial burden and toxicities faced by responding patients with improved quality of life.

\section{\#307 A PROOF-OF-CONCEPT SEVERITY SCREENING TOOL FOR OPERATIONAL SAFETY REPORTS IN RADIATION ONCOLOGY}

P Beidler*, M Nguyen, K Lybarger, J Kang, E Ford. University of Washington, Seattle, WA

10.1136/jim-2022-WRMC.303

Purpose of Study While incident learning systems in radiation oncology allow departments to track and improve patient safety, reviewing and analyzing these reports remains a timeconsuming human task. Here, we present a data-driven classifier to identify high-severity reports for urgent/scrutinous review.

Methods Used We utilized a corpus of 7,094 free-text operational safety reports from the University of Washington Department of Radiation Oncology from 2012-2021. Each report was labeled with a Near Miss Risk Index (NMRI) as part of typical clinical workflow. NMRI scores ranged from $0-4$, with 0 , 1 , and 2 considered low-severity, and 3 and 4 considered high-severity. Approximately $15 \%$ of the reports are labeled high-severity.

Two neural network-based approaches were used to predict severity. As a baseline, we implemented a Long Short-Term Memory network with self-attention (LSTM-A) using word vectors pre-trained on PubMed texts and clinic notes. Our second model uses a compressed version of the popular BERT, DistillBERT, which was pretrained on Wikipedia text and English literature. Transformer models, like DistillBERT, use transfer learning to leverage large corpora of unannotated text and achieve state-of-the-art performance on many natural language processing (NLP) tasks. 
Abstract \#307 Table 1 Performance metrics on test set

\begin{tabular}{lll} 
& AUC & F1 \\
\hline LSTM-A & 0.77 & 0.45 \\
DistillBERT & 0.72 & 0.45 \\
\hline
\end{tabular}

Models were trained to predict the ordinal NMRI scores using a regression objective function, to take advantage of the ordinal scores. To assess the models as a clinical screening tool, performance was evaluated as a binary classification task where predicted scores were mapped to low- and high-severity labels. Regression training improved performance compared with binary classification training.

The reports were split into train (70\%), validation (15\%), and test $(15 \%)$ sets. Model hyperparameters were tuned using the training and validation sets to maximize area under the receiver operating characteristic curve (AUC), and final performance was evaluated on the withheld test set.

Summary of Results Results from each approach are reported in table 1. Although DistillBERT is a more sophisticated architecture, both models performed similarly.

While neither model completely generalized to the test set, both are shown to have predictive value. Since NMRI scores were annotated by many individuals over 9 years, scores may vary between raters and over time, limiting model performance.

Conclusions This work presents two candidate architectures for a severity screening tool for incident reports in radiation oncology. We show AUC scores up to 0.77, demonstrating that these architectures have the potential to identify safetycritical reports and reduce latency of interventions. Future work includes incorporating radiation oncology-specific text and experimenting with BERT variants that are pretrained on clinical text.

\section{\#308 INVESTIGATING BARRIERS TO RADIATION ONCOLOGY TREATMENT FOR AMERICAN INDIAN AND ALASKA NATIVE PATIENTS}

D Marashi* ${ }^{*}$ M Greer, L Moriguchi Halasz. University of Washington School of Medicine, Seattle, WA

\subsection{6/jim-2022-WRMC.304}

Purpose of Study American Indian/Alaska Native (AI/AN) citizens comprise $2 \%$ of the population, both in the United States and Washington State. Among this population, cancer is the leading cause of death for females and the second for males. Radiation therapy (RT) is recommended for about $50 \%$ of cancer patients. Unfortunately, few data are available regarding the barriers to RT treatment for AI/AN populations. Our objective is to characterize the barriers $\mathrm{AI} / \mathrm{AN}$ patients experience while accessing cancer care at the University of Washington Medical Center, and their affiliated hospitals, and to determine which barriers (if any) are captured and addressed in the electronic health record.

Methods Used Levesque et al. describe a ' $5 \mathrm{~A}$ ' theoretical framework - approachability, acceptability, availability, affordability, and appropriateness - that considers supply-side factors (e.g., health systems, institutions, organizations, providers) alongside demand-side factors (e.g., individual, household, community, population), as a framework to describe the complex process patients experience utilizing healthcare. We conducted an analysis based on this framework using $36 \mathrm{AI} / \mathrm{AN}$ patient charts, focusing on their radiation oncology and social work notes from the last five years. All data were double coded. Quotes describing each barrier were used to support the coding process, and these were referred to to resolve any discrepancies between coders.

Summary of Results When looking specifically at the supplyside dimensions of access to oncologic services, availability/ accommodation (25.0\%) and affordability (16.6\%) appeared as the most common barriers to care. In contrast, a very small percentage of patient charts mentioned acceptability as a barrier to care $(1.0 \%)$. Most chart descriptions did not feature demand-side abilities of patients or populations to access services $(5.0 \%)$. Illustrative vignettes were also organized to represent the different types of barriers discovered in patient charts.

Conclusions AI/AN communities find it challenging to access health services (either the physical space or healthcare providers) in a timely manner, and potentially lack the economic resources to pay for healthcare services without catastrophic expenditures. Our review also uncovered the supply-side dominance in terms of dimensions of health care access. In other words, despite evidence suggesting individual-level interventions are more effective at improving access for patients, there was still an emphasis on services provided rather than selfmanagement and health literacy approaches. It is not known whether this reflects that providers do not address demandside dimensions of access or simply do not record it in the chart. Regardless, the findings suggest efforts to address all dimensions of access may be important to diminishing health care disparities.

\section{\#309 CONCURRENCE OF COCCIDIOIDOMYCOSIS AND MALIGNANCY}

J Slaton, R Sharma*, M Mistry, N Dhillon, L Moosavi, A Heidari, R Johnson, R Kuran, R Polineni, E Cobos. Kern Medical Center, Bakersfield, CA

\subsection{6/jim-2022-WRMC.305}

Purpose of Study Coccidioidomycosis (CM) is endemic to Southwestern USA with over 150000 cases per year. Concurrence of $\mathrm{CM}$ and malignancy has been reported sporadically but the interaction and association is yet to be studied. This study is designed to identify and describe cases with CM and malignancy from a single center located in highly endemic area of Coccidioides in California.

Methods Used A retrospective chart review following IRB approval at our institution was conducted. The inclusion criteria was patients greater than or equal to 18 years of age, positive CM serology and positive diagnosis of malignancy on biopsy. Pediatric age group, inmates, pregnant females, human immunodeficiency virus patients and patients with incomplete medical records were excluded from this study.

Summary of Results Twelve total patients were included in this study. Characteristics of cancer by location of $\mathrm{CM}$ are displayed in table $1.5 / 12$ patients presented with associated diabetes mellitus. 11/12 patients underwent therapeutic treatment with fluconazole and one patient didn't receive antifungal therapy. 11 patients underwent surgical resection for cancer, one patient had sero-reactivaton of $\mathrm{CM}$ post fluconazole 


\begin{tabular}{|c|c|c|c|}
\hline Characteristics & $\begin{array}{l}\text { Pulmonary } \\
(\mathrm{n}=9)\end{array}$ & $\begin{array}{l}\text { Extrapulmonary } \\
\text { Dissemination } \\
(n=2)\end{array}$ & $\begin{array}{l}\text { Lymphatic } \\
\text { Dissemination } \\
(n=1)\end{array}$ \\
\hline Colorectal Cancer & 3 & 0 & 0 \\
\hline Prostate Cancer & 2 & 0 & 0 \\
\hline Renal Cell Carcinoma & 1 & 2 & 0 \\
\hline Melanoma & 1 & 0 & 1 \\
\hline Gynecologic Cancer & 2 & 0 & 0 \\
\hline Gastric Cancer & 1 & 0 & 0 \\
\hline Cancer preceded CM onset & 5 & 0 & 1 \\
\hline $\mathrm{CM}$ preceded cancer onset & 3 & 2 & 0 \\
\hline Simultaneous onset of $\mathrm{CM}$ and cancer & 1 & 0 & 0 \\
\hline
\end{tabular}

failure and 1 patient succumbed to life. Common cancer types noted with concurrence $\mathrm{CM}$ infection in this study are colorectal, prostate, gynecologic, renal cell, gastric and skin. Onset of cancer prior to $\mathrm{CM}$ infection and vice versa was noted in almost equal number of patients. Only one case demonstrated simultaneous onset of cancer and CM. Extrapulmonary dissemination of CM was noted only among renal cell carcinoma and melanoma patients.

Conclusions Coexistence of coccidioidomycosis and cancer is not common but when it occurs, the interaction between the two conditions is not well understood and management is formidable. A close collaboration between oncology and infectious diseases teams is paramount.

\section{\#310 OUTCOME OF PLANNED CHECKPOINT INHIBITOR DISCONTINUATION IN METASTATIC NON-MELANOMA SOLID TUMOR PATIENTS IN A COMMUNITY ONCOLOGY PRACTICE: A CASE SERIES}

${ }^{1}$ RD Lopez-Flores*, 1,2,3W Samlowski, 'L Perez. 'University of Nevada Reno School of Medicine, Reno, NV; ${ }^{2}$ University of Nevada Las Vegas Kirk Kerkorian School of Medicine, Las Vegas, NV; ${ }^{3}$ Comprehensive Cancer Centers of Nevada, Henderson, NV

\subsection{6/jim-2022-WRMC.306}

Purpose of Study Checkpoint inhibitor (CKI) therapy has markedly altered the survival of patients with many solid tumors. In addition to melanoma, it appears that $10-40 \%$ of patients with other tumor types can achieve durable remissions. The duration of treatment or whether treatment can be safely stopped to maintain a complete response is controversial. Based on melanoma-derived data, we tested whether CKI treatment could be safely discontinued in patients with other solid tumors.

Methods Used A retrospective analysis was performed in adults with metastatic non-melanoma solid tumors treated with CKIbased therapy. Patients with solid tumors who achieved a complete remission on 2 sequential scans at least 3 months apart were identified from our computerized patient database. Patient data was analyzed for patient characteristics, as well as progression-free, and overall survival.

Summary of Results Sixty-nine non-melanoma solid tumor patients were treated with CKI-based regimens in our clinic and 15 achieved a complete remission (21.7\%). Five patients were female $(35.7 \%)$ and the remaining nine were male (64.3\%). A 100\% PFS was reported for these patients who achieved CR with over 20 months of median follow up after elective treatment discontinuation. Median OS was not reached. CKI toxicity primarily included acute toxicity with rare, chronic unresolved toxicity over 6-month duration in two patients.

Conclusions In this retrospective case series, all patients remained in complete remission after treatment discontinuation. We hypothesized that appropriate selection of patients for early treatment discontinuation may decrease their economic burden related to ongoing treatment, limit potential toxicity, and improve quality of life.

\section{\#311 NOVEL FLOW CYTOMETRY ANTIGENS TO DIFFERENTIATE FOLLICULAR LYMPHOMA AND DIFFUSE LARGE B-CELL LYMPHOMA}

L Schwarz* , J Fromm. University of Washington School of Medicine, Seattle, WA

\subsection{6/jim-2022-WRMC.307}

Purpose of Study Flow cytometry can aid in the identification of blood cancers, including Non-Hodgkin's Lymphomas. Its ability to quantify the antigen expression of cells is often used to differentiate between similar neoplasms. However, there is currently a dearth of antigens which differentiate Diffuse Large B-cell Lymphoma (DLBCL) and Follicular Lymphoma (FL). We evaluated the ability of novel antigens including CD32, CD38, CD40, CD44, CD71, and CD75 to discriminate between the two lymphomas.

Methods Used Tissue samples had been previously collected, immunohistochemically and morphologically categorized, and cryopreserved in long-term liquid $\mathrm{N}_{2}$ storage. DLBCL and FL samples were identified using an internal database, thawed,


CD38/CD75/CD71/CD19/CD5, and run on a BD LSR II Flow Cytometer. Benign follicular hyperplasia (FH) samples were also processed and run as a negative control. Populations were isolated and analyzed using WoodList, an internal software program. Median fluorescence intensity (MFI) for each antigen, forward scatter (FSC), and side scatter (SSC) were collected for neoplastic cells in DLBCL and FL samples and for all B-cells in FH. Values were exported to Excel and used to create two-tailed boxplots using the maximum, minimum, median, and first (Q1) and third (Q3) quartile values. Antigens were considered 'successful' in differentiating the two lymphomas if there was no overlap between Q3 of one neoplasm and Q1 of another.

Summary of Results While there were apparent differences between FL and DLBCL (both germinal and non-germinal center) in the expression levels of CD32, CD38, CD40, CD44, and CD75, there was significant overlap in the plots for each of these antigens. For CD71 however, FL was adequately differentiated from both germinal and non-germinal center DLBCL. Both forward scatter and side scatter differentiated low-grade but not intermediate-grade FL from DLBCL. Incidentally, CD44 and CD32 showed the ability to separate non-germinal center from germinal center DLBCL. However, that data is limited by the low sample size of non-germinal DLBCL $(n=5)$. 
Conclusions CD71 was the sole antigen which successfully discriminated FL from both germinal and non-germinal center DLBCL in isolation. However, combining parameters, such as FSC and CD71, may better separate these two lymphomas. Further research with a more robust sample size is needed to confirm the ability of CD44 and CD32 to differentiate between germinal and non-germinal center DLBCL.

\section{$\# 312$ FREQUENCY OF ACUTE LEUKEMIA IN PEDIATRIC PATIENTS WITH BELL PALSY}

${ }^{1} \mathrm{CM}$ Tanjï, ${ }^{2,3}$ LG Yamamoto. ${ }^{1}$ Hawaii Pacific Health, Honolulu, $\mathrm{HI}_{;}{ }^{2}$ University of Hawai'i at Manoa, Honolulu, HI; ${ }^{3}$ Kapi'olani Medical Center for Women and Children, Honolulu, HI

\subsection{6/jim-2022-WRMC.308}

Purpose of Study A recent study identified a $0.6 \%$ rate of acute leukemia in children with facial palsy. In response, the primary objective of our study was to confirm if there is a relationship between Bell Palsy and acute leukemia in children younger than 19. If found, obtaining a Complete Blood Count (CBC) screening upon Bell Palsy diagnosis would be in order and reconsideration of corticosteroid treatment, which has been shown to interfere with leukemia diagnosis, if the CBC is suspicious. If not found, this would cast serious doubt on whether a true relationship exists.

Methods Used A retrospective chart review was conducted $(n=513)$ of patients $<19$ years old with Bell Palsy between April 7, 1995 to June 4, 2021. Data was collected using the hospital billing system and an electronic medical record from 4 community hospitals in Hawai'i. Statistical analyses were conducted to describe the patient demographic.

Summary of Results $53 \%$ of the patients with Bell Palsy were female and the remaining $47 \%$ were males, with around $67 \%$ of patients in the $6-12$ and $13-<19$ years age groups. Of the 513 pediatric patients with Bell Palsy, one patient developed leukemia, leading to an association of $0.19 \%$. However, the patient's leukemia diagnosis was 3.5 years after their initial Bell Palsy encounter, suggesting these two events might not be medically related.

Conclusions Our data is unable to confirm an association between Bell Palsy and acute leukemia.

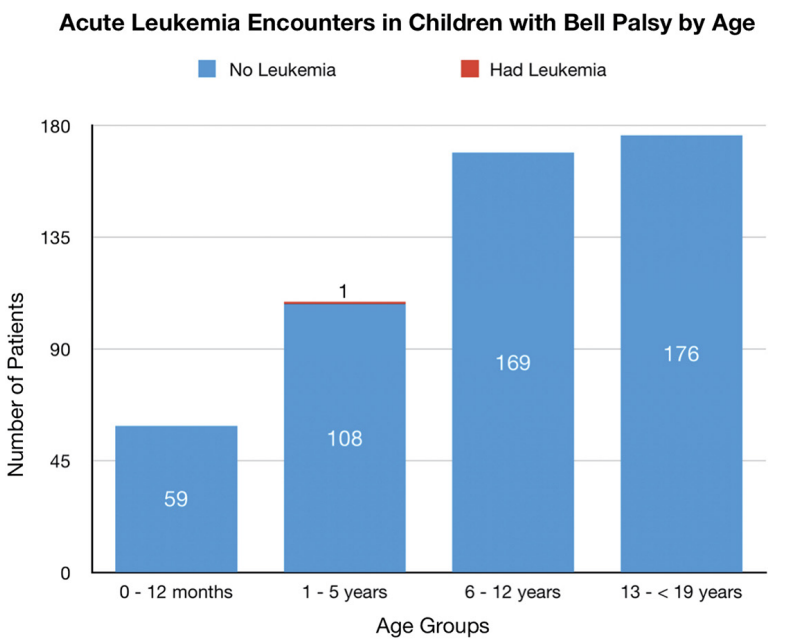

Abstract \#312 Figure 1 Among the 513 patients, only one patient with Bell Palsy was diagnosed with acute leukemia.

\section{Infectious diseases II}

\section{Concurrent session}

\author{
10:15 AM
}

Friday, January 21, 2022

\section{\#313 3-AMINOIMIDAZO[1,2-A] PYRIDINE DERIVTIVES WITH ANTIMICROBIAL ACTIVITY AGAINST STAPHYLOCOCCUS AUREUS}

${ }^{1} \mathrm{~L}$ Johar*, ${ }^{2} \mathrm{E}$ Bentley, ${ }^{2} \mathrm{D}$ Vosburg, ${ }^{1} \mathrm{H}$ Szurmant. ${ }^{1}$ Western University of Health Sciences College of Osteopathic Medicine of the Pacific, Pomona, CA; ${ }^{2}$ Harvey Mudd College, Claremont, $C A$

\subsection{6/jim-2022-WRMC.309}

Purpose of Study According to the World Health Organization (WHO), antimicrobial resistance is a serious public health issue on a global scale. Nationally, the US Centers for Disease Control and Prevention (CDC) estimate that 3 million infections are caused by antibiotic-resistant bacteria, resulting in approximately 35,900 deaths annually. Antimicrobial resistances occur when bacteria are forced to evolve due to widespread exposure to antimicrobial drugs. One way to address antimicrobial resistance is to identify novel antimicrobial compounds that can target essential microbial cellular processes. Among a diverse library of novel heterocycles produced in one of our laboratories, we previously identified one, a 3-Aminoimidazo $[1,2-a]$ pyridine with moderate antimicrobial activity against methicillin resistant Staphylococcus aureus (MRSA). To identify the substituents of the compound necessary for killing activity and to potentially improve the initial hit compound, a medicinal chemistry approach was pursued.

Methods Used 3-aminoimidazo[1,2-a]-heterocycles were synthesized using a green, microwave-assisted Groebke-BlackburnBienaymé multicomponent reaction. Compounds were screened for their ability to inhibit growth of MRSA. For screening purposes, we utilized the Kirby-Bauer disk diffusion assay, according to Clinical Laboratory Standards Institute protocols. To this end, bacterial suspensions with a density equivalent to a 0.5 McFarland standard were evenly spread over fresh agar plates. Paper disks containing $100 \mu \mathrm{g}$ of the respective compounds, vancomycin as a positive control, or no compound as a negative control were prepared. Compound and control disks were evenly distributed on the agar plates and plates were incubated for $12 \mathrm{~h}$ at $37^{\circ} \mathrm{C}$ to allow growth of the bacterium. Compounds with antimicrobial activities were identified by a visible ring of growth inhibition around the respective paper disks.

Summary of Results A series of 3-aminoimidazo[1,2-a]-heterocycles were synthesized and subjected to antimicrobial screening. In addition to the previously identified active compound, a second compound was found to exhibit similar moderate antimicrobial activity against MRSA. The active compounds share 4-chlorophenyl and tert-butyl substituents and varied core heterocyclic structures. Equipped with this knowledge, further medicinal chemistry efforts are underway to improve the activity of these compounds.

Conclusions Compounds similar to the two candidates with antimicrobial activities have also been identified to have antiinflammatory, anticancer, and anti-fibrosis effects. Thus 3-Aminoimidazo[1,2-a]pyridines and related structures have 
demonstrated a wide range of biological activities, including antimicrobial effects. Further efforts are underway to select for improved killing activity and move towards cytotoxicity studies.

\section{\#314 THE ANTIBIOTIC SPECTRUM OF NOVEL METHIONYL- TRNA SYNTHETASE INHIBITORS}

${ }^{1,2} \mathrm{CE}$ Liston*, ${ }^{1} \mathrm{~N}$ Molasky, ${ }^{1} \mathrm{~A}$ Mushtaq, ${ }^{1} \mathrm{JR}$ Gillespie, ${ }^{1,2}{ }^{2} \mathrm{~F}$ Buckner. ${ }^{1}$ University of Washington, Seattle, WA; ${ }^{2}$ University of Washington School of Medicine, Seattle, WA

\subsection{6/jim-2022-WRMC.310}

Purpose of Study As antibiotic resistance continues to increase worldwide, patient care and outcomes will decline. Therefore, it is necessary to investigate alternative biochemical pathways in bacterial lifecycles and select new drug targets for novel inhibitory compounds. One target of interest is the MethionyltRNA Synthetase (MetRS) enzyme which is critical in protein synthesis. Typically, Gram-positive bacteria express type-1 MetRS and Gram-negative express type-2 MetRS. The purpose of this study is to explore the efficacy of novel MetRS inhibitors on type-1 MetRS enzymes compared to control antibiotics in Gram-positive and Gram-negative bacteria.

Methods Used All preparations for bacterial growth and cultivation followed the standardized guidelines outlined by the Clinical Laboratory Standards Institute. Minimum Inhibitory Concentration (MIC) assays were performed in triplicate and repeated at least twice. The selected Gram-positive strains were non-anthracis Bacillus ssp., Listeria monocytogenes, Staphylococcus epidermidis, and the Gram-negative bacterial strains were Serratia marcescens, Pseudomonas aeruginosa, and Burkholderia cepacia. Each strain was streaked from frozen stock to strain-appropriate agar and incubated at $37^{\circ} \mathrm{C}$ for 24 hours. Single colony inocula were collected from the plate and cultured to the exponential growth phase in strain-specific broth. The culture was adjusted to MacFarland 0.5 standard and quantified through the absorbance reader $\left(\mathrm{OD}_{600}\right)$. The microbroth dilution was completed in 96-well plates in a 2fold dilution using novel MetRS inhibitory compounds ChemIDs: 2541, 1986, 1717, 2536, 2144, 2093, 2596 and control antibiotics. The MacFarland adjusted bacteria were added to the plates. Negative and positive controls were established in each plate and after an additional 18-24 hours of incubation at $37^{\circ} \mathrm{C}$, the MIC90 was calculated from the data generated by the absorbance reader.

Summary of Results The novel MetRS inhibitors demonstrated variable yet potent inhibitory effect against type-1 MetRS enzymes in the Gram-positive strains. The MICs ranged from $<0.0312-0.25 \mu \mathrm{g} / \mathrm{ml}$. In most cases these MICs were more efficacious than the control antibiotics. As predicted, the compounds showed little activity in the type-2 MetRS enzymes in Gram-negative bacteria.

Conclusions In vitro testing showed potent activity against a variety of Gram-positive bacteria species. Parallel studies not reported here demonstrated in vivo activity in mouse models of MRSA infection. The compounds have excellent potential for development as selective agents for treating Gram-positive infections.

\section{\#315 THE WALR PROTEIN OF GRAM-POSITIVE PATHOGENS AS A TARGET FOR THE DEVELOPMENT OF NEW ANTIMICROBIALS}

${ }^{1} \mathrm{~L}$ Johar*, ${ }^{2} \mathrm{C}$ Majdi, ${ }^{3} \mathrm{E}$ Badarau, ${ }^{3} \mathrm{~J}$ Dessolin, ${ }^{2} \mathrm{Z}$ Benfodda, ${ }^{1} \mathrm{H}$ Szurmant. ${ }^{1}$ Western University of Health Sciences, Pomona, CA; ${ }^{2}$ Universite de Nimes, Nimes, France; ${ }^{3}$ Université de Bordeaux, Bordeaux, France

10.1136/jim-2022-WRMC.311

Purpose of Study So-called 'two component systems' (TCS) are the primary means by which bacteria sense and respond to their environment. They regulate important decisions such as virulence factor expression, antimicrobial resistance development, sporulation, motility to name a few. Grampositive bacteria have a single system, the WalRK system, that is essential for viability. TCS comprise a sensor histidine kinase that auto-phosphorylates and then activates an associated response regulator/transcription factor protein by phosphoryl group transfer. Since similar proteins are not found in the animal kingdom, TCS in general and the WalRK system in particular, have been suggested as excellent targets for anti-infective agents. Past drug discovery efforts have primarily focused on the WalK histidine kinase, but multiple arguments can be made that the WalR response regulator is the better target. Utilizing a combined virtual and experimental approach, we aim to chemically target the WalR protein.

Methods Used For screening of virtual compound libraries, we utilized existing crystal structures of various WalR protein fragments to model a full-length WalR protein and define the DNA-binding site. Molecular dynamic docking simulations along with the Life Chemicals compound library were utilized to virtually identify chemical hit compounds. Standard molecular cloning and affinity purification techniques were utilized for WalR overexpression. Isothermal titration calorimetry is used for the quantification of protein/small molecule affinities.

Summary of Results A 17,000-compound library was reduced to 4354 molecules with a previously identified ability to bind to DNA-polymerases. These compounds were virtual screened for their propensity to bind to the DNA-binding surface of the WalR DNA-binding domain. Eight hit molecules were predicted to strongly associate with the defined DNA-binding interface. Of these, three compounds shared a thiazolo[4,5-d] pyridazine core. To experimentally validate the ability of these compounds to bind to WalR we cloned, expressed, and purified to homogeneity two WalR constructs comprising either the full-length or the isolated DNA-binding domain. While the full-length protein proved largely insoluble, the isolated DNA-binding domain could be purified to homogeneity at high yields. Utilizing this construct, isothermal titration calorimetric experiments are underway to validate the hit compounds.

Conclusions Compounds that inhibit the activity of the essential WalR transcription factor could serves as antibiotics against numerous important human pathogens, including but not limited to Staphylococci, Streptococci, Bacilli and Listeria. Our research is a step forward towards identifying such molecules. 
\#317 COVID-19, MENTAL HEALTH STATUS, AND ADHERENCE TO PUBLIC HEALTH GUIDELINES AMONG THE ELDERLY POPULATION IN UNITED STATES

${ }^{1} \mathrm{JY}$ Hwang ${ }^{*},{ }^{1} \mathrm{C}$ Lee, ${ }^{1} \mathrm{~T}$ Nguyen, ${ }^{2} \mathrm{~T}$ Buck, ${ }^{2} \mathrm{~K}$ Mun, ${ }^{2} \mathrm{TT}$ Vanteru, ${ }^{1} \mathrm{D}$ Baral, ${ }^{2} \mathrm{D}$ Tirschwell, ${ }^{1}$ A Kim. 'Washington State University Elson S Floyd College of Medicine, Spokane, WA; ${ }^{2}$ University of Washington School of Medicine, Seattle, WA

\subsection{6/jim-2022-WRMC.312}

Purpose of Study Do-not-resuscitate (DNR) orders allow patients, per their wishes, to decline cardiopulmonary resuscitation (CPR) in a cardiac or respiratory arrest event. DNR status has been linked with severe illness, advanced age, poor disease prognosis, and deteriorating health status with impending death. Conversely, DNR status has not been associated with a reduction in priority of care in the intensive care units. In the current COVID-19 pandemic, patients and families are often faced with a need to make a DNR decision with limited information. Some hospital systems had to impose a unilateral DNR policy, resulting in unprecedented, unresolved ethical dilemmas. This study further investigates the characteristics and outcomes of hospitalized COVID-19 patients in association with DNR status.

Methods Used An observational study of hospitalized patients with COVID-19 was conducted in the greater Seattle area from January 2020 to December 2020. Data was collected through review of the electronic medical record as part of the national American Heart Association COVID-19 registry. Inclusion criteria for DNR included patients with any documentation of a DNR order, partial DNR order, or advanced directive related to restricting attempts to resuscitate prior to or after arrival to the hospital. Physician Orders for Life-Sustaining Treatment form, Do Not Attempt Resuscitation form, and the Medical Orders for Scope of Treatment form were all considered as documentation of DNR. We performed a chi-square test of independence and one-way ANOVA analysis.

Summary of Results Of the total 396 hospitalized COVID-19 patients in our registry, DNR status was available for 368 patients. Of the 368 patients, 114 (31\%) were deemed to be DNR. The average age of patients who were deemed DNR was 72 years, while the average age for those who were not DNR was 55 years $(\mathrm{F}(1,366)=73.37, \mathrm{p}<.001)$. Patients with comorbidities were more likely to be DNR (34\%) compared to patients with no past medical history $(18 \%)\left(\chi^{2}=6.603, \mathrm{df}=1, \mathrm{p}=0.010\right)$. With regard to mortality, 69 out of the 114 patients with a DNR order expired (61\%), while 11 out of the 254 patients without a DNR order expired $(4 \%)\left(\chi^{2}=142.768, \mathrm{df}=1, \mathrm{p}<.001\right)$. Of the total 80 expired patients, $86 \%$ were DNR. There was no statistically significant difference in the average length of hospitalization between the two groups $(\mathrm{F}(1,366)=1.44$, $\mathrm{p}$ $=0.2311$ ).

Conclusions Our data suggest that DNR is associated with increased mortality, age, and comorbidities. However, there were no statistically significant associations observed between sex and length of hospitalization. These results potentially reflect the overwhelming burden our healthcare system has faced during the COVID-19 pandemic when resources have been substantially limited.
A Nguyen*, K Schrode, M Shaheen. Charles Drew University of Medicine and Science, Los Angeles, $C A$

10.1136/jim-2022-WRMC.313

Purpose of Study The COVID-19 virus has led to widespread economic, health, and social changes. Elderly affected population experience serious disease complications and isolation from their social groups. These complications may cause psychological stressors on the elderly. This study aims to elucidate if the COVID-19 pandemic has contributed to a lessened mental health status for the elderly with positive COVID-19 diagnoses or symptoms.

Methods Used We analyzed data from NHAT's COVID-19 Supplemental Questionnaire. The outcome variables were sleep disturbance, loneliness, depression, and anxiety, and adherence to public health guidelines. Chi-square and multiple logistic regression tests were performed.

Summary of Results Of the 3,107 elderly participants, $2.1 \%$ had COVID-19, 3.3\% had COVID-19 symptoms, $17.5 \%$ had more sleep, 23\% were depressed, 28\% had anxiety, 19\% felt lonely, and the majority follow the public health guidelines. No significant association was found between test positive for COVID-19 and the outcome variables $(p>0.05)$ except in having worse sleep and being more likely to follow public health guidelines $(\mathrm{p}<0.05)$. Participants with mild/moderate symptoms were more likely to feel lonely than those with no symptoms $(p<0.05)$. Those with severe COVID-19 symptoms were more likely to have depression and follow public health guidelines relative to those with no symptoms $(\mathrm{p}<0.05)$.

Conclusions COVID-19 positive was associated with adherence to public health guidelines. Loneliness and depression were associated with COVID-19 symptoms. Those diagnosed with COVID-19 had a higher likelihood of sleep issues and adherence to public health guidelines. Resources are needed to help the elderly manage the changing mental health status associated with future pandemics.

\section{\#318 A COMMUNITY TRAINING PROGRAM ON CORONAVIRUS VACCINES HESITANCY FOR THE PREVENTION AND ELIMINATION OF COVID-19: A PROGRAM TO HELP ACHIEVE HERD IMMUNITY}

${ }^{1} \mathrm{R}$ Sadeghi*, ${ }^{2} \mathrm{M}$ Sadeghi, ${ }^{1} \mathrm{E}$ McGhee. ${ }^{1}$ Charles Drew University of Medicine and Science, Los Angeles, CA; ${ }^{2}$ University of Southern California, Los Angeles, CA

\subsection{6/jim-2022-WRMC.314}

Purpose of Study The highly contagious severe acute respiratory syndrome 2 (SARS-CoV-2) has prompted a global effort to find solutions for eradicating the coronavirus disease 19 (COVID-19) pandemic. Full FDA approval and Emergency Use Authorization (EUA) has been granted for three COVID19 vaccines; however, underserved communities continue to be impacted by the virus through comorbidities and health disparities literacy. Providing underserved populations a 
community educational program will help with the complexities of understanding vaccines. This study aims to minimize vaccine hesitancy, increase the uptake of COVID-19 vaccines, and promote herd immunity.

Methods Used A COVID-19 vaccine educational program was created for a diverse audience to enhance the knowledge in underserved communities on the benefits of vaccines to prevent diseases. The training module materials presented in the program provided information on the background and significance of vaccines to the COVID-19 pandemic. Following the training, the participants completed demographics, test review questions, and a one-page survey feedback questionnaire. SPSS statistical software was used for descriptive One-Way ANOVA analysis for the data calculations.

Summary of Results After examining the results from 103 participants, an exemplary average score of $98.4 \%$ was observed for the review section. The mean average response on the Likert-scale survey questions was 'Strongly Agree' for comprehending new information about COVID-19 vaccines. One-Way ANOVA produced statistically significant $p$ values $\leq 0.05$ by SPSS. Additionally, 84 participants agreed to educate 10 additional individuals about the benefits of vaccines, expanding the population of the study to be inclusive of 840 participants.

Conclusions The community engagement educational training program demonstrated a decrease in vaccine hesitancy and promoted the uptake of COVID-19 vaccines in underserved communities, fundamentally assisting the elimination and prevention of the COVID-19 pandemic by contributing to herd immunity.

\section{\#319 EFFECTS OF GEOGRAPHY, SOCIODEMOGRAPHICS AND POLITICAL LEANING ON COVID-19 CASE-FATALITY RATE IN WEST COAST STATES}

JS Gerken*, D Kuivinen, D Zapata, I Zapata. Rocky Vista University College of Osteopathic Medicine, Parker, CO

\subsection{6/jim-2022-WRMC.315}

Purpose of Study The COVID-19 pandemic, caused by the SARS-Cov-2 virus, has impacted nearly every aspect of scientific research. Many factors such as comorbidities, socioeconomic descriptors along with political associations have been associated to fatality rates. One specific factor that has continuously suspected to have a potential impact on COVID-19 fatality rates is Vitamin D deficiency. Few studies have evaluated the effect of environmental, geographic, or sociodemographic variables together on COVID-19 casefatality rate. Our study evaluates COVID-19 case-fatality rate on a per-county basis of the Pacific Coast states using comorbidity, sociodemographic and political leaning data in conjunction with geographic variables such as average precipitation, air pollution, average temperature, latitude and elevation which have been shown to correlate to Vitamin D deficiency.

Methods Used This analysis evaluates the effect sizes of each particular factor described using Generalized Additive Models (GAM). This type of model was selected to accommodate for non-parametric evaluations of continuous predictors. Estimates obtained through these models then are standardized and sorted by their effect size and direction to allow for fair interpretation with a standardized context.
Summary of Results Our analysis showed that geographic and environmental variables associated with Vitamin D levels did not affect COVID-19 case-fatality rates; however, factors such as Medicare flu vaccination rate, voting Democrat during the 2020 Presidential Election, and being uninsured were shown to be the most important factors in reducing COVID-19 case fatality rate. Comorbidities, disproportionate higher females in a county, mental health, and voting Republican in the 2020 Presidential Election significantly increased COVID-19 case fatality rates.

Conclusions The effect of Vitamin D deficiency, indirectly measured through geographic variables, was not supported by our results. However, there were other clinically significant factors such as comorbidities, socioeconomic, and political leaning factors that influenced COVID-19 case fatality rates.

This work was presented in part at Rocky Vista University Appreciation Day (virtual) on October 15, 2021

\section{\#320 THERAPEUTIC ANTICOAGULATION IN HOSPITALIZED PATIENTS WITH COVID-19}

${ }^{1} \mathrm{C}$ Lee*, ${ }^{*} \mathrm{~T}$ Nguyen, ${ }^{1} \mathrm{JY}$ Hwang, ${ }^{2} \mathrm{~T}$ Buck, ${ }^{2} \mathrm{~K}$ Mun, ${ }^{2} \mathrm{IT}$ Vanteru, ${ }^{1} \mathrm{D}$ Baral, ${ }^{2} \mathrm{D}$ Tirschwell, ${ }^{1}$ A Kim. 'Washington State University Elson S Floyd College of Medicine, Spokane, WA; ${ }^{2}$ University of Washington School of Medicine, Seattle, WA

\subsection{6/jim-2022-WRMC.316}

Purpose of Study Throughout the COVID-19 pandemic, providers observed increased rates of thrombosis and inflammation among COVID-19 patients which affected multiple organs and led to complications such as lung failure, myocardial infarction, and stroke. Furthermore, studies have shown increased D-dimer levels to be associated with worse outcomes in COVID-19 patients. A spectrum of anticoagulants is currently being used in COVID-19 patient management as needed to combat this hypercoagulable state. Current treatment guidelines and literature investigating the effects of different anticoagulants in COVID-19 patients are continuing to evolve. Here, we describe a preliminary study that investigated the effects of therapeutic anticoagulation on the outcomes of hospitalized COVID-19 patients.

Methods Used From a national registry developed by the American Heart Association, we performed a retrospective observational cohort study of hospitalized COVID-19 patients who were admitted to one of four hospitals in the Greater Seattle area from January 2020 to December 2020. Diagnosis of COVID-19 was made either clinically using hospital specific criteria, or by polymerase chain reaction testing. Medications given for therapeutic anticoagulation include parenteral unfractionated heparin, subcutaneous low molecular weight heparin full therapeutic dose, argatroban, bivalirudin, direct oral anticoagulant, and warfarin. Mortality was quantified by the number of patients who died during hospitalization or were discharged to hospice care.

Summary of Results Out of 396 hospitalized COVID-19 patients, 57 patients received one or more anticoagulants for therapeutic use. For $89 \%$ of those patients (51 out of 57), therapeutic anticoagulation was indicated due to pre-hospitalization anticoagulant use or development of myocardial infarction, pulmonary embolism, or deep vein thrombosis during hospitalization. Of those who received therapeutic anticoagulation, the survival rate was $67 \%$ (38 out of 57 ). On the contrary, out of the 339 patients who did not receive any type of therapeutic anticoagulation, the survival rate was 79\% (267 
out of 339). This difference of higher survival rate and lower mortality rate in patients who did not receive therapeutic anticoagulation was not statistically significant $(p=0.066)$.

Conclusions Our preliminary findings indicate that therapeutic anticoagulation does not affect mortality in hospitalized COVID-19 patients. However, therapeutic anticoagulation may be necessary in high-risk patients who are more susceptible to a COVID-19 induced hypercoagulable state to reduce complications or death resulting from thromboembolic events. Further investigation is necessary with additional analyses performed to control for confounding by indication and other prognostic factors.

\section{\#321 SHORT-COURSE ANTIBIOTICS FOR TREATMENT OF UNCOMPLICATED COMMUNITY ACQUIRED PNEUMONIA IN PEDIATRIC PATIENTS}

${ }^{1} \mathrm{YN}$ Manely*, 'M Arunachalam, ${ }^{1} \mathrm{~T}$ Hoang, ${ }^{1} \mathrm{E}$ Kang, ${ }^{1} \mathrm{H}$ Lee, ${ }^{1} \mathrm{~J}$ Parikh, ${ }^{1} \mathrm{C}$ Palomino, 1,2B Afghani. 'UC Irvine School of Medicine, Irvine, CA; ${ }^{2}$ Children's Hospital of Orange County, Orange, $C A$

\subsection{6/jim-2022-WRMC.317}

Purpose of Study Several studies of adults have shown that a 5 -day course of antibiotics is as effective as a 10-day course in treatment of community acquired pneumonia (CAP). However, the effectiveness of short-course therapy in pediatric patients with CAP has not been well documented. The main goal of this study was to investigate the literature for studies that compare the effectiveness of short-course vs long course antibiotics in treatment of pediatric CAP.

Methods Used We conducted a literature search using search engines such as Google Scholar, SciHub, and PubMed. Key words used included: 'pediatric' 'children' 'pneumonia' 'shortcourse' 'long-course' and 'treatment'. Only studies of pediatric patients with uncomplicated community acquired pne]'monia that included a control group and used high-dose amoxicillin as part of the treatment a were included in our review.

Summary of Results We found 4 studies that satisfied our inclusion criteria. All four studies excluded patients diagnosed with severe or complicated pneumonia and those with underlying conditions. Please see table 1 for summary of the results. The age of children in the studies ranged from 2 months to 18 years. The investigators measured cure rates and/or relapse rates during the follow-up period of 14 to 30 days. The studies showed that a short-course of 5-7 days was not inferior to a 10-14 days of treatment.The design was different in various studies as 3 of the studies required chest radiograph as well as clincial evidence of pneumonia while one study was based on clinical diagnosis of pneumonia.

Conclusions The results from our literature review show that a 5-7 day antibiotic course is non-inferior to a longer course of 10 to 14 days in treatment of previously healthy children with uncomplicated community acquired pneumonia. Larger studies are warranted to determine whether these results are reproducible across different populations or non-trail conditions.

\section{Morphogenesis and malformations}

\section{Concurrent session}

\section{0:15 AM}

Friday, January 21, 2022

\section{\#322 SIN3A IS REQUIRED FOR EPIGENETIC REGULATION OF DIAPHRAGM AND LUNG DEVELOPMENT}

G Stokes, D McCulley*. UCSD, University of California San Diego, La Jolla, CA, La Jolla, CA

\subsection{6/jim-2022-WRMC.318}

Purpose of Study One of the most common and severe congenital malformations is congenital diaphragmatic hernia (CDH) which occurs in 1 out of 3500 live births with a mortality rate of $20-50 \%$. Although abnormal diaphragm formation is the hallmark of the disease, the high mortality rate is due to abnormal lung and pulmonary vascular development causing lung hypoplasia and pulmonary hypertension. Despite the frequency and severity of $\mathrm{CDH}$, little is known about the underlying developmental and genetic mechanisms responsible for the disease. Our hypothesis is that a core group of genes is required for diaphragm, lung, and pulmonary vascular development and that pathogenic variants in these genes are responsible for failure of diaphragm formation as well as defects in lung and pulmonary vascular development in

Abstract \#321 Table 1 Studies comparing short and long-course treatment of uncomplicated pneumonia in children

\begin{tabular}{|c|c|c|c|c|c|c|}
\hline $\begin{array}{l}\text { First Author, Year of } \\
\text { Publication, Location } \\
\text { of study }\end{array}$ & Patient Types: & $\begin{array}{l}\text { Duration of } \\
\text { Short and Long } \\
\text { Treatment }\end{array}$ & $\begin{array}{l}\text { Number of Subjects in Short Term } \\
\text { Group, Age Range }\end{array}$ & $\begin{array}{l}\text { Follow Up } \\
\text { Period }\end{array}$ & $\begin{array}{l}\text { Outcome in Short-Course vs Long- } \\
\text { Course Antibiotics }\end{array}$ & P-value \\
\hline Pernica 2021, Canada & $\begin{array}{l}\text { Emergency } \\
\text { room patients }\end{array}$ & $\begin{array}{l}5 \text { days vs } 10 \\
\text { days }\end{array}$ & $\begin{array}{l}\text { 5-day course, } N=126,10 \text {-day course: } \\
N=126 \text {, Age range } 6 \mathrm{~m} \text { to } 10 \text { yrs }\end{array}$ & 14-21 days & Clinical Cure: $85.7 \%$ vs. $84.1 \%$ & $P=N S$ \\
\hline Greenberg 2013, Israel & Hospitalized & 3 vs 5 vs 10 days & $\begin{array}{l}\text { 3-day course: } 10,5 \text {-day course: } N=56,10 \\
\text { day course: } N=42, \text { Age: } 6-59 \text { months }\end{array}$ & 30 days & $\begin{array}{l}\text { Failure rate: } 3 \text { day vs } 5 \text { days vs } 10 \text { days: } \\
40 \% \text { vs } 0 \% \text { vs } 0 \%\end{array}$ & $\begin{array}{l}P \text { value }<0.001 \text { for } 3 \\
\text { days vs } 5 \text { days and } 10 \\
\text { days but } p \text { value NS } \\
\text { for } 5 \text { vs } 10 \text { days }\end{array}$ \\
\hline Ginsburg 2020, Malawi & $\begin{array}{l}\text { Hospitalized } \\
\text { and discharged }\end{array}$ & 3 vs 5 days & $\begin{array}{l}\text { 3-day course: } N=1497,5 \text { day course: } \\
N=1503 \text {, Age: } 2 \text { to } 59 \text { months }\end{array}$ & 21 days & $\begin{array}{l}\text { Treatment failure on or before day } 6 \text { : } \\
5.9 \% \text { vs } 5.2 \% \text {, Relapse before day } 14 \text { : } \\
6.9 \% \text { vs } 5.8 \%\end{array}$ & $P=N S$ \\
\hline $\begin{array}{l}\text { Same } 2020 \text {, United } \\
\text { States }\end{array}$ & $\begin{array}{l}\text { Hospitalized } \\
\text { and discharged }\end{array}$ & $\begin{array}{l}5-7 \text { days vs } 8-14 \\
\text { days }\end{array}$ & $\begin{array}{l}\text { 5-7 day group: } N=167,8-14 \text { day group: } \\
N=270 \text {, Age: } 6 \text { months- } 18 \text { years }\end{array}$ & 30 days & Failure rate: $3 \%$ vs $6 \%$ & $P=N S$ \\
\hline
\end{tabular}

NS=Not significant 
patients. To investigate this hypothesis, we are studying the role that genetic mutations identified in patients with $\mathrm{CDH}$ play in diaphragm, lung, and pulmonary vascular development. In this study we investigated the role played by SIN3A, a gene containing pathogenic sequence variants identified by whole genome sequencing in patients with $\mathrm{CDH}$, in diaphragm, lung, and pulmonary vascular development.

Methods Used To investigate the role of SIN3A, we used tissue and cell-specific deletion in diaphragm and lung mesenchymal progenitor cells in mice. The diaphragm and lung phenotypes associated with loss of SIN3A were characterized using whole mount, brightfield, and immunofluorescence imaging. Pulmonary vascular defects associated with loss of SIN3A were characterized by fluorescence imaging as well as echocardiography and right heart catheterization. The genetic mechanisms responsible for abnormal lung and pulmonary vascular development were studied using gene expression analysis with RNA collected from whole lung and sorted lung mesenchymal cells.

Summary of Results Loss of SIN3A in somatic mesoderm derived skeletal muscle and lateral plate mesoderm derived mesothelium resulted in abnormal diaphragm development and $\mathrm{CDH}$ in mice. In the developing lungs, loss of SIN3A resulted in failure of alveologenesis and pulmonary hypertension. Lung defects in SIN3A deficient mice were evident at late embryonic stages with failure of mesenchymal cell differentiation, decreased cell cycling, and increased DNA damage. Loss of SIN3A was associated with an imbalance of histone acetylation that was restored by embryonic inhibition of histone acetyltransferase.

Conclusions These data demonstrate the importance of genetic evaluation in structural malformations such as $\mathrm{CDH}$. In the case of SIN3A loss of function, impaired epigenetic regulation of gene expression in the diaphragm and lungs resulted in failure of diaphragm formation as well as lung and pulmonary vascular defects common among patients with $\mathrm{CDH}$. These defects were reversed by restoring the balance of histone acetylation.

\section{\#323 PATTERN OF LIMB INVOLVEMENT IN CONSTRICTION BAND SYNDROME}

${ }^{1}$ A Starcevich*, ${ }^{2} \mathrm{~L}$ Demetri, ${ }^{3} \mathrm{E}$ Peterson, ${ }^{1,3} \mathrm{MC}$ Manske, ${ }^{1,3} \mathrm{MA}$ James. ${ }^{1}$ University of California Davis School of Medicine, Sacramento, CA; ${ }^{2}$ University of California San Francisco, San Francisco, CA; ${ }^{3}$ Shriners Hospitals for Children Northern California, Sacramento, CA

\subsection{6/jim-2022-WRMC.319}

Purpose of Study Constriction band syndrome (CBS) is an uncommon congenital condition causing limb deformity, with varying clinical presentation. We sought to characterize the pattern of limb involvement in CBS by analyzing a large cohort of patients.

Methods Used We performed a retrospective review of all patients with CBS presenting to our tertiary medical center between January 1, 1998 and December 31, 2018. Patients were identified by ICD-9 and ICD-10 codes. Medical records were reviewed for demographic data and associated conditions. Clinical photographs and radiographs were reviewed to determine the pattern of limb involvement.

Summary of Results 138 children with CBS were identified. Upper and lower extremities were affected equally $(80 \%$ and $79 \%$, respectively). The most prevalent feature was distal limb amputation (89\%) followed by constriction bands (79\%) and

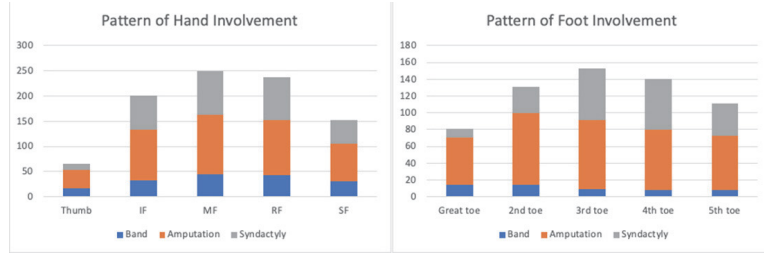

Abstract \#323 Figure 1 Pattern of hand and foot involvement

acrosyndactyly (68\%). Amputations were characterized by hypoplasia and deformed appearance of the amputated bone on radiographs. There was a strong predilection for involvement of central digits of the hands and feet with sparing of the thumb/great toe and small finger/toe. Although most features of CBS were limited to the distal extremities, some children had limb amputations proximal to the digits (17\%) and proximal constriction bands (39\%). The average number of involved extremities was 2.5 limbs per child, however $33 \%$ had involvement of only one limb. Children with involvement of a single limb were significantly less likely to have an associated diagnosis compared to children with multiple involved limbs (36\% vs. 56\%, p=0.047). The most common associated conditions were clubfoot (34\%) and craniofacial anomalies (12\%).

Conclusions Children with CBS can be categorized as having either mild or severe involvement based on the number of involved limbs and associated conditions. This characterization may provide implications for prognosis and treatment options. Future investigation of prenatal risk factors may further elucidate the etiology of this heterogenous condition.

\section{\#324 BIALLELIC VARIANTS IN GPX4 ARE ASSOCIATED WITH A SPECTRUM OF SKELETAL ANOMALIES, HYPOTONIA, AND NEURODEGENERATION AND MAY INCREASE SUSCEPTABILITY TO FERROPTOSIS}

${ }^{1,2} \mathrm{~K}$ Wigby ${ }^{*},{ }^{3,4} \mathrm{R}$ Saneto, ${ }^{1,2} \mathrm{~J}$ Friedman, ${ }^{5} \mathrm{H}$ Liu, ${ }^{5} \mathrm{~B}$ Stockwell. ${ }^{1}$ University of California San Diego, La Jolla, CA; ${ }^{2}$ Rady Children's Institute for Genomic Medicine, San Diego, CA; ${ }^{3}$ Seattle Children's Hospital, Seattle, WA; ${ }^{4}$ University of Washington, Seattle, WA; ${ }^{5}$ Columbia University, New York, NY

10.1136/jim-2022-WRMC.320

Case Report Biallelic loss of function variants in GPX4 are associated an exceedingly rare pattern of malformation of Sedaghatian-type Spondylometaphyseal Skeletal Dysplasia (SSMD), brain malformations, hypotonia, and neonatal lethality. We report three long-term survivors in two unrelated families identified by whole exome sequencing to harbor a homozygous variant in GPX4 (c.455 G>A, p. R152H, based on NP_001354761.1, the mature short form of GPX4) and who extend the phenotype to include other skeletal anomalies and neurodegeneration.

Proband 1 (family 1) presented with IUGR, microcephaly (4 SD), hypotonia, hypoplastic optic nerves, and bilateral auditory neuropathy. Skeletal survey demonstrated metaphyseal cupping and irregularity of all long bones. Spine series showed cervical platyspondyly. Serial brain MRIs revealed cerebellar atrophy and supratentorial white matter atrophy. Probands 2 and 3 (family 2) presented with infantile hypotonia and gross motor delays. Skeletal surveys revealed brachycephaly, proximal tibial metaphyseal flaring, and coxa valga. Now age 13 years, proband 2 has intractable epilepsy and has diffuse 
supratentorial and infratentorial volume loss on serial brain MRIs. Proband 3 has bilateral auditory neuropathy.

GXP4 encodes for a phospholipid glutathione peroxidase that is critical for eliminating peroxidized phospholipids in membranes. It thus prevents ferroptosis, a type of regulated cell death driven by iron-dependent lipid peroxidation. Animal knockouts of Gpx4 are embryonic lethal; adult conditional Gpx4 knockout mice develop seizures, ataxia and neuronal loss [Yoo et al, 2012]. Computational and cellular modeling from patient-derived fibroblasts revealed a $40 \%$ reduction in activity via destabilization of secondary protein structure which in turn disrupts the active catalytic site. This data suggests that the p. $\mathrm{R} 152 \mathrm{H}$ variant is a hypomorphic allele that produces sufficient enzymatic function to likely allow for survival beyond infancy but may result in increased susceptibility to ferroptosis culminating in cell death, tissue damage, and neurodegeneration.

\section{\#325 EXPANDING THE PHENOTYPE OF PIK3C2A RELATED SYNDROME: REPORT OF TWO SIBLINGS WITH NOVEL FEATURES AND GENOTYPE}

${ }^{1} \mathrm{C}$ Galarreta Aima*, ${ }^{2} \mathrm{DR}$ Blair, ${ }^{2} \mathrm{~A}$ Slavotinek. 'Valley Children's Hospital, Madera, CA; ${ }^{2}$ University of California San Francisco, San Francisco, CA

\subsection{6/jim-2022-WRMC.321}

Case Report Tiosano et al. (2019) identified a novel syndrome characterized by short stature, coarse facial features, ocular and skeletal abnormalities in five individuals with homozygous loss-of-function mutations in PIK3C2A. This report describes a pair of siblings of Hispanic ethnicity with novel PIK3C2A truncating variants and a phenotype consistent with original report.

The siblings, a 5-year-old boy and 2-year-old girl, share a strikingly similar facial gestalt with coarse appearance, sparse eyebrows, prominent epicanthal folds, broad nasal bridge, prominent nasal tip and full lips. Both have sparse hair, two hair whorls, broad distal phalanges, 5th finger clinodactyly, persistence of fetal pads and flat feet. The boy is short (height $\mathrm{Z}$ score -2.5), while the sister has normal height ( $\mathrm{Z}$ score 1.3). Both have developed gibbus deformity, more severe in the boy who has hypoplasia of L2 vertebral body. He has significant ocular abnormalities, including bilateral congenital cataracts, nystagmus, esotropia and high myopia, and mild to moderate, right-sided hearing impairment that has been presumed to be sensorineural loss. The sister had an uncomplicated perinatal course, whereas the boy had significant respiratory depression at birth and developed persistent pulmonary hypertension in the neonatal period. Both children have dental decay and global developmental delay. The sister had GBS sepsis at 4-weeks-old and was found to have proteinuria which persisted at 2 years of age with $\mathrm{UPr} / \mathrm{Cr}$ ratio range 3.4-6.5. Plasma albumin and renal ultrasound were normal. Exome sequencing analysis of both children and their mother revealed two novel PIK3C2A variants in the siblings, c.4381delC (p.Arg1461Glufs*31) and c.1555C>T (p. Arg519Ter).

Previously reported features present in these patients include short stature, congenital cataract, hearing loss, coarse facial features and scoliosis. In contrast to brother and prior patients, the sister had normal stature and she also exhibits persistent proteinuria which has not been reported. This report brings to 7 the number of patients reported with PIK3C2A-related syndrome, confirms core clinical features, establishes intra-familial variability and expands the phenotype to include proteinuria.

\section{\#326 ROHHAD MIMICKED BY A TUBB2B NOVEL VARIANT}

R Garg*, A Tam, J Shieh. University of California San Francisco, San Francisco, CA and University of California San Francisco, San Francisco, CA

\subsection{6/jim-2022-WRMC.322}

Purpose of Study Although many individuals have been diagnosed with ROHHAD [rapid-onset obesity, hypothalamic, autonomic dysregulation, neuroendocrine tumor] since this condition was first defined in 2007, the genetic basis remains unknown. Here we describe individuals with $\mathrm{ROH}-$ HAD-like presentation that underwent medical genetics evaluations. We review clinical characteristics of tubulinopathies and query whether they have overlapping features with ROHHAD.

Methods Used Two individuals referred to the genetics clinic for ROHHAD had trio whole exome sequencing performed. One of the individuals was found to have a novel variant in $T U B B 2 B$ gene. A literature search from PubMed on TUBB2B resulted in 66 articles. 16 were excluded based on a lack of clinical information. In total, 50 articles were reviewed for clinical features that have been described for TUBB2B. Prior studies on ROHHAD were reviewed.

Summary of Results Trio exome sequencing revealed a de novo missense variant in TUBB2B c.421G $>$ A (p.Gly141Ser) at a highly conserved amino acid residue in the patient with earlyonset obesity (BMI 48 by 1 year life) and additional features. A second unrelated patient had non-diagnostic exome. The individual with $T U B B 2 B$ variant is a 2 -year-old female with severe obesity, developmental delay, hypotonia, obstructive sleep apnea (OSA), hypertension, ocular motor apraxia, hypothyroidism, colitis, clostridium difficile infection, and anemia. The hypothalamic-pituitary axis was tested by ACTH level and stimulation testing and was not diagnostic. Variants in $T U B B 2 B$ are typically associated with brain malformations and characterized by a wide spectrum of features. It is possible that early onset obesity may be a rare presentation of TUBB2B tubulinopathies since the sequencing of 74 obesity genes did not yield an additional diagnostic variant.

Conclusions A novel TUBB2B variant was identified in a patient with brain MRI findings and severe obesity. TUBB2B has been reported to associated with developmental delay in $92 \%(45 / 49)$, motor impairment in $89 \%$ (32/36), microcephaly in $86 \%(44 / 51)$, impaired muscular tone in $87 \%(21 / 24)$, ocular features in $74 \%(17 / 23)$, seizures in 68\% (34/50), behavior abnormalities 61\% (8/13), and craniofacial dysmorphology in $71 \%(5 / 7)$ of the cases. OSA was reported in one case. An association of tubulinopathies with rapid-onset obesity would be worth considering. There are numerous examples of genetic conditions with both neurodevelopmental and obesity phenotypes. Possible overlap in ROHHAD-like phenotypes and tubulinopathies could involve the central nervous system, but in general, the TUBB2B condition can be discerned by genetic testing and imaging, distinguishable from classical ROHHAD, which would need tumor screening. Further studies may help elucidate the full spectrum of tubulinopathies and their clinical presentations. 
\#327 BARTH SYNDROME: AT THE CONFLUENCE OF CARDIOLOGY, DYSMORPHOLOGY, AND BIOCHEMICAL GENETICS

S Tahata*, CG Tise, BJ Floyd, K Cusmano-Ozog. Stanford University, Stanford, CA

\subsection{6/jim-2022-WRMC.323}

Purpose of Study Barth syndrome is an X-linked disorder that presents with dilated cardiomyopathy and/or left ventricular non-compaction, 3-methylglutaconic aciduria (3-MGA), neutropenia, and characteristic facial features in young males with hemizygous pathogenic variants in TAFAZZIN (OMIM: 300394). The finding of 3-MGA in patients with cardiomyopathy may be suggestive of Barth syndrome but is not specific to this disorder, and in some cases may misdirect the diagnostic work-up in patients with 3-MGA secondary to other etiologies. We present our institutional experience with the diagnosis and management of Barth syndrome, as well as the role of 3-MGA in the diagnostic evaluation of patients with cardiomyopathy.

Methods Used Retrospective chart review of probands with Barth syndrome evaluated at Lucile Packard Children's Hospital from January 1, 2003 to the present.

Summary of Results Seven males were diagnosed with Barth syndrome via molecular genetic testing. Cardiac findings at presentation (median age: 2 months, range: neonate to 15 years) included dilated cardiomyopathy with left ventricular non-compaction $(n=5)$ and cardiac arrest $(n=2)$. Urine organic acid analysis noted 3-MGA in 6 of 7 patients, though most elevations were mild. Molecular diagnosis of Barth syndrome occurred via gene panel $(n=3)$, single gene or familial variant testing $(n=2)$, or unspecified genetic testing $(n=2)$, with a median time to molecular diagnosis of 4 months (range: 2 weeks to 9 years). In 4 patients, cardiac function improved over time; 2 patients required cardiac transplant; and 2 patients (including 1 transplanted patient) died from cardiac causes. Median follow-up was 9 years (range: 3 weeks to 24 years).

Conclusions Among the 7 individuals with Barth syndrome, the most common features included dilated cardiomyopathy, left ventricular non-compaction, and 3-MGA. Although 3MGA is a classically described finding in patients with Barth syndrome, elevated 3-MGA levels are not always present. Furthermore, as 3-MGA can also be seen secondary to acute clinical states and/or mitochondrial dysfunction, reliance on 3MGA in boys with cardiomyopathy may promote premature narrowing of the differential diagnosis and ultimately delay diagnosis of other conditions. Further investigation into the prevalence of 3-MGA in other metabolic and genetic disorders, especially those with cardiac involvement, is warranted.

\section{\#328 EXPANDING THE PHENOTYPE OF SPARC-RELATED OSTEOGENESIS IMPERFECTA}

${ }^{1} \mathrm{~B}$ Nightingale, ${ }^{2} \mathrm{AJ}$ Schildt*, ${ }^{2} \mathrm{D}$ Stevenson. 'Lucile Salter Packard Children's Hospital at Stanford, Palo Alto, CA; ${ }^{2}$ Stanford University, Stanford, CA

\subsection{6/jim-2022-WRMC.324}

Purpose of Study Osteogenesis imperfecta (OI) is a skeletal disorder typically associated with defects in collagen proteins, the majority due to autosomal dominant pathogenic variants in COL1A1 and COL1A2. However, OI is increasingly found to be genetically heterogeneous with other inheritance patterns observed. Recently, biallelic variants in SPARC, encoding for secreted protein, acidic, cysteine-rich (SPARC), have been reported in rare cases of autosomal recessive OI (type XVII). Literature review identified 6 cases. Here, we describe an individual who was found to have an earlier reported SPARC homozygous missense variant c.787G >A (p.Glu263Lys).

Methods Used Case report with chart and literature review. Summary of Results The proband is a 6yo male born to consanguineous parents. Early on, he had global hypotonia with gross and fine motor delays and remains non-ambulatory. $\mathrm{He}$ had bilateral conductive hearing loss but normal speech development. MRI of the brain showed periventricular white matter volume loss. He did not have dentinogenesis imperfecta though did have multiple caries. At 6 months he sustained a femur fracture and subsequently developed $>10$ fractures requiring multiple surgeries. He developed severe thoracolumbar levoscoliosis with multilevel compression fractures. $\mathrm{He}$ also had thoracic cage deformity with bony fusion of several ribs, and generalized osteopenia. Molecular testing identified a homozygous variant in SPARC, and his brother with history of fracture after minor trauma was found to be heterozygous for the same variant.

Conclusions Our patient shares features common in the previously described cases such as early neuromuscular concerns, scoliosis, long bone and vertebral compression fractures, and delayed motor milestones, suggesting these are consistent across SPARC-related OI. Interestingly, our patient displayed white matter changes on MRI, also found in two other cases. However, our case experienced fractures at a younger age with a more severe course compared to most reports. He also had bony fusion of several ribs and hearing loss, which have not been reported in SPARC-related OI. His family history raises the possibility of mild manifestations for heterozygous carriers. Overall, our patient provides further insight into the variation of SPARC-related OI and expands on the phenotype of individuals with pathogenic variants in SPARC.

\section{\#329 WORTH THE EFFORT: IMPROVING DISCOVERY AND CARE THROUGH A TEAM APPROACH TO AN UNUSUAL CASE OF GORLIN SYNDROME}

${ }^{1} \mathrm{~V}$ Taliercio*, ${ }^{1,2}$ J zhao, 'S Boyden, 'L Botto, 'D Viskochil. 'University of Utah Health, Salt Lake City, UT; ${ }^{2}$ ARUP Laboratories, Salt Lake City, UT

\subsection{6/jim-2022-WRMC.325}

Case Report Born with cleft lip and palate, $\mathrm{MH}$ later showed odontogenic keratocytes, calcification of the falx cerebri, nonunion of the posterior elements of T3 vertebrae, palmar pits, and intermittent exotropia, thus meeting clinical criteria for Gorlin syndrome (GS). She had no basal cell carcinomas (BCC) or family history of BCC. She was also found to have bilateral hypoplasia of the semicircular canals with unilateral sensorineural hearing loss, as well as Kallman syndrome (hypogonadotropic hypogonadism $(\mathrm{HH})$ with anosmia), none of which are an established component of GS. Despite the clinical diagnosis of GS, sequencing and deletion/duplication analysis of the PTCH1 and SUFU genes and genomic SNP microarray were negative. Because of the complexity of the phenotype and the lack of molecular confirmation, she was enrolled in the Undiagnosed Disease Program and was evaluated by our team of clinicians, molecular geneticists, and data scientists. 
Whole genome sequencing (WGS) from the clinical laboratory identified a deep intronic PTCH1 variant $(\mathrm{c} .3+314 \mathrm{G}>\mathrm{T})$, present also in her healthy father and sibling, which was considered to be likely benign. However, when we reanalyzed the WGS data using two structural variant callers, RUFUS and Smoove, we detected signals suggesting a $38.3 \mathrm{Mb}$ de novo balanced inversion inv(9)(q22.32q34.2). One of the breakpoints was located in intron 2 of the PTCH1 gene. As a result, this inversion is predicted to result in either a truncated protein or mRNA subject to nonsense-mediated decay. This finding was confirmed by karyotyping, FISH analysis of metaphase chromosomes, and Sanger sequencing. Notably, the initial WGS also identified a maternally-inherited VUS in the CHD7 gene (c.8077G>C; p.Gly2693Arg), which is associated with CHARGE syndrome. The latter finding is likely to account for bilateral hypoplasia of the semicircular canals (not a known feature of GS), and possibly also for Kallman syndrome (reported in several cases of CHD7-associated conditions).

MH either has a blended phenotype due to the combined effect of de novo PTCH1 disruption and an inherited CHD7 variant or she represents an expanded phenotype of Gorlin syndrome. Pursuing and completing the diagnostic evaluation helped a) discover an unusual molecular etiology for GS; b) show the value of using novel tools to identify structural variants with WGS; c) provide more precise clinical management and counseling to the proband and her family.

This work was part UDN (U01HG007672)

\section{Neonatology general IV}

\section{Concurrent session}

\section{0:15 AM}

\section{Friday, January 21, 2022}

\section{\#330 MATERNAL MARIJUANA USE DURING PREGNANCY AND BREASTFEEDING: ASSESSING IN-HOSPITAL OUTCOMES OF EARLY PRETERM INFANTS}

${ }^{1} \mathrm{MA}$ Chang ${ }^{*},{ }^{2} \mathrm{~S}$ Curtis, ${ }^{2} \mathrm{NL}$ Davis. ${ }^{1}$ University of Southern California Keck School of Medicine, Los Angeles, $C{ }^{2}{ }^{2}$ University of Maryland School of Medicine, Baltimore, MD

\subsection{6/jim-2022-WRMC.326}

Purpose of Study Legalization and decriminalization of marijuana has been associated with increased use in women of child-bearing age. Since Tetrahydrocannabinol (THC) has been shown to pass into breastmilk, concern exists for effects of exposure prenatally and postnatally via mother's milk (MM). Our neonatal intensive care unit (NICU) allows MM provision for infants regardless of THC status, though many will restrict MM use given concerns about effects on the neonate. Our objective was to evaluate differences in outcomes between neonates who did vs. did not have exposure to THC prenatally and postnatally via MM.

Methods Used We performed a retrospective medical record review of early preterm ( $<34$ weeks gestational age, GA) neonates born 9/2014-12/2020 and admitted to our Level IV NICU. Our institution conducts universal urine toxicology and substance use screens on admitted mothers. We compared maternal and neonatal characteristics in four cohorts: 1) THC positive moms providing $\mathrm{MM}, 2$ 2) THC positive moms who provided no MM, 3) THC negative moms who provided MM, 4) THC negative moms who provided no MM. We performed multivariable analyses to assess the effect of THC and MM status on significant outcomes.

Summary of Results We identified 763 early preterm neonates, of whom 59\% $(n=448)$ were non-Hispanic Black and $31 \%$ $(n=233)$ were non-Hispanic White. Seventeen percent $(n=130)$ of mothers tested positive for THC. THC positive mothers were more likely to be late to prenatal care (PNC) while those who did not provide $\mathrm{MM}$ were more likely to receive no PNC $(p<0.0001)$. There were no significant differences between the 4 cohorts in regards to growth restriction, oxygen requirement at 36 weeks, necrotizing enterocolitis, and ventilator requirement. However, we did find increased incidence of intraventricular hemorrhage (IVH) in infants who were THC exposed $(p=0.0015)$, though the largest incidence overall and of grade III/IV IVH was in THC exposed infants without MM. We therefore performed logistic regression for predictors of IVH in early preterms and found that when controlling for variables such as birth weight, neither THC status $(p=0.18)$ nor $\mathrm{MM}$ status $(\mathrm{p}=0.79)$ significantly predicted IVH in early preterms. The only significant predictors were antenatal steroids, delivery mode, and birth GA.

Conclusions Providing MM from THC positive women to early preterm neonates is controversial as longer term effects of this exposure are unknown. In our study, we found no evidence that providing MM from THC positive mothers was detrimental to the health of this early premature population through hospital discharge. A better understanding of longer term perinatal outcomes associated with THC exposure would inform appropriate interventions to improve clinical outcomes and safely encourage MM provision for early preterm infants.

\section{\#331 LATE PRETERM ANTENATAL STEROID USE AND INFANT OUTCOMES IN A SINGLE CENTER}

MD Wahl*, C McEvoy, M Go. Oregon Health and Science University School of Medicine, Portland, OR

\subsection{6/jim-2022-WRMC.327}

Purpose of Study Antenatal steroids (AS) have been shown to lower respiratory complications in late preterm infants (LPI) and is now part of the management of pregnancies at risk for delivery between 34 0/7- 36 6/7 weeks gestational age (GA). We aim to determine our center's adherence to the published Antenatal Betamethasone for Women at Risk for Late Preterm Delivery (ALPS) trial (PMID26842679) and assess neonatal outcomes related to its use.

Methods Used We conducted a retrospective chart review of singleton mother-LPI dyads who delivered in our center from January 2019 to December 2020. We excluded those with major congenital anomalies. Data on AS use, maternal characteristics and neonatal outcomes were recorded and analyzed.

Summary of Results There were 175 mother-infant dyads included, $92(53 \%)$ received AS and 83 (47\%) did not receive AS (No AS) prior to delivery. Maternal characteristics between the two groups such as age, race, ethnicity, delivery mode, smoking status, gestational hypertension and preeclampsia were similar. The incidence of preterm premature rupture of membranes was $66 \%$ in the AS group compared to $52 \%$ in the No AS group $(p=0.02)$. Diabetes was present in $20 \%$ of 
the women in the AS group, 11\% of which had pregestational diabetes (an exclusion criterion in the ALPS trial). We found 90\% adherence to the ALPS protocol within our center. The AS group had a mean GA of 35.5 weeks at delivery and an average time from admission to delivery of 58.2 hours, compared to the No AS group (36.1 weeks, $p<0.05$ and 19.96 hours, $p=0.01$, respectively). Neonatal outcomes such as birth weight, need for respiratory support, hypoglycemia, NICU admission and hospital length of stay, were similar between the two groups when controlled for GA.

Conclusions We continue to see some variation in the administration of AS in the late preterm gestation. In our study, AS administration did not improve neonatal outcomes such as the need for respiratory support, NICU admission, and overall hospital length of stay, although we are underpowered for these clinical results. Our study reflects that there remains variation in the approach to women with diabetes delivering during the late preterm period. We recommend that more studies are needed to evaluate the impact of AS use in women with diabetes, as well as overall long-term impact of AS on late preterm infants.

\section{\#332 THE INFLUENCE OF MATERNAL-CHILD INTERACTIONS DURING EARLY CHILD DEVELOPMENT AND THE IMPACT ON ANTERIOR CINGULATE CORTEX VOLUMES}

S Markee* , J Phillips, D Novak, J Lowe. University of New Mexico Hospital, Albuquerque, NM

\subsection{6/jim-2022-WRMC.328}

Purpose of Study Studies investigating differences in regional brain volumes in children during early childhood are limited. Many theories suggest the anterior cingulate cortex (ACC) is at the center of the brain's self-regulatory system playing a key role in learning, emotion, impulsivity and decision making. The development and functional organization of the ACC is likely an essential step in the cerebral maturation that underlies cognitive, social and emotional development, which good parenting aims to foster. Our aim is to investigate the volume of the ACC identified with MRI and developmental outcomes in children at 18 months. We hypothesize that the volume of the ACC will be larger in children who received more positive directing behaviors from their mothers during play and that ACC volumes will be larger in children with higher cognitive and language scores as measured by the Bayley Scales of Infant Development (BSID-III).

Methods Used Healthy term infants were recruited in Albuquerque, NM between 2010-2012 as part of a longitudinal study of parenting and child development. MRI scans were manually traced for volumes of the ACC $(n=22)$. The BSID-III measured cognitive and language development. The Maternal Attention Directing Manual (S. Landry 2000) was used to code six minute free-play videos of maternal-child dyads, focusing on positive and negative maternal directing behaviors. Summary of Results Spearman correlation indicated a significant positive association between left-sided ACC volumes and maternal brief nonverbal commands $(p=0.043)$. Increased frequency of redirect commands, a negative maternal directing behavior, was associated with smaller right ACC volumes $(p=0.007)$; increased duration of redirect commands was negatively associated with both right-sided ACC volumes $(p=0.004)$ and total ACC volumes $(p=0.035)$. We also found a significant negative association between redirecting behavior and BSID-III language scores $(\mathrm{p}=0.018)$. Partial correlation controlling for maternal income maintained a significant negative association with redirecting commands, however nonverbal brief commands were no longer significant. No significance was seen between ACC volumes and positive maternal behaviors or BSID-III scores.

Conclusions We demonstrate a significant negative correlation between the right and total ACC volumes in children whose mothers used more redirecting commands, a form of negative maternal directing behavior. A negative impact was also found between BSID-III language scores and maternal redirecting behavior. Overall, these results suggest that maternal redirecting may have a negative impact on ACC volumes and early child language development.

\section{\#333 LONG-TERM OUTCOMES IN TERM INFANTS EXPOSED TO DELAYED CORD CLAMPING}

K Tedesco*, JY Massoumi, O Okolo, M Chu, S Sakhamuru, L Barton, R Cayabyab, R Ramanathan, J Keel. Los Angeles County University of Southern California Medical Center, Los Angeles, CA

\subsection{6/jim-2022-WRMC.329}

Purpose of Study AAP, ACOG and NRP have recommended delayed cord clamping (DCC) in term and preterm deliveries. DCC has established short and long-term benefits. Studies have shown that DCC in term infants is associated with improved hematologic status, neurodevelopmental outcomes and decreased risk of iron deficiency anemia. The purpose of the study is to compare long-term outcomes of term infants with immediate cord clamping (ICC) vs. delayed cord clamping (DCC).

\begin{tabular}{|c|c|c|c|}
\hline & $\begin{array}{l}\text { Immediate Cord } \\
\text { Clamping } n=34\end{array}$ & $\begin{array}{l}\text { Delayed Cord } \\
\text { Clamping } n=28\end{array}$ & $\begin{array}{l}\text { P- } \\
\text { value }\end{array}$ \\
\hline 9-12 Months of age: & $60(55,60)$ & $58(50,60)$ & 0.46 \\
\hline \multicolumn{4}{|l|}{ Communication* } \\
\hline 9-12 Months of age: Gross & $55(50,60)$ & $58(48,60)$ & 0.82 \\
\hline \multicolumn{4}{|l|}{ Motor* } \\
\hline 9-12 Months of age: Fine & $60(50,60)$ & $55(50,60)$ & 0.41 \\
\hline \multicolumn{4}{|l|}{ Motor* } \\
\hline 9-12 Months of age: & $55(45,60)$ & $60(50,60)$ & 0.45 \\
\hline \multicolumn{4}{|l|}{ Problem Solving* } \\
\hline \multirow[t]{3}{*}{ 9-12 Months of age: Social* } & $55(45,60)$ & $60(45,60)$ & 0.40 \\
\hline & Immediate Cord & Delayed Cord & P-value \\
\hline & Clamping $n=16$ & Clamping $n=12$ & \\
\hline 18-24 Months of age: & $50(35,58)$ & $48(40,50)$ & 0.71 \\
\hline \multicolumn{4}{|l|}{ Communication* } \\
\hline 18-24 Months of age: Gross & $60(55,60)$ & $60(55,60)$ & 0.76 \\
\hline \multicolumn{4}{|l|}{ Motor* } \\
\hline 18-24 Months of age: Fine & $58(50,60)$ & $53(40,60)$ & 0.41 \\
\hline \multicolumn{4}{|l|}{ Motor ${ }^{*}$} \\
\hline 18-24 Months of age: & $53(40,60)$ & $40(38,55)$ & 0.22 \\
\hline \multicolumn{4}{|l|}{ Problem Solving* } \\
\hline 18-24 Months of age: & $55(48,60)$ & $48(35,55)$ & 0.16 \\
\hline Social* & & & \\
\hline
\end{tabular}

${ }^{*}$ Median (25th, 75th percentile) 


Demographics and Hemoglobin levels at Birth Between Groups
\begin{tabular}{|c|c|c|c|}
\hline & Immediate cord clamping $\mathrm{n}=83$ & Delayed cord clamping $\mathrm{n}=76$ & P value \\
\hline Birth weight $(\mathrm{g})^{*}$ & $3320(2980,3750)$ & $3385(3050,3700)$ & 0.99 \\
\hline Gestational age $(\mathrm{wks})^{*}$ & $39(38,40)$ & $39(38,40)$ & 0.93 \\
\hline Cesarean section, $\mathrm{n}(\%)$ & $41(49.4)$ & $26(34.7)$ & 0.06 \\
\hline Hispanic Race $\mathrm{n}(\%)$ & $22(26.51)$ & $36(48)$ & 0.02 \\
\hline Maternal Pre-eclampsia, $\mathrm{n}(\%)$ & $12(14.5)$ & $4(5.3)$ & 0.07 \\
\hline Hemoglobin at 24 hours of life $(\mathrm{g} / \mathrm{dL})^{*}$ & $16.3(14.7,18)$ & $17.7(16.1,19.1)$ & $<0.01$ \\
\hline
\end{tabular}
"Median (25th percentile, 75th percentile)

Methods Used Retrospective study of term infants born at LAC+USC Medical Center and admitted to the neonatal intensive care unit between 2016-2019. Demographics, clinical data, duration of cord clamping and neurodevelopmental outcomes were collected from electronic medical records and paper records. Infants with $\mathrm{ABO}$ incompatibility were excluded. Ages and stages questionnaire was used to assess communication, gross and fine motor, problem solving and social skills at 9-12 months and 18-24 months of age. Data was analyzed with Wilcoxon Rank Sum and Chi Square tests. Summary of Results There were 174 infants included in the study, however only 62/174 (36\%) and 28/174 (16\%) were assessed for neurodevelopmental outcomes at 9-12 months and 18-24 months of age respectively. Demographics were not different between the two groups except for Hispanic race. Hemoglobin level was significantly higher at birth in infants with DCC. (Table 1). Median neurodevelopmental scores in any domains of ASQ at 9-12 and 18-24 months (table 2) and median hemoglobin levels at 9-12 months of age were not significantly different between ICC and DCC $[12.45(11.7,12.8)$ vs. $12.2(11.6,13.1) \mathrm{p}=0.84]$. No infant was treated with iron.

Conclusions Our preliminary data showed that delayed cord clamping in term infants resulted in a higher hemoglobin at birth. However, at 9-12 months of age, there was no difference in the hemoglobin levels between groups. Neurodevelopmental scores at 9-12 months and at 18-24 months were similar. No infant was diagnosed to have iron deficiency anemia. These results need to be verified in a larger sample size.

\section{\#334 IMPACT OF MATERNAL CHRONIC ILLNESS AND PLACENTAL ABNORMALITIES ON NEONATAL OUTCOMES IN VERY LOW BIRTH WEIGHT INFANTS}

K Ramm* ${ }^{*}$ A Hisey, NS Nanduri, Y Shao, C Marquez, L Barton, R Ramanathan, M Biniwale. LAC+USC Medical Center, Los Angeles, CA

\subsection{6/jim-2022-WRMC.330}

Purpose of Study Placental abnormalities and maternal chronic illnesses are often associated with adverse neonatal outcomes including small for gestational age (SGA) and stillbirth; however, there is limited information regarding its impact on morbidities in very low birth infants. This study aims to identify the impact of maternal factors, specifically chronic illness and placental abnormalities, on short term neonatal outcomes in VLBW infants.

Methods Used The data on VLBW infants from LAC+USC medical center between the years of 2009 to 2021 was retrospectively collected from electronic medical records after receiving IRB approval. Maternal factors included placental abnormalities, such as placenta previa, abruption, and placenta accreta and maternal chronic illnesses, such as sickle cell disease, hypertension, diabetes mellitus, hepatitis, cardiomyopathy, asthma, lupus erythematosus, obesity, and cancer. Neonatal outcomes associated with required resuscitation and ventilation were tested for significance using SPSS software version 28.

Summary of Results Of 383 VLBW infants, $48 \%$ were born to mothers with a chronic illness and $6.6 \%$ of these infants were born to mothers with placental abnormalities. Infants born to mothers with chronic illness were likely to be delivered by $\mathrm{c}$ section $(88 \%$ vs $73 \% \mathrm{p}<0.01)$ and were found to be SGA (34\% vs $23 \% \mathrm{p}=0.09)$. Only significant neonatal morbidity associated with the VLBW infants born to mothers with chronic illness was patent ductus arteriosus (PDA) $(75 \%$ vs $65 \% \mathrm{p}=0.02$ ). Maternal illness did not increase the risk for any other NICU morbidities. Infants delivered due to placental factors were more likely to need resuscitation at the time of delivery $(67 \%$ vs $36 \% \mathrm{p}=0.002)$, invasive ventilation including high frequency ventilation and surfactant therapy. There was no statistically significant impact on long-term morbidities including bronchopulmonary dysplasia or abnormal MRI related to maternal illness or placental abnormality.

Conclusions Placental abnormalities were shown to significantly affect a VLBW infant's need for resuscitation and ventilation after birth; however, maternal chronic illnesses cause growth restriction in VLBW infants and predisposes them to have PDA. These conditions do not seem to affect long term neonatal outcomes.

\section{Nephrology and hypertension}

\section{Concurrent session}

\section{0:15 AM}

Friday, January 21, 2022

\section{\#335 ANALYSIS OF HUMORAL AND CELLULAR IMMUNE RESPONSES TO SARS COV-2 VACCINATION (BNT162B2) IN IMMUNOCOMPROMISED RENAL ALLOGRAFT RECIPIENTS}

SC Jordan*, B Shin, T Gadsden, A Vo, N Ammerman, R Zhang. Cedars-Sinai Medical Center, Los Angeles, CA and Cedars-Sinai Medical Center, Los Angeles, CA

\subsection{6/jim-2022-WRMC.331}

Purpose of Study The SARS-CoV-2 pandemic has resulted in more than 4 million deaths worldwide. Treatments have had limited efficacy, thus the last best hope is development of 
effective vaccines. Currently available vaccines, including Pfizer (BNT162b2), demonstrated $>90 \%$ efficacy against ancestral SARS-CoV-2 infection. However, this did not include an assessment of efficacy in immunocompromised individuals and was dependent on Spike RBD-IgG detection only. Recent data also demonstrate that immunocompromised transplant recipients have increased mortality after SARS-CoV-2 infection which makes efforts for the development of effective vaccine strategies more prescient.

Methods Used Here, we report on an assessment of (Spikespecific IgG) and CD4+/CD8 + T-cell responses to BNT162b2 in immunocompromised kidney transplant patients compared to normal controls. We also analyzed the impact of immunosuppressive regimens on immune responses to BNT162b2 assessing patients on Tacrolimus + mycophenolate + prednisone $(\mathrm{Tac}+)$ v. patients maintained on belatacept + mycophenolate + prednisone $($ Bela +$)$. Sixty-one kidney transplant recipients $>1 \mathrm{M}$ post 2 nd dose of the BNT162b2 mRNA vaccine had determinations of Spike-Receptor Binding Domain (RBD)-specific IgG levels and analysis of Spike-specific CD4+/ $\mathrm{CD} 8+\mathrm{T}$-cell immune responses. Responses were compared to 41 age-matched healthy individuals (non-immunocompromised) controls. Fresh whole blood was collected in sodium heparinized tubes for T-cell stimulation assay. Plasma obtained was stored at $-80^{\circ} \mathrm{C}$ for SARS-CoV-2 Spike RBD IgG analysis.

Summary of Results Spike-RBD IgG responses were detected in $88 \%$ of normal individuals, but only $21 \%$ of $\mathrm{Tx}$ patients responded at $2 \mathrm{M}$ post-vaccination. No healthy controls showed CD4+ T-cell reactivity to Spike proteins prior to vaccination. However, there was a significant response at 1month post-vaccination. CD4+T-cell responses post 2 nd dose of BNT162b2 were reduced in Tx patients (93\% (38 of 41) positive in healthy controls vs $31 \%$ (16 of 52 ) $\mathrm{n} \mathrm{Tx}$ recipients, $\mathrm{p}<0.0001)$. After 2-3 months post 2nd BNT162b2, $\sim 62 \%$ of transplant recipients showed positive CD4+ T cell responses. CD8 + Spike-specific T-cells were detected in $56 \%$ healthy controls and $37 \% \mathrm{Tx}$ recipients 1 month post-2nddose of the BNT162b2 vaccination $(\mathrm{P}=\mathrm{NS})$. No differences were seen by immunosuppression type (Bela+ v. Tac + ).

Conclusions In summary, we found a profound deficiency in SARS-CoV-2 Spike-RBD IgG responses after BNT162b2 vaccination $(21 \%$ Tx v. 93\% Normals) This is consistent with other reports. However, SARS-CoV-2 Spike-specific CD4+/ $\mathrm{CD} 8+\mathrm{T}$-cell responses were seen in $47 \%$ of $\mathrm{Tx}$ patients. Analysis of T-cell responses to SARS-CoV-2 vaccination augments our ability to more properly define the scope and duraibility of immunity as T-cell immunity likely provides protection from a devastating disease.

\section{\#336 LEFT VENTRICULAR HYPERTROPHY, ACTIVATION OF MAMMALIAN TARGET OF RAPAMYCIN (MTOR) AND SUPPRESSED AUTOPHAGY IN THE HEART BEFORE HYPERTENSION IN MICE WITH POLYCYSTIC KIDNEY DISEASE (PKD)}

OA Oto*, D Pokhrel, D Atwood, C Edelstein. University of Colorado Health, Aurora, CO

\subsection{6/jim-2022-WRMC.332}

Purpose of Study To determine cardiac structure and function, blood pressure (BP) and mTOR signaling in the heart in $\mathrm{Pkd} 1^{\mathrm{RC} / \mathrm{RC}}(\mathrm{RC})$ mice.

Methods Used RC mice represent a hypomorphic PKD1 gene knock in matching a human disease variant, PKD1 p.
R3277C. 120 day old RC mice were studied that have a kidney cyst density (\% of kidney \pm SEM) of $13.8 \pm 2.8$. Serial blood pressure measurements were taken in conscious animals using a noninvasive tail-cuff system. mTOR and autophagy proteins were determined in the heart by immunoblot analysis.

Summary of Results See table 1. Heart weight corrected for body weight $(\mathrm{HW} / \mathrm{BW})$ was increased in RC mice. There was cardiac hypertrophy in RC mice as evidenced by increase in both intra-ventricular septum (IVS) and left ventricular wall (LVW) thickness. On echocardiogram there was increased LV mass, decreased ratio of peak velocity of early to late filling of mitral inflow (E/A ratio) indicating grade 1 diastolic dysfunction and decreased LV diastolic volume in RC mice. Blood pressure was not increased in RC mice. Increased mTOR signaling resulting in suppressed autophagy are processes that are known to influence cardiac hypertrophy Polycystin (PC-1), the gene product of the $\mathrm{Pkd} 1$ gene, is known to modulate the mTOR pathway. We hypothesized that PKD mice that also have the gene defect in the heart should have upregulation of mTORC1/2 in the heart. Thus, as it was unlikely that increased BP was contributing to the cardiac phenotype, we next looked at mTOR signaling and autophagy. In 70 day old mice, a time point before cardiac hypertrophy, there was an increase in mTORC1 (pAktThr308) and mTORC2 (pAktSer473) in the heart in RC mice. In addition, at 70 days old, there was suppressed autophagic flux in the heart in RC mice as evidenced by decreased LC3-II (a marker of autophagosomes) and no increase in LC3-II with bafilomycin.

\begin{tabular}{llll} 
Abstract \#336 Table 1 & Mean values & & \\
\hline & WT & RC & P value \\
\hline HW/BW & 0.47 & 0.53 & $\leq 0.001$ \\
IVS $(\mu \mathrm{m})$ & 1045 & 1286 & $\leq 0.05$ \\
LVW $(\mu \mathrm{m})$ & 956 & 1218 & $\leq 0.01$ \\
LV mass $(\mathrm{mg} / \mathrm{g})$ & 1.2 & 1.5 & $\leq 0.05$ \\
E/A ratio & 1.5 & 1.2 & $\leq 0.05$ \\
LV Diast Vol $(\mu \mathrm{L})$ & 71 & 57 & $\leq 0.05$ \\
Systolic/Diastolic/Mean BP (mm & $116 / 88 /$ & $109 / 84 /$ & NS \\
Hg) & 97 & 92 & \\
\hline
\end{tabular}

$\mathrm{NS}=$ not significant

Conclusions There is increased heart weight, cardiac hypertrophy, increased LV mass, grade 1 diastolic dysfunction, activation of mTORC1/2 and suppressed autophagy in the heart in $\mathrm{RC}$ mice before the onset of hypertension

In conclusion, factors other than hypertension like increased mTORC1/2 signaling or suppressed autophagy may play a role in causing cardiac hypertrophy in PKD mice.

\section{\#337 ANTINEUTROPHIL CYTOPLASMIC AUTOANTIBODY NEGATIVE PAUCI-IMMUNE GLOMERULONEPHRITIS IN A YOUNG FEMALE WITH SYSTEMIC LUPUS}

S Garcia*, R Jariwal, N Sekhon, P Pierre, M Sukkar, J Bhandohal, S Mishra, S Eppanapally. Kern Medical Center, Bakersfield, CA

10.1136/jim-2022-WRMC.333 
Purpose of Study Lupus associated glomerulonephritis (GN) typically presents as deposits of preformed immunoglobulins and complement within the glomeruli of the kidney. While, crescentic pauci-immune glomerulonephritis (CrGN) is commonly an anti-neutrophil cytoplasmic autoantibodies (ANCA) associated vasculitis causing rapidly progressive and necrotizing CrGN in the absence of immune complexes. We present the case of a systemic lupus erythematosus (SLE) patient with proteinuria and ANCA negative serology which was found to have pauci-immune GN on renal biopsy.

Methods Used A 23 year old woman with Systemic Lupus Erythematosus presented with generalized body aches, fatigue, and shortness of breath for 3 days. Physical examination revealed tachycardia, tachypnea, and bilateral costovertebral angle tenderness. Further testing revealed elevated Partial Thromboplastin Time and D-dimer. Labs also revealed acute kidney injury with nephrotic range proteinuria. Urine microscopy revealed granular casts of acute tubular necrosis and dysmorphic red blood cell cast leading to a possible diagnosis of proliferative glomerulonephritis or membranous nephropathy. A renal biopsy was performed revealing active and chronic pauci-immune crescentic glomerulonephritis characterized by $20 \%$ acute, $5 \%$ subacute fibrocellular and $20 \%$ remote fibrous crescents. There was $33 \%$ global glomerulosclerosis and mild-to-moderate tubulointerstitial scarring.

Summary of Results Pauci immune is a form of GN lacking immune reaction products except for minimal accumulation of fibrin and presents as a renal limited vasculitis. Distinguished by absence of circulating basement membrane antibodies and negative finding on renal immunofluorescence. The likelihood of a patient to have SLE in conjunction with ANCA associated pauci-immune $\mathrm{GN}$ is $2 \%$.

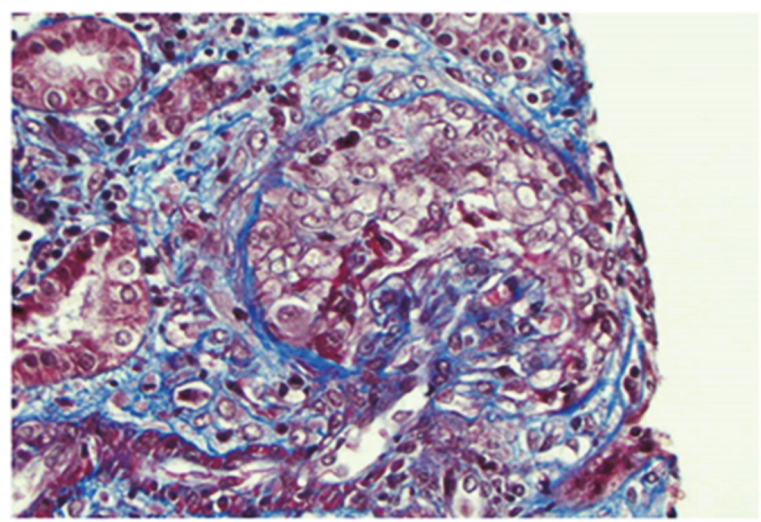

Abstract \#337 Figure 1 Glomerulus with cellular crescents (acute glomerular crescent)

Conclusions ANCA negative pauci-immune $\mathrm{CrGN}$ is an uncommon presentation of kidney damage in the setting of SLE. Despite source, progression of disease ends with end stage renal failure in ANCA positive/ANCA negative $\mathrm{CrGN}$ and LN. We believe ANCA negative CrGN warrants further investigation as it may be considered a separate entity from ANCA positive CrGN, but with no change in current management aside from extrarenal systemic considerations for ANCA positive pauci-immune GN.
\#338 EATING DISORDERS, LAXATIVE ABUSE, URINARY TRACT INFECTION, AND NEPHROLITHIASIS: A CASE REPORT AND REVIEW OF LITERATURE

H Luo*, DM Vigil. University of New Mexico Health Sciences Center, Albuquerque, NM

10.1136/jim-2022-WRMC.334

Case Report Eating disorders are characterized by a persistent disturbance of eating that impairs health or psychosocial functioning. Renal stones are listed among the complications of eating disorders; however, very few cases have been reported.

We present a case of emphysematous pyelonephritis and nephrolithiasis associated with bulimia and laxative abuse. 49year-old woman with a history of bulimia, laxative abuse, urinary tract infections, nephrolithiasis, post-traumatic stress disorder was referred to nephrology for her chronic kidney disease and nephrolithiasis. Patient was diagnosed bulimia and laxative abuse when she was 15 years old, which continued into adulthood. At age 37, she was diagnosed with left kidney hydronephrosis requiring a left ureteral stent. At age 46, she developed a $12 \mathrm{~mm}$ left kidney stone complicated by emphysematous pyelonephritis, requiring an emergent ureteral stent. In our clinic the following labs and imaging were obtained: Creatinine $1.31 \mathrm{mg} / \mathrm{dl}$ (eGFR $44 \mathrm{ml} / \mathrm{min}$ ), Potassium $2.9 \mathrm{mEq} /$ 1, HCO3 $25 \mathrm{mEq} / \mathrm{l}$, Calcium $9.2 \mathrm{mg} / \mathrm{dl}$, Po4 $4.3 \mathrm{mg} / \mathrm{dl}, \mathrm{Mg}$ $2.1 \mathrm{mg} / \mathrm{dl}$. Urinalysis had RBCs, WBCs, and LCE. Urine culture grew Escherichia coli. CT scan showed an atrophic left kidney and a $13 \mathrm{~mm}$ staghorn calculus. Urology performed left ureteroscopy, laser lithotripsy and ureteral stent exchange. Stone analysis notable for mixed ammonium hydrogen urate/ carbonate apatite. Repeat CT scan demonstrated 2 small left non-obstructing lower pole nephroliths. 24-hour urorisk panel showed hypocitraturia and was started on potassium citrate for stone prevention. She was referred to an eating disorder specialist and a dietitian. Her clinical course continues to wax and wane with potassium ranging 2.4 to $4.2 \mathrm{mEq} / \mathrm{l}$, and HCO3 18 to $33 \mathrm{mEq} / \mathrm{l}$.

Eating disorders and laxative abuse often lead to extracellular fluid volume depletion, acid base disorders, hypokalemia, and hypocitraturia, all of which predispose the formation of kidney stones. We present this complex case of nephrolithiasis and pyelonephritis associated with an eating disorder and laxative abuse, attempting to explain the potential mechanisms of formation of staghorn ammonium renal stone involving hypocitraturia and frequent urinary tract infections.

\section{\#339 ARE RENAL SPARING PROTOCOLS SAFE AND EFFECTIVE IN LIEU OF RANDOMIZED TRIALS IN HEART TRANSPLANT?}

${ }^{1} \mathrm{~J} \mathrm{Hu}{ }^{*},{ }^{2} \mathrm{~T}$ Singer-Englar, ${ }^{2} \mathrm{~N}$ Patel, ${ }^{2} \mathrm{~S} \mathrm{Kim},{ }^{2} \mathrm{M}$ Hamilton, ${ }^{2} \mathrm{~J}$ Kobashigawa. ${ }^{1}$ Western University of Health Sciences College of Osteopathic Medicine of the Pacific-Northwest, Lebanon, OR; ${ }^{2}$ Cedars-Sinai Smidt Heart Institute, Los Angeles, CA

\subsection{6/jim-2022-WRMC.335}

Purpose of Study The calcineurin inhibitors (CNIs), including tacrolimus and cyclosporine, have revolutionized outcomes of heart transplant (HTx) patients but unfortunately have many side effects, which include nephrotoxicity, hypertension, and malignancy. It has been recently demonstrated through two large, randomized trials, the SCHEDULE Trial and the 
MANDELA Trial, that weaning of CNIs has resulted in an increase of rejection episodes. Most recently, many HTx centers have been using a modified CNI wean protocol in order to prevent further nephrotoxicity and to avoid kidney dialysis. It appears that an earlier $(<2$ years) renal sparing protocol (RSP) may have more problems compared to later initiation of the protocol (2-5 years after transplant). Therefore, we reviewed our experience in CNI weaning.

Methods Used Between 2010 and 2020, we assessed 34 HTx patients who underwent our RSP beginning after 6 months post-HTx and divided them into those intiated $<2$ year and between 2-5 years after heart transplant. Our protocol for CNI weaning is to decrease the CNI by one-half for 2 weeks, then by an additional one-half for 2 weeks, and then stop. Meanwhile, patients are also started on a proliferation signal inhibitor (PSI), mostly sirolimus, beginning at $1 \mathrm{mg} /$ day and the dose is increased accordingly to maintain a PSI level between 4 and $8 \mathrm{ng} / \mathrm{mL}$. Endpoints included subsequent freedom from any treated rejection (ATR, including acute cellular rejection $[\mathrm{ACR}]$, antibody-mediated rejection [AMR], and biopsy-negative rejection [BNR]), survival, and freedom from cardiac dysfunction (hemodynamic compromise rejection [HCR], defined as left ventricular ejection fraction $\leq 40 \%$ ) in the 1 year following RSP initiation. As CNI wean has also been demonstrated to have beneficial aspects, we also assessed the need for antihypertensive medications, diabetes in terms of hemoglobin A1c, and glomerular filtration rate (GFR).

Summary of Results $89 \%$ of patients were successfully weaned off CNI at $<2$ years and $94 \%$ at $2-5$ years after transplant. However, RSP was not sustained in $50 \%$ of RSP $<2$ year patients and in $31 \%$ of $2-5$ year patients. The reasons included rejection, medication intolerance, and death. GFR for both groups was markedly improved at 1-year post RSP initiation. There was no difference between groups in anti-hypertension medications or hemoglobin A1c levels.

\begin{tabular}{llll} 
Abstract \#339 Table 1 & & & \\
\hline Endpoint & $\begin{array}{l}\text { RSP } \\
\text { initiation } \\
\text { ( } \mathbf{n}=18)\end{array}$ & $\begin{array}{l}\text { RSP initiation 2-5 } \\
\text { years after HTx } \\
\text { ( } \mathbf{n}=16)\end{array}$ & $\begin{array}{l}\text { p- } \\
\text { value }\end{array}$ \\
& $88.9 \%$ & $93.8 \%$ & 1.000 \\
\hline CNI wean successful & $50.0 \%$ & $31.3 \%$ & 0.315 \\
RSP discontinued early & $16.7 \%$ & $0.0 \%$ & 0.230 \\
RSP discontinued due to ACR & $0.0 \%$ & $6.3 \%$ & 0.471 \\
RSP discontinued due to BNR & $16.7 \%$ & $12.5 \%$ & 1.000 \\
RSP discontinued due to death & $11.1 \%$ & $0.0 \%$ & 0.487 \\
RSP discontinued due to medication & & & \\
intolerance & $5.6 \%$ & $12.5 \%$ & 0.591 \\
RSP discontinued due to proteinuria & $1.1 \pm 1.2$ & $1.1 \pm 1.0$ & 0.899 \\
Antihypertensive medications at baseline & & 0.174 \\
Antihypertensive medications 1 year after & $1.3 \pm 1.2$ & $0.8 \pm 0.8$ & 0.146 \\
RSP initiation & & & 0.962 \\
Hemoglobin A1c at baseline & $5.9 \pm 0.6$ & $6.6 \pm 1.1$ & \\
Hemoglobin A1c 1 year after RSP & $5.8 \pm 0.7$ & $5.8 \pm 0.5$ & 0.982 \\
initiation & & & 0.293 \\
GFR at baseline & $36.9 \pm$ & $36.8 \pm 15.6$ & \\
\hline GFR 1 year after RSP initiation & 14.0 & & $53.7 \pm$ \\
& 14.0 & $51.3 \pm 20.4$ & \\
\hline
\end{tabular}

Conclusions CNI weaning at a large, single center appears to be safe and efficacious for select patients but sustaining RSP continues to be a challenge. Further development of other RSPs is warranted.

\section{\#340 TRIPLE WHAMMY CAUSING NEPHRONOPHTHISIS AND ATYPICAL OPHTHALMOLOGIC \& NEUROLOGIC MANIFESTATIONS}

J Froud*, A Egense, SP Shankar. UC Davis Children's Hospital, Sacramento, CA

\subsection{6/jim-2022-WRMC.336}

Case Report We report on an adolescent male with end-stage renal disease, septo-optic dysplasia (SOD), degenerative high myopia, and developmental delays (DD) with 2q13 homozygous deletion and $17 \mathrm{q} 12$ duplication. Nephronophthisis (NPH) is a genetically heterogeneous cystic renal disorder progressing to end-stage renal disease. Mutations in NPHP1 at $2 \mathrm{q} 13$ cause Joubert syndrome (JS) and NPH. JS is characterized by abnormal brain MRI (molar tooth sign), hypotonia, DD, and other multi-systemic features in $<30 \%$ of cases. The $17 \mathrm{q} 12$ microduplication syndrome is characterized by variable features including intellectual disability, hypotonia, seizures, autism, microcephaly, structural brain anomalies (focal cortical dysplasia, periventricular leukomalacia), and eye anomalies such as strabismus, astigmatism, amblyopia, cataract, coloboma, and microphthalmia.

Case Presentation An 11-year-old boy was referred to genetics for molecularly confirmed NPH. He also had DD, learning disabilities, congenital nystagmus, SOD, amblyopia, degenerative high myopia, and hypothyroidism. A brain MRI in infancy showed heterotopia, lissencephaly, and hypoplastic septum pellucidum. Family history was non-contributory. He was diagnosed with stage 3 chronic kidney disease at age 11 when he presented with polydipsia. Renal biopsy confirmed NPH. A nephronophthisis genetic testing panel revealed homozygous NPHP1 deletion. Given his clinical features were unusual for NPHP1 disorder alone, a chromosomal microarray (CMA) was performed. This identified a homozygous $110 \mathrm{~kb}$ deletion at 2q13 involving the NPHP1, MALL, and SMIM37 genes and an additional pathogenic $1.45 \mathrm{Mb}$ duplication at $17 \mathrm{q} 12$. Parental genetic testing was declined.

Discussion We describe the first reported case, to the best of our knowledge, of a patient with a dual diagnosis of NPH due to homozygous NPHP1 deletion and the $17 \mathrm{q} 12$ microduplication syndrome with unique ocular and neurologic findings. Although JS was considered as a potential diagnosis, SOD, degenerative high myopia, and absence of typical findings such as molar tooth sign made JS unlikely, prompting additional genetic evaluation. Homozygous deletions in NPHP1 account for approximately $21 \%$ of all NPH cases and are not typically associated with brain and eye involvement. The $17 \mathrm{q} 12$ duplication syndrome is the likely cause of his DD and learning disabilities. However, his specific MRI and eye findings have not been reported in $17 \mathrm{q} 12$ duplication. Neither NPHP1 deletion nor $17 \mathrm{q} 12$ duplication are known to be associated with SOD. This individual is an example of multiple genetic variations leading to a unique and complex phenotype. Future investigations and similar cases are needed to better understand the genotype/phenotype correlations and interactions. 
\#341 EMERGING ANTICOAGULANTS FOR THE THROMBOPROPHYLAXIS OF ATRIAL FIBRILLATION IN PATIENTS WITH CHRONIC KIDNEY DISEASE

${ }^{1} \mathrm{~K}$ Maii, ${ }^{2} \mathrm{~V}$ Lu, ${ }^{1} \mathrm{M}$ Vu, ${ }^{1} \mathrm{E}$ Jiang, ${ }^{1} \mathrm{~S}$ Noh, ${ }^{1} \mathrm{~K}$ Chen, ${ }^{1} \mathrm{~S}$ Fuchs. ${ }^{1}$ Western University of Health Sciences, Pomona, CA; ${ }^{2}$ Albert Einstein College of Medicine and Montefiore Medical Center, Bronx, NY

\subsection{6/jim-2022-WRMC.337}

Purpose of Study The mainstay treatment for atrial fibrillation (Afib) thromboprophylaxis is anticoagulants. However, their indication in patients with concomitant end stage renal disease (ESRD) remains complex due to increased risks of bleeding and drug-drug interactions. While the American heart association (AHA) guidelines only recommends warfarin in this context, the European Heart Rhythm Association's (EHRA) guideline holds no recommendations currently but promotes an personalized approach factoring in patient preferences and clinical situations. This review aims to bridge this contrast and limitation by investigating novel therapeutic options that may allow for safe anticoagulation in patients with Afib and ESRD. Methods Used We performed a comprehensive literature search for novel anticoagulants studied in the context of Afib and CKD. The databases Pubmed, ScienceDirect and Googler scholars were utilized with articles limited to a publication date of 2010 onward. Further exclusion criteria included all drugs before 2018 that are FDA approved for use in ESRD and already reviewed in the current US and European national guidelines.

Summary of Results The four anticoagulants we found matching our inclusion criteria includes Betrixaban, Fondaparinux, Tecafarin and Ichorcumab. Tecarfarin has most of the benefits associated with warfarin but with less drug-drug interaction and a decrease kidney dependent metabolism. Fondaparinux and betrixaban have both demonstrated advantages in the context of lower dosage control and a lack of need for laboratory monitoring. Studies have also demonstrated a reduction in duration of hospital stay with Fondaparinux. Ichorcumab has also demonstrated the potential as a safer alternative for those at higher risks of bleeding.

Conclusions Our review suggests there are multiple novel anticoagulants that may diversify the current EHRA and AHA recommendations for patients with concomitant Afib and ESRD. Although these therapies have demonstrated non-inferiority to warfarin, higher level trials are needed to further establish their therapeutic index and efficacy in the context of Afib and ESRD.

\section{Neuroscience II}

\section{Concurrent session}

\section{0:15 AM}

\section{Friday, January 21, 2022}

\section{\#342 WHITE MATTER METABOLITE RATIOS PREDICT COGNITIVE OUTCOME IN PEDIATRIC TRAUMATIC BRAIN INJURY}

'L Berger*, 'B Bartnik-Olsen, ${ }^{2} S$ Ashwal. 'Loma Linda University School of Medicine, Loma Linda, CA; ${ }^{2}$ Loma Linda University Medical Center, Loma Linda, CA
Purpose of Study The rapid acceleration-deceleration motion in traumatic brain injury (TBI) generates widespread mechanical shearing forces resulting in diffuse axonal injury and downstream metabolic consequences. This mechanism predisposes regions with high axon density (i.e. white matter (WM) to greater burden of injury. Metabolite changes are well described in adult TBI, however less is known about the longitudinal consequences in the pediatric population. The purpose of this study was to evaluate the prognostic ability of global WM and GM metabolite ratios following pediatric TBI and their relationship to 12 month neuropsychological assessments of IQ, attention, and memory.

Methods Used 3D proton magnetic resonance spectroscopic imaging (MRSI) in pediatric patients with complicated mild (cMild), moderate, and severe TBI was acquired acutely (6-18 days) and 12-months post injury and compared to agematched normal developing adolescents. A global linear regression model, co-registering MRSI metabolite maps with 3D high resolution magnetic resonance images was used to identify longitudinal white and gray matter metabolite ratio changes.

Summary of Results Acutely, GM NAA/Cr, WM NAA/Cr and WM NAA/Cho ratios were significantly lower in all TBI groups, compared to controls (ANCOVA, $\mathrm{p} \leq 0.02$; figure A, B). GM NAA/Cho was reduced only in the severe TBI group ( $\mathrm{p} \leq 0.001)$. At 12 months, all metabolite ratios normalized to control levels in each of the TBI groups (figure C, D). Acute GM and WM NAA ratios were strongly correlated to 12month assessments of IQ, attention, and memory (Pearson correlation with FDR, $\mathrm{q}<0.001$ ).
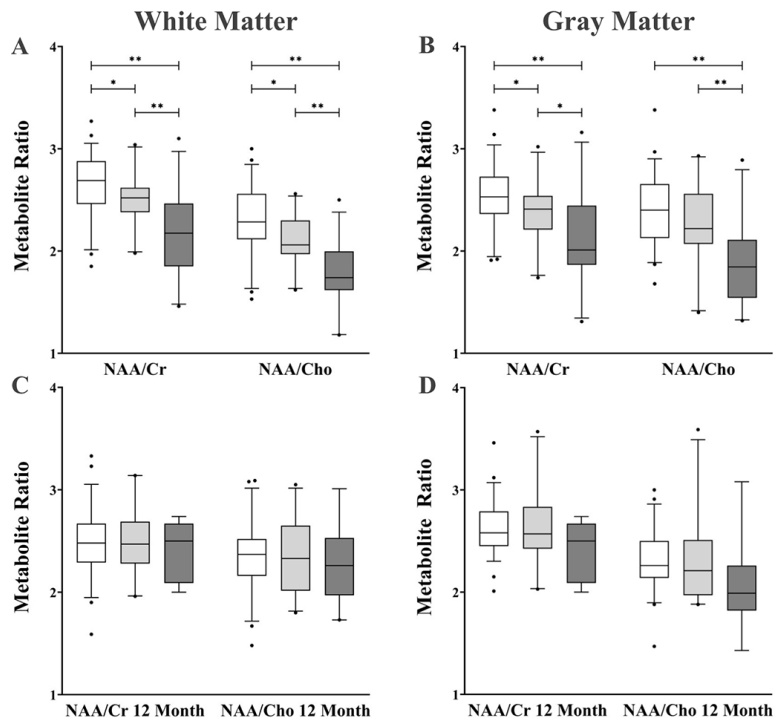

Abstract \#342 Figure 1 Acute $(A, B)$ and 12 month $(C, D)$ white matter (WM) and gray matter (GM) NAA/Cr and NAA/Cho ratios for control (white), mild/moderate TBI (light gray), and severe TBI subjects (dark gray)

Conclusions These findings suggest that whole brain GM and WM metabolite ratios reflect longitudinal changes in neuronal metabolism following TBI, which can be used to predict neuropsychological outcome in pediatric patients. 


\section{\#343 METABOLIC-DRIVEN ANALYTICS OF PYRUVATE TREATMENT AFTER TBI DETECT MAJOR BENEFITS IN ANTI-OXIDANT AND ANTI-INFLAMMATORY PATHWAYS}

${ }^{1} \mathrm{~N}$ Golovachev*, 'L Siebold, ${ }^{1} \mathrm{~J}$ Tan, ${ }^{2} \mathrm{~N}$ Harris, ${ }^{1} \mathrm{~B}$ Bartnik-Olsen. 'Loma Linda University School of Medicine, Loma Linda, CA; ${ }^{2}$ University of California Los Angeles David Geffen School of Medicine, Los Angeles, CA

\subsection{6/jim-2022-WRMC.339}

Purpose of Study The purpose of this study was to elucidate the metabolic pathways affected by ethyl pyruvate (EP) given 24 hours after experimental traumatic brain injury (TBI).

Methods Used 18 male rats were randomized into vehicletreated controlled cortical impact $(\mathrm{CCI} ; \mathrm{N}=9)$ or ethyl pyruvate-treated CCI $(\mathrm{N}=9)$ groups. Moderate to severe CCI injury was induced in the left parietal cortex following a $2.0 \mathrm{~mm}$ cortical deformation via pneumatic piston. The vehicle group (CCI Veh) was injected with $0.1 \mathrm{M}$ phosphatebuffered saline (PBS) and the EP treatment group (CCI EP) was injected with EP (40mg/kg, i.p.) at 0, 1, 3 and 6 hours post-injury. At 24 hours post-injury, rats were euthanized by decapitation under deep isoflurane anesthesia and tissue samples were collected from the left cortex and frozen/powdered in liquid nitrogen. Samples were shipped to Metabolon, Inc., (Durham, NC) and prepared for analyses of global metabolic profiles using Ultrahigh Performance Liquid Chromatography-Tandem Mass Spectroscopy (UPLC-MS/MS) or Gas Chromatography-Mass Spectroscopy (GC-MS). MetaboAnalyst 5.0, GraphPad Prism (Version 9.2), and Sketch (Version 75) were used for statistical analyses and figure composition.

Summary of Results Sample analysis produced 380 detected metabolites. Cystine, ascorbate (vitamin C), nicotinamide ribonucleotide (NMN), adenosine 5'-monophosphate (AMP), and adenosine from the ipsilateral cortex were all significantly altered following EP treatment 24 hours post-injury (greater than 2-fold change compared to vehicle-treated animals). The top 15 metabolites with the greatest importance from random forest analysis, along with a heatmap, are depicted in figure 1. The top 15 altered pathways associated with EP treatment are depicted in table 1 , with pyruvate metabolism and TCA cycle metabolism demonstrating the lowest Holm corrected pvalue. Other significant metabolic pathways include purine, cysteine and methionine, and glutathione metabolism.

Conclusions This dosing regime of EP alters metabolic pathways associated with anti-oxidation, anti-inflammation, and metabolism that may contribute to its neuroprotective effects.
Abstract \#343 Table 1 Significantly altered pathways after EP treatment 24 hours after TBI. Pathways with $>1$ metabolite hit and $>0$ impact score. Table sorted by Holm $p$ value. A metabolite hit is defined as a significantly altered metabolite in the pathway of interest. The pathway impact is calculated as the sum of the importance measures of the matched metabolites normalized by the sum of the importance measures of all metabolites. AcetylCoA is seen as a key metabolite present in multiple pathways.

\begin{tabular}{|c|c|c|c|}
\hline Enriched Pathways & Holm p & Impact & Metabolite Hits \\
\hline Pyruvate metabolism & 0.0056112 & 0.15397 & Acetyl-CoA; Phosphoenolpyruvate \\
\hline Citrate cycle (TCA cycle) & 0.0056112 & 0.03668 & Acetyl-CoA; Phosphoenolpyruvate \\
\hline Fatty acid elongation & 0.01706 & 0.03668 & Acetyl-CoA \\
\hline Fatty acid degradation & 0.01706 & 0.18092 & Acetyl-CoA \\
\hline beta-Alanine metabolism & 0.01706 & 0.05597 & Acetyl-CoA \\
\hline $\begin{array}{l}\text { Valine, leucine and } \\
\text { isoleucine degradation }\end{array}$ & 0.01706 & 0.02836 & Acetyl-CoA \\
\hline Propanoate metabolism & 0.01706 & 0.01269 & Acetyl-CoA \\
\hline Fatty acid biosynthesis & 0.01706 & 0.00213 & Acetyl-CoA \\
\hline Glycolysis/Gluconeogenesis & 0.01706 & 0.24574 & $\begin{array}{l}\text { Acetyl-CoA; 2-Phospho-D-glycerate; } \\
\text { Phosphoenolpyruvate }\end{array}$ \\
\hline Purine metabolism & 0.017575 & 0.05989 & $\begin{array}{l}\text { Adenosine 5'-monophosphate; } \\
\text { Adenosine }\end{array}$ \\
\hline $\begin{array}{l}\text { Glyoxylate and } \\
\text { dicarboxylate metabolism }\end{array}$ & 0.017575 & 0.00794 & Acetyl-CoA; 2-Phospho-D-glycerate \\
\hline $\begin{array}{l}\text { Cysteine and methionine } \\
\text { metabolism }\end{array}$ & 0.017586 & 0.09592 & L-Cystine; L-Cysteine \\
\hline $\begin{array}{l}\text { Glycine, serine and } \\
\text { threonine metabolism }\end{array}$ & 0.026461 & 0.00241 & 2-Phospho-D-glycerate; L-Cysteine \\
\hline Glutathione metabolism & 0.051481 & 0.25939 & 2-Phospho-D-glycerate; L-Cysteine \\
\hline $\begin{array}{l}\text { Nicotinate and } \\
\text { nicotinamide metabolism }\end{array}$ & 0.054464 & 0.03158 & Nicotinamide D-ribonucleotide \\
\hline
\end{tabular}

\section{\#344 METHYLPREDNISOLONE INDUCES INCREASED NEURONAL TISSUE SPARING AFTER SPINAL CORD INJURY IN A RODENT MODEL}

M Solorio*, T Prasse, J Bergquist, ZZ Khaing, CP Hofstetter. University of Washington, Seattle, WA

\subsection{6/jim-2022-WRMC.340}

Purpose of Study Currently, there is no universally accepted neuroprotective drug for traumatic spinal cord injuries (tSCI) in the clinic. Our objective is to identify a treatment to use during the acute phase aimed at limiting neuronal tissue loss after SCI. Previous studies have shown that

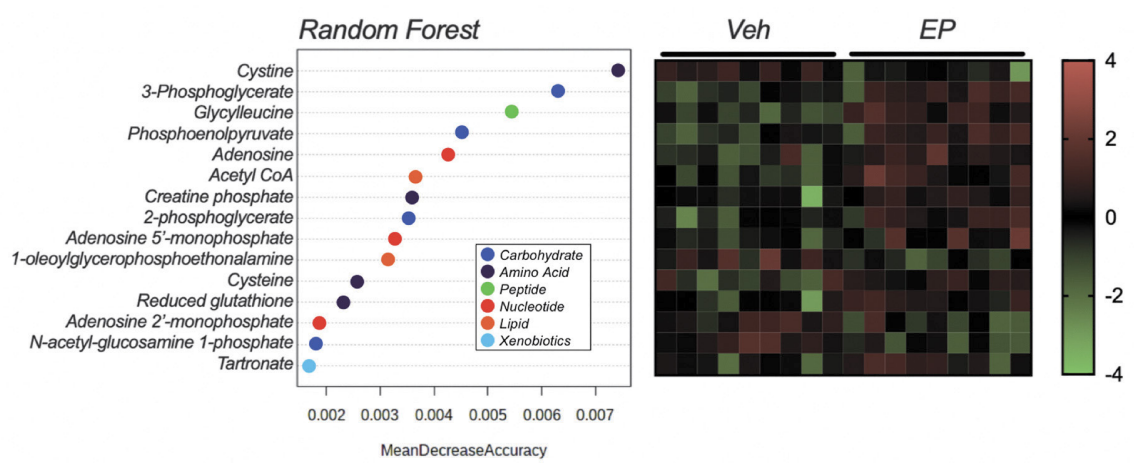

Abstract \#343 Figure 1 The top 15 metabolites with the greatest importance from random forest analysis, along with a heatmap 
methylprednisolone (MPSS) has neuroprotective effects, possibly through its anti-inflammatory properties. Here we analyze the effects of acute MPSS administration on tissue sparing after a cervical tSCI in a rodent model.

Methods Used We used 16 adult female rats randomized into three different groups: laminectomy only $(n=3)$, SCI only $(n=8)$, SCI+MPSS treatment $(n=5)$. They underwent a longitudinal incision over $\mathrm{C} 3-\mathrm{C} 7$, then a laminectomy to expose the spinal cord at the C5 level. The rats that were in the SCI only group and the SCI+MPSS group, underwent a midline injury using an Infinite Horizon impactor at $200 \mathrm{kdyn}$. Rats that were in SCI+MPSS treatment group received one immediate post injury $(60 \mathrm{mg} / \mathrm{kg}$ body weight) dose and three additional intravenous boluses (30 mg/kg body weight) of MPSS every two hours after injury. After the SCI, all animals had behavioral testing using functional locomotor (FL) scoring done 7, 10 and 14 (days post injury) dpi. Tissue sparing analysis was performed by measuring the lesion area of cross-sectional slices after immunohistochemistry (IHC) staining, which visualized anti-GFAP and anti-CD68.

Summary of Results All the injured animals showed forelimb motor function improvement over time after injury. However, there was no statistically significant difference in between any of the injured groups. Tissue sparing analysis showed that animals in the SCI+MPSS group had a significantly higher mean tissue sparing percentage of $86 \%$ [ \pm 14.2]. Animals in the SCI only group had a mean tissue sparing percentage of $76 \%$ $[ \pm 18.5]$ at the lesion area $(p<0.0001)$. Lesion area analysis also showed a significantly larger area of damage detected in SCI only animals with a mean difference of $\left[0.82 \mathrm{~mm}^{2}\right]$ compared to animals in SCI+MPSS group ( $p<0.0001$ ).

Conclusions These results show promising neuroprotective properties of MPSS becoming effective by promoting acute tissue sparing when given immediately after SCI, which implies increased neuronal function. Although there was no statistically significant difference in behavioral analysis between treatment groups, this is most likely due to the comparably short survival time and a similar extent of injury in all groups. More research is needed to identify new strategies to minimize the systemic side effects of MPSS and optimize the local effective dose.

\section{\#345 ATYPICAL PARSONAGE-TURNER SYNDROME INVOLVING CRANIAL NERVES 8, 9, 10, 11 AND THE PHRENIC NERVE}

${ }^{1} \mathrm{C}$ Munhall ${ }^{*}{ }^{2} \mathrm{~K}$ Heiland. ${ }^{1}$ The University of Arizona College of Medicine Phoenix, Phoenix, $A Z ;{ }^{2}$ HonorHealth, Scottsdale, $A Z$

10.1136/jim-2022-WRMC.341

Case Report Parsonage-Turner syndrome, also commonly referred to as Neuralgic Amyotrophy (NA), is a condition leading to symptoms centered around the brachial plexus. It often arises as abrupt unilateral shoulder pain, followed by paresthesias and progressive weakness. Despite unclear etiology, NA has been associated with postinfectious, postsurgical, post-vaccination, and posttraumatic states. Patients are usually treated with pain management and physical therapy and usually exhibit good long-term recovery.

Although classicaly involving the brachial plexus, cases have been documented involving a variety of other nerves. A 2016 review in the journal of Neurology and Neurophysiology found only 22 cases with cranial nerve involvement reported in the English literature to that date. CN 10 was most commonly involved and presented most often as a laryngeal nerve palsy. The authors noted phrenic involvement in 6 out of 22 cases, three of which were asymptomatic and found via chest $\mathrm{x}$-ray. This case report highlights a unique presentation of NA which exhibited involvement of cranial nerves $(\mathrm{CN})$ 8, 9, 10, and 11 as well as phrenic nerve involvement.

Case A 70 year old man with a history of moderate bilateral sensorineural hearing loss (SNHL) vaccination. Flexible laryngoscopic exam demonstrated a sluggish true left vocal fold with impaired adduction. Imaging of the chest and neck showed no lesion impinging on $\mathrm{CN} 10$, but demonstrated diaphragmatic paralysis confirmed with fluoroscopy.

The patient followed up several months later reporting new dysphagia and microaspiration. The patient was noted to have velopharyngeal insufficiency with reflux of liquids into the nasopharynx, left soft palate paresis, and right uvular deviation suggesting impairment of left $\mathrm{CN} 9$ in addition to $\mathrm{CN}$ 10. Audologic evaluation revealed worsening of left SNHL from moderate to severe to now profound, representing involvement of $\mathrm{CN}$ 8. A neurologist additionally noted weakness of the left trapezius muscle consistent with a CN 11 deficit.

After 2 years of ongoing dysphonia and microaspiration despite speech therapy, the patient received a left true vocal fold injection which improved his dysphonia and aspiration risk. The patient noted ongoing dysphagia, and subsequent transesophageal manometry demonstrated absent esophageal peristalsis consistent with left $\mathrm{CN} 10$ dysfunction. The involvement of left $\mathrm{CN} \mathrm{8,9,10,11,} \mathrm{and} \mathrm{phrenic} \mathrm{nerve} \mathrm{in}$ addition to brachial plexopathy was a uniquely atypical presentation of NA. The atypical involvement of multiple cranial nerves in a variety of neurologic syndromes, including NA, Bell's palsy, Guillan-Barré, and many others should be a clinical consideration for physicians when evaluating patients with multiple cranial nerve palsies.

\section{\#346 SCREEN TIME AND CHANGES TO OCULO- PHYSIOLOGICAL MEASUREMENTS}

K Inouye* ${ }^{*}$ P Davey. Western University of Health Sciences, Pomona, CA

\subsection{6/jim-2022-WRMC.342}

Purpose of Study Due to the COVID-19 pandemic, the average amount of time spent in front of a screen has spiked considerably. Prior studies suggested that reading standardized text/ font from an iPad causes measurable physiological and visual changes compared to paper. This is particularly evident if digital devices used at nighttime are leading to sleep disturbances. What is not known is if the use of digital devices during the day leads to measurable physiological changes compared to reading print media.

Methods Used We recruited 30 volunteers to evaluate whether these changes could be observed in 30 minutes using a cognitively demanding reading task. These volunteers were asked to do a total of 3 readings: iPad in well-lit room, paper in welllit room, and iPad in dark room. Physiological and visual measurements were taken before and after readings, and patients were asked to fill out a dry eye questionnaire before and after each visit. 
Summary of Results Our results showed a significant increase in dry eye symptoms across all readings $(p<0.05)$, although there was no significant difference between symptoms for iPad and paper when reading in a well-lit room $(p=0.89)$. We also noted decreased blood pressure across all three readings, increased contrast sensitivity with paper and decreased contrast sensitivity with iPad in a well-lit room, and decreased blue cone sensitivity with iPad in a dark room.

Conclusions Based on the parameters measured, we can surmise that 30 minutes of reading a cognitively demanding reading task is not enough time to evoke the previously observed physiological response to screens, although the visual changes were consistent. The results of the dry eye symptom questionnaire suggest that for shorter periods, ambient light might alleviate ocular fatigue that was noted when reading from the iPad in a dark room.

\section{Surgery IV}

\section{Concurrent session}

\section{0:15 AM}

\section{Friday, January 21, 2022}

\section{\#347 TIME OF ONSET AND DIAGNOSIS OF VOICE DISORDERS IN PATIENTS WITH CHRONIC COUGH}

${ }^{1} \mathrm{JF}$ Kim*, ${ }^{2,3} \mathrm{~T}$ Murry, ${ }^{2} \mathrm{P}$ Krishna, ${ }^{3} \mathrm{R}$ Saab, ${ }^{2} \mathrm{~B}$ Crawley. ${ }^{1}$ Loma Linda University School of Medicine, Loma Linda, $C A ;{ }^{2}$ Loma Linda University Adventist Health Sciences Center, Loma Linda, $C A ;{ }^{3}$ Drexel University College of Medicine, Philadelphia, PA

\subsection{6/jim-2022-WRMC.343}

Purpose of Study Chronic cough (CC), defined as cough lasting at least eight weeks, is a complaint of up to $46 \%$ of patients referred to specialist clinics. Previously, studies have reported a relationship between CC and voice disorders. However, studies have not reported on the specific diagnosis of the voice disorder or on the time relationship between the patients' first voice complaint and the voice diagnosis made by an otolaryngologist. The data in this study examine complaints of voice changes (hoarseness) and the length of time before a diagnosis of the voice disorder.

Methods Used The first complaint of hoarseness or voice changes in 105 patients with a diagnosis of CC was identified from medical records between January 2015 and February 2020. The diagnoses of the voice disorders were determined following referral to an otolaryngologist. Inferential statistics were used to determine differences in gender, age, types of voice disorders, and the time between voice complaint and the voice diagnosis. Common comorbidities were also documented.

Summary of Results Of the 105 patients, 18 males and 87 females, data were available for 88 patients to determine that the average time between complaint of hoarseness and diagnosis of a specific voice disorder was 32.3 months (Range: 1180 months). There was no difference in age between males and females $(p=0.05)$. For the group, the most common diagnoses were neurogenic voice disorders, namely, vocal fold paresis $(n=47)$ and vocal fold atrophy $(n=28)$. Common comorbidities included allergies, gastroesophageal reflux, and airway disorders.
Conclusions This study adds to the complexity of treating chronic cough. The results of the present study suggest that neurogenic voice disorders are the most common voice diagnoses in patients with CC. Other treatments that failed to resolve the CC were explored prior to referral to an otolaryngology clinic. In reviewing the medical records, we found that patients were often referred to other specialties for treatment of gastroesophageal reflux, allergies, and various airway disorders despite their initial voice complaints. This study points out the need for team management, including an otolaryngologist, in patients experiencing hoarseness, dysphonia, or other common voice complaints along with the primary complaint of cough. The range of voice disorders and comorbidities in the cohort also demonstrates the complexity of diagnoses of chronic cough.

\section{\#348 SEQUENTIAL INTRARTICULAR CORTICOSTEROID AND HYALURONIC ACID PROVIDES SIGNIFICANT KNEE OSTEOARTHRITIS SYMPTOM IMPROVEMENT}

${ }^{1} \mathrm{BS}$ Andrew*, ${ }^{2} \mathrm{TJ}$ Robinson, ${ }^{1} \mathrm{~J}$ McGinley. 'University of Washington School of Medicine, Seattle, WA; ${ }^{2}$ University of Wyoming College of Arts and Sciences, Laramie, WY

\subsection{6/jim-2022-WRMC.344}

Purpose of Study Pain related to knee osteoarthritis (OA) is a common and growing presentation to medical facilities. Intraarticular hyaluronic acid (HA) injections along with intraarticular corticosteroid injections represent minimally invasive and cost-effective treatment options. In rare cases, the use of hyaluronic acid has resulted in pseudo sepsis. Our study evaluated the efficacy of combined corticosteroid and HA injection in treatment of pain associated with knee OA. In addition, we hypothesize that corticosteroid preceding $\mathrm{HA}$ injections can reduce the incidence of pseudo sepsis.

Methods Used A retrospective chart review was conducted to identify patients with pain related to knee OA treated with corticosteroid and HA (177 patients, average age 75 years, 80 females and 97 males) as well as corticosteroid alone (296 patients, average age 65 years, 216 females and 80 males). Under sonographic guidance, a corticosteroid (Group \#1 - 40 mg Depo-Medrol, 25 patients, 40 mg Kenalog, 152 patients; Group \#2 - 40 mg Depo-Medrol, 51 patients, 40 mg Kenalog, 241 patients, $4 \mathrm{mg}$ Dexamethasone, 1 patient) was injected into the knee joint. In Group \#1, under sonographic guidance, HA (Synvisc-One ${ }^{\circledR} 48 \mathrm{mg}$ ) was injected 10 \pm 8 days following the corticosteroid. A $0-10$ patient selfreported pain scale was used as the primary outcome. For statistical analysis a repeated measures mixed effects model was used to fit the percent pain reduction values. Secondary outcomes included adverse events (pseudo sepsis) and additional treatments. Pain scores were collected prior to treatment and at 1 month, 3 months, 6 months, and 12 months post-treatment.

Summary of Results Reported pain level was reduced at all time points in both groups compared to pre-treatment levels. The overall percent reduction in pain was greater in Group 1 compared to Group 2 at all recorded time points. At one year post treatment, there was an average of $45 \%$ pain reduction of Group 1 compared to $34 \%$ in Group 2. No adverse events were reported.

Conclusions Combined intraarticular corticosteroid and HA injections demonstrated improvement in pain reduction 
compared to corticosteroid alone up to one year post treatment. In addition, the combined therapy resulted in no reported cases of pseudo sepsis. Based on our study, combined corticosteroid and HA injection represents a safe and effective treatment option in patients suffering from knee OA pain.

\section{\#349 RURAL GENERAL SURGERY CHALLENGES AND RESOURCE LIMITATIONS IN WASHINGTON STATE}

KC Larson*, V Lobova, S Lewis, AS Hoppe. Washington State University Elson S Floyd College of Medicine, Spokane, WA

\subsection{6/jim-2022-WRMC.345}

Purpose of Study Rural general surgeons operate in an environment that differs significantly from colleagues practicing in urban or academic settings. Challenges experienced by this surgical subgroup will be unique and may not be elucidated unless intentionally identified. Understanding their challenges provides insight into their research priorities and how we can best support this distinct group of surgeons going forward. As an initial starting point, we seek to form connections with and among these surgeons through personal interviews and site visits. Our end goal is creating a consortium of rural general surgeons for Washington state.

Methods Used We conducted a pilot mixed-methods study to explore the challenges and research priorities of rural general surgeons in Washington State. Critical access hospitals (CAH) were identified by the Washington State Department of Health having met the criteria outlined by federal designation under the Rural Hospital Flexibility Program. Surgeons practicing at CAHs were identified using online facility directories and through connections via faculty and CEOs/CMOs. Communication included email, phone calls, and mailed letters. Once identified, part 1 consisted of virtual, semi-structured interviews and surveys. Part 2 included comprehensive in-person interviews and site visits to the facility. Part 3 will include creating a consortium of rural general surgeons and further analysis of the qualitative data.

Summary of Results We contacted 79 rural general surgeons in Washington resulting in 25 interviews, 17 completed surveys, and 3 in-person site visits. From the interviews, Washington rural general surgeons identified the following areas of concern: blood bank and operating room supply limitations, adequacy of resident training for solo rural practice, limited staff and nurse training, minimal peer support, restricted medical subspecialty consultation, and high surgeon attrition due to high turnover of both surgeons and staff.

Conclusions We established a myriad of systematic healthcare concerns regarding providing quality rural general surgery care in the state of Washington. We hope to utilize this research to raise awareness of the current challenges and investigate solutions to mitigate the challenges in rural general surgery.

\section{\#350 COMPARISON OF MULTIPORT VERSUS SINGLE PORT VIDEO ASSISTED THORACOSCOPIC SURGERY FOR PEDIATRIC PNEUMOTHORAX}

MA Sacks*, L Goodman, YS Mendez, FA Khan, A Radulescu. Loma Linda University Medical Center, Loma Linda, CA

10.1136/jim-2022-WRMC.346
Purpose of Study The purpose of this study was to compare the operative outcomes of single port and multiport video assisted thoracoscopic surgery(VATS) for primary spontaneous pneumothorax (PSP).

Methods Used After Institutional Board Review (IRB) approval, a retrospective study of a single surgeon's experienced using the thoracoscopic technique during a two-year period reviewed thoracoscopic cases for PSP in children ( $<18$ years). Seven cases classified into three traditional multiport VATS (MPVATS) and four single port VATS (SP-VATS). Times were compared between initial chest tube placement, surgery, chest tube removal, discharge, and follow up. Morphine Milligram Equivalents (MME) were calculated for opioids using the opioid conversion guide by the Center for Disease Control.

Summary of Results Seven patients were included in the study. The median age was 14.88 years [range 12-17 years], but similar between the groups. Both groups had predominant male presence $67 \% \mathrm{MP}$ and $75 \%$ SP. The average time from chest tube placement to surgery was 3.6 days (MP 4.32 vs SP 3.06, $\mathrm{p}=0.21$ ). Operating time averaged 1.2 hours and was similar between the groups: MP 1.04 vs SP 1.33 hours $(\mathrm{p}=0.09)$. The estimated blood loss was higher for MP $5.33 \mathrm{ml}$ vs SP $2.25 \mathrm{ml}(\mathrm{p}=0.11)$. Intraoperative pain medications were delivered weight-based including intravenous acetaminophen and intradermal $0.25 \%$ bupivacaine hydrochloride and epinephrine. MME were similar intraoperative and postoperative until chest tube removal. The chest tube removal occurred at 5.54 vs 3.59 days MP vs SP respectively ("p $<0.05)$. Length of stay was 10.46 vs 8.33 days for MP and SP $(p=0.30)$. In an average of 10 months follow up, one SP patient had a recurrent pneumothorax after chest tube removal, requiring replacement of chest tube, and one MP patient required an additional surgery for contralateral PSP.

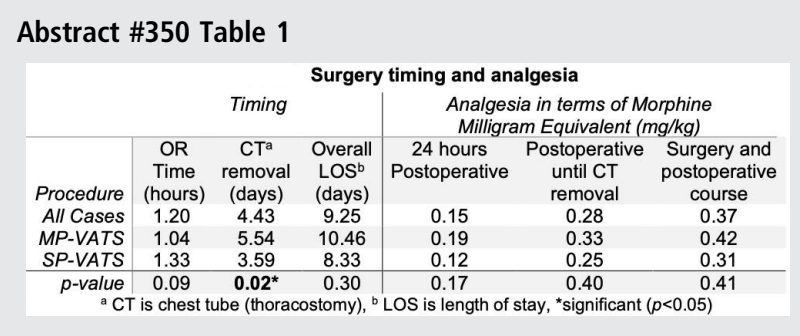

Conclusions In this small case series, outcomes were not significantly different for single port when compared with traditional multiport VATS for PSP. This provides a convincing basis to expand the usage for this technique and analyze with a larger group.

\section{\#351 FAMILY PRESENCE IN THE OPERATING ROOM}

${ }^{1}$ SK Kamra* ${ }^{2} \mathrm{Z}$ Quinonez, ${ }^{3} \mathrm{~K}$ Pyke-Grimm, ${ }^{2,3} \mathrm{D}$ Char. ${ }^{1}$ Pomona College, Claremont, CA; ${ }^{2}$ Stanford Medicine, Stanford, CA; ${ }^{3}$ Lucile Salter Packard Children's Hospital at Stanford, Palo Alto, $C A$

10.1136/jim-2022-WRMC.347

Purpose of Study Care locations are becoming more fluid, with patients often moving between emergency room (ER), imaging, operating room (OR), and intensive care unit (ICU) for 
resuscitations and diagnostic procedures which will inform acute decisions. Locations like the ICU and ER often have spaces that can be rapidly converted from bedspace into operating rooms. With family presence (FP) already allowed in some of these locations, should (and if so in what contexts) families be allowed to be present in the OR similar to how they are being allowed in these other care locations?

Congenital Heart Disease (CHD) is the most common type of birth defect leading to critical illness and death in the US. Given the complexity of disease, these children repeatedly undergo invasive diagnostic and therapeutic procedures. Families often want to be present to support their chidren through these procedures, and want as much information as they can gather to inform difficult clinical care choices. We assessed the perceptions of clinicians caring for children with $\mathrm{CHD}$ about $\mathrm{FP}$ in the OR.

Methods Used We conducted semi-structured interviews of 7 clinicians (anesthesiologists, nurses, and surgeons) at a highvolume pediatric cardiac surgical center. We discussed previous experience with and understanding of FP, perceptions toward FP in the OR, and support needed for implementing FP to the OR. Responses were analyzed using grounded theory and a codebook was developed.

Summary of Results Several significant themes emerged including: the role of family member emotions on FP success; potential benefit of FP on reducing patient anxiety and fear, with concomitant reduction in need for sedation medications; whether FP is needed in contexts when the patient is unconscious; and, if FP might distract clinicians. Clinicians also expressed the need for structural changes to support FP, particularly cultivating necessary staff who could explain what is going on to the families and policy for who is responsible for the family if they are present during procedures.

Conclusions This pilot study suggest potential benefits of FP in OR for children with CHD: 1) enhancing family understanding of clinical care to inform decision-making; 2) reducing patient anxiety and need for anesthetics or restraints; and, 3) end-of-life or serious situations where family perceptions of grief may be positively impacted by FP. However, these benefits are mitigated by possible harms: 1) medical-legal concerns will need to be addressed so clinicians feel comfortable being observed; and, 2) distractions to the care team by FP, including self-consciousness, lack of training managing families' emotions, and the possibility that certain families' emotional states may worsen patient and team distress with consequent impact on clinicians and critical clinical workflows.

\section{\#352 CASE REPORT: ANESTHESIA MUMPS FOLLOWING OPERATIVE REPAIR OF A TRAUMATIC ORTHOPEDIC INJURY}

${ }^{1} \mathrm{H}$ Hajeh*, ${ }^{2} \mathrm{~J}$ Miller, ${ }^{1} \mathrm{M}$ Gill, ${ }^{1} \mathrm{~J}$ Bhandohal. ${ }^{1}$ Kern Medical Center, Bakersfield, CA; ${ }^{2}$ Ross University School of Medicine, Miramar, FL

\subsection{6/jim-2022-WRMC.348}

Case Report A 25-year-old male presented to the emergency department after sustaining a gunshot wound to the left elbow. On presentation, the patient endorsed elbow pain and physical exam revealed swelling and bleeding from the site of injury. X-ray imaging showed a comminuted, intra-articular fracture of the distal humerus with fragments of shrapnel. Patient was sent to the operating room for open reduction



Abstract \#352 Figure 1

and internal fixation, ulnar nerve neuroplasty and fragmented bullet extraction. The surgery lasted 2 hours with no operative complications. After 8 hours postoperatively, the patient complained of swelling of the right cheek. He denied any pain or loss of sensation other than mild 'soreness and tension'. Physical examination showed a right parotid swelling with $9.3 \mathrm{~cm}$ craniocaudal and $6.2 \mathrm{~cm}$ ventrodorsal dimensions.

Facial nerve assessment was normal with no restriction in the temporomandibular joint movement. The enlargement was firm, mobile, with no warmth nor erythema. No tenderness was elicited on palpation. Immunization records weren't available and the patient was unable to recall his vaccination history including Mumps, Measles or Rubella. Mumps antibodies in serum test showed Mumps IgG antibodies concentration of 27.70 $\mathrm{AU} / \mathrm{mL}$ and $\operatorname{IgM}$ antibodies titre of $<1: 20$ denoting immunity and no acute infection. Of note, the patient denied any past postsurgical complications including similar parotid enlargements. A clinical diagnosis of "anesthesia mumps" was made and the patient was managed with supportive care. On postoperative day two, the parotid gland remained swollen, however markedly decreased in size from the day prior. The patient remained otherwise asymptomatic, denying pain or discomfort. With this significant clinical improvement, the patient was discharged before complete resolution of the swelling due to the benign nature of the condition. He was scheduled for a follow up in the clinic, however the patient cancelled the appointment. A phone call appointment was arranged and the patient endorsed reduction in the size of the swelling and complete resolution 2 days after discharge.

Conclusion Although cases of anesthesia mumps are usually benign with no treatment required, they remain stressful for the patient and the physician alike if the benign nature of the condition wasn't recognized. This warrants spreading awareness among physicians on this case and assuring the patients that such conditions are benign and complete resolution is expected.

\section{\#353 UNUSUAL CASE OF PRIMARY SMALL BOWEL BEZOAR CAUSING INTUSSUSCEPTION}

${ }^{1}$ MM Won*, ${ }^{2}$ MA Sacks, ${ }^{1}$ RM Leigh, ${ }^{2}$ L Goodman, ${ }^{2} E$ Tagge, ${ }^{2}$ A Radulescu. ' Loma Linda University School of Medicine, Loma Linda, CA; ${ }^{2}$ Loma Linda University Children's Hospital, Loma Linda, CA

\subsection{6/jim-2022-WRMC.349}

Case Report Intussusception, the prolapse of one section of intestine into another, is a common cause of small bowel obstruction in pediatric patients. Bezoars are concretions of ingested foreign material. Trichobezoars, bezoars made of hair, more commonly occur in female pediatric patients. If gastric 
trichobezoars grow to sufficient size, 'Rapunzel syndrome' may occur as the bezoar extends into the intestines, creating lead points for intussusception to occur. Rarely, hair passes completely through the stomach and forms a trichobezoar within the small bowel. This obstruction can also create lead points and cause intussusception. This is the third recorded case of intussusception due to a primary intestinal bezoar.

Case Presentation We present the case of an 8-year-old male with a preliminary diagnosis of appendicitis. Upon further imaging, bowel obstruction related to a small bowel to small bowel intussusception was discovered to be the probable cause. Diagnostic laparoscopy revealed an ileo-ileal intussusception caused by an ileal bezoar, with subsequent evacuation of the causative bezoar.

Conclusion This is the second reported case of an isolated ileal trichobezoar causing intussusception. While intussusception secondary to a trichobezoar most commonly occurs due to Rapunzel syndrome, it is still possible for a intestinal trichobezoar to be present without an associated gastric trichobezoar.

\section{\#354 COMPLEX LOWER LEFT LEG DEFORMITY CORRECTION WITH PRECICE SYSTEM: A CASE REPORT AND REVIEW OF THE LITERATURE}

${ }^{1} \mathrm{M}$ Porter* ${ }^{*}{ }^{1,2} \mathrm{AE}$ Telis, ${ }^{1} \mathrm{MG}$ Johnston. ${ }^{1}$ Washington State University Elson S Floyd College of Medicine, Spokane, WA; ${ }^{2}$ Providence Orthopedics and Sports Medicine, Spokane, WA

\subsection{6/jim-2022-WRMC.350}

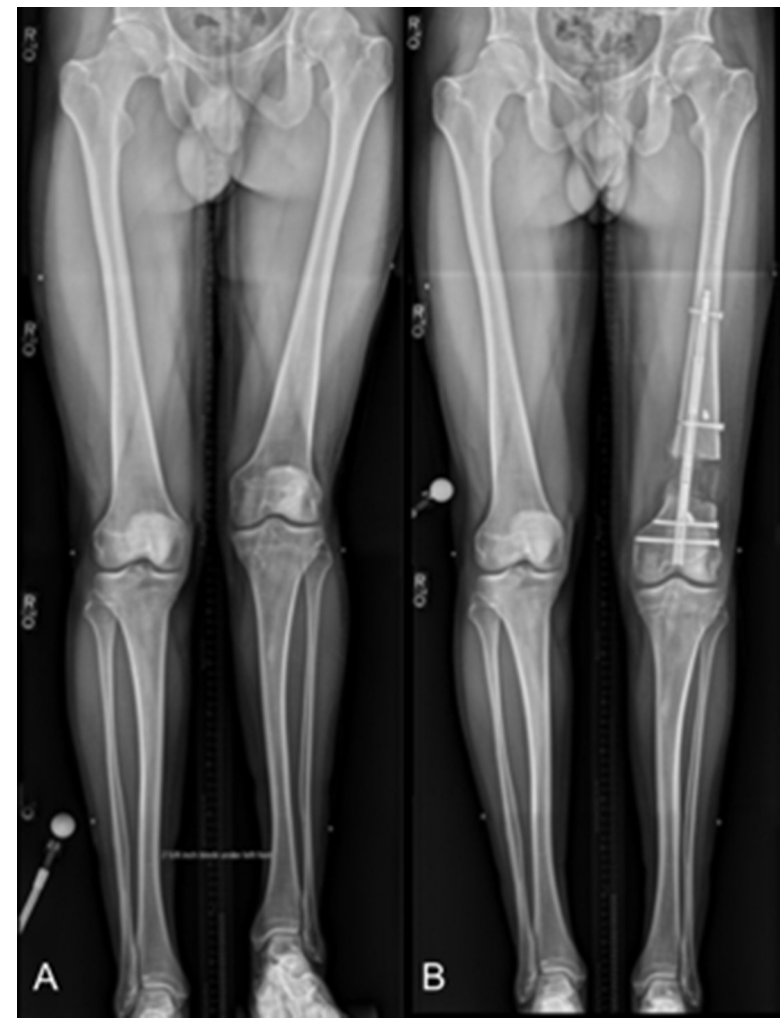

Abstract \#354 Figure 1 A: Lower Extremity X-Ray standing scanogram on blocks, 2-5/8-inch block under left foot date 11/19/2019; B: Lower Extremity X-Ray 2 months status post-surgery 03/16/2020.
Case Report Limb length discrepancies (LLD) pose a challenge to the orthopedic community with their multivariate etiologies; ranging from congenital malformation to acquired conditions that include bone loss from open fractures, nonunion, osteomyelitis, tumor, malunion, bone debridement due to infection, or comminution. Limb lengthening as a corrective measure occurs via distraction osteogenesis, a process entailing cutting and slowly separating the bone via a device called a distractor, allowing for the bone healing process to fill the gap over time. While historical Ilizarov principles of lengthening at a rate of $1 \mathrm{~mm}$ per day continue to be viable, the complications and inconveniences associated with Ilizarov method external fixation devices drove surgeons to develop alternative corrective interventions. To address this issue, Paley et all developed the PRECICE (Nuvasive) system, a magnetic intramedullary (MI) lengthening nail and the corresponding technique that would allow for internally based limb lengthening. We report one patient with history of complex left lower limb discrepancy with evidence of a combination of post-traumatic and congenital etiology presenting with genu valgum. With these indications, the patient underwent a corrective osteotomy of the left distal femur with retrograde placement of a PRECICE MI lengthening nail system. The patient had successful LLD correction with robust healing 9 months follow-up.

\section{\#355 A MASSIVE PHYLLODES TUMOR OF THE BREAST \\ ${ }^{1} \mathrm{~F}$ Moshtagh Sisan*, ${ }^{2} \mathrm{~J}$ Davis. 'Western University of Health Sciences, Pomona, CA;} ${ }^{2}$ Arrowhead Regional Medical Center, Colton, CA

\subsection{6/jim-2022-WRMC.351}

Case Report Phyllodes tumors are fibroepithelial neoplasms that make up less than $1 \%$ of breast tumors with an average size of $6 \mathrm{~cm}$. They usually arise in the third and fourth decade of life in women and the mainstay of treatment is surgical excision. Here, we present a rare case of a highly vascularized benign phyllodes tumor that weighted $7.98 \mathrm{~kg}$.

Methods Used Retrospective case report

Summary of the results A 55-year-old female without family history of breast or ovarian cancer presented for evaluation of a non-tender growing mass in her right breast. She initially noted the mass after a mechanical injury to her right breast two years prior to presentation. On physical examination, a firm non-mobile mass was palpated. There were not any skin changes or nipple discharge.

Ultrasound of the right breast revealed a large heterogeneous lobulated mass measuring $27.5 \times 21 \mathrm{~cm}$, replacing a great portion of her right breast. CT chest with contrast demonstrated a large soft tissue density mass measuring $25 \mathrm{~cm}$. Core needle biopsy was completed, and showed highly cellular spindle cell proliferation composed of fusiform cells with hyperchromatic nuclei and increased mitotic figures.

The Immunohistochemistry panel revealed that the lesional cells were positive with antibodies directed against Vimentin+, SMA $\mathrm{f}+$, and GATA3 $\mathrm{f}+$. Patient underwent a right breast mastectomy. Tumor was found to be massive with weight of $7.98 \mathrm{Kg}$ and highly vascularized with venous hypertension. This led to a high amount of blood loss for which the patient received 2 units of packed red blood cells. 
Gross examination of the mastectomy specimen showed multiple focal areas of ulceration, necrosis and cystic areas occasionally filled with hemorrhagic gelatinous material.

Morphological evaluation revealed spindle tumor cells arranged in long sweeping fascicles with herringbone pattern and storiform areas consistent with a benign Phyllodes tumor. Discussion Phyllodes tumors are usually painless tumors with an average size of $6 \mathrm{~cm}$. However, as seen in our patient, these tumors can rarely be larger than $20 \mathrm{~cm}$. Etiology of these tumors remains unknown. Trauma has been previously hypothesized to be involved in development of phyllodes breast tumors through possible fibroblast growth stimulators such as endothelin-1. Our patient with a phyllodes tumor reported a breast injury preceding her diagnosis of breast cancer. Most reports of phyllodes tumors of the breast endorse surgical resection of the neoplasm as a primary treatment without focusing on intraoperative complications. During our surgery, we encountered a highly vascular tumor that led to the need for blood transfusion after implementing clamping and electrocautery technique.

\section{\#356 ROLE FOR SERIAL PET-CT SCANS IN FOLLOW-UP OF HPV-ASSOCIATED HEAD AND NECK CANCERS}

${ }^{1} \mathrm{C}$ Munhall*, ${ }^{2} \mathrm{~K}$ Heiland, ${ }^{2} \mathrm{~J}$ Newell. ${ }^{1}$ The University of Arizona College of Medicine Phoenix, Phoenix, AZ; ${ }^{2}$ HonorHealth, Scottsdale, AZ

\subsection{6/jim-2022-WRMC.352}

Case Report Human papillomavirus (HPV) is a sexually transmitted infection (STI) acquired through oral, skin-to-skin, or genital contact. The CDC estimates 79 million Americans are infected with HPV, making it the most prevalent STI in the country. This virus persists in $10 \%$ of people, in the epithelial linings of the oropharynx and anogenital tract. Although HPV may persist asymptomatically, it has the potential for oncogenesis.

Increasing HPV prevalence has led to a rise in related cancers. In 2018, head and neck squamous cell cancer (HNSCC) was the sixth most common cancer worldwide, with nearly 900,000 new cases and approximately 450,000 related deaths. The incidence of HNSCC is expected to increase by $30 \%$ by 2030. While tobacco and alcohol are important risk factors for HNSCC, increasing rates of HNSCC in the U.S. are primarily due to oropharyngeal HPV infection. The diagnosis, management, and surveillance of HPV-driven HNSCC and related cancers is thus an increasingly relevant clinical consideration. In this case, we present a patient who developed a concomittant HPV-driven anal cancer, atypical after HNSCC, detected and treated early through serial PET-CT surveillance. Case A 70-year-old female with a history of cervical dysplasia status post hysterectomy presented to an otolaryngologist with globus sensation and oropharyngeal swelling. Exam demonstrated a left tonsillar mass and left neck lymphadenopathy. The tonsillar mass was biopsied and found to be p16-positive squamous cell carcinoma, indicating HPV involvement. Computed tomography (CT) of the neck demonstrated two enlarged lymph nodes. She was treated with surgical resection of the mass and ipsilateral neck dissection. Serial whole-body positron emission tomography (PET-CT) scans showed no evidence of recurrence or metastases, but scans just more than 2 years after treatment revealed a second primary cancer (SPC) of the anus. This anal cancer also demonstrated p16 positivity.
The patient was treated with chemoradiation therapy with a complete response.

Discussion The rising prevalence of HPV infection raises the likelihood of SPCs in patients with a history of HPV-driven cancers. While approximately $90 \%$ of local recurrences occur within two years of initial treatment, this does not account for SPC development. Patients with primary HNSCC have a high risk of developing SPCs of the head and neck and rarely metastases. SPCs of the anogenital tract following HNSCC are less common, but people with any HPV-driven primary exhibit 7-fold increased risk for a SPC development. This paper hopes to highlight early detection and treatment of a SPC of the anus through whole-body PET-CT surveillance.The role of such scans in evaluating for local recurrence, metastases, and SPCs is an important question in managing patients with HPV-driven HNSCC.

Joint plenary session

WAFMR, WAP, WSCl, AND WSPR

Friday, January 21, 2022

\section{1:30 PM - 4:15 PM}

\section{\#357 UPDATED CLINICAL NODAL STAGING SYSTEM FOR P16 + OROPHARYNGEAL SQUAMOUS CELL CARCINOMA ASSOCIATED WITH LOSS OF PROGNOSTIC DATA}

${ }^{1} \mathrm{~K} V \mathrm{~V}^{*},{ }^{2} \mathrm{C}$ Ladbury, ${ }^{2} \mathrm{~A}$ Amini. ${ }^{1}$ Western University of Health Sciences, Pomona, $\mathrm{CA} ;{ }^{2} \mathrm{City}$ of Hope, Duarte, $C A$

\subsection{6/jim-2022-WRMC.353}

Purpose of Study Due to human papillomavirus (HPV) associated oropharyngeal squamous cell carcinoma (OPSCC) having significantly improved prognosis, the American Joint Committee on Cancer (AJCC) introduced a distinct staging system for p16+ OPSCC in its 8th edition. The clinical nodal staging system removed multiple pathologic factors present in the 7 th edition, including nodal quantity, and extracapsular extension (ECE). This study aimed to characterize whether the simplification of the staging system resulted in the loss of prognostic value by using the National Cancer Database (NCDB).

Methods Used The NCDB was queried for patients diagnosed with p16+ OPSCC. Patients with no staging information, metastatic disease, who did not receive definitive surgery or radiation, or had unknown follow-up were excluded. The prognostic impact of nodal size, nodal quantity, nodal laterality, and ECE on overall survival (OS) were assessed using survival analysis with the Kaplan Meier method, univariable, and multivariable Cox proportional hazards regression.

Summary of Results A total of 21,868 patients met the inclusion criteria. On Kaplan Meier analysis, patients with more than one positive lymph node had significantly inferior OS ( $<<0.001 ; 5$-yr OS: $82 \%$ vs $86 \%)$. Patients with ECE also had inferior outcomes ( $\mathrm{p}<0.001 ; 5$-yr OS: $82 \%$ vs $75 \%)$. A largest nodal size of $>6 \mathrm{~cm}$ was also associated with inferior OS ( $\mathrm{p}<0.001) ; 5$ yr OS: $66 \%$ vs $82 \%)$. Lastly, patients with contralateral or bilateral nodal involvement also had inferior OS ( $p<0.001 ; 5$ yr OS: $71 \%$ vs $84 \%$ ). On multivariable Cox regression, having more than one positive node $(\mathrm{p}<0.001$; HR [95\%CI]: 1.17 [1.07-1.28]), ECE $(p<0.001 ;$ HR $[95 \%$ 
$\mathrm{CI}]: 1.20$ [1.04-1.38]), and a node $>6 \mathrm{~cm} \quad(\mathrm{p}<0.001 ; \mathrm{HR}$ [95\%CI]: 1.52 [1.30-1.79]) remained associated with inferior overall survival. There was no longer a significant difference among nodes $<3 \mathrm{~cm}$ and between $3-6 \mathrm{~cm} \quad(\mathrm{p}=0.56)$. Node laterality and was also no longer significant $(\mathrm{p}=1.00)$. Other factors associated with OS included age, Charlson Deyo score, insurance type, T stage, and lymphovascular invasion (LVSI).

Conclusions Although the incorporation of p16+ status in OPSCC staging was an important addition to the AJCC 8th edition, the associated simplifications in the current staging system result in loss of valuable prognostic information in nodal staging, including nodal quantity and ECE, which could limit appropriate risk stratification of patients with node-positive OPSCC. Further work evaluating the impact of the updated nodal staging for p16+ OPSCC prognostication and treatment selection is warranted.

\section{\#358 TELEMEDICINE INCREASES ACCESS TO PEDIATRIC WEIGHT MANAGEMENT AMID THE COVID-19 PANDEMIC}

${ }^{1} \mathrm{~K}$ Foos*, ${ }^{2} \mathrm{M}$ Vallabhan, ${ }^{1} \mathrm{~J}$ Page-Reeves, ${ }^{1} \mathrm{~S}$ Negrete, ${ }^{1} \mathrm{~A}$ Kong. ${ }^{1}$ University of New Mexico Health Sciences Center, Albuquerque, NM; '2University of New Mexico Hospital, Albuquerque, NM

\subsection{6/jim-2022-WRMC.354}

Purpose of Study From January to March 2020, the COVID19 pandemic emerged in the US and NM forcing healthcare practices to reimagine patient care. NM shelter-in-place orders started in March. Initially following these orders, telephone visits were the only option for healthcare services. The Healthy and Fit Children's Clinic (H\&FCC) at UNM leveraged telemedicine for medical management of their pediatric patients with overweight and obesity through partnership with established telemedicine clinics, transitioning within one month of the pandemic's onset to a $100 \%$ virtual healthcare model. This study assessed this transition in relation to four objectives: patient volume, billing/reimbursement, no-show rates, and patient satisfaction.

Methods Used Improvement science methodology guided the project throughout. The clinic partnered with the HRSAfunded telemedicine project (\#H2ARH3037) to implement telemedicine in the care and prevention of childhood obesity. Telemedicine clinic encounters were billed through UNM hospital. Multiple iterative convergent PDSA cycles informed step by step telemedicine implementation workflow. Information was collected from scheduled clinic visits and no shows/cancellations, billing and reimbursement, and a six-question patient satisfaction survey about their clinic visits.

Summary of Results Following telemedicine implementation (April-August 2020), the median number of patients seen per week at H\&FCC increased by $120 \%$ and no-shows declined overall by $20 \%$, which indicated a sustained positive shift above the established pandemic baseline (Jan-March 2020). Telemedicine clinic visits were billed at $\$ 122$ to $\$ 177$, an increase from $\$ 24$ to $\$ 35$ for telephone visits, leading to a $74 \%$ increase in total monthly median billing from April 2020 which indicates a sustained positive shift above the established baseline, surpassing even pre-pandemic billing (April 2019March 2020.) Over the study period, satisfaction surveys were completed by 26 patients ages 7-15 years old, eight of whom were monolingual Spanish speakers. These patients rated satisfaction with clinic visits $\geq 90 \%$ across all six questions compared to $83 \%$ previous global provider rating for in-person visits.

Conclusions With rising rates of childhood obesity exacerbated by substantial pandemic weight gain among youth greater than pre-pandemic rates (Woolford et. al, 2021), finding an effective model for consistent feasible healthcare is crucial for accessible quality care continuity particularly for the more vulnerable underserved populations seen in NM. This study demonstrated that telemedicine has potential to improve patient outcomes, improve patient satisfaction, and receive reimbursement for services. Future steps should assess a hybrid clinic model to maximize benefits of face-to-face visits with convenience and reliability of telemedicine

\section{\#359 A CROSS-SECTIONAL STUDY OF THE NEUROPSYCHIATRIC PHENOTYPE OF CACNA1C-RELATED DISORDER}

${ }^{1} \mathrm{RJ}$ Levy ${ }^{*},{ }^{2} \mathrm{~K}$ Timothy, ${ }^{1} \mathrm{~J}$ Bernstein, ${ }^{3} \mathrm{~S}$ Pasca. ${ }^{1}$ Stanford University School of Medicine, Stanford, CA; ${ }^{2}$ Timothy Syndrome Foundation, Salt Lake Clty, UT; ${ }^{3}$ Stanford University, Stanford, $C A$

\subsection{6/jim-2022-WRMC.355}

Purpose of Study CACNA1C encodes the alpha subunit of the L-type calcium channel Cav1.2, which is highly expressed in brain and heart. Gain of function pathogenic variants in specific regions of CACNA1C cause Timothy syndrome (TS) with cardiac long QT syndrome, facial features, and syndactyly as well as high rates of epilepsy, autism spectrum disorder, and intellectual disability. Work from our lab found Cav1.2 is involved in cell fate specification, activity-dependent signaling, and neuronal migration, but it is unclear how this cellular pathophysiology causes neuropsychiatric symptoms. A broader spectrum of CACNA1C-related disorder is now recognized that includes isolated cardiac disease, isolated neurologic deficits, and TS. We initiated a comprehensive survey of CACNA1C-related disorder to better define the neuropsychiatric and developmental phenotype and thus better inform research into the role of calcium channels in neural development.

Methods Used Caregivers of and individuals with CACNA1Crelated disorder completed an IRB-approved online survey on developmental milestones, neuropsychiatric symptoms and diagnoses, and medications trialed.

Summary of Results 17 participants completed the survey, including two parents with mosaicism. The mean age at diagnosis was 7 years (standard deviation 10.9y). 7 participants have TS type 1 with syndactyly and 2 participants have TS type 2 without syndactyly. The most common CACNA1C variant was c.1216G $>$ A p.G406R in exon $8 \mathrm{~A}$ or 8 (which causes TS1 or TS2, respectively) in 5 participants and 7 additional variants were reported. The most common neuropsychiatric symptoms were developmental delay in $88 \%$, hypotonia in $65 \%$, incoordination in $65 \%$, autism spectrum disorder in $53 \%$ (autistic features in 77\%), epilepsy in 35\%, depression or anxiety in $12 \%$, and schizophrenia in 6\%. 4 participants tried medication for epilepsy or psychiatric symptoms.

Conclusions CACNA1C-related disorder encompasses a spectrum of cardiac and neuropsychiatric symptoms. In our cohort, there was a significantly increased incidence of developmental delay, neurologic symptoms such as hypotonia, incoordination, and epilepsy, and psychiatric symptoms including autistic features and mood disorders. These findings indicate the key role of Cav1.2 in brain development and the clinical 
importance of screening and treating neuropsychiatric symptoms in this disorder. Future directions clinically include cohort expansion and deep phenotyping of neuropsychiatric symptoms. We are also developing 3D neural culture models of CACNA1C variants to understand the role of calcium flux in neuronal migration and cortical development.

\section{\#360 SYSTOLIC DYSFUNCTION AT THE TIME OF DIAGNOSIS CORRELATES WITH AMYLOID BURDEN AND PREDICTS MORTALITY IN TRANSTHYRETIN CARDIAC AMYLOIDOSIS}

${ }^{1} \mathrm{~S}$ Bukhari ${ }^{*},{ }^{2} \mathrm{~V}$ Malayala, ${ }^{2} \mathrm{Z}$ Shahid. ${ }^{1}$ Temple University, Philadelphia, $P A ;{ }^{2}$ Jeanes Hospital, Philadelphia, PA

\subsection{6/jim-2022-WRMC.356}

Purpose of Study Transthyretin cardiac amyloidosis (ATTR-CA) is generally considered to be associated with diastolic heart failure; however, systolic heart failure (HFrEF) can also be seen, particularly in patients with advanced disease. The mechanisms and prognostic implications of systolic dysfunction in patients with ATTR-CA have not been systematically studied, which we tried to explore in our study.

Methods Used In our prospective registry, the diagnosis of ATTR-CA was based on positive a PYP scintigraphy and negative serum studies for AL amyloidosis. Patients were classified as ATTR-rEF (ejection fraction, $\mathrm{EF}<40 \%$ ) or ATTR-pEF $(\mathrm{EF}>40 \%)$ based on cardiac magnetic resonance imaging (CMR). CMR was used to measure global extracellular volume (ECV) for quantification of amyloid burden. KaplanMeier survival analysis and adjusted cox proportional hazard analysis were performed.

Summary of Results Of the 124 ATTR-CA patients (mean age $79.9 \pm 7.4,87 \%$ men, 90\% Caucasian), 51 (41\%) were ATTR-rEF. Compared to ATTR-pEF, ATTR-rEF patients were more symptomatic ( NYHA-FC $\geq 3,61 \%$ vs $26 \%, \mathrm{p}<0.001$ ), had lower prevalence of obstructive coronary artery disease (CAD) $(37 \%$ vs $55 \%, p=0.05)$, worse mean diastolic dysfunction (3 vs $2.15, \mathrm{p}<0.01)$, lower tricuspid annular plane



systolic excursion (TAPSE $<1.7,59 \%$ vs $25 \%$, p $<0.001$ ) and worse renal function ( creatinine, $1.63 \pm 0.85$ vs $1.27 \pm$ $0.55 \mathrm{mg} / \mathrm{dl}, \mathrm{p}<0.01)$. On CMR, ATTR-rEF group had higher ECV compared to ATTR-pEF (mean ECV, 62\% vs 51\%, $\mathrm{p}<0.01$ ). Over a mean follow up period of 1.5 years, 27 (22\%) patients died. ATTR-rEF was associated with higher mortality compared to ATTR-pEF (35\% vs $12 \%, p=0.002$; HR 3.7, 95\%CI 1.62-8.63, p<0.01). On Cox proportional hazard model adjusting for TAPSE and creatinine, reduced EF was an independent predictor of mortality (HR 3.02, 95\% CI $1.30-7.10, p=0.01)$. When divided into $\mathrm{EF} \geq 50 \%$, EF 41$49 \%$ and $\mathrm{EF} \leq 40 \%$, there was stepwise increase in risk of mortality $(p<0.01)$.

Conclusions HFrEF is present in more than one-third of patients with ATTR-CA at the time of diagnosis, and is an independent predictor of mortality in ATTR-CA. ATTR-rEF patients also have higher ECVs, indicative of higher amyloid burden and potentially more advanced disease.

\section{Behavior and development II}

\section{Concurrent session}

\section{8:00 AM}

\section{Saturday, January 22, 2022}

\section{\#361 EFFECTS OF EXERCISE ON REALIGNING A DISRUPTED MOLECULAR CLOCK}

${ }^{1} \mathrm{ET}$ Scholten*, ${ }^{2} \mathrm{E}$ McCoy, ${ }^{2} \mathrm{EE}$ Schmitt. 'University of Washington School of Medicine, Seattle, WA; ${ }^{2}$ University of Wyoming, Laramie, WY

\subsection{6/jim-2022-WRMC.357}

Purpose of Study Approximately $18 \%$ of the US population work irregular shifts. This can lead to a circadian misalignment of our body's internal molecular clock. Our clock is critical for biological processes such as hormone secretion, body temperature regulation, and sleep/wakefulness cycles. Shift work is a constant in our society so it is valuable to study mechanisms that could mitigate its negative effects. Previous research has shown that voluntary exercise has promising effects on regulatory gene frequency in peripheral tissues in mice. Therefore, the purpose of this study was to determine the effect of forced treadmill exercise on circadian rhythm realignment.

Methods Used 24 ( $n=12$ males, $n=12$ females)C67/B1 fourmonth-old mice were used in this experiment. The 4 experimental conditions $(n=3)$ included: 1) disrupted + exercise (DE), 2) disrupted + sedentary (DS), 3) non-disrupted + exercise (NDE), and 4) non-disrupted + sedentary (NDS). The disrupted groups were circadian disrupted in a separate vivarium by changing their $12 \mathrm{hr}$ wake and sleep cycle by one hour every day. Pre and post dual-energy X-ray absorptiometry (DEXA) measurements were also recorded on all mice to determine changes in body composition of the experimental groups. The gastrocnemius and soleus skeletal muscles (SM) were extracted, RNA was isolated, then qRT-PCR was performed on key circadian clock genes (Bmal, Per2, Clock) to

\section{Abstract \#360 Figure 1}


determine the effect of exercise on realignment of the molecular clock.

Summary of Results Analysis of body composition showed that the males in the DE, DS, NDE, and NDS all lost percent body fats of $14.4 \%, 9.7 \%, 10.35 \%$, and $11.3 \%$, respectively. Analysis of the female mice displayed a decrease in the DE and DS groups of $3.3 \%$ and $11.5 \%$, respectively, while NDE and NDS groups of female mice both increased in percent body fat of $19.75 \%$ and $10.65 \%$, respectively. Analysis of key circadian clock genes in the SM of male mice showed no significant differences in Per2 or Bmal expression between groups, yet there was a trend toward significance $(p=0.09)$ in Clock gene expression in DE mice. In the SM of female mice, there was no change in Per2 expression, but there was a trend towards significance $(p=0.07)$ in Bmal expression in the DS group, and significance $(\mathrm{p}=0.01)$ in Clock expression in the DE group.

Conclusions Our results demonstrate that exercise does play a role in the re-entrainment of a misaligned molecular clock when examining circadian genes. The results also reveal sexdifferences in gene expression, which is an important factor when considering an exercise intervention protocol for individuals that work the night shift or have a disrupted clock. We will continue to analyze gene expression in other key peripheral tissues (heart, lung, kidney) to continue to identify positive responses to an exercise protocol to re-align a disrupted circadian rhythm.

\section{\#362 DEVELOPMENT AND PILOT STUDY OF A BOOKLET TO SUPPORT CONVERSATIONS OF QUALITY OF LIFE AND GOALS OF CARE AMONG PARENTS EXPECTING A BABY WITH A FETAL ANOMALY}

${ }^{1} \mathrm{M}$ Coquillette, ${ }^{2} \mathrm{H}$ Nsier*, ${ }^{2} \mathrm{AG}$ Dempsey. ${ }^{1}$ Children's Hospital Colorado, Aurora, CO;

${ }^{2}$ University of Colorado Denver School of Medicine, Aurora, CO

\subsection{6/jim-2022-WRMC.358}

Purpose of Study To develop a booklet supporting conversations of quality of life and goals of care with parents expecting a baby with a fetal anomaly. Aims: 1) assess acceptability, feasibility, demand, implementation, and practicality; 2) evaluate and refine the booklet; and 3) evaluate participants' qualitative feedback regarding preliminary satisfaction with the booklet.

Methods Used A mixed-methods study involving $\mathrm{N}=20$ expectant mothers presenting in a fetal care center at a children's hospital, approached at 30-36 weeks gestation. At enrollment, participants completed a demographic and background questionnaire and were given the booklet. The booklet was adapted for fetal care from an existing aid used by surrogate decision-makers, with input from multidisciplinary healthcare staff at the study site. Participants were sent surveys 2weeks after enrollment and 4-weeks after delivery. Outcome measures included characteristics of recruited and enrolled parents, refusal rate for participation, completion rate of

\section{Abstract \#362 Table 1 Study aims and related participant quotes}

\begin{tabular}{|c|c|c|c|}
\hline Study Aim & $\begin{array}{l}\text { Question/Prompt (Question } \\
\text { Type) }\end{array}$ & Participant Quotes (2-Week Survey) & Participant Quotes (4-Week Survey) \\
\hline \multirow{2}{*}{$\begin{array}{l}\text { Aim 1: Assess feasibility, } \\
\text { acceptability, demand, } \\
\text { implementation, and } \\
\text { practicality }\end{array}$} & $\begin{array}{l}\text { Who did you talk to about the } \\
\text { questions? (Multiple choice) }\end{array}$ & 'My partner' (50\%) 'Alone' (38\%) 'My family' (13\%) & 'Alone' (100\%) \\
\hline & $\begin{array}{l}\text { When is the best time to give } \\
\text { the guide to parents? (Multiple } \\
\text { choice) }\end{array}$ & $\begin{array}{l}\text { 'Soon after fetal diagnosis' (33\%) 'At a follow-up appointment' } \\
(33 \%) \text { 'At the delivery planning meeting' }(33 \%)\end{array}$ & This question was not asked on the 4-week survey. \\
\hline \multirow[t]{3}{*}{$\begin{array}{l}\text { Aim 2: Evaluate and } \\
\text { refine the booklet and its } \\
\text { processes }\end{array}$} & $\begin{array}{l}\text { The guide helped me to... } \\
\text { (Multiple choice) }\end{array}$ & $\begin{array}{l}\text { 'Prepare for my baby's hospital stay' }(71 \%) \text { 'Identify family } \\
\text { strengths' }(71 \%) \text { 'Identify what is most important to us in our } \\
\text { baby's care' }(57 \%) \text { 'Have difficult but important conversations' } \\
(43 \%) \text { 'Cope or lower stress' }(29 \%) \text { 'Talk to friends and family' } \\
(29 \%) \text { 'Talk to the medical team' ( } 29 \%) \text { 'Understand my baby's } \\
\text { condition' (14\%) }\end{array}$ & $\begin{array}{l}\text { 'Identify family strengths' }(75 \%) \text { 'Understand by baby's } \\
\text { condition' ( } 25 \%) \text { 'Identify what is most important to us in our } \\
\text { baby's care' }(25 \%) \text { 'Talk to friends and family' ( } 25 \%)\end{array}$ \\
\hline & $\begin{array}{l}\text { The problems of the guide } \\
\text { are... (Multiple choice) }\end{array}$ & 'Topics are too stressful' (60\%) 'Too long' (20\%) & 'Too long' (25\%) \\
\hline & $\begin{array}{l}\text { What topics are missing? (Free } \\
\text { response) }\end{array}$ & None reported & 'None, each person is looking for different things' \\
\hline \multirow[t]{2}{*}{$\begin{array}{l}\text { Aim 3: Evaluate } \\
\text { preliminary responses of } \\
\text { participants to the booklet }\end{array}$} & $\begin{array}{l}\text { Please give any comments } \\
\text { about problems of the guide or } \\
\text { how we can improve it (Free } \\
\text { response) }\end{array}$ & $\begin{array}{l}\text { 'I wish it talked more about what to expect during the birth and } \\
\text { time in the CICU' 'Topics or the way they are worded could be } \\
\text { triggering to certain people' }\end{array}$ & None reported \\
\hline & $\begin{array}{l}\text { Please give any other comments } \\
\text { about the guide (Free response) }\end{array}$ & $\begin{array}{l}\text { 'Helped to ask more informed questions to our care team' 'There } \\
\text { was good information to think about and address in the book' } \\
\text { 'Even though it makes her [the mother] nervous, it helps prepare } \\
\text { her for the future' 'The guide helped me come up with a plan } \\
\text { and helped me understand the importance of being aware of any } \\
\text { change' 'Good for those that like to journal and write down their } \\
\text { feelings' 'I didn't find any problems in the guide; it just really } \\
\text { helps you think deeply' }\end{array}$ & $\begin{array}{l}\text { 'I got this really close to delivery, so I had already thought all } \\
\text { this through. Would have been great to get this when we got } \\
\text { the diagnosis' 'We were pretty prepared, though I can see it } \\
\text { may be helpful for families who are not' 'It helped her [the } \\
\text { mother] talk to her family and identify strengths and } \\
\text { weaknesses'. }\end{array}$ \\
\hline
\end{tabular}

For multiple choice questions, the rate of endorsement is listed next to each response (\%) 
surveys, and qualitative feedback about parent experience using the study booklet and parent perception of booklet utility.

Summary of Results Expectant parents expressed willingness and interest in these topics and using this booklet, with 20/21 recruited parents enrolling in the study. Feedback survey response rates were low; $50 \%$ of mothers returned at least one feedback survey. Responding participants reported multiple benefits and few concerns with the booklet. At 2-weeks, $100 \%$ of respondents indicated they had looked at the booklet and $75 \%$ of respondents at 4 -weeks indicated they looked at the booklet after the baby's birth. At 2-weeks, participants indicated moderate satisfaction $(78 / 100$ on a visual analog scale) and moderate helpfulness $(63 / 100$ on a visual analog scale). At both 2 and 4-weeks, participants indicated they would recommend this booklet to other parents in the fetal care center. Participants' qualitative and quantitative responses about the booklet are listed in table 1 .

Conclusions The results of this mixed-methods study suggest this parenting population is interested in engaging in conversations about quality of life and goals of care pre-delivery, even with low-resource tools. Attrition was high, likely due to the stress of parenting a medically complex child. Yet, a majority indicated it helped prepare them for their infant's hospital stay and identify family strengths and care goals. Most indicated they would recommend the booklet to other parents of medically complex children. Future study is needed to assess this intervention style's impact on parent mental health, parenting experience, and ability to engage in medical decision-making for their child. Future studies should investigate the efficacy of ongoing interventions given with clinician facilitation/guidance rather than a one-time intervention.

\section{\#363 IMPACT OF HARM REDUCTION TREATMENT WITH OR WITHOUT PHARMACOTHERAPY ON CONCURRENT SUBSTANCE USE AMONG PEOPLE EXPERIENCING HOMELESSNESS AND ALCOHOL USE DISORDER}

${ }^{1} \mathrm{~N}$ Mostofi ${ }^{*},{ }^{1,2} \mathrm{SE}$ Collins. 'University of Washington School of Medicine, Seattle, WA; ${ }^{2}$ Washington State University, Pullman, WA

\subsection{6/jim-2022-WRMC.359}

Purpose of Study A prior randomized controlled trial showed that behavioral harm reduction treatment for alcohol use disorder (HaRT-A) - with or without pharmacotherapy (i.e., extended-release naltrexone) - was effective in improving alcohol outcomes and quality of life for people experiencing homelessness and alcohol use disorder. Because nearly $80 \%$ of the sample also reported polysubstance use at baseline, this secondary study tested whether HaRT-A also positively impacted other substance use outcomes.

Methods Used In the parent study, 308 adults with current alcohol dependence and homelessness were randomized to receive either HaRT-A plus intramuscular injections of $380 \mathrm{mg}$ extended-release naltrexone (XR-NTX; HaRT-A + XR-NTX), HaRT-A plus placebo (HaRT-A + placebo), HaRT-A alone, or community-based supportive services as usual (control). In this secondary study, we used multilevel mixed-effects models to detect changes in substance use before and after exposure to HaRT-A. For less prevalent behaviors, outcomes included any past-month use (cocaine, methamphetamine, opioids), and for more prevalent outcomes (i.e., polysubstance use and cannabis use), outcomes were past-month frequency (i.e., number of days of use).

Summary of Results Compared to control participants, HaRT-A (with or without XR-NTX) participants showed significantly reduced 30-day frequency of cannabis use (IRR $=0.59,95 \%$ $\mathrm{CI}=0.40-0.86, \mathrm{p}=.006)$ and polysubstance use $(\mathrm{IRR}=0.64$, $95 \% \mathrm{CI}=0.43-0.98)$. No other significant changes in prevalence or frequency of use were detected.

Conclusions Compared to services as usual, receipt of HaRT-A is associated with reduced cannabis and polysubstance use frequency. These findings suggest that the benefits of HaRT-A may extend beyond its impact on alcohol and quality of life outcomes to positively reshape individuals' overall substance use patterns. Future studies are needed to further investigate the efficacy of harm reduction treatment for polysubstance use and to develop guidelines for polysubstance use treatment.

\section{\#364 EXPANDING BEHAVIORAL PHENOTYPES IN SEX CHROMSOME TRISOMY WITH PARENT REPORTED CHARACTER STRENGTHS}

${ }^{1,2}$ TG Thompson, ${ }^{3} \mathrm{~J}$ Rubin*, ${ }^{1,2} \mathrm{~N}$ Tartaglia. ${ }^{1}$ Children's Hospital Colorado, Aurora, CO; ${ }^{2}$ University of Colorado Denver School of Medicine, Aurora, CO; ${ }^{3}$ University of North Carolina at Chapel Hill College of Arts and Sciences, Chapel Hill, NC

\subsection{6/jim-2022-WRMC.360}

Purpose of Study Children with sex chromosome trisomy (SCT; XXY/Klinefelter syndrome, XYY, XXX) are known to have increased risks for behavioral health problems including deficits in social cognition, inattention, and increased rates of anxiety and depression. However, a more balanced description of the phenotype that includes strengths is lacking. Limited data on the strengths of school-aged children with SCT has documented profiles of extraordinary kindness, creativity, and perseverance. To date, there has been no holistic description of behaviors, including the assets, of young children with SCT. This study aimed to describe parent reported behavior and strengths in 18-36 month old children with SCT.

Methods Used Data were collected for the eXtraordinarY Babies longitudinal natural history study. Parents rated behaviors of their children with prenatally identified SCT $(n=140)$ on the Child Behavior Checklist (CBCL) at ages $18 \mathrm{~m}(\mathrm{n}=67)$, $24 \mathrm{~m}(\mathrm{n}=103)$, and $36 \mathrm{~m}(\mathrm{n}=54)$. Descriptive statistics portrayed Internalizing (IB), Externalizing (EB), and Total Problem (TPB) behaviors at each time point. One-sample T-tests were used to compare the sample to the norming population $(\mathrm{M}=50$, $\mathrm{SD}=10)$; one-way ANOVA compared groups $(\mathrm{XXY}=97$, $\mathrm{XYY}=13, \mathrm{XXX}=30$ ). Parents provided free-text responses to the CBCL question: 'Please describe the best things about your child'. Positive psychology content analysis was used to code qualitative responses for character strengths.

Summary of Results Results for the pooled sample revealed relatively low rates of $\operatorname{IB}(18 \mathrm{~m}=39.59 ; \quad 24 \mathrm{~m}=41.34$; $36 \mathrm{~m}=44.70), \mathrm{EB}(18 \mathrm{~m}=41.34 ; 24 \mathrm{~m}=43.00 ; 36 \mathrm{~m}=44.96)$, and $\operatorname{TPB}(18 \mathrm{~m}=40.53 ; 24 \mathrm{~m}=42.43 ; 36 \mathrm{~m}=45.72)$; all significantly lower than the norming population at all time points $(\mathrm{p}<.05)$. At $36 \mathrm{~m}$, boys with XYY showed significantly higher EB than boys with $\mathrm{XXY}(59.00 \mathrm{v} 43.16 ; \mathrm{p}=.012)$; small sample size in $\mathrm{XYY}$ limits interpretation. There were no other group differences. Parents described their children's strengths effusively; the most frequent codes were loving, happy, sweet, humorous, curious. 
Conclusions This study expands the SCT phenotype by providing a more balanced description of behavior. Results indicate that toddlers with SCT show fewer problem behaviors than the general population and parents describe many strengths of character in their young children, including loving, happy, and sweet demeanors. Future analyses will examine how behavior and strengths profiles in SCT develop over the lifespan and how they relate to a variety of psychosocial and developmental outcomes. A more thorough understanding of the phenotype, including a robust understanding of assets, can improve clinical practice and inform development of interventions.

\section{\#365 ADVERSE CHILDHOOD EXPERIENCES (ACE) AND CO- MORBIDITIES AMONG ADULTS WITH DIABETES MELLITUS: META-ANALYSIS OF THE ASSOCIATION BETWEEN ACE AND DIABETIC DEPRESSION}

M Malwane*, J Abad, M Riddle, M Thompson. University of Nevada Reno, Reno, NV

\subsection{6/jim-2022-WRMC.361}

Purpose of Study Adverse childhood experiences (ACEs) are associated with increased risk of diabetes in adulthood. However, the impact of ACEs on clinical outcomes among adults with diabetes is not well understood. We aim to elicit a better underestanding of which diabetes co-morbidities have been linked to ACEs from the current literature.

Methods Used In this systematic review, we assessed the literature on relationships between ACE and diabetes clinical outcomes/co-morbidities. We searched Embase, PubMed, and ProQuest through August 12th, 2020 using keywords related to ACEs and autoimmune disease or diabetes. We scanned references of retrieved articles, and two authors independently

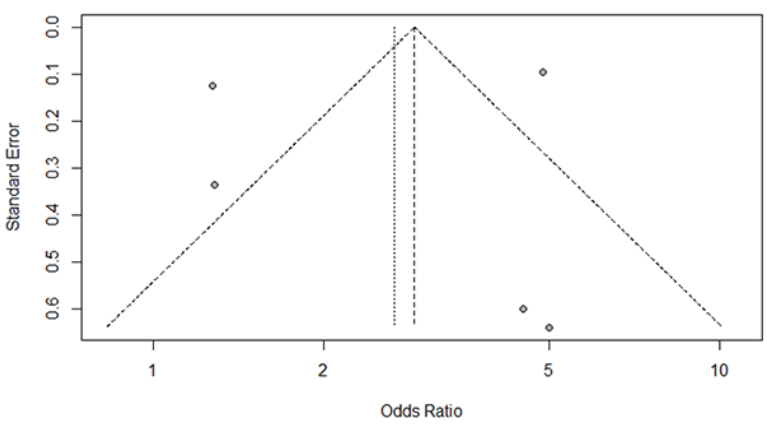

Abstract \#365 Figure 1 evaluated article eligibility, extracted data, and assessed quality. We used meta-analytic methods for outcomes that were in included in at least four studies to estimated pooled odds ratios (ORs) using random effects models and the inverse variance method for pooling.

Summary of Results Our search located 458 articles, of which 13 studies were eligible for inclusion, comprising a total of 16,118 individuals. Most studies used clinical samples $(\mathrm{n} / \mathrm{N}=8$ / 11) of African American or white patients $(n=5, n=3)$ with type II diabetes (Type $\mathrm{I}=18.2 \%$; pooled= 27.3\%). Depression was the most studied co-morbidity $(n=6)$, followed by cardiovascular disease, obesity, $\mathrm{HbA} 1 \mathrm{c}$, and post-traumatic stress disorder (each were assessed twice). The pooled OR for depression among diabetic patients with moderate/high $(\geq 2)$ relative to low (0-1) ACE exposure, was 2.67 (95\% CI: 1.16$6.12 ; p=0.0537, \mathrm{k}=5)$ with high heterogeneity $\left(\mathrm{I}^{2}=0.95\right)$.

Conclusions We found a strong association between ACEs and depression among diabetics: additional research is needed before pooled estimates on other clinical outcomes can be calculated. Potential benefits of ACE-informed psychotherapeutic interventions for diabetic patients with high ACEs merits investigation.

\section{\#366 ADVERSE CHILDHOOD EXPERIENCES AND DIABETES IN ADULTHOOD: AN UPDATED SYSTEMATIC REVIEW AND META-ANALYSIS}

J Abad*, M Malwane, M Thompson, M Riddle. University of Nevada Reno, Reno, NV

\subsection{6/jim-2022-WRMC.362}

Purpose of Study There is growing evidence that adverse childhood experiences (ACEs) increase the risk of diabetes in adulthood through inflammatory and behavioral pathways: previous meta-analyses require updating and exploration of sub-groups to explain heterogeneity of effect in the observed literature.

Methods Used We searched PubMed, Embase, and ProQuest through August 12, 2020, using PRISMA guidelines and key words related to ACEs, autoimmune disease, and diabetes. We included English language peer-reviewed articles and graduate dissertations reporting associations between ACE and diabetes on subjects $\geq 18$ years old. Two authors independently screened studies for inclusion, extracted data, and assessed quality. For studies that estimated the association between $\geq 4$ ACEs and diabetes, we used random effects meta-analysis to calculate the pooled odds ratio (OR), and meta-regression methods to test sources of heterogeneity among pooled effect estimates.

$\begin{array}{lrl}\text { Study } & \text { TE } & \text { se TE } \\ & & \\ \text { Elm 2020 } & 1.50 & 0.5996 \\ \text { Itoop 2020 } & 0.25 & 0.3354 \\ \text { McCurley 2019 } & 0.24 & 0.1254 \\ \text { Powers 2016 } & 1.61 & 0.6391 \\ \text { Roy 2011 } & 1.58 & 0.0960\end{array}$

Random effects model Heterogeneity: $I^{2}=95 \%, \tau^{2}=0.7461, p<0.0 \uparrow$
Odds Ratio

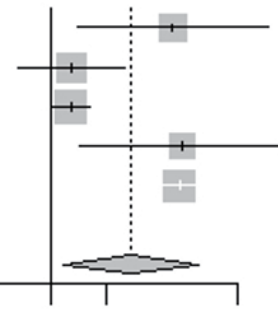

0.512
OR

95\%-Cl Weight

\begin{tabular}{|c|c|}
\hline ti & \\
\hline$[0.6$ & \\
\hline & \\
\hline & \\
\hline [4. & \\
\hline
\end{tabular}

$2.67[1.16 ; 6.12] 100.0 \%$

$\begin{array}{lllll}0.1 & 0.5 & 1 & 2 & 10\end{array}$

\section{Abstract \#365 Figure 2}





\section{Abstract \#366 Figure 1}

Summary of Results Of 458 unique articles, 51 were eligible for the systematic review and 14 ( $N=261,966$ individuals) for the quantitative synthesis. Relative to none, exposure to $\geq 4$ ACEs increased the odds of diabetes by $15 \%(\mathrm{OR}=1.15,95 \%$ $\mathrm{CI}=1.07-1.24, \quad \mathrm{I}=87.2 \%, \mathrm{p}=0.0004)$. Subgroup analyses showed OR estimates were lower among studies with higher proportions of female $(\beta=-0.025, \mathrm{se}=0.011, \mathrm{p}=0.018)$ but not Caucasian $(\beta=-0.0015$, se $=0.001, \quad p=0.137)$ subjects. From a study design perspective, signifcant heterogeneity was explained by the number of questions used to assess ACE $(\beta=0.007, \mathrm{se}=0.003, \mathrm{p}=0.013)$ but not each additional covariate modeled $(\beta=-0.027, \mathrm{SE}=0.0154, \mathrm{p}=0.0797)$.

Conclusions Our results support a significant association between ACEs and diabetes in adulthood, highlighting the potential benefits of ACE screening and interventions to help reduce diabetes prevalence, particularly for men.

\section{\#367 EATING TO HONOR YOUR BODY AND LIFE: RECIPES AND INFORMATION FOR THE BLACKFEET COMMUNITY IN MONTANA}

K Jones*. University of Washington School of Medicine, Seattle, WA

\subsection{6/jim-2022-WRMC.363}

Purpose of Study On the Blackfeet Reservation, food insecurity and chronic disease are prevalent and reduce residents' life expectancies. When American Indians were forcibly relocated, their communities began to rely on processed foods provided by the government, and over more than a century, cooking with processed foods rather than traditional foods was adopted. This project's goal was to offer culturally-responsive nutrition education and recipes.

Methods Used An asset-based, community engagement approach was utilized during the development of this project, and involved consulting Southern Piegan Health Center, the
Blackfeet Food Access and Sustainability Team (FAST), the Food Delivery Program on Indian Reservations (FDPIR), and staff at Blackfeet Community Hospital (BCH). These organizations do valuable work on nutrition education and improving the food environment on the reservation, and the goal was to supplement their efforts. The project was influenced by conversations with patients about nutrition as well as by exposure to the local grocery stores and food environment. It was also shaped by literature on nutrition interventions on reservations.

Summary of Results Average FDPIR food boxes meet a relatively strong Healthy Eating Index value, indicating that healthy choices are available to community members who use FDPIR. Participants may need help choosing these options or tips for utilizing those foods. Additionally, research on another reservation found a need for skill-based nutrition education that addresses time and monetary constraints. So, a nutrition guide highlighting healthy tips was made, and 4 recipes were compiled - 2 simple ways to enliven canned vegetables, and 2 Indigikitchen recipes using traditional Blackfeet foods. Nutrition guides were placed in exam rooms at $\mathrm{BCH}$, where patients often sit waiting for providers. Guides and recipes were provided to partners at FAST Blackfeet, the Blackfeet FDPIR, and $\mathrm{BCH}$.

Conclusions Using an asset-based approach enhanced this project's outcome. The materials were shared with organizations that work in the community and can display and distribute them at their food pantries. The recipes can be made available in proximity to the ingredients they require. Feedback from community partners, such as the type of seasoning preferred by community members, was utilized. Examining how the resources are received by the community is the next step.

\section{\#368 ASSESSMENT OF VOLUNTEER AND CAREER FIREFIGHTER FITNESS IN A RURAL SETTING}

CD Johnson*, R Faulkner, M Jette, A Hall, R Duerr. Western University of Health Sciences College of Osteopathic Medicine of the Pacific-Northwest, Lebanon, OR

\subsection{6/jim-2022-WRMC.364}

Purpose of Study Lebanon, Oregon is a rural town served by the Lebanon Fire District, which relies on both career and volunteer firefighters. Through the WesternU Lebanon Emergency Alliance we were presented with a unique opportunity to assess the physical fitness and health of both career and volunteer firefighters. In the literature reviewed, a selfreported study of 230 firefighters, volunteer members were seen to have higher rates of obesity compared to career firefighters which calls for better preventative care and health screenings for volunteers (Yoo et al., 2009). The Lebanon Fire District employs double the amount of volunteers compared to careers spread over a large rural region. Our study was designed to determine the difference in health risks, if any, that exist between career and volunteer firefighters in a rural community.

Methods Used A randomly selected sample of volunteer and career firefighters employed by the Lebanon Fire District were given a questionnaire developed to assess self-reported personal health measures including exercise frequency, flexibility, and diet. After completing the survey, each participant took part in a fitness assessment that was proctored by a trained 
medical student or career firefighter. Data collected included BMI, muscle strength, flexibility, and aerobic capacity. Participants' scores were paired with survey responses. Inclusion criteria were all fire-fighters in Lebanon, Oregon. Any high-risk participant, defined as an individual with any one of the following conditions: COPD, asthma, diabetes, angina, renal disease, shortness of breath with mild exertion, palpitations, tachycardia and known heart murmur, required physician or paramedic supervision during their fitness assessment. This study was approved by the Western University of Health Sciences Institutional Review Board.

Summary of Results Within the Lebanon Fire District there are approximately 36 career firefighters and 64 volunteer firefighters including a cohort of 12 medical students through the WesternU Lebanon Emergency Alliance (WLEA). Of the firefighters employed by the Lebanon Fire District, 55 individuals completed the survey and fitness assessment. Career VO2 max was 44 and volunteer VO2 max was 42 with a difference of 2 (P value 0.07).

Conclusions In our small pilot study, we found that career firefighters overall had better flexibility and VO2 max as compared to volunteer firefighters, but volunteer firefighters had lower BMIs compared to career firefighters. This study may be limited in that it was primarily self-reported and there were twice as many volunteer firefighters tested compared to career firefighters. Further study with a greater number of participants is indicated.

\section{Diversity, equity and inclusion III}

\section{Concurrent session}

\section{8:00 AM}

\section{Saturday, January 22, 2022}

\section{\#369 THE PRIORITY OF GENDER \& RACIAL DISPARITY IN NEUROLOGY JOURNALS: A BIBLIOMETRIC ANALYSIS, 2016-2021}

${ }^{1} S$ Mohammed* , ${ }^{2}$ S Siddiqi. 'California University of Science and Medicine, San Bernardino, CA; ${ }^{2}$ Arrowhead Regional Medical Center, Colton, CA

\subsection{6/jim-2022-WRMC.365}

Purpose of Study Medicine and the field of neurology are not immune to gender and racial disparities present more broadly in society. Even within academia there remains a persistent lack of representation of women and racial minorities, but with the recent spotlight on discrimination it is more necessary than ever that prevailing disparities are acknowledged by medical journals and reflected in their publication priorities. This bibliometric study assesses whether social justice trends have influenced the number of articles published that discuss gender and racial disparity in the past 5 years within the top three leading neurological journals.

Methods Used The leading journals in neurology were chosen based on the SCImago Journal Rank Indicator, which measures the impact or influence of a journal, and total citations in the past 5 years. The three highest neurology journals based on those two parameters were The Lancet Neurology, Nature Reviews Neurology, and Alzheimer's and Dementia. Online archives of each of these journals were used and a search was conducted using the key words 'gender', 'race', and 'disparity', to find articles related to such topics. Data was collected from September 2016 to August 2021 by counting the number of articles discussing gender or racial disparity for each month. The data was then processed into charts to provide a visual representation of how important the journal deems topics related to gender and racial inequality.

Summary of Results The results indicated that although there was a slight overall increase in coverage of the aforementioned topics, it was negligible compared to the broad impact of current social justice movements. Between the three leading journals, Nature Reviews Neurology had the fewest publications on both gender and racial disparity. Our data suggests an inadequate publication priority for scholarly work on gender and racial disparity in the chosen journals, over the latest five years reviewed.

Conclusions Our assumption is that any trend towards more publications related to gender and racial disparities are reflective of the publication priorities of, and thus the perceived relevance to, the editorial boards of the chosen journals. As physicians whose job is to advance and better healthcare for all our patients, it is necessary that the journals we subscribe and read in the field also reflect the topics that are pertinent today. There appears to be a disconnect between current events and leading journals in neurology. We believe that this bibliometric study, albeit limited by a relatively small sample size, reflects the larger lack of diversity and coverage on socially relevant topics in leading neurology journals. This study can be replicated to other journals and other fields as well to establish a broader view of changes or the lack thereof in medicine.

\section{\#370 RACIAL/ETHNIC DIFFERENCES IN ACCESS TO FORMAL HOME CARE AND ITS RELATION TO COGNITION AND ACTIVITY OF DAILY LIVING FUNCTIONS}

T Layne*, M Shaheen, K Schrode. Charles Drew University of Medicine and Science, Los Angeles, $C A$

10.1136/jim-2022-WRMC.366

Purpose of Study Elderly individuals prefer to age in their home. Formal Home Care Services (FHCS) helps to support an individual's ability to age at home. Studies have illustrated that FHCS are associated with improved Activity of Daily Living (ADL) functioning and cognition, however, there is a disparity in accessing FHCS among lower socioeconomic groups. Little research exists in the U.S. that endeavor to understand the factors associated with the lack of access to FHCS and its impacts amongst the disadvantaged groups. We aim to examine access to FHCS among African American (AA) and Latino American (LA) geriatric populations and its effect on ADLs, IADLs and cognition.

Methods Used We analyzed data related to receiving FHCS for 1,552 subjects $\geq 65$ years old from the National Health and Aging Trends Study (NHATS). The outcomes included cognition, depression, injuries, mobility, and ADL function. Data was analyzed using chi square test and multiple logistic regression.

Summary of Results $25 \%$ of study subjects received FHCS [Hispanics (37\%) and African Americans (36\%) compared to Whites $(24 \%),(\mathrm{p}<0.05)]$. There was no significant difference between FHCS recipients and facility care recipients in ADLs, 
IADLs, cognition and depression $(\mathrm{p}>0.05)$. FHCS recipients were more likely to have higher injuries/hospital stays since baseline as well as an increased need for ADL assistance compared to facility care recipients $(p<0.05)$.

Conclusions Although most Medicare beneficiaries who received home care were minorities, further analysis and investigation is needed to understand the factors associated with the poorer care outcomes observed in those who received home care.

\section{\#371 INTERVIEWING SPANISH SPEAKING PARENTS ABOUT COMMUNICATION IN THE NICU}

E Batton*, L Catalan, M Freeman, C Ramos, S Hurst, K Marc-Aurele. University of California San Diego, La Jolla, CA

\subsection{6/jim-2022-WRMC.367}

Purpose of Study To explore from whom and in what manner parents with Limited English Proficiency (LEP) receive information about their baby and to assess parent satisfaction with communication in the NICU.

Methods Used We conducted in-person interviews of Spanish speaking NICU parents using a semi-structured interview format. Parents of newborns admitted to the NICU for at least one week who identified Spanish as their preferred language were eligible. Interviews were performed by one of two bilingual providers. All interviews were audio-taped and transcribed. Transcripts were reviewed for errors by certified bilingual Spanish speakers and then translated to English. A directed content comparison approach was taken to identify contextualized segments from each interview that correspond to targeted questions. We collected newborn demographic data, length of stay, and SNAPPE II (Score for Neonatal Acute Physiology-Perinatal Extension II), a predictor of neonatal mortality.

Summary of Results Fourteen interviews were conducted. Average gestational age at birth was 33 weeks, 4 days [25,5 $41,2]$ and $43 \%$ were female. Eighty-eight percent of SNAPPEII scores at 12 hours of life were between 0 to 20 (low). The average length of stay was 45 days [9-120 days]. At the time of the interview, the infants were 32 days old on average [3118 days]. The average interview duration was 8 minutes (522 minutes). Parents primarily received information from nurses and reported rarely speaking with doctors. They were more likely to receive information when they were present in the NICU and when they asked. Parents feared that interpreters were inaccurate, but no parent reported ever refusing an interpreter. They reported difficulty expressing doubts to the medical team. Parents often experienced a delay in communication, which was perceived to be as a result of not speaking English. Parents expressed variable satisfaction with the frequency of information received but almost all reported being extensively involved in their child's care. Parents requested more bilingual providers be available in addition to electronic interpreters.

Conclusions Parents with LEP face unique challenges communicating in the NICU. Our findings can inform neonatal quality initiatives to decrease delays and burdens for families with LEP to receive timely and good communication. Future studies are needed to determine the best strategies to facilitate communication with Spanish speaking families.

\section{\#372 HOW IMPORTANT IS IT TO BE IN A COMMUNITY THAT REFLECTS YOUR RACIAL IDENTITY? EXPLORING THE EXPERIENCES OF BLACK, INDIGENOUS AND PEOPLE OF COLOR (BIPOC) STUDENTS IN RURAL \& UNDERSERVED CLINICAL SETTINGS}

B Cedeno Betancourt Sil*, T Keys. University of Washington School of Medicine, Seattle WA

\subsection{6/jim-2022-WRMC.368}

Purpose of Study Medical educators have recently begun to research clinical learning environments as experienced by BIPOC Medical students. The limited results demonstrate that BIPOC students regularly experience mistreatment and discrimination, often as microaggressions. This qualitative study aims to explore how the type of clinical settings, particularly rural and urban underserved locations impact BIPOC medical student's experiences.

Methods Used Current self-identified BIPOC University of Washington medical students who participated and completed the Rural/Urban Opportunities Program within the last three years were invited to participate in a 90-minute focus group discussion via Zoom. The sessions were facilitated by a current medical student who utilized guided questions to explore the experiences of BIPOC students in urban/rural clinical learning environments. The guided questions were developed by the research team, and utilized in all five focus groups. From 8/4 - 9/1, 2021, five focus groups were conducted with two to four participants per focus groups and one individual interview. There was a total of 12 participants. All focus groups were transcribed and are being reviewed for thematic content. Preliminary themes were identified by the research team.

Summary of Results BIPOC students expressed general apprehensions of being placed in a rural clinical setting, more so than students who were placed in an urban underserved clinical setting, with BIPOC preceptors that serve BIPOC patients. Students placed with either a BIPOC preceptor, or within a BIPOC community tended to have a more positive clinical experience. However, being placed in an urban underserved setting is not necessarily a protector against microaggressions in the clinical setting. Students placed in a rural community with non-BIPOC preceptors who acknowledged possible microaggressions had a positive clinical experience. In general, BIPOC medical students preferred urban underserved clinical experiences over rural clinical experiences due to the belief that a rural clinical setting would result in a greater number of microaggressions.

Conclusions We found that microaggressions can take place in both urban and rural underserved clinical settings. More research needs to be done to further understand the experiences of BIPOC students to improve diversity in medical education. Immediate systemic changes include acknowledgments of the experiences BIPOC students could encounter in Rural/ Underserved clinical settings, and an avenue for safely bringing the experiences up to preceptors and faculty. 
\#373 THE IMPACTS OF RACE ON PREGNANCY OUTCOME IN WOMEN OF COLOR: OSTEOPATHIC MEDICAL STUDENTS' PERSPECTIVE

I Adesina*, V Liederbauer, B Novak, AL Nelson, E Guenther. Western University of Health Sciences, Pomona, CA

\subsection{6/jim-2022-WRMC.369}

Purpose of Study The main goal of this study is to evaluate the perceptions and attitudes of medical students about the impact race has on access to quality of care as well as pregnancy outcomes in non-Hispanic Black women compared to other races.

Methods Used A voluntary, anonymous 17-question survey was emailed to students at Western University of Health Sciences in Pomona, CA. Responses to most questions were rated on a 5 -point Likert scale from disagree strongly to strongly agree. There was also an open-ended question for participants to share their thoughts about these issues. IBM SPSS Statistics 27 was used to run descriptive statistics and conduct chi-square analysis (a p value $<0.05$ was considered statistically significant). Responses from Caucasian students were compared to those who declared other races. This abstract reports the results for four of the main questions relating to these outcomes.

Summary of Results The response rate was 21\% (250) from the 1200 invited students. Racially, $48.0 \%$ were white, and $67 \%$ were female. First-year students made up $39.6 \%$ of the population with subsequent years in declining proportions: $23.7 \%, 20.8 \%$, and $15.7 \%$ respectively. Over $85 \%$ of respondents believed that race is a strong predictor of pregnancy outcome; $66 \%$ disagreed with the statement that health problems of women of color are primarily due to personal choices they make. Over $87 \%$ of respondent agreed the color of a person's skin can influence the quality of medical care that a person receives.

Conclusions Even though studies show that Black women have maternity mortality rate 3-4 times higher than non-Hispanic White women, $15 \%$ of respondents did not recognize the impact that race had on maternal outcomes. One-third of students did not disagree with the statement that Black women are primarily responsible for their own poor health. Importantly, one in seven students was not aware of the increased mistrust women of color have of the medical system. The findings demonstrate remaining education gaps that despite limited response rate, may serve as a baseline to measure the impact of more extensive curriculum changes to teach medical students more about equality and diversity in the future.

\section{\#374 VARYING RATES OF DISABILITY EXPOSURE REPORTED ON CORE ROTATIONS WHILE OSTEOPATHIC MEDICAL STUDENTS REPORT SUPPORT FOR FORMAL DISABILITY TRAINING}

${ }^{1} \mathrm{KM}$ Lucara*, ${ }^{1} \mathrm{~S}$ Henderson, ${ }^{1} \mathrm{M}$ Blair, ${ }^{2} \mathrm{AL}$ Nelson, ${ }^{1} \mathrm{E}$ Guenther. ${ }^{1}$ Western University of Health Sciences College of Osteopathic Medicine of the Pacific-Northwest, Lebanon, OR; ${ }^{2}$ Western University of Health Sciences College of Osteopathic Medicine of the Pacific, Pomona, $C A$

\subsection{6/jim-2022-WRMC.370}

Purpose of Study In the 2014 US Census, $17.6 \%$ of adults were severely disabled. People living with a disability have self-reported higher rates of dissatisfaction regarding unmet needs in their healthcare experience and there are no standards in the Commission on Osteopathic College Accreditation curricula for treating those with disabilities. Without the accreditation standards, we sought to quantify the exposure to caring for patients with disabilities that 3rd and 4th year osteopathic medical students (OMS) get while on clinical rotations, to investigate how prepared students felt they were to care for this population. We also studied the attitudes of OMS towards formal disability training.

Methods Used An anonymous, beta-tested, IRB-approved survey and clarifying disability definitions were distributed electronically to all 3rd and 4th year OMS on clinical rotations in the spring. Descriptive statistics were calculated for each question based on the denominator of individuals who responded to the specific question.

Summary of Results 600 surveys were distributed, and 59 responded with 40 completed. $51 \%$ of students reported exposure to caring for people with disabilities prior to medical school. 30\% reported being exposed to greater than 20 patients with disabilities on rotations. Reported exposure rates to caring for patients with disabilities differed by core rotation: internal medicine (86\%), psychiatry (84\%), family medicine $(79 \%)$, pediatrics $(77 \%)$, osteopathic manipulative medicine (76\%), surgery (53\%), and obstetrics and gynecology $(36 \%)$. Students reported the most comfort with patients with physical/ambulatory disabilities (85\%) and the least comfort with sensory difficulties (50\%). Using the Likert-scale, 68\% reported feeling probably $(4 / 5)$ or definitely $(5 / 5)$ prepared to care for patients with disabilities. However, $68 \%$ also felt the current curriculum probably or definitely did not fully prepare them and thought formal disability training should be added to pre-clinical curriculum.

Conclusions In this pilot study, experience with the care of individuals with disabilities was observed on all core medical rotations, but some offered minimal exposure. Overall, students were in strong support of implementing formal training in caring for patients with disabilities and the majority felt the current curriculum did not prepare them to care for these patients.

\section{\#375 AN ASSESSMENT OF ACCESS TO TOPICAL CALCINEURIN INHIBITORS FOR PEDIATRIC VITILIGO}

${ }^{1} \mathrm{H}$ Munzing ${ }^{*},{ }^{1,2,3} \mathrm{H}$ Brandling-Bennett. 'University of Washington School of Medicine, Seattle, WA; ${ }^{2}$ Seattle Children's Hospital, Seattle, WA; ${ }^{3}$ UW Medicine, Seattle, WA

\subsection{6/jim-2022-WRMC.371}

Purpose of Study Vitiligo is an autoimmune disease that presents with depigmented patches of skin and hair. Untreated childhood vitiligo can have lasting psychosocial effects on patients and their caregivers. Many insurance companies, Medicaid in particular, consider vitiligo to be a 'cosmetic disease' without considering the substantial psychosocial health implications. Absence of an FDA-approved therapy for repigmentation of vitiligo offers further challenge, though off-label therapy with topical calcineurin inhibitors (TCIs) is a first-line therapy. This study examined access to TCIs among patients with Medicaid insurance compared with private insurance.

Methods Used A retrospective cohort study was conducted at Seattle Children's Hospital's ( $\mathrm{SCH}$ ) which examined the medical records of patients (180) that were <18-years-old, seen between January 2010 and December 2020, had at least two 
medical visits with a diagnosis of vitiligo, and were prescribed treatment with tacrolimus or pimecrolimus (TCI). Their records were reviewed for prescribed treatment plan, ability to access the TCI medications, and insurance type. Associations of Medicaid vs. private insurance and quality of TCI access were studied. Chi-squared statistical testing was used to determine significant differences between treatment access in Medicaid versus private-insurance patients.

Summary of Results Patients with Medicaid represented 66.9\% of the study cohort while private insurance accounted for 33.1\%. Of the Medicaid patients, 50.4\% experienced 'good access' (no insurance issues reported) to the prescribed TCI compared to $89.5 \%$ of private-insurance patients. 'Challenging access' (received medication but experienced: denials initially or during treatment, limited access to more potent TCIs, or necessity to pay out-of-pocket) was reported for $41.7 \%$ of Medicaid patients and $7.0 \%$ of private-insurance patients. 'No access' was experienced by $7.8 \%$ and $3.5 \%$ of patients with Medicaid and private insurance, respectively. There is a statistically significant difference in the ability to appropriately access the prescribed treatment for vitiligo for private insurance versus Medicaid patients ( $p \leq 0.001$ ). Of the 29 documented prior authorization denials, 18 referenced 'cosmetic' conditions for the coverage denial.

Conclusions Insurance carriers frequently consider vitiligo to be a cosmetic condition, limiting access to prescribed TCI medications for pediatric patients. Inequity in access to prescribed treatment exists for patients with Medicaid versus private insurance coverage. Medicaid patients are much more likely to experience insurance denial, lapses in treatment coverage, out-of-pocket cost, and limited coverage of stronger TCI formulations.

\section{\#376 TWENTY YEARS ON-HAS PATIENT-CENTERED CARE BEEN EQUALLY WELL INTEGRATED AMONG MEDICAL SPECIALTIES?}

S Lim*, A Khorrami, RJ Wassersug. The University of British Columbia Faculty of Medicine, Vancouver, $B C$, Canada

\subsection{6/jim-2022-WRMC.372}

Purpose of Study The concept of 'patient-centered care' (PCC) was endorsed by a US Institute of Medicine 2001 publication, now cited $>5000$ times. PCC emphasizes autonomy of patients, prioritizing their subjective assessment of healthcare needs. In this study, we assessed how 6 medical specialties-pediatrics, OB-GYN, orthopedics, radiology, dermatology, and neurosurgery-have engaged with PCC plus two derivative concepts: 'person-centered care' (PeCC) and 'family-centered care' (FCC) over the past 20 years. We hypothesize that large differences in use of these terms reflect gender differences among physicians within the disciplines. Specifically, female dominated fields will have greater engagement with PCC, PeCC and FCC.

Methods Used Reference to PCC, PeCC and FCC in the medical literature for the 6 specialities was used as a proxy for clinical engagement of the concepts in those disciplines. We gathered our sample by cross referencing $\mathrm{MeSH}$ terms of PCC, PeCC \& FCC and the medical subspecialty in PubMed, CINAHL, and PsycInfo. In each specialty, a Pearson correlation test elucidated the change in number of publications of each concept versus percent of female physicians.
Summary of Results There is significant correlation (all $p$ $<.00001$ ) between reference to PCC in the literature and the number of women in each field except neurosurgery $(p>0.5)$. Pediatrics showed the most extensive reference to PCC followed by OB-GYN, with a significant difference between all disciplines $(p<0.001)$. When correcting for the number of papers published in the different fields, reference to PCC is $\sim 18 \mathrm{X}$ more common in pediatrics than in neurosurgery. PeCC is used in pediatrics and OBGYN far more than any other specialty. PCC use grew exponentially each year until 2016, when the trend started to flatten/decline. Conversely, PeCC references have only climbed. If this continues, within 3 years, PeCC will overtake PCC as the dominant care centricity term in academic literature. FCC is exclusively used by pediatrics.

Conclusions Differences in engagement with PCC, PeCC, and FCC appear to reflect true differences in face-to-face interactions within the disciplines, with high uptake in person-oriented specialties (pediatrics \& OBGYN) and low uptake in technique-oriented specialties (surgery). Our data show these differences correlate with the percentage of females in each specialty. Over the last 20 years, order of PCC uptake in the fields has not changed; the female bias has persisted. Our results do not endorse nor reject PCC/PeCC/FCC ideology. Rather, they recognize stereotypical cognitive differences between men and women. Particularly, Baron-Cohen et al (2013) have shown women are more attentive to patients' facial expressions and underlying emotional needs. Thus, as gender proportions continue to shift, we anticipate changes to medical culture. Ideal care will embrace both stereotypical male \& female modes of medical practice.

\section{Endocrinology and metabolism III}

\section{Concurrent session}

\section{8:00 AM}

\section{Saturday, January 22, 2022}

\section{\#377 SENSITIVITY AND SPECIFICITY OF THE MONTREAL COGNITIVE ASSESSMENT FOR DETECTING CLINICALLY SIGNIFICANT COGNITIVE IMPAIRMENT IN OLDER ADULTS WITH TYPE 1 DIABETES}

R Kudrna*, J Choe, L Fonseca, N Chaytor. Washington State University Elson S Floyd College of Medicine, Spokane, WA

\subsection{6/jim-2022-WRMC.373}

Purpose of Study The Montreal Cognitive Assessment (MoCA) is a screening test used to detect cognitive impairment. Data for using MoCA in older adults with type 1 diabetes (T1D) is limited despite increased risk for cognitive impairment in this population.

Methods Used Older adults with T1D were administered a battery of neuropsychological measures and the MoCA. Clinically significant cognitive impairment was defined as 2 or more test performances $\geq 1.5$ SD below demographically corrected normative data. Receiver operating curve (ROC) analysis was performed and the Youden index was used to select an optimal MoCA cutoff score. 
Abstract \#377 Table 1 Summary of results

\begin{tabular}{llll}
\hline MoCA Score & Sensitivity & Specificity & Youden Index \\
\hline$<25$ & 0.479 & 0.829 & 0.308 \\
$<26$ & 0.604 & 0.714 & 0.318 \\
$<27$ & 0.750 & 0.610 & 0.360 \\
$<28$ & 0.865 & 0.419 & 0.285 \\
\hline
\end{tabular}

Summary of Results 201 older adults with T1D (mean age = 68.3, 47\% female, 57\% with bachelor's degree or higher, $92 \%$ non-Hispanic white) completed both the neuropsychological test battery (47.8\% impaired) and MoCA (mean = 25.55, SD $=3.1$. ROC area under the curve was 0.745 [95\% CI 0.678 $-0.812, p<0.05]$. The standard cutoff score of $<26$ resulted in sensitivity of 0.604 and specificity of 0.714 , while a cutoff score of $<27$ resulted in a sensitivity of 0.750 and specificity of 0.610 . The Youden indices for these cutoff values are 0.318 and 0.360 respectively. Minimally acceptable sensitivity (i.e., $>0.80$ ) was obtained when using a cutoff score of $<28$, while $>0.80$ specificity was obtained with a cutoff score of $<25$.

Conclusions The MoCA appears to have moderate overall utility as a cognitive screening instrument in older adults with T1D. Use of the standard cutoff score of $<26 / 30$ may not adequately detect those requiring further evaluation $(60 \%$ sensitivity). The optimal MoCA cutoff score (based on Youden's Index) for detecting cognitive impairment defined by neuropsychological testing was $<27 / 30$. A score of $<28$ resulted in acceptable sensitivity, but was accompanied by low specificity (42\%), necessitating further cognitive evaluation.

\section{\#378 SIDE EFFECT SYNERGISM BETWEEN METFORMIN AND GLP-1 RECEPTOR AGONISTS - AND A SOLUTION}

JM Miles*. University of Kansas Medical Center, Kansas City, KS

\subsection{6/jim-2022-WRMC.374}

Case Report Metformin (MET) is the most widely prescribed medication for type 2 diabetes (T2DM), but it has unfortunate gastrointestinal (GI) side effects (chiefly diarrhea, in 30\% or more of patients) that limit its use in some individuals. MET raises circulating concentrations of glucagon-like peptide-1 (GLP-1), increases satiety, and in some studies delays gastric emptying. Roughly half of an oral dose of MET is not absorbed; and is thought to be responsible for bile acid malabsorption. GLP-1 receptor agonists (GLP-1RAs) are often used in combination with MET and have their own GI side effects; as many as $10-20 \%$ of subjects receiving GLP-1RAs experience nausea and/or diarrhea. We report 6 patients with type 2 diabetes who developed GI side effects when taking MET and a GLP-1RA in combination. Included were 4 males and 2 females, $70 \pm 2$ years old, with BMI $34 \pm 3 \mathrm{~kg} / \mathrm{m} 2$ and hemoglobin A1c 7.5 $\pm 0.8 \%$. All were taking MET 2000-2500 $\mathrm{mg} /$ day in two divided doses before meals or otherwise on an empty stomach, with no GI side effects. When exenatide ER $2 \mathrm{mg}$ weekly was added to MET, a 79 y.o. woman developed severe diarrhea, nausea and vomiting. These symptoms resolved completely when she started taking both doses of MET immediately after eating (PC). Initiation of semaglutide $0.25 \mathrm{mg}$ weekly resulted in intolerable nausea in a 68 y.o. woman; her symptoms disappeared with PC administration of MET, and she subsequently had no side effects on the $0.5 \mathrm{mg}$ dose. A 68 y.o. man was given dulaglutide $0.75 \mathrm{mg} /$ week and tolerated it without difficulty, but when the dose was increased to $1.5 \mathrm{mg} /$ week he developed severe diarrhea and nausea that resolved completely when he took a $48 \mathrm{~h}$ holiday from MET; symptoms did not recur when he resumed MET immediately PC. A 64 y.o. man tolerated dulaglutide $0.75 \mathrm{mg} /$ week but had abdominal cramping and diarrhea with the 1.5 mg dose. There was complete remission of symptoms with PC administration of MET, and he eventually tolerated $3.0 \mathrm{mg} /$ week with no GI side effects. When a 70 y.o. man increased the dose of dulaglutide from $1.5 \mathrm{mg} /$ week to $3.0 \mathrm{mg} /$ week he began having near-daily diarrhea. He started taking MET on a full stomach and eventually was able to take $4.5 \mathrm{mg}$ dulaglutide with no adverse GI effects. A 69 y.o. man began having diarrhea and abdominal discomfort when the dose of dulaglutide was increased from $0.75 \mathrm{mg} /$ week to $1.5 \mathrm{mg} /$ week. With PC administration of MET, he eventually was able to take 4.5 $\mathrm{mg} /$ week with no GI side effects. Conclusions: These 6 cases demonstrate side effect synergism between GLP-1RAs and MET that resolved when MET was taken PC. When diarrhea occurs with initiation of a GLP-1RA, it may thus be an indirect effect mediated via MET, since GLP-1RAs do not cause bile acid malabsorption. After-meal administration of MET allows some patients to tolerate a full dose of both medications, with potential greater benefit in the treatment of T2DM.

\section{\#379 METFORMIN INDUCED LACTIC ACIDOSIS: A CASE REPORT}

${ }^{1} \mathrm{P}$ Chan*, ${ }^{2} \mathrm{E}$ Deemer, ${ }^{2} \mathrm{~L}$ Moosavi, ${ }^{2} \mathrm{~A}$ Heidari. ${ }^{1}$ Ross University School of Medicine, Miramar, FL; ${ }^{2}$ Kern Medical Center, Bakersfield, CA

\subsection{6/jim-2022-WRMC.375}

Purpose of Study To present a rare case of metformin induced lactic acidosis (MALA)

Methods Used Retrospective study

Summary of Results Metformin is the most commonly prescribed and preferred initial drug therapy for type 2 diabetes. Metformin is the only FDA-approved biguanide, due to its lower risk for lactic acidosis. Although rare, metformin-associated lactic acidosis (MALA) has a mortality rate of $31 \%$, which underscores the importance of early diagnosis and treatment.

We report on a 75 -year-old female with diabetes mellitus type 2 with HbA1c $13.6 \%$ and hypertension presented to the emergency room complaining of 2 episodes of clear, watery diarrhea, nausea, and 2 episodes of nonbloody nonbilious emesis for 2 days. Labs were significant for acute kidney injury (AKI) with creatinine of $5.65 \mathrm{mg} / \mathrm{dL}$, anion gap metabolic acidosis $(\mathrm{Na}$ of $129 \mathrm{mmol} / \mathrm{L}, \mathrm{Cl}$ of $96 \mathrm{mmol} / \mathrm{L}, \mathrm{HCO} 3$ of $20 \mathrm{mmol} / \mathrm{L}$, and corrected anion gap of $19 \mathrm{mmol} / \mathrm{L}$ ), and severe lactic acidosis of $8.4 \mathrm{mmol} / \mathrm{L}$. Over 3 days, the lactic acid levels were labile at $8-9 \mathrm{mmol} / \mathrm{L}$, despite aggressive fluid resuscitation. Computerized tomography (CT) abdomen/pelvis, retroperitoneal ultrasound, comprehensive stool panel, blood cultures, and wound cultures were negative for infection, while urinalysis and culture were positive for yeast. Patient's kidney function progressively worsened with oliguria, requiring 
hemodialysis (HD). After $2 \mathrm{HD}$ sessions, the patient's lactic acidosis and anion gap metabolic acidosis resolved.

Of note, the patient was admitted and treated 1 week prior for intractable nausea/vomiting, acute kidney injury, and urinary tract infection. At discharge, the patient's labs showed blood urea nitrogen of $18 \mathrm{mg} / \mathrm{dL}$ and creatinine of $0.86 \mathrm{mg} /$ dL. She was discharged with ciprofloxacin $500 \mathrm{mg}$ twice daily, Metformin $500 \mathrm{mg}$ twice daily, and lisinopril $2.5 \mathrm{mg}$ daily.

Conclusions Metformin is a first-line diabetes medication with a well-known, rare, side effect of lactic acidosis. As a diagnosis of exclusion with a high mortality risk, it is imperative physicians can quickly identify and treat. The treatment for MALA is $\mathrm{HD}$ or continuous veno-venous hemofiltration (CVVH) and should be initiated urgently to prevent further morbidity or mortality.

Ultimately, this patient's rapid decline following metformin initiation illustrates the importance of early recognition and treatment of MALA.

\section{\#380 VITAMIN D STATUS AND CYSTIC FIBROSIS RELATED DIABETES: A RETROSPECTIVE CHART REVIEW}

${ }^{1} \mathrm{~T}$ Gunawardana*, ${ }^{2} \mathrm{Y}$ Peng, ${ }^{2} \mathrm{M}$ Wu, ${ }^{2} \mathrm{~J}$ Alvarez, ${ }^{2} \mathrm{~V}$ Tangpricha. ${ }^{2}$ Emory University, Atlanta, $G A ;{ }^{2}$ Emory University School of Medicine, Atlanta, GA

\subsection{6/jim-2022-WRMC.376}

Purpose of Study Cystic fibrosis, caused by a mutation of the cystic fibrosis transmembrane conductance regulator (CFTR) gene, often results in chronic infection and retention of mucus in the lungs. This drastically decreases the functionality of epithelial cells and has been found to be comorbid with organ dysfunctions such as the pancreas, liver, and even vas deferens. Pancreatic disorder in patients with CF has been associated with decreased absorption of fat-soluble vitamins, including A, D, E, and $\mathrm{K}$, but its relationship with vitamin D hasn't been reported [1]

1. Bertolaso, et al. J. Pediatr. Gastroenterol. Nutr. 2014; 58.4: 443

Methods Used In this retrospective chart review of a longitudinal cohort study of patients identified from the Emory Clinic Data Warehouse, it was attempted to determine the relationship between vitamin D levels and the onset of CFRD. Vitamin D levels were measured via serum 25-hydroxyvitamin D $(25(\mathrm{OH}) \mathrm{D})$, with measurements taken between January 1st, 2008, and December 31st, 2012. Patients included as part of the study were CF patients in the Emory Clinic and Hospital from 2002-2012, and were stratified based on decreased vitamin D levels. Log-rank (Mantel-Cox) Tests compared the relative risk of time to CFRD onset by vitamin D status, while Chi-square tests assessed the association between the development of CFRD and vitamin D status.

Summary of Results The chi-square tests concluded that 25 $(\mathrm{OH}) \mathrm{D}<20 \mathrm{ng} / \mathrm{mL}$ and CFRD development are not independent events (P0.03*), and Log-rank (Mantel-Cox) Test showed a significant hazard ratio between time to CFRD onset and vitamin $\mathrm{D}$ status stratified by deficiency at $25(\mathrm{OH}) \mathrm{D}<20 \mathrm{ng} /$ $\mathrm{mL}$ (95\%CI: 1.2, 2.7, P<0.05**).

Conclusions Adults with CF and 25(OH)D levels below 20ng/ $\mathrm{mL}$ were at an increased risk of developing CFRD overall, while those below $30 \mathrm{ng} / \mathrm{mL}$ didn't show similar correlations. As such, concentrations of $25(\mathrm{OH}) \mathrm{D}$ above $20 \mathrm{ng} / \mathrm{mL}$ may decrease risk of progression to CFRD, in those with CF.
\#381 VITAMIN D, C- REACTIVE PROTEIN, AND TRIGLYCERIDEGLUCOSE INDEX AS POTENTIAL BIOMARKERS FOR DETECTION OF DIABETIC KIDNEY DISEASE

DS Ward*, M Shaheen, K Schrode. Charles Drew University of Medicine and Science, Los Angeles, $C A$

\subsection{6/jim-2022-WRMC.377}

Purpose of Study Diabetic kidney disease (DKD) as a cause of end-stage kidney disease is increasing over time. DKD is projected to increase by $54 \%$ in the US. Glomerular filtration rate (GFR) and Creatine are known biomarkers to detect DKD. This study aims to examine vitamin D and C-reactive protein (CRP) and Triglyceride-Glucose index (T-G index) as biomarkers for the detection of DKD. Find potential biomarkers to aid in the accuracy of the diagnosis of diabetic kidney disease

Methods Used We analyzed data from The National Health and Nutrition Examination Survey 2001-2010. We classified DKD by GFR or albumin-creatinine ratio. Biomarkers tested were vitamin D, CRP, and T-G index. We analyzed the data using multiple logistic regression and produced a receiver operating curve (ROC) to determine the accuracy of the predictions adjusting for the confounding variables. Data were analyzed using SAS9.4 accounting for the design and sample weight.

Summary of Results Of the 1,691 subjects, 54\% had DKD by GFR, $6 \%$ had DKD by albumin-creatinine ratio, $52 \%$ had high-risk CRP levels, 74\% had high-risk T-G index, and 2\% had low vitamin D.

Subjects with high-risk vitamin D, high-risk CRP, and the combined high-risk CRP and T-G index were 5, 3, 2.6, 7 times more likely to have DKD (by albumin-creatinine ratio) respectively relative to the other groups $(\mathrm{p}<0.05)$. These biomarkers can predict subjects with DKD with $67 \%$ to $68 \%$ accuracy.

Conclusions CRP, T-G index, and Vitamin D biomarkers can be used to detect patients with DKD. Longitudinal prospective large sample studies would further the study for possible use in the early detection of DKD.

\section{\#382 THE DEVELOPMENT OF FOURNIER'S GANGRENE IN A PATIENT WITH A HISTORY OF SCROTAL ABSCESS AND UNDERLYING TYPE 2 DIABETES TREATED WITH A SODIUM-GLUCOSE COTRANSPORTER-2 INHIBITOR}

S Sevilla*, J McCaleb, A Alkasir, D Currie. University of Nevada Reno, Reno, NV

\subsection{6/jim-2022-WRMC.378}

Case Report Fournier's gangrene (FG) is a life threatening disease characterized by necrotizing fasciitis of the scrotal and perineal areas. Sodium-glucose cotransporter-2 (SLGT2) inhibitors are a widely approved antihyperglycemic therapy that have been associated with an increased risk of FG. We present a case involving a diabetic patient developing FG several years after initiation of empagliflozin along with a thorough review of the literature.

Case Presentation This case involves a 72 year old Native American male veteran who was initially treated for a left scrotal abscess in 2005. It was during this admission that the veteran was first diagnosed with type 2 diabetes mellitus and started on glipizide. Metformin, glargine, and saxagliptin were sequentially added to his regimen. In 2019, he was started on 
empagliflozin after an A1c of 10.6. Three years later, he was admitted for polymicrobial perineal and scrotal necrotizing fasciitis including part of the bulbar urethra. The extent of his infection required serial debridements and split thickness skin grafts to salvage exposed areas of the left testicle and penile shaft. The veteran was under inpatient care for a total of 45 days during which time empagliflozin was stopped. Subsequent outpatient follow up revealed progressive wound healing and stabilization of his A1c.

Discussion FG is a rapidly progressive disease with a reported mortality rate of $20 \%$ to $40 \%$. SLGT2 inhibitors are a relatively new class of blood sugar lowering medications that work by decreasing glucose reabsorption in the proximal tubule. The glycosuria that is facilitated by this mechanism increases the risk of genitourinary infections. In 2018, the FDA sent out a warning regarding rare occurrences of FG with the use of SLGT2 inhibitors. Although a following meta analysis of randomized controlled trials showed no difference in risk of FG between SGLT2 inhibitors and controls, the number of events was small and led to a wide confidence interval. Thus, an increase in FG risk cannot be ruled out. Further data is needed to support the development of refined guidelines for prescribing SLGT2 inhibitors. A comprehensive history and physical, including past genital infections requiring surgical intervention, may better risk stratify patients for the development of FG prior to initiation of empagliflozin. Alternative treatment options outside of class, such as GLP-1 agonists, may be favorable in the setting of a history of such infections.

Conclusion There is a substantial number of reported cases involving FG and the use of SLGT2 inhibitors in diabetic patients. We intend to add to the pool of data and increase prescriber awareness in order to decrease the incidence and preserve the quality of life of these patients. The implementation of refined drug use criteria for SLGT2 inhibitors may be beneficial.

\section{Genetics II}

\section{Concurrent session}

\section{8:00 AM}

\section{Saturday, January 22, 2022}

\section{\#383 CREATING A ZEBRAFISH MODEL USING CRISPR TO MODEL PHENOTYPIC CHARACTERISTICS OF CNKSR2- RELATED DISEASE}

${ }^{1} \mathrm{SL}$ Cole*, ${ }^{2} \mathrm{~S}$ Nishizaki, ${ }^{1} \mathrm{~L}$ Aki Higa, ${ }^{2} \mathrm{M}$ Dennis, ${ }^{1} \mathrm{JJ}$ Shen. ${ }^{1}$ UC Davis, Sacramento, CA; ${ }^{2}$ University of California Davis, Davis, CA

\subsection{6/jim-2022-WRMC.379}

Purpose of Study The field of genetics is rapidly evolving and more patients are receiving genetic diagnoses for their conditions. Greater understanding of the underlying pathophysiology of genetic diseases is important for improving clinical management and investigating treatment options for affected individuals. Pathogenic variants in Connector Enhancer of Kinase Suppressor of RAS2 (CNKSR2) cause an X-linked condition characterized by epilepsy, sleep disturbances, and developmental delay particularly affecting speech. Even though this is a very rare condition with only $\sim 40$ patients identified worldwide, there is more information known clinically compared to our knowledge about the pathogenic mechanism of disease. We aim to use a zebrafish model to provide further insights.

Methods Used We are establishing a zebrafish model to evaluate CNKSR2-related disease. Due to a genome duplication event there are two paralogues, Cnksr2a and Cnksr2b, that are being disrupted individually and in combination, to determine the extent to which the human disease can be recapitulated. Embryos are injected with knockout DNA designed using CRISPR technology. Once hatched, several tests are performed using FishInspector to evaluate for dysmorphology, and Zebrabox to assess for seizures. FishInspector software is used to measure characteristics of wildtype zebrafish against the CNKSR2 disease model. Features evaluated include head size, eye size and spacing. For seizure evaluation, zebrafish are evaluated first in a normal state and then taken through procedures to reduce the seizure threshold. The movement and activities during each cycle are compared between wild type and the disease model.

Summary of Results At a few days of life the zebrafish were evaluated for phenotypic differences in dysmorphology and movement. Preliminary findings indicate that loss of one of the paralogues results in a statistically significant larger head size (macrocephaly) and that the CNKSR2 disease model zebrafish appear to exhibit more activity consistent with seizures compared to wild type.

Conclusions Studying human disease through zebrafish allows for comprehensive phenotyping of rare genetic conditions, and this model represents a valuable translational medicine tool in investigating future treatments such as gene therapies.

\section{\#384 MATERNALLY INHERITED PTCHD1 VARIATION CAUSING NEURODEVELOPMENTAL DISORDER IN A FEMALE}

${ }^{1,2} \mathrm{M}$ Neu ${ }^{*},{ }^{3} \mathrm{~K}$ Bowling, ${ }^{3} \mathrm{G}$ Barsh, ${ }^{2} \mathrm{~B}$ Korf, ${ }^{3} \mathrm{G}$ Cooper, ${ }^{2} \mathrm{~A}$ Hurst. ${ }^{1}$ Stanford University School of Medicine, Stanford, $C A ;{ }^{2}$ The University of Alabama at Birmingham School of Medicine, Birmingham, AL; ${ }^{3}$ HudsonAlpha Institute for Biotechnology, Huntsville, AL

10.1136/jim-2022-WRMC.380

Purpose of Study This study aims to expand the knowledge of PTCHD1-related neurodevelopmental disorder as a potential cause of developmental delay/intellectual disability and autism spectrum disorder in females.

Methods Used Genetic workup included MECP2 single gene sequencing, chromosomal microarray, short stature panel, Russell-Silver syndrome methylation testing, intellectual disability panel, genome sequencing, and $\mathrm{X}$-inactivation testing.

Summary of Results Genetic workup including MECP2 single gene sequencing, chromosomal microarray, short stature panel, and Russell-Silver syndrome methylation testing were negative. An intellectual disability panel yielded 5 variants of uncertain significance (VUS) that were not compelling. Genome sequencing yielded two likely pathogenic variants per ACMG standards: p.R90* in SCO2 (explanatory for family history of myopia) and p.Y478* in PTCHD1, both maternally inherited. This variant in PTCHD1 was not observed in large-scale databases and variation in PTCHD1 has previously been reported in association with neurodevelopmental disorders. However, PTCHD1-associated neurodevelopmental disorder is inherited in an X-linked fashion, so we sought to determine if a skewed $\mathrm{X}$-inactivation could cause expression in the proband and not 
in the mother. This X-inactivation testing revealed a ratio of 100:0, consistent with a highly skewed pattern with the maternal X chromosome active while the paternal X chromosome is inactive.

Conclusions Variation in PTCHD1 has previously been reported in association with a broad phenotype of autism and developmental delay/intellectual disability (DD/ID), however this X-linked disease is rarely reported in females. Here we report a case in which a truncating variant in PTCHD1 was inherited from an unaffected mother to an 8-year old female proband with $\mathrm{DD} / \mathrm{ID}$, and for which $\mathrm{X}$-inactivation studies demonstrated significant skewing towards the truncated allele. This case highlights the utility of genome sequencing and Xinactivation studies to diagnose challenging cases of X-linked disease in females that may otherwise be overlooked.

\section{\#385 CALCULATION OF BRAIN ORGANOIDS GLUCOSE CONSUMPTION. A CRITICAL FIRST STEP IN UNDERSTANDING ORGANOID METABOLISM}

L Forero*, AE Lee, LM Bird, C Snethlage, A Muotri. University of California San Diego, La Jolla, CA

\subsection{6/jim-2022-WRMC.381}

Purpose of Study Cortical organoids are in-vitro models of the human cerebral cortex. They are derived from human subjects, cultured as pluripotent stem cells, and then differentiated into self-organizing neuronal tissue. They offer a unique way to explore neuroscience in an accessible and flexible environment free of the constraints of in-vivo testing. However, their fidelity as a model for the human brain is still not entirely clear. With the goal of future use in translational research, further study is needed to compare the metabolism and physiology of organoids to functional human brain tissue. The goal of this study was to find the average glucose consumption of an invitro organoid and compare this with the glucose consumption of an in-vivo human brain.

Methods Used We will be using 232 established mature brain organoids derived from a healthy subject divided into six wells with $3 \mathrm{~mL}$ each of standard neurobasal media. We will estimate weight per organoid based on previously established average values. We will measure at Time A the glucose within three $1 \mathrm{~mL}$ samples of the organoid media using a YSI analyzer. Approximately 48 hours later, at Time B, we will take another three samples from the other three wells and again assess the glucose content via YSI analyzer. We will then analyze the glucose consumed by calculating glucose per 1 gram of tissue per day. Finally, we will compare this to known values of human cortical glucose consumption.

Summary of Results The average glucose organoid consumption per organoid was $0.035 \mathrm{mg}$ of glucose in 24 hours. On average, an adult human brain consumes 120 grams of glucose in 24 hours. Estimating an average human brain weight of 1300 grams, we estimated that an adult human brain consumes 90 $\mathrm{mg}$ of glucose per gram of brain tissue per day. Per our findings, organoids consumed on average $26 \mathrm{mg}$ of glucose per gram of brain organoid tissue per day.

Conclusions We have performed an experiment revealing the average glucose consumption of a mature cortical organoid, something that has never before been published in the medical literature. We have discovered that organoids consume approximately $1 / 3$ of the glucose that the average human brain consumes. This information will help establish further translational potential as well as in any future experiments with organoids involving metabolism.

\section{\#386 NEONATAL LETHALITY AND GENETIC MODIFIERS IN A NEW MOUSE MODEL OF NIEMANN-PICK DISEASE, TYPE C}

${ }^{1,2,3} \mathrm{JL}$ Rodriguez-Gil* , ${ }^{1} \mathrm{DE}$ Watkins-Chow, ${ }^{3} \mathrm{FM}$ Platt, ${ }^{1} \mathrm{WJ}$ Pavan. ${ }^{1} \mathrm{NHGRI}$, Bethesda, MD; ${ }^{2}$ Stanford University School of Medicine, Stanford, CA; ${ }^{3}$ University of Oxford Medical Sciences Division, Oxford, UK

\subsection{6/jim-2022-WRMC.382}

Purpose of Study Niemann-Pick Disease, Type C (NPC) is a fatal neurodegenerative disorder that exhibits intracellular accumulation of unesterified cholesterol in late endosomes/ lysosomes and marked accumulation of glycosphingolipids in neuronal tissue. NPC disease is extremely heterogeneous in the timing of clinical presentation (prenatal to adulthood) and severity. Currently there are no FDA-approved therapies that effectively increase lifespan or slow disease progression. For rare disorders such as NPC where sample size is limited, model organisms have played an essential role in the identification of genetic modifiers. We hypothesize that strain-specific variants between inbred mice will have an impact on disease severity, enabling us to identify potential genetic modifiers of NPC disease.

Methods Used We generated a new mouse model for NPC1 harboring a novel allele (Npc1 $1^{\text {em1Pav }}$ ) using CRISPR/Cas9-mediated gene targeting.

Summary of Results Neonatal lethality: During characterization of this mouse model, a significant reduction of viable homozygotes was observed $(10.5 \%$ vs. $25 \%, p<0.0001)$. Interestingly, the expected genotype frequency was observed at E19.5 (25\%) suggesting Npc1 mutants exhibit a previously uncharacterized lethal phenotype postnatally. We confirmed that the majority of Npc1 mutants died shortly after birth and showed signs of respiratory insufficiency. Subsequent electron microscopy analysis of lung tissues showed atypical surfactant morphology.

Genetic modifiers: We also identified strain-specific QTL affecting lifespan. Interestingly, Npc1 mutants on a B6J genetic background had a more severe visceral pathology of foam cell accumulation than Npc1 mutants on a BALB/cJ background. These underlying pathological changes translated into B6J mutants having a significantly shorter lifespan (70 days) than mutants on a BALB/cJ background (84 days; $\mathrm{p}<0.0001$ ), suggesting strain-specific modifiers contribute to disease severity. QTL analysis of backcross N2 mutants detected significant linkage to markers on chromosomes $1 \quad(\mathrm{LOD}=5.57)$ and 7 $(\mathrm{LOD}=8.91)$.

Conclusions The generation of our new NPC1 mouse model facilitated the first analysis of neonatal lethality in an NPC1 model organism. This was also associated with abnormal lung surfactant pathology. A similar phenomenon has been reported in NPC patients with pre/perinatal onset. This model may serve as the first animal model for neonatal- onset NPC1. Furthermore, our study is also the first to identify genomic regions in Npc1 mutant mice containing potential modifier variants associated with changes in lifespan. Identification of modifiers will contribute to our understanding of the highly variable phenotype observed in NPC patients and advance our efforts to improve patient therapy. 
A Renck*, JA Morales, C Lee. Stanford University School of Medicine, Stanford, CA

\subsection{6/jim-2022-WRMC.383}

Purpose of Study Hyperammonemia is a documented phenomenon in patients with fibrolamellar hepatocarcinoma (FH). There are several proposed mechanisms for hyperammonemia in these patients, including intrahepatic shunting, increased cell breakdown from chemotherapy initiation, decreased expression of the OTC gene in tumor cells undergoing treatment with chemotherapy, or a paraneoplastic process disrupting the urea cycle. We hypothesize that the etiology of hyperammonemia in patients with $\mathrm{FH}$ is due to acquired ornithine transcarbamylase (OTC) deficiency.

Methods Used Chart review, biochemical laboratory analysis, and literature review.

Summary of Results Here we describe a 9-year-old girl with newly diagnosed fibrolamellar hepatocarcinoma $(\mathrm{FH})$ with secondary liver failure, hyperammonemia, renal insufficiency with acute kidney injury, gastrointestinal (GI) bleed, and large portal vein thrombosis. Physical exam demonstrated evidence of portal congestion, including dilated veins on the abdomen as well as an enlarged liver. Ammonia level peaked at 370 umol/ $\mathrm{L}$ prior to initiation of continuous renal replacement therapy (CRRT). Biochemical laboratory analysis while on CRRT included plasma amino acids with normal glutamine, normal acylcarnitine profile, and significantly elevated orotic acid in the urine. She was experiencing persistent hyperammonemia ranging from 200-250 umol/L despite CRRT, so she was treated with intravenous arginine and Ammonul (sodium phenylacetate and sodium benzoate). These medications are used in the treatment of primary OTC deficiency, and they produced a decrease in ammonia to normal levels in our patient. Other medication options, including lactulose, rifaximin, and other oral medications were not able to be given due to her ongoing GI bleed. Repeat plasma amino acids were sent once CRRT was discontinued and showed elevated glutamine.

Conclusions Our patient demonstrated biochemical findings (hyperammonemia, elevated glutamine, significantly elevated urine orotic acid) and response to treatment that were suggestive of acquired OTC deficiency. Acquired OTC deficiency is defined by decreased OTC activity that is independent of an OTC disease-causing variant. This evidence supports the hypothesis that acquired OTC deficiency is the etiology of hyperammonemia in FH. Surjan et al. hypothesized that the molecular mechanism is related to increased expression of a fusion protein that activates expression of Aurora kinase A. Aurora kinase A then upregulates c-Myc expression, an oncogene that targets ODC. ODC in turn catalyzes the decarboxylation of ornithine in polyamine synthesis, shunting ornithine away from the urea cycle and inhibiting OTC activity. In order to investigate this proposed mechanism, we intend to conduct further studies to measure polyamine synthesis, a downstream effect of the aforementioned mechanism.

\section{\#388 CHARACTERIZING DECIDUAL AND FETOPLACENTAL GENE EXPRESSION IN PREGNANCY RELATED BULK RNA SEQUENCING DATA}

${ }^{1} \mathrm{LA}$ Chen*, ${ }^{2} \mathrm{~A}$ Rajput, ${ }^{3} \mathrm{G}$ Huynh, ${ }^{2} \mathrm{~S}$ Nair, ${ }^{4} \mathrm{~S}$ Lapehn, ${ }^{1,4} \mathrm{~A}$ Paquette. ${ }^{1}$ University of Washington School of Medicine, Seattle, WA; ${ }^{2}$ University of Washington, Seattle, WA; ${ }^{3}$ Institute for Systems Biology, Seattle, WA; ${ }^{4}$ Seattle Children's Research Institute, Seattle, WA

\subsection{6/jim-2022-WRMC.384}

Purpose of Study Analyzing the transcriptome of the maternalfetal interface can provide insight into placental development and pregnancy complications such as spontaneous preterm birth. Placental and decidual gene expression is cell-type specific, so a molecular dissection of cell and tissue types may improve our understanding of the maternal-fetal interface and reveal differences related to disease. We compiled cell and tissue type specific genes from single cell RNA sequencing (scRNA) studies and investigated relative gene expression in bulk RNA sequencing data of the decidua and placenta.

Methods Used We performed a literature search of placenta publicly available scRNA studies published after 2015. We compiled the results of these studies into a cell specific gene list which described genes uniquely expressed in placental and decidual cells. We tested the accuracy of this gene list by using it to differentiate between paired chorionic villous trophoblast (CVT) and decidua basalis (DB) samples from publicly available bulk RNA dataset GSE73714. We then evaluated the ratios of decidual/placental gene expression in other publicly available bulk RNA datasets from the placenta and decidua using t-tests.

Summary of Results We compiled 2,158 genes from 5 scRNA studies, with 1,103 genes from 9 cell types reported as placenta specific, and 1,208 genes from 9 cell types reported as decidua specific. Many genes reported as placenta-specific in scRNA sequencing datasets were highly expressed in both CVT and DB samples from GSE73714. We evaluated the ratios of decidual-placental gene expression in 5 bulk RNA datasets, containing 17 decidua-related and 29 placenta-related samples total. In a dataset containing matched DB and CVT samples, DB samples had a higher decidua-specific gene expression than samples taken from CVT (t-test, $\mathrm{p}<0.05$ ).

Conclusions We curated a gene list of placental and decidual specific genes using scRNA studies which differentiated between paired DB and CVT samples. Our findings can be used to study cell composition changes in pregnancy complications. For example, placenta samples from spontaneous preterm births often have upregulated immune signaling genes, and our results could help characterize immune cells associated with those genes. Our study also highlights research gaps including a lack of specificity in scRNA datasets and limited decidual scRNA data. More research on decidual tissue is needed to better understand the maternal side of the maternal-fetal interface. 
Healthcare delivery research III

\section{Concurrent session}

\section{8:00 AM}

\section{Saturday, January 22, 2022}

\section{\#389 EVALUATION OF THE EFFICACY OF UNIVERSITY OF NEVADA, RENO SCHOOL OF MEDICINE'S PRE- MATRICULATION PROGRAM, MEDICAL FIRST-YEAR INTENSIVE TRANSITION}

A Wybrecht* E Etter, B Janes. University of Nevada Reno School of Medicine, Reno, NV

\subsection{6/jim-2022-WRMC.385}

Purpose of Study University of Nevada, Reno School of Medicine's (UNR Med) Medical First-Year Intensive Transition (MedFIT) is a two-week orientation program that introduces matriculating students to the academic and extracurricular rigors of medical school. The established program includes a curriculum of lectures intended to introduce students to a normal week of medical school culminating in a low-stakes, professorwritten lecture exam and anatomy practical. In addition, students are placed into groups and introduced to their Pack Mentor, a 2nd year medical student that serves as a longitudinal peer mentor. Previous research on UNR Med's MedFIT has evaluated the curriculum structure through qualitative feedback with student perceptions. However, there is a lack of quantitative data analyzing the real efficacy of each session through objective assessment.

Methods Used A survey was used to evaluate the subjective efficacy of the MedFIT program as well as the objective assessment of their gained knowledge from the program, both pre- and post-MedFIT in July 2021. The survey contained a mix of Likert-style and free response questions for the perceived efficacy and receptivity to various sessions. Additionally, multiple choice questions were included to assess true efficacy through student's retention of the information. The data collected will be evaluated using a paired t-test to compare preand post-MedFIT survey scores of each question. Longitudinal retention of this material and relevance to performance in medical school will be evaluated with a post Block 1 and Block 2 survey in early October and December 2021, respectively.

Summary of Results From preliminary analysis of our results, students viewed the lectures, mock lecture exam, and anatomy practical as the most helpful in preparing them for medical school. The nutrition lecture and student panels were deemed least helpful. We saw large improvements between our pre- and post-medFIT survey scores in students' confidence to find reputable medical sources, understand the Medical Student Performance Evaluation, and which extracurriculars are available to get involved in. Scores in comfortability regarding the Pack Mentor mentorship program or how to manage stress in medical school lacked significant change. Our largest improvements in actual efficacy include information on student body leadership positions as well as supplemental training sessions with a $51 \%$ and $49 \%$ increase in scores, respectively.

Conclusions Preliminary results indicate that MedFIT is effective at presenting salient administrative information and policies while acclimating students to medical school but that the Pack Mentor program could be an area for improvement in the future. Potential solutions might be adding in more structured interactions to allow for cohesiveness across all groups and more time for the incoming students to meet their classmates through social wellness activities.

\section{\#390 ANALYSIS OF ATTITUDES ON SOCIAL MEDIA TOWARDS INTIMATE PARTNER VIOLENCE SCREENING}

${ }^{1} \mathrm{~A}$ Cheng ${ }^{*},{ }^{1} \mathrm{~J}$ Lai, ${ }^{1,2} \mathrm{AL}$ Nelson, ${ }^{1} \mathrm{M}$ Fraix. ${ }^{1}$ Western University of Health Sciences, Pomona, $C A ;{ }^{2}$ University of California Los Angeles, Los Angeles, CA

10.1136/jim-2022-WRMC.386

Purpose of Study Intimate partner violence (IPV) is a preventable public health issue that affects millions of Americans each year. We sought to determine if women and men believe that current protocols are effective and are able to provide IPV survivors with resources for their safety. We also wanted to learn about the experiences and views of the general public on IPV screening. While there have been some studies to suggest that the majority of patients support or agree with IPV screening, there are few current studies that look into patients' attitudes towards IPV screening efficacy, benefit, or frequency.

Methods Used In this IRB approved study, we conducted an analysis of the materials posted on Reddit, an anonymous social media platform. Posts containing the key search words in either the original post or comments were selected for analysis. Exclusion criteria included posts that were created over 5 years ago and posts or comments that did not pertain to IPV or IPV screening. To analyze common attitudes and perceptions, posts and comments were categorized into common themes, which were generated throughout the data collection process and were created when three similar comments were found. Frequencies of themes were compared to identify the most salient themes across all posts. Two-sample $\mathrm{Z}$ tests of proportions were conducted to compare proportions between perceptions of IPV survivors versus non survivors.

Summary of Results Out of 17 posts containing 3,908 comments only 450 pertained to IPV or IPV screening. Though the majority $(61.7 \%)$ of posts had a generally positive view of IPV screening, there were some concerning salient themes such as 'screened in front of significant other, family member, abuser' and 'upset at delivery or method of screening'. Out of the total number of comments that indicated screening status, or whether or not they were screened, there were more that indicated they were screened $(73.7 \%)$ than not screened (26.3\%). In general, most posters think that IPV screening is helpful (81.2\%). However, a significantly greater proportion of posters who indicated they were survivors of IPV believe screening is harmful $(59.5 \%)$ than posters who indicated they have not experienced IPV (0\%). There was no significant difference in positive and negative views between pre-COVID-19 and post-COVID-19 comments.

Conclusions This study shows that the general population has a generally positive view of IPV screening. The negative themes identified and discrepancies in perceptions between IPV survivors and who have not experienced IPV may indicate that there are gaps in how IPV screening is being conducted. 
\#391 THE QUALITY OF YOUTUBE VIDEOS ON RADIOTHERAPY AND PROSTATECTOMY FOR PROSTATE CANCER

${ }^{1} \mathrm{~N}$ Wong*, ${ }^{2} \mathrm{P}$ Ingledew. ' $S t$. George's University School of Medicine, St. George, Grenada; ${ }^{2}$ British Columbia Cancer Agency, Vancouver, BC, Canada

\subsection{6/jim-2022-WRMC.387}

Purpose of Study Prostate cancer is the 3rd leading cause of death among Canadian men with radiotherapy and prostatectomy as options for each disease stage. Studies show that information about treatments and management of side effects are the most needed information for cancer patients. With $\sim 22$ billion people visiting YouTube and many cancer patients using it as a source for cancer related information, it is prudent to understand the quality of information available. This study aims to describe the quality of YouTube videos with specific focus on radiotherapy and prostatectomy and the management of treatment side effects.

Methods Used Videos were selected by searching phrases such as 'Prostate Cancer Radiotherapy or Prostatectomy side effects and/or management'. The first 50 videos were recorded. A rating tool derived from similar tools for the evaluation of websites was adapted to analyse the videos for: currency, attribution, content, coverage and accuracy. DISCERN was used to score attribution and content. Materials from NCCN, UpToDate and cancer.ca were used to develop a consensus document and to evaluate accuracy and coverage of the information presented. Two raters were involved in the review to ensure consistency.

Summary of Results Of the videos analyzed, video length ranged from one minute to one hour long and the dates of creation from 2012 to 2021. Videos were led by physicians $(74 \%)$, patients $(16 \%)$ or other health professionals $(8 \%)$. Of the presenters, physician Video Popularity Index $=23.5$ while patient presented videos $=61.4$. 57\% Radiotherapy videos described the procedure or the risks/benefits of the procedure while $33 \%$ provided a description of both the procedure and described risks/benefits. 53\% Prostatectomy videos described the procedure or the risks/benefits of the surgery while $33 \%$ provided both a procedural description and risk/benefits. $83 \%$ Radiotherapy videos covered side effects, $60 \%$ covered quality of life, and $70 \%$ covered special considerations. In comparison, $89 \%$ prostatectomy videos covered side effects, $75 \%$ covered quality of life, and $78 \%$ covered special considerations. No misinformation was found in radiotherapy videos, however $13 \%$ had missing or ill-elaborated information for side effects. Similarly, no misinformation was found in prostatectomy videos but $19 \%$ did not discuss the procedure in depth.

Conclusions This study provides a description of online resources available to prostate cancer patients. While most of the information is accurate, not all videos cover quality of life, or could be improved to better explain technical aspects of treatment \& management of side effects. This information can be helpful for physicians and patients to navigate educational needs, improve patient understanding and increase patient independence in effective coping and management of side effects.
\#392 WHY ARE SOME PARENTS AGAINST OR UNSURE ABOUT VACCINATING THEIR CHILDREN AGAINST COVID-19?

${ }^{1,2} \mathrm{M}$ Berra*, ${ }^{1,2} \mathrm{M}$ Kung, ${ }^{3} \mathrm{~K}$ He, ${ }^{1,2} \mathrm{~B}$ Afghani. ${ }^{1}$ University of California Irvine, Irvine, $C A$; ${ }^{2}$ Children's Hospital of Orange County, Orange, CA; ${ }^{3}$ Children's Hospital of Los Angeles, LoS Angeles, CA

\subsection{6/jim-2022-WRMC.388}

Purpose of Study The COVID-19 pandemic continues to evolve. Increasing community vaccination rates will be an important factor in curbing the spread of the virus. As children return to schools and other activities, they represent a large group of potential vectors. It is important to understand what concerns parents possess regarding vaccinating their children so that we may help address these hesitations.

Methods Used An anonymous electronic survey was distributed via REDCap to parents of children who were seen in the outpatient or inpatient setting in an academic center between September 2020 and September 2021. Parents were asked to qualify their willingness to vaccinate their children against COVID-19 by choosing 'yes', 'no', or 'not sure'. They then had the opportunity to share an open-ended response explaining their answer. These responses were then analyzed for common themes among those who responded 'no' or 'not sure'.

Summary of Results Of 189 parents who completed surveys, $15 \%$ responded 'no' and 30\% responded 'not sure' when asked if they would vaccinate their children against COVID19. When asked an open-ended question about their hesitation, 42 wrote the reason for their concern. The figure 1 below shares the common themes expressed in these responses. Some responses included multiple themes and thus were attributed more than once in the final totals. The most common themes among those who were unsure was the fear of side effects (56\%), and the most common them among those not wanting the vaccine was the need for more information and research about the vaccine (47\%).

Conclusions It is crucial to be aware of the parents' concerns regarding the COVID-19 vaccine. Our study suggests that the most common reasons for hesitancy are fear regarding the potential side effects and the lack of adequate research and data. Knowledge about the determinants of hesitancy provides insight as to the most important topics to focus when educating families who struggle to make a decision. As the COVID19 pandemic continues to evolve, larger studies are warranted to identify reasons for vaccine hesitancy in different populations.

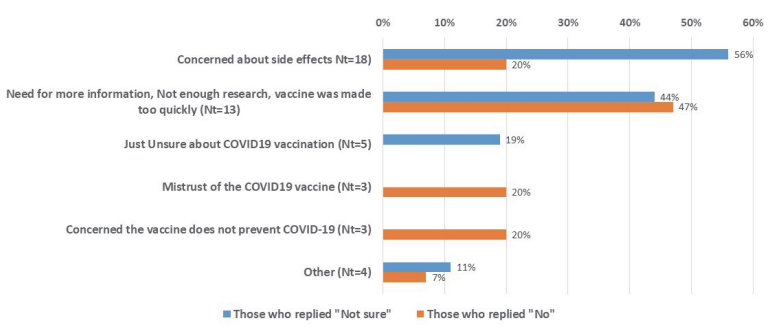

Abstract \#392 Figure 1 
\#393 STRATEGIES TO IMPROVE ALCOHOL USE DISORDER POST-DISCHARGE TREATMENT ENGAGEMENT IN MCCALL, IDAHO

N Massey*. University of Washington School of Medicine, Seattle, WA

\subsection{6/jim-2022-WRMC.389}

Purpose of Study Discussions with local healthcare workers from McCall, a small resort town of 3,000 nestled in the mountains of central Idaho, regarding public health issues highlighted concerningly high rates of alcohol abuse within the community. These observations were supported by the St. Luke's 2019 Community Health Needs Report that reported 25\% of the population engaged in binge drinking in 2016 compared to the national average of $16 \%$. Further investigation highlighted the ways a culture of substance abuse, rising costs of living, and a significant shortage of treatment options contribute to high rates of alcohol use within McCall.

Methods Used An asset-based approach was selected to investigate possible interventions to address alcohol use disorder (AUD). Input from staff at St. Luke's McCall pointed to Recovery Oriented Community (ROC), the only local resource helping individuals with AUD. ROC is led by a group of peer-recovery coaches (PRC), individuals with lived experience in recovery that are focused on providing recovery coaching and resource navigation. Initial discussions with ROC centered around existing ideas to begin introducing hospitalized patients with AUD to PRC. This concept aims to help patients connect with recovery resources after discharge. A literature review of available data was selected to begin investigating the viability and efficacy of such a system.

Summary of Results Research thus far has demonstrated the feasibility of the proposed system and shown encouraging results including improved post-discharge treatment engagement and lower rates of alcohol use following hospitalization. This information was delivered to ROC in an effort to bolster existing discussions with St. Luke's to develop specific policies that incorporate PRC in hospital care for patients with AUD.

Conclusions The asset-based approach used for this project allowed opportunities to assist ROC through providing evidence and support for ongoing efforts to address AUD in McCall. Most meaningfully, this project was done in a practical and relatively expeditious manner with little initial capital. Future work with ROC will focus on educating providers on the benefit of PRC, the development of a strong relationship between these providers and ROC, and ongoing research into the efficacy of this system.

\section{\#394 SUPPORT FOR PEOPLE WITH DEMENTIA AND THEIR CAREGIVERS IN RAVALLI COUNTY, MONTANA USING AN ASSET-BASED APPROACH}

JM Starke*. University of Washington School of Medicine, Seattle, WA

\subsection{6/jim-2022-WRMC.390}

Purpose of Study Ravalli County, Montana has an older population, with $26.5 \%$ being 65 or older compared to just $19.3 \%$ of the state of Montana. Hospice staff note that many people move to Ravalli County to retire for the rural lifestyle and outdoor recreation. In this study, an asset-based approach was used to identify a need in Ravalli County for more support for people with dementia and their caregivers. A review of literature found that Memory Cafes are a realistic intervention.

Methods Used The Ravalli County Health Assessment was reviewed to identify epidemiological factors unique to the county. Next, interviews with a primary caregiver and hospice staff were conducted to learn about specific needs. Additionally, an interview was conducted with the Ravalli County Council on Aging (RCCOA) to recognize existing assets in the community and where there is need for more growth. Finally, literature was reviewed to identify a realistic intervention.

Summary of Results A primary caregiver and hospice staff noted that there is a need for more support for caregivers of the elderly population. The director of RCCOA informed of a plethora of services they offer to the elderly and said there is a specific need for more support for people with dementia and their caregivers. They also noted that RCCOA has resources to expand services in the community. A qualitative study from London found that Memory Cafes, which are a weeklymonthly gathering in the community for people with dementia and their caregivers, provides socialization, decreases isolation, normalizes living with dementia, and supports caregivers of people with dementia.

Conclusions People with dementia and their caregivers in Ravalli County, Montana could better be supported through the implementation of Memory Cafes. RCCOA has the resources and connections in the community to organize this service, making Memory Cafes a realistic intervention for Ravalli County. The asset-based approach was successful because it worked from the perspective of both community members and community organizations as well as the literature to identify a need and solution. Further research is needed to understand how to address barriers to implementing Memory Cafes, especially geographic barriers considering Ravalli County has a low population density.

\section{\#395 IMPROVING OUTREACH FOR UNHOUSED INDIVIDUALS EXPERIENCING OPIOID DEPENDENCE IN SHORELINE, WASHINGTON}

AN Phan*. University of Washington, Seattle, WA

10.1136/jim-2022-WRMC.391

Purpose of Study Unhoused individuals are one of the communities most affected by substance use disorders, but few seek treatment due to stigma, negative attitudes about treatment, lack of motivation, or other challenges such as finances, treatment location, hours of operation of the clinics, etc. Shoreline, a city located in King County, Washington, has been identified by International Community Health Services (ICHS) as a community with a high large number of patients who suffer from opioid abuse. This report analyzes a plausible method for improving outreach for opioid abuse in unhoused individuals that can be implemented by community health partners in Shoreline.

Methods Used Therapeutic Health Services (THS), one of the community health partners addressing opioid abuse located in Shoreline, has made efforts in improving outreach by partnering with programs that house homeless individuals. Different outreach method studies were analyzed in this public health report and peer-recovery was chosen as the most potentially successful outreach method to implement into programs such as THS. One of the studies analyzed was an intervention 
implemented as part of the Illinois Opioid State Targeted Response project. This study implemented those who were at least 1 year in stable recovery to contact populations that were considered 'hidden'. The method used to determine efficacy in this study was determined by the percentage of those that showed up to a linkage meeting.

Summary of Results The results of the Illinois Opioid State Targeted Response project showed that of those who showed up, 92\% agreed to have the linkage manager schedule them a treatment intake appointment and of those, $86 \%$ initiated treatment for an overall treatment initiation rate of $47 \%$. This is significantly better than the overall treatment initiation rate of $28 \%$ found in prior SAMHSA-sponsored 12 site HIV risk reduction projects.

Conclusions Because of the work already underway to expand outreach, implementing peer recovery workers as part of outreach projects is something that can very realistically be done. Additionally, THS has already implemented peer-recovery albeit part of counseling rather than outreach, so this is a plausible method of expanding outreach. Next steps would include seeking out potential candidates and gathering resources needed for implementation of this program.

\section{\#396 AN ANALYSIS OF ORAL HISTORIES: EXPERIENCES OF NON-PHYSICIAN HEALTHCARE WORKERS DURING THE COVID-19 PANDEMIC IN PORT TOWNSEND, WASHINGTON}

K Sexton*. University of Washington School of Medicine, Seattle, WA

\subsection{6/jim-2022-WRMC.392}

Purpose of Study The COVID-19 pandemic affected healthcare communities from the institutional level to the personal level. Current research focuses on the experiences of physicians during the COVID-19 pandemic; however, few studies have investigated the professional and personal impact of COVID19 on non-physician employees of healthcare centers. Additionally, many of the narratives surrounding the COVID-19 pandemic have been centered around large urban institutions; there has been little information discussing the impact of the pandemic on rural communities. The objective of this study is to gain insight into the perspectives of non-physician healthcare workers in a rural town and bring awareness to their experiences.

Methods Used A deductive qualitative study of semi-structured interviews was conducted with employees from a healthcare center in a rural town. Placement site was determined by University of Washington School of Medicine's Office of Rural Programs. A total of five non-physician employees of Jefferson Healthcare Medical Center in Port Townsend, WA were interviewed. Interviews were conducted from June 2021 through August 2021. Interview questions were formed through multiple sessions with colleagues of the National Humanities Center and the University of Washington School of Medicine. As transcripts were reviewed, themes were identified and analyzed.

Summary of Results Analysis revealed the following themes: (1) impact of vaccination on personal and professional identities; (2) communal responsibility throughout the COVID-19 pandemic; and (3) consequences of institutionally implemented safety measures. Additional themes centered around the emotions related to COVID-19, change, and uncertainty.
Conclusions This study brought insight into the experiences of non-physician healthcare workers in a rural town during the COVID-19 pandemic. Utilizing open ended questions with focused listening can give a voice to those in the community. The themes identified demonstrate the importance of recognizing the diverse experiences of community members and the value of community in a rural environment. Lastly, there is much to be gained from using a narrative-based interview style within and outside research settings.

\section{Neonatology general V}

\section{Concurrent session}

\section{8:00 AM}

\section{Saturday, January 22, 2022}

\section{\#397 IMPROVING SCREENING, DIAGNOSIS, AND, MANAGEMENT OF TRANSIENT HYPOGLYCEMIA IN A LEVEL III NEONATAL INTENSIVE CARE UNIT}

W Hulse*, K Schulte, V Eickelkamp-Marron, H Redder, B Chan, J Davidson, C Torr, T DuPont, P Grubb. University of Utah Health, Salt Lake City, UT

\subsection{6/jim-2022-WRMC.393}

Purpose of Study The physiologic transition from fetal to neonatal glucose homeostasis has a nadir by $2-4$ hours of life before a self-recovery. There is emerging evidence that treatment of transient hypoglycemia with intravenous (IV) dextrose may negatively impact neurodevelopmental outcomes. We explored our neonatal intensive care unit (NICU) care practices and identified practice patterns that suggest an over-diagnosis and over-treatment of normal physiologic events. We aimed to modify practice patterns to optimize screening, diagnosis, and management of transient hypoglycemia.

Methods Used The quality improvement population was neonates born $\geq 35$ weeks gestational age at the University of Utah NICU. Infants excluded are those with congenital anomalies, hypoxic-ischemic encephalopathy, mechanical ventilation, or vasopressor infusion. Interventions were obtaining the first blood glucose measurement at 2 hours of life, standardizing the blood glucose concentration for intervention to $<45 \mathrm{mg} / \mathrm{dL}$, and providing the first enteral feed by 2 hours of life. Process measures were time to first blood glucose measurement, the number of infants receiving an IV dextrose infusion or bolus, time to first enteral feed, and total hypoglycemic events in the first 24 hours of life.

Summary of Results Pre-intervention $n=40$ and post-intervention $n=153$. Mean time from birth to first blood glucose measurement increased from $49.5(\mathrm{SD} \pm 17.8)$ to 122 minutes $(\mathrm{SD} \pm 37.1) \quad(\mathrm{p}<0.001)$. IV dextrose infusion rate decreased from $97.5 \%$ of admissions to $41.2 \%$ ( $p<0.01$ ), with a decreased rate of boluses from $32.5 \%$ of patients to $5.8 \%$ $(p<0.001)$. Time to first enteral feed decreased significantly (per XmR chart) from 15.8 hours of life to 3.2 hours of life $(\mathrm{p}<0.001)$. Blood glucose measurements $<45 \mathrm{mg} / \mathrm{dL}$ in the first 24 hours of life had a non-statistically significant rise from $10.1 \%$ to $13.9 \%$.

Conclusions Our practice pattern changes better identified hypoglycemia that persistent beyond the physiologic nadir 
while preventing treatment of normal physiologic events. This evidence shows that early enteral feeds over IV dextrose infusions can provide initial management of transient hypoglycemia without a significant rise in total hypoglycemic events within the first 24 hours of life.

\section{\#398 INVESTIGATING RELATIONS BETWEEN THE NICU SPEECH ENVIRONMENT AND WEIGHT GAIN IN INFANTS BORN VERY PRETERM}

M Chan Morales*, K Kumar, VA Marchman, M Scala, KE Travis. Stanford University, Stanford, CA

\subsection{6/jim-2022-WRMC.394}

Purpose of Study Children born preterm, especially those born very preterm ( $<32$ weeks gestational age, GA) are at risk for adverse neurodevelopmental outcomes, including language and related learning disorders. Adverse language outcomes in preterm children have been attributed, in part, to the minimal amounts speech exposure that neonates are exposed to while in the neonatal intensive care unit (NICU). Experimental studies that directly expose preterm infants to maternal speech sounds in the NICU find significant improvements in health factors relevant for neurodevelopment. However, few studies have examined whether natural variations in the speech environment of the NICU are related to short-term health outcomes in preterm infants. Such data are important for optimizing the speech environment of the NICU. The purpose of this study was to examine relations between the NICU speech environment and rate of weight gain during hospitalization, an important determinant of neurodevelopmental and physical health outcomes for preterm infants.

Methods Used Participants were infants born very preterm $<32$ weeks GA in the NICU at Stanford's Lucile Packard Children's Hospital $(n=20)$. The auditory environment of each infant was assessed at 32-36-week post-menstrual age (PMA) using a speech-counting device known as a Starling, which continuously estimates the amount of speech spoken near to the infant per 5 minute interval. Speech rates were averaged for each infant over the entire 4-week period. Average rates of weight gain (Average grams/kilo/day) were ascertained via electronic medical chart review over the same period. Zero-order correlations and linear regressions controlling for the time infant spent in an incubator versus an open crib and starting weight at 32 weeks PMA were performed to examine associations between speech counts and average rates of weight gain.

Summary of Results Results indicated that infants who heard more speech, on average, gained weight significantly more quickly than those who heard less speech $(r(20)=0.64, p<$ .01). Importantly, speech rate accounted for more than $40 \%$ additional variance after covariates $\left(\mathrm{r}^{2}\right.$-change $=.43, F(1,16)$ $=13.6, p<.001)$. Analyses further exploring rates of visitation and caloric intake as possible confounders did not reduce the size of the effects.

Conclusions The present findings suggest that enhancing the amount of speech exposure that preterm infants experience in the NICU may be beneficial for promoting physical growth, an important determinant of neurodevelopment. As such, NICU policies should be modified to increase speech exposure.
\#399 LIMITED ENGLISH PROFICIENCY AND HISPANIC VERY LOW BIRTHWEIGHT INFANT HEALTH OUTCOMES

${ }^{1} \mathrm{~J}$ Feister*, ${ }^{2} \mathrm{P}$ Kan, ${ }^{1} \mathrm{H}$ Lee. ${ }^{1}$ Lucile Salter Packard Children's Hospital at Stanford, Palo Alto, $C A ;{ }^{2}$ Stanford University, Stanford, CA

10.1136/jim-2022-WRMC.395

Purpose of Study Limited English proficiency (LEP) has been shown to impact patient care. There is a gap in knowledge on the impact of LEP on NICU patients. The primary aim of this study was to a.) characterize the prevalence of LEP in Hispanic families with VLBW infants and b.) determine the association between LEP to various outcomes related to preterm birth.

Methods Used This was a cohort study comparing outcomes in patients with primarily Spanish-speaking families (a proxy for LEP) to patients with primarily English-speaking families. Data from the California Perinatal Quality Care Collaborative with linkage to Office of Statewide Health Planning and Development database were used. Inclusion criteria were VLBW infants (birthweight $<1500 \mathrm{~g}$ ) of Hispanic ethnicity born at participant hospitals from 2009-2012 with documented primary language as either Spanish or English. Exclusion criteria were infants with major congenital anomalies or early mortality. Multivariable regression models accounting for infant factors (birthweight, gestational age) and maternal factors (age, parity, insurance status, and education) were created and odds ratios (OR) were estimated.

Summary of Results Of 7,020 infants who met inclusion criteria, $28 \%$ of families had LEP. Infants of families with LEP were more likely to receive human milk at discharge [crude OR 1.25 (95\% CI 1.12-1.39), aOR 1.55 (1.36-1.77)] less likely to have necrotizing enterocolitis [crude OR 0.74 (0.58$0.94)$, aOR $0.62(0.47-0.82)]$, and more likely to have a hospital readmission within the first year of life (crude OR 1.21 (1.09-1.34), aOR $1.00(0.88-1.12)]$. There were no significant differences in in-hospital mortality, need for oxygen at discharge, retinopathy of prematurity, prolonged length of stay, or high risk infant follow-up clinic referrals.

Conclusions LEP is common among Hispanic families in California with VLBW infants. Infants with LEP are more likely to receive breast milk at discharge and less likely to have NEC than those from families who identify English as their primary language. Families with LEP may be more likely to be recent immigrants, a group that is sometimes demonstrated to have better infant health outcomes than their native born counterparts. Infants of families with LEP were more likely be readmitted to the hospital, suggesting that LEP may be a surrogate marker of re-admission risk, potentially highlighting communication barriers.

\section{\#400 BONE QUALITY ASSESSMENT IN PRETERM INFANTS}

A Tarrell*, S Malone Jenkins, M Grinsell, B Yoder. University of Utah Health Hospitals and Clinics, Salt Lake City, UT

\subsection{6/jim-2022-WRMC.396}

Purpose of Study Diagnosis of metabolic bone disease (MBD) in preterm infants is challenging. The standard for diagnosis is dual energy $\mathrm{x}$-ray absorptiometry (DXA) to measure bone mineral content (BMC), which has barriers limiting its use. Quantitative ultrasound (QUS) is an alternative mode of assessing bone status via measuring speed of sound (SOS). 
SOS is determined by bone cortical thickness, density, microstructure, and elasticity. The purpose of this study is to determine the relationship between BMC, SOS, and growth parameters in preterm infants.

Methods Used In this observational prospective study, preterm infants born at $\leq 32$ weeks gestational age (GA) or birth weight $(\mathrm{BW}) \leq 1800 \mathrm{~g}$ underwent both $\mathrm{SOS}$ and BMC measurements. BMC (Hologic) and SOS (Omnisense 8000P) were assessed $\leq 7$ days of each other at either discharge or 40 weeks corrected GA (CGA). Absolute values were assessed for both tests as well as standardized z-scores for SOS. Pearson correlations $\left(\mathrm{R}^{2}\right)$ and linear regression were used to determine the relationship between demographics, growth parameters and the two modalities.

Summary of Results 41 preterm infants with a mean GA of 29.8 weeks $(\mathrm{SD} \pm 2.2)$ and mean BW $1315 \mathrm{~g}( \pm 429)$ had bone quality assessment at a mean CGA 37.7 weeks ( \pm 2.1 ). SOS was positively correlated with birth GA $\left(\mathrm{R}^{2} 0.261, \mathrm{p}<\right.$ $0.05)$, BW $\left(\mathrm{R}^{2} 0.090, \mathrm{p}=0.057\right)$, and birth head circumference $\left(R^{2} 0.101, p<0.05\right)$. SOS was negatively correlated with CGA at the time of the test $\left(\mathrm{R}^{2} 0.240, \mathrm{p}<0.05\right)$. BMC did not correlate with birth GA, BW, or head circumference. However, BMC positively correlated with CGA, weight, and length at the time of the test $\left(\mathrm{R}^{2} 0.342,0.794,0.239\right.$ and $\mathrm{p}$ $<0.05$ respectively). SOS and BMC were weakly inversely correlated $\left(\mathrm{R}^{2} 0.163, \mathrm{p}<0.05\right)$.

Conclusions SOS and BMC poorly correlate to each other in preterm infants assessed close to term GA. SOS, but not BMC, correlated with birth GA and BW. SOS showed an inverse correlation with advancing postnatal age, with z-scores decreasing from birth to several weeks of age. BMC showed a positive correlation with advancing postnatal age. Given these findings, and the availability to perform repeated SOS at the bedside, we speculate that SOS may be a safer alternative and offer more information about bone health status in growing preterm infants compared to BMC. More research is needed to better understand the roles of SOS and BMC in the assessment of preterm infant bone health.

\section{\#401 FACTORS ASSOCIATED WITH ABNORMAL BRAIN MAGNETIC RESONANCE IMAGING IN VERY LOW BIRTH WEIGHT INFANTS}

NS Nanduri* , A Hisey, C Marquez, L Barton, M Biniwale, R Ramanathan. Keck Hospital of USC, Los Angeles, CA

\subsection{6/jim-2022-WRMC.397}

Purpose of Study Preterm very low birth weight infants (VLBW) are at greater risk of neurodevelopmental morbidity due to impaired brain development. Though it is clinically difficult to predict the outcome of these infants when discharged from the NICU, abnormal brain MRI findings may predict long-term consequences. Existing research shows very limited diagnostic testing done on these infants to assess neurodevelopment in the NICU. Term-equivalent MRI may indicate the need for further developmental assessments and close follow up. There is no consensus on how factors associated with various intensive care interventions link to abnormal MRI findings when these infants reach term gestation. This study aims to evaluate these factors associated with abnormal MRI findings in preterm VLBW infants.

\begin{tabular}{|c|c|c|c|}
\hline & $\begin{array}{l}\text { Brain MRI } \\
\text { normal (\%) }\end{array}$ & $\begin{array}{l}\text { Brain MRI } \\
\text { abnormal (\%) }\end{array}$ & $P$ value \\
\hline Intubation in delivery room & 40.7 & 59.3 & 0.034 \\
\hline Vaginal delivery & 32.3 & 67.7 & 0.008 \\
\hline Highest pCO2 $>50$ in first 24 hours of life & 36.3 & 63.7 & 0.033 \\
\hline Invasive ventilation in first 24 hours & 39 & 61 & $<0.001$ \\
\hline $\begin{array}{l}\text { Invasive synchronized intermittent } \\
\text { mandatory ventilation (SIMV) }\end{array}$ & 42 & 57.9 & 0.046 \\
\hline High frequency oscillatory ventilation & 30.9 & 69.1 & 0.003 \\
\hline High frequency jet ventilation & 23.4 & 76.6 & $<0.001$ \\
\hline Patent ductus arteriosus & 40.9 & 59.1 & 0.004 \\
\hline
\end{tabular}

Methods Used Retrospective data of preterm VLBW infants $(<1500$ g) born between 2009 and 2021 at LAC + USC Medical Center was evaluated for MRI brain findings. Institutional IRB approval was obtained. An abnormal MRI was defined as abnormalities documented by pediatric neuroradiologists, including hemorrhage, periventricular leukomalacia, infarct or ischemia. Maternal factors, resuscitation at delivery, and early neonatal factors were analyzed for significant association.

Summary of Results Out of 356 infants matching inclusion criteria, 191 (52.3\%) had abnormal brain MRIs. Lower gestational age $(\mathrm{p}<0.001)$ correlated strongly with abnormal MRI. Infants delivered by spontaneous vaginal delivery as well as those intubated in the delivery room and with low 5 min Apgar scores were more likely to have abnormal MRI. VLBW infants with higher ventilation and oxygen requirements within the first 24 hours of life were at higher risk for developing abnormal MRI. Infants requiring invasive ventilation support beyond 24 hours of life were also more likely to have abnormal MRI, including those requiring high frequency oscillatory ventilation and jet ventilation. Incidence of abnormal MRI in infants with hemodynamically significant patent ductus arteriosus (PDA) was also significantly higher. These infants were also more likely to have initial low hematocrit $(\mathrm{p}=0.003)$ and severe intraventricular hemorrhage on cranial ultrasound $(\mathrm{p}=0.016)$. NICU duration of stay was longer for infants with abnormal MRI $(p<0.001)$. Over $50 \%$ of abnormal MRI findings were related to cerebellar abnormalities.

Conclusions VLBW infants with factors including need for intubation in the delivery room, higher ventilation and oxygen requirement in the first 24 hours of life, longer invasive ventilation, hemodynamically significant PDA, low hematocrit and abnormal cranial ultrasound predict abnormal brain MRI findings performed at term.

\section{\#402 USE OF ELECTRONIC TEXT MESSAGING OF NRP PRINCIPLES TO PEDIATRIC TRAINEES \& IMPACT ON RETENTION}

${ }^{1} \mathrm{~F}$ Eskandar-Afshari*, ${ }^{1} \mathrm{MP}$ Martes Gomez, ${ }^{1} \mathrm{~S}$ Liu, ${ }^{1} \mathrm{~K}$ Tedesco, ${ }^{2} \mathrm{AM}$ Yeh, ${ }^{2} \mathrm{R}$ Ramanathan, ${ }^{2}$ M Biniwale. ' LAC+USC Medical Center, LA, CA; ${ }^{2}$ USC Keck School of Medicine, LA, CA

\subsection{6/jim-2022-WRMC.398}

Purpose of Study Pediatric trainees have decreased exposure to neonatal resuscitation. The purpose of this study was to 


\section{WHAT ARE THE INDICATIONS FOR PULSE OXIMETRY?}

- When resuscitation is anticipated

- To confirm your perception of persistent central cyanosis

- When supplemental oxygen is administered

- When positive pressure ventilation is required

\section{WHERE SHOULD THE PULSE OXIMETER BE PLACED AND WHAT OXYGEN SATURATIONS SHOULD BE TARGETED?}

The pulse oximeter should be placed on the right hand or wrist to obtain a pre-ductal saturation (see image on right).

\section{WHAT ARE THE TARGET PRE-DUCTAL SATURATIONS AFTER BIRTH?}

\begin{tabular}{|cc|}
\hline Pre-ductal $\mathrm{Spo}_{2}$ Target \\
$1 \mathrm{~min}$ & $60 \%-65 \%$ \\
$2 \mathrm{~min}$ & $65 \%-70 \%$ \\
$3 \mathrm{~min}$ & $70 \%-75 \%$ \\
$4 \mathrm{~min}$ & $75 \%-80 \%$ \\
$5 \mathrm{~min}$ & $80 \%-85 \%$ \\
$10 \min$ & $85 \%-95 \%$ \\
\hline
\end{tabular}

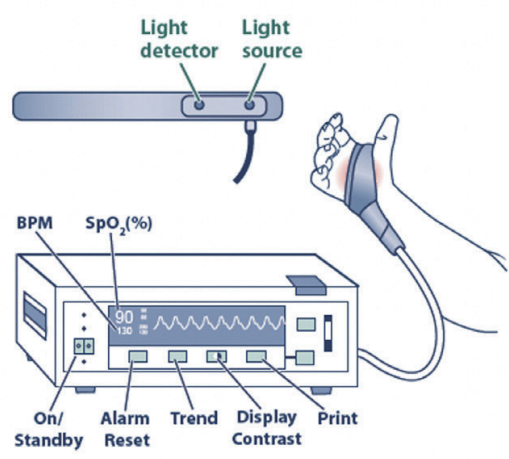

Abstract \#402 Figure 1 NRP Content pearl example

provide distance learning via text messaging of NRP principles 8 th edition and assessing retention and integration of NRP skills among pediatric residents.

Methods Used This is a prospective, randomized-controlled study including Pediatrics and Medicine-Pediatrics residents. An initial survey and quiz were completed to assess baseline knowledge and confidence in neonatal resuscitation. Twenty participants were included and randomized to control group, no text messages, and intervention group, receiving biweekly NRP content pearls (Image 1) for 6weeks. Subsequently, there was a 6 -week period of no contact. All participants completed a repeat quiz at 6-weeks and 12-weeks to assess their knowledge retention. Two high fidelity simulations were completed by all participants who were scored on skills, knowledge and teamwork using a validated tool.

Summary of Results Of the 20 study participants, 16 were Pediatric residents and 4 were Medicine-Pediatrics residents. Thirty-five percent were PGY-1, 40\% were PGY-2 and $25 \%$ were PGY-3. Fifty percent had less than three resuscitations for term infants and $70 \%$ had less than three resuscitations for preterm infants in the last year. Majority agreed that they needed more knowledge and experience in neonatal resuscitation. Seventy percent stated that they are not confident in leading a neonatal resuscitation. As for the initial quiz scores, Pediatric PGY-1, 2, and 3 scored $72.5 \%$, $69.25 \%$, and 69\%, respectively. Medicine-Pediatrics PGY-1 and 2 scored $63.4 \%$ and $80 \%$, respectively. Questions missed were about secondary apnea, pre-birth questionnaire, volume of intravenous/intraosseous flush to be given and the chronologic sequence of transitional physiology after birth.

Conclusions Based on our initial results, it is evident that Pediatric trainees do not get enough experience, lack knowledge, and do not feel comfortable leading a neonatal resuscitation. With ongoing text messaging of NRP content pearls, we hope to show an increase in Pediatric trainees' retention of knowledge and confidence level in caring for neonates requiring resuscitation at birth.

\section{\#403 COMPARISON OF PROCALCITONIN AND HIGH SENSITIVITY C-REACTIVE PROTEIN AS SCREENING TESTS IN NEONATES FOR EARLY ONSET SEPSIS AND LATE ONSET SEPSIS}

A Vachhani* , A Chambliss, M Durand, R Ramanathan, R Cayabyab. LAC+USC Medical Center, Keck School of Medicine of USC, Los Angeles, CA

10.1136/jim-2022-WRMC.399

Purpose of Study Early detection of both early onset sepsis (EOS) and late onset sepsis (LOS) in newborns is difficult as the first signs of infection are often nonspecific. A laboratory test to rapidly identify these infants would add value in improving outcomes and limiting unnecessary antibiotic exposure. The purpose of this study was to compare the utility of procalcitonin (PCT) and high sensitivity CRP (hsCRP) as screening tests for EOS and LOS.

Methods Used Retrospective study of infants admitted to the neonatal intensive care unit from August 2018-February 2021. Infants with two paired PCT and hsCRP values obtained 1224 hours apart and with blood, urine or cerebrospinal fluid culture results were included. EOS workup was performed at $\leq 72$ hours of life while LOS workup at $>72$ hours of life. Infants on antibiotics at the time of workup and those diagnosed with presumed infection, but negative cultures were excluded. Infants were classified as non-infected or infected (positive culture). Data were analyzed with Fisher-exact test or Chi Square and Wilcoxon rank sum where appropriate. Diagnostic value was determined by calculating sensitivity, specificity, positive predictive value (PPV) and negative predictive value (NPV) using cutoff of $10 \mathrm{mg} / \mathrm{L}$ and $1.0 \mathrm{ng} / \mathrm{mL}$ for hsCRP and PCT respectively.

Summary of Results A total of 359 infants received workups for EOS and 126 infants for LOS. There were 12 infants (3\%) who had confirmed EOS while 21 (17\%) with confirmed LOS. Demographics and outcomes of infants are shown in table 1. The sensitivity, specificity, PPV and NPV for serial measurements of PCT and hsCRP are shown in table 2. For EOS, subsequent measurements of both biomarkers led to 
Abstract \#403 Table 1 Demographics between groups

\begin{tabular}{|c|c|c|c|c|c|c|}
\hline & EOS Workup & & & LOS Workup & & \\
\hline & $\begin{array}{l}\text { Non-infected Infants } \\
\mathrm{N}=275\end{array}$ & $\begin{array}{l}\text { Infants with Confirmed Infection } \\
\mathrm{N}=12\end{array}$ & P-value & $\begin{array}{l}\text { Non-infected Infants } \\
\mathrm{N}=80\end{array}$ & $\begin{array}{l}\text { Infected with Confirmed Infections } \\
\mathrm{N}=21\end{array}$ & $\overline{\text { P-value }}$ \\
\hline Gestational Age ${ }^{\star} \wedge$ & $34.9(33.1,37.6)$ & $39.2(37.3,40.2)$ & $<0.01$ & $37.1(31.4,39.4)$ & $38.2(31.1,39.5)$ & 0.86 \\
\hline Birth Weight $(\mathrm{g})^{* \wedge}$ & $2285(1850,3120)$ & $2915(2238,3365)$ & 0.24 & $2780(1500,3320)$ & $2632(1455,3285)$ & 0.74 \\
\hline Small for Gestational Age ${ }^{{ }^{\wedge}}$ & $32(12)$ & $1(8)$ & 1.0 & $6(9)$ & $2(12)$ & 0.67 \\
\hline Male sex ${ }^{+}$ & $142(52)$ & $10(83)$ & 0.04 & 41 (57) & $12(60)$ & 1.0 \\
\hline C-section $^{+^{\wedge}}$ & $174(63)$ & $4(33)$ & 0.06 & $28(44)$ & $8(53)$ & 0.58 \\
\hline Length of stay (days)* & $13(6,22)$ & $14(12,19)$ & 0.40 & $3(2,28)$ & $14(7,31)$ & $<0.01$ \\
\hline Survival to Discharge ${ }^{+}$ & $268(97)$ & $12(100)$ & 1.0 & $72(100)$ & $19(75)$ & 0.22 \\
\hline
\end{tabular}

* Median (25th percentile, 75 th percentile) $+\mathrm{N}(\%) \wedge$ Some missing data in LOS patients Note: Demographic for infants with multiple LOS admission were included only once in analysis

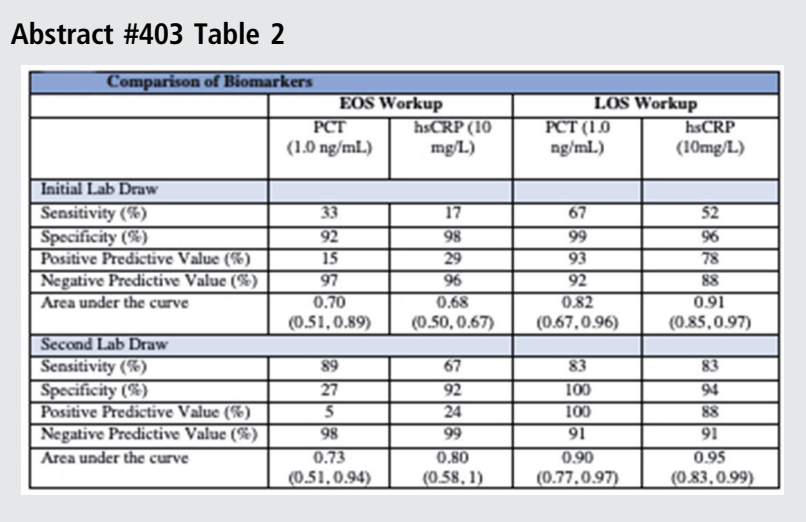

an increase in sensitivity, but a decrease in PPV while in LOS sensitivity and PPV increased. The areas under the ROC curve were higher for both biomarkers for LOS in comparison to EOS (table 2).

Conclusions Our preliminary findings indicate that PCT and hsCRP may have better diagnostic value in patients for LOS in comparison to EOS. Previous studies suggest a natural rise in levels on serial measurements in the first 72 hours in all neonates influencing its diagnostic value and presence of peripartum and maternal risk factors can also influence levels after birth in the absence of infection.

\section{Neonatology - perinatal biology II}

\section{Concurrent session}

\section{8:00 AM}

\section{Saturday, January 22, 2022}

\section{\#404 MULTIOMICS LONGITUDINAL MODELING OF PREECLAMPTIC PREGNANCIES}

${ }^{1} \mathrm{C}$ Espinosa, ${ }^{1} \mathrm{I}$ Maric*, ${ }^{*} \mathrm{~K}$ Contrepois, ${ }^{2} \mathrm{M}$ Moufarrej, 'IS Stelzer, ${ }^{1} \mathrm{D}$ Feyaerts, ${ }^{3} \mathrm{X}$ Han, ${ }^{1}$ A Tang, ${ }^{1} \mathrm{RJ}$ Wong, ${ }^{1} \mathrm{GL}$ Darmstadt, ${ }^{1}$ VD Winn, ${ }^{1} \mathrm{GM}$ Shaw, ${ }^{1} \mathrm{DA}$ Relman, ${ }^{2} \mathrm{SR}$ Quake, ${ }^{1} \mathrm{MS}$ Angst, ${ }^{1} \mathrm{M}$ Snyder, ${ }^{1} \mathrm{DK}$ Stevenson, ${ }^{1} \mathrm{~B}$ Gaudilliere, ${ }^{1} \mathrm{~N}$ Aghaeepour. ${ }^{1}$ Stanford University School of Medicine, Stanford, CA; ${ }^{2}$ Stanford University, Stanford, $C A ;{ }^{3}$ University of the Pacific - San Francisco Campus, San Francisco, CA
Purpose of Study Preeclampsia is a complex disease of pregnancy of unclear pathophysiology. Specific complex changes in a woman's physiology precede diagnosis of preeclampsia. Understanding these changes at different levels of biology can be enabled by simultaneous application of multiple assays. We developed prediction models for preeclampsia risk by analyzing six omics datasets from a longitudinal cohort of pregnant women.

Methods Used We performed a multiomics analysis of the transcriptome, proteome, metabolome, lipidome, and microbiome from blood, urine and vaginal samples collected longitudinally during pregnancy at the Lucile Packard Children's Hospital at Stanford University. Our discovery and validation cohort included 33 and 16 women, respectively. Prediction models were built for each omics set using the elastic net machine learning method and their integration was performed using stacked regression. Immune system mass cytometry features and available clinical variables were subsequently integrated with the model.

Summary of Results The multiomics model predicting which women will develop preeclampsia had high accuracy (area under the receiver operating characteristics curve (AUC) of 0.94; 95\% confidence interval (CI): $[0.90,0.99])$. A prediction model using only ten urine metabolites provided an accuracy equivalent to that of the model using the complete metabolomic dataset and was validated using an independent cohort (AUC $=0.87 ; 95 \% \mathrm{CI}:[0.76,0.99])$. Univariate analysis further confirmed statistical significance of proteins and metabolites chosen by the prediction model. Several proteins identified as biomarkers in our model have previously been associated with preeclampsia (e.g., LEP, VEGF-A) further validating our findings. Integration with clinical variables further improved prediction accuracy of the urine metabolome model (AUC $=0.90 ; 95 \%$ CI: $[0.80,0.99]$, validated). Several biological pathways were identified to be associated with preeclampsia. Integration with the immune system data confirmed known pathological alterations associated with preeclampsia and suggested novel associations between the immune and proteomic dynamics.

Conclusions While further validation in larger populations is necessary, these encouraging results will serve as a basis for a simple, early diagnostic test for preeclampsia. 
\#405 DISCOVERY AND VERIFICATION OF EXTRACELLULAR MIRNA BIOMARKERS FOR NON-INVASIVE PREDICTION OF PREECLAMPSIA IN ASYMPTOMATIC WOMEN

N Do*. University of California San Diego, La Jolla, CA

\subsection{6/jim-2022-WRMC.401}

Purpose of Study Preeclampsia is a pregnancy complication characterized by new-onset high blood pressure and protein in the urine. It is the most common cause of preterm birth and the second most common cause of maternal mortality. For patients with risk factors such as chronic hypertension, diabetes, or prior affected pregnancy, daily low dose aspirin administered between 12-28 weeks of gestation has been shown to decrease preeclampsia risk. However, many patients that develop preeclampsia do not have known risk factors. Thus, effective and non-invasive screening for high-risk pregnancies may be clinically useful. This study explores extracellular miRNAs (exRNAs) in blood and other biofluids as identifiers of preeclampsia risk. In a previous study, we identified candidate miRNA biomarkers for the prediction of preeclampsia. Our aim is to validate these biomarkers of interest in an independent set of patients in a larger cohort.

Methods Used To perform this validation, we isolated the exRNA from maternal serum from a new cohort of subjects using the Plasma/Serum Circulating and Exosomal RNA Purification Kit (Slurry format, Norgen Biotech). We then used small RNA sequencing to quantify the RNA in those samples. We will also use qPCR to specifically quantify the two miRNAs comprising our top bivariate candidate biomarker, hsamiR-516b-5p/hsa-miR-155-5p, which we showed in our original study to be correlated with preeclampsia risk.

Summary of Results exRNA of adequate quality was extracted from the maternal serum samples. The acquired sequencing data showed adequate quality for the large majority of exRNA samples, as measured by several metrics, including: total sequencing reads; percentage of miRNAs as compared to all RNA biotypes in the sample; and miRNA complexity, which indicates the number of detected distinct miRNA sequences in each sample.

Conclusions Going forward, we will perform data analysis to determine whether the candidate miRNAs from the original cohort are confirmed in the validation cohort. If the performance of the biomarkers of interest is validated, this will

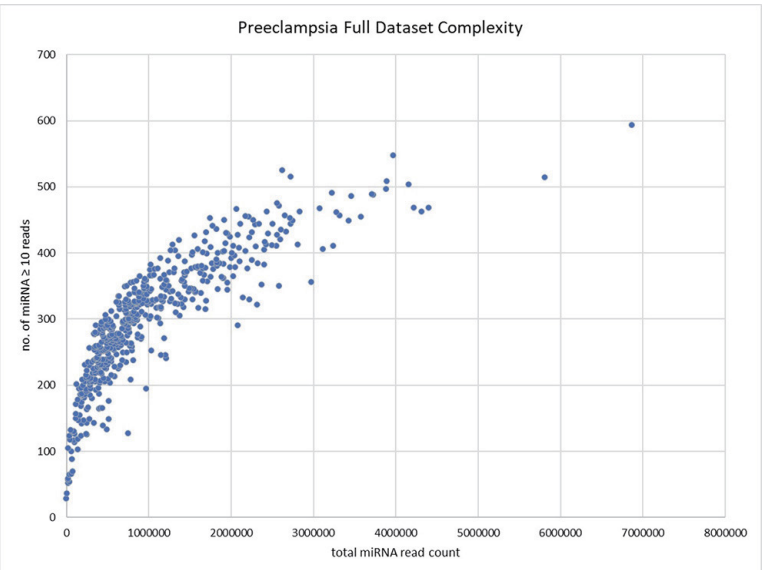

Abstract \#405 Figure 1 Complexity refers to the number of miRNAs with at least 10 or more read counts support their further development as clinical biomarkers for prediction of preeclampsia.

\section{\#406 BREASTFEEDING DIFFICULTY INCREASES DEPRESSIVE SYMPTOMS AND IMPAIRS MATERNAL-INFANT BONDING: A CONTENT ANALYSIS OF MATERNAL BREASTFEEDING NARRATIVES}

${ }^{1}$ EA Wright*, ${ }^{1} \mathrm{~A}$ Mehta, ${ }^{1} \mathrm{AL}$ Nelson, ${ }^{2} \mathrm{H}$ Stohl, ${ }^{2} \mathrm{M}$ Economidis. ${ }^{1}$ Western University of Health Sciences College of Osteopathic Medicine of the Pacific, Pomona, CA; ${ }^{2}$ Harbor-UCLA Medical Center, Torrance, CA

10.1136/jim-2022-WRMC.402

Purpose of Study Protection against postpartum depression (PPD) is a commonly cited maternal benefit of breastfeeding ${ }^{1}$. However, one study found that negative breastfeeding experiences may actually increase depressive symptoms instead of reducing them. The purpose of this study is to evaluate women's self-reported associations between breastfeeding and PPD $^{2}$. Methods Used An electronic search was conducted on Reddit including original user posts with keywords 'breastfeeding' and 'depression'. Relevant posts and corresponding comments ( $\mathrm{n}=$ 584) were extracted from February 2013 to July 2021 and subject to qualitative content analysis.

Summary of Results Narratives most frequently described difficulty breastfeeding as the cause of new onset mental health symptoms (53\%); however, mothers breastfeeding without difficulty were also susceptible (20\%). Among mothers reporting exacerbated pre-existing mental health symptoms, a similar greater proportion described difficulty breastfeeding (17\%), but still others experienced no difficulty (6\%). Common words included in these posts were 'guilt' (17\%), 'fail' (14\%), 'pressure' (7\%), 'shame' (6\%), and 'alone' (6\%), highlighting the emotional impact of breastfeeding difficulty. Only 3\% of posts described breastfeeding as improving mental health symptoms. Other themes yielded from this study included weaning-induced mental health symptoms (17\%) and decreased milk supply due to mental health symptoms (2\%). A concerningly large proportion of posts described a lack of knowledge (23\%). Finally, among posts mentioning bonding ( $\mathrm{n}=99$ ), $58 \%$ reported that breastfeeding inhibited infant bonding, versus only $1 \%$ enhanced bonding-contradicting the prevailing idea that breastfeeding stimulates bonding ${ }^{3}$. After switching to formula, 63\% reported increased bonding, $8 \%$ no change, and $6 \%$ decreased bonding.

Conclusions Mothers struggling to breastfeed may not experience the classically described benefits of breastfeeding, instead exhibiting increased depressive and other mental health symptoms and impaired maternal-infant bonding. These findings highlight the need for enhanced education on breastfeeding and mental health relationships, and adjustment of practices to meet the diversity of maternal experiences.

\section{\#407 MULTIOMICS MODELING OF PRETERM BIRTH IN LOW- AND MIDDLE-INCOME COUNTRIES}

CE Espinosa Bernal ${ }^{*}$, I Maric, DK Stevenson, N Aghaeepour. Stanford University School of Medicine, Stanford, CA

10.1136/jim-2022-WRMC.403

Purpose of Study Preterm birth (PTB) is the leading cause of death in children under five years of age across the globe. 
Efforts to improve our understanding of PTB are hindered by its complex etiologies. Epidemiologic associations between PTB and maternal clinical history, demographic characteristics, and social determinants have been extensively described, but have been limited in inference owing to a lack of attendant biological data.

Methods Used This work employed multiomic profiling and multivariate modelling to investigate the biological signatures of some of these maternal covariates and gain insight into the epidemiological signature of PTB. Maternal covariates and plasma samples were collected during early pregnancy from a multinational cohort of 13,841 pregnant women (PTB rate of $11.4 \%$ ) across four low- and middle-income countries. Plasma samples from 231 participants were further analyzed to generate proteomics, metabolomics, and lipidomics datasets.

Summary of Results An epidemiological model for the prediction of PTB achieved moderate accuracy (AUROC $=0.70$ ), highlighting the difficulty of the predictive task. Machine learning-based multiomics models had strong performance for the prediction of multiple relevant objectives, including time to delivery (Pearson's $\mathrm{R}=0.64$ ) and maternal covariates such as age $(\mathrm{R}=0.57)$, gravidity $(\mathrm{R}=0.53)$, and body mass index (BMI, $\mathrm{R}=0.80$ ). The biological signature for time to delivery included fetal-associated proteins such as alpha-fetoprotein and immune proteins like PD-L1. Maternal age had a strong negative correlation with type IX collagen conserved across our multinational cohort. Gravidity had an age-independent association with levels of endothelial NOS and inflammatory chemokine CXCL13, an observation further validated on an independent Stanford cohort $(n=17)$. BMI positively correlated with adipocyte hormone leptin and structural fatty acid protein FABP4.

Conclusions These results simultaneously identify novel signatures for maternal covariates impacting PTB and shed light on potential biological and socioeconomic interventions that generalize across multiple populations.

\section{\#408 EARLY PREDICTION OF GESTATIONAL DIABETES MELLITUS BY IN-VIVO MAGNETIC RESONANCE IMAGING}

B Lee*, K Sung, C Janzen, S Vangala, SU Devaskar. University of California Los Angeles, Los Angeles, CA

\subsection{6/jim-2022-WRMC.404}

Purpose of Study Gestational diabetes (GDM) is a risk factor for the development of cardiovascular and metabolic disease in both mothers and their offspring, and central obesity is associated with the development of GDM. Detection of GDM in early gestation may improve health outcomes of at-risk mothers and babies. Our group and others have developed invivo magnetic resonance imaging (MRI) modalities suitable for monitoring pregnancies during early gestation. We hypothesized that early gestation MRI will predict the subsequent development of GDM.

Methods Used 200 women were recruited in the first trimester of pregnancy. We recorded the pregnancy course through four visits (at 11-14 weeks, 19-22 weeks, 36 weeks, and at delivery), and conducted chart reviews to gather clinical outcomes on the mother-baby dyad. Two MRIs were prospectively performed on each subject, at 14-16 weeks and 19-22 weeks. Using imaging software, placental volume was obtained by

\begin{tabular}{|c|c|c|c|}
\hline Race & $\begin{array}{l}\text { GDM group } \\
\text { (n,\% of total GDM } \\
\text { group) }\end{array}$ & $\begin{array}{l}\text { Control group } \\
\text { ( } \mathrm{n}, \% \text { of total control } \\
\text { group) }\end{array}$ & Total \\
\hline White, non-Hispanic & $9(41 \%)$ & $81(48 \%)$ & 90 \\
\hline Hispanic & $4(18 \%)$ & $33(20 \%)$ & 37 \\
\hline Asian & $9(41 \%)$ & $42(25 \%)$ & 51 \\
\hline Black or African American & 0 & $12(7 \%)$ & 12 \\
\hline $\begin{array}{l}\text { American Indian or Alaska } \\
\text { Native }\end{array}$ & 0 & $1(1 \%)$ & 1 \\
\hline Total & 22 & 169 & 191 \\
\hline
\end{tabular}

tracing placental regions of interest in three axes. Fat content was measured from the L1 through L5 vertebrae. Subcutaneous fat area ratio (SFAR) was derived by assessing the subcutaneous fat area normalized to the whole trunk area; visceral fat area ratio (VFAR) was derived by assessing the visceral fat area normalized to the whole visceral area.

Summary of Results A total of 191 subjects were analyzed (table 1). 169 women (88\%) did not develop GDM, forming our control group. 22 women (12\%) developed GDM.

SFAR did not differ between the GDM and control groups in either the first or the second MRI scans. In contrast, mean VFAR, as analyzed by the two-tailed T-test, was higher in the GDM group versus the control group, at both the first $(p<0.0001)$ and second MRI $(p=0.002)$. Receiver operating characteristic curves (ROC) for VFAR, using the Wilcoxon rank-sum test, revealed an area under the curve (AUC) of $0.895(\mathrm{p}<0.001)$ and $0.806(\mathrm{p}=0.003)$ for the first and second MRIs, respectively. In contrast the SFAR ROC AUCs were not predictive.

Mean placental volumes, as analyzed by two-tailed T-test, did not differ between the GDM and control group at the first MRI, but were significantly larger in GDM vs controls ( $p$ $=0.01$ ) at the second MRI. ROC AUCs for placental volumes were not predictive at the first, but predictive at the second MRI (AUC $=0.6517 ; \mathrm{p}=0.03$ ).

Conclusions Visceral fat area and placental volume in early gestation may be predictive of the subsequent development of GDM. We speculate such early predictions set the stage for undertaking streamlined clinical trials towards developing timely interventions in improving outcomes for mothers and children.

Supported by NIH grant U01-HD087221.

\section{Case reports II}

\section{Concurrent session}

\section{0:15 AM}

Saturday, January 22, 2022

\section{\#409 PERIANAL PSEUDOVERRUCOUS PAPULES AND NODULES PRESENTING AS SUSPECTED CHILD ABUSE}

JP Wang * ${ }^{*}$ K Shea. LAC+USC Medical Center/USC, Los Angeles, CA

10.1136/jim-2022-WRMC.405 
Case Report Due to their sensitive location, perianal lesions often alert clinicians to potential sexual abuse. Perianal pseudoverrucous papules and nodules (PPPN), first described in patients with urostomies in 1979 under the name chronic papillomatous dermatitis, are often mistaken for condyloma acuminata due to their wart-like appearance. Early recognition and treatment of PPPN would lessen parental distress and avoid unnecessary workup for children who suffer from this illness.

Case Description The subject is a four-year-old Hispanic male who was admitted to the pediatric ward for perianal lesions, rectal pain, and itching. The patient's lesions first appeared 8 months prior to this admission, gradually becoming larger and more numerous. The lesions were preceded by a history of constipation, minimally responsive to MiraLax, and subsequent frequent and loose stools with stool-holding behaviors.

The subject was then prescribed topical mupirocin, bacitracin, lidocaine, and oral Keflex at multiple outside ED visits.

The patient was referred for inpatient management of his lesions by DCFS. He was diagnosed with severe constipation and encopresis. Dermatology clinically diagnosed PPPN without a biopsy. He underwent bowel disimpaction and was placed on an aggressive bowel regimen.

Patient was discharged on hydrocortisone, ketoconazole, and zinc oxide for four months with significant improvement of lesions. Patient was toilet trained six months after discharge with complete resolution of lesions.

Conclusion PPPN present with multiple well-demarcated and dome-shaped papules due to constant irritations. They have smooth surfaces and are about $2-10 \mathrm{~mm}$ in diameter. Their friable textures have a tendency to ulcerate and are prone to secondary infections.

Definitive diagnosis is made by skin biopsy, useful in cases with challenging clinical course that mimic other conditions. However, most cases are diagnosed clinically.

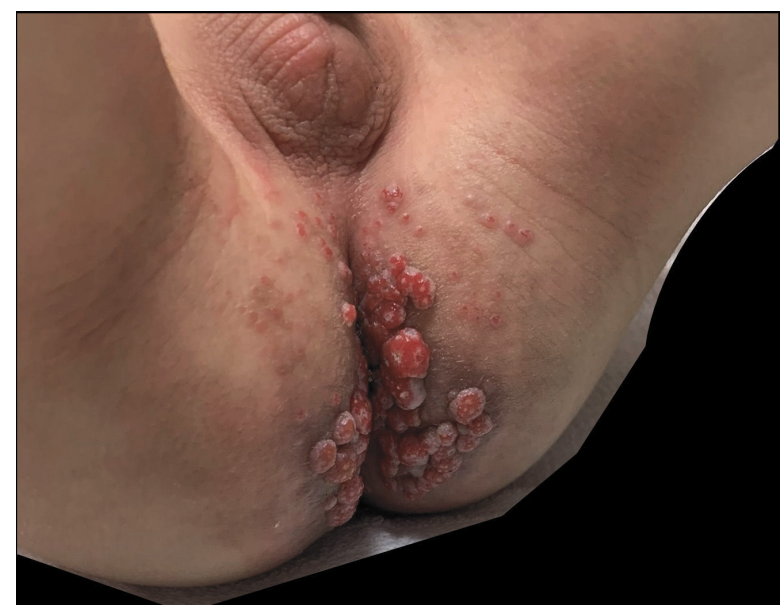

Abstract \#409 Figure 1 Skin exam showed multiple 3-8mm papules and nodules at the perianal area

The mainstay of treatment targets the inciting factors, including diaper use, encopresis, and colostomy bag. Therapy should be aimed to restore the skin barrier, eliminate any source of irritations, and treat superimposed infections.
\#410 LATE PRETERM INFANT WITH POSTNATAL DIAGNOSIS OF RENAL TUBULAR DYSGENESIS

${ }^{1} S$ Gaffar*, ${ }^{2}$ P Arora, ${ }^{1} \mathrm{R}$ Ramanathan. 'Los Angeles County University of Southern California Medical Center, Los Angeles, CA; ${ }^{2}$ Children's Hospital of Los Angeles, Los Angeles, CA

\subsection{6/jim-2022-WRMC.406}

Case Report A 2025-gram male infant was born to a 23-yearold multiparous woman by cesarean section for breech presentation at 34 gestational weeks. Rupture of membranes occurred 65 hours prior, with meconium-stained fluid. Pregnancy was complicated by limited prenatal care and polysubstance abuse (tetrahydrocannabinol and methamphetamines). Serologic testing was unremarkable except for unknown gonorrhea, chlamydia, and group B streptococcus.

$\mathrm{He}$ was intubated and given surfactant soon after delivery for labored breathing. Chest radiographs revealed pneumothoraces requiring bilateral chest tube placement. Empiric antibiotics were started as his clinical condition deteriorated with escalating ventilator requirements and hypotension requiring multiple inotropes. He became oliguric with hyponatremia $(126 \mathrm{mEq} / \mathrm{L})$, elevated creatinine $(3.78 \mathrm{mg} / \mathrm{dL})$, and non-cystic parenchymal disease on renal ultrasound. Renal failure was managed with strict fluid balance per nephrology; 1 week later, peritoneal dialysis commenced. Subsequent whole exome sequencing identified two ACE gene mutations, confirming renal tubular dysgenesis (RTD).

Since the patient was not considered a transplant candidate, a supportive care plan was formulated to optimize future transplant candidacy. Presently, he continues to receive peritoneal dialysis, gastrostomy tube feeds, and developmental therapy.

Discussion RTD is acquired in an autosomal recessive manner or from exposure to substances causing renal hypoperfusion. Immunohistochemistry detects epithelial membrane antigen but not CD-10, illustrating absence of proximal tubules. This adds to known glomerular and ductal hypoplasia. By these mechanisms, antenatal reduction of glomerular filtration manifests as intrauterine oligohydramnios.

Another hallmark of RTD is refractory hypotension arising from renin-angiotensin dysfunction. Fresh frozen plasma, intravenous hydrocortisone or fludrocortisone, and vasopressin infusion provide transient circulatory support. Glucocorticoids stimulate hepatic transcription of the renal tubular growth factor angiotensinogen. This degree of postnatal hypotension supports the postulation that antenatal hydrocortisone may improve neonatal outcomes.

RTD is a rare disease that starts in fetal life with defective tubule development. Perinatal oligohydramnios evolves into acute renal insufficiency, respiratory failure, and refractory hypotension in the neonatal period. Prenatal molecular genetic testing means that RTD is no longer relegated to exclusive postmortem diagnosis and may have a higher incidence than we think.

\section{\#411 A RARE CASE OF COFFIN-SIRIS SYNDROME CAUSED BY SOX4 MUTATION WITH NEW PHENOTYPIC FEATURES}

${ }^{1,2,3} \mathrm{AE}$ Lee* ${ }^{*}{ }^{1,2,3} \mathrm{~J}$ Friedman, ${ }^{1,3} \mathrm{~J}$ Lenberg, ${ }^{1,2,3} \mathrm{~K}$ Wigby. ${ }^{1}$ Rady Children's Hospital San Diego, San Diego, CA; ${ }^{2}$ University of California San Diego, La Jolla, CA; ${ }^{3}$ Rady Children's Institute for Genomic Medicine, San Diego, CA

10.1136/jim-2022-WRMC.407 
Case Report De novo missense variants involving the HMG domain of SOX4 were recently reported to cause a CoffinSiris like syndrome characterized by global developmental delays, distinctive craniofacial features, hypotonia and 5th finger clinodactyly (OMIM \# 184430). To date, only four cases have been reported in the literature (Zawerton et al, 2019). We report on a case of a young girl with global developmental delay, hypotonia, distinctive facies, cardiac anomalies, sensorineural hearing loss secondary to hypoplastic cochlear nerves and striking stereotypies, who was identified on whole genome sequencing to have a novel heterozygous de novo variant in SOX4 (c. 199A $>$ G, p.Met67Val). The proband was the product of a $302 / 7$ week uncomplicated pregnancy. At birth she was noted to have a peri-membranous VSD. At eight months of age she was markedly hypotonic and poorly responsive to sound, and was found to have underdeveloped cochlear nerves. She presented to our genetics service at 19 months of age where she was noted to have pre-auricular pits, small stature, and severe developmental delay. Family history was non-contributory Chromosomal microarray showed only $24 \mathrm{Mb}$ of homozygosity consistent with distant consanguinity. A congenital hypotonia panel was non-diagnostic. Duo whole genome sequencing with maternal sample identified the SOX4 variant, later confirmed to be de novo. Coffin-Siris syndrome is a rare pattern of malformation characterized by growth deficiency, microcephaly, coarse facial features, intellectual disability and hypoplastic fifth finger and toenails. Of those, the majority are caused by ARID1B, which is a gene encoding the subunits of the SWI/SNF complex which is essential in chromatin remodeling. Interestingly, SOX11 is another known cause of Coffin-Siris. SOX11, together with SOX4 and SOX12 and forms the SOXC complex, with highly conserved identical DNA-binding domains, giving insight to the mechanism of pathology which we elaborate on. Individuals with SOX4 variants appear to have relatively mild dysmorphology including anteverted nares, wide mouth with a cupid bow, and posteriorly rotated ears. Our proband extends this phenotype with unique features of hypoplastic cochlear nerves and severe stereotypies which we hypothesize are related to her severe hearing loss.

\section{\#412 PERITONEAL TUBERCULOSIS MIMICKING OVARIAN CANCER}

${ }^{1} \mathrm{~A}$ Cox, ${ }^{2} \mathrm{C}$ D'Assumpcao*, ${ }^{2} \mathrm{~A}$ Froush, ${ }^{2} \mathrm{~A}$ Heidari, ${ }^{2} \mathrm{AA}$ Ramzan. ${ }^{1}$ Ross University School of Medicine, Miramar, FL; ${ }^{2}$ Kern Medical Center, Bakersfield, CA

\subsection{6/jim-2022-WRMC.408}

Case Report Diagnosis of peritoneal tuberculosis may be challenging. This form of extrapulmonary infection may present as abdominal and/or pelvic masses with pain, bloating, and discomfort. The clinical manifestations are similar to ovarian cancer, including elevation in tumor marker CA-125, peritoneal seeding and lymphadenopathy. Multiple diagnostic procedures are sometimes needed to make a definitive diagnosis.

Methods Used Retrospective case review following IRB approval.

Summary of Results A 50-year-old Hispanic woman presented with generalized abdominal discomfort, bloating, fatigue, unintentional weight loss and night sweats. CT imaging revealed an $11 \times 9 \times 9 \mathrm{~cm}$ cystic pelvic mass associated with large volume loculated ascites, diffuse lymphadenopathy and peritoneal implants. She had an elevated CA-125 of 583 units $/ \mathrm{mL}$. Paracentesis was non-diagnostic. Intraperitoneal biopsies revealed diffuse granulomatous inflammation with foreign body-like multinucleated cells and no evidence of malignancy. Acid-fast stain and acid-fast bacilli (AFB) smear and culture were negative. Chest $\mathrm{x}$-ray showed bilateral hilar adenopathy and a granuloma in left upper lung, with subsequent positive QuantiFERON gold. Antituberculosis therapy was initiated with Isoniazid, rifampin, ethambutol, and pyrazinamide (RIPE). After 5 months of RIPE therapy, the CA-125 normalized and there was dramatic radiographic improvement in lymphadenopathy and peritoneal disease burden. The pelvic mass persisted and she underwent bilateral salpingo-oophorectomy. Histopathology resulted as a serous cystoadenoma with necrotizing granulomatous inflammation. The AFB smear and mycobacterium tuberculosis complex PCR were positive. The patient recovered post-operatively and was re-initiated on RIPE therapy. Mycobacterial culture and sensitivity results are pending.

Conclusion Peritoneal tuberculosis can be mistaken for ovarian cancer and thorough diagnostic evaluation is essential to ensure appropriate management and treatment.

\section{\#413 CLOSTRIDIUM PARAPUTRIFICUM IN 46-YEAR-OLD MALE WITH LIVER DISEASE}

${ }^{1} \mathrm{C}$ D'Assumpcao*,${ }^{1,2} \mathrm{~K}$ Dao, ${ }^{1} \mathrm{~A}$ Heidari. ${ }^{1}$ Kern Medical Center, Bakersfield, CA; ${ }^{2}$ Ross University School of Medicine, Miramar, FL

\subsection{6/jim-2022-WRMC.409}

Case Report Clostridium paraputrificum is an anaerobic spore forming gram positive bacilli that is part of normal human gastrointestinal flora. Less than $1 \%$ of all clostridium infections in the literature are C. paraputrificum. We report a 46 year old male with alcoholic liver disease and lower gastrointestinal hemorrhage who was found to have C. paraputrificum bacteremia.

Method Retrospective case report.

Case Presentation A 46-year-old male with heavy alcohol abuse, hypertension and congestive heart failure presented with two weeks of worsening abdominal pain that progressed to bright red blood per rectum. He was found to be in septic shock at presentation to the emergency department. $\mathrm{He}$ received aggressive fluid resuscitation and vasopressor support. He was diagnosed with liver cirrhosis complicated by splenomegaly and ascites shortly after admission. Ascitic fluid analysis supported diagnosis of spontaneous bacterial peritonitis. $\mathrm{He}$ was started in ceftriaxone. He eventually developed acute oliguric kidney injury due to shock and hepatorenal syndrome. He was placed on intermittent dialysis. Admission blood cultures grew C. paraputrificum. Metronidazole was added. Upper and lower endoscopy found large internal hemorrhoids vessels associated with rectal varices requiring banding. $\mathrm{He}$ continued to requiring blood transfusions daily. He was a poor candidate for colorectal surgery and outpatient dialysis. Patient and family ultimately agreed with hospice care.

Conclusion C. paraputrificum is a rarely reported cause of anaerobic septicemia. Translocation from the gastrointestinal tract is the usual path and mucosal damage should be investigated. Cirrhosis portends a poorer prognosis. 
\#414 THE NEUROSYPHILIS AND COCCIDIOIDOMYCOSIS CONUNDRUM

J Nguyen, VE Espinoza*, A Shah, G Black, K Radicic, R Kuran, A Heidari. Kern Medical Center, Bakersfield, CA

\subsection{6/jim-2022-WRMC.410}

Case Report Neurosyphilis is an infection of the central nervous system caused by Treponema pallidum that can occur following initial infection. Early forms of neurosyphilis affect cerebrospinal fluid, meninges, and vasculature while latter forms affect the brain and spinal cord parenchyma. We describe a case of neurosyphilis complicated by fluconazole toxicity during treatment of pulmonary coccidioidomycosis. A 26-year-old man with poorly controlled type 1 diabetes mellitus, miliary pulmonary coccidioidomycosis, heart failure with reduced ejection fraction, left eye cranial nerve III palsy and right cranial nerve VII palsy presented to the emergency department for weakness and syncope. Physical exam was remarkable for equal pupils unreactive to light or accommodation bilaterally, point tenderness of bilateral iliac crest, and left CNIII and right CNVII palsies. MRI brain with and without contrast showed slightly diffuse increased enhancement. CXR showed left perihilar and right upper lobe infiltrates. Transesophageal echocardiogram showed an ejection fraction of $30 \%$. Lumbar puncture (LP) performed showed WBC 5, RBC 0, glucose 48, protein 254 with cocci IgG WR CF $1: 4$ and an opening pressure of $14 \mathrm{~cm}$. Due to supratherapeutic fluconazole level in the setting of disseminated cocci, fluconazole was discontinued and Cresemba was started with noted improvement of weakness. Neurology recommended repeat lumbar puncture with flow cytometry and cytology for carcinomatosis meningitis, oligoclonal bands and IgG synthesis rate in addition to RPR, VLDR and FTA-ABS. Repeat LP showed WBC 3, RBC 2, glucose 78 and protein 135.9 with opening pressure $30 \mathrm{~cm}$ of $\mathrm{H} 2 \mathrm{O}$. Labs showed nonreactive RPR and VDRL with reactive FTA-ABS and positive syphilis antibodies. Furthermore, cerebrospinal fluid studies showed albuminocytologic dissociation consistent with neurosyphilis. A diagnosis of late latent syphilis with bilateral posterior subcapsular cataract, and CNIII and CNVII palsy was made and the patient was then started on Penicillin $G$ for treatment of neurosyphilis. Neurosyphilis caused by $T$. pallidum is rare due to spontaneous resolution in cases without an inflammatory response. This case demonstrates a unique case of late latent syphilis consistent with neurosyphilis due to history of bilateral posterior subcapsular cataract at age 18 with cranial nerves III and VII palsy.

\section{\#415 A CASE OF RAOULTELLA PLANTICOLA BACTEREMIA IN AN IMMUNOCOMPROMISED MALE}

${ }^{1} \mathrm{~A}$ Garcia*, ${ }^{1} \mathrm{~J}$ Oberndorf, ${ }^{2} \mathrm{~J}$ Tsai, ${ }^{3} \mathrm{~N}$ Mangat, ${ }^{3} \mathrm{~A}$ Heidari, ${ }^{3} \mathrm{~S}$ Mishra, ${ }^{3} \mathrm{~K}$ Radicic. ${ }^{1}$ Ross University School of Medicine, Miramar, $\mathrm{FL}^{2}{ }^{2}$ American University of the Caribbean School of Medicine BV, Cupecoy, Sint Maarten (Dutch part); ${ }^{3}$ Kern Medical Center, Bakersfield, CA

\subsection{6/jim-2022-WRMC.411}

Case Report Raoultella Planticola formerly called Klebsiella planticola is a gram-negative aerobic rod that belongs to the Enterobacteriaceae family and is often established in aquatic habitat and soil. The published cases infected with $R$. planticola are scarce. It rarely causes infection in an immunocompetent host. In contrast, in immunocompromised patients, inoculation of $R$. planticola can surpass innate and adaptive host response leading to bacteremia. Here we describe a case of $R$. planticola bacteremia in a patient on chemotherapy who presented with fever with a recent dog bite to his right lower extremity as a potential source.

Methods A single patient case report was conducted after IRB approval.

Case Presentation A 41-year-old male with right knee osteosarcoma who was receiving infusions of Cyclophosphamide and Mesna presented to the emergency department (ED) with fever and chills for 3 days. He was bitten by his friend's dog on the medial aspect of his right posterior calf resulting in a bleeding wound one month prior while working in the garden. One day later, he developed regional swelling and pain associated with fever which progressively worsened for almost two weeks, followed by complete self-resolution. While in ED, he was tachycardic and febrile to $39.4{ }^{\circ} \mathrm{C}$. Imaging was unremarkable. The site of the dog bite was well healed and had no tenderness, fluctuance, erythema, or warmth. Blood cultures were drawn from peripheral and his chemotherapy port and he was started on broad-spectrum antibiotics with vancomycin and cefepime. After two days, the blood culture from peripheral grew gram negative rods resembling enterics and cefepime was replaced by meropenem. The patient became afebrile. Blood cultures identified as $R$. planticola, sensitive to ceftriaxone. Antibiotic therapy was switched to ceftriaxone on hospital day 4. Repeat blood cultures were negative and the patient remained afebrile for greater than 24 hours. He was discharged with at-home infusions with Ceftriaxone for 14 days.

Conclusion $R$. planticola is an emerging infection, particularly in oncology patients. Clinicians should be aware and include it in their differential diagnosis of any unidentified gram negative infection in immunocompromised host as failure to treat in a timely manner could result in a fatal outcome.

\section{Healthcare delivery research IV}

\section{Concurrent session}

\section{0:15 AM}

\section{Saturday, January 22, 2022}

\section{\#416 APPLICATION OF EXPLAINABLE MACHINE LEARNING IN ANALYZING SAFETY AND QUALITY REPORTS FROM A CLINICAL UNIT}

M Nguyen*, P Beidler, J Kang, K Lybarger, E Ford. University of Washington, Seattle, WA

\subsection{6/jim-2022-WRMC.412}

Purpose of Study Accurate assessment of safety-related reports in the healthcare setting is essential for improving care quality, optimizing workflow, and preventing future safety incidents. At present such systems rely on a manual review process, which can be very time-consuming and difficult to standardize. The purpose of this study is to develop an explainable machine learning (ML) model for automatically identifying the severity of incident reports and streamlining quality improvement processes. We hypothesized that high severity incident reports can be automatically identified using ML methods. 
Methods Used This study utilizes 7,095 operational quality reports collected in the radiation oncology department at the University of Washington from 2012-2021. Each report was assigned a near-miss risk index score (0-4) as part of ongoing clinical practice, rather than a separate annotation process. For this experimentation, the reports were assigned binary labels of high-severity (score of 0-2) and low-severity (score of 3-4), and the data was split into a training, validation, and test set with a 70-15-15 ratio. We chose Random Forest as the pilot model because this model has historically performed well in text classification tasks. The input features to the Random Forest model were term frequency-inverse document frequency vectors, where each dimension denotes the importance of a word. Model performance was evaluated using the area under the receiver operating characteristic curve (AUC), which integrates the diagnostic ability of a binary classifier at all operating points, and $\mathrm{F} 1$, which is the harmonic mean of precision and recall. We then used the popular SHapley Additive exPlanations (SHAP) package to understand which words in the safety reports are most indicative of report severity.

Summary of Results Our Random Forest model achieved an AUC of 0.66 and F1 of 0.42 on the withheld test set. The dataset was annotated over a 9-year period by different clinicians as a part of clinical workflow, resulting in increased variance in scoring that negatively impacts training and prediction performance. Our results compare favorably to the manual rating process where the interrater agreement of this dataset was 37.6\% (Mullen et al, 2015). The SHAP interpretability analysis indicates that the top 10 most predictive words are 'field', 'shift', 'use', 'treatment', 'therapist', 'schedule', 'isocent', 'catch', 'wrong', and 'cm', in descending order of importance.

Conclusions This study shows that ML is a viable method to analyze and score operational quality reports in the clinical healthcare setting. We present a pilot prediction model with an AUC of 0.66 and F1 of 0.42 , proving that a suitable detection alert tool to triage safety-related reports for timely review. To our knowledge, this approach has not been attempted to classify reports' severity and fills a critical unmet need as a quality improvement tool in healthcare.

\section{\#417 DIAGNOSIS OF BRAIN AND BONE CANCERS USING BIOMEDICAL IMAGING AND ARTIFICIAL INTELLIGENCE APPLICATIONS}

${ }^{1,2} \mathrm{KH}$ Miao*${ }^{1,2} \mathrm{JH}$ Miao. ${ }^{1} \mathrm{NYU}$ School of Medicine, New York, NY; ${ }^{2}$ Cornell University, New York, NY

\subsection{6/jim-2022-WRMC.413}

Purpose of Study According to the CDC, greater than 100 million people in the United States are affected by cancers, such as those of the brain and bone. With early detection and diagnosis, patients' cancers can be better managed with improved outcomes. Thus, diagnosing early is critical for improving their quality of life. Artificial intelligence applications and imaging in cancer diagnosis include expediency in resource-limited areas globally, such as triage, to increase cancer detection while aiding medical professionals.

Methods Used In this research project, an artificial intelligence model is developed using machine learning, imaging, and patient data to aid diagnosis of brain and bone cancers to increase detection and diagnostic accuracy. The model was also built using artificial intelligence algorithms to diagnose cancer in patients. Clinical data from patients was used to develop, train, and test the model. To train it, $55 \%$ of the patient data was randomly selected while the remaining $45 \%$ was utilized for testing its diagnosis capabilities.

Summary of Results In diagnosing cancer in patients, the machine learning model was able to achieve an overall diagnostic accuracy of $81 \%$ for brain cancers and $83 \%$ for bone cancers compared to previously published methods that ranged in $51 \%-72 \%$ accuracy.

Conclusions Thus, artificial intelligence algorithms and imaging can be used to help medical professionals for diagnosing bone and brain cancers in patients, such as in resource-limited areas globally in triage cases. Furthermore, artificial intelligence can be used to aid cancer detection, improving outcomes and quality of life.

\section{\#418 DIAGNOSING MELANOMA AND METASTASES USING DEEP NEURAL NETWORKS, ARTIFICIAL INTELLIGENCE, AND MEDICAL IMAGING}

JH Miao*. Cornell University, New York, NY

\subsection{6/jim-2022-WRMC.414}

Purpose of Study Cancer is one of the most common causes of death globally, impacting millions of patients worldwide. Melanomas cause a large number of deaths because of metastases. Early and accurate cancer screening is important to improve patient outcomes and potentially save lives. Detecting melanoma and metastases with high accuracy and precision is thus essential. Computer-aided detection (CAD) systems have been developed around the world to help aid healthcare professionals. In this research, a computer-aided diagnosis using artificial intelligence and medical imaging is applied to create a machine learning model to evaluate the presence of melanoma and metastases in patients.

Methods Used In this research, a deep convolutional neural network model was designed using artificial intelligence and developed to help detect the presence of melanoma in imaging scans. The machine learning model was applied to a clinical patient dataset containing over 2000 patients. Data from $60 \%$ of the patients was used to train the machine learning model, and the remaining $40 \%$ of the patient data was used for testing performance.

Summary of Results The deep learning model achieved a $83.8 \%$ sensitivity and $84.3 \%$ specificity in detecting melanoma cancer and metastases in patients.

Conclusions Therefore, computer-aided diagnoses using artificial intelligence can help detect melanoma cancer and metastases in radiologic imaging to aid patient outcomes. Especially in areas where there are fewer healthcare resources and medical professionals present, the machine learning model can help aid melanoma cancer diagnosis in underserved populations.

\section{\#419 FINANCIAL ASSESSMENT OF A HYBRID HUMAN AND ARTIFICIAL INTELLIGENCE WORKFLOW FOR FOLLOW-UP RECOMMENDATIONS FROM RADIOLOGY}

B Roth*, R Kampalath, K Nakashima, S Shieh, T Bui, R Houshyar. University of California Irvine, Irvine, $C A$

10.1136/jim-2022-WRMC.415 
Purpose of Study Many radiology interpretations indicate the need for follow up imaging, and adherence to these recommendations is often incomplete. Lack of follow up on these recommendations may result in poor patient outcomes. At our institution we deploy a hybrid workflow utilizing a combination of natural language processing (NLP) software to tag radiology exams and a quality and safety nurse to handle subsequent communication and documentation with referring medical providers or patients. In this study, we assess the financial costs and benefits of our institution's hybrid workflow to determine the overall financial feasibility of this system.

Methods Used After Institutional Review Board approval, the total number of radiology scans with follow-up imaging recommendations between February 1, 2020 and January 1, 2021 were pulled for inclusion in this study. Mammography patients were excluded due to a separate, predefined screening protocol. Reports flagged as 'overdue' by the mPower FollowUp Recommendation Algorithm (Nuance Communications Inc., Burlington, MA) and/or the quality and safety nurse were included to determine revenue. Revenue was calculated based on the Medicare 2020 and 2021 values for patients who had overdue clinically indicated follow-up exams, whose ordering providers were notified by the nurse, and who then completed the necessary imaging. Personnel cost of a quality and safety nurse was calculated by determining the percentage of time the nurse spent on follow-ups and multiplying this number by the average national salary of a quality and safety nurse.

Summary of Results A total of 3,011 patients were flagged as overdue. After careful review of the charts, 327 were missing orders for follow up recommended imaging. Of these 84 patients completed follow up outside of our institution or declined follow up. Quality nurse interventions led to 244 patients completing their follow-up imaging at our institution. The follow-up imaging of these patients accounted for $\$ 35,106.90$ of revenue, which represents a conservative, generalizable value based on the 2020 and 2021 Medicare national rates. The quality and safety nurse spent about $60 \%$ of her time on follow-ups and therefore, personnel cost was estimated at $\$ 26.326 .80$.

Conclusions Our cost and revenue estimates are based off of national averages and as such will vary significantly by location. However, our analysis suggests that a hybrid human and NLP workflow for follow-up recommendations from radiology would most likely not cause financial losses for institutions. Additionally, this workflow acts as a safety net to ensure patients receive adequate follow up imaging and markedly mitigates risks from litigation.

\section{\#420 SYSTEM DYNAMICS MODELING AS AN INTERVENTION PLANNING TOOL TO PROMOTE INSTITUTIONAL DELIVERY: LEARNING FROM WEST CHAMPARAN IN BIHAR, INDIA}

${ }^{1} \mathrm{C}$ Lee*, ${ }^{2} \mathrm{~V}$ Ward, ${ }^{2} \mathrm{~S}$ Abdalla, ${ }^{2,3} \mathrm{KM}$ Mehta, ${ }^{2} \mathrm{~S}$ Carmichael, ${ }^{4} \mathrm{M}$ Singh, ${ }^{4} \mathrm{~S}$ Das, ${ }^{4} \mathrm{~K}$ Ranjan, ${ }^{4} \mathrm{~S}$ Sonthalia, ${ }^{4} \mathrm{~T}$ Mahapatra, ${ }^{2} \mathrm{GL}$ Darmstadt. ${ }^{1}$ Universitetet $i$ Agder Fakultet for Samfunnsvitenskap, Kristiansand, , Norway; ${ }^{2}$ Stanford University School of Medicine, Stanford, CA; ${ }^{3}$ University of California, San Francisco, San Francisco, CA; ${ }^{4}$ Care India, New Delhi, India

\subsection{6/jim-2022-WRMC.416}

Purpose of Study Women in India are encouraged to deliver in health facilities through the Janani Suraksha Yojana (JSY) conditional cash-transfer scheme provided to pregnant women and accompanying frontline health workers (Accredited Social Health Activists, ASHAs). Despite additional governmental efforts catalyzed by a statewide technical-support program led by CARE India and funded by the Bill and Melinda Gates Foundation, institutional delivery rates (IDRs) among Scheduled Caste and Tribe women in West Champaran district remained 10-15 percentage points lower for multiparous than primiparous women in 2014-19. The study aims to understand the major causal mechanism that caused the disparities of the IDRs among marginalized women with increasing parities, identify high-impact intervention points, and design effective interventions.

Methods Used We integrated, analyzed, and synthesized population survey and administrative data, and background literature to formulate a system dynamics (SD) simulation model to explore patterns, generate insights, and inform future policy design to equitably increase IDRs. SD models map and quantify bidirectional relationships between components in systems and the impacts on outcome variables over time.

Summary of Results Modelling and synthesis revealed antenatal counselling from ASHAs at home appeared to motivate firsttime pregnant women to deliver at a facility. However, multiparous women were more dissuaded by JSY payment delays, experience of below-expectation quality of care during past institutional deliveries, transportation problems, and lack of caregiving support at home for other children while giving birth at facilities. Over successive pregnancies, women probably become less adherent to counselling and ASHAs interact with them with reduced intensity.

Conclusions Improving quality of care, transportation-availability and antenatal counselling, and increasing and regularizing JSY payments seem important to increase IDRs among marginalized women. The current model revealed JSY payment improvement, transportation availability, making facility workforce and facility capacity functional fast, increasing ASHA work performance through nighttime street lighting, namebased tracking of beneficiaries, and reducing ASHA capacity shortages quickly, concurrently, may lead to a modest $(4 \%)$ increase in IDR among primiparous women. This intervention strategy may contribute to $18 \%, 6 \%$, and $14 \%$ increases in IDRs among women with parity two, three and four plus, respectively. Further model development is warranted.

\section{\#421 GENDER, TELEMEDICINE, AND COVID-19: PREFERENCES FOR UTILIZING CARE AMONGST DIVERSE, LOW-INCOME HOUSING AUTHORITY RESIDENTS}

II Pasumarthi* ${ }^{*}{ }^{1} \mathrm{~T}$ Hollar, ${ }^{2} \mathrm{~N}$ Alety, ${ }^{3} \mathrm{E}$ Solano, ${ }^{3} \mathrm{M}$ Edman, ${ }^{3} \mathrm{G}$ Ochoa. ${ }^{1}$ Nova Southeastern University, Fort Lauderdale, FL; ${ }^{2}$ The George Washington University Milken Institute of Public Health, Washington; ${ }^{3}$ YMCA of South Florida, Fort Lauderdale, FL

\subsection{6/jim-2022-WRMC.417}

Purpose of Study The COVID-19 pandemic has led to a significant increase in the utilization of telemedicine, or virtual visits with a healthcare provider through the use of technology such as phones, tablets, or computers. However, opportunities remain to explore the varying preferences, by gender, of diverse, low-income individuals toward this platform, particularly during the time of COVID-19.

Methods Used As part of a COVID-19 testing and education initiative conducted by the YMCA of South Florida, in partnership with the Housing Authority of the City of Fort 
Lauderdale and the Broward County Housing Authority, housing authority residents, in Broward County, the 17th largest county in the US by population, residents were surveyed regarding their preferences of accessing care through telemedicine (phones, tablets, or computers) or through face-to-face interactions. We performed secondary data analysis of survey data collected by the YMCA of South Florida. This analysis involved descriptive statistics for describing the respondents and their preferences, Chi-square for detecting significant differences between males and females for preferring to utilize telemedicine rather than face-to-face exchanges with respondents' health care providers, and logistic regression to determine the odds of males' or females' likelihood of preferring accessing care via telemedicine.

Summary of Results Twenty-five percent $(25.70 \%, \mathrm{n}=140)$ of respondents preferred to visit their physician through the use of telemedicine rather than attending in-person. A significantly higher percentage of males $(37.5 \%, \mathrm{n}=40)$ reported a preference for utilizing telemedicine than females $(21.0 \%, \mathrm{n}=100)$ $\left(X^{2}[1, \mathrm{n}=140]=4.07, p=.04\right)$. Males were found to have had two times the odds of preferring telemedicine (aOR 2.26, 95\% CI 1.01-5.03) as compared to females.

Conclusions These findings could shape the way clinical healthcare providers offer telemedicine services to their patients. Since diverse housing authority resident males have a preference towards telemedicine, it is worthwhile that health care providers serving this vulnerable population during COVID-19 offer options for patients to access care through telemedicine. Future research can be done to assess the reason for this gender difference in the preference for telemedicine, as well as to understand if there are specific platforms patients prefer when accessing telemedicine (phone, computer, etc.).

\section{\#422 A REVIEW OF THE LEVEL OF EVIDENCE IN POINT OF CARE ULTRASOUND WITHIN THE PICU}

${ }^{1}$ A Walls*, ${ }^{1}$ A Willyerd, ${ }^{2}$ E Su. ${ }^{1}$ Phoenix Children's Hospital, Phoenix, AZ; ${ }^{2}$ The University of Texas Health Science Center at Houston John P and Katherine G McGovern Medical School, Houston, TX

\subsection{6/jim-2022-WRMC.418}

Purpose of Study To review the last decade of point of care ultrasound research and determine the level of evidence provided as it pertains to the pediatric intensive care unit.

Methods Used A retrospective review of the current literature was performed utilizing the following publicly available databases: Pubmed, Scopus and Cochrane Databases regarding bedside point of care ultrasound in the pediatric population. Each data source was queried for the following inclusion criteria terms: 'pediatric', 'ultrasound', 'bedside', 'intensive care' and 'critical care'. Data was collected from 2011 to 2020. Each article was subcategorized into: Case Report, Case Series, Cohort Study, Systematic Review, Meta-Analysis and Randomized Controlled Trial and provided a level of evidence (LOE) based on the respective study design. Exclusion criteria included studies performed in the neonatal intensive care unit, studies that included adults and anesthesia regional pain investigations that involved point of care ultrasound. Statistical analysis was performed on Minitab 5.0 (State College, PA).

Summary of Results A total of 122 publications met our inclusion criteria and 31 met exclusion criteria. The overall LOE in the pediatric ultrasound literature did not improve significantly from 2011 to $2020(\mathrm{P}=0.321)$ as per ANOVA calculations. However, the overall year over year number of pediatric publications increased each year except for 2015 and 2017 (0\%,-35\%), respectively. The average LOE increased each year except for 2013, 2017 and 2018. The most frequent publication type was found to be the retrospective cohort study with a frequency of 31 out of the 121 (26\%), reported studies. There were 11 Randomized Control Trials, 14 Systematic Reviews and Meta-Analysis which accounted for Level 1 and Level 2 evidence, respectively. The year over year change in Level 1 and Level 2 evidence did not significantly change over the study period $(\mathrm{P}=0.643)$.

Conclusions The literature regarding the use of point of care ultrasound in the critical care setting remains limited. Our investigation reveals no statistically significant improvement in the LOE over the past decade. Despite this trend, there is obvious increased interest in point of care ultrasound, as the sheer volume of articles tended to increase year over year during the study period. Our community should continue with investigations which fall into higher LOE categories in order to aid in clinical decision making at bedside and assist in the creation of practice guidelines.

\section{Hematology and oncology III}

\section{Concurrent session}

\section{0:15 AM}

\section{Saturday, January 22, 2022}

\section{\#423 SICKLE CELL PAIN CRISIS COMPLICATED BY OPIOID INDUCED HYPERALGESIA, TREATED WITH LOW DOSE KETAMINE INFUSION}

F Venter*, R Dunn, J Bhandohal, S Mishra, E Cobos. Kern Medical Center, Bakersfield, CA

\subsection{6/jim-2022-WRMC.419}

Case Report PURPOSE OF STUDY: Sickle Cell Disease (SCD) can present with Acute Vaso-occlusive Crisis (VOC) and neuropathic pain, involving central sensitization, peripheral injury, and hyperalgesia. Management of VOC is challenging and requires IV fluids, oxygen and significant doses of intravenous (IV) opioids. However, this can result in opioid induced hyperalgesia $(\mathrm{OIH})$. Few papers have explored the use of low dose Ketamine for pain control. This case review shows a 36year-old female with VOC in pain, worsened with increasing doses of IV opioids. OIH was suspected and treated with a low dose ketamine infusion resulting in a dramatic resolution of her pain.

Methods This is a single case review after IRB approval.

Summary of Results A 36-year-old African American female with lifelong SS genotype SCD was admitted for VOC worsening over 4 days and unalleviated by her prescribed breakthrough pain medications. Treatment was started with fluids and increasing doses of IV opioids. By day 5, the pain became unbearable, suggesting $\mathrm{OIH}$ in the setting of concomitant VOC. She was treated with low dose Ketamine infusion at $0.1 \mathrm{mg} / \mathrm{kg} / \mathrm{hr}$, increased to $0.2 \mathrm{mg} / \mathrm{kg} / \mathrm{hr}$ with dramatic resolution of her pain. Once IV ketamine and opioids were discontinued, patient's pain was comfortably managed only on oral maintenance MS Contin for the following 48 hours. 
Conclusion $\mathrm{OIH}$ is postulated to develop when the central glutamate transporter system is inhibited, increasing the amount of glutamate available to excitatory N-Methyl-Daspartic acid (NMDA) receptors. This allows for cross talk of the neural mechanisms for pain and tolerance. Morphine targets NMDA receptors. When administered, it is neurotoxic and causes apoptotic cell death of the dorsal horn, thus sensitizing the neurons. Ketamine is a very potent inhibitor of NMDA receptors and can block the neurotoxic properties of morphine. After ketamine is discontinued, the patient does not revert back to their $\mathrm{OIH}$ state, thus allowing for the safe return to longer-acting opioids. Few publications have reported on the use of low-dose ketamine to manage pain during VOC. It could be considered as an adjunct analgesic agent during VOC episodes in patients that endorse persistent severe pain despite receiving high-dose opioid therapy.

\section{\#424 IMMUNE THROMBOCYTOPENIA FOLLOWING COVID-19 VACCINE}

S Prasad*, M Adebayo, R Jariwal, G Petersen, E Cobos. Kern Medical Center, Bakersfield, $C A$

\subsection{6/jim-2022-WRMC.420}

Purpose of Study Several vaccines have been developed and are being administered against severe acute respiratory syndrome coronavirus 2. Common side effects include fever, chills, headache, myalgia, and soreness at the injection site. However, some rare adverse effects have also been reported. Cases of thrombocytopenia following COVID-19 vaccination have been reported since the initiation of mass vaccinations. We present a case of thrombocytopenia presenting with petechiae and mucosal bleeding which developed as an adverse response after first-dose administration of the Moderna COVID-19 vaccine and was refractory to first-line therapy. Treatment options for refractory thrombocytopenia are discussed.

Methods Used Retrospective case study.

Summary of Results A 58-year-old Hispanic male presented to our hospital for acute onset of mucosal bleeding, petechiae and easy bruising. He denied any previous history of spontaneous bleeding or easy bruising. Patient had received his first dose of the Moderna COVID-19 vaccine three weeks prior to the development of spontaneous bleeding. Physical examination was notable for diffuse petechiae along the arms, legs, and abdomen along with numerous oral lesions and gingival bleeding. He was noted to have a platelet count of $3 \times 10^{9} / \mathrm{L}$ with all other cell lines within normal limits. After a comprehensive and exhaustive workup of all well-known precipitants of immune thrombocytopenic purpura returned negative, a diagnosis of idiopathic thrombocytopenic purpura was made. Patient was treated with first-line therapy of platelet transfusion, high dose steroids, and intravenous immunoglobulin (IVIG) multiple times but platelet count remained refractory. We then treated the patient with romiplostim - a thrombopoietin receptor agonist - and fostamatinib - an inhibitor of spleen tyrosine kinase. The platelet count improved and his symptoms were resolved.

Conclusions Since the mechanism of COVID-19 vaccine induced thrombocytopenia is unclear, it is important to acknowledge that some patients may not respond to the standard accepted treatment with steroids and IVIG. Cases similar to ours may benefit from therapy with romiplostim and fostamatinib when first-line therapy is unsuccessful.

\section{\#425 CASE SERIES OF DIFFUSE LARGE B CELL LYMPHOMA PRESENTING AS PELVIC MASS}

R Sharma*, A Heidari, E Cobos, G Petersen, AA Ramzan, R Polineni. Kern Medical Center, Bakersfield, CA

\subsection{6/jim-2022-WRMC.421}

Purpose of Study Primary diffuse large B-cell lymphoma (DLBCL) of the pelvis is rare with a non-specific clinical presentation. Although management of this tumor is standardized with high curative rates, the prognosis depends on timely and accurate diagnosis and therapy. Presenting with non-specific clinical symptoms, a multidisciplinary approach is recommended to diagnose and treat this disease. In this case series we present two patients that presented to our institution with primary DLBCL of the pelvis initially suspected as gynecologic malignancy.

Methods Used This study was approved by the Institutional Review Board of Kern Medical. A retrospective review of both the patient's record was performed. Literature search was conducted on PubMed and Google Scholar. The following search terms were applied: diffuse large B-cell lymphoma of the pelvis, pelvic tumors, R-CHOP.

Summary of Results A 27-year-old woman presented with intractable abdominal pain, distention and unintentional weight loss. She had large bilateral ovarian masses, peritoneal carcinomatosis and metastatic adenopathy. A CT guided biopsy of a peritoneal implant was performed. A 58-year-old woman presented with a large pre-sacral mass and inguinal adenopathy. An inguinal lymphadenectomy was performed. Pathology for both patients revealed large B-cells positive for CD45+, $\mathrm{CD} 20+, \mathrm{TCL} 1+, \mathrm{CD} 4+$, consistent with diffuse large b cell lymphoma (DLBCL). Both patients were treated with systemic chemoimmunotherapy with monoclonal antibody against CD20 (rituximab) in combination with Cyclophosphamide, Doxorubicin, Vincristine, Prednisone (R-CHOP). One patient completed therapy and currently has no evidence of disease and the other patient has been receiving therapy.

Conclusions Even though the incidence of DLBCL is on the rise, pelvis as the primary location remains rare. Given a high rate of cure and entirely different approach to treatment for lymphoma as compared to gynecological malignancies it is important to remember this condition in the differential diagnosis. A multidisciplinary approach between gynecology oncology, medical oncology and pathology would result in early diagnosis treatment and cure.

\section{\#426 EXTRAMEDULLARY MYELOMA OF LIVER MASQUERADING AS GALLBLADDER CARCINOMA}

${ }^{1} S$ Kolagatla*, ${ }^{1} \mathrm{~F}$ Smith, ${ }^{2} \mathrm{M}$ Julapelly, ${ }^{1} \mathrm{~J}$ Piercy, ${ }^{1} \mathrm{~N}$ Moka. ${ }^{1}$ Appalachian Regional Healthcare, Lexington, KY; ${ }^{2}$ Hurley Medical Center, Flint, MI

\subsection{6/jim-2022-WRMC.422}

Case Report Multiple myeloma (MM) is a malignant clonal proliferation of plasma cells in the bone marrow. Extramedullary myeloma (EMM) refers to soft tissue or visceral proliferation of clonal plasma cells can occur approximately in $15 \%$ of 
patients with MM. Organs involved by EMM may include skin, central nervous system, and viscera. Our case is EMM initially diagnosed as a gallbladder carcinoma.

A 76-year-old male found to have IgG Kappa MM with normal cytogenetics and 40\% involvement of bone marrow.

Offered transplant after CR from bortezomib (V), dexamethasone (D) and thalidomide but patient declined. Subsequent relapse treated with carfilzomib and later daratumumab regimen.

Kappa light chains (K) increased but bone marrow negative. PET and MRI of liver with contrast confirmed liver mass and retroperitoneal lymphadenopathy but the gall bladder not visualized. The liver mass was suspected to be a gallbladder carcinoma.
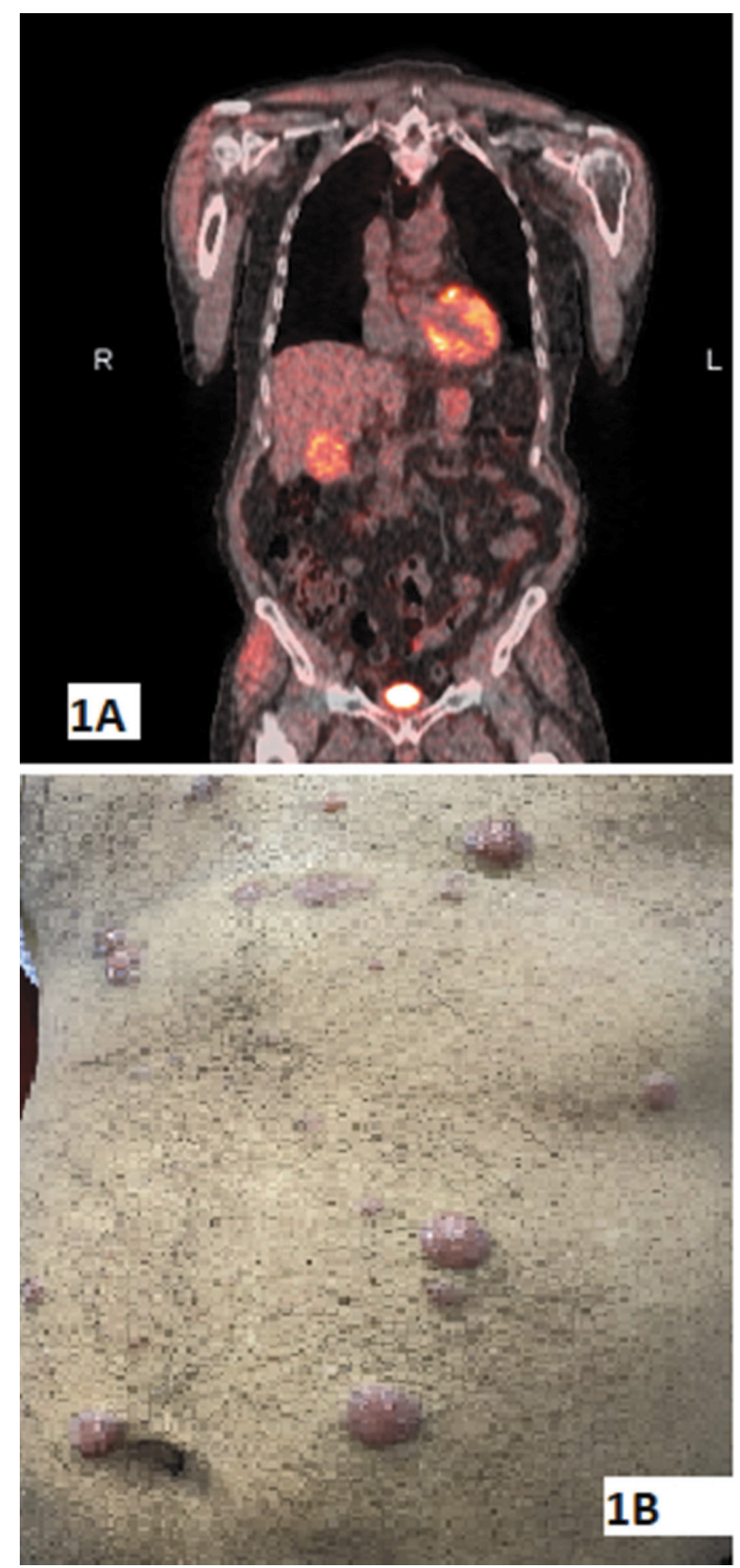

Abstract \#426 Figure 1
CT guided biopsy of the liver mass showed proliferation of plasma cells. Given the rapid progression of $\mathbf{K}$ received V, D, cyclophosphamide and etoposide without cisplatin.

Intitial regression of the liver lesions and $\mathbf{K}$ but immediate worsening. Developed rapidly evolving non-painful, firm cutaneous nodules over his anterior upper abdomen and chest (figure 1B). Skin lesion biopsy showed plasma cells. He is currently on elotuzumab $\mathbf{V}$ and $\mathbf{D}$, but his kappa light chains continue to rise.

EMM suspected in patients with MM who have localized to a particular organ or Light chain escape. EMM can mimic a primary malignancy such as gallbladder carcinoma as in this case. EMM is aggressive with $<6$ month survival. Number or prior lines of therapy are associated with higher risk of extramedullary relapse. Lenolidamide and prior allogenic transplant lowered the risk of EMM. No prospective studies defining an optimal treatment strategy for exramedullary myeloma. Vemurafenib for BRAF mutation or immune therapies with CAR-T cells have shown promising results in a limited number of EMM patients.

\section{\#427 SUPERIOR VENA CAVA SYNDROME SECONDARY TO ADULT T-CELL LYMPHOBLASTIC LYMPHOMA}

V Bustamante*, Z Alsadek, O Morgan, S Mishra, K Radicic. Kern Medical Center, Bakersfield, CA

\subsection{6/jim-2022-WRMC.423}

Case Report A 31-year-old Latino male presented with sharp right-sided chest and upper quadrant pain radiating to his back, worse with movement. History revealed non-productive cough, fatigue, subjective fevers, and night sweats. Patient had decreased right lower breath sounds. CT angiography with contrast revealed moderate effusion with perihilar compressive atelectasis and diffuse lymphatic adenopathy. Thoracentesis revealed exudative effusion with $81 \%$ lymphocytes. CT-guided mediastinal lymph node core biopsy showed adult T-cell lymphoblastic lymphoma, staining positive for CD3, BCL2, CD1a, TDT, and CD10.

Repeat CT angiography revealed severe SVC narrowing to $7 \mathrm{~mm}$. Patient was started on hyper-CVAD regimen (Cyclophosphamide, Mesna, Vincristine, Doxorubicin/Adriamycin, Dexamethasone). During bone marrow biopsy, SVC syndrome was noted. While prone, his right upper extremity and face swelled, followed by dyspnea and cough. Despite repositioning, symptoms reemerged with a positive Pemberton sign.

SVC syndrome, arising from conditions blocking blood flow through the SVC, can be a substantial contributor to mortality. In $>90 \%$ of cases, SVC syndrome is caused by malignancy (e.g. T-cell lymphoblastic lymphoma). Obstruction can occur via direct tumor invasion or external compression of the vessel. Moreover, disease rate varies depending on etiology; our patient developed symptoms within 12 days, favoring malignancy. Upon getting a bone marrow biopsy, our patient showed the classic findings of SVC syndrome.

Once the patient was stable, a work-up was done for malignancy-associated SVC syndrome. Nearly all cases of SVC syndrome are diagnosed clinically, but certain diagnostic tests 
and procedures are helpful. MRI and CT scans provide information regarding possible etiologies and, therefore, direct the approach to a tissue diagnosis. Tissue evaluation with immunohistochemistry remains the mainstay in diagnosis of lymphomas. Our patient's immunohistochemistry was positive for tumor markers discovered in only a subset of tumors, directing towards treatment with combination chemotherapy instead of local measures (e.g. radiation therapy, percutaneous vascular procedures).

Therapeutic maneuvers for clinically stable patients such as head elevation can reduce hydrostatic pressure and relieve edema. For symptomatic patients, radiation therapy and intravascular stent placement are recommended. Nonetheless, initial management should be led by the severity of symptoms and the underlying malignancy. The primary therapy for T-cell lymphoblastic lymphoma is hyper-CVAD chemotherapy, which our patient received.

While the severity of symptoms differs widely, SVC syndrome may be fatal. Given the sporadic presentation and aggressive nature of T-cell lymphoblastic lymphoma, early recognition and identifying when to intervene are critical factors for positive patient outcome.

\section{\#428 VINBLASTINE INDUCED PARALYTIC ILEUS IN A YOUNG FEMALE WITH HODGKIN LYMPHOMA}

${ }^{1} \mathrm{G}$ Malolot*, ${ }^{1} \mathrm{P}$ Chan, ${ }^{2} \mathrm{H}$ Aboaid, ${ }^{2} \mathrm{~N}$ Raza, ${ }^{2} \mathrm{R}$ Polineni. ${ }^{1}$ Ross University School of Medicine, Miramar, FL; ${ }^{2}$ Kern Medical Center, Bakersfield, CA

\subsection{6/jim-2022-WRMC.424}

Purpose of Study A rare presentation of paralytic ileus induced from Vinblastine treatment.

Methods Used Retrospective Study

Summary of Results 31-year-old female with Hodgkin lymphoma stage II presented with progressive diffuse non-radiating abdominal pain, nausea, and vomiting. Patient's symptoms started a few days after initiation of second cycle of ABVD (Adriamycin, Bleomycin, Vinblastine, Dacarbazine) therapy. Laboratory studies were remarkable for anemia and leukopenia. Abdominal X-ray was significant for gaseous distention mostly prominent in the colon just beneath the left hemidiaphragm. Further imaging studies with Computed tomography (CT) abdomen and pelvis revealed colonic gaseous distention with moderate residual fecal material and moderate small bowel fluid-filled distention. Given high suspicion for paralytic ileus induced by Vinblastine, the patient was treated with prokinetic agent metoclopramide, a stimulant laxative, and vinblastine was continued .Patients symptoms resolved with conservative management.

Conclusions Paralytic ileus is a common clinical condition that is associated with autonomic neuropathy subsequently leading to decreased bowel peristalsis. Most are often caused postoperative but a small niche of the population undergoing chemotherapy treatment with vinca alkaloids vincristine and vinblastine are susceptible with this condition. Toxicity profile of Vinblastine affecting the gastrointestinal system seems to be dose-related.

Metabolism of Vinblastine is processed by the hepatic cytochrome P450 3A. Majority of the pharmacokinetic interactions are due to previously administered drugs that are metabolized same as Vinblastine. After coadministration effects can lead to potent enzyme inducers or inhibitors. There are abundant drugs out in the market that can interfere with Vinblastine metabolism which can potentially aggregate increasing drugrelated toxicity.

The importance of this case illustrates that early recognition of Vinblastine induced paralytic ileus can lead to better outcomes and shorter hospital courses as treatment as mentioned above is different based on the cause of paralytic ileus .

\section{\#429 CARCINOSARCOMA OF DUODENUM AND PANCREASES SUCCESSFULLY TREATED BY WHIPPLE'S PROCEDURE}

${ }^{1} \mathrm{P}$ Chan*, 'G Malolot, ${ }^{2} \mathrm{~N}$ Raza, ${ }^{2} \mathrm{R}$ Polineni. 'Ross University School of Medicine, Miramar, FL; ${ }^{2}$ Kern Medical Center, Bakersfield, CA

\subsection{6/jim-2022-WRMC.425}

Purpose of Study A rare presentation of carcinosarcoma of duodenum and pancreas treated with Whipple procedure and adjuvant chemotherapy.

Methods Used Retrospective Study

Summary of Results 53-year-old female incidentally found to be anemic with hemoglobin of $7.7 \mathrm{~g} / \mathrm{dL}$ and liver function test which showed alkaline phosphatase of 679 unit/L, aspartate transaminase of 129 unit/L, alanine transaminase of 220 unit/L, and bilirubin of $1.6 \mathrm{mg} / \mathrm{dL}$ on regular lab workup ordered by surgeon prior to elective lipoma removal. Due to anemia, transaminitis, and hyperbilirubinemia, computerized tomography (CT) scan of abdomen and pelvis were performed which was remarkable for 5 centimeter ulcerative mass in the second part of duodenum without evidence of metastasis. An Esophagogastroduodenoscopy (EGD) was performed which revealed non-obstructive circumferential ulcerated mass with biopsy finding may represent a sarcomatoid carcinoma or carcinosarcoma. Malignant cells stain positive with AE-1/AE-3, CD10, CA 19-9, and CK7. Patient acutely developed worsening abdominal pain and was hospitalized with gastric outlet obstruction and underwent Whipple's procedure. Patient had a carcinosarcoma with $60 \%$ sarcomatous component on $40 \%$ adenocarcinoma component in the primary tumor. However, there were 8 out of 43 lymph nodes involved predominantly with adenocarcinoma. Final pathology was indicative for staged III carcinosarcoma of the duodenum and pancreas requiring adjuvant chemotherapy of Folfirinox for six months.patients' follow up imaging were unremarkable and the patient has no evidence of disease.

Conclusions Carcinosarcomas are a rare malignant tumors that consists of a mixture of two components, the carcinomatous and sarcomatous elements.Commonest sites include uterus, head and neck and are extremely rare in the duodenum. Localized tumors located in the region that can potentially obstruct gastric outlet flow such as in this case ought to be resected. Whipple's procedure has shown an efficacious role in the treatment plan. Although, given that there are no direct guidelines on treatment for such a rare carcinosarcoma, this case highlights the importance of timely diagnosis, resection, and adjuvant therapy considering there is a high risk for recurrence. 


\section{Immunology and rheumatology II}

\section{Concurrent session}

\author{
10:15 AM
}

\section{Saturday, January 22, 2022}

\section{\#430 B-CELL INTERFERON- $\beta$ CORRELATES WITH LUPUS NEPHRITIS IN SYSTEMIC LUPUS ERYTHEMATOSUS}

${ }^{1} \mathrm{FK}$ Alduraibi* ${ }^{1}{ }^{1} \mathrm{H}$ Fatima, ${ }^{1} \mathrm{~W}$ Chatham, ${ }^{1} \mathrm{H}$ Hsu, ${ }^{1,2} \mathrm{~J}$ Mountz. ${ }^{1}$ The University of Alabama at Birmingham, Birmingham, AL; ${ }^{2} V A$ Clinic Birmingham, Birmingham, AL

\subsection{6/jim-2022-WRMC.426}

Purpose of Study Early diagnosis of lupus nephritis (LN) can be challenging since some patients do not exhibit overt clinical manifestations until advanced stages. B cell interferon-beta (IFN $\beta$ ) correlates with development of B cell autoreactive phenotype. The objective of the present study is to determine if elevated IFN $\beta$ in circulating B cells can be a useful indicator for the development of more severe histopathologic features of LN.

Methods Used Flow cytometry was used to quantitate intracellular IFN $\beta$ in naive $(\operatorname{IgD}+\mathrm{CD} 27-) \mathrm{CD} 19+\mathrm{B}$-cells in the peripheral blood mononuclear cells (PBMCs) of a cross-sectional cohort $(\mathrm{N}=80)$ of patients with systemic lupus erythematosus (SLE), 33 of whom had lupus nephritis. Serologic and clinical manifestations of LN included anti-DNA, anti-Sm, C3, C4, and urine protein/creatinine ratio were determined. The correlation of B-cell IFN $\beta$ with lupus nephritis classification and histopathological findings, light, electron microscopy, and immunofluorescence (IF) for deposition of $\operatorname{IgM}$, IgG, IgA, $\mathrm{C} 1 \mathrm{q}$, and $\mathrm{C} 3$ was determined in 23 of the 33 patients for whom renal biopsy data was available.

Summary of Results LN was identified in $41 \%$ of our cohort of 80 SLE patients. Naïve B-cell IFN $\beta$ was positively associated with the development of LN but not cutaneous disease. Higher levels of B-cell IFN $\beta$ also correlated with higher levels of circulating anti-dsDNA, anti-Sm, and the urinary protein/ creatinine ratio. Biopsy examination revealed that proliferative LN lesions (Class III, IV with or without V) characterized by significantly elevated endocapillary hypercellularity, fibrous crescent, and fibrocellular crescent were significantly associated with high B-cell IFN $\beta$. Surprisingly, IgG, IgA, IgM, C3, and $\mathrm{C} 1 \mathrm{q}$ deposition in the kidney was not correlated with $\mathrm{B}$-cell IFN $\beta$.

Conclusions Our results suggest that B-cell IFN $\beta$ can be used in combination with other clinical diagnostic markers to assist in identifying patients who are at high risk of developing advanced LN.

\section{\#431 IDENTIFICATION OF MESENTERIC LYMPH NODE COMMENSALS INVOLVED IN NEONATAL CD4 T CELL ACTIVATION}

${ }^{1} \mathrm{RE}$ Temme*, ${ }^{2} \mathrm{~J}$ Schwensen, ${ }^{2} \mathrm{M}$ Shenoy, ${ }^{2} \mathrm{M}$ Koch. ${ }^{1}$ University of Washington School of Medicine, Seattle, WA: ${ }^{2}$ Fred Hutchinson Cancer Research Center, Seattle, WA

\subsection{6/jim-2022-WRMC.427}

Purpose of Study Maternal antibodies have an important role in neonatal immune development, particularly in regard to gut-associated lymphoid tissue and the microbiota. Neonatal mice born to antibody-deficient (uMT-/-) mothers show both an elevation in CD4 T-cell activation and increase in microbial growth in mesenteric lymph nodes (mLN) compared to pups born to wild type mothers. These results are supported by experimental data from germ-free mice that show significantly lower CD4 activation. It is therefore hypothesized the microbiota is responsible for stimulating this observed immune response. The goal of this project was to identify differences in commensals colonizing mLNs of pups born to uMT-/mothers versus antibody-sufficient mothers (uMT \pm ) in order to find a candidate microbe that could be eliciting the observed CD4 T cell expansion phenotype. This is important because understanding the mechanism in which adaptive immune responses work in response to microbiota can be used to develop more effective immune-modulating therapeutic interventions.

Methods Used We compared neonatal mice born to uMT-/dams and $\mathrm{uMT} \pm$ dams; all pups are able to produce antibodies but the uMT-/- pups did not receive antibodies via breast milk from their mothers. At day 21 of age, the mLNs were harvested, homogenized, and placed in an anaerobic chamber. Each mLN was divided equally and plated on two selective growth media. When colonies formed, they were individually restreaked on the same media, then underwent 16S PCR amplification, DNA purification, and sent for sequencing. The resulting sequences were matched to bacterial species via $16 \mathrm{~S}$ sequence identity using BLAST and the bacterial specimens were frozen for future use.

Summary of Results Sequencing revealed 60 possible bacterial species in both groups. Further broken down, $34(57 \%)$ of these species were shared between uMT-/- and uMT \pm , while $21(34 \%)$ were unique to uMT-/- and $5(8 \%)$ were unique to $\mathrm{uMT} \pm$. Bacterial species in the shared group fell largely into the Staphylococcus and Lactobacillus genuses with a few in the Parabacteroides genus. In the uMT-/- unique group, Bifidobacterium, Escherichia, Muribaculum/Duncaniella, Bacteroides, and Streptococcus genuses were identified. The uMT \pm unique bacteria were in the Enterococcus genus.

Conclusions Results from this project along with previous data support that receiving antibodies during the neonatal interval does affect microbial diversity, with the majority of unique bacterial species growing from uMT-/- mLNs. It's possible one of these unique species is responsible for the CD4 $\mathrm{T}$ cell expansion phenotype observed in past experiments but further investigation is required to determine the mechanism behind how the microbiota is eliciting this $\mathrm{T}$ cell response.

\section{\#432 RHEUMATOID ARTHRITIS AMONG MINERS IN NEW MEXICO, UTAH, AND COLORADO}

P Blanc*, L Trupin, G Schmajuk, E Yelin. University of California San Francisco, San Francisco, CA

10.1136/jim-2022-WRMC.428

Purpose of Study Our previous population-based studies have shown that coal miners in Appalachia have a 2-3-fold increased odds of rheumatoid arthritis (RA), consistent with the association between RA and silica reported in other settings. We wished to ascertain if RA risk was similarly elevated in mineral extraction workers in the western US with likely 
Abstract \#432 Table 1 Rheumatoid arthritis (RA) associated with silica exposure: multivariate logistic regression including smoking, race/ethnicity, and age

\begin{tabular}{lllll}
\hline $\begin{array}{l}\text { RA Definition } \\
\text { (models exclude RA } \\
\text { by other definition } \\
\text { only) }\end{array}$ & $\begin{array}{l}\text { Underground } \\
\text { hard rock } \\
\text { mining }\end{array}$ & $\begin{array}{l}\text { Underground } \\
\text { soft rock mining } \\
\text { (including coal) }\end{array}$ & $\begin{array}{l}\text { Surface } \\
\text { mining/ore } \\
\text { processing }\end{array}$ & $\begin{array}{l}\text { Silica from } \\
\text { non-mining } \\
\text { sources }\end{array}$ \\
\hline RA + glucocorticoids & $2.97(1.40$, & $8.80(3.55,21.83)$ & $4.34(2.27$, & $3.15(1.75$, \\
(model $n=1165)$ & $6.33)$ & & $8.29)$ & $5.68)$ \\
RA + DMARDs (model & $1.42(0.58$, & $4.47(1.59,12.57)$ & $2.46(1.24$, & $2.91(1.73$, \\
$n=1170)$ & $3.49)$ & & $4.89)$ & $4.88)$
\end{tabular}

Cells are odds ratios ( $95 \%$ confidence intervals). Referent $=$ no silica exposure. DMARD $=$ disease modifying anti-rheumatic drug.

exposure to silica in hard rock or soft rock (predominantly coal) mining.

Methods Used We conducted a population-based, random digit dial telephone survey of 2000 men aged $\geq 50$ years in counties in Colorado, New Mexico, and Utah selected due to increased silicosis mortality mapped by the National Institute for Occupational Safety and Health. Questionnaire items assessed underground hard rock, underground coal or other soft rock mining; surface mining; other occupations involving silica exposure; cigarette smoking; and reported diagnosis of RA from a health care provider. We defined RA to require reported treatment with either corticosteroids or disease modifying anti-rheumatic drugs (DMARDS). We used multivariable logistic regression to estimate the odds of RA associated with mining employment and other silica exposure, adjusted for age, race and ethnicity (White non-Hispanic vs. all others), and smoking history (current, former, never).

Summary of Results Respondent demographics: mean age, 68.6 \pm 10.1 years; $82.6 \%$ White non-Hispanic; $49.4 \%$ ever smokers. Exposures included: 138 (6.9\%) underground hard rock; $65(3.3 \%)$ underground coal and other soft rock; 164 (8.2\%) surface mining and processing; and 397 (19.9\%) non-mining silica exposure. Outcomes: RA with corticosteroids 89 (4.5\%); RA with DMARD 94 (4.7\%). Exposure-associated risk is shown in table 1.

Conclusions Hard rock underground miners, surface miners, and those exposed to silica from non-mining sources are at increased risk of RA, but odds of RA were particularly elevated among soft rock underground miners.

\section{\#433 LIPOSOMAL FORMULATION OF REDUCED GLUTATHIONE DECREASES BACTERIAL LOAD AND ALTERS THE IMMUNE RESPONSE IN MYCOBACTERIUM TUBERCULOSIS INFECTION}

${ }^{1} \mathrm{~N}$ Kachour, ${ }^{1} \mathrm{~A}$ Beever, ${ }^{2} \mathrm{~J}$ Owens, ${ }^{2} \mathrm{R}$ Cao, ${ }^{3} \mathrm{~A}$ Kalloli, ${ }^{3} \mathrm{R}$ Kumar, ${ }^{1} \mathrm{~K}$ Sasaninia, ${ }^{1} \mathrm{C}$ Vaughn, ${ }^{1} \mathrm{M}$ Singh, ${ }^{1} \mathrm{E}$ Truong, ${ }^{2} \mathrm{C}$ Khatchadourian ${ }^{*},{ }^{2} \mathrm{C}$ Sisliyan, ${ }^{2} \mathrm{~K}$ Zakery, ${ }^{4} \mathrm{~W}$ Khamas, ${ }^{3} \mathrm{~S}$ Subbian, $1,2 \mathrm{~V}$ Venketaraman. 'Western University of Health Sciences, Graduate College of Biomedical Sciences, Pomona, CA; ${ }^{2}$ Western University of Health Sciences College of Osteopathic Medicine of the Pacific, Pomona, $C A ;{ }^{3}$ Rutgers University Public Health Research Center at New Jersey Medical School, Newark, NJ; ${ }^{4}$ Western University of Health Sciences College of Veterinary Medicine, Pomona, CA

10.1136/jim-2022-WRMC.429
Purpose of Study Reduced form of Glutathione (GSH) has direct antimycobacterial activity at physiological concentrations and inhibits the growth of Mycobaterium tuberculosis (Mtb) within monocytes. This experiment aims to elucidate the effects of a liposomal formulation of GSH (L-GSH) on the levels of free radicals, inflammatory cytokines, and granuloma formation.

Methods Used C57BL6 (WT) mice (11 males and 11 females) were infected with approximately $1000 \mathrm{CFU}$ of H37Rv strain of $M t b$. Treated groups included mice treated with either $40 \mathrm{mM}$ L-GSH (40LGSH) or $80 \mathrm{mM}$ L-GSH (80LGSH) administered via drinking water. Control groups were administered mock treatment in the form of plain drinking water.

Summary of Results Mtb infection increased free radical production as measured by Malondialdehyde (MDA) and diminished the levels of reduced and total forms of GSH in the lungs. Treatment with 40LGSH resulted in a significant increase in the levels of both reduced form of glutathione at 2 weeks, 4 weeks and 8 weeks post-infection and total glutathione at 4 weeks and 8 weeks post-infection in lung lysates. 40LGSH treatment showed significant increase in the levels of reduced and total forms of glutathione in the plasma at 4 weeks post-infection. 80LGSH treatment significantly increased the levels of reduced and total forms of glutathione in the lung lysates at 8 weeks post-infection. Both treatments resulted in a reduction of MDA levels in lung tissue and plasma. LGSH treatments reduced the lung area involved in granuloma formation as well as granuloma size and complexity. 40LGSH treatment decreased the bacterial burden in the lungs significantly at 4 weeks post-infection, in the spleen at 2, 4 and 8 weeks post-infection and in the liver at 4-weeks post-infection. Both treatments significantly decreased the levels of IL- 6 at 4 weeks post-infection and IL-10 at 8 weeks post-infection, and significantly increased the levels of IL-2 at 2- and 8-weeks post-infection in lung lysates. 40LGSH treatment significantly increased the levels of Interferon gamma at 2-, 4- and 8weeks post-infection in lung lysates.

Conclusions 40LGSH was more effective in increasing the levels of reduced and total forms of GSH in the lung and plasma. L-GSH treatment altered cytokine profile and reduced bacterial load, granulomatous response, and free radical levels in mice. Thus, L-GSH may be implicated as an adjuvant to standard therapy in those infected with $M t b$.

\section{\#434 CREATION AND ANALYSIS OF A TRANSITION PROCESS AND TRANSFER REGISTRY: A COLLABORATIVE EFFORT OF PEDIATRIC AND ADULT RHEUMATOLOGISTS}

${ }^{1} \mathrm{~A}$ Freifeld*, ${ }^{2} \mathrm{KD}$ Nowicki, ${ }^{2} \mathrm{P}$ Pan, ${ }^{3} \mathrm{~J}$ Zell, ${ }^{2} \mathrm{~K}$ Moore. ${ }^{1}$ University of Colorado Denver, Denver, CO; ${ }^{2}$ Children's Hospital Colorado, Aurora, CO; ${ }^{3}$ University of Colorado Health, Aurora, $\mathrm{CO}$

\subsection{6/jim-2022-WRMC.430}

Purpose of Study It is known that the period of transfer from pediatric to adult rheumatology is associated with poor outcomes. Reliably identifying and tracking patients before and after transfer are a prerequisite for determining risk factors and developing interventions to improve outcomes. We sought to create a streamlined transition process utilizing a registry and to identify risk factors for transfer failure. 


\begin{tabular}{ll} 
Abstract \#434 Table 1 & Patient population, N=23 \\
\hline Sex & \\
Female & $19(83 \%)$ \\
Race & \\
Asian/Pacific Islander & $1(4 \%)$ \\
Black & $3(13 \%)$ \\
Hispanic & $11(48 \%)$ \\
White, Non-Hispanic & $6(26 \%)$ \\
Other & $2(9 \%)$ \\
Insurance & \\
Medicaid & $13(56 \%)$ \\
Military & $2(9 \%)$ \\
Commercial & $8(35 \%)$ \\
Rheumatology Diagnosis & \\
JIA & $13(56 \%)$ \\
Spondyloarthritis & $3(13 \%)$ \\
SLE & $3(13 \%)$ \\
Vasculitis & $2(9 \%)$ \\
JDM & $1(4 \%)$ \\
Other & $3(13 \%)$ \\
Rheumatology Medications & \\
Mean number at last pediatric visit & 2.5 \\
Biologics & $11(48 \%)$ \\
JAK & $2(9 \%)$ \\
MTX/LEF & $11(48 \%)$ \\
MMF/AZA & $5(22 \%)$ \\
HCQ & $3(13 \%)$ \\
Steroids & $6(26 \%)$ \\
\hline & \\
\hline &
\end{tabular}

Methods Used This is a descriptive cohort with a plan to transfer from pediatric to adult rheumatology within the University of Colorado. Collaborating pediatric and adult rheumatologists created a transfer log to streamline tracking of patients and information sharing. Patients were retrospectively identified for inclusion over a 14 month period. We captured demographics, rheumatologic disease, disease activity, medications, hospital use, insurance status, and compliance with care plans. Data was collected by chart review and managed using REDCap.

Summary of Results Twenty-three patients were identified with plans to transfer to adult rheumatology (table 1). 70\% completed a first visit in adult rheumatology with an average of 137 days from the last pediatric rheumatology visit. 30\% had their first adult visit within 4 months of their last pediatric visit. In subgroup analysis, patients who did not transfer within 4 months waited a mean of 346 days before the first adult visit. Five patients never established with adult rheumatology and had a longer interval between their last 2 pediatric rheumatology visits compared to those who transferred (313 \pm 136 days vs, $170.3 \pm 155$ days, $p=0.07)$. Disease complexity, number of medications, and disease activity were similar between groups.

Conclusions We examined the outcomes of 23 pediatric patients with a plan to transfer care to an adult rheumatology clinic. Interestingly, disease complexity and insurance status did not appear to predict time to transfer of care. Ongoing analysis will allow for the further development of metrics for defining a successful transfer, identification of risk factors for poor transfer outcomes, and process improvement interventions.
Neonatology general VI

\section{Concurrent session}

10:15 AM

\section{Saturday, January 22, 2022}

\section{\#435 CORRELATION OF MATERNAL AND CORD BLOOD SARS- COV-2 IMMUNOGLOBULIN LEVELS IN COVID-19 DURING PREGNANCY}

G Rojas*, M Aslam, C Uy. University of California IIvine, Irvine, CA

\subsection{6/jim-2022-WRMC.431}

Purpose of Study COVID-19 caused by the SARS-CoV-2 virus has led to a worldwide pandemic but the impact of SARSCoV-2 infection during pregnancy and delivery with subsequent neonatal effects still remains unclear. It is known that pregnant women are at higher risk of viral infections given an alteration in immune response, it is therefore likely that many would have had asymptomatic COVID-19 infection at some point during pregnancy. Studies have shown that maternal antibodies can be detected in umbilical cord blood as early as the first trimester and are a reliable source of identification of maternal past and recent infections at the time of delivery. As there is potential of in-utero transmission, it is crucial to determine the significance of a positive serology test at the time of delivery.

Objectives To evaluate the presence SARS-COV-2 antibodies in cord blood of infants born to mothers with active COVID-19 infection. The goal of this case control study is to provide a better estimate of the incidence of COVID-19 infection in pregnant women admitted to UC Irvine Medical Center, to better correlate maternal antibody levels via cord blood validation of antibodies to SARS-CoV-2, and to determine neonatal effects of maternal COVID-19 infection. We hypothesize there is a correlation between umbilical cord blood and maternal blood SARS-CoV-2 antibody levels, and that in utero vertical transmission of SARS-COV-2 is possible.

Methods Used The study was approved by the Institutional IRB. The pregnant mothers admitted to labor and delivery floor with or without Covid-19 positive status were enrolled using informed consent. Maternal blood obtained at the time of enrollment and cord blood collected at delivery. The relevant clinical data from mother infant dyad was also collected. All samples were analyzed using a coronavirus antigen microarray containing immunologically significant antigens from SARS-CoV-2 which can detect SARS-CoV-2 immunoglobulin levels. Our research is still ongoing, but to date, eighteen paired maternal-cord blood samples were analyzed.

Summary of Results Of the 18 patients enrolled into the study, 16 were confirmed SARS-COV-2 RT PCR positive. Maternal and neonatal characteristics were similar between Covid-19 positive and negative patients. Our microarray test showed comparable results between maternal and cord blood in detection of SARS-CoV-2 IgM and IgG levels.

Conclusions SARS-CoV-2 immunoglobulins levels in cord blood correlate with maternal levels and cord blood can be used an easy non-risky source to detect maternal and neonatal Covid-19 infection. Further enrollment and data collection is underway to confirm this association. 


\section{\#436 THE IMPACT OF CORONAVIRUS DISEASE 2019 ON PREGNANCY AND INFANT CHARACTERISTICS IN NEW MEXICO}

${ }^{1} \mathrm{~T}$ Emery*, ${ }^{1} \mathrm{KN}$ Baillie, ${ }^{2} \mathrm{~J}$ Maxwell, ${ }^{2} \mathrm{H}$ Ko. ${ }^{1}$ University of New Mexico School of Medicine, Albuquerque, NM; ${ }^{2}$ University of New Mexico Health Sciences Center, Albuquerque, NM

\subsection{6/jim-2022-WRMC.432}

Purpose of Study Severe Acute Respiratory Syndrome Coronavirus-1 (SARS CoV-1) and Middle East Respiratory Syndrome (MERS), two illnesses caused by a coronavirus, have been shown to affect maternal and neonatal morbidity and mortality. Coronavirus disease 2019 (COVID-19) has been shown to affect vasculature including placental changes such as microcalcifications and thrombi formation. Significant remodeling of the placenta occurs at the end of the first trimester and into the second trimester. Insults during this crucial period can affect placental size and functionality. These changes may contribute to intrauterine fetal growth restriction (IUGR). We explored the relationship between 1) antenatally-acquired maternal COVID-19 infection and fetal growth and 2) the timing of antenatal COVID-19 infection and neonatal birth weight.

Methods Used A retrospective chart review was completed using the University of New Mexico (UNM) Data Warehouse and the electronic medical record system. ICD-10 codes were used to identify those that had a positive pregnancy test and positive COVID screening test between 3/1/2020 and 3/24/ 2021 at UNM Hospital. Individuals who had a positive pregnancy test but a negative or undocumented COVID screening test without symptoms were included for comparison as a control group. Chi-square analysis was used to compare categorical data.

Summary of Results To date, 240 maternal charts have been reviewed with three excluded due to pregnancy termination and 237 neonatal charts have been reviewed. Thirty-four women were identified as COVID-19 positive during pregnancy, 41 women with negative testing, one woman who declined testing, and the remainder with no testing documented and no symptoms reported. Twenty-two women were identified as having COVID-19 infection in the third trimester of pregnancy, with $9 \%$ of these infants being small for gestational age. The infants born to women who were negative for COVID-19 were small for gestational age in $12 \%$ of the births. Interestingly, the incidence of delivery via cesarean section was $12 \%$ in the 41 deliveries to COVID-19 negative women. Of those positive, 27\% had cesarean section deliveries, a nearly significant increase $(\mathrm{p}=0.06)$.

Conclusions The charts reviewed to date did not reveal any significant difference in infant growth parameters at birth in women who tested negative or positive for COVID-19 during pregnancy. However, there was an increased incidence of cesarean section deliveries in women with COVID-19 infection during pregnancy. Most women were not symptomatic, so this does not seem to be related to illness. Additional investigations are required to further delineate if this relationship persists.
\#437 DEBRIEFING PERFORMANCE IN A SIMULATION-BASED TRAINING PROGRAM IN NEONATAL RESUSCITATION: SIMULATING SUCCESS

${ }^{1,2} \mathrm{BN}$ Lieu*${ }^{*}{ }^{1,2} \mathrm{H}$ Lee, ${ }^{1,2} \mathrm{M}$ Bennett, ${ }^{1,2} \mathrm{~K}$ Padua. ${ }^{1}$ Stanford, Stanford, CA; ${ }^{2}$ California Perinatal Quality Care Collaborative, Stanford, CA

\subsection{6/jim-2022-WRMC.433}

Purpose of Study Neonatal resuscitation is a complex procedure that requires the finesse of an multidisciplinary team. The California Perinatal Quality Care Collaborative (CPQCC) and the Center for Advanced Pediatric Perinatal Education (CAPE) implemented an on-site simulation-based training program called Simulation Success at 15 NICUs over 15-months. Simulation and debriefing present an opportunity for the team to reduce errors and identify latent safety threats. There is a gap in knowledge on real-life debriefing practice for neonatal simulation. This project analyzes the debriefing performance of the NICUs through the course of the program.

Methods Used The debrief videos were anonymized and scored according to CAPE Real-Time Debriefing Evaluation tool. The fifteen sites were randomly assigned into four groups (G1, G2, G3, G4). Performance was evaluated through the ratio of trainee responses to the sum of instructor questions and instructor statements (TR:IQ+IS). A goal TR:IQ+IS was set to be greater than 3:1. Least squares linear regression was applied to the ratios, yielding a line of best fit for each group.

Summary of Results 82 total videos were including in the analysis. There were 22, 28, 17, and 15 videos for G1, G2, G3, and G4, respectively. The ratios of G1, G2, and G4 reveal no substantial trends and lie within the same order of magnitude, approaching a slope of zero. However, G3 illustrates a significant rate of change that is one order of magnitude larger than the others. All linear regressions yield a line that lie under the target ratio.

Conclusions Variations in debriefer and trainee attendance at the sites may contribute to the lack of trends in G1, G2, and G4. The absence of significant declining debrief performance
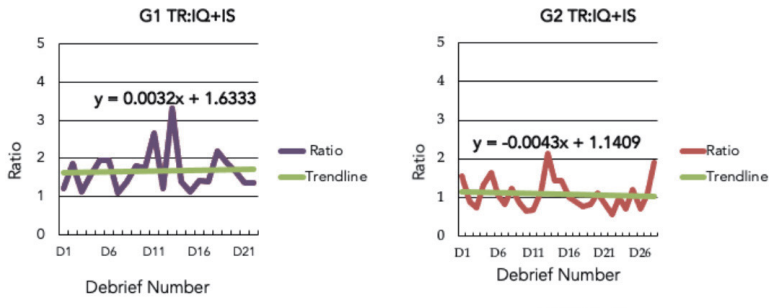

G3 TR:IQ+IS
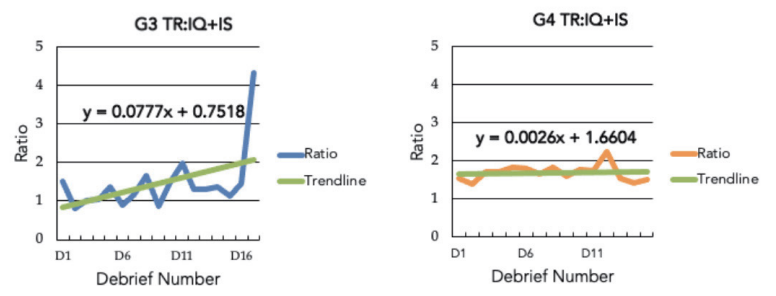

Abstract \#437 Figure 1 TR:IQ+IS ratios and trendlines per group 
highlights the potential for sustaining debriefing in simulationbased training. G3's trend suggests increased engagement in trainees over time. Lack of ratios near 3:1 hint that this target may be difficult to achieve in practice. Moving forward, evaluation of the simulations in tandem with debriefs may be telling, especially in the context of qualitative briefing performance and correlation to patient outcomes.

\section{\#438 TIME TO POSITIVITY IN BLOOD CULTURES IN A LEVEL IV NICU}

${ }^{1} \mathrm{Y}$ Nishihara*, ${ }^{*} \mathrm{C}$ MacBrayne, ${ }^{2} \mathrm{~A}$ Prinzi, ${ }^{1} \mathrm{~J}$ Zenge, ${ }^{1} \mathrm{~T}$ Grover, ${ }^{1} \mathrm{~S}$ Parker. ${ }^{1} \mathrm{C}$ hildren's Hospital Colorado, Aurora, CO; ${ }^{2}$ University of Colorado, Denver, $\mathrm{CO}$

\subsection{6/jim-2022-WRMC.434}

Purpose of Study In the Neonatal Intensive Care Unit (NICU) setting, subtle clinical deterioration of the infant, combined with the lack of specificity in clinical signs to identify true infection often triggers an evaluation for sepsis - where cultures are obtained, and empiric antibiotics are initiated.

Limiting the duration of antibiotic exposure has potential benefits in curtailing antimicrobial resistance and reducing unwanted adverse effects.

We aimed to determine the time to positivity (TTP) of blood cultures in a free-standing level IV NICU over a 6-year period, with the goal to reassess our antimicrobial practice in the NICU.

Methods Used Data were extracted from the Children's Hospital Colorado data warehouse for all patients admitted to the NICU, who had a positive blood culture between January 2013 to December 2018. These patient's charts were reviewed for both microbiologic and clinical data. TTP was calculated based on date and time culture was collected, compared to the date and time growth was first reported. Micro-organisms were categorized into absolute pathogens, potential pathogens (e.g., CoNS, other strep), common contaminants, yeast and other less frequently identified organisms.

Summary of Results A total of 314 positive blood cultures were identified from 270 individuals. The mean gestational age was 34.59 weeks with an average birthweight of $2356 \mathrm{~g}$. Overall TTP median and interquartile range (IQR) was 21.16 $(14.39,25.48)$ hours. The median (IQR) TTP for gram-positive absolute pathogens and gram-negative absolute pathogens were $16.32(12.97,22.38)$ and $12.55(11.25,14.08)$ hours, respectively. Of the 314 positive culture results, 299 (95\%) were initiated with antibiotics; 131 (41\%) were later deemed as contaminant, and treatment discontinued. Central line associated bacterial infection was documented in 35 cases (11\%). Death within 4 weeks of culture positivity was recorded in 25 (9.2\%) cases.

Conclusions The majority of gram-positive and gram-negative pathogens were identified within $24 \mathrm{~h}$ of blood culture collection. A substantial number of cases were later categorized as contaminants, highlighting the importance of correct sterile technique when obtaining cultures. These findings highlight opportunities for antimicrobial stewardship to limit antibiotic exposure in the NICU. The high mortality within 4 weeks of blood culture positivity warrants further study.

\section{\#439 MATERNAL AND PERIPARTUM RISK FACTORS THAT AFFECT C-REACTIVE PROTEIN AND PROCALCITONIN IN NON-INFECTED NEWBORNS DURING THE FIRST 72 HOURS OF LIFE}

A Vachhani*, A Chambliss, M Durand, R Ramanathan, R Cayabyab. LAC+USC Medical Center, Keck School of Medicine of USC, Los Angeles, CA

\subsection{6/jim-2022-WRMC.435}

Purpose of Study Early detection of infection is difficult as the first signs of infection are nonspecific. Providers often rely on biomarkers to help identify these infants including high sensitivity c-reactive protein (hsCRP) and procalcitonin (PCT). Maternal and peripartum risk factors may influence hsCRP and PCT making levels difficult to interpret. The purpose of this study was to compare the effect of maternal and peripartum risk factors on hsCRP and PCT levels in non-infected infants during the first 72 hours of life.

Methods Used Retrospective study of infants admitted to the neonatal intensive care unit from August 2018-February 2021 who were worked up for early onset sepsis. Infants with three paired PCT and hsCRP values and blood, urine or cerebrospinal fluid culture were included. Biomarker levels were drawn at 0,1, 2 time points at $12-24$ hours intervals from birth. Demographics and laboratory results were collected. Infants were classified as non-infected and included in the study if cultures were negative and prolonged antibiotics were not continued for presumed infection. Maternal risk factors included pre-eclampsia and diabetes mellitus (DM). Peripartum risk factors included vaginal delivery, need for positive pressure ventilation (PPV) in the delivery room, presence of meconium-stained amniotic fluid (MSAF) and diagnosis of chorioamnionitis. Data was analyzed with Wilcoxon-rank sum test.

Summary of Results A total of 275 infants were classified as non-infected. The median (IQR) gestational age was 34.9 (33.1, 37.6) weeks and median (IQR) birth weight was 2285 $(1850,3120)$ grams. One hundred one $(37 \%)$ of infants were born by vaginal delivery. Serial labs were drawn at time points $0,1,2$ corresponding to median (IQR) hour of life of 1 (1, 2), $13(12,20)$, and $32(25,40)$ hours respectively. Maternal diagnosis of DM did not affect hsCRP or PCT (data not shown). Peripartum risk factors such as MSAF, chorioamnionitis and PPV at birth affected hsCRP, but not PCT. Only maternal diagnosis of pre-eclampsia affected PCT on the second timepoint (table 1).

Conclusions Our preliminary findings suggest that maternal and peripartum risk factors affect hsCRP more than PCT, particularly chorioamnionitis and presence of MSAF. These risk factors contributed to rise in hsCRP levels after birth making it difficult to interpret levels in infants screened for infection. Our study showed a natural rise and fall of PCT in noninfected infants, regardless of exposure to risk factors within 72 hours of life. PCT may be a more specific biomarker to screen infants at risk for infection immediately after birth in comparison to hsCRP. However, further studies are necessary to establish time-based reference ranges for PCT to increase clinical utility. 
Abstract \#439 Table 1 Risk factors affecting biomarkers

\begin{tabular}{|c|c|c|c|c|c|c|}
\hline & \multicolumn{3}{|c|}{ hsCRP (mg/L) } & \multicolumn{3}{|c|}{ PCT (ng/mL) } \\
\hline & $\begin{array}{l}\text { Risk } \\
\text { Factor } \\
\text { Present }\end{array}$ & $\begin{array}{l}\text { Risk Factor } \\
\text { Not } \\
\text { Present }\end{array}$ & $\begin{array}{l}\mathrm{P} \text { - } \\
\text { value }\end{array}$ & $\begin{array}{l}\text { Risk } \\
\text { Factor } \\
\text { Present }\end{array}$ & $\begin{array}{l}\text { Risk Factor } \\
\text { Not } \\
\text { Present }\end{array}$ & $\begin{array}{l}\text { P- } \\
\text { Value }\end{array}$ \\
\hline $\begin{array}{l}\text { Meconium } \\
\text { Stained Amniotic } \\
\text { Fluid^^}\end{array}$ & $\mathrm{N}=22$ & $\mathrm{~N}=250$ & & $\mathrm{~N}=22$ & $\mathrm{~N}=250$ & \\
\hline Time 0 Lab Draw & $\begin{array}{l}0.3 \\
(0.2,0.7)\end{array}$ & $\begin{array}{l}0.2 \\
(0.2,0.3)\end{array}$ & 0.23 & $\begin{array}{l}0.25 \\
(0.14 \\
0.44)\end{array}$ & $\begin{array}{l}0.19 \\
(0.15,0.32)\end{array}$ & 0.36 \\
\hline Time 1 Lab Draw & $\begin{array}{l}5.1 \\
(0.7 \\
10.7)\end{array}$ & $\begin{array}{l}1.0 \\
(0.5,2.4)\end{array}$ & 0.01 & $\begin{array}{l}2.23 \\
(0.99 \\
4.7)\end{array}$ & $\begin{array}{l}2.58 \\
(0.84,7.86)\end{array}$ & 0.72 \\
\hline Time 2 Lab Draw & $\begin{array}{l}4.8 \\
(0.9,9.8)\end{array}$ & $\begin{array}{l}1.2 \\
(0.5,4.3)\end{array}$ & 0.04 & $\begin{array}{l}1.73 \\
(0.56 \\
2.96)\end{array}$ & $\begin{array}{l}2.05 \\
(0.83,6.55)\end{array}$ & 0.49 \\
\hline Chorioamnioitis & $\mathrm{N}=26$ & $\mathrm{~N}=249$ & & $\mathrm{~N}=26$ & $\mathrm{~N}=249$ & \\
\hline Time 0 Lab Draw & $\begin{array}{l}0.3 \\
(0.3,0.5)\end{array}$ & $\begin{array}{l}0.2 \\
(0.2,0.3)\end{array}$ & $<0.01$ & $\begin{array}{l}0.25 \\
(0.15 \\
0.84)\end{array}$ & $\begin{array}{l}0.19 \\
(0.15,0.33)\end{array}$ & 0.06 \\
\hline Time 1 Lab Draw & $\begin{array}{l}9.0 \\
(0.9 \\
20.8)\end{array}$ & $\begin{array}{l}1.0 \\
(0.5,2.1)\end{array}$ & $<0.01$ & $\begin{array}{l}4.50 \\
(1.67 \\
15.15)\end{array}$ & $\begin{array}{l}2.46 \\
(0.84,7.53)\end{array}$ & 0.09 \\
\hline Time 2 Lab Draw & $\begin{array}{l}8.0 \\
(1.7,30)\end{array}$ & $\begin{array}{l}1.1 \\
(0.5,3.9)\end{array}$ & $<0.01$ & $\begin{array}{l}1.73 \\
(0.56 \\
2.96)\end{array}$ & $\begin{array}{l}2.03 \\
(0.81,6.22)\end{array}$ & 0.58 \\
\hline Pre-Eclampsia^^ & $\mathrm{N}=71$ & $\mathrm{~N}=203$ & & $\mathrm{~N}=71$ & $\mathrm{~N}=203$ & \\
\hline Time 0 Lab Draw & $\begin{array}{l}0.2 \\
(0.2,0.3)\end{array}$ & $\begin{array}{l}0.2 \\
(0.2,0.3)\end{array}$ & 0.27 & $\begin{array}{l}0.21 \\
(0.16 \\
0.31)\end{array}$ & $\begin{array}{l}0.19 \\
(0.14,0.34)\end{array}$ & 0.91 \\
\hline Time 1 Lab Draw & $\begin{array}{l}1.0 \\
(0.5,3.7)\end{array}$ & $\begin{array}{l}1.0 \\
(0.5,2.8)\end{array}$ & 0.87 & $\begin{array}{l}1.88 \\
(0.67 \\
4.71)\end{array}$ & $\begin{array}{l}2.77 \\
(0.92,9.6)\end{array}$ & 0.05 \\
\hline Time 2 Lab Draw & $\begin{array}{l}1.9 \\
(0.8,5.8)\end{array}$ & $\begin{array}{l}1.3 \\
(0.5,4.4)\end{array}$ & 0.24 & $\begin{array}{l}2.15 \\
(0.66 \\
4.84)\end{array}$ & $\begin{array}{l}1.99 \\
(0.84,7.14)\end{array}$ & 0.48 \\
\hline Vaginal Delivery & $\mathrm{N}=101$ & $\mathrm{~N}=174$ & & $\mathrm{~N}=101$ & $\mathrm{~N}=174$ & \\
\hline Time 0 Lab Draw & $\begin{array}{l}0.2 \\
(0.2,0.4)\end{array}$ & $\begin{array}{l}0.2 \\
(0.2,0.3)\end{array}$ & $<0.01$ & $\begin{array}{l}0.22 \\
(0.15 \\
0.6)\end{array}$ & $\begin{array}{l}0.19(0.14, \\
0.27)\end{array}$ & 0.10 \\
\hline Time 1 Lab Draw & $\begin{array}{l}1.3 \\
(0.7,5.4)\end{array}$ & $\begin{array}{l}1.0 \\
(0.4,1.6)\end{array}$ & $<0.01$ & $\begin{array}{l}3.30 \\
(1.25 \\
7.09)\end{array}$ & $\begin{array}{l}2.24(0.81, \\
8.18)\end{array}$ & 0.61 \\
\hline Time 2 Lab Draw & $\begin{array}{l}1.8 \\
(0.7,5.9)\end{array}$ & $\begin{array}{l}1.1 \\
(0.5,3.7)\end{array}$ & 0.12 & $\begin{array}{l}2.50 \\
(0.87 \\
5.7)\end{array}$ & $\begin{array}{l}1.85(0.74 \\
3.7)\end{array}$ & 0.90 \\
\hline $\begin{array}{l}\text { Use of Positive } \\
\text { Pressure at Birth }\end{array}$ & $\mathrm{N}=162$ & $\mathrm{~N}=113$ & & $N=162$ & $\mathrm{~N}=113$ & \\
\hline Time 0 Lab Draw & $\begin{array}{l}0.2 \\
(0.2,0.3)\end{array}$ & $\begin{array}{l}0.3 \\
(0.2,0.4)\end{array}$ & $<0.01$ & $\begin{array}{l}0.20 \\
(0.16 \\
0.31)\end{array}$ & $\begin{array}{l}0.18 \\
(0.14,0.48)\end{array}$ & 0.54 \\
\hline Time 1 Lab Draw & $\begin{array}{l}1.0 \\
(0.5,1.6)\end{array}$ & $\begin{array}{l}1.3 \\
(0.6,5.8)\end{array}$ & 0.02 & $\begin{array}{l}2.53 \\
(0.84 \\
8.21)\end{array}$ & $\begin{array}{l}2.65 \\
(1.01,7.11)\end{array}$ & 0.64 \\
\hline Time 2 Lab Draw & $\begin{array}{l}1.2 \\
(0.6,4.1)\end{array}$ & $\begin{array}{l}1.7 \\
(0.5,7.5)\end{array}$ & 0.29 & $\begin{array}{l}2.30 \\
(0.98 \\
6.87)\end{array}$ & $\begin{array}{l}1.55 \\
(0.59,5.61)\end{array}$ & 0.08 \\
\hline
\end{tabular}

Data are shown as median $(\mathrm{IQR}) \wedge$ Some missing data

\section{$\# 440$ \\ EXPERIENCE WITH PARENTAL LEAVE DURING PEDIATRIC FELLOWSHIP: THE FELLOW PERSPECTIVE}

N Dyess*, B Weikel, J Barker, T Garrington, TA Parker. University of Colorado Denver School of Medicine, Aurora, $\mathrm{CO}$

\subsection{6/jim-2022-WRMC.436}

Purpose of Study To describe the experience of parental leave during pediatric fellowship training from the fellows' perspective.

Methods Used A national web-survey was sent to fellows at ACGME-accredited pediatric fellowship programs, either directly or through their program.

Summary of Results Of the 1003 fellows who opened the survey, 761 (76\%) provided information on their experience with parental leave during fellowship training. 43\% reported having children, $26 \%$ planned on having a/another child during fellowship, and $16 \%$ are unsure. Of those with children, 59\% have 1 child and 27\% have 2 children. Approximately half of those with children reported having at least one child during fellowship. Of the 56\% who were not planning on having a child during fellowship, only $14 \%$ stated their program's parental leave policy (PLP) was a deterrent. The majority of respondents felt that fellowship (26\%) or post-fellowship (25\%) was the best time to have children. $89 \%$ of respondents did not consider PLP when ranking fellowship programs.

Of respondents who do not have children, 56\% experienced colleagues who had or adopted a child during fellowship. 24\% reported feeling unduly burdened by their colleague's decision to have a child during fellowship and $13 \%$ felt their colleagues should have delayed childbearing. Of these, $47 \%$ felt that an alternative PLP may have alleviated these feelings.

Of respondents who had children during fellowship, daycare and a fellow's partner were the primary means of childcare while the fellow is at work. $81 \%$ of fellows breastfed their children during fellowship for an average of 9 months. $82 \%$ of respondents who had a child during fellowship stated they did not experience significant financial difficulties. 56\% reported receiving adequate PLP information prior to their leave and $36 \%$ reported they did not. $15 \%$ of respondents reported feeling discrimination at work while pregnant. $35 \%$ of respondents who had children during fellowship are having to extend their fellowship end date due to their parental leaves, and $34 \%$ took unpaid weeks to construct their parental leaves (average of 4 weeks). Regardless, $87 \%$ of respondents do not wish they would have delayed childbearing until after fellowship.

Conclusions Many pediatric fellows have children or are planning on having children during fellowship. Areas where a trainee's experience with parental leave during pediatric fellowship could improve include increased clarity and transparency of PLP to better guide a fellow's family planning, allowance of parental leave without having to extend training, increased paid leave to minimize unpaid leave utilization, increased advocacy on the parent fellow's behalf to eradicate discrimination, and increased protection of co-fellows not planning on having children in fellowship. 
$\# 441$ SHORT AND LONG TERM OUTCOMES IN LATE PRETERM INFANTS EXPOSED TO DELAYED CORD CLAMPING COMPARED TO IMMEDIATE CORD CLAMPING

O Okolo*, JY Massoumi, K Tedesco, M Chu, S Sakhamuru, L Barton, R Ramanathan, R Cayabyab. University of Southern California Keck School of Medicine, Los Angeles, CA

\subsection{6/jim-2022-WRMC.437}

Purpose of Study AAP, ACOG, and NRP have recommended delayed cord clamping (DCC) for term and preterm deliveries for at least 30-60 seconds after birth. It has been established that DCC provides short term and long-term benefits. In preterm infants, DCC is associated with improved transitional circulation, higher hemoglobin, decreased need for blood transfusion, and lower incidence of necrotizing enterocolitis. The purpose of this study is to compare short-term and longterm outcomes in late preterm infants (LPT) exposed to delayed cord clamping vs. immediate cord clamping (ICC).

Methods Used Retrospective study of preterm infants born at $34^{0 / 7}-36^{6 / 7}$ weeks of gestation and admitted to the neonatal intensive care unit (NICU) between 2018-2020. Demographics, cord clamping information, laboratory values, clinical and neurodevelopmental data were extracted from electronic medical records and the neonatal database. Age and Stages Questionnaire was used to evaluate neurodevelopment at 18 months chronologic age (CA). Data was analyzed with ChiSquare or Fisher Exact Test and Wilcoxon Rank Sum test where appropriate.

Abstract \#441 Table 1 Demographics, short- and long-term outcomes between the two groups

Immediate Cord Immediate Cord P Value

Clamping $n=50 \quad$ Clamping $n=50$

\begin{tabular}{|c|c|c|c|}
\hline \multicolumn{4}{|l|}{ DEMOGRAPHICS } \\
\hline Birth Weight $(\mathrm{g})$ * & $2560(2360,2890)$ & $2230(2068,2525)$ & $<0.001$ \\
\hline Gestational Age (wks) * & $35.8(35.1,36.3)$ & $34.7(34.1,35.1)$ & $<0.01$ \\
\hline Male gender, $\mathrm{n}(\%)$ & $29(58)$ & $25(50)$ & 0.42 \\
\hline Hispanic Race, n (\%) & $29(55)$ & $24(48)$ & 0.09 \\
\hline Cesarean Section, n (\%) & $38(76)$ & $33(66)$ & 0.27 \\
\hline Maternal Preeclampsia, n (\%) & $7(14)$ & $11(22)$ & 0.30 \\
\hline Maternal Gestational Diabetes, n (\%) & $11(22)$ & $13(26)$ & 0.64 \\
\hline APGAR score $1 \mathrm{~min}$ * & $7(3,8)$ & $8(7,8)$ & 0.03 \\
\hline APGAR score $5 \mathrm{~min}$ * & $8(6,9)$ & $9(8,9)$ & 0.03 \\
\hline \multicolumn{4}{|l|}{ SHORT TERM OUTCOMES } \\
\hline $\begin{array}{l}\text { Hemoglobin at } 24 \text { Hours of Life (gm/ } \\
\mathrm{dL}) \text { * }\end{array}$ & $15.8(14.3,17.8)$ & $16.3(15,18.1)$ & 0.23 \\
\hline Phototherapy, n (\%) & $16(33)$ & $17(34)$ & 0.89 \\
\hline $\begin{array}{l}\text { PRBC Transfusion within } 24 \text { hours of } \\
\text { life, } n(\%)\end{array}$ & $3(6)$ & $0(0)$ & 0.08 \\
\hline $\begin{array}{l}\text { Temperature on admission to NICU } \\
\left({ }^{\circ} \mathrm{C}\right){ }^{*}\end{array}$ & $36.8(36.6,37.1)$ & $36.6(36.4,36.8)$ & $<0.01$ \\
\hline $\begin{array}{l}\text { Hypothermia on admission to NICU, } \\
n(\%)\end{array}$ & $2(4.1)$ & $13(26)$ & $<0.01$ \\
\hline \multicolumn{4}{|l|}{ NEURODEVELOPMENTAL OUTCOMES } \\
\hline \multicolumn{4}{|l|}{ AT 18-24 MONTHS OF AGE } \\
\hline Communication * & $40(25,50)$ & $35(30,45)$ & 0.93 \\
\hline Gross Motor * & $60(60,60)$ & $57.5(55,60)$ & 0.48 \\
\hline Fine Motor * & $55(45,60)$ & $50(50,60)$ & 0.93 \\
\hline Problem Solving * & $55(45,60)$ & $52.5(35,55)$ & 0.52 \\
\hline Personal Social * & $60(55,60)$ & $55(45,60)$ & 0.31 \\
\hline
\end{tabular}

*median (25th percentile, 75 th percentile)
Summary of Results There were 156 infants admitted to the NICU during the study period. Preliminary analysis included 50 infants exposed to ICC and 50 infants exposed to DCC. There were 19/100 (19\%) infants followed up for neurodevelopment at 18 months. Infants exposed to DCC were significantly smaller, immature and with lower APGAR scores at 1 and 5 minutes compared to infants in the ICC group. Infants exposed to DCC had significantly lower median temperature on admission to the NICU and a higher rate of hypothermia defined as temperature less than $36.5^{\circ} \mathrm{C}$. One infant in the DCC group had a temperature less than $36^{\circ} \mathrm{C}$. Median hemoglobin levels at 18-36 hours of life and rate of phototherapy were not significantly different between groups. No infant exposed to DCC compared to $3(6 \%)$ infants in ICC received packed red blood cell transfusion within the first 24 hours of life. Median hemoglobin values at 12 months CA and median ASQ scores at 18 months of CA were similar in both groups. (Table 1).

Conclusions Our preliminary data showed that DCC compared to ICC in LPT infants did not result in a higher hemoglobin at birth and at 1 year of age, and neurodevelopmental outcomes at 18 months CA was similar. DCC in immature and smaller infants can be a risk factor for hypothermia, therefore, it is important to follow recommendations by NRP to prevent this adverse effect. Data collection is continuing to increase sample size and verify these findings.

Neonatology pulmonary III

\section{Concurrent session}

\section{0:15 AM}

\section{Saturday, January 22, 2022}

\section{\#442 HIGHER WEIGHT FOR LENGTH IS ASSOCIATED WITH INCREASED SEVERITY IN BRONCHOPULMONARY DYSPLASIA IN PRETERM INFANTS}

R Kalikkot, K Harvey, KC Hart, P Alur*. The University of Mississippi Medical Center, Jackson, MS

\subsection{6/jim-2022-WRMC.438}

Purpose of Study Bronchopulmonary dysplasia (BPD) causes significant morbidity and mortality in infants. Obesity negatively impacts lung function in children and adolescents. There are no studies correlating weight for length (W/L) or the body mass index (BMI) in preterm infants with higher Respiratory Severity Scores (RSS) in BPD. W/L is accurate in predicting fat-free mass and fat mass in premature infants. Our aim was to explore with a prospective observational study if higher W/L z-scores at 33-36 weeks of gestation correlate with higher RSS at $36 \& 40$ weeks corrected gestational age in preterm infants with BPD.

Methods Used Supported by NIH (Award \# 1U54GM115428). Preterm infants born@ @ 30 weeks requiring respiratory support @ 30-33 weeks for $>2$ days were enrolled. Weight, length, and head circumference were measured weekly. Data on W/L z-scores \& BMI were collected. RSS was calculated as per the STOP-ROP trial. Data were analyzed using descriptive and inferential statistics. Linear 
mixed model regression was used to study the relationship between the continuous variables with potential covariates.

Summary of Results We enrolled 81 preterm infants. Maternal and neonatal demographics are in the figure. RSS was significantly higher in grade 3 BPD- 1.0 vs 0.23 in grade 1 BPD ( $p$

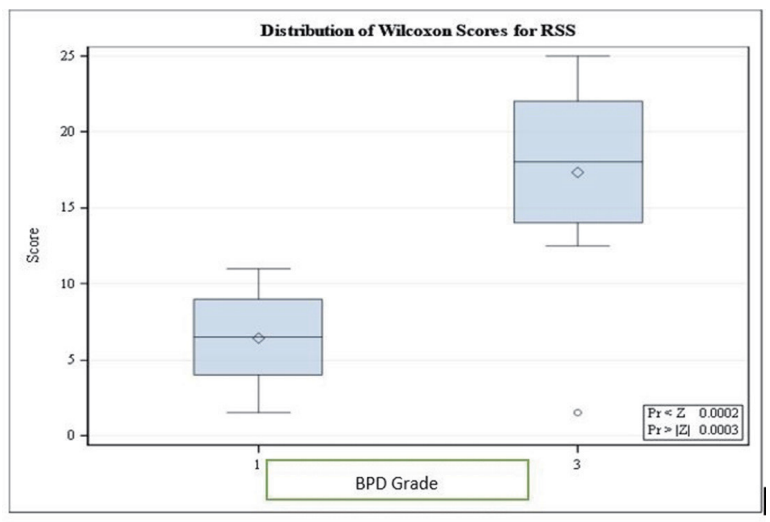

Abstract \#442 Figure 1

\begin{tabular}{llll} 
Abstract \#442 Table 1 & & \\
\hline $\begin{array}{l}\text { Weight/Length } \\
\text { Category }\end{array}$ & $\begin{array}{l}\text { Mean RSS-40 } \\
\text { weeks }\end{array}$ & SD & $\begin{array}{l}\text { P } \\
\text { Value* }\end{array}$ \\
\hline 50 percentile (N=11) & 0.09 & 0.27 & 0.01 \\
\hline
\end{tabular}

Abstract \#442 Table 2
\begin{tabular}{|l|l|}
\hline Maternal Demographics & Means \\
\hline African American & $80 \%$ \\
\hline Diabetes & $16.20 \%$ \\
\hline Pre-eclampsia & $46.05 \%$ \\
\hline Antenatal steroids & $74 \%$ \\
\hline PROM & $31.65 \%$ \\
\hline BMI & $35.22 \pm 10.19$ \\
\hline Neonatal Demographics & Mean \pm SD \\
\hline Females & $59 \%$ \\
\hline Mean birth weight (grams) & $0.906 \pm 0.24$ \\
\hline Mean gestational age (weeks) & $26.8 \pm 1.77$ \\
\hline Duration of Ventilation (days) & $19.41 \pm 28.37$ \\
\hline Mean Wt/L z-scores @ 36 weeks CGA & $(-0.32 \pm 1.03)$ \\
\hline Mean BMI @ 36 weeks CGA & $12.29 \pm 1.36$ \\
\hline Mean RSS @ 36 weeks CGA & $0.27 \pm 0.45$ \\
\hline Mean calories (Kcal/kg/d) & $115.2 \pm 16.8$ \\
\hline Mean protein (g/kg/d) & $3.75 \pm 0.59$ \\
\hline Postnatal Steroids & $26.00 \%$ \\
\hline
\end{tabular}

$<0.0001$ ) (figure 1). For every unit increase in $\mathrm{Wt} / \mathrm{L} \mathrm{Z}$ score, RSS increased by 0.069 ( $\mathrm{p}<0.0001$ ). RSS significantly correlated with $\mathrm{W} / \mathrm{L} \mathrm{Z}$ scores, percentiles, and BMI $(\mathrm{P} \leq 0.0001)$ even after including postnatal steroids, gestational age, and sex in the regression model. The results remained significant after excluding infants with postnatal steroids. The mean RSS @ 40 weeks in $\mathrm{W} / \mathrm{L} \leq 50$ was $0.0 .09 \pm 0.27$ compared to 0.47 \pm 0.37 in $>50$ percentile category $(\mathrm{p}=0.01)$.

Conclusions Our study is the first to show that higher BMI and W/L may adversely affect respiratory severity in BPD infants. Hence, an optimal W/L ratio should be maintained to avoid additional burdens in infants with BPD.

\section{\#443 NOVEL APPLICATION OF NEONATAL POINT-OF-CARE ULTRASOUND: ULTRASOUND-GUIDED LESS INVASIVE SURFACTANT ADMNISTRATION}

${ }^{1} \mathrm{~J}$ Velasquez*, ${ }^{2} \mathrm{R}$ Ramanathan, ${ }^{2} \mathrm{~A}$ Yeh. ${ }^{1} L A C+$ USC Medical Center, LA, CA; ${ }^{2}$ Keck School of Medicine of USC, LA, CA

\subsection{6/jim-2022-WRMC.439}

Purpose of Study Point-of-care ultrasound (POCUS) has become a very useful tool in guiding bedside clinical management. This study aims to describe a novel and innovative application of neonatal POCUS: Ultrasound-guided Less Invasive Surfactant Administration (LISA).

Methods Used This is a single-center, prospective observational study conducted in a level III NICU. In our unit, LISA is performed routinely in newborns on noninvasive respiratory support needing surfactant therapy. A 16 GA x 5.25, BD Angiocath is adapted to deliver the surfactant. The catheter insertion was performed as per standard unit protocol. As part of the study protocol, POCUS was used to identify and confirm catheter position inside the trachea, along with video laryngoscopy in all cases to ensure the catheter passing through the vocal cords.

POCUS was performed with the linear transducer (GE LOGIQ e) positioned transversely over the suprasternal notch. The trachea can be identified in the midline position as a hyperechoic air-mucosa interface with reverberation artifacts. A palpable anatomic landmark allows for a simpler, more feasible, and reproducible technique while ensuring, based on anatomy, the visualization of the catheter in the correct position



Abstract \#443 Figure 1 
Abstract \#443 Table 1 Patient characteristics

\begin{tabular}{|c|c|c|c|c|c|c|c|c|c|c|}
\hline No. & $\begin{array}{l}\text { Antenatal } \\
\text { steroids }\end{array}$ & $\begin{array}{l}\text { Antenatal } \\
\text { steroids indicated }\end{array}$ & $\begin{array}{l}\text { Mode of } \\
\text { delivery }\end{array}$ & $\begin{array}{l}\text { Delivery room } \\
\text { interventions }\end{array}$ & $\begin{array}{l}\text { Gestational } \\
\text { age (weeks) }\end{array}$ & $\begin{array}{l}\text { Birth weight } \\
\text { (grams) }\end{array}$ & Gender & $\begin{array}{l}\text { Mode of } \\
\text { ventilation }\end{array}$ & $\begin{array}{l}\text { FiO2 prior to } \\
\text { surfactant }\end{array}$ & $\begin{array}{l}\text { Indication for Surfactant } \\
\text { Replacement therapy }\end{array}$ \\
\hline 1 & No & No & NSVD & PPV & $41+1 / 7$ & 2945 & Female & NIPPV & $30 \%$ & MAS \\
\hline 2 & Yes & Yes & C-section & PPV & $30+3 / 7$ & 1415 & Male & NIPPV & $30 \%$ & RDS \\
\hline 3 & Yes & Yes & C-section & PPV & $32+1 / 7$ & 1800 & Female & NIPPV & $25 \%$ & RDS \\
\hline 4.a & Yes & Yes & C-section & PPV & $32+2 / 7$ & 1910 & Male & NIPPV & $35 \%$ & RDS \\
\hline 4.b & Yes & Yes & C-section & PPV & $32+2 / 7$ & 1910 & Male & NIPPV & $40 \%$ & RDS \\
\hline 5 & Yes & Yes & C-section & PPV & $30+3 / 7$ & 1455 & Female & NIPPV & $45 \%$ & RDS \\
\hline 6 & Yes & Yes & C-section & PPV & $30+3 / 7$ & 1165 & Female & NIPPV & $40 \%$ & RDS \\
\hline 7 & Yes & Yes & C-section & PPV & $32+6 / 7$ & 1360 & Female & NIPPV & $40 \%$ & RDS \\
\hline 8 & No & No & NSVD & PPV & $37+2 / 7$ & 2900 & Female & NIPPV & $25 \%$ & IDM-related RDS vs TTN \\
\hline 9 & Yes & Yes & C-section & PPV & $27+4 / 7$ & 980 & Female & NIPPV & $50 \%$ & RDS \\
\hline 10 & Yes & Yes & C-section & PPV & $27+0 / 7$ & 957 & Male & NIPPV & $30 \%$ & RDS \\
\hline
\end{tabular}

(midline, inside the trachea, in between the vocal cords and above the carina).

Summary of Results We studied 10 patients with 11 surfactant administrations. The mean gestational age was $31+0 / 7$ weeks, with a mean birth weight of 1688 grams. The catheter was detected in all patients by using POCUS. Visualization of the catheter and the surfactant instillation were obtained in all cases. Although the small size of the patients made the procedure more challenging, interfering in some cases with the person inserting the laryngoscope, rotation of the probe indicator allows the insertion of the laryngoscope without changing the location of the trachea.

Conclusions This is the first description of ultrasound-guided less invasive surfactant administration to the best of our knowledge. It is a simple, feasible, and reproducible POCUS technique that allows for catheter confirmation inside the trachea when video laryngoscopy is unavailable

\section{\#444 IS EARLY LIFE ACETAMINOPHEN EXPOSURE RELATED TO AN INCREASED RISK OF BPD?}

'S McKenna*, 'CJ Wright, 'L Sherlock, 'D Riebel, ${ }^{2} E$ Jensen. ${ }^{1}$ University of Colorado Anschutz Medical Campus, Aurora, CO; ${ }^{2}$ The Children's Hospital of Philadelphia, Philadelphia, PA

\subsection{6/jim-2022-WRMC.440}

Purpose of Study Acetaminophen(APAP) is commonly administered to preterm infants and is increasingly used to treat the patent ductus arteriosus(PDA). Preclinical models demonstrate that the developing lung is susceptible to APAP-induced injury. Whether early life APAP exposure contributes to lung injury in preterm neonates is unknown.

Methods Used We performed a systematic literature search on PUBMED to identify randomized controlled trials evaluating APAP for prevention or treatment of a PDA. Relevant trial and clinical data including treatment group rates of BPD were abstracted. Random effects meta-analysis and meta-regression according to the mean gestational age(GA) of trial participants were performed.

Summary of Results Of 196 identified manuscripts, 12 trials with 1001 subjects were included in the final analyses (figure 1). Mean GA ranged from 25.3 to 33.6. The outcome of BPD, although variably defined, was reported for 930
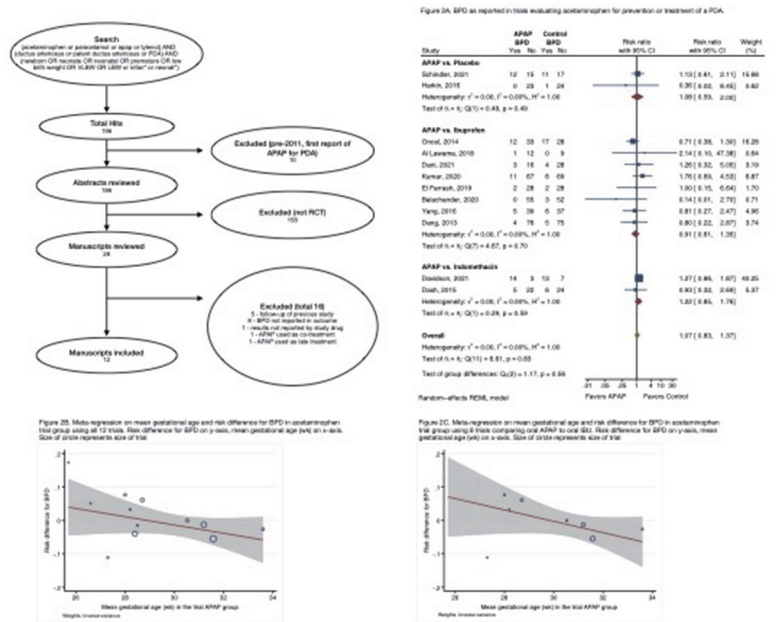

Abstract \#444 Figure 1 and 2

subjects. Averaged across all trials, APAP exposure did not significantly increase the risk of BPD [figure 2A; RR 1.07 $(0.83,1.37)]$. However, meta-regression demonstrated a possible inverse relationship between GA and BPD risk. For each week decrease in mean study GA, the risk difference for developing BPD with APAP exposure increased by $1.2 \%$ (range $3 \%$ to $-0.5 \% ; \mathrm{p}=0.17$; figure $2 \mathrm{~B}$ ). When limited to the 8 studies that compared oral APAP to oral ibuprofen, this weekly risk increased to $1.7 \%$ (range $4.0 \%$ to -0.6 ; $\mathrm{p}=.14$; figure $2 \mathrm{C}$ ). These results bordered on statistical significance, but the power to detect true differences at lower gestational age was limited by the low number of trial participants born $<28$ wks. Among all 12 reviewed trials, only 127 subjects born $<28$ wks were randomized to APAP. The 8 trials comparing oral APAP to ibuprofen randomized only 68 subjects $<28$ wks to APAP.

Conclusions Data from published RCTs show no significant effect of APAP on BPD risk in preterm infants. However, a possible and concerning inverse association between the risk of BPD with APAP exposure by GA suggests possible adverse effects among the least mature infants. More data are needed to determine whether early life APAP exposure in extremely premature infants is safe. 


\section{\#445}

PREDICTORS AND OUTCOMES OF LATE-PRETERM NEONATES NEEDING RESPIRATORY SUPPORT IN THE DELIVERY ROOM

A Filkins*, FB Wertheimer, R Ramanathan, M Biniwale. Los Angeles County University of Southern California Medical Center, Los Angeles, CA

\subsection{6/jim-2022-WRMC.441}

Purpose of Study The etiology and management of respiratory distress in late preterm neonates is not as well described as in the very preterm population. This study identified factors associated with delivery room (DR) positive pressure ventilation (PPV) support in the late preterm population.

Methods Used Preterm neonates with a gestational age of 3436 weeks who were admitted to LAC+USC from 2017-2020 were included in this retrospective review. DR ventilatory support, as well as maternal history, initial lab results, and comorbid diagnoses were abstracted. Study variables were analyzed with Chi-squared and t-tests.

Summary of Results Approximately 37\% [167/460] of the neonates required PPV in the DR and 92\% $(n=153 / 167)$ were placed on nasal cannula. Nasal continuous positive airway pressure (NCPAP) mode was most utilized $(n=132)$, followed by high flow nasal cannula (HFNC) $(n=89)$, nasal intermittent positive pressure ventilation (NIPPV) $(n=105)$, and intubation $(n=18)$. Face masks were only utilized in preparation for intubation. the DR included pre-eclampsia $(16.4 \%$ [45/230] vs $24.5 \%$ [39/159], $p=0.044)$, placenta previa $(2.2 \%[6 / 274]$ vs $9.3 \%$ $[15 / 161], p=0.002)$, and placental abruption $(1.1 \%[3 / 274]$ vs $4.9 \%$ [8/162], $\mathrm{p}=0.023)$. In our study, chorioamnionitis, prolonged rupture of membranes $>18$ hours, substance abuse, and meconium aspiration were not significantly associated with the need for PPV. Neonatal factors are listed in table 1.

Air leaks on initial X-ray $(0 \%$ [0/279] vs $2.4 \%$ [4/164], $\mathrm{p}=0.018$, respiratory distress syndrome (RDS) $(0.4 \%[1 / 279]$ vs $14.6 \%$ [24/164], $\mathrm{p}=0.001)$, and sepsis $(1.8 \%$ [5/278] vs $5.5 \%$ [9/164], $\mathrm{p}=0.047)$ were significant comorbidities associated with the use of DR PPVsupport in late preterm neonates.

Conclusions Late preterm neonates who required PPV support in the DR tended to be younger, with lower APGAR scores, and higher $\mathrm{PCO}_{2}$. These infants were more likely to need invasive ventilatory support at 24 hours. Maternal conditions compromising fetal perfusion were associated with respiratory support requirements while maternal infections were not. Neonates who received PPV support in the DR were more likely to have air leaks on initial X-ray, RDS and sepsis.
Maternal factors associated with the requirement of PPV in

\#446 SURVEY OF CONTINUOUS POSITIVE AIRWAY PRESSURE
(CPAP) PRACTICES AMONG NEONATAL PROVIDERS IN
THE UNITED STATES

R Mamidi*, K MacDonald, M Go, C McEvoy. Oregon Health and Science University, Portland, OR

\subsection{6/jim-2022-WRMC.442}

Purpose of Study To evaluate practice variation in the initiation, management, and discontinuation of neonatal nasal CPAP (nCPAP) among the Neonatal-Perinatal section of the American Academy of Pediatrics (AAP). We hypothesized large variations in clinical practice particularly with regards to discontinuation of CPAP and use of chinstraps.

Methods Used A web-based IRB approved survey consisting of 25 questions was administered to the AAP Neonatal-Perinatal section. It inquired about the NICU size, level, and location, and evaluated approaches for initiation and management of CPAP, and methods and post-menstrual ages used to wean infants born at $<32$ weeks gestation off CPAP. It also assessed the use of chinstraps and rates of nipple feeding while on CPAP. Data were analyzed using descriptive statistics.

Summary of Results Overall, $857 / 3700$ (23\%) of providers (718 neonatologists and 98 neonatal fellows) consented to the survey. The majority (83\%) worked in a NICU with $>20$ beds and $94 \%$ in a level 3 or higher. $822 / 830$ (99\%) used CPAP with $300(36 \%)$ primarily using bubble CPAP in infants $<32$ weeks gestation; 229 (27\%) using ventilator driven CPAP and $262(31 \%)$ using a combination of these. In terms of the primary interface applied: $297 / 781$ (38\%) used short binasal prongs; $61(8 \%)$ used long binasal prongs; 106 (14\%) used a nasal mask; 295 (38\%) used a combination of these interfaces. There was wide variation in: 1) the maximum CPAP setting applied to an infant $<32$ weeks gestation in the first 72 hours of life with $46 / 795(6 \%)$ using $5 \mathrm{cmH} 2 \mathrm{O} ; 258$ (32\%) using 6 $\mathrm{cmH} 2 \mathrm{O} ; 244$ (31\%) using $7 \mathrm{~cm} \mathrm{H} 2 \mathrm{O} ; 216$ (27\%) using $8 \mathrm{~cm}$ $\mathrm{H} 2 \mathrm{O} ; 31$ (4\%) using $\geq 8 \mathrm{~cm} \mathrm{H} 2 \mathrm{O} ; 2$ ) the typical minimum CPAP setting prior to stopping or weaning CPAP, responses were: $224 / 794$ (28\%) decreased to $4 \mathrm{cmH} 2 \mathrm{O} ; 493(62 \%)$ to

Abstract \#446 Table 1 Percent of responses for preferred method of weaning off nCPAP

\begin{tabular}{ll}
\hline Preferred Method to Wean off nCPAP & $\begin{array}{l}\% \text { of Responses } \\
\text { Favoring this Method }\end{array}$ \\
\hline Directly to room air & $37 \%$ \\
Wean to high or low flow nasal cannula and then room air & $59 \%$ \\
Increased time per day off CPAP until off & $5 \%$ \\
\hline
\end{tabular}

Abstract \#445 Table 1 Neonatal factors associated with the requirement of PPV in the DR

\begin{tabular}{lllllll}
\hline & $\begin{array}{l}\text { Gestational age } \\
\text { (weeks) }\end{array}$ & $\begin{array}{l}\text { Birth weight } \\
(\mathbf{g})\end{array}$ & $\begin{array}{l}\mathbf{5} \text { min } \\
\text { APGAR }\end{array}$ & $\begin{array}{l}\text { First blood } \\
\mathbf{p H}\end{array}$ & $\begin{array}{l}\text { First blood } \\
\text { gas C02 }\end{array}$ & $\begin{array}{l}\text { Invasive ventilation } \\
\text { at 24 hours (\%) }\end{array}$ \\
\hline No respiratory support in DR & 35.5 & 2585 & 8.9 & 7.16 & 44.8 & 0 \\
Respiratory support in DR & 35.1 & 2716 & 7.6 & 7.27 & 52.4 & 0.4 \\
P-value & $<0.001$ & 0.020 & $<0.001$ & 0.464 & $<.7$ \\
\hline
\end{tabular}


$5 \mathrm{cmH} 2 \mathrm{O}$ and $44(6 \%)$ to $6 \mathrm{cmH} 2 \mathrm{O} ; 3)$ the methods to wean off CPAP (see table 1). When asked when CPAP is discontinued or weaned, $69 \%$ responded when specific stability criteria were achieved; $22 \%$ at a specific PMA (5\% at $>30$ weeks; $85 \%$ at $>32$ weeks; $11 \%$ at $>34$ weeks), and $8 \%$ answered other. Greater than $65 \%$ of responses endorsed that chinstraps improve CPAP efficacy; however, less than $11 \%$ routinely applied a chinstrap. When asked regarding barriers to their use, $27 \%$ responded there were no barriers; $20 \%$ responded no sign of leak at the mouth; 15\% reported CPAP bubbling is adequate; $10 \%$ did not have the equipment. $78 \%$ of responses (614/789) never allowed infants to nipple on CPAP. 54\% of providers worked in a NICU without guidelines for CPAP initiation and $64 \%$ without guidelines for CPAP discontinuation.

Conclusions There are significant practice variations across neonatology caregivers with regards to CPAP initiation, management, and discontinuation. Further evidence-based research is needed in relation to respiratory outcomes to optimize CPAP strategies.

\section{Neuroscience III}

\section{Concurrent session}

\section{0:15 AM}

\section{Saturday, January 22, 2022}

\section{\#447 INTRADISCAL CONCENTRATED BONE MARROW ASPIRATE INJECTIONS REDUCE PAIN IN PATIENTS WITH LUMBAR DEGENERATIVE DISC DISEASE}

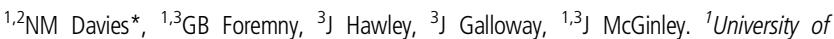
Washington School of Medicine, Seattle, WA; ${ }^{2}$ University of Idaho, Moscow, ID; ${ }^{3}$ The McGinley Clinic, Casper, WY
}

\subsection{6/jim-2022-WRMC.443}

Purpose of Study Low back pain, often caused by degenerative disc disease (DDD), is one of the most common reasons for primary care and orthopedic office visits. It's also among the costliest medical issues affecting our healthcare system. Concentrated bone marrow aspirate (BMAC) injections, along with conservative management, represent a cost-effective and minimally invasive treatment option. Our hypothesis is that BMAC injections, along with conservative care, can provide short and long-term pain reduction in patients with DDD.

Methods Used A retrospective chart review was conducted for 17 patients with DDD who received a BMAC injection along with conservative care in our clinic over the past 6 years. Each patient underwent a procedure where $60 \mathrm{ml}$ of bone marrow was aspirated from the posterior iliac crest, concentrated to $10 \mathrm{ml}$, and then injected into the degenerated disc. Patients were instructed to limit activity to non-weight bearing for 3 weeks post-procedure and partial weight-bearing with a $5 \mathrm{lb}$. lifting limit for an additional 3 weeks. All NSAIDs were held 10 days prior to and 3 months following the procedure. A 0-10 patient self-reported pain scale was used as the primary outcome. Secondary outcomes included adverse events and additional treatments. Pain scores were collected prior to the initial treatment and post-treatment at 3 weeks, 6 weeks, 3 months, 6 months, 12 months, 18 months, 24 months, and
36 months. Comparisons were analyzed using a paired-samples sign test.

Summary of Results 17 patients (11 male, 6 female) with ages ranging from $27-80$ years old $(49.7 \pm 16.4$ years $)$ were included in the study with an average follow-up time of 18.2 \pm 16.3 months. Reported pain levels were significantly reduced at 3 months post-injection compared with baseline (13 patients; $\Delta-3.3$ points; $\mathrm{p}=<0.001$ ). Additionally, pain continued to decrease up to 3 years post-injection ( 7 patients; $\Delta-4.5$ points from baseline; $\mathrm{p}=0.016)$. No adverse events were reported. No patients elected for surgery following treatment or received repeat BMAC injections during the 36month follow-up period.

Conclusions Patients with DDD who were treated with BMAC, along with conservative care, experienced a significant improvement in long-term pain levels without major adverse events related to the treatment. This represents a viable minimally invasive treatment option in patients with painful DDD.

\section{\#448 CHOROID THICKNESS AND VASCULARITY AS A BIOMARKER TO ASSESS AGE RELATED MACULAR DEGENERATION}

${ }^{1} \mathrm{~S}$ Lee*, ${ }^{2} \mathrm{RK}$ Wang. ' University of Washington, Anchorage, WA; ${ }^{2}$ University of Washington, Seattle, WA

\subsection{6/jim-2022-WRMC.444}

Purpose of Study Earlier detection of ophthalmic conditions can lead to improved prognosis and pursuit of necessary treatment. Finding a biomarker that can be easily screened for and correlated to the presence of early ophthalmic conditions, e.g. age related macular degeneration (AMD), would greatly benefit patients. This study focuses on the changes in choroidal thickness and vascularity measurements as parameters for decreased blood flow supplying the eye. We postulate that the measurement of choroidal thickness and vascularity can serve as a novel biomarker in monitoring ophthalmic disease in patients.

Methods Used This research employed optical coherence tomography (OCT) imaging for the purpose of this study. For analysis of the choroid, each patient underwent a total of 8 OCT scans, 4 OCT scans each of OS and OD eyes. Images then underwent an automatic segmentation algorithm for visualization of the choroidal layer; follow-up manual corrections were made for improved accuracy. Results were defined by two parameters, a choroidal thickness value and choroidal vascularity index, which were then represented using a colorcoded thickness and vessel imaging maps with corresponding numerical averaged values.

Summary of Results Of the 29 patients, 26 patients produced useful OCT results. Results were split into left sided or right sided results for choroidal changes. The thickness measurement showed great variation with values ranging from 137.66$308.78 \mu \mathrm{m}$ (OS) and $126.77-274.03 \mu \mathrm{m}$ (OD) eye, whereas the vascularity measurement had a narrower range of $0.56-0.67$ OS and 0.56-0.67 OD. To define values outside of normal physiological differences in choroidal thickness between the left and right eyes, the mean choroidal thickness (MCT) was defined as a range of $-36.01 \mu \mathrm{m}-46.38 \mu \mathrm{m}$; this defines any OD MCT greater than 46.38 or OS MCT greater than 36.01 as abnormal. This is according to a previous study which defined the normal bounds of physiologic interocular 
difference. Of the 26 cases, three cases fell outside of normal physiologic MCT interocular differences with values as follows: case $3247(58.64 \mu \mathrm{m}$, OS > OD), case $5721(38.55 \mu \mathrm{m}$, OS > OD) and case 7051 (74.46, OD > OS). The remaining 23 cases had MCT interocular differences within normal physiologic bounds. Each case was then matched to the patient diagnoses for further evaluation.

Conclusions The 3 cases with abnormal interocular MCT values presented with the following diagnoses: wet AMD (OS) and subclinical MNV (OD), dry AMD with GA (OS) and dry AMD with drusen (OD), and a right sided orbital AV fistula. Given the few cases which displayed abnormal MCT values, any significant correlation between choroid thickness as a biomarker for AMD or other ophthalmic conditions would seem premature. As the sample population remains small, increased recruitment of participants will be pursued for further study.

\section{\#449 A CLOUD-BASED PIPELINE TO PROCESS VERY PRETERM INFANT DIFFUSION MRI AND AUTOMATED FIBER QUANTIFICATION TRACTOGRAPHY}

${ }^{1}$ LE Rickerich*, ${ }^{1} \mathrm{D}$ Sproul, ${ }^{2} \mathrm{~L}$ Bruckert, ${ }^{1} \mathrm{G}$ Lerma-Usabiaga, ${ }^{2} \mathrm{SE}$ Dubner, ${ }^{2} \mathrm{KE}$ Travis. ${ }^{1}$ Stanford University, Stanford, CA; ${ }^{2}$ Stanford University School of Medicine, Stanford, CA

\subsection{6/jim-2022-WRMC.445}

Purpose of Study Preterm infants are at high risk for white matter (WM) injury detectable using advanced diffusion MRI (dMRI) methods. WM microstructural metrics derived from tractography of dMRI relate to neonatal illness and clinical outcomes. Preparing and processing dMRI data, identifying WM tracts, and extracting microstructural metrics is complex and data-intensive. Our goal was to implement a fully reproducible, cloud-based pipeline to process dMRI, perform tractography, and extract microstructural metrics in a database of infants born at $<32$ weeks and imaged at near-term gestational age.

Methods Used The processing pipeline utilizes Flywheel, a cloud-based data processing platform. Analyses are implemented as Dockerized containers or gears. Aligned T1weighted $(\mathrm{T} 1 \mathrm{w})$ anatomical images are segmented using Infant Freesurfer. Infant Freesurfer output and a template identifying anatomical regions of interest (ROIs) are fed together into a processing gear to create ROIs in each infant subject space. $\mathrm{dMRI}$ data is preprocessed and the anatomical $\mathrm{T} 1 \mathrm{w}$ data, brain mask (distinguishing brain from non-brain), output markers, and dMRI data are used to generate individual tract profiles and extract WM metrics.
Summary of Results To test our pipeline, we pre-selected a cohort of infants born $<28$ weeks gestation $(\mathrm{N}=55$; mean gestational age $=26.5$ (1.2) weeks; mean postmenstrual age at scan $=37.1(1.8)$ weeks) with both $\mathrm{T} 1 \mathrm{w}$ and dMRI scans, intended for analysis in a future study. We segmented and extracted fractional anisotropy (FA) and mean diffusivity (MD) values for 6 six corpus callosum segments. T-tests assessed consistency of different approaches to segmenting tracts (wholebrain tractography, WBT, versus region of interest-to-region of interest, ROI-to-ROI).

31 subjects $(56.4 \%)$ successfully ran through the pipeline. The remaining 24 subjects were likely unsuccessful due to computational resource allocation issues. Within each corpus callosum segment, values were generated for over $90 \%$ of subjects. Mean FA and MD are shown in the table 1. Results suggest variability for some tracts. Mean FA differed between WBT and ROI in the posterior CC. Our results were consistent with published studies with regard to both WBT and ROI-to-ROI tractography.

Conclusions We successfully implemented a reproducible pipeline to analyze preterm infant dMRI data. The next steps are to verify the anatomical accuracy of our ROI approaches, resolve resource allocation issues and process remaining subjects, and expand the pipeline to include other major cerebral and cerebellar tracts. Successful implementation of the pipeline will enable reproducible analyses to address questions relevant to clinicians and researchers surrounding neonatal brain development in the context of preterm birth.

\section{\#450 ASSESSING CONCUSSION EDUCATION IN ALLOPATHIC MEDICAL AND PHYSICAL THERAPY PROGRAMS}

${ }^{1,2}$ EA Tangog * ${ }^{*}, 2 \mathrm{~W}$ Cheung, ${ }^{1,2} \mathrm{~L}$ McKay, ${ }^{3} \mathrm{~T}$ Alsky, ${ }^{1} \mathrm{D}$ Baron. ${ }^{1}$ Western University of Healt Sciences, Pomona, CA; ${ }^{2}$ Western University of Health Sciences College of Osteopathic Medicine of the Pacific, Pomona, CA; ${ }^{3}$ The University of Arizona College of Medicine Tucson, Tucson, AZ

\subsection{6/jim-2022-WRMC.446}

Purpose of Study Our previous study assessed the state of concussion education and students' interest in the subject across osteopathic medical schools in the country. The results from the first study suggested that osteopathic medical students expressed a desire to obtain a more formal concussion education in their respective programs. This study evaluates the same parameters regarding concussion symptoms, diagnosis and management, while also expanding the study population to include students from allopathic medical and physical therapy programs across the country.

\begin{tabular}{|c|c|c|c|c|c|c|c|c|c|}
\hline Tract & $n$ & $\begin{array}{l}\text { WBT: FA mean } \\
\text { (SD) }\end{array}$ & $\begin{array}{l}\text { ROI: FA mean } \\
\text { (SD) }\end{array}$ & $\begin{array}{l}\text { WBT: MD mean } \\
\text { (SD) }\end{array}$ & $\begin{array}{l}\text { ROI: MD mean } \\
\text { (SD) }\end{array}$ & $\begin{array}{l}\text { FA t-test } \\
\text { statistic }\end{array}$ & $\begin{array}{l}\text { FA t-test } p \text { - } \\
\text { value }\end{array}$ & $\begin{array}{l}\text { MD t-test } \\
\text { statistic }\end{array}$ & $\begin{array}{l}\text { MD t-test } p \text { - } \\
\text { value }\end{array}$ \\
\hline CC-Occipital & 31 & $0.13(0.06)$ & $0.17(0.07)$ & $1.46(0.21)$ & $1.52(0.19)$ & -2.27 & 0.03 & -1.19 & 0.23 \\
\hline CC-Post. & 31 & $0.11(0.04)$ & $0.14(0.05)$ & $1.48(0.17)$ & $1.57(0.25)$ & -2.12 & 0.04 & -1.69 & 0.1 \\
\hline \multicolumn{10}{|l|}{ Parietal } \\
\hline CC-Sup. & 28 & $0.12(0.05)$ & $0.15(0.05)$ & $1.56(0.42)$ & $1.56(0.16)$ & -2.55 & 0.01 & -0.88 & 0.38 \\
\hline \multicolumn{10}{|l|}{ Parietal } \\
\hline CC-Motor & 28 & $0.14(0.04)$ & $0.15(0.03)$ & $1.51(0.26)$ & $1.56(0.16)$ & -1.95 & 0.06 & -0.86 & 0.39 \\
\hline CC-Sup. Frontal & 28 & $0.16(0.03)$ & $0.17(0.04)$ & $1.57(0.28)$ & $1.65(0.22)$ & -1.32 & 0.19 & -1.11 & 0.27 \\
\hline CC-Ant. Frontal & 30 & $0.15(0.04)$ & $0.17(0.04)$ & $1.55(0.24)$ & $1.62(0.20)$ & -1.63 & 0.11 & -1.23 & 0.22 \\
\hline
\end{tabular}


Methods Used An electronic survey was distributed to current allopathic medical and physical therapy students from all years of training in the United States. The same survey from the first study was used with an addition of two questions, making the total 16 questions. Four questions corresponded to participant demographics, six questions evaluated knowledge in concussion diagnosis and treatment, and four questions gauged any prior experience with concussion education. The two new questions allowed participants to select their gender and differentiate which program they were in. Data were collected in aggregate.

Summary of Results Based on the preliminary data collection of 338 responses, about $50 \%$ of both allopathic medical and physical therapy students correctly identified the definition of a concussion. Furthermore, more than half of responders identified that they have not received any formal training or education on concussions and request to see official education on concussion pathophysiology, symptoms, and treatment integrated into their program's curriculum.

Conclusions While data collection is still ongoing, the preliminary results of our study suggest that some allopathic medical and physical therapy programs lack the inclusion of this topic in their curriculums. Furthermore, participants identify that receiving concussion education in their preclinical years is essential, valuable, and applicable to any setting of medical care. Overall, our data support a want and need of a comprehensive concussion education in both programs across the country.

\section{\#451 ACUTE MONOCULAR VISUAL LOSS AFTER SINUS SURGERY}

P Parikh*, OM Dumitrascu. Mayo Clinic Arizona, Scottsdale, AZ

10.1136/jim-2022-WRMC.447

Postoperative monocular vision loss is caused by ischemic optic neuropathy (ION) or central retinal artery occlusion (CRAO). While brain MR-DWI (diffusion-weighted imaging) is not routinely used for diagnosis, recent studies have shown restricted diffusion of the optic nerve $(\mathrm{ON})$ or retina may indicate acute CRAO. Here, we present a case of acute CRAO to highlight this neuroimaging finding and further therapy options.

Case-Report A 71-year-old male without vascular risk factors underwent maxillary bilateral antrostomy and septoplasty for chronic sinusitis. 20-30 minutes upon awakening, he reported acute painless left eye vision loss. Visual acuity (VA) was bare light perception (left) and 20/25 (right). Facial CT was negative for pathology. Fundoscopy showed retinal whitening, cherry-red spot, narrowed arterioles with scattered segmentation, and no hemorrhage. Brain MRI showed left ON diffusion restriction and increased T2 signal (figure 1), concerning for posterior ION; however, proximal CRAO was more likely given the clinical exam. Patient received oral aspirin, intraarterial verapamil and alteplase, IV acetazolamide and dexamethasone, and topical Alphagan. At 6-month follow-up, left eye VA was no light perception. Despite extensive workup, no other etiology was found.

Discussion We present a case of proximal CRAO post-sinus surgery in which brain MR-DWI helped with lesion localization. The outcome was dismal despite therapy. Both ION and CRAO can cause postoperative vision loss. PION has normal ophthalmoscopic findings, and ON DWI due to cytotoxic edema has $81-100 \%$ sensitivity and $86-100 \%$ specificity. CRAO has retinal edema, segmentation of arterioles, and a cherry-red spot. ON DWI sensitivity and specificity are 55\%/70-100\%, per a recent study. In our case, the ON diffusion restriction was caused by CRAO in its narrowest part, where it enters the ON. A similar reported case of post-sinus surgery acute CRAO underwent therapy with hyperbaric oxygen (HBO) with visual improvement. American Academy of Ophthalmology recommends emergent stroke workup and antiplatelet; there is no level I data to support $\mathrm{HBO}$ or other treatments for CRAO. In conclusion, we highlight the combination of retinal ischemic features on fundoscopy and proximal ON diffusion restriction on MRDWI to be diagnostic of proximal CRAO. Future studies should evaluate the accuracy and utility of MR-DWI and $\mathrm{HBO}$ in acute CRAO.

\section{Published only - not presented}

\section{\#452 CLINICAL VIABILITY OF BIOMARKERS FOR TOXIC STRESS: AN INVESTIGATION}

${ }^{1,2}$ I Pasumarthi*. ' Nova Southeastern University, Fort Lauderdale, FL; ${ }^{2}$ Office of the California Surgeon General, Sacramento, CA

\subsection{6/jim-2022-WRMC.448}

Purpose of Study The toxic stress response is a biological process that may be caused by the prolonged activation of the stress response due to exposure to cumulative adversity. This process can disrupt biological systems through neuroendocrine, immunologic, and epigenetic mechanisms. The toxic stress

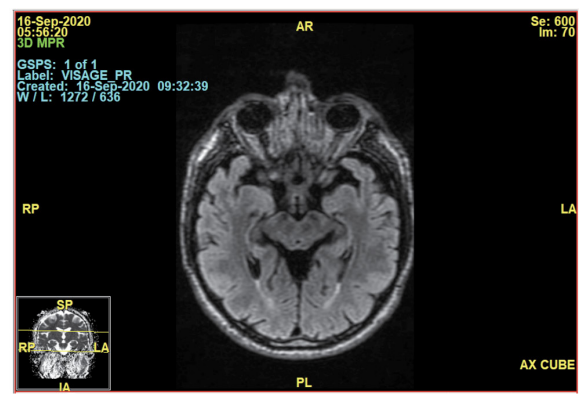

B. Corresponding increase in $\mathrm{T} 2$ signal in left optic nerve 
response has been correlated with acute and chronic illnesses, including some of the leading causes of death such as heart disease, suicide, and cancer. Early clinical identification of the toxic stress response and associated health conditions is imperative to limit potentially lifelong negative health effects. The use of clinical biomarkers stands to be a promising method of early identification of the toxic stress response. However, research around clinical biomarkers is still in its nascency and there has been little to assess the clinical viability of these biomarkers. The objective of this study is to determine the potential clinical viability of biomarkers for the toxic stress response.

Methods Used A literature review was conducted to aggregate biomarkers for the toxic stress response. Biomarkers were then scored based on invasiveness (non-invasive and invasive) and definitiveness in diagnosing the toxic stress response (not definitive, semi-definitive, and mostly definitive). Biomarkers with a low invasive score and a high definitive score were considered to have high clinical viability.

Summary of Results Biomarkers considered primary mediators of the toxic stress response, such as cortisol, epinephrine, and norepinephrine, were considered among the most clinically viable biomarkers with low invasive scores and fairly definitive scores. Some promising biomarkers with currently low clinical viability include telomere shortening and infant eye-tracking.

Conclusions This research could indicate which biomarkers might be suitable for the development of clinical guidance and which might need further research before more widespread acceptance. Areas for future research include identifying novel biomarkers for the toxic stress response and expanding the body of literature and the strength of the evidence surrounding these biomarkers.

\section{\#453 FULMINANT HEPATIC FAILURE SECONDARY TO WILSONS DISEASE}

${ }^{1}$ VK Narang*, ${ }^{2} \mathrm{H}$ Truong, ${ }^{1} \mathrm{~K}$ Grewal, ${ }^{1} \mathrm{~L}$ Moosavi, ${ }^{2} \mathrm{I}$ Kalha, ${ }^{1} \mathrm{~S}$ Mishra, ${ }^{1} \mathrm{~K}$ Radicic. ${ }^{1}$ UCLAKern Medical, Bakersfield, CA; ${ }^{2}$ Ross University School of Medicine, Miramar, FL

\subsection{6/jim-2022-WRMC.449}

Case Report Wilson Disease (WD) is a rare genetic disorder of impaired copper excretion causing accumulation of copper in vital organs including the liver, brain, and cornea. Diagnosis usually occurs between the ages of 5 and 35 years. Patients typically present with cirrhosis, neurological symptoms, and Kayser-Fleischer rings. Rarely, WD can present as fulminant hepatic failure necessitating liver transplant. Here, we describe a 36-year-old male with alcohol abuse with acute liver failure initially deemed secondary to alcohol. However, biochemical findings were highly suggestive of WD.

Methods Retrospective single case review after IRB approval Case Report A 36-year-old male with a history of alcohol abuse presented to the hospital with bilateral lower extremity edema. He had no known prior liver disease and had stopped alcohol 2 months ago. Initial workup showed acute liver failure with elevated liver function tests (LFTs), and imaging findings of decompensated cirrhosis. His mentation declined, and he developed tremors and hallucinations. He was treated with diuretics, thiamine, and Ativan for alcohol withdrawal. Despite one week of treatment, his LFTs remained elevated, and his mentation declined. Further workup revealed ceruloplasmin of
$13 \mathrm{mg} / \mathrm{dL}$. Initial 24-hour urinary copper excretion was unobtainable due to his mentation, but after penicillamine challenge it was $111 \mathrm{mcg} / 24 \mathrm{hr}$. He was noted to have possible KayserFleischer rings but slit-lamp exam could not be performed due to accessibility. Patient had a Leipzig score of 4, suggestive of WD. Genetic testing for ATP7B mutation was negative. Brain MRI revealed significant cerebral and cerebellar atrophy without parenchymal involvement. Liver biopsy was unobtainable due to elevated INR. After penicillamine, he had significant improvement in cognition and motor function. He was accepted to a liver transplant center.

Conclusions A history of alcohol abuse in a patient with liver failure usually leads to the suspicion of alcohol as the etiology. This case highlights the importance of a full workup to avoid anchoring bias. Our patient had a low ceruloplasmin and a high $24 \mathrm{hr}$ urinary copper excretion which may suggest WD. Liver biopsy would be needed for confirmation. Early recognition and liver transplant evaluation are imperative in these patients

\section{\#454 COVID-19 RELATED LIFESTYLE CHANGE IS MULTIDIMENSIONAL}

${ }^{1} \mathrm{~K}$ Morris*, ${ }^{2} \mathrm{M}$ Bouchonville, ${ }^{1} \mathrm{~S}$ Fanchiang. ${ }^{1}$ Charles Drew University of Medicine and Science, Los Angeles, CA; ${ }^{2}$ University of New Mexico School of Medicine, Albuquerque, NM

\subsection{6/jim-2022-WRMC.450}

Purpose of Study Both, negative and positive lifestyle changes have been reported during the COVID-19 pandemic and many focused on particular areas of lifestyle. However, few studies have implemented multidimensional lifestyle measures. The work of cultural neuroscience has suggested that through self-reflection, a general pattern of self-enhancement motivation will be elicited regardless of the cultural background. We proposed that implementing a multidimensional lifestyle measure (the Short Multidimensional Inventory Lifestyle Evaluation - Confinement) with additional dimensions: Lifelong Learning, Nature/Environmental Connection, and Sense of Safety to explore its potential for clinical use, would lead to self-reflection beyond conventional measures to facilitate positive lifestyle change for health promotion and chronic illness management.

Methods Used We conducted a cross-sectional, anonymous online 40-item multidimensional survey focusing on lifestyle using social-media recruitment as a pilot. People 18 years or older were target survey respondents.

Summary of Results Among participants $(n=37), 75 \%$ were female; more than more 50\% were from suburban areas, and about a third of them lived in urban areas. The results indicated changes in six dimensions with positive changes in Physical Activity, Lifelong Learning, and Sense of Safety but worse changes in Social Support and Screen Time. Whereas about equal proportion of the respondents reported positive and negative changes in Nature/Environmental Connection dimension. More than $50 \%$ of respondents indicated that taking this lifestyle survey gave insight into their lifestyle and $64 \%$ of them would do periodic lifestyle surveys or something similar to this multidimensional lifestyle survey for selfreflection.

Conclusions All three additional dimensions of the multidimensional lifestyle survey reflected lifestyle changes due to the COVID-19 pandemic beyond Physical Activity, Social 
Support, and Screen Time dimensions. The proposed multidimensional lifestyle evaluation may be useful for self-reflection and further studies will be necessary to determine if the expanded lifestyle evaluation is superior for activating behavioral change.

\section{\#455 ANALYSIS OF TREATMENT GUIDELINES FOR HYPEREMESIS GRAVIDARUM AND NAUSEA/VOMITING OF PREGNANCY}

A Morrow*, R McCann, B Brooks. Rocky Vista University College of Osteopathic Medicine, Ivins, UT

\subsection{6/jim-2022-WRMC.451}

Purpose of Study Hyperemesis gravidarum (HG) is the most severe form of nausea and vomiting of pregnancy (NVP), or morning sickness. $2 \%$ of pregnancies in the United States are affected by HG. The condition is characterized by severe vomiting in pregnant women, especially during the first trimester, often leading to hypovolemia and weight loss. The standard of care for HG and NPV is commonly ineffective. We hypothesize that based on patient experience, the current treatment guidelines for HG are not clinically effective. Our objective is to identify the efficacy of the management protocol that is currently in place for NVP and $\mathrm{HG}$.

Methods Used A survey was designed based on diagnostic criteria as well as standard demographic identifiers and the most common medications used in the treatment of $\mathrm{HG}$ and was distributed to NPV and HG user groups on social media.

Summary of Results In our study, the vast majority of women diagnosed with HG trialed at least five medications, most of which were ineffective or had severe side effects.

Conclusions The data presented in this research provides insight into the suffering that patients with these diagnoses face day-to-day. Establishing this gap in treatment can facilitate the development of effective treatments that will provide relief for thousands of women.

This work was presented in part at Rocky Vista University Appreciation Day (virtual) on October 15, 2021

\section{\#456 ACUTE PROMYELOCYTIC LEUKEMIA OF THE EYE}

R Dunn*, F Venter, S Mishra. Kern Medical Center, Bakersfield, CA

\subsection{6/jim-2022-WRMC.452}

Case Report Acute promyelocytic leukemia(APML) often presents with systemic symptoms such as weakness, fatigue, infections, ecchymoses and bleeding. It is usually associated with the translocation of $\mathrm{t}(15 ; 17)$ causing a defect in the Retinoic Acid Receptor (RAR). However, this case reviews a 37 year old male previously treated for Acute Myeloid Leukemia (AML) who presented with APML of the eye, confirmed by biopsy. This case stresses the importance of including a fundoscopic eye exam for possible ocular involvement - which is not uncommon in APML and may easily be missed

Methods IRB approval was obtained, and single case review was done.

Summary of Results A 37-year-old Hispanic male presented with progressively worsening generalized weakness, fatigue, shortness of breath and easy bruising for 1 week. Upon admission, patient was hemodynamically stable and afebrile. Labs were concerning for complete blood count with differential showing meylocytes at $0.2 \times 10^{\wedge} 3 / \mathrm{mcL}$, promylocytes of $0.1 \times 10^{\wedge} 3 / \mathrm{mcL}$, and blasts of $0.1 \times 10^{\wedge} 3 / \mathrm{mcL}$. D-Dimer was also elevated at greater than 5000 fibrinogen equivalent units/ mL. Peripheral smear was obtained which showed blast cells with Auer rods, suggesting APML. Diagnosis was confirmed with flow cytometry showing CD117 positive atypical/immature myeloid cells. FISH analysis showed $\mathrm{t}(15 ; 17)$ translocation. Patient was started on All-trans Retinoic acid (ATRA) $60 \mathrm{mg}$ BID on with remission of APML. However, the patient developed left orbital swelling and pain on treatment day 7. CT Maxillofacial w/Contrast was obtained showing a rimenhancing fluid collection. Ophthalmology consult was attempted but could not be done due to accessibility. Patient underwent biopsy of the eye at higher center and was consistent with APML. Patient was re-admitted at our facility and restarted on ATRA and intrathecal chemotherapy with great response.

Conclusion Acute leukemias generally present with systemic presentations like fever, body aches, fatigue and bleeding in APML. Ocular involvement can be due to direct infiltration of the leukemia or secondary to some blood abnormality like anemia, thrombocytopenia, or leukocytosis. Leukemic retinopathies are present in around $35.4 \%$ of leukemia patient and may present manifest after induction of chemotherapy. Clinicians should be vigilant to look for ocular involvement to prevent blindness.

\section{\#457 REPARATION OF THE ION GRADIENTS: THE ROLE OF ION CHANNELS AND THEIR POTENTIAL TO GUIDE THE CLINICAL MANAGEMENT OF EXTERNALLY INDUCED SKIN WOUNDS}

${ }^{1} \mathrm{~K} \mathrm{Mai}^{*},{ }^{2} \mathrm{M}$ Zhao. ${ }^{1}$ Western University of Health Sciences, Pomona, CA; ${ }^{2}$ University of California Davis, Sacramento, CA

\subsection{6/jim-2022-WRMC.453}

Purpose of Study The skin holds unique ion gradients that play a vital role in the process of wound healing. While the physiology behind this mechanism remains unclear, recent innovations regarding cutaneous ion channels may provide a better understanding of this biophysiological process. This is paramount because while molecular channel immunohistology has been utilized in forensic pathology to differentiate wound age postmortem, the same has not yet been reviewed for ion channels in skin wounds. Thus, a deeper comprehension of ion channels is pertinent in not only understanding ion gradient associated wound healing but would also provide a clinical significance in its potential to differentiate wound stage and wound types.

Methods Used A comprehensive literature search was used to search for over 100 studies involving ion channels in the context of skin wounds. Journal articles were reviewed from Pubmed, ScienceDirect, and Google Scholar. Inclusion criteria limited the review to ion channels studied in the context of ion gradients, wound stage determination, and types of skin wounds.

Summary of Results Calcium, potassium, water, sodium, and chloride gradients have been found in the skin allowing for the identification of nine channels/pumps matching our inclusion criteria. These include Kcnj8, Kcnh2, TRVP1, TRVP2, 
TRVP3, TRVP4, Orai1, Na+/K+ ATPase, and AQP3 channels. Kcnh2 and Kcnj8 were found in association with the homeostatic phase of wound healing, AQP3 channels in association with the proliferative phase, and TRVP2 in association with the remodeling/maturation phase. Ion channels also have potential for differentiating different wound types. These include TRVP3 and AQP3's association with burn wounds and Orai1's association with UVB-induced wounds.

Conclusions Potential channels involved in the reparation of ion gradients following externally induced skin wounds were identified based on their variability and timing of expression following the event. These characteristics provided further evidence to our proposed feedback relationships between the gradients and the notion that they may play a clinical role in differentiating wound stages and types. However, there has yet been a channel identified as a main contribution to the Inflammatory stage. Instead, a combination of decreased $\mathrm{Na}+$ / $\mathrm{K}+, \mathrm{Kcnh} 2$, and $\mathrm{Kcnj} 8$ expression may allow for differentiation of this stage. The ability to differentiate wounds would provide improved diagnostic accuracy in the clinical context and allow for the personalization of treatment options. Given the role of ion fluxes and resultant wound electric fields in wound healing, temporal and spatial expression of ion channels and pumps is expected to offer a significant understanding of the powerful mechanism of electric signaling in wound healing.

\section{\#458 NECROTIZING FASCIITIS: A POTENTIAL NIDUS FOR HEPATORENAL SYNDROME}

${ }^{1} \mathrm{~K}$ Mai*${ }^{*}{ }^{1} \mathrm{~K}$ Chen, ${ }^{1} \mathrm{R} \mathrm{Li},{ }^{2} \mathrm{P}$ Flores. ${ }^{1}$ Western University of Health Sciences, Pomona, CA; ${ }^{2}$ Hemet Global Medical Center, Hemet, CA

\subsection{6/jim-2022-WRMC.454}

Case Report Studies proposed bacterial translocation from the GI track as a potential source of hepatorenal syndrome (HRS). While this has been reported in a few cases, they occurred in the setting of liver trauma associated endogenous infections. Here we report a case of HRS following the onset of necrotizing fasciitis (NF). Overall, suggesting that in the absence of liver trauma, spread from skin wounds may contribute to the onset of HRS.

A female patient presents with bleeding from NF extending from her bilateral flanks to her upper thighs and a history of alcoholic cirrhosis. Kidney functions were originally within normal limits, however, reached $4.40 \mathrm{mg} / \mathrm{dL}$ by day 18 .

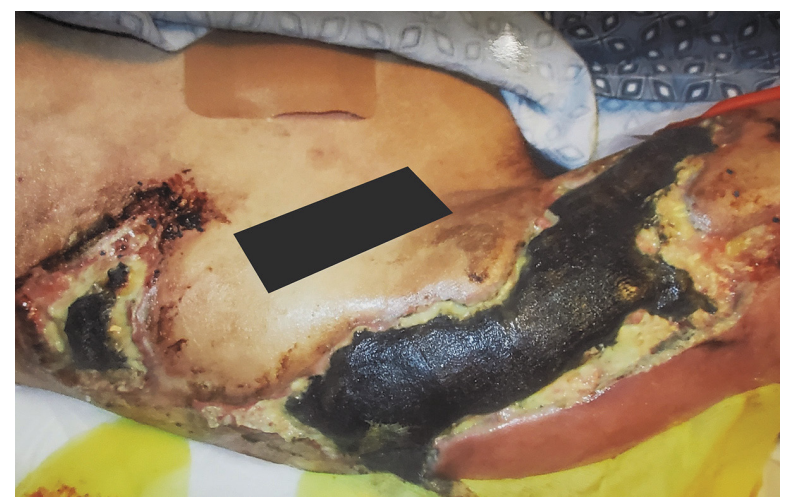

\section{Abstract \#458 Figure 1}

Despite trials of albumin, midodrine and withholding diuretics, creatinine levels continually rose, suggesting type 1 HRS. NF was also identified through cultures isolating Enterococcus Faecalis, Staphylococcus Aureus and Klebsiella Oxytoca. CT scan further demonstrated skin interruptions, ulcers and anasarca affected regions. Routine wound care, surgical debridement, and antibiotic treatment with ceftriaxone and daptomycin was utilized.

Aside from NF, there were no other identifiable causes of HRS. The patient's bleeding event on admission occurred closely to her onset of HRS. This suggests that hematogenous/contiguous spread of cutaneous infections or bacterial products may be responsible for the onset of HRS in those with preexisting liver diseases. We suggest early utilization of barrier therapies and antimicrobial dressings. This case further questions whether only skin wounds close to the kidneys contribute to HRS or if distal wounds may also be influential.

\section{\#459 A RARE CASE OF ACUTE PANCREATITIS DUE TO EPSTEIN BARR VIRUS INFECTION}

${ }^{1} \mathrm{M}$ Tu*, ${ }^{1} \mathrm{~V}$ Marquez, ${ }^{2} \mathrm{H}$ Ipalawatte. ${ }^{1}$ Clinica Sierra Vista, Bakersfield, CA; ${ }^{2}$ Ross University School of Medicine - Barbados Campus, Bridgetown, Barbados

\subsection{6/jim-2022-WRMC.455}

Case Report A common early childhood and adolescence infection Epstein Barr Virus (EBV), presents with infectious mononucleosis, upper respiratory tract infection, diarrhea, and abdominal complaints. We report a case of EBV infection with atypical manifestation of acute pancreatitis, in a healthy 17year-old (yo) patient.

Presentation A 17 yo male with a history of fatty liver disease presented to our emergency room with a 1-day history of severe left upper and lower epigastric pain. The patient was normotensive and afebrile, with a soft nondistended abdomen and LUQ, epigastric and LLQ tenderness with no guarding, rebound tenderness, no bruising noted. Laboratory data revealed a total white blood cell count of $13.1 \times 103$ / $\mathrm{mcL}$, hemoglobin of $15.0 \mathrm{~g} / \mathrm{dl}$, platelet count of 240 $\mathrm{x} 10^{\wedge} 3 / \mathrm{mcL}$, prothrombin time of $15.4 \mathrm{~s}$, international normalized ratio (INR) of 1.23 , aspartate aminotransferase of $46 \mathrm{U} / \mathrm{l}$, alanine aminotransferase of $104 \mathrm{U} / \mathrm{l}$, alkaline phosphatase of $148 \mathrm{U} / \mathrm{l}$, total bilirubin of $1.2 \mathrm{mg} / \mathrm{dl}$, and lipase of 2,431 U/1 (normal range: 0-160 U/l). Computed tomography (CT) of the abdomen and pelvis revealed acute necrotizing pancreatitis with a small unorganized acute necrotic collection, edematous pancreas, and hepatomegaly. Magnetic resonance imaging (MRI) showed necrotizing pancreatitis with moderate peripancreatic, perisplenic, and perinephric inflammatory fluid with no drainable fluid collection identified.No biliary ductal dilatation. No evidence of cholelithiasis or choledocholithiasis. Hepatomegaly. Splenomegaly suggesting underlying portal hypertension. On hospital day 2 patient had a temperature max of $38.6 \mathrm{C}$, tachycardic in the $120 \mathrm{~s}$, tachypneic in the 30 s. SIRs criteria were met and septic workout was initiated and the patient started on Meropenem, with pancreatic fluid as a possible source of infection. Patient was treated for pancreatitis with guideline direct medical therapy. Viral hepatitis profiles (A, $\mathrm{B}, \mathrm{C})$, HIV RNA, and monospot test were all negative. The diagnosis of EBV infection was made by the positive result 
of EBV immunoglobulin $\mathrm{G}(\mathrm{IgG})$ (VCA, viral capsid antigen) 129.00 unit $/ \mathrm{mL}$. Labs additionally showed CMV antibody IgG 3.10 unit $/ \mathrm{mL}$

Discussion EBV-induced acute pancreatitis is a rare condition, the sooner an etiology can be deduced, the more options that a patient has in terms of therapy. Our patient has EBVinduced acute pancreatitis, a condition often not seen in conjunction. He did not exhibit any symptoms of infectious mononucleosis at the time of presentation, which is commonly associated with EBV infection. He deviates from many common associations. 1s. With this case, we wanted to emphasize the importance of being vigilant, have broad differentials and early intervention based on clinical suspicion. For the best possible patient outcomes.

\section{\#460 TACKLING SEVERE INTRAVENTRICULAR HEMORRHAGE IN A SINGLE CENTER LEVEL IV NICU}

EF Squire*, J Reiss, L Bain. Stanford University School of Medicine, Stanford, CA

\subsection{6/jim-2022-WRMC.456}

Purpose of Study Premature infants $<32 \mathrm{w}$ gestational age are at increased risk of severe (grade 3,4) intraventricular hemorrhage (IVH) due to immature cerebral vasculature and decreased autoregulation of cerebral blood flow. Severe IVH is associated with increased long-term morbidity and mortality in this population. There are numerous modifiable risk factors and protective factors in the antenatal, intrapartum, and postnatal time periods that are associated with decreased rates of severe IVH. This quality improvement study examines modifiable risk factors of severe IVH at a single center level IV NICU, which noted a recent increase in severe IVH rates following several years of sustained reduction after implementation of a 'Brain Care Bundle' targeted at IVH prevention. A retrospective chart review of all inborn babies $<32$ w gestational age from 2010 to 2020 who were diagnosed with either grade 3 or grade 4 IVH during their NICU stay demonstrated that some IVH risk reduction strategies such prenatal steroid use and elimination of the use of sodium bicarbonate have been consistently used over time, however other practices such as maintaining midline head positioning for the first 3 days of life and delayed umbilical cord clamping showed variability among patients. Inconsistent use of best practices provides an opportunity to use quality improvement strategies to reenforce elements of our Brain Care Bundle and reduce severe IVH rates once again. Our goal is to reduce severe IVH rates in inborn infants $<32$ weeks GA by $50 \%$ from $10 \%$ to $5 \%$ over the next 2 years consistent with rates in this population across other California NICUs. We will do this by re-educating and re-enforcing our current Brain Care Bundle for NICU providers and identifying ways to improve adherence to IVH reduction strategies. We also plan to analyze additional data, specifically
CO2 levels in the first week of life, in hopes of adding novel interventions to our Brain Care Bundle. We will then monitor provider compliance with our updated Brain Care Bundle as well as overall rates of severe IVH to determine the impact of our interventions.

Methods Used As above

Summary of Results As above

Conclusions As above

\section{\#461 PROTECTIVE MECHANISM OF BERBERINE ON SPINAL CORD INJURY IN RATS}

${ }^{1,2} \mathrm{H}$ Duan, ${ }^{1} \mathrm{C}$ Hao, ${ }^{1} \mathrm{X}$ Yang ${ }^{*}$. ${ }^{1}$ The First Hosptal of Shanxi Medical University, Taiyuan, China; ${ }^{2}$ Shanxi Provincial Key Laboratory of Brain Science and Neuropsychiatric Diseases, Taiyuan, China

\subsection{6/jim-2022-WRMC.457}

Purpose of Study It is an important cause of neurological dysfunction for secondary inflammation after spinal cord injury. At present, there are relatively few effective drugs as an inflammatory inhibitor of spinal cord injury. Berberine is a common inflammatory inhibitor of traditional Chinese medicine extract, so we tried to explore the therapeutic significance of this drug for spinal cord injury.

Methods Used The SD rats were randomly divided into three groups: sham operation group $(n=30)$, non-intervention $\operatorname{group}(n=30)$, and Berberine intervention $\operatorname{group}(n=30)$. The T10 SCI model was established by Allen's percussion method.BBB score,SEP and MEP latency and amplitude were used to evaluate hind limb function. The water content of spinal cord was measured. The spinal cell structure was observed by HE and Nissl staining and electron microscopy. The motor function was detected by SEP(Somatosensory Evoked Potential) and MEP (motor evoked potential. BBB (Basso-Beattie-Bresnahan) score was used to evaluate the hind limb function of rats.

Summary of Results The BBB score of berberine in SCI model was significantly lower than that in sham operation group from postoperative day 1 to 31 . Hindlimb function of Berberine intervention group began to recover faster than the nonintervention group on day $10(\mathrm{p}<0.05)$. The latency of SEP and MEP after spinal cord injury was significantly longer than that of sham operation group $(p<0.05)$, but there was no significant difference in the latency of SEP and MEP between the two groups $(p>0.05)$. The amplitude of SEP and MEP in rats after spinal cord injury was significantly lower than that in the sham operation group $(\mathrm{P}<0.05)$. In addition, after berberine treatment, the degree of spinal cord edema in rats decreased to some extent $(\mathrm{p}<0.05)$.

Conclusions Berberine can promote the recovery of injured spinal cord to some extent, which may be related to its inflammatory inhibition. 